NUREG/CR-6143

SAND93-2440

Vol. 6, Part 1

\title{
Evaluation of Potential Severe Accidents During Low Power and Shutdown Operations at Grand Gulf, Unit 1
}

Evaluation of Severe Accident Risks for

Plant Operational State 5 During a Refueling Outage

Main Report and Appendices

Manuscript Completed: December 1994

Date Published: March 1995

Prepared by

T. D. Brown, L. N. Kmetyk, D. Whitehead, J. Forester, ${ }^{1}$ L. Miller, J. Johnson ${ }^{2}$

Sandia National Laboratories

Albuquerque, NM 87185

Prepared for

Division of Systems Technology

Office of Nuclear Regulatory Research

U.S. Nuclear Regulatory Commission

Washington, DC 20555-0001

NRC Job Code L1679

1Science Applications International Corporation, 2109 Air Park Road, S.E., Albuquerque, NM 87106

2GRAM, Inc., Albuquerque, NM 87112 
This Page is intentionally left blank. 


\section{DISCLAIMER}

This report was prepared as an account of work sponsored by an agency of the United States Government. Neither the United States Government nor any agency thereof, nor any of their employees, make any warranty, express or implied, or assumes any legal liability or responsibility for the accuracy, completeness, or usefulness of any information, apparatus, product, or process disclosed, or represents that its use would not infringe privately owned rights. Reference herein to any specific commercial product, process, or service by trade name, trademark, manufacturer, or otherwise does not necessarily constitute or imply its endorsement, recommendation, or favoring by the United States Government or any agency thereof. The views and opinions of authors expressed herein do not necessarily state or reflect those of the United States Government or any agency thereof. 


\section{DISCLAIMER}

Portions of this document may be illegible in electronic image products. Images are produced from the best available original document. 


\begin{abstract}
Traditionally, probabilistic risk assessments (PRAs) of severe accidents in nuclear power plants have considered initiating events potentially occurring only during full power operation. Recent studies and operational experience have, however, implied that accidents during low power and shutdown could be significant contributors to risk. In response to this concern, in 1989 the Nuclear Regulatory Commission (NRC) initiated an extensive program to carefully examine the potential risks during low power and shutdown operations. Two plants, Surry (pressurized water reactor) and Grand Gulf (boiling water reactor), were selected as the plants to be studied. The program consists of two parallel projects being performed by Brookhaven National Laboratory (Surry) and Sandia National Laboratories (Grand Gulf). The program objectives include assessing the risks of severe accidents initiated during plant operational states other than full power operation and comparing the estimated risks with the risk associated with accidents initiated during full power operation as assessed in NUREG-1150. The scope of the program is that of a Level-3 PRA.
\end{abstract}

The subject of this report is the PRA of the Grand Gulf Nuclear Station, Unit 1. The Grand Gulf plant utilizes a 3833 MWt BWR6 boiling water reactor housed in a Mark III containment. The Grand Gulf plant is located near Port Gibson, Mississippi. The regime of shutdown analyzed in this study was plant operational state (POS) 5 during a refueling outage, which is approximately Cold Shutdown as defined by Grand Gulf Technical Specifications. The entire PRA of POS 5 is documented in a multi-volume NUREG report (NUREG/CR-6143). The internal events accident sequence analysis (Level 1) is documented in Volume 2. The Level 1 internal fire and internal flood analyses are documented in Volumes 3 and 4, respectively. The Level 1 analysis of accidents initiated by seismic events is documented in Volume 5 and was performed for the NRC by Future Resources Associates, Inc. The accident progression and risk analyses (Level-2/3) for traditional internal events are documented in Volume 6, and a summary of the results for all analyses is documented in Volume 1.

The analysis documented in this volume of the report is the Level $2 / 3$ analysis of the traditional internal events. Plant damage states, which define the configuration of the plant and its systems at the onset of core damage for the accident scenarios developed in the Level 1 analysis, were used to define the interface between the Level 1 and Level $2 / 3$ analyses. In the Level 2/3 analysis, the possible progressions of the accident following the onset of core damage were delineated and the amount of radioactive material released to the environment was estimated. Based on the amount of radioactive material released to the environment, health effects to the general public were estimated. In addition to the offsite consequences, a scoping analysis of the potential doses and dose rates within the site were also estimated. The final product of the analysis was the integration of the accident frequencies with the consequences of the accidents to form an expression for aggregate risk.

The mean early fatality risk of the Grand Gulf plant resulting from internal events for POS 5 during a refueling outage is $1.4 \mathrm{E}-08$ per calendar year, and the 5 th and 95 th percentiles are 3.7E-11 and 3.9E-08 per calendar year, respectiveily. Similarly, the mean total latent cancer fatality risk is $3.8 \mathrm{E}-03$ per calendar year, and the 5 th and 95 th percentiles are $4.3 \mathrm{E}-04$ and $1.2 \mathrm{E}-02$ per calendar year, respectively. The risk from POS 5 is not insignificant compared to the risk from full power operation. In fact, although the mean risk values from the two studies are similar, the mean risk values from POS 5 are actually greater than the full power risk values. Hence the full power risk distributions by themselves do not completely characterize the risks associated with the operation of this plant. To accurately characterize the plant's risk, results from this study suggest that it may be necessary to include other modes of operation in addition to the full power mode of operation. 
This page is intentionally left blank. 


\section{Contents}

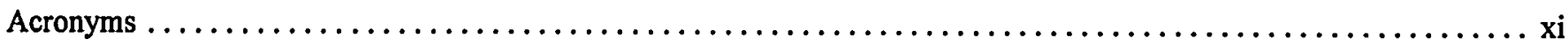

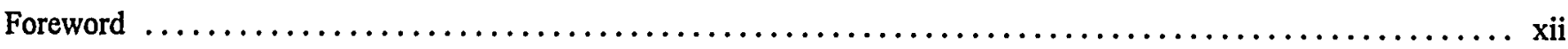

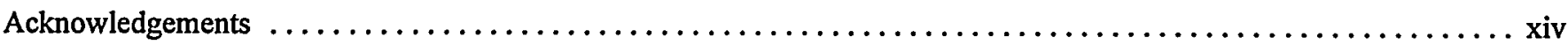

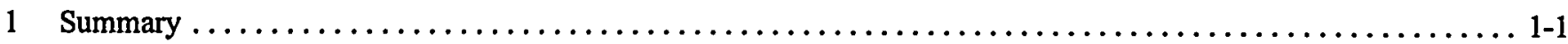

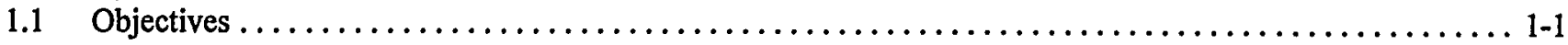

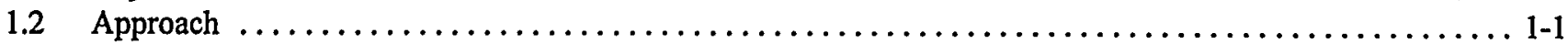

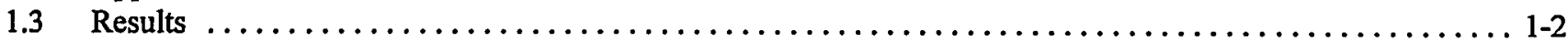

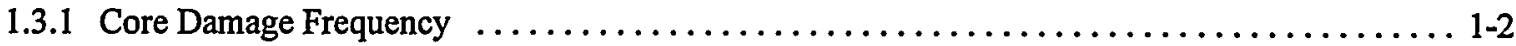

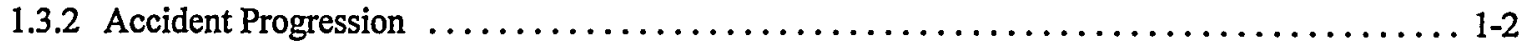

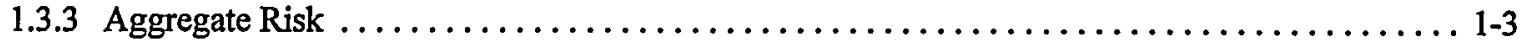

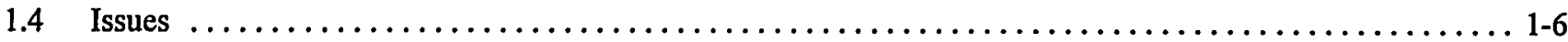

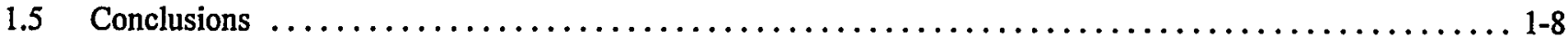

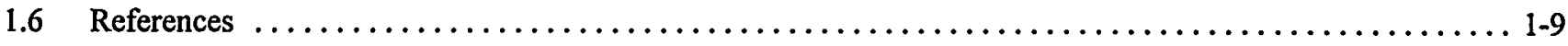

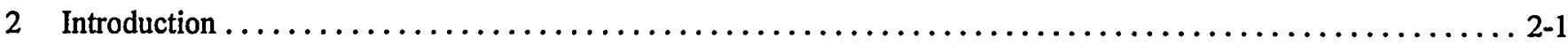

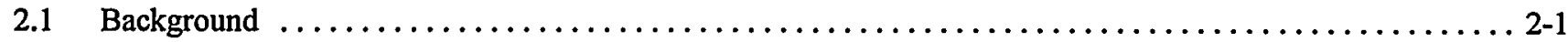

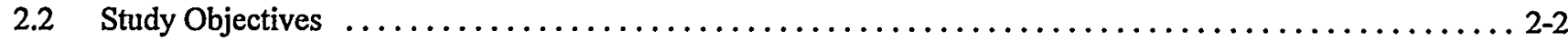

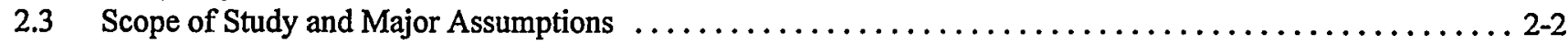

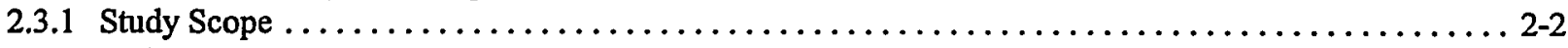

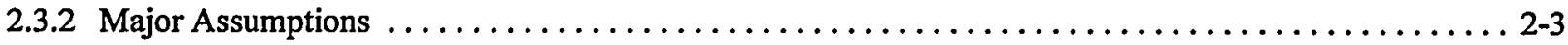

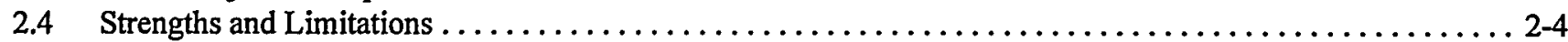

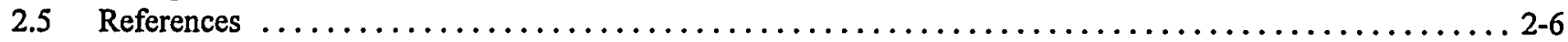

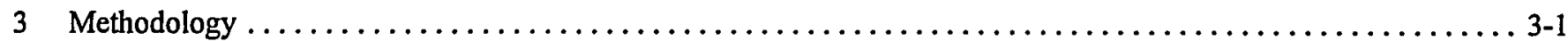

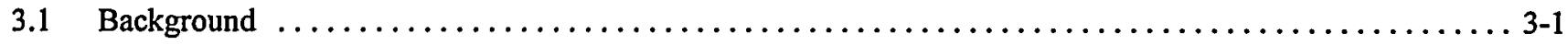

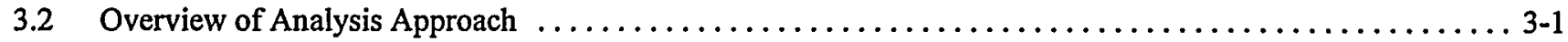

3.2.1 Representation of Risk . . . . . . . . . . . . .

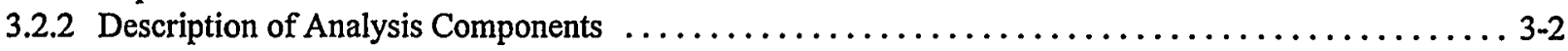

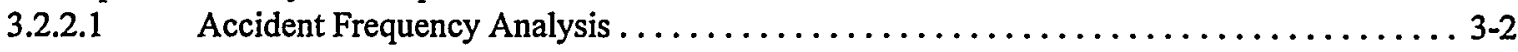

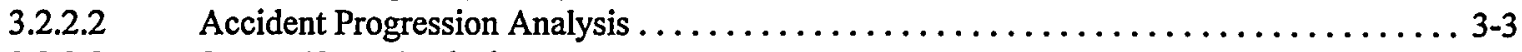

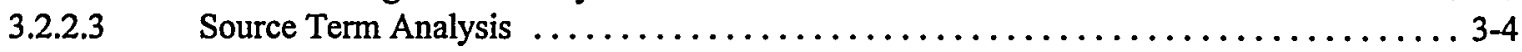

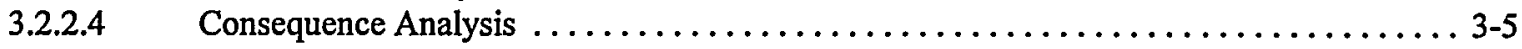

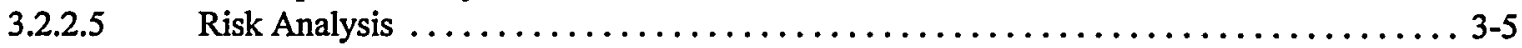

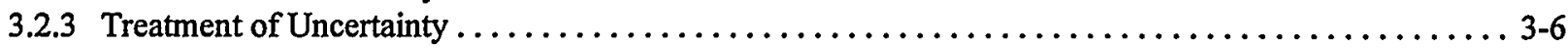

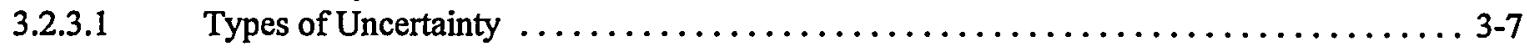

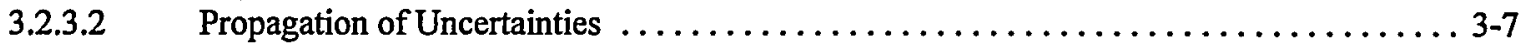

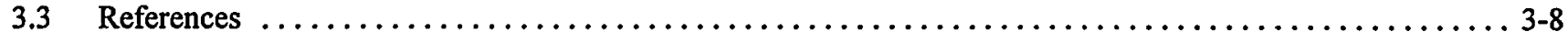

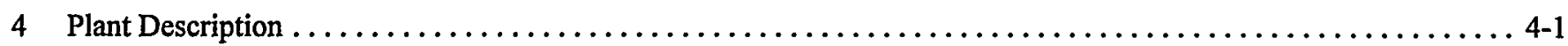

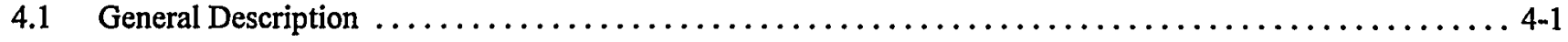

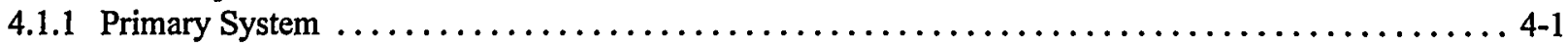

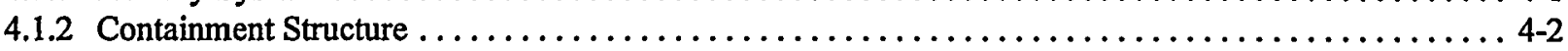

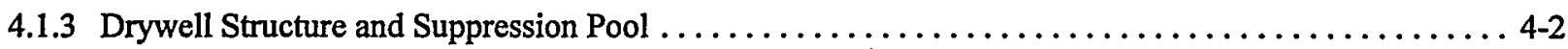

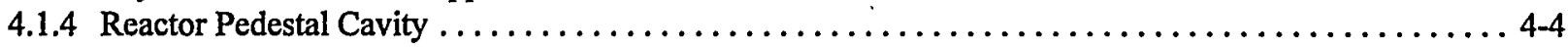

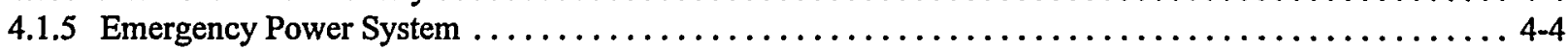

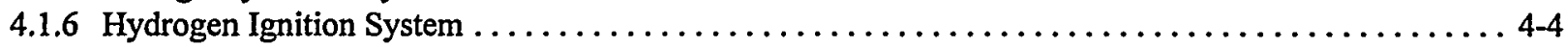

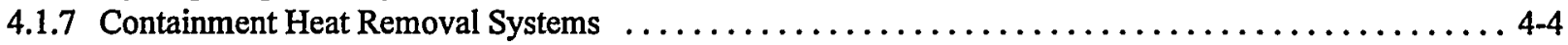

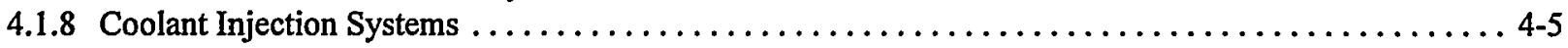

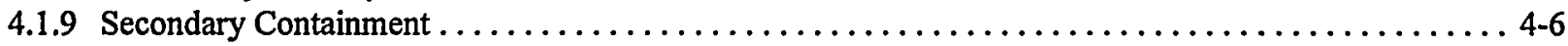




\section{Contents (Continued)}

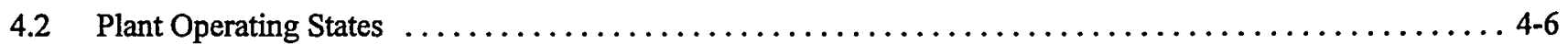

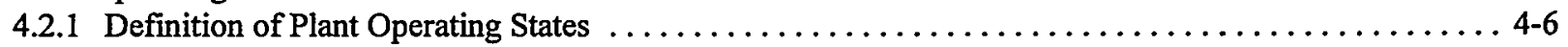

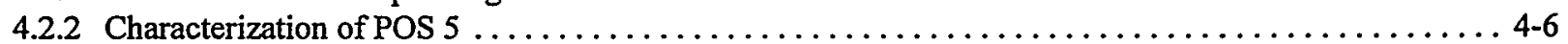

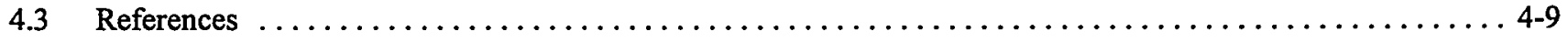

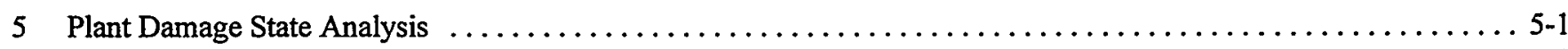

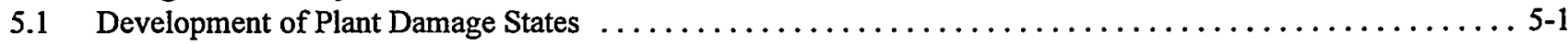

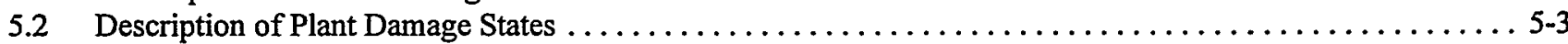

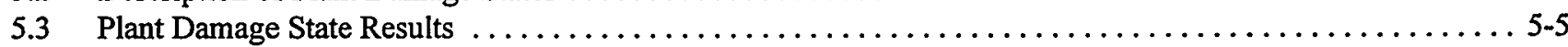

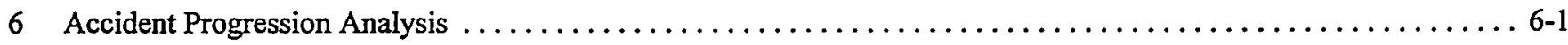

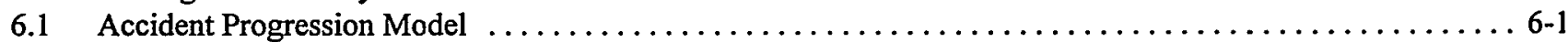

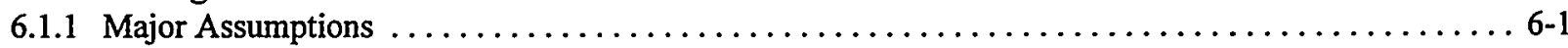

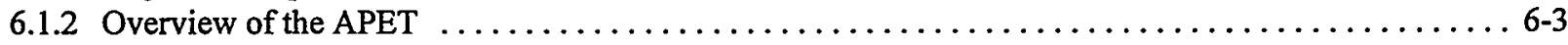

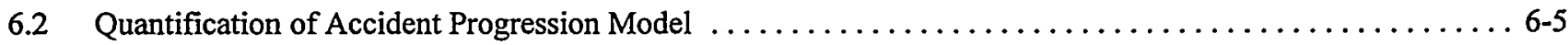

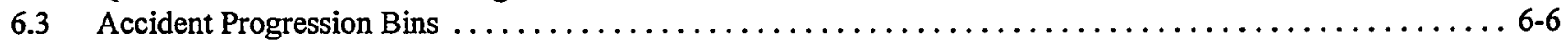

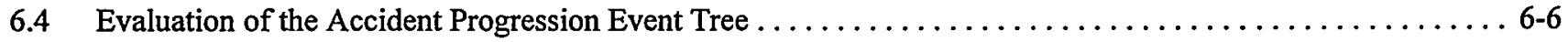

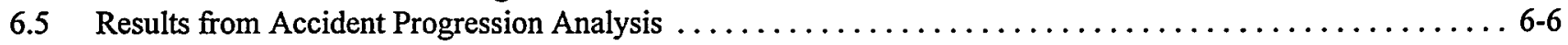

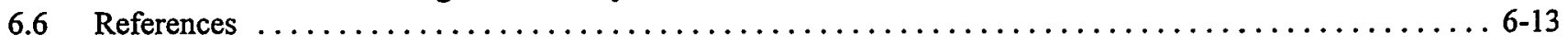

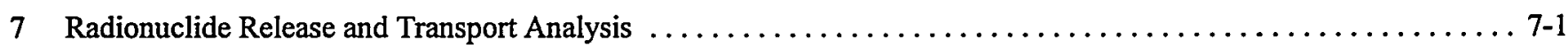

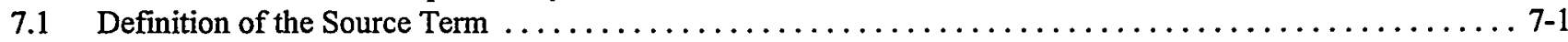

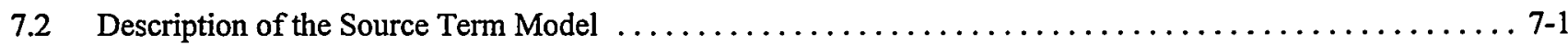

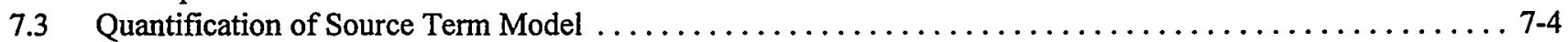

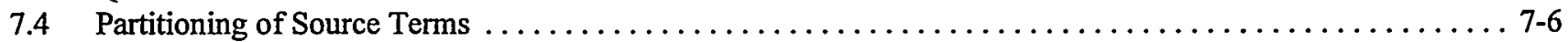

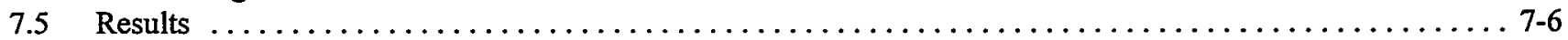

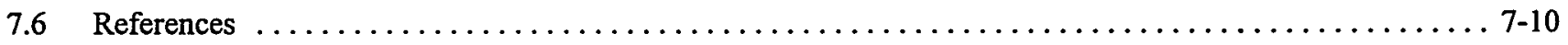

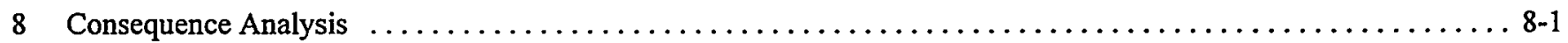

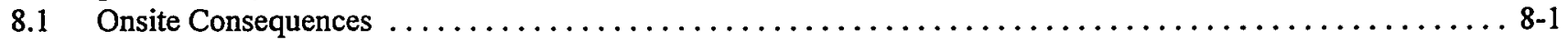

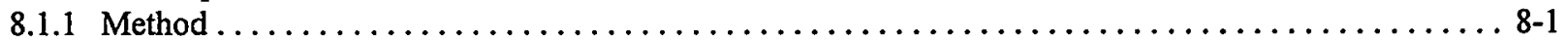

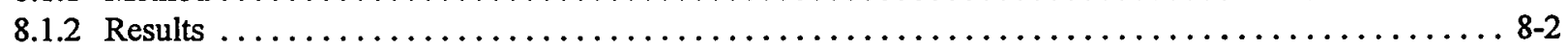

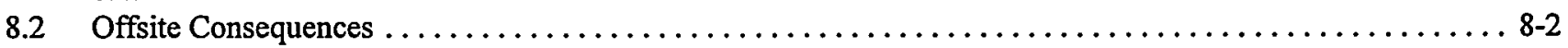

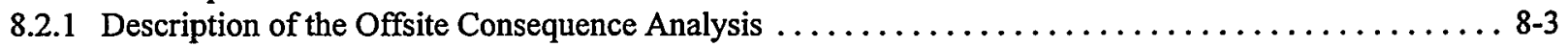

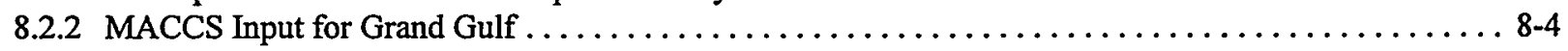

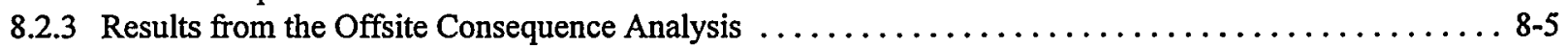

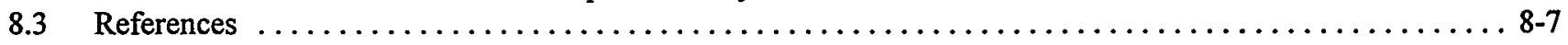

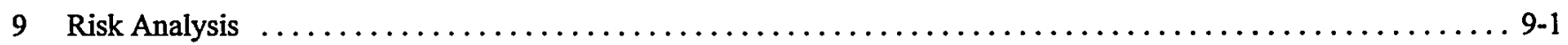

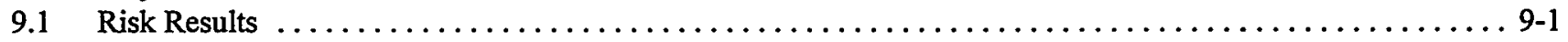

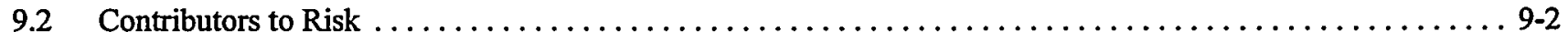

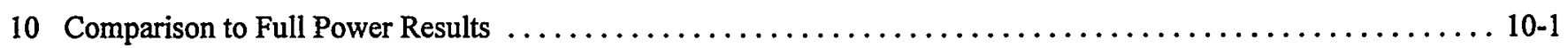

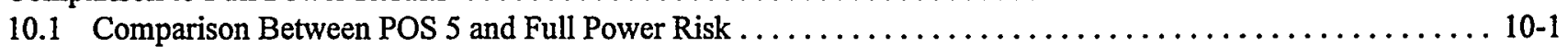

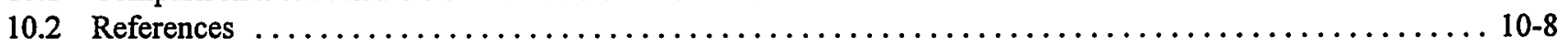

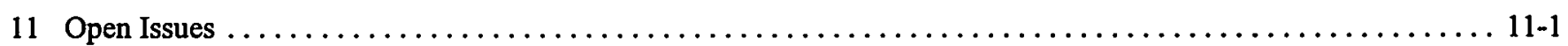




\section{Contents (Continued)}

Appendix A Supporting Information for the Plant Damage State Analysis $\ldots \ldots \ldots \ldots \ldots \ldots \ldots \ldots \ldots \ldots \ldots \ldots$ A-1

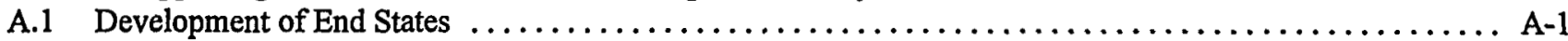

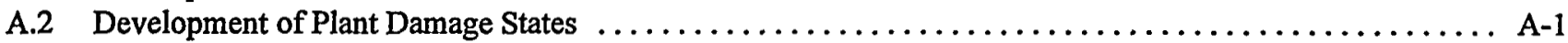

A.3 Comparison Between Level 1 and Level 2/3 PDS Frequencies $\ldots \ldots \ldots \ldots \ldots \ldots \ldots \ldots \ldots \ldots \ldots$ A-2

Appendix B Supporting Information for the Accident Progression Analysis $\ldots \ldots \ldots \ldots \ldots \ldots \ldots \ldots \ldots \ldots \ldots \ldots \ldots \ldots$

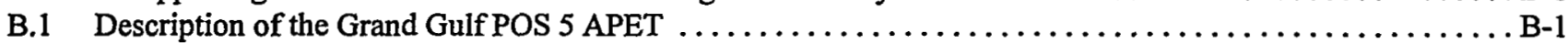

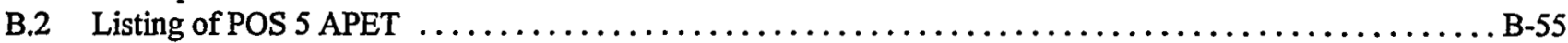

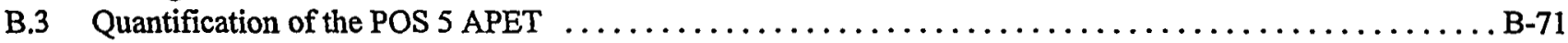

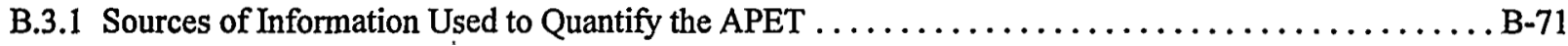

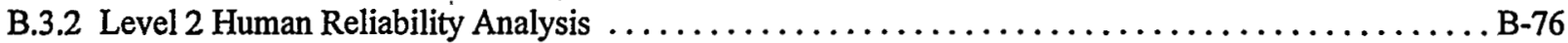

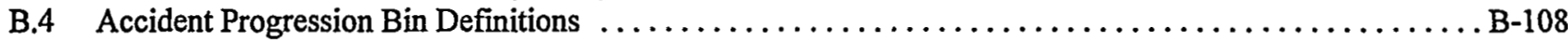

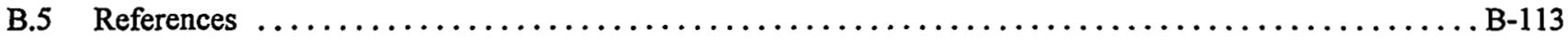

Appendix $C$ Supporting Information for the Source Term Analysis $\ldots \ldots \ldots \ldots \ldots \ldots \ldots \ldots \ldots \ldots \ldots \ldots \ldots \ldots \ldots \ldots \ldots$

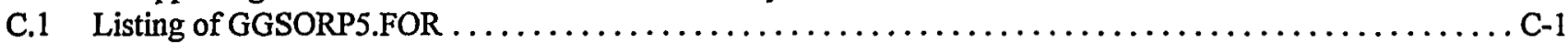

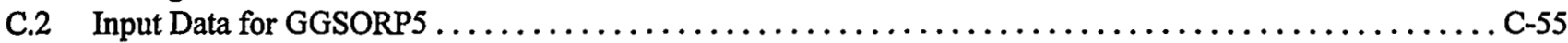

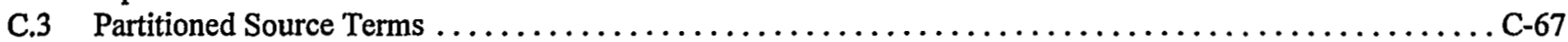

Appendix D Supporting Information for the Consequence Analysis $\ldots \ldots \ldots \ldots \ldots \ldots \ldots \ldots \ldots \ldots \ldots \ldots \ldots \ldots \ldots \ldots \ldots \ldots$

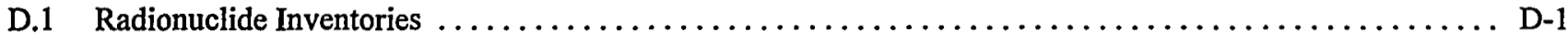

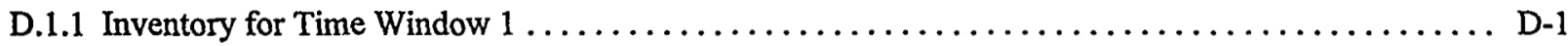

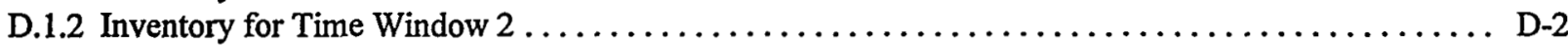

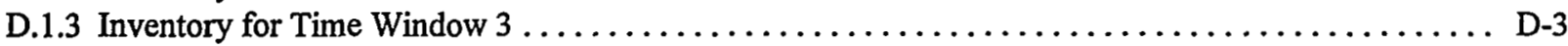

D.2 Listing of Code Used to Estimate Onsite Consequences $\ldots \ldots \ldots \ldots \ldots \ldots \ldots \ldots \ldots \ldots \ldots \ldots \ldots \ldots \ldots \ldots \ldots \ldots$

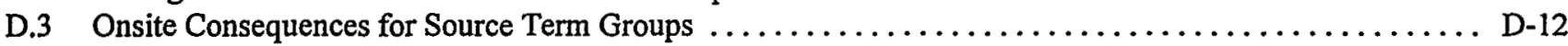

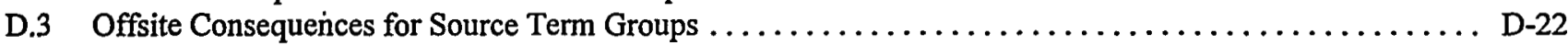

Appendix E Supporting Information for the Risk Analysis $\ldots \ldots \ldots \ldots \ldots \ldots \ldots \ldots \ldots \ldots \ldots \ldots \ldots \ldots \ldots \ldots \ldots \ldots \ldots \ldots$

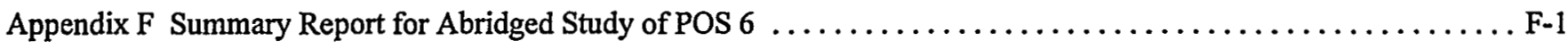




\section{List of Figures}

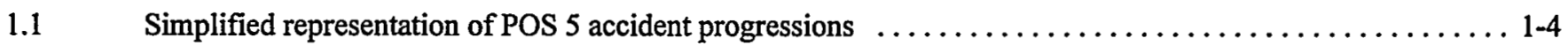

Comparison of Grand Gulf full power and POS 5 early fatality risk $\ldots \ldots \ldots \ldots \ldots \ldots \ldots \ldots \ldots \ldots \ldots$

Comparison of Grand Gulf full power and POS 5 total latent cancer risk $\ldots \ldots \ldots \ldots \ldots \ldots \ldots \ldots \ldots 1-7$

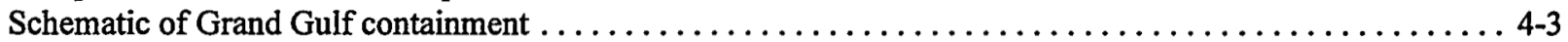

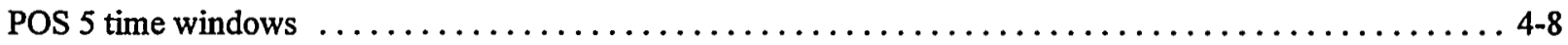

Simplified representation of POS 5 accident progressions $\ldots \ldots \ldots \ldots \ldots \ldots \ldots \ldots \ldots \ldots \ldots \ldots \ldots, 8$

Simplified representation of LOCA PDS group accident progression $\ldots \ldots \ldots \ldots \ldots \ldots \ldots \ldots \ldots \ldots, 6-9$

Simplified representation of Station Blackout PDS group accident progression $\ldots \ldots \ldots \ldots \ldots \ldots \ldots \ldots .6,9$

Simplified representation of Other PDS group accident progression $\ldots \ldots \ldots \ldots \ldots \ldots \ldots \ldots \ldots \ldots \ldots, 6 \ldots \ldots$

Probability of containment closure and probability of core damage arrest $\ldots \ldots \ldots \ldots \ldots \ldots \ldots \ldots \ldots, 6-11$

Probability of containment failure given that containment is closed before core damage $\ldots \ldots \ldots \ldots \ldots, 6-12$

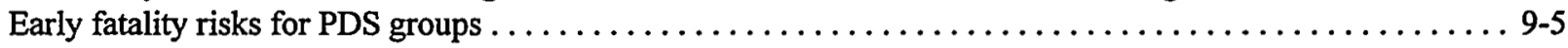

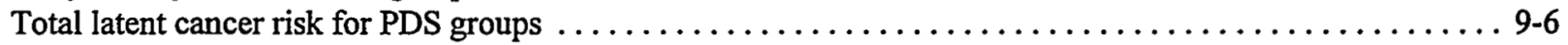

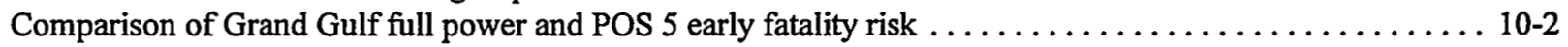

Comparison of Grand Gulf full power and POS 5 total latent cancer risk $\ldots \ldots \ldots \ldots \ldots \ldots \ldots \ldots \ldots$

Comparison of Grand Gulf full power and POS 5 population dose within 50 miles of the site risk . . . . . 10-4

Comparison of Grand Gulf full power and POS 5 population dose within 1000 miles of the site risk ..... 10-5

Comparison of Grand Gulf full power and POS 5 core damage frequencies $\ldots \ldots \ldots \ldots \ldots \ldots \ldots \ldots \ldots$ 


\section{List of Tables}

1.1 Core damage frequency for POS 5 and fractional contributions to the core damage frequency

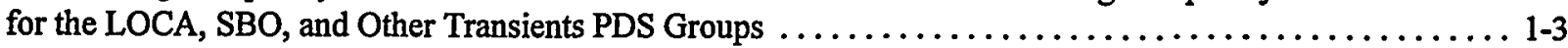

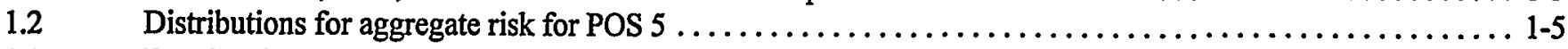

1.3 Fractional contribution to aggregate risk for the LOCA, SBO, and Other Transients PDS groups .........

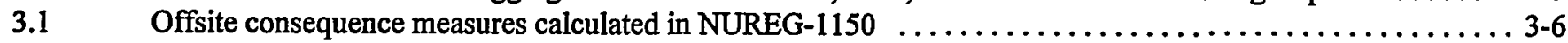

4.1 Relationship between operating conditions and plant operating states $\ldots \ldots \ldots \ldots \ldots \ldots \ldots \ldots \ldots \ldots \ldots, 4-7$

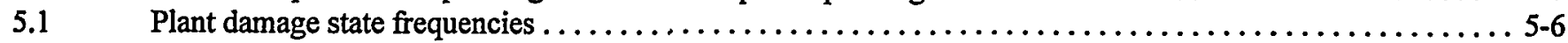

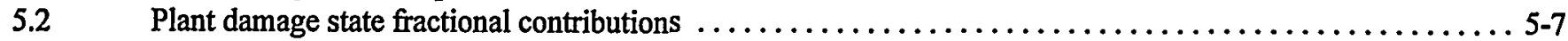

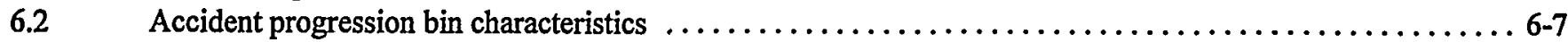

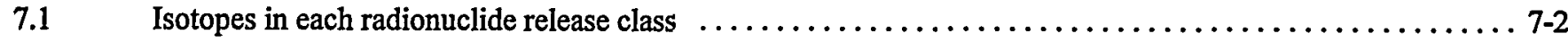

7.2 Sources of information used to quantify the source term parameters $\ldots \ldots \ldots \ldots \ldots \ldots \ldots \ldots \ldots \ldots, 7-7$

8.1-1 Mean parking lot doses and dose rates predicted by Wilson model $\ldots \ldots \ldots \ldots \ldots \ldots \ldots \ldots \ldots \ldots \ldots .8,2$

8.1-2 Mean parking lot doses and dose rates predicted by Ramsdell model $\ldots \ldots \ldots \ldots \ldots \ldots \ldots \ldots \ldots \ldots . . \ldots \ldots$

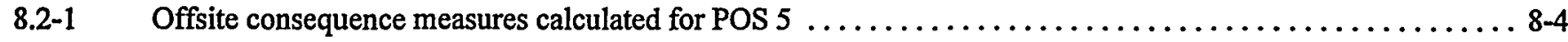

8.2-2 Site-specific input data for Grand Gulf MACCS calculations $\ldots \ldots \ldots \ldots \ldots \ldots \ldots \ldots \ldots \ldots \ldots \ldots \ldots ., 8,5$

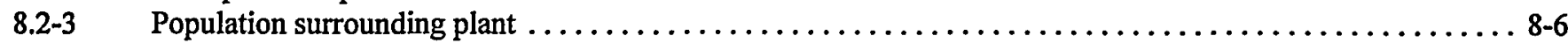

8.2-4 Shielding factors used for Grand Gulf MACCS calculations $\ldots \ldots \ldots \ldots \ldots \ldots \ldots \ldots \ldots \ldots \ldots \ldots \ldots .6 \ldots$

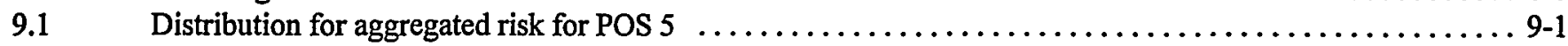

9.2 Mean fractional contributions to aggregate risk for the LOCA, SBO, and Other Transient PDS groups ..... 9-3

9.3 Fractional contribution to aggregate risk for Time Windows 1,2 , and $3 \ldots \ldots \ldots \ldots \ldots \ldots \ldots \ldots .4 .4 \ldots \ldots$

9.5 Mean Fractional Contributions of Plant Damage States to Risk $\ldots \ldots \ldots \ldots \ldots \ldots \ldots \ldots \ldots \ldots \ldots \ldots, 9.7$

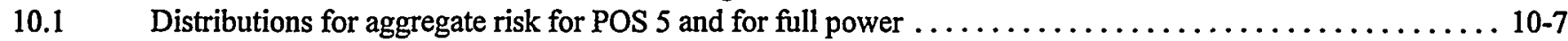

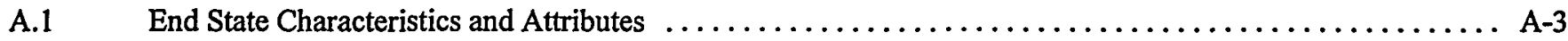

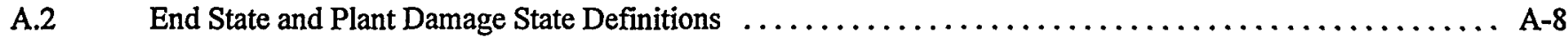

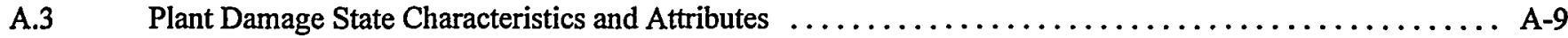

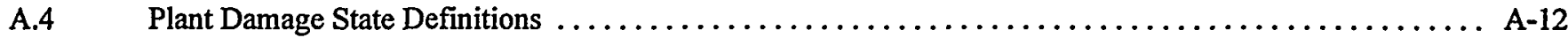

A.5 Comparison between Level 1 and Level 2 Plant Damage State Results $\ldots \ldots \ldots \ldots \ldots \ldots \ldots \ldots$ A-13

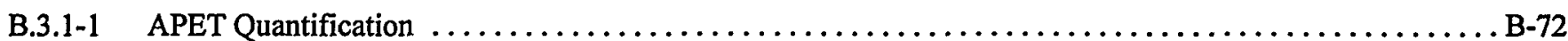

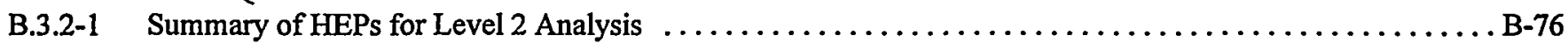

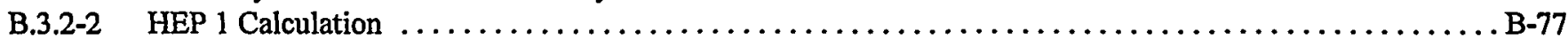

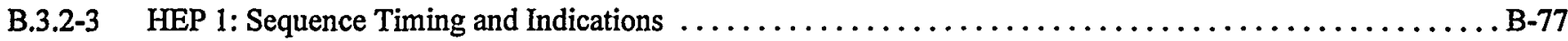

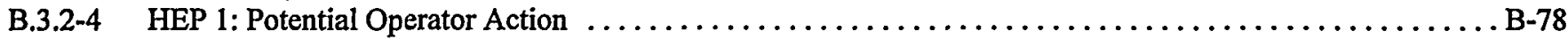

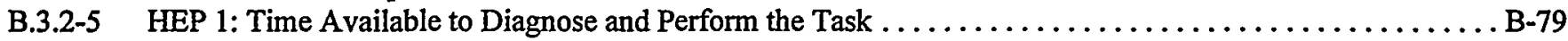

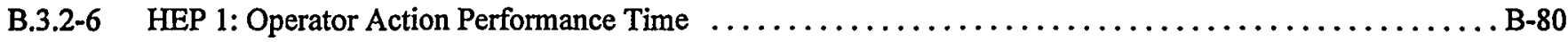

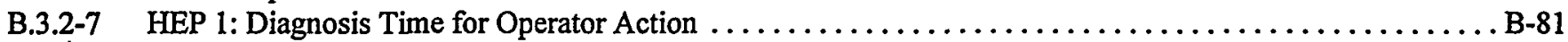

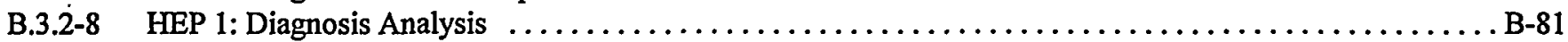

B.3.2-9 HEP 1: Post Diagnosis Action Time Identification per Step 10, Table 8-1 of ASEP HRAP ........... B-82

B.3.2-10 HEP 1: Post Diagnosis Stress-Level Identification per Step 10, Table 8-1 of ASEP HRAP ........... B-83

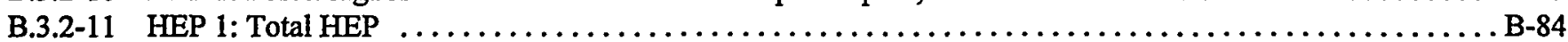

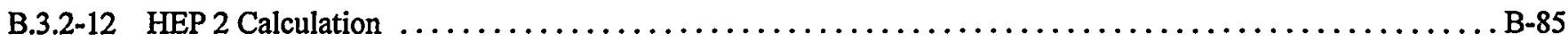

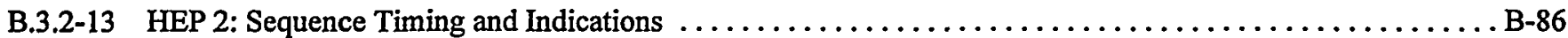

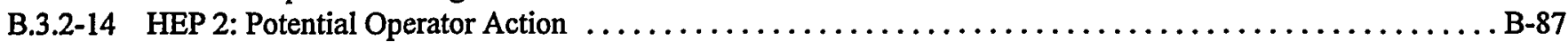

B.3.2-15 HEP 2: Time Available to Diagnose and Perform the Task $\ldots \ldots \ldots \ldots \ldots \ldots \ldots \ldots \ldots \ldots \ldots \ldots \ldots \ldots \ldots \ldots$

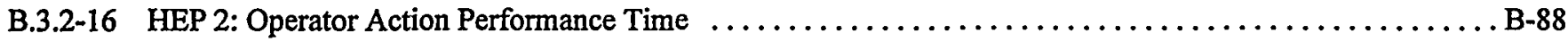

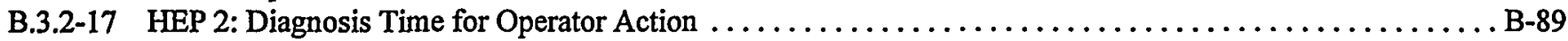

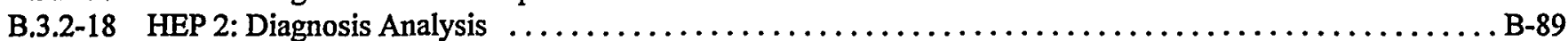

B.3.2-19 HEP 2: Post Diagnosis Action Time Identification per Step 10, Table 8-1 of ASEP HRAP ........... B-90

B.3.2-20 HEP 2: Post Diagnosis Stress-Level Identification per Step 10, Table 8-1 of ASEP HRAP ............ B-91

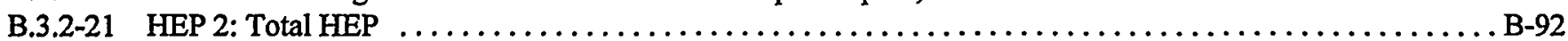




\section{List of Tables (continued)}

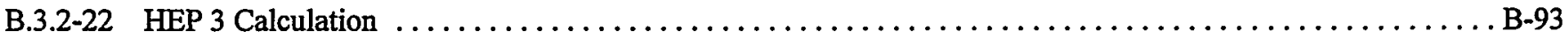

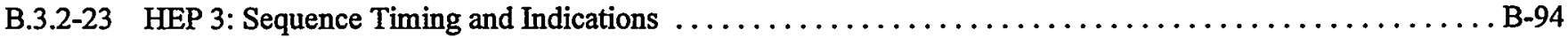

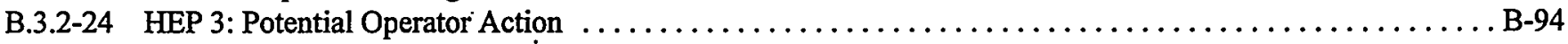

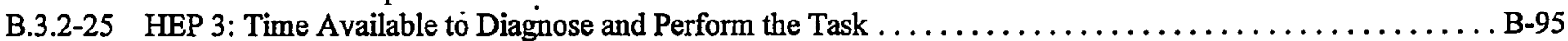

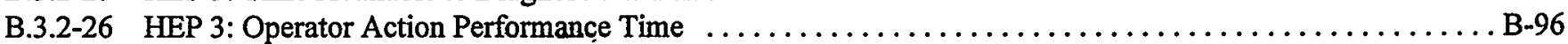

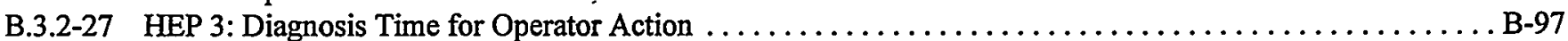

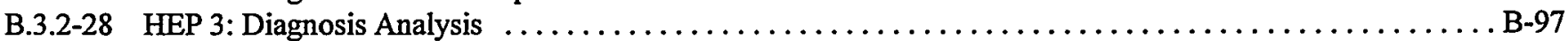

B.3.2-29 HEP 3: Post Diagnosis Action Time Identification per Step 10, Table 8-1 of ASEP HRAP . . . . . . . . . . B-98

B.3.2-30 HEP 3: Post Diagnosis Stress-Level Identification per Step 10, Table 8-1 of ASEP HRAP . . . . . . . . . . . B-99

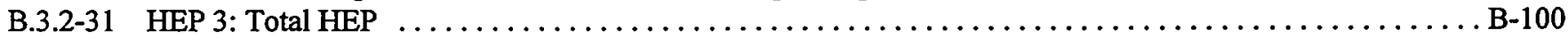

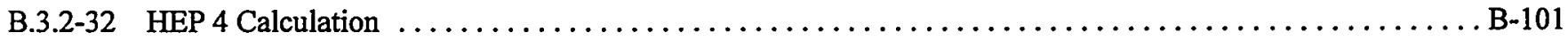

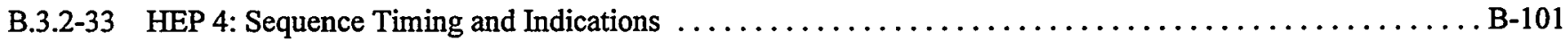

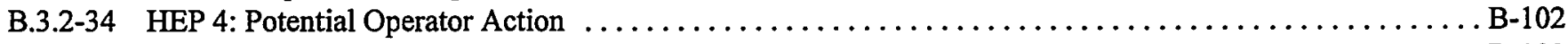

B.3.2-35 HEP 4: Time Available to Diagnose and Perform the Task .......................... B-103

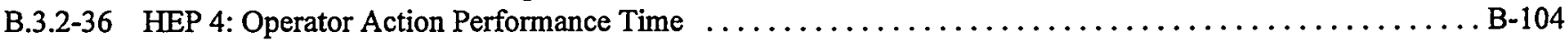

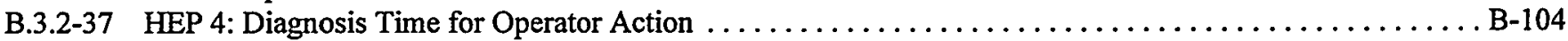

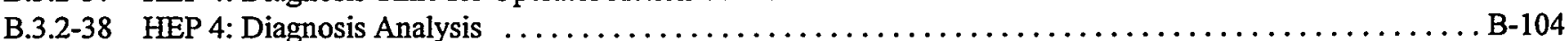

B.3.2-39 HEP 4: Post Diagnosis Action Time Identification per Step 10, Table 8-1 of ASEP HRAP . . . . . . . . B B-105

B.3.2-40 HEP 4: Post Diagnosis Stress-Level Identification per Step 10, Table 8-1 of ASEP HRAP ........... B-106

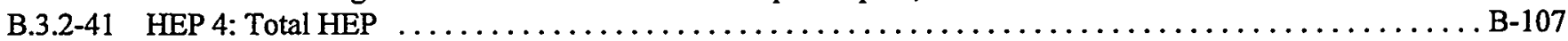

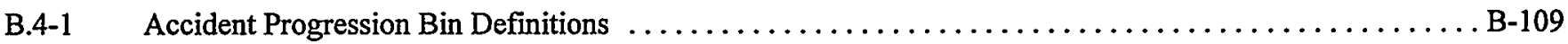

C.3-1 Source Terms for Partitioned Source Term Groups $\ldots \ldots \ldots \ldots \ldots \ldots \ldots \ldots \ldots \ldots \ldots \ldots \ldots \ldots \ldots \ldots \ldots \ldots \ldots \ldots$

D.3-1 Dose and Dose Rates Calculated Using Wilson/Reg Guide Model $\ldots \ldots \ldots \ldots \ldots \ldots \ldots \ldots \ldots \ldots$ D-12

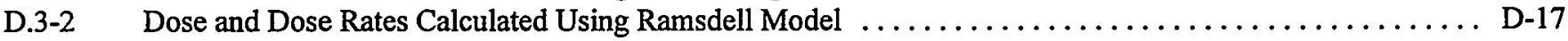

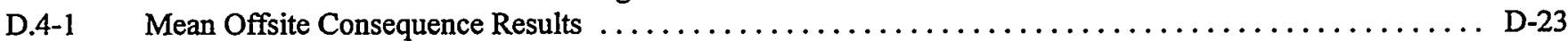

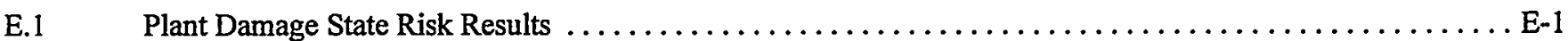


ADHR Auxiliary Decay Heat Removal

ADS Automatic Depressurization System

APB Accident Progression Bin

APET Accident Progression Event Tree

BNL Brookhaven National Laboratory

BWR Boiling Water Reactor

$\mathrm{CCI}$ Core-Concrete Interaction

CD Core Damage

CDS Condensate

CNMT Containment

CRD Control Rod Drive

CS Containment Sprays

CVS Containment Venting System

DF Decontamination Factor

DCH Direct Containment Heating

ECCS Emergency Core Cooling Systems

EOP Emergency Operating Procedures

EPS Emergency Power System

ES End State

ESF Engineered Safety Feature

FCI Fuel-Coolant Interaction

HEP Human Error Probability

HIS Hydrogen Igniter System

HPCS High Pressure Core Spray

HPME High Pressure Melt Ejection

HRA Human Reliability Analysis

LHS Latin Hypercube Sampling

LOCA Loss-of-Coolant Accident

LPCI Low Pressure Coolant Injection

LPCS Low Pressure Core Spray

LP\&S Low Power and Shutdown

MSIV Main Steam Isolation Valve

NRC Nuclear Regulatory Commission

OC Operating Condition

PDS Plant Damage State

POS Plant Operating State

PRA Probabilistic Risk Assessment

PWR Pressurized Water Reactor

RCIC Reactor Core Isolation Cooling

RHR Residual Heat removal

RPV Reactor Pressure Vessel

SBO Station Blackout

SDC Shutdown Cooling (Systems)

SGTS Standby Gas Treatment System

SP Suppression Pool

SPC Suppression Pool Cooling

SPMU Suppression Pool Makeup

SRV Safety Relief Valve

SSW Standby Service Water Crosstie

STG Source Term Group

VB Vessel Breach 


\section{Foreword}

\section{(NUREG/CR-6143 and 6144) \\ Low Power and Shutdown Probabilistic Risk Assessment Program}

Traditionally, probabilistic risk assessments (PRA) of severe accidents in nuclear power plants have considered initiating events potentially occurring only during full power operation. Some previous screening analyses that were performed for other modes of operation suggested that risks during those modes were small relative to full power operation. However, more recent studies and operational experience have implied that accidents during low power and shutdown could be significant contributors to risk.

During 1989, the Nuclear Regulatory Commission (NRC) initiated an extensive program to carefully examine the potential risks during low power and shutdown operations. The program includes two parallel projects performed by Brookhaven National Laboratory (BNL) and Sandia National Laboratories (SNL), with the seismic analysis performed by Future Resources Associates. Two plants, Surry (pressurized water reactor) and Grand Gulf (boiling water reactor), were selected as the plants to be studied.

The objectives of the program are to assess the risks of severe accidents due to internal events, internal fires, internal floods, and seismic events initiated during plant operational states other than full power operation and to compare the estimated core damage frequencies, important accident sequences and other qualitative and quantitative results with those accidents initiated during full power operation as assessed in NUREG-1150. The scope of the program includes that of a level-3 PRA.

The results of the program are documented in two reports, NUREG/CR-6143 and 6144. The reports are organized as follows:

For Grand Gulf:

NUREG/CR-6143 - Evaluation of Potential Severe Accidents During Low Power and Shutdown Operations at Grand Gulf, Unit 1

Volume 1: Summary of Results

Volume 2: $\quad$ Analysis of Core Damage Frequency from Internal Events for Plant Operational State 5 During a Refueling Outage

Part 1: Main Report

Part 2: Internal Events Appendices A to $\mathrm{H}$

Part 3: Internal Events Appendices I and J

Part 4: Internal Events Appendices $\mathrm{K}$ to $\mathrm{M}$

Volume 3: Analysis of Core Damage Frequency from Internal Fire Events for Plant Operational State 5 During a Refueling Outage

Volume 4: $\quad$ Analysis of Core Damage Frequency from Internal Flooding Events for Plant Operational State 5 During a Refueling Outage

Volume 5: Analysis of Core Damage Frequency from Seismic Events for Plant Operational State 5 During a Refueling Outage

Volume 6: Evaluation of Severe Accident Risks for Plant Operational State 5 During a Refueling Outage

Part 1: Main Report

Part 2: Supporting MELCOR Calculations 


\section{Foreword (Continued)}

\section{For Surry:}

NUREG/CR-6144 - Evaluation of Potential Severe Accidents During Low Power and Shutdown Operations at Surry Unit-1

Volume 1: $\quad$ Summary of Results

Volume 2: Analysis of Core Damage Frequency from Internal Events During Mid-loop Operations

Part 1: Main Report

Part 2: Internal Events Appendices A to D

Part 3: Internal Events Appendix E

Part 4: Internal Events Appendices $\mathrm{F}$ to $\mathrm{H}$

Part 5: Internal Events Appendix I

Volume 3: Analysis of Core Damage Frequency from Internal Fires During Mid-loop Operations

Volume 4: Analysis of Core Damage Frequency from Internal Floods During Mid-loop Operations

Volume 5: Analysis of Core Damage Frequency from Seismic Events During Mid-loop Operations

Volume 6: Evaluation of Severe Accident Risks During Mid-loop Operations Part 1: Main Report

Part 2: Appendices 


\section{Acknowledgements}

The authors wish to thank the NRC project manager, Chris Ryder, for his support, interest, and thoughtful management of the project. We would also like to thank the MELCOR development team at Sandia for making the modifications to the code that enabled us to analyze accidents at conditions other than full power. Finally, we would like to thank Tim Peterson (Tech Reps, Inc.) for editing and preparing this document for publication. 


\section{Summary}

Traditionally, probabilistic risk assessments (PRAs) of severe accidents in nuclear power plants have only considered accidents initiated during full power operation. Some previous screening analyses, which were performed for other than full power modes of operation, suggest that risks during low and off power modes of operation were small relative to those occurring during full power operation. However, recent studies and operational experiences indicate that low power and shutdown accident risks may be significant. Although the power of the reactor core is much less in off power conditions than at full power, the technical specifications allow for more equipment to be inoperable in off power conditions (e.g., under certain conditions the containment can be open).

In response to concerns about risk during low power and shutdown conditions, the U.S. Nuclear Regulatory Commission's Office of Nuclear Regulatory Research (NRC RES) has undertaken a two-phase project to analyze the frequencies, consequences, and risk of accidents occurring during modes of operation other than full power.

Phase 1 of the project was completed in September of 1991 [Whitehead et al., 1991]. This phase involved a coarse screening of potential accidents that could occur at a Boiling Water Reactor (BWR) while operating at other than full power. This coarse analysis was adopted as a means of obtaining, in a relatively short time, some estimate of the potential for accidents during low power and shutdown conditions. The BWR examined was the Grand Gulf Nuclear Power Station, a single unit 1250 MWe (net) BWR 6 power plant with a Mark III containment, located near Port Gibson, Mississippi.

The coarse screening analysis indicated that risk during these conditions cannot be shown to be insignificant by a screening analysis. Hence the NRC decided to conduct a follow-on detailed analysis. Subsequently, the NRC decided to first perform a detailed analysis consisting of a Level 3 PRA on one of the off power conditions. Based on trends indicated in the results of the coarse screening analysis, plant operational state (POS) 5 (consisting mainly of Cold Shutdown Operating Condition) was selected for detailed analysis. (Note that POSs are artificial subdivisions of the time that plants spend in low power and shutdown conditions. This concept was developed during Phase 1 of this project to allow the analyst to better represent the plant as it transitions from power to nonpower operation.) This report presents the results of the detailed analysis of the Grand Gulf facility in POS 5 during a refueling outage.

A companion project involving the Surry Pressurized Water
Reactor (PWR) is being conducted by Brookhaven National Laboratory.

This volume of the report, Volume 6, presents the Level 2/3 portion of the Level 3 PRA that was performed on POS 5.

\subsection{Objectives}

The purpose of this study was to perform an analysis of potential accidents that could occur at Grand Gulf while the plant is in POS 5 during a refueling outage. Only accidents initiated from traditional internal events were analyzed in this study (i.e., accidents initiated by internal fires or floods were not included). The specific objectives used to achieve this purpose are listed below:

- $\quad$ For POS 5, perform a characterization of the accident progressions following core damage and estimate the consequences that result from these accidents.

- Determine quantitatively the risk and estimate the uncertainty in risk for this mode of operation.

- $\quad$ Compare the risk associated with POS 5 to the risk associated with full power operation.

- $\quad$ Provide an assessment of the potential for a radioactive release to cause onsite consequences.

\subsection{Approach}

The risk associated with POS 5 was determined in the Level 2 and 3 portions of the PRA using a simplified form of the NUREG-1150 methodology [U.S. NRC, 1990]. The Level $2 / 3$ portion of the PRA is concerned with the progression of postulated accidents following the onset of severe core damage and the estimation of the consequences that result from the release of any radioactive material. As such, it consists of the following constituent analyses: plant damage state (PDS) analysis, accident progression analysis, source term analysis, consequence analysis, and risk analysis. A brief summary of the approach used in each of the constituent analyses is provided below.

Plant Damage State Analysis: PDSs were developed to define the interface between the accident frequency analysis (Level 1) and the accident progression analysis (Level 2). Core damage accidents that have similar plant 
and system configurations at the onset of core damage are grouped together; each group is called a plant damage state.

Accident Progression Analysis: Based on the configuration of the plant defined by the PDSs, event tree techniques were used to delineate the accident progressions following the onset of core damage. The accident progressions define the status of the containment and other features of the plant that are used to mitigate the accident during the various phases of the accident; they also identify phenomena that may impact the release of radioactive material. The accident progression event tree (APET) developed in this study is similar in concept to the APETs developed in NUREG-1150; however, it is not as detailed. Compared to the NUREG-1150 APETs, the POS 5 APET included fewer questions (i.e., top events), addressed issues in less detail, and did not use formal expert judgment procedures to quantify the APET.

Source Term Analysis: Source terms, which characterize the type and amount of radioactive material releases from the plant, were estimated for accident progression groups using the parametric approach developed in the NUREG1150 study [Jow et al., 1993]. The parametric expression was quantified, to the extent possible, using information from the NUREG-1150 full power analysis of Grand Gulf [Harper et al., 1992]. The source terms were then combined into a manageable number of source term groups using a partitioning algorithm first developed in the NUREG-1150 study [Iman et al., 1990] and then modified in the full power study of the LaSalle plant [Brown et al., 1992].

Consequence Analysis: Offsite consequences were estimated for each source term group using the MACCS code. The emergency response assumption used in this study are the same as those used in the NUREG-1150 Grand Gulf plant analysis [USNRC, 1990; Brown et al., 1990]. In addition to offsite consequences, this study also included a scoping analysis of onsite consequences.

Risk Analysis: The risk results reported in this study are estimates of aggregate risk, which is the sum over all accident scenarios of the product of the accident frequency with its consequence. The aggregate risk results calculated in this study account for the amount of time, on average, that the plant is in POS 5 during a typical calendar year (i.e., the plant is in POS 5 for only a small fraction of the year-approximately $3 \%$ ). The risk calculated in this study is not the risk attributable to one year of operation in POS 5. All risk results presented in this report are on a per calendar year basis.

A limited uncertainty analysis (which included variables from the PDS, accident progression, and source term analyses) was also performed. In contrast to NUREG1150 , formal expert opinion techniques were not used in this study to quantify the accident progression and source term models. Where appropriate, however, distributions developed in NUREG-1150 were used in this study. For events that could not be quantified using existing distributions, new distributions were developed by the project staff.

To analyze the potential accidents that can occur during POS 5 it was necessary to divide POS 5 into three distinct time regimes (also called time windows): (1) from entry into POS 5 to 24 hours after shutdown, (2) from 24 hours after shutdown to entry into POS 6 (POS 6 begins approximately 94 hours after shutdown and roughly corresponds to the refueling mode of operation), and (3) POS 5 again after core alterations (this last time regime starts approximately 40 days after shutdown and lasts for approximately 10.4 days). For each time window the appropriate core power and radionuclide inventory was used to estimate the timing of the accident and its potential consequences.

\subsection{Results}

\subsubsection{Core Damage Frequency}

For discussion purposes, the core damage scenarios identified in the Level 1 analysis can be combined into the following three PDS groups (12 PDSs were actually evaluated in the accident progression analysis): loss of coolant accidents (LOCAs), Station Blackouts (SBOs), and Other Transients. The total core damage frequency and the fractional contributions to the core damage frequency for these three groups are provided in Table 1.1. The LOCA PDS group is the dominant contributor to the core damage frequency, followed the SBO PDS group and the Other Transients PDS group.

\subsubsection{Accident Progression}

A simplified representation of the APET that addresses the major aspects of the accident is shown in Figure 1.1. (The actual APET included 59 top events or questions). Figure 1.1 combines the results from all the accidents and is conditional on the occurrence of core damage; the values displayed in these figures are mean conditional probabilities. From the simplified tree presented in Figure 1.1 , it can be seen that in the most likely accidents in POS 5 
Table 1.1 Core damage frequency for POS 5 and fractional contributions to the core damage frequency for the LOCA, SBO, and Other Transients PDS groups

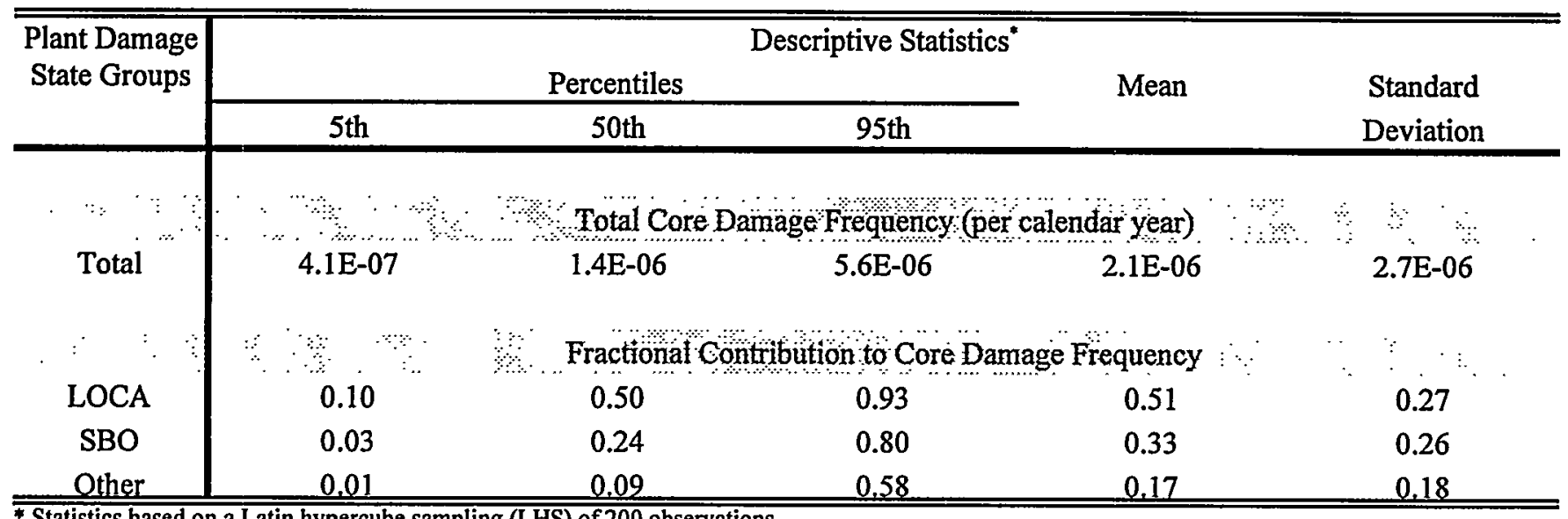

* Statistics based on a Latin hypercube sampling (LHS) of 200 observations.

the containment is open, the suppression pool is bypassed, and the vessel fails. For the cases where the vessel fails, there is a significant probability that the core debris will either be quenched in a flooded cavity or the interactions between the core debris and the concrete structures beneath the vessel, the core-concrete interaction (CCI), will occur in a flooded cavity. For the former, the releases associated with $\mathrm{CCI}$ are prevented. In the latter case, the radioactive releases are scrubbed by the water in the flooded cavity, which helps reduce the source term to the environment. If the containment is closed prior to core damage, it is predicted to either fail or to be vented after core damage because containment heat removal is not available in these accidents. Venting the containment late in the accident is the most likely scenario. For the accidents identified in POS 5 , the containment sprays were never available after the onset of core damage.

\subsubsection{Aggregate Risk}

Table 1.2 presents the offsite risk results for the following six measures: early fatalities, total latent cancer fatalities, population dose within 50 miles of the site, population dose within 1000 miles of the site, average individual early fatality risk within 1 mile of the site, and average individual latent cancer risk within 10 miles of the site.

Many factors can affect the magnitude and severity of the release and in turn affect risk. Factors associated with POS 5 accidents that tend to increase risk include the following:

In many of the accidents the containment equipment hatch was open during the entire accident. An open equipment hatch provides a path for radionuclides to escape from the containment to the auxiliary building and then out into the environment.

- Two plant features that can be used to attenuate the release of radioactive aerosols are the suppression pool and the containment sprays. In both the LOCA and the SBO PDSs, the radioactive material released from the damaged fuel bypassed the suppression pool. The containment sprays were not available in any of the POS 5 accidents.

- In many of the accidents, core cooling was not restored early in the accident, thus precluding any possibility of arresting the core damage process before vessel failure. When the vessel fails, the core debris in the vessel is released into the reactor cavity, allowing for possible CCIs. Significant amounts of radioactive material can be released during this ex-vessel phase of the accident.

A number of factors associated with these POS 5 accidents also tend to decrease risk. These factors are listed below:

- Although in many of the accidents the containment equipment hatch is open, the suppression pool is bypassed, and the containment sprays are unavailable, the releases pass through the auxiliary building before escaping into the environment. Because of its large volume and surface area, the auxiliary building provides a location for the radionuclides to be attenuated by deposition and thereby reduce the source term to the environment. 


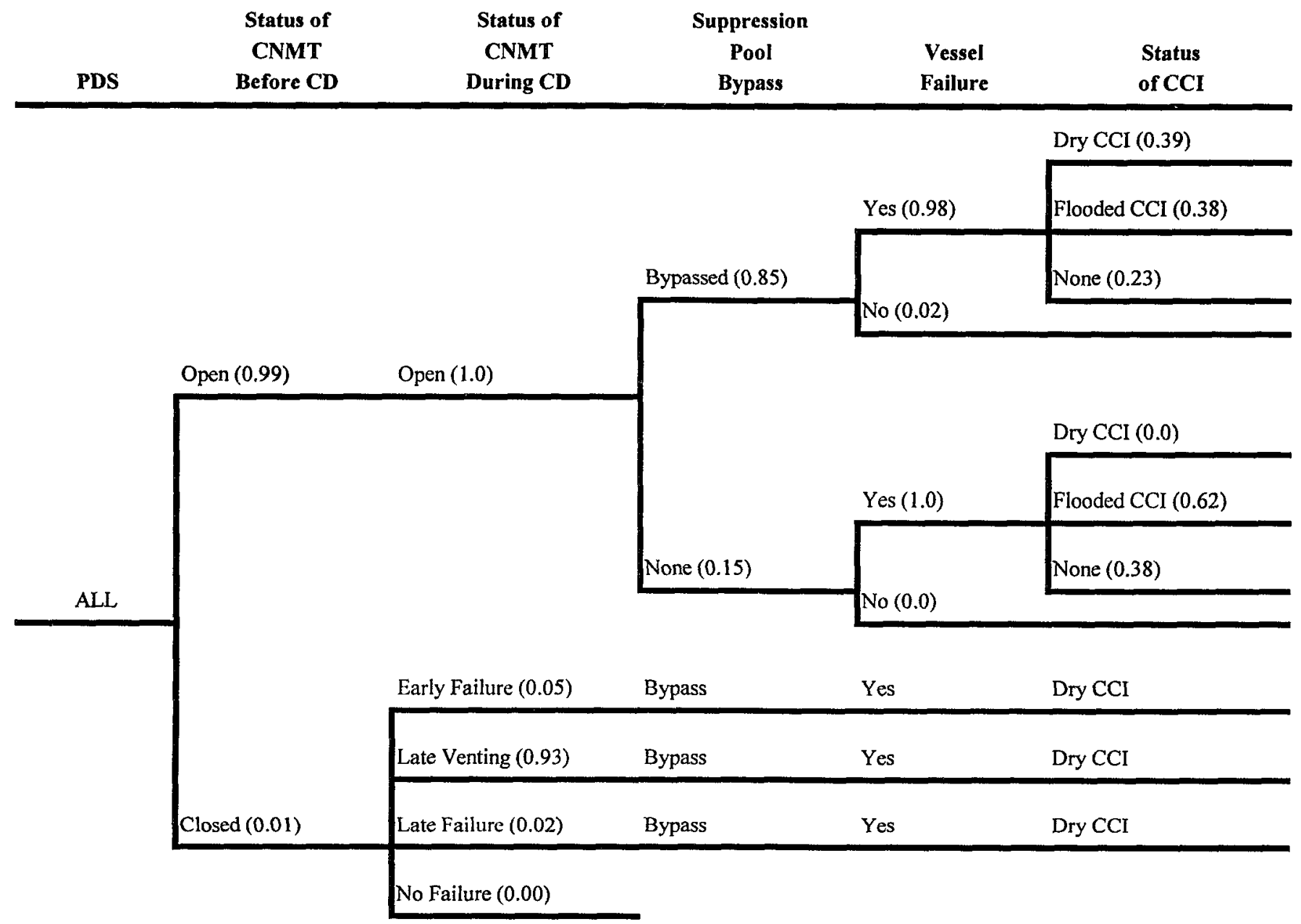

Figure 1.1 Simplified representation of POS 5 accident progressions

- The accidents delineated for these shutdown conditions progress sufficiently slowly that there is typically a considerable amount of time available for the public to respond to the accident and evacuate before exposure to the release. This is primarily important for the early health effects consequence measures, which are more strongly affected by the time available for evacuation.

- $\quad$ Radioactive decay has reduced the radioactive potential of these shutdown accidents relative to the inventory that is present immediately after the reactor is shut down. This factor is primarily important for early health effects, which are more strongly affected by the shorter lived radionuclides. This effect is much less noticeable for latent health effects, which are more strongly affected by the longer lived isotopes.

- The population around the Grand Gulf plant is relatively low. Although many factors influence the magnitude of the consequences, in general, for a given release, a smaller population correlates with a smaller number of fatalities. Of the four Mark III plants in the United States, Grand Gulf has the fewest number of people living within 50 miles of the plant, according to the 1990 census data. The Mark III plant with the greatest number of people living within 50 miles of the site has a population that is more than an order of magnitude greater than the Grand Gulf 50 mile population. 
Table 1.2 Distributions for aggregated risk for POS 5

(all values are per calendar year; population doses are in person-rem)

\begin{tabular}{|c|c|c|c|c|c|}
\hline \multirow{3}{*}{$\begin{array}{c}\text { Consequence } \\
\text { Measures }\end{array}$} & \multicolumn{5}{|c|}{ Descriptive Statistics ${ }^{*}$} \\
\hline & \multicolumn{3}{|c|}{ Percentiles } & \multirow[t]{2}{*}{ Mean } & \multirow{2}{*}{$\begin{array}{c}\text { Standard } \\
\text { Deviation }\end{array}$} \\
\hline & 5 th & 50th & 95th & & \\
\hline Early Fatality Risk & $3.7 \mathrm{E}-11$ & $2.8 \mathrm{E}-09$ & $3.9 \mathrm{E}-08$ & $1.4 \mathrm{E}-08$ & $5.4 \mathrm{E}-08$ \\
\hline Total Latent Cancer Risk & 4.3E-04 & $1.9 \mathrm{E}-03$ & $1.2 \mathrm{E}-02$ & $3.8 \mathrm{E}-03$ & 7.7E-03 \\
\hline Population Dose within 50 miles of the plant & $1.3 \mathrm{E}-01$ & $5.3 \mathrm{E}-01$ & $3.1 \mathrm{E}+00$ & $9.9 \mathrm{E}-01$ & $1.9 \mathrm{E}+00$ \\
\hline Population Dose within 1000 miles of the plant & $9.9 \mathrm{E}-01$ & $4.4 \mathrm{E}+00$ & $2.8 \mathrm{E}+01$ & $8.7 \mathrm{E}+00$ & $1.8 \mathrm{E}+01$ \\
\hline Individual Early Fatality Risk-- 0 to 1 mile & $4.2 \mathrm{E}-13$ & $2.7 \mathrm{E}-11$ & $3.0 \mathrm{E}-10$ & $9.6 \mathrm{E}-11$ & $3.4 \mathrm{E}-10$ \\
\hline Individual Latent Cancer Risk-- 0 to 10 miles & $2.5 \mathrm{E}-10$ & $9.4 \mathrm{E}-10$ & $4.9 \mathrm{E}-09$ & $1.6 \mathrm{E}-09$ & $2.4 \mathrm{E}-09$ \\
\hline
\end{tabular}

* Statistics are based on an LHS sample of 200 observations.

To place the risks from POS 5 into context, they were compared to the risks from full power operation as estimated in the NUREG-1150 Grand Gulf plant analysis [USNRC, 1990; Brown et al., 1990]. In Figures 1.2 and 1.3 , the early fatality and total latent cancer fatality risks from full power operation and POS 5 are presented. This comparison shows that the risks from POS 5 are not insignificant compared to the risks from full power operation. In fact, although the mean risk values from the two studies are similar (i.e., not differing by more that a factor of 5), the mean risk values from POS 5 are actually greater than the full power risk values.

Table 1.3 provides the fractional contributions to the early fatality risk and the total latent cancer risk for the following three PDS groups: LOCAs, SBOs, and Other Transients. The fractional contributions to the population dose risk measures (not shown in Table 1.3 for brevity) are similar to the fractional contributions to the total latent cancer risk measure. From Table 1.3 it can be seen that, on average, the SBO PDS group is the dominant contributor to the total early fatality risk. Because a large amount of overlap exists among the three distributions, as is evident from the descriptive statistics provided in Table 1.3, on any given observation (an observation is one particular trial in the many trials made in a Monte Carlo type analysis) the contribution from the three groups can vary. That is, for one observation the SBO group may be dominant, whereas for another observation the LOCA group may be the dominant group. On average, however, the $\mathrm{SBO}$ is the dominant contributor. The SBO PDS group's large contribution to early fatality risk can be attributed to its relatively high contribution to the core damage frequency coupled with the fact that the containment equipment hatch is open, the suppression pool is bypassed, and the auxiliary building fails early in these accidents. Combined, these factors cause the SBOs to have relatively high risk values. The LOCA PDS group, however, is not a dominant contributor to early fatality risk even though it is a dominant contributor to the core damage frequency. This situation occurs primarily because the dominant contributors to the LOCA core damage frequency are LOCA accidents that are initiated while the plant is in Time Window 3 (i.e., PDS3-1). Numerous factors can potentially reduce the number of early fatalities that occur when the accident is initiated in Time Window 3 relative to the other time windows. These factors include the following conditions: (1) Radioactive decay has reduced the inventory of short-lived radionuclides that are important to early health effects. (2) Because of the lower decay heat the accidents progress more slowly, allowing more time for the population to evacuate. (3) The release is spread out over a longer time which, helps reduce the concentration of radionuclides in the environment. For these reasons Time Window 3 is a negligible contributor to early fatality risk.

For latent cancer health effects, the LOCA and SBO PDS groups are, on average, the dominant contributors to risk. Because the radionuclides that are important to the latent health effects tend to have long half lives, these risk measures are not particularly sensitive to the time of accident occurrence relative to shutdown . Latent cancers primarily depend on the total amount of radioactive material released, not on the time it was released (i.e., early in the accident versus late in the accident). Because latent cancers are not strongly dependent on the timing characteristics of the accident (i.e., start of release or release duration), the latent cancer risk will depend on the likelihood of the accident and on the total amount of radioactive material released. In all of the core damage 


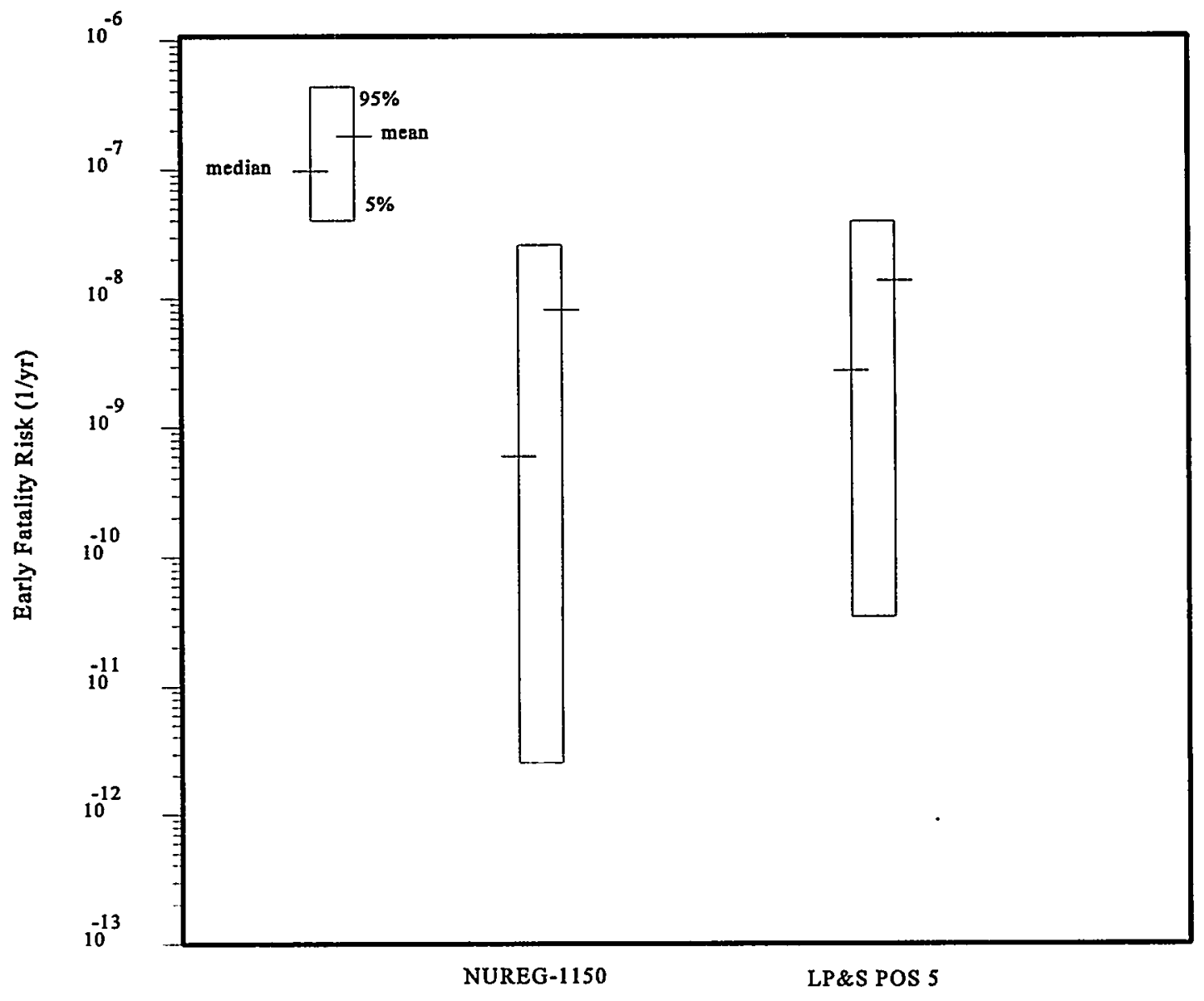

Figure 1.2 Comparison of Grand Gulf full power and POS 5 early fatality risk

accidents delineated in this study, the containment is either open at the start of the accident or fails during the accident, and in most of the accidents the core damage process is not arrested in the vessel. Thus, although the timing of the accident may vary, when the uncertainty in the source term is considered, all the accidents will result in roughly similar releases of radioactive material to the environment. Thus, as can be seen in Tables 1.1 and 1.3, the mean fractional contribution to latent cancer risks tends to be roughly similar to the mean fractional contribution to the core damage frequency for each of the PDS groups. The fractional contributions from the LOCA and Other Transient groups tend to be less than their fractional contributions to the core damage frequency because for these PDSs portions of the release are scrubbed by either the suppression pool or the pool formed by flooding the containment. The fractional contribution from the SBO
PDS group tends to be greater than the fractional contribution to the core damage frequency because for these accidents the containment is open at the start of the accident, the auxiliary building fails early in the accident, the vessel nearly always fails, CCI nearly always occurs, and the releases are rarely scrubbed by water. Therefore the releases associated with the SBO tend to be large relative to the other accidents analyzed in this study.

\subsection{Issues}

The study presented in this volume is for a single POS (POS 5) and, as such, assesses the risk associated only with POS 5. This study does not attempt to assess the risk with the entire low power and shutdown regime of operation. 


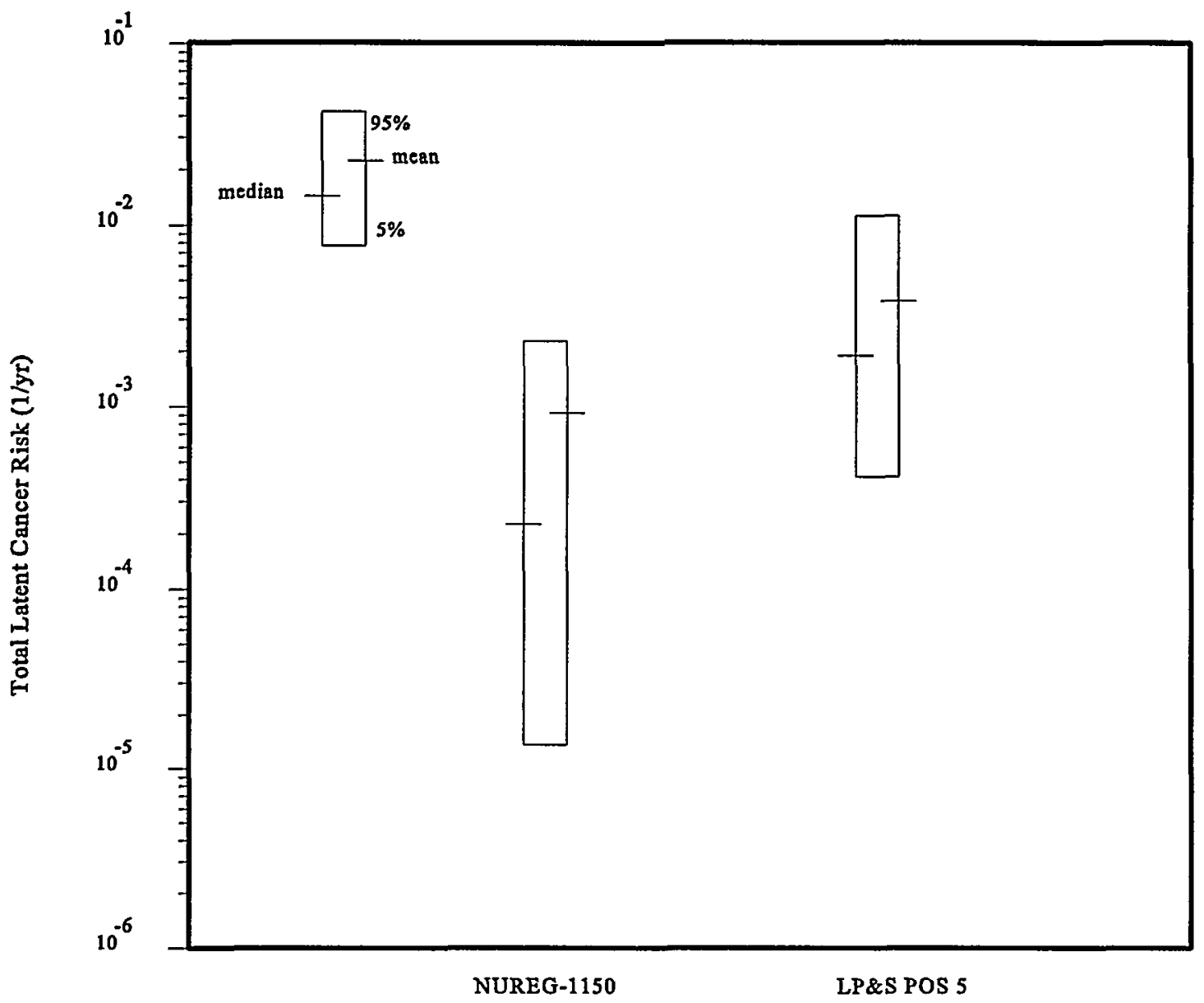

Figure 1.3 Comparison of Grand Gulf full power and POS 5 total latent cancer risk

Although the Level 1 screening study and other qualitative insights suggest that POS 5 is the risk-dominant mode of shutdown, no detailed study has been performed on the other POSs to confirm this finding.

Note that reducing the risk in one POS by changing when equipment is available and unavailable can shift the risk to another POS. Because this study only addresses the risk associated with one POS, the affect of these changes on overall risk (i.e., risk across all the POSs) cannot currently be quantitatively assessed.

Only accidents initiated from traditional internal events were analyzed in this study. Hence the risk calculated for POS 5 is not complete because it does not include accidents initiated by internal fires or floods, nor does it include accidents initiated by seismic events.
Because only a single plant was analyzed, these results cannot be considered generic and applicable to a population of plants. The plant and system models used in this study are based on the Grand Gulf plant as it operates in a selected mode of operation. Thus, although some insights may be applicable to other plants, in general the results from this study should not be arbitrarily applied to other plants or conditions. The model used to develop the progression of the accidents after the onset of core damage is in part based on the Grand Gulf Emergency Operating Procedures and other procedures and practices at the plant. Changes in these procedures and practices can certainly affect the progression of the accident and the ultimate risk of the POS. Similarly, because the offsite consequences are sensitive to the site characteristics and surrounding region (e.g., weather, population, land usage), for a given release of radioactive material, the consequences can be expected to vary from one site to the next. 
Table 1.3 Fractional contributions to aggregate risk for the LOCA, SBO, and Other Transients PDS groups

\begin{tabular}{|c|c|c|c|c|c|}
\hline \multirow{3}{*}{$\begin{array}{c}\text { Plant Damage } \\
\text { State Groups }\end{array}$} & \multicolumn{5}{|c|}{ Descriptive Statistics ${ }^{*}$} \\
\hline & \multicolumn{3}{|c|}{ Percentiles } & \multirow[t]{2}{*}{ Mean } & \multirow{2}{*}{$\begin{array}{l}\text { Standard } \\
\text { Deviation }\end{array}$} \\
\hline & 5th & 50 th & 95th & & \\
\hline & \multicolumn{5}{|c|}{ Fractional Contribution to Early Fatality Risk } \\
\hline LOCA & 0.001 & 0.04 & 0.72 & 0.16 & 0.24 \\
\hline $\mathrm{SBO}$ & 0.08 & 0.87 & 1.00 & 0.73 & 0.30 \\
\hline \multirow[t]{2}{*}{ Other } & 0.001 & 0.04 & 0.61 & 0.12 & 0.18 \\
\hline & \multicolumn{5}{|c|}{ Fractional Contribution to Total Latente Cancer Fatality Risk } \\
\hline LOCA & 0.04 & 0.38 & 0.90 & 0.42 & 0.27 \\
\hline SBO & 0.04 & 0.41 & 0.90 & 0.45 & 0.28 \\
\hline Other & 0.01 & 0.06 & 0.55 & 0.13 & 0.17 \\
\hline
\end{tabular}

${ }^{*}$ Statistics are based on an LHS sample of 200 observations.

\subsection{Conclusions}

The following conclusions can be drawn from this study:

- Without many plant features available to mitigate a release, the potential exists for a large release of radioactive material if core damage should occur. For the most likely accidents the containment is open, the suppression pool is bypassed, and the containment sprays are not available.

- In the event that the containment is closed prior to the onset of core damage, it is always predicted to fail because containment heat removal was not available in the analyzed accidents.

- $\quad$ The risks from POS 5 are not insignificant compared to the risks from full power operation. Hence the full power risk distributions by themselves do not completely characterize the risks associated with the operation of this plant. To accurately characterize the plant's risks, results from this study suggest that it may be necessary to include other modes of operation in addition to the full power mode. This inclusion could have important implications for assessments that rely on the total risk from a plant, such as when comparisons are made to the safety goals.
Although only a simplified scoping study of the onsite consequences was performed, the possible onsite consequences of an accident during shutdown could be significant, particularly because in many of the accidents the containment remains open, allowing for an early release of radioactive material. 


\subsection{References}

[Whitehead et al., 1991]

[USNRC, 1990]

[Jow et al., 1993]

[Harper et al., 1992]

[Iman et al., 1990]

[Brown et al., 1992]

[Brown et al., 1990]
D. W. Whitehead et al., "BWR Low Power and Shutdown Accident Frequencies Project, Phase 1 - Coarse Screening Analysis", Vol. 1, Draft Letter Report, Sandia National Laboratories and Science and Engineering Associates, Inc. November 23, 1991 update, Copy available in the NRC Public Document Room.

U. S. Nuclear Regulatory Commission, "Severe Accident Risks: An Assessment for Five U. S. Nuclear Power Plants," NUREG-1150, Vols. 1-3, December 1990-January 1991.

H.-N. Jow et al., "XSOR Code User's Manual," NUREG/CR-5360, SAND89-0943, Sandia National Laboratories, November 1993.

F. T. Harper et al., "Evaluation of Severe Accident Risks: Quantification of Major Input Parameters: Experts' Determination of Source Term Issues," NUREG/CR-4551, Vol. 2, Rev. 1, Part 4, Sandia National Laboratories, June 1992.

R. L. Iman et al., "PARTITION: A Program for Defining the Source Term/Consequence Interface in the NUREG-1150 Probabilistic Risk Assessments," NUREG/CR-5262, SAND88-2940, Sandia National Laboratories, May 1990.

T. D. Brown et al. "Integrated Risk Assessment for the LaSalle Unit 2 Nuclear Power Plant," NUREG/CR-5305, SAND90-2765, Vols. 1-2, Sandia National Laboratories, August 1992.

T. D. Brown et al., "Evaluation of Severe Accident Risks: Grand Gulf Unit 1," NUREG/CR-4551, SAND86-1309, Vol. 6, Rev. 1, Sandia National Laboratories, December 1990. 
Summary 


\section{Introduction}

\subsection{Background}

The safety of commercial nuclear plants during full power operation has been previously assessed in many probabilistic safety assessment studies. The U.S. Nuclear Regulatory Commission (NRC) has been an active participant in these studies, which include the landmark Reactor Safety Study [USNRC, 1975], the five plant studies performed as part of the NUREG-1150 study [USNRC, 1990], and the LaSalle plant analysis performed under the RMIEP/PRUEP programs [Payne, 1992; Brown et al., 1992]. Furthermore, all licensees are required to perform an individual plant examination, which also assesses the safety of the plant during full power operation.

Recent events at several nuclear power generating stations, recent safety studies, and operational experience, however, have highlighted the need to assess the safety of plants during low-power and shutdown modes of operation. In contrast to full power operation, very little information exists on the safety of plants during the low-power and shutdown modes of operation. In the past, the assumption has been that full power operation is the risk-dominant mode of operation because the decay energy is greatest at the time of shutdown and then decays as a function of time. Thus the rationale was that during shutdown modes of operation the decay heat would be sufficiently low to allow ample time to respond to any abnormal event that may threaten the core cooling function. Furthermore, given the unlikely event that a release occurred, radioactive decay would lessen the radiological potential of the release. This argument's weakness is that the technical specifications allow for more equipment to be inoperable in off-power conditions. Thus, while there may be more time to respond to an accident during shutdown, many of the systems that are relied $\phi n$ to mitigate an accident during power operation may not be available during shutdown.

To gain a better understanding of the risk significance of low-power and shutdown modes of operation, the Office of Nuclear Regulatory Research at the NRC has undertaken a two phase program to analyze the frequencies, consequences, and risk of accidents occurring during modes of operation other than full power. To investigate the likelihood of severe core damage accidents during offpower conditions, probabilistic risk assessments (PRAs) were performed for two nuclear plants: Unit 1 of the Grand Gulf Nuclear Station, which is a BWR-6 Mark III boiling water reactor (BWR), and Unit 1 of the Surry Power Station, which is three loop, subatmospheric, pressurized water reactor (PWR). This report will discuss the analysis performed on the BWR.

The analysis of the BWR was conducted at Sandia National Laboratories. The analysis of the PWR was performed at Brookhaven National Laboratory. The LP\&S PWR analysis is reported in NUREG/CR-6144 and will not be discussed further in this report. The present report describes and discusses the results of the BWR analysis. Volume 1 is a summary of the BWR study. Volumes 2 through 5 present the accident frequency analysis (i.e., Level 1) performed under FIN L1923. Volume 6 presents the Level 2/3 analyses performed under FIN L1679. Part 1 of Volume 6 presents and discusses the accident progression, radionuclide release and transport, consequence, and risk analyses. Part 2 of Volume 6 presents the deterministic code calculations performed with the MELCOR code [Summers et al., 1991] that were used to support the development and quantification of the PRA models.

Phase 1 of the project was completed in September of 1991 [Whitehead et al., 1991]. This phase involved a coarse screening of potential accidents that could occur at a BWR while operating at other than full power; it was adopted as a means of obtaining, in a relatively short time, some estimate of the potential for accidents during low-power and shutdown conditions. The coarse screening analysis indicated that risk during these conditions cannot be shown to be insignificant by a screening analysis. Hence, the NRC decided to perform follow-on detailed analyses. The NRC decided to perform first a detailed analysis consisting of a Level 3 PRA on one of the off-power conditions. Based on trends indicated in the results of the coarse screening analysis, plant operational state (POS) 5 (consisting mainly of Cold Shutdown Operating Condition) was selected for detailed analysis. (Note: Plant operational states are artificial subdivisions of the time plants spend in low-power and shutdown conditions. The divisions were developed during Phase 1 of this project to allow the analyst to better represent the plant as it transitions from power operation to nonpower operation.) This report presents the results of the detailed analysis of the Grand Gulf facility in POS 5 during a refueling outage.

Several reasons exist for performing a Level 3 PRA and producing estimates of risk, as opposed to only estimating the likelihood of core damage. The configuration of the plant during shutdown can be very different from the configuration during full power, which can lead to drastically different accident progressions and releases of 
radioactive material. Hence it is not sufficient to determine the frequency of core damage during shutdown and then infer the risk based on PRA results from full-power operation. If the core damage frequency associated with shutdown is less than the full-power core damage frequency, for example, it is not valid to infer that the risk will also be less, because the consequence could be higher during shutdown. Also, to be able to assess the safety of shutdown modes of operation, it will be useful to determine the relative importance of shutdown risk as compared to full-power operation risk. The risks associated with the operation of these two plants at full power were characterized in the NUREG-1150 study [USNRC, 1990].

\subsection{Study Objectives}

The purpose of this study was to perform an analysis of potential accidents that could occur at Grand Gulf while the plant is in POS 5 during a refueling outage. Specific objectives used to achieve this purpose included:

- For POS 5, perform a characterization of the accident progressions following core damage and estimate the consequences that result from these accidents.

- Determine quantitatively the risk and estimate the uncertainty in risk for POS 5.

- Compare the risk associated with POS 5 to the risk associated with full power operation.

- Provide an assessment of the potential for a radioactive release to cause onsite consequences.

This study will address the following types of questions:

- What are the characteristics of accident progressions for the selected regime of shutdown? Are there any significant differences between these progressions and progressions typical of full power accidents? What are the dominant phenomena?

- What is the risk associated with this regime of operation and how does it compare to full power operation? What are the risk-significant configurations? Can anything be done to reduce this risk?

- What is the potential for releases from these accidents to cause onsite consequences? Is the plant in a particularly vulnerable configuration (i.e., containment open, large numbers of people in the vicinity of the plant)?

\subsection{Scope of Study and Major Assumptions}

\subsubsection{Study Scope}

The study reported in this volume is the Level $2 / 3$ portion of a Level 3 PRA that was performed to investigate the risk associated with a selected regime of shutdown. The Level $2 / 3$ portion of the PRA is concerned with the progression of postulated accidents following the onset of severe core damage and the estimation of the consequences that result from the release of any radioactive material. As such, it consists of the following constituent analyses: plant damage state (PDS) analysis, accident progression analysis, source term analysis, consequence analysis, and risk analysis. The Level 2/3 portion of this PRA utilized a simplified form of the NUREG-1150 methodology; that is, simplified models were used to perform the accident progression analysis and only a limited uncertainty analysis was performed. This analysis is focused; that is, the models developed and used in this analysis (e.g., accident progression event tree and parametric source term model) are specific to accidents represented by the PDSs. No effort was made to make the models general, that is, applicable to any type of postulated accident or other plants. The advantage of the focused approach is that resources are spent on the accidents of concern; the disadvantage is that, if a different type of accident is postulated after the models are constructed, the models must be modified. Other points that define the scope of the analysis include:

- The consequence analysis includes the traditional offsite assessment (i.e., similar to the offsite consequence assessment performed in the NUREG-1150 plant studies) and will also include a scoping assessment of onsite consequences.

The simplified uncertainty analysis will only include those issues thought to be the most important to risk and only issues from the accident progression and source term analyses. (The uncertainty in the core damage frequency will be propagated through the analysis via the uncertainty in the plant damage states). For many of these issues, distributions developed during the NUREG-1150 project will be used. If an applicable distribution from an existing study does 
Introduction

not exist, the project staff will develop the appropriate distribution. Formal expert elicitation techniques (i.e., the use of panels of experts from a variety of organizations and companies) will not be used to select issues, nor will they be used to develop distributions.

- Only one plant was selected for analysis in this study: Unit 1 of the Grand Gulf Nuclear Station.

While the low-power and shutdown modes of operation encompass many plant and system configurations, only the cold shutdown regime of operation during a refueling outage was investigated in detail in this study. This regime of shutdown is referred to as POS 5. This mode of operation was selected because of its importance in the Level I coarse screening analysis [Whitehead et al., 1991] and a qualitative assessment of its importance relative to the other modes of operation. For more discussion of the rationale used to select POS 5 for this study, see Section 3 of Volume 2, Part 1 of this report.

Only accidents initiated by traditional internal events were analyzed (i.e., accidents initiated by internal fire, internal flood are not included in this study).

Abridged Analysis of POS 6: An abridged PRA of POS 6 (i.e., refueling mode of operation prior to fuel movement) was performed in the spring of 1992. POS 6 was selected because the containment and vessel are both open during this mode of operation. This plant configuration was of particular interest to the NRC. The scope of the abridged PRA of POS 6 was considerably narrower than the analysis of POS 5. The POS 6 analysis relied on very simplified plant damage state, accident progression, and uncertainty analyses. Compared to the POS 5 analysis, the POS 6 accident progression analysis considered fewer issues and addressed them in less detail. Since the PDSs were based on results from the Level 1 coarse screening study [Whitehead et al., 1991], which only grouped core damage sequences into categories and did not provide frequency estimates, the POS 6 results were conditional on the occurrence of the PDS, and therefore estimates of risk were not calculated. The abridged analysis of POS 6 was very limited in scope and detail; it did, however, provide insights into the impact of the plant configuration on the progression of the accident and the resulting consequences. For the sake of completeness, this study is presented in Appendix F. Note that the scope, level of detail, methods and assumptions used in the analysis of POS 6 are very different from those used in the analysis of POS 5.

\subsubsection{Major Assumptions}

During the course of the study it was necessary to make a number of assumptions to keep the analysis manageable. Many of these assumptions, if changed, could have a significant impact on the results. While many assumptions are itemized in the individual analysis chapters, a list of the more significant assumptions is presented below. The major assumptions used in the Level 1 analysis are listed in Volume 2 of this report.

- During POS 5 the plant is in the cold shutdown mode of operation with the vessel head attached. The variability of plant configuration and system availability during POS 5 can be adequately captured by dividing the cold shutdown mode of operation into 3 segments or time windows.

- $\quad$ Each time window has a characteristic decay heat load and radionuclide inventory.

- Accidents assigned to a particular time window are assumed to initiate at the start of the time window.

- Core damage is defined in the Level 1 analysis as the start of fuel heatup. For consistency this same definition was used in the Level 2/3 analysis.

- $\quad$ The mission time for the Level 1 analysis was 24 hours. Thus sequences for which core damage did not occur within 24 hours from the start of the accident were dropped from the analysis.

- To restore offsite power to the plant if power is lost prior to or during the accident, dc power from the station batteries is required. Therefore, if the station batteries deplete prior to the restoration of offsite power, it is assumed that offsite power cannot be restored during the accident.

- At the start of the accident the reactor pressure vessel head vent is open. This vent can be closed prior to core damage if ac power is available.

- The containment and drywell are both open at the start of the accident. The containment can only be closed if offsite ac power is available; containment 
closure must be completed prior to the onset of core damage. The drywell is assumed to remain open throughout the accident.

-

If the containment fails, it is assumed to fail above the auxiliary building roof, thereby allowing radioactive releases to bypass the auxiliary building and enter the environment directly. The enclosure building that surrounds the portion of the containment above the auxiliary building roof is estimated to offer essentially no attenuation to the release. This assumption is consistent with the assumption used in the Grand Gulf plant analysis performed as part of the NUREG-1150 study, which is described in detail in Volume 6 of NUREG/CR-4551 [Brown et al., 1990].

- If the containment is closed prior to the onset of core damage and then subsequently vented after the onset of core damage, it is assumed that the vent stays open throughout the accident. Although the emergency operating procedures (EOPs) direct the operators to close the containment when its pressure drops below a certain pressure, without containment heat removal the containment will have to be vented again later in the accident when the pressure again rises above the vent pressure. No attempt was made in this analysis to model the opening and closing of the containment vent. Furthermore, because the availability of the containment purge system during POS 5 is not being modeled (and is not required by the technical specification), it is assumed that the containment will be vented directly to the environment and will not pass through the containment purge system with its associated filters and charcoal beds.

- If the containment equipment hatch and/or personnel locks are open, the airborne radioactive material will enter the auxiliary building prior to being released to the environment. The auxiliary building is assumed to fail on a 5 psi overpressure. Neither the standby gas treatment system nor the ventilation system are modeled. Thus it is assumed that no engineered features are available to attenuate the release in the auxiliary building, and the only attenuation that the release will experience in the building is that caused by natural processes (e.g., natural deposition).

- The Grand Gulf Emergency Operating Procedures are applicable after the onset of core damage, and the operators will continue to follow them.
- No operator actions were modeled that would require operators to enter the containment or auxiliary building following onset of core damage.

- Recovery of coolant injection after the onset of core damage is only considered if (1) injection systems were not available prior to core damage but became available following core damage, or (2) conditions occur following the onset of core damage that would cause the operators to use a system previously available but not used prior to core damage.

\subsection{Strengths and Limitations}

As with any study, this study has it strengths and limitations; awareness of both allows this study to be placed in its proper context with its results used appropriately. The strengths are listed first, followed by the limitations.

The strengths of this study include:

- The analysis is a Level 3 PRA that accounts for the progression of the accident following the onset of core damage, the release of radioactive material from the core and its transport through the primary system and the containment, and the transport of the radioactive material in the environment and the resulting health effects. The PRA techniques allow the many possible accident types to be delineated and systematically evaluated.

- The PRA techniques used in this study are based on the well proven methodology developed in the NUREG-1150 program; the NUREG-1150 methodology was extensively peer reviewed prior to its publication in 1990.

- Risk results are calculated.

- The analysis includes a detailed coupling with the accident frequency analysis via the plant damage state analysis.

- An estimate of the uncertainty in risk that results from the uncertainties associated with input parameters to the accident progression analysis and source term analysis is calculated and displayed.

- The study includes a limited assessment of onsite consequences. Doses and dose rates within the 
site boundary that result from a radioactive release are estimated.

- $\quad$ A Human Reliability Analysis (HRA) was performed to quantify the human error probabilities (HEPs) associated with key operator actions during the progression of the accident following the onset of core damage.

- $\quad$ MELCOR calculations were used to support the development and quantification of accident frequency, accident progression, and source term analyses. Wherever possible, consistent calculations were used to quantify the Level 1 and Level $2 / 3$ models.

The limitations of this study include:

- As with any study, the results and conclusions are only as strong as the information base available at the time of the study. There are several areas in the PRA where the information base for accidents initiated during shutdown is limited because in the past the focus has been on accidents initiated while the plant is at full power. For example, much of the data used in this study to model the release and transport of radioactive material during the accident are based on the information base developed for accidents initiated while the plant is at full power.

- These results cannot be considered generic and applicable to a population of plants because only a single plant was analyzed. The plant and system models used in this study are based on the Grand Gulf plant as it functions in a selected mode of operation. Thus, while some insights may be applicable to other plants, in general the results from this study should not be arbitrarily applied to other plants or conditions. The model used to develop the progression of the accidents after the onset of core damage is, in part, based on the Grand Gulf Emergency Operating Procedures and other procedures and practices at the plant. Changes in these procedures and practices can certainly affect the progression of the accident and the ultimate risk of the POS. Similarly, since the offsite consequences are sensitive to the site characteristics and surrounding region (e.g., weather, population, land usage), for a given release of radioactive material, the consequences can be expected to vary from one site to the next.
- Only a single POS was analyzed, and therefore the risk of the entire low-power and shutdown regime of operation was not assessed.

- $\quad$ Although progression of the accident following core damage is assumed to be adequately modeled for the intended purposes, the accident progression model is not as detailed as the models developed in the NUREG-1150 study or the PRUEP study.

- Similar to the NUREG-1150 plant studies, the uncertainties in the values for input parameters to the consequence models were not characterized. The only uncertainty included in the consequence assessment was the stochastic variability in the weather at the time of the accident; this uncertainty was only accounted for in the offsite consequence assessment.

Formal expert elicitation techniques were not used to quantify the uncertainty in input parameters. Distributions for important parameters were either developed by the project staff or obtained from existing PRAs (primarily NUREG-1150).

\section{Vol. 6, Part 1}


Introduction

\subsection{References}

[USNRC, 1975]

[USNRC, 1990]

[Payne, 1992]

[Brown et al., 1992]

[Summers et al., 1991]

[Whitehead et al., 1991]

[Brown et al., 1990]
U.S. Nuclear Regulatory Commission, "Reactor Safety Study - An Assessment of Accident Risks in U.S. Commercial Nuclear Power Plants," WASH-1400 (NUREG75/014), October 1975.

U.S. Nuclear Regulatory Commission, "Severe Accident Risks: An Assessment for Five U.S. Nuclear Power Plants," NUREG-1 150, Vols. 1-3, December 1990-January 1991.

A. C. Payne, Jr., "Analysis of the LaSalle Unit 2 Nuclear Power Plant: Risk Methods Integration and Evaluation Program (RMIEP)," NUREG/CR-4832, SAND92-0537, Vol. 1, Sandia National Laboratories, July 1992.

T. D. Brown et al. "Integrated Risk Assessment for the LaSalle Unit 2 Nuclear Power Plant," NUREG/CR-5305, SAND90-2765, Vols. 1-2, Sandia National Laboratories, August 1992.

R. M. Summers et al., "MELCOR 1.8.0: A Computer Code for Nuclear Reactor Severe Accident Source Term and Risk Assessment Analyses," NUREG/CR-5531, SAND900364, Sandia National Laboratories, January 1991.

D. W. Whitehead et al., "BWR Low Power and Shutdown Accident Frequencies Project, Phase 1 - Coarse Screening Analysis", Vol. 1, Draft Letter Report, Sandia National Laboratories and Science and Engineering Associates, Inc. November 23, 1991 update, Copy available in the NRC Public Document Room.

T. D. Brown et al., "Evaluation of Severe Accident Risks: Grand Gulf Unit 1," NUREG/CR-4551, SAND86-1309, Vol. 6, Rev. 1, Sandia National Laboratories, December 1990. 


\section{Methodology}

\subsection{Background}

The probabilistic risk assessment (PRA) performed in this study utilizes methods developed and applied in the NUREG-1150 study [USNRC, 1990] and the PRUEP study [Brown et al., 1992]. A primary objective of the NUREG1150 study was to provide a current assessment of the risks of five nuclear power plants of different designs and to provide quantitative estimates of the risk uncertainties. To achieve this objective, an analytical PRA framework was developed that allowed accidents to be modeled at a level of detail consistent with the state of knowledge and that also allowed uncertainties in important physical and chemical phenomena to be characterized. Areas where significant state-of-the-art advancements were made included the consistent and comprehensive treatment of uncertainty in all areas of the PRA, the development of detailed accident progression and source term models, and the development and application of expert judgment techniques to assess the likelihood and nature of rare and complex phenomena associated with severe core damage accidents. NUREG-1150 was peer reviewed, and when it was published in 1990 it represented the state-of-the-art in Level 3 PRA. Although improvements have been made to the PRA models, and additional data on severe accidents have been acquired since publication of NUREG-1150, the general methodology was judged to represent the current state-of-the-art in Level 3 PRA. Therefore, its framework was used, to the extent possible, in this study.

The objectives of the NUREG-1150 study led to an analysis approach based on the following ideas:

- general and relatively fast-running models for the individual analysis components,

- well defined interfaces between the individual analysis components,

- use of Monte Carlo techniques in conjunction with an efficient sampling procedure to propagate uncertainties, and

- use of expert panels to develop defensible distributions for important phenomenological issues (as explained later, this aspect of the NUREG-1150 approach was not used in the present study).

An overview of the analysis approach is presented in Section 3.2. A simplified version to the NUREG-1150 approach was applied to study the risk of plant operating state (POS) 5. The significant differences between the NUREG-1150 methods and the methods employed in this study are discussed where appropriate.

\subsection{Overview of Analysis Approach}

Because the NUREG-1150 methods are described in detail elsewhere [Ericson et al., 1990; Gorham et al., 1994], this section will only provide a brief overview of these methods. Much of this discussion has been extracted from Volume 1 of NUREG/CR-4551 and a related journal article [Breeding et al., 1992].

The NUREG-1 150 plant studies are integrated PRAs that can be characterized as consisting of six analysis components. The first component is the accident frequency analysis, which determines the likelihood and nature of accidents that result in a loss of cooling to the fuel and that subsequently lead to fuel damage. This state of the core is referred to as core damage. The second component is the accident progression analysis, which determines the progression of the accident following the onset of fuel damage and addresses the response of engineered barriers, such as the reactor pressure vessel and the containment, to loads that occur during the accident. The third component is the radioactive material release and transport analysis (also called the source term analysis), which determines the amount of radioactive material released during the accident and its subsequent transport and deposition in the engineered environment (e.g., containment). The fourth component is the consequence analysis, which determines the transport of radioactive material outside the plant and estimates the health effects and costs associated with the release of this radioactive material. The fifth component is the risk analysis, which assembles the results of the preceding analysis components into an overall expression of risk. The sixth and final component is the uncertainty analysis, which estimates the uncertainty in the risk results caused by uncertainty in the characterization of important physical and chemical phenomena. Measures of uncertainty in risk are obtained by repeating many times the calculations just indicated, with different values for important parameters selected randomly by a form of Monte Carlo sampling. This procedure provides a distribution of risk estimates that is a measure of the uncertainty in risk.

The representations of risk used in this report are defined in the next section. Following the definition of risk is a description of the individual analysis components and a description of the treatment of uncertainty in the PRA. 


\subsubsection{Representation of Risk}

Two representations of risk are used in this report. The first definition is the ordered set of triples [Kaplan and Garrick, 1981] of the form:

$$
R=\left\{\left(s_{i}, f_{i}, o_{i}\right), i=1, \ldots . n S\right\}
$$

where

$$
\begin{aligned}
& s_{i}=\text { a scenario (i.e., accident) that leads to an outcome } \\
& \text { (i.e., consequence) of interest, } \\
& f_{i}=\text { frequency (units: } \mathrm{yr}^{-1} \text { ) for scenario } i, \\
& o_{i}=\text { outcome associated with scenario } i \\
& n S=\text { number of scenarios under consideration. }
\end{aligned}
$$$$
\text { and }
$$

The objective of a probabilistic risk assessment is the determination of the triples that constitute the set $R$. Combined, the accident frequency and accident progression analyses define the accident scenarios, $\mathrm{s}_{\mathrm{i}}$, and the frequency of each scenario, $f_{i}$. Similarly, the source term analysis and the consequence analysis combine to determine the outcome or consequence of the accident scenario, $o_{i}$. When complicated technical systems such as nuclear power plants are analyzed, the number of scenarios can be quite large (i.e., thousands); at this point it is neither practical to present the risk results in this form nor reasonable to expect one to draw conclusions or make decisions based on this representation. In such cases it is convenient to collapse the set of triples into a value for aggregate risk, which is defined as

$$
r=\sum_{i=1}^{n S} f_{i} o_{i}
$$

Although aggregate risk is appealing because it is a summary measure of the order set of triples, it is important to recognize that information is lost when the order set of triples is converted into an expression for aggregate risk. To assess the importance of various contributors to aggregate risk, it will often be necessary to examine the constituents of the risk triple. Thus both representations of risk are employed in a PRA.

The term risk is often used loosely and may refer to any number of possible products of the PRA, for example, the core damage frequency or the probability of containment failure. To avoid ambiguity, in this volume the term risk - will refer to aggregate risk (i.e., defined in equation 3.2), where the outcome of interest, $o_{i}$, represents the offsite consequences that result from the release of radioactive material from the plant. Because various measures of offsite consequences exist (e.g., early fatalities, total latent cancer fatalities, population dose within 50 miles of the plant, etc.), there will be an equal number of risk measures. The various consequence measures used in this study are discussed in Section 3.2.2.4.

\subsubsection{Description of Analysis Components}

\subsubsection{Accident Frequency Analysis}

The accident frequency analysis uses event tree and fault tree techniques (1) to identify the combinations of events that can lead to core damage and (2) to estimate their frequencies of occurrence. On a system level, these combinations of events are labeled sequences. On an individual fault level (e.g., failures of specific pumps and valves), these combinations of events are called cut sets. The cut sets of interest are those that contain no more faults than those required to cause core damage. These cut sets are denoted minimal cut sets. The cut sets are identified by means of fault trees, and the minimal cut sets are sorted into accident sequences by means of event trees. The frequency of an accident sequence is obtained by combining the frequency of the initiating event with the sum of the probabilities of all the minimal cut sets in the sequence. The accident frequency analysis that was performed as part of this program is discussed in detail in Volumes 2 through 5 of this report.

Typically, there are too many sequences to individually analyze in the subsequent analyses. Furthermore, within the resolution of the PRA, many of these sequences will result in similar progressions following core damage; therefore, it would be redundant to individually analyze each sequence. Instead, accident sequences that provide a similar set of initial and boundary conditions for the subsequent accident progression analysis are grouped into a plant damage state (PDS). The PDSs form the interface between the accident frequency analysis and the accident progression analysis. The number of PDSs that are defined for a given analysis depends on the diversity of accident sequences and the resolution desired in the subsequent analyses. In some cases the definition of the PDS does not correspond exactly to the accident sequence definitions, so it may be necessary to place some minimal cut sets from a sequence in one PDS and the remaining minimal cut sets in 
another PDS. The frequency of a PDS is the sum of the frequencies of the minimal cut sets that it contains.

\subsubsection{Accident Progression Analysis}

The purpose of the accident progression analysis is to represent the progression of the accident from the time of core damage until the completion of the release of radioactive material from the containment. This analysis models the response of the radioactive barriers (e.g., reactor pressure vessel and containment) to the stresses placed on them during the various phases of the accident. The accident progression analysis uses an event tree called an accident progression event tree (APET) to determine the possible ways that an accident might evolve from each PDS. Each different progression is represented by a different path through the APET. The definition of each PDS provides enough information to define the initial conditions for the APET. Past observations, experimental data, and mechanistic code calculations are used in the development of the model for the accident progression that is embodied in the APET. These same sources of information were utilized when determining the probabilities at the branch points in the APET.

The APET developed for POS 5 is similar in concept and structure to the APETs developed in the NUREG-1150 study; however, it is not as detailed. When compared to the NUREG-1150 APETs, the POS 5 APET included fewer questions (i.e., top events), addressed issues in less detail (e.g., hydrogen combustion phenomena), and did not use formal expert judgment procedures to quantify the APET. Some of this simplification was possible because the configuration of the plant during POS 5 precluded or minimized the need to address certain issues (e.g., after the drywell equipment hatch has been removed, it is no longer necessary to determine the structural response of the drywell to loads that occur during the accident). In other cases, it was necessary to make simplifying assumptions to keep the study manageable. Although the analysis of POS 5 did not exploit formal expert judgment techniques to quantify issues (e.g., branch point probabilities), it made extensive use of distributions developed in NUREG-1150 for the accident progression analysis. Many of these NUREG-1150 distributions were generated using formal expert judgment techniques.

In the NUREG-1150 plant studies, the APET was evaluated for each PDS individually; therefore, the results from these individual analyses were conditional on the occurrence of the PDS that was evaluated. The analysis of POS 5 adopted the approach used in the PRUEP study [Brown et al., 1992]: a single APET was developed for all the various PDSs, and it was only evaluated once. In this case the APET analysis is conditional on the occurrence of core damage, not on any particular PDS. This approach was taken because issues that affected many PDSs could be easily treated in a consistent manner, the truncation of individual accident progression paths could be treated in a consistent manner across all PDSs, and the management of output files was simplified.

The APET was quantified using information from: (1) the Level 1 analysis (see Volumes 2 through 5 of this report), (2) a human reliability analysis performed for this study to determine human error probabilities associated with operator actions during core damage, (3) MELCOR calculations performed specifically for this study (see Volume 6, Part 2 of this report), and (4) data from the NUREG-1150 Grand Gulf plant study [Brown et al., 1990]. For those events that were judged by the project staff to be important to risk, and for which existed a large amount of uncertainty about the value to assign to the branch probability, a distribution of probabilities was assigned to the branch. No rigorous analytical process was used to select these events. Rather the events were selected based on prior experience and results from existing PRAs. For the remaining events that were either judged to be less important or for which the branch probability was not believed to be uncertain, a single value was used.

The interface between the accident progression and source term analysis is defined through accident progression bins (APB). Because of the large number of questions in a typical APET, and because many of these questions have more than two branches, there are far too many paths through the tree to permit each path to be considered in the subsequent source term and consequence analyses. Furthermore, many of the progressions (i.e., event paths) developed with the APET will lead to similar source terms (i.e., within the resolution of the analysis). Therefore, to avoid performing redundant calculations, similar accident progression paths are collected into groups called APBs. Each APB defines a set of unique initial and boundary conditions for the source term analysis.

The codes EVNTRE [Griesmeyer et al., 1989] and PSTEVNT [Higgins, 1989] were used to evaluate the APET and process the results. The products of the accident progression analysis are the accident progression bin definitions, their associated probabilities conditional on core damage, and an expression of the uncertainty in these probabilities. 


\subsubsection{Source Term Analysis}

The source term analysis models the release and transport of radioactive material from the fuel and core debris to the environment; it is performed so that the radiological severity of the accident can be assessed. The product of this analysis is a collection of parameters, referred to as the source term, that characterize the type and amount of radioactive material released from the containment, the start and duration of the release, and the location of the release. In this context, containment is generalized to include the region where engineered barriers are available to attenuate a release before it enters the environment (e.g., auxiliary building that surrounds the containment building); the environment is the region beyond the containment. The inputs to the source term analysis are the APBs defined in the accident progression analysis. The APBs describe the configuration of the plant, the status of systems that can be used to mitigate the release, and the occurrence of phenomena that can impact the source term. A source term is calculated for each APB. Although the source term analysis follows the accident progression analysis, the two are actually intimately coupled, as the release and transport of the radioactive material occurs during the accident progression and is affected by phenomena that occur during this phase of the accident.

Source terms for the various APBs were estimated using the parametric expressions developed in the NUREG-1 150 study. These parametric expressions are implemented in a set of codes collectively known as XSOR [Jow et al., 1993; Cybulskis et al., 1989]. These codes are similar in approach; however, a different code is developed for each plant analysis in order to reflect the features unique to each plant. The parametric code GGSOR [Jow et al., 1993; Brown et al., 1990] was modified to reflect the different plant configuration and the different possible release paths during shutdown. In this approach a simple parametric model, based on results from mechanistic code calculations and other sources of information, is created and used to calculate a source term for each APB. Parametric codes use a combination of parameters, which represent the physics of the accident at a very general level, to estimate the release to the environment. For example, a parameter can be used to represent the fraction of a particular radionuclide in the fuel that is released to the vessel. Another parameter can then be used to represent the fraction of the radionuclide in the vessel that is released to the containment. The parametric approach is not meant to be a substitute for detailed calculations. Rather it is a framework for integrating the results of these codes together with experimental results and expert judgment.
The parametric approach was selected for the following reasons:

- The code is relatively fast running and therefore can be used to estimate source terms for each APB.

- The approach is amenable to uncertainty analysis.

- The NUREG-1150 XSOR code for Grand Gulf already exists; thus the only modifications needed are those required to account for the plant configuration during shutdown.

- To a large extent, the XSOR code data base already exists (some modifications/additions were made to the data base to account for unique phenomena and the plant configuration during shutdown).

Although this approach also has several disadvantages, which include very little physics explicitly included in the model and a very coarse manner of characterizing the timing of the release, its advantages make it a good choice when the uncertainty in the source term is to be characterized.

The XSOR model was quantified using the following sources of information:

- Wherever appropriate, distributions developed during the NUREG-1150 study were used [Harper et al., 1992]. MELCOR calculations were used to identify areas where full-power accidents are similar enough to shutdown accidents that the use of full- power data was reasonable.

- MELCOR calculations were used to determine timing information (e.g., release times and release durations) and the energy associated with the release.

The uncertainties in important input parameters were characterized and propagated through the XSOR model to develop an expression for the uncertainty in the resulting source term. Although no new source term issues were quantified with formal expert judgment techniques, an internal "Source Term Advisory Group" was formed to provide guidance on the use of existing methods and data, and to review the source term issues being treated and identify any new issues that may be important to shutdown accidents. This guidance was directed at an earlier study (see Appendix F) and was factored into this analysis. 
Because the parametric approach results in a source term for every APB, it is impractical to estimate consequences for each source term individually. Instead, the source terms must be collapsed into a manageable number of groups. Source terms with a similar potential to cause early and chronic health effects are collected into a source term group (STG); a single source term is then defined (e.g., the mean of the group of source terms) for each STG. The STGs were created using the PARTITION algorithm that was first developed during the NUREG-1150 study [Iman et al., 1990] and then modified in the PRUEP program [Brown et al., 1992]. Consequences for the STG are estimated using this representative source term. The source term groups form the interface between the source term analysis and the consequence analysis.

The product of this analysis is the mean source term associated with each source term group.

\subsubsection{Consequence Analysis}

Whereas the source term analysis is the analysis of the release and transport of radioactive material from the fuel and core debris to the environment, the consequence analysis is the analysis of the transport of this material through the environment, the health effects, and the costs that result from the release of this radioactive material. Consequences that would accompany a core damage accident are typically divided into two categories: offsite consequences and onsite consequences. The offsite consequence analysis predicts the health effects to the public and the economic impacts associated with the dispersal of radioactive materials into the environment beyond the site boundary. The onsite consequence analysis is confined to the region within the site boundary. As such, onsite consequences include health effects to personnel working at the plant at the time of the accident as well as the cost of replacement power, capital loss, and cleanup of the reactor facility. The consequence assessment for most commercial reactor PRAs is focused on the assessment of offsite consequences because the regulations promulgated and enforced by the NRC emphasize protection of the public. Thus most of the Level 3 PRAs that are performed, including the NUREG-1150 PRAs, include only an assessment of offsite consequences. Because of this emphasis on offsite consequences, methods to perform offsite consequence assessments are available, have been subjected to considerable review, and have been applied in many PRAs. This is not the case for onsite consequence assessments. Thus the consequence analysis for this study includes both a traditional offsite consequence analysis and a limited scope onsite consequence analysis.

\section{Offsite Consequences}

The offsite consequences to the general public were estimated using the MACCS code [Chanin et al., 1990; Jow et al., 1990; Rollstin et al., 1990]. This code models the transport and dispersion of plumes of radioactive material released from the plant. As the plumes travel through the atmosphere, material is deposited on the ground. Various pathways through which the general population can be exposed are considered. Emergency response and protective action guides are also considered as means for mitigating the extent of the public exposure. For this study the inputs to MACCS, aside from the source terms and the core inventory, were the same as those used in the NUREG1150 Grand Gulf study [Brown et al., 1990; Sprung et al., 1990]. The same offsite consequence measures that were calculated and reported in NUREG-1150, which are listed in Table 3-1, were also calculated in this study.

\section{Onsite Consequences}

In this limited onsite analysis, health effects are not calculated. Instead, doses and dose rates are estimated for a range of distances out to the site boundary using building wake effect correlations (also referred to as parking lot doses). For comparative purposes, two sets of correlations were used. The first correlation was developed by Ramsdell [1990] whereas the second set uses a combination of models developed by Wilson and the NRC [Wilson, 1984; USNRC, 1982]. The calculations were performed for two sets of weather scenarios: one is stable and the other is unstable. For simplicity, the directional dependence of the weather is ignored. The dose rate is based only on the immersion exposure path whereas the dose is based on the inhalation exposure path as well as the immersion exposure path. The total dose is a 50 -year committed dose. Two exposure times are assumed: the passage of the entire plume, and a 15 minute exposure. The products of this analysis include, for each source term group, the dose rate for each plume segment, the total dose received by a receptor that is exposed to the entire plume, and the total dose received by a receptor that is only exposed to the first 15 minutes of the plume.

\subsubsection{Risk Analysis}

Risk to the general public was calculated using the aggregate risk definition. In this calculation, the core damage frequency, the conditional probabilities of the source term groups, and the offsite consequences associated with each source term group are combined. The fractional contribution to aggregate risk from selected PDSs and 
Table 3.1 Offsite consequence measures calculated in NUREG-1150

\begin{tabular}{||l|l||}
\hline \hline Consequence Measure & \multicolumn{1}{|c|}{ Description } \\
\hline Early fatalities & $\begin{array}{l}\text { Number of fatalities occurring within 1 year of the accident because of } \\
\text { early exposure (i.e., exposure incurred within seven days of the accident). }\end{array}$ \\
\hline $\begin{array}{l}\text { Total latent cancer } \\
\text { fatalities }\end{array}$ & $\begin{array}{l}\text { Number of latent cancer fatalities caused by both early and chronic } \\
\text { exposure (i.e., chronic exposure is that incurred more than seven days after } \\
\text { the accident). }\end{array}$ \\
\hline $\begin{array}{l}\text { Population dose within 50 } \\
\text { miles }\end{array}$ & $\begin{array}{l}\text { Population dose, expressed in effective dose equivalents for whole body } \\
\text { exposure (person-rem), caused by early and chronic exposure pathways } \\
\text { within 50 miles of the reactor. Because of the nature of the chronic } \\
\text { pathways models, the actual exposure from food and water consumption } \\
\text { may take place beyond 50 miles (e.g., food and water originating within } 50 \\
\text { miles of the plant may be consumed by people located beyond 50 miles) }\end{array}$ \\
\hline $\begin{array}{l}\text { Population dose within } \\
\text { entire region }\end{array}$ & $\begin{array}{l}\text { Population dose, expressed in effective dose equivalents for whole body } \\
\text { exposure (person-rem), because of early and chronic exposure pathways } \\
\text { within the surrounding region. }\end{array}$ \\
\hline $\begin{array}{l}\text { Individual early fatality } \\
\text { probability within one mile }\end{array}$ & $\begin{array}{l}\text { Probability of dying within one year for an individual within one mile of } \\
\text { the site exclusion boundary (i.e., ef/pop, where ef is the number of early } \\
\text { fatalities within one mile of the exclusion boundary, and pop is the } \\
\text { population within one mile of the exclusion boundary). }\end{array}$ \\
\hline 10 miles & $\begin{array}{l}\text { Probability of dying from cancer for an individual within ten miles of the } \\
\text { plant (i.e., cf/pop, where cf is the number of cancer fatalities caused by } \\
\text { direct exposure in the resident population within ten miles of the plant, and } \\
\text { pop is the population size within ten miles of the plant). The calculation } \\
\text { inhoes not include ingestion but does include integrated groundshine and } \\
\text { inhalation exposure. }\end{array}$ \\
\hline \hline
\end{tabular}

APBs was also determined. Because of the scoping nature of the onsite consequence analysis, an analogous calculation was not performed to estimate onsite risk. The products of this analysis include the following risk measures: early fatality risk, total latent cancer risk, population dose within 50 miles, population dose within 1000 miles, individual early fatality risk within 1 mile, and individual latent cancer risk within 10 miles. The uncertainty in each of these measures was also characterized.

\subsubsection{Treatment of Uncertainty}

An important and distinguishing feature of the NUREG1150 plant study was its consistent and comprehensive treatment of the uncertainties in the PRA and its quantitative estimates of the uncertainties in aggregate risk. The types of uncertainties addressed in the PRA and the propagation of these uncertainties through the constituent analysis are discussed in the next two subsections. 


\subsubsection{Types of Uncertainty}

The NRC Probabilistic Risk Assessment (PRA) Working Group [USNRC, 1994] has defined two general types of uncertainties that are present in PRA calculations. As described in NUREG-1489, these two types include (1) stochastic uncertainty caused by the inherent variability in some measurable physical quantity and (2) state-ofknowledge uncertainties that result from a lack of complete information about systems, phenomena, and processes. The latter can be further subdivided into parameter, model, and completeness uncertainties. Parameter uncertainty results from the lack of knowledge about the correct inputs to models being used in the analysis. Model uncertainty occurs because perfect models cannot be constructed. Completeness uncertainty refers to uncertainty that all significant phenomena and relationships have been considered (e.g., whether all accident scenarios that could significantly affect the determination of risk have been identified). NUREG-1489 provides a more detailed discussion of these types of uncertainties.

In this study, as in the NUREG-1150 study and consistent with the definitions provided in NUREG-1489, the project staff chose to model uncertainty as consisting of two parts: (1) stochastic uncertainty and (2) state-of-knowledge uncertainty. Because this study uses simplified models and treats issues at a general level, the distinction between stochastic processes and state-of-knowledge processes is not always clear.

Both types of uncertainties exist throughout the PRA. In the accident frequency and accident progression analyses, stochastic uncertainty is expressed in fault trees and event trees. The trees account for alternative outcomes that are expected to vary from one accident to the next in a random manner. Although state-of-knowledge uncertainty contains several components (parameter, model, and completeness), only the uncertainty in the input parameters were explicitly considered in this PRA. For example, in the accident progression analysis, state-of-knowledge uncertainty is represented by the values assigned to the inputs (e.g., branch probabilities) to the trees. Similar to NUREG-1150, the source term analysis performed for this study included the state-of-knowledge uncertainty associated with the uncertainty in the values for the parameters in the parametric code. State-of-knowledge uncertainties were not addressed in the consequence analysis. Only the stochastic uncertainty caused by weather variability was explicitly represented. The calculation of aggregate risk combines the results from all the individual accident scenarios and, as such, eliminates the display of stochastic uncertainty. In this study, the distribution of aggregate risk values that is calculated from the PRA represents the stateof-knowledge uncertainty that arises from the uncertainty in the input values to the PRA models.

\subsubsection{Propagation of Uncertainties}

To evaluate the state-of-knowledge uncertainties, the parameters that represent the events and phenomena thought to be most important to the uncertainty in risk were represented by distributions rather than fixed values or point estimates. The issues selected for such treatment were chosen by the project staff. No rigorous analytical process was used to select these issues. Rather the issues were selected based on prior experience, results from existing PRAs, uncertainty in the issue, anticipated contribution to uncertainty in risk, and interest within the reactor safety community. For many of these issues, distributions used in the NUREG-1150 study were applied to this study. If an applicable distribution from an existing study did not exist, the project staff developed the appropriate distribution. The use of formal expert elicitation techniques (i.e., the use of panels of experts from a variety of organizations and companies) was not used in this study.

The propagation of these uncertainties in the PRA was accomplished by using a modified form of Monte Carlo simulation known as Latin hypercube sampling (LHS). In simple terms, the uncertainty was addressed by performing the accident frequency, accident progression, and source term analysis many times (e.g., 100) with different sets of input values selected with LHS [Iman et al., 1984]; a value for aggregate risk was calculated for each set of inputs, and the collection of risk values resulted in a distribution of risk. The LHS form of Monte Carlo simulation was selected because:

- it creates a mapping from analysis input to analysis results,

- it allows consideration of essentially any variable that can be supplied to a model as input or generated as an output,

- $\quad$ it will operate in the presence of large uncertainties and discontinuities,

- $\quad$ it is possible to incorporate correlations between variables, and

- $\quad$ it is easy to implement. 


\subsection{References}

[USNRC, 1990]

[Brown et al., 1992]

[Ericson et al., 1990]

[Gorham et al., 1993]

[Breeding et al., 1992]

[Kaplan and Garrick, 1981]

[Griesmeyer et al., 1989]

[Higgins, 1989]

[Summer et al., 1991]

[Brown et al., 1990]

[Jow et al., 1993]

[Cybulskis et al., 1989]

[Harper et al., 1992]
U.S. Nuclear Regulatory Commission, "Severe Accident Risks: An Assessment for Five U. S. Nuclear Power Plants," NUREG-1150, Vols. 1-3, December 1990-January 1991.

T. D. Brown et al., "Integrated Risk Assessment for the LaSalle Unit 2 Nuclear Power Plant," NUREG/CR-5305, SAND90-2765, Vols. 1-2, Sandia National Laboratories, August 1992.

D. M. Ericson et al., "Analysis of Core Damage Frequency: Internal Events Methodology," NUREG/CR-4550, SAND86-2084, Vol. 1, Rev. 1, Sandia National Laboratories, January 1990.

E. D. Gorham et al., "Evaluation of Severe Accident Risks: Methodology for the Containment Source Term, Consequence, and Risk Integration Analyses," NUREG/CR-4551, SAND86-1309, Vol. 1, Rev. 1, Sandia National Laboratories, December 1993.

R. J. Breeding et al., "Summary Description of the Methods Used in the Probabilistic Risk Assessment for NUREG-1 150," Nuclear Engineering and Design, 135 (1992) 127.

S. Kaplan and B. J. Garrick, "On the Quantitative Definition of Risk, Risk Analysis, 1 (1981) 11-27.

J. M. Griesmeyer et al., "A Reference Manual for the Event Progression Analysis code (EVNTRE)," NUREG/CR-5174, SAND88-1607, Sandia National Laboratories, September 1989.

S. J. Higgins, "A User's Manual for the Postprocessing Program PSTEVNT," NUREG/CR-5380, SAND88-2988, Sandia National Laboratories, November 1989.

R. M. Summers et al., "MELCOR 1.8.0: A Computer Code for Nuclear Reactor Severe Accident Source Term and Risk Assessment Analyses," NUREG/CR-5531, SAND900364, Sandia National Laboratories, January 1991.

T. D. Brown et al., "Evaluation of Severe Accident Risks: Grand Gulf Unit 1," NUREG/CR-4551, SAND86-1309, Vol. 6, Rev. 1, Sandia National Laboratories, December 1990.

H.-N. Jow et al., "XSOR Code User's Manual," NUREG/CR-5360, SAND89-0943, Sandia National Laboratories, November 1993.

P. Cybulskis et al., "Assessment of the XSOR Codes," NUREG/CR-5346, BMI-2171, Battelle Columbus Division, November 1989.

F. T. Harper, "Evaluation of Severe Accident Risks: Quantification of Major Input Parameters: Experts' Determination of Source Term Issues," NUREG/CR-4551, Vol. 2, Rev. 1, Part 4, Sandia National Laboratories, June 1992. 
[Iman et al., 1990]

[Chanin et al., 1990]

[Jow et al., 1990]

[Rollstin et al., 1990]

[Sprung et al., 1990]

[Ramsdell, 1990]

[Wilson, 1994]

[USNRC, 1982]

[USNRC, 1994]

[Iman et al., 1984]
R. L. Iman et al., "PARTITION: A Program for Defining the Source Term/Consequence Interface in the NUREG-1150 Probabilistic Risk Assessments," NUREG/CR-5262, SAND88-2940, Sandia National Laboratories, May 1990.

D. I. Chanin et. al., "MELCOR Accident Analysis Consequence Code System," NUREG/CR-4691, SAND86-1562, Sandia National Laboratories, Vol 1, February 1990.

H-N Jow et. al., "MELCOR Accident Analysis Consequence Code System," NUREG/CR-4691, SAND86-1562, Sandia National Laboratories, Vol 2, February 1990.

J. A. Rollstin et. al., "MELCOR Accident Analysis Consequence Code System," NUREG/CR-4691, SAND86-1562, Sandia National Laboratories, Vol 3, February 1990.

J. L. Sprung et al., "Evaluation of Severe Accident Risks: Quantification of Major Input Parameters, MACCS Input," NUREG/CR-4551, SAND86-1309, Vol. 2, Rev. 1, Part 7, Sandia National Laboratories, December 1990.

J. V. Ramsdell, Jr., "Diffusion in Building Wakes for Ground-Level Releases," Atmospheric Environment, Vol. 24B, No. 3, 377-388, (1990).

Wilson in Atmospheric Science and Power Production, Ed. Randerson, D., DOE/TIC27601, 299, (1984).

U.S. Nuclear Regulatory Commission, Regulatory Guide 1.145, Revision 1, November 1982.

U.S. Nuclear Regulatory Commission, "A Review of NRC Staff Uses of Probabilistic Risk Assessment," NUREG-1489, March 1994.

R. L. Iman, "A FORTRAN 77 Program and User's Guide for the Generation of Latin Hypercube and Random Samples for Use with Computer Models," NUREG/CR-3624, SAND83-2365, Sandia National Laboratories, 1984. 
Methodology 


\section{Plant Description}

Section 4.1 provides a general description of the Grand Gulf primary system, containment, and the important systems that can be used to mitigate an accident. Section 4.2 describes the configuration of the plant as modeled in the Level $2 / 3$ analysis.

\subsection{General Description}

The Grand Gulf Nuclear Station, Unit 1 utilizes a Mark III containment design to house a BWR/ 6 boiling water reactor (BWR). The station is operated by Energy Operations Inc. Unit 1 was constructed by Bechtel Corporation and began commercial operation in July 1985. The plant is located on the east bank of the Mississippi river in southwestern Mississippi, about 6 miles northwest of Port Gibson, Mississippi. The nearest large city is Jackson, Mississippi, approximately 55 miles northeast of the plant.

Because of the importance of the following features to the progression of an accident following the onset of core damage, the subsections that follow will discuss in greater detail these features of the plant:

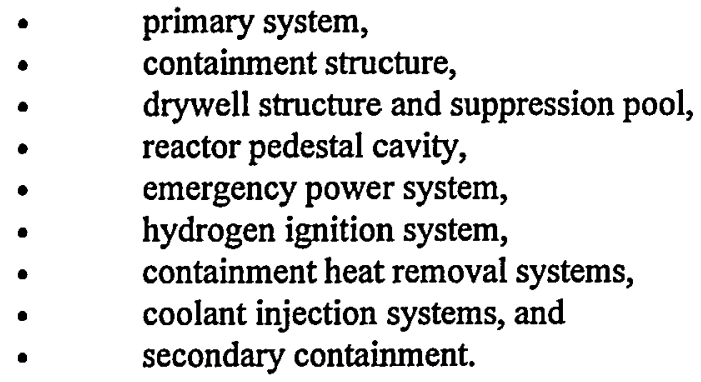

Much of the material provided in the following subsections was extracted from the Grand Gulf UFSAR [SERI, 1992] and from Volume 6 of NUREG/CR-4550 [Drouin et al., 1989].

\subsubsection{Primary System}

The nuclear reactor of Grand Gulf Unit 1 is a $3833 \mathrm{MWt}$ BWR-6 single-cycle forced-circulation BWR designed and supplied by the General Electric Company. In the Mark III design, the reactor pressure vessel (RPV) is founded on the reactor pedestal located in the drywell. The RPV contains the core, jet pumps, steam separators, and steam dryers. The vessel has an internal diameter of 20 feet, 11 inches and an internal height of 73 feet. It is fabricated of low alloy steel and is clad internally with stainless steel (except for the top head, nozzles, and nozzle weld zones, which are unclad). The reactor vessel design pressure and temperature are $1250 \mathrm{psig}$ and $575^{\circ} \mathrm{F}$, respectively. The nominal pressure and temperature in the steam dome are 1040 psia and $549{ }^{\circ} \mathrm{F}$, respectively. The reactor is cooled by water that enters the lower portion of the core and boils as it flows upward around the fuel rods. The steam leaving the core is dried by the steam separators and dryers located in the upper portion of the reactor vessel. The steam is then directed to the turbine through four main steam lines. Each steam line contains two isolation valves in series (i.e., main steam line isolation valves, MSIVs), one on each side of the containment barrier. Following reactor isolation, the steam in the vessel is directed to the suppression pool via a series of tailpipes. A safety relief valve (SRV) forms the boundary between the main steam line and the tailpipe. To help disperse the steam in the pool, the tailpipe is fitted with a quencher, which is located near the bottom of the suppression pool. Following closure of the MSIVs, 20 SRVs and the associated piping are available for pressure relief. Eight of these valves are connected to the automatic depressurization system (ADS), which is designed to rapidly depressurize the primary system to a pressure at which the low pressure injection systems can provide coolant to the core. The SRVs are located in the drywell, and drywell pressures of approximately $100 \mathrm{psi}$ will prevent the valves from opening.

The reactor core is arranged as an upright circular cylinder composed of essentially two components: fuel assemblies and control rods. The core contains 800 fuel assemblies. Each fuel assembly consists of a Zircaloy-4 fuel channel and the fuel rods (the number of fuel rods and water rods vary, depending on the fuel design). The fuel channel provides a fixed flow path for the boiling coolant, serves as a guiding surface for the control rods, and protects the fuel during handling operations. A fuel rod consists of slightly enriched $\mathrm{UO}_{2}$ pellets sealed in a Zircaloy- 2 cladding tube. The reactivity of the core is controlled by cruciform control rods dispersed throughout the lattice of fuel assemblies. The control rods, which consist of $B_{4} C$ in stainless steel tubes surrounded by a stainless steel sheath, enter the core from the bottom and are positioned by individual control rod drives. The core has an equivalent diameter of approximately 16 feet and an active fuel height of 12.5 feet.

The RPV includes a 2-inch vent line. One end of this line is attached to the top of the vessel head; the other end discharges into the sump located in the reactor cavity directly below the vessel. This line remains closed and is not used during normal operation; it is opened during cold shutdown. Although the vent line is small, the status of this 
line (i.e., opened or closed) can impact the time available to respond to a core damage accident, and if core damage occurs it can also impact the magnitude of the release of radioactive material to the environment.

\subsubsection{Containment Structure}

The Grand Gulf plant uses a Mark III containment. The general arrangement of the containment is displayed in Figure 4.1. The containment is a cylindrical reinforced concrete structure with a steel liner and a hemispherical dome. The containment encloses both the drywell and the suppression pool. During normal operation, the drywell and containment communicate through passive vents in the suppression pool. In addition to the passive vents, vacuum breakers between the containment and the drywell allow the containment atmosphere to be vented into the drywell if the drywell pressure drops below the containment pressure. An important feature of the Mark III containment is its large free volume $\left(1.4 \times 10^{6} \mathrm{ft}^{3}\right.$, not including drywell volume), which allows it to have a low design pressure (15 psig). The internal design temperature is $185^{\circ} \mathrm{F}$. The assessed mean failure pressure of the containment is 55 psig [Harper, 1994]. Because of its large volume, the Grand Gulf containment is not inerted. Hydrogen control is accomplished by means of the hydrogen ignition system (HIS). The HIS is designed to deliberately burn the hydrogen at low concentrations, so the accompanying containment pressurization is negligible. The ultimate heat sink is comprised of mechanical draft cooling tower structures.

Personnel can enter the containment through 3 penetrations: the equipment hatch, upper personnel lock, and lower personnel lock. The equipment hatch is a 19-foot-diameter steel pressure seating hatch. The center line of the equipment hatch penetration is located at an elevation of 172 feet, 3 inches The hatch is attached from inside the containment with 20 bolts. To maintain tightness along the mating surfaces, the hatch uses two compression seals (gasket concept) around its periphery. The hatch is stored inside the containment in a storage bin above the opening. Offsite ac power is required to move and position the hatch. Each personnel airlock consists of a cylindrical steel shell with steel bulkheads at each end and two steel doors in the bulkheads that open toward the reactor. Sealing of each door is accomplished by two continuous-inflatable seals that surround the door edge. When the door is closed, the seals inflate outwardly from the door and impinge against a smooth stainless steel sealing surface. The normal operating pressure of the airlock inflatable seals is 60 psig. The airlock doors are 6 feet, 8 inches high and 3 feet, 6 inches wide. The center-line elevation of the upper lock is 212 feet, 8 inches The center-line elevation of the lower lock is 124 feet, 8 inches, which is approximately 13 feet above the nominal suppression pool level.

If the containment pressure cannot be maintained below the primary containment pressure limit, the containment vent system (CVS) can be used to reduce the containment pressure. The vent path is a 20 -inch-diameter purge exhaust line, which is part of the containment ventilation and filtration system. This line includes four air-operated dampers, which are normally closed. The CVS discharges to the roof of the auxiliary building. Containment venting requires instrument air for opening the air-operated dampers. The dampers also require power from Divisions 1 and 2 of emergency ac power for operation of the solenoids. The emergency operating procedures require containment venting when the containment pressure exceeds 20 psig.

\subsubsection{Drywell Structure and Suppression Pool}

In the Mark III design, the drywell and suppression pool are completely surrounded by the containment structure. The drywell structure is a cylindrical reinforced concrete structure with a flat roof and a steel drywell head. The drywell contains the reactor vessel, the SRV valves, the control rod drive (CRD) housings and the recirculation pumps. The drywell has a free volume of 27,000 cubic feet $\left(2.7 \times 10^{5} \mathrm{ft}^{3}\right)$, a design pressure of $30 \mathrm{psid}$, and an internal design temperature of $330^{\circ} \mathrm{F}$. The assessed mean failure pressure of the drywell structure is 85 psid [Harper, 1994].

The drywell volume communicates with the containment volume through the vapor suppression pool. The suppression pool serves as a heat sink during accident conditions. Passive horizontal vents in the drywell wall allow steam and noncondensibles released in the drywell to pass into the suppression pool, where the steam is condensed and the noncondensibles are released into the containment atmosphere. The suppression pool consists of two regions. The first region is located in the containment (i.e., wetwell) and is bounded on one side by the containment wall and on the other side by the drywell wall. The second region is in the drywell and is bounded on one side by the drywell wall and on the other side by the weir wall. The passive horizontal vents in the drywell wall connect the two regions of the pool. There are a total of 135 vents (three rows of vents, each row has 45 vents); each vent has a nominal diameter of 2.33 feet. The suppression pool has a nominal volume of 136,000 cubic feet. 
Plant Description

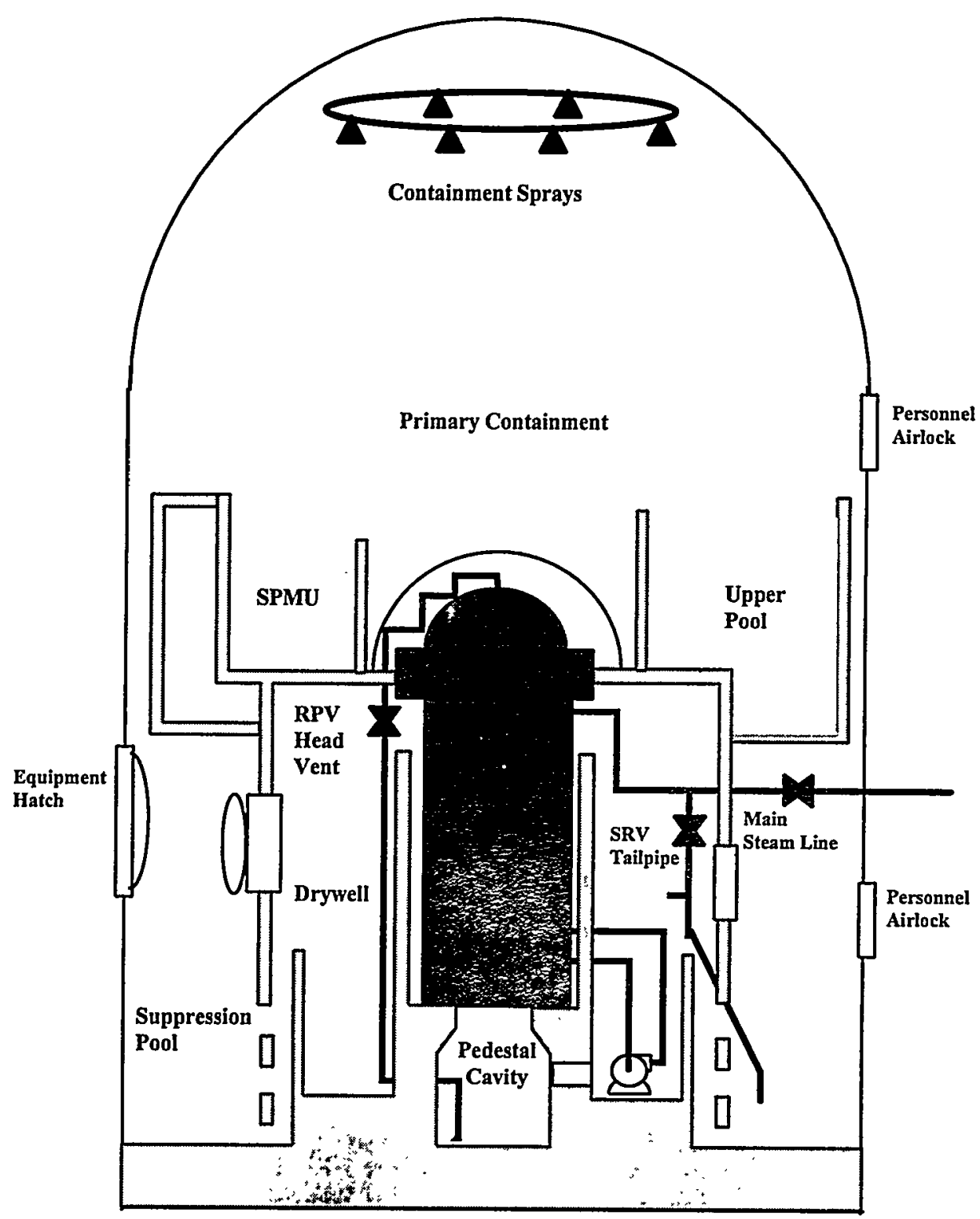

Figure 4.1 Schematic of Grand Gulf containment 
If the drywell pressure drops below the containment pressure, vacuum breakers located in the drywell wall and powered by emergency ac power will open and allow the pressure in the two volumes to equilibrate.

Personnel can access the drywell through two penetrations: the drywell equipment hatch and drywell personnel lock. The drywell equipment hatch is approximately 10 feet in diameter, and its center line is located at an elevation of 122 feet, 4 inches. The drywell personnel lock is similar in design to the containment personnel locks. The center line elevation of the drywell personnel lock is 120 feet.

\subsubsection{Reactor Pedestal Cavity}

The reactor pedestal cavity is located directly below the RPV. The upper section of the cavity is formed by the 5.75-foot-thick pedestal wall, and the lower section of the cavity is recessed into the drywell floor. The pedestal cavity is essentially a right cylinder with a diameter of 21.17 feet and a depth of approximately 28 feet. The upper section of the cavity contains CRD housings. The major pedestal penetrations are the CRD piping penetrations at the top of the pedestal and the CRD removal opening, which is a 3- by 7-foot doorway located 9.5 feet above the cavity floor. It is estimated that the cavity can contain all the core debris released at the time of vessel failure. Thus direct attack of the drywell wall by core debris is not an issue at Grand Gulf, as it is for the Mark I containments.

When the drywell is flooded to the top of the weir wall, a water depth of 22.8 feet can be established in the cavity. Water can enter the cavity from either (1) the vessel, following failure of the bottom head of the RPV, or (2) from the drywell. Water can enter the drywell during a loss-of-coolant accident (LOCA) or through overflow from the suppression pool. Water in the drywell can enter the reactor cavity via two paths. The first pathway is through the drywell floor drains-four 4-inch drains in the drywell floor that connect to the equipment drain sump in the pedestal. The second pathway is through a door in the pedestal, which is located 3.33 feet above the drywell floor. The potential for large amounts of water to settle in the cavity has two major implications. First, a large quantity of water in the cavity has the potential to cool the core debris released from the vessel and thereby prevent the erosion of concrete by the core debris. The water will also retain a portion of the radionuclides released from the core debris in the event that it is not quenched. Second, when the core debris breaches the vessel, water in the cavity creates the possibility of large fuel-coolant interactions (FCIs).

\subsubsection{Emergency Power System}

The emergency power system (EPS) consists of the ac and dc power divisions required by the Engineered Safety Features (ESF) to safely shut down the plant. Both ac and dc are divided into three separate divisions. Divisions 1 and 2 are for the majority of the ESF, while Division 3 is dedicated to the high-pressure core spray system and its required support systems. The ac divisions normally receive power from one of three offsite sources through the ESF transformers. In addition, each ESF $4.16 \mathrm{kV}$ bus has a standby diesel generator, which is available to supply bus loads during loss of normal ac power.

\subsubsection{Hydrogen Ignition System}

The Grand Gulf containment utilizes a hydrogen ignition system (HIS) to control the accumulation of hydrogen during accident conditions. In the core region is an abundant supply of zirconium (i.e., fuel cladding, channel boxes) which, at the elevated temperatures typical of core damage accidents, readily reacts with steam to produce hydrogen. The HIS functions to prevent the buildup of large quantities of hydrogen inside the containment during accident conditions. This task is accomplished by igniting, via a spark, small amounts of hydrogen before large amounts accumulate. The HIS consists of 90 glow plugs (General Motors ac division, Model 7G), 45 powered by each ac power division. The HIS is manually actuated. The igniters are located throughout the containment and drywell volumes. The Grand Gulf Emergency Procedures specify that the HIS is not to be used after hydrogen levels exceed $9 \%$.

\subsubsection{Containment Heat Removal Systems}

Suppression pool cooling (SPC) and the containment spray (CS) system are two modes of the residual heat removal (RHR) system. The RHR system is a two-train system with motor-operated valves and pumps. Both trains have two heat exchangers in series downstream from the pump. The function of SPC is to remove decay heat from the suppression pool during accident conditions. The SPC system takes suction from the suppression pool, cools the water by passing the water through heat exchangers (with service water on the shell side), and returns the water to the suppression pool. The SPC system is manually initiated and controlled. The function of the CS system is to suppress the pressure in the containment during accidents. This task is accomplished by taking suppression pool water, 
passing it through a heat exchanger, and distributing the water as fine droplets into the containment atmosphere via a series of spray headers in the containment dome. The drywell contains no spray headers. Both the SPC and the CS modes of the RHR system require ac power.

\subsubsection{Coolant Injection Systems}

Many systems in a BWR can be utilized to supply coolant to the core. The high pressure core spray (HPCS) system and the reactor core isolation cooling (RCIC) system can be used when the reactor pressure is high. The control rod drive (CRD) system can be used as a backup source of high pressure injection. The low pressure core spray (LPCS) system and the low pressure coolant injection (LPCI) system can be used when reactor pressure is low. Additional systems that can be aligned and used as alternate sources of low pressure injection include the service water cross-tie (SSW cross-tie), condensate, and firewater systems.

The function of the HPCS system is to provide coolant to the reactor vessel during accidents when the pressure in the vessel is high. The HPCS system consists of a single train with motor-operated valves and a motor-driven pump, which are powered by Division 3 emergency power. The pump is capable of delivering $550 \mathrm{gpm}$ against a reactor pressure of $1177 \mathrm{psig}$, and full flow of $7115 \mathrm{gpm}$ against a reactor pressure of $200 \mathrm{psig}$. Suction is taken from either the condensate storage tank or the suppression pool.

The RCIC system consists of a single train with motoroperated valves and a turbine-driven pump. The RCIC pump can deliver $825 \mathrm{gpm}$ at any reactor pressure greater than $200 \mathrm{psig}$. Suction is taken from either the condensate storage tank or the suppression pool. The coolant is supplied to the core via the feedwater line. Steam from the vessel is used to drive the turbine. The technical specifications do not require the RCIC system to be available during cold shutdown; therefore it was not modeled in this analysis.

The CRD hydraulic system can be used as a backup source of high pressure injection. This system includes two pumps that together can achieve a flow rate of approximately 238 gpm with the reactor at $1103 \mathrm{psia}$. The CRD pumps take suction from the condenser hotwell makeup/reject line. CRD pump A requires Division 1 ac power; CRD pump $B$ requires Division 2 ac power.

The function of the LPCS system is to provide coolant to the reactor vessel during accidents in which the vessel pressure is low. The LPCS system is a single-train system consisting of motor-operated and manual valves and a motor-driven pump. The LPCS pump is rated at $7115 \mathrm{gpm}$ with a discharge head of $319 \mathrm{psig}$. The source of water for the LPCS pump is the suppression pool. The LPCS system is powered by the Division 1 emergency power.

The function of the LPCI system is to provide coolant to the reactor vessel during accidents in which system pressure is low. The LPCI system is but one mode of the RHR system and as such shares components with other modes. The LPCI system is a three-train system consisting of motor-operated valves and motor-driven pumps. Trains $A$ and $B$ have two heat exchangers each in series downstream from the pump. Train $C$ is injection-dedicated and has no heat exchangers. The LPCI pump suction source is the suppression pool. Train A is powered by Division 1 emergency power. Trains $\mathrm{B}$ and $\mathrm{C}$ are powered by Division 2 emergency power.

The SSW cross-tie system is used as a source of coolant makeup during accidents where normal sources of emergency injection have failed. The SSW cross-tie system uses SSW pump B to inject water into the reactor via the LPCI system Train B injection lines. SSW pump B is a motor-driven pump and takes suction from the cooling tower basins. Both the SSW Train B and the LPCI system Train B are powered by Division 2 emergency power. The system must be manually aligned and manually actuated.

The condensate system has three condensate pumps and three condensate booster pumps. Each pump is motordriven and rated at $9170 \mathrm{gpm}$. The condensate system takes suction from the condensate storage tank and injects coolant into the vessel through the feedwater line. The condensate pumps are powered by non-safety buses.

The firewater system can be used as a backup source of low pressure injection. The firewater system is a three-train system consisting of one motor-driven pump and two diesel-driven pumps. The pumps feed into a common header that supplies water to the fire hoses. The pumps take suction from two 300,000-gallon water storage tanks. The fire hoses are connected via an adapter to various test connections in the auxiliary building. These connections feed into various injection systems, and water can then be injected through the systems' injection valve. The firewater system can supply approximately 320 GPM at a vessel pressure of 0 psig; the shutoff head is approximately 92 psig. The operator is required to align the system and to start the diesel-driven pumps, which do not require ac power from the emergency power system. 


\subsubsection{Secondary Containment}

The Grand Gulf plant utilizes a secondary containment that completely encloses the primary containment. The secondary containment provides a method for controlling the unlikely release of radioactive materials from the primary containment. Two buildings form the secondary containment. The auxiliary building - which contains safety systems, fuel storage and shipping equipment, and necessary auxiliary support systems-surrounds the lower portions of the containment. The enclosure building encloses the upper portion of the containment above the auxiliary building roof and provides a boundary for the standby gas treatment system, which maintains a negative pressure in the volume between the containment and the enclosure building to ensure that leakage of radioactive materials from the containment is filtered prior to release to the environment in the unlikely event of a LOCA.

\subsection{Plant Operating States}

During full power operation, the technical specification rigorously defines the configuration of the plant and its associated systems to ensure that essentially no equipment important for mitigating an accident is unavailable for an extended period of time. This includes both core cooling and containment systems. During shutdown, the technical specifications allow much more latitude in the availability of systems. Furthermore, the configuration of the plant changes during shutdown to allow for maintenance and refueling (e.g., systems are taken off line for maintenance, and the reactor vessel is opened to replace the fuel).

Because technical specifications are not as prescriptive during shutdown as they are during full power, and because of the need to perform maintenance on systems and to alter the configuration of the plant to refuel, the configuration of the plant and the availability of accident mitigation systems vary drastically from one mode of operation to the next. To accommodate this variability, regimes of operation, or Plant Operating States (POSs), were defined.

\subsubsection{Definition of Plant Operating States}

A POS is defined as a plant condition for which the status of the plant systems (operating, standby, unavailable) can be specified with sufficient accuracy to model subsequent accident events. A POS is not identical to a Mode or Operating Condition (OC) as defined in the technical specifications [USNRC, 1984]; however, POS definitions are based on OCs. Using the OCs as a starting point, seven
POSs were defined. The relationship between the OCs and the POSs is provided in Table 4.1. A des-cription of the process used to identify and characterize a POS is provided in Appendix A of Volume 2 of this report.

\subsubsection{Characterization of POS 5}

POS 5 is rigorously defined as Cold Shutdown (OC 4) and Refueling (OC 5) only to the point where the vessel head is off. POS 5 can be entered either coming down from power or going back up to power.

For the purposes of delineating accident scenarios and estimating consequences, it was necessary to divide POS 5 into time segments or time windows. During a refueling outage (RFO) the plant can be in POS 5 for an extended time; the event that initiates the accident can occur any time during this period. Because the decay heat load from the core decreases with time, the amount of accident-response time available to the operators will depend on the occurrence time of the event that initiates the accident during POS 5. Furthermore, the radionuclide inventory also changes with time, and therefore the radiological potential of the accident will also change with time. Because of this time-dependency, the duration the plant is in POS 5 is divided into time windows. A unique decay heat level and radiological inventory are then assigned to each window. To keep the calculations manageable, only three time windows were defined. In POS 5 there are two natural time segments: the first corresponds to the time the plant is in POS 5 as it is coming down from power prior to refueling; the second corresponds to the time the plant again enters POS 5 after refueling. Between these two POS 5 segments, the plant is in POS 6 and POS 7. Because, on average, about 36 days elapse between the first and second POS 5 time segments, the decay heat and radionuclide inventory for the first time segment will be significantly different from the second segment. The first segment is further subdivided to account for the availability of an alternate source of decay heat removal. The Alternate Decay Heat Removal System (ADHRS) can be used to remove decay heat from the core after the reactor has been shut down for at least 24 hours. Thus the first segment is divided to distinguish the time in POS 5 preceding 24 hours after shutdown from the time in POS 5 following 24 hours after shutdown.

The time after shutdown when the plant enters POS 5 and the time spent in POS 5 are based on Grand Gulf refueling outage data. Although information was available for the first four RFOs, only data from the second, third, and fourth 
Table 4.1 Relationship between operating conditions and plant operating states

\begin{tabular}{|c|c|}
\hline Operating Condition & Plant Operating State \\
\hline 1. Power Operation & \multirow{2}{*}{$\begin{array}{l}\text { 1. Vessel pressure from rated conditions to } 500 \text { psig and } \\
\text { thermal power not greater than } 15 \% \text {; core coolant at } \\
\text { any temperature }\end{array}$} \\
\hline 2. Startup & \\
\hline \multirow{3}{*}{$\begin{array}{l}\text { 3. Hot Shutdown } \\
\text { (core coolant temperature greater than } 200^{\circ} \mathrm{F} \text { ) }\end{array}$} & 2. Vessel pressure from rated conditions to $500 \mathrm{psig}$ \\
\hline & 3. Vessel pressure from 500 psig to above $100 \mathrm{psig}$ \\
\hline & 4. Vessel pressure less than $100 \mathrm{psig}$ and RHR/SDC on \\
\hline 4. Cold Shutdown & $\begin{array}{l}\text { 5. Until vessel head is detensioned } \\
\text { (includes part of Operating Condition 5) }\end{array}$ \\
\hline \multirow{2}{*}{$\begin{array}{l}\text { 5. Refueling (vessel head detensioned or removed, } \\
\text { temperature of core coolant no greater than } 140^{\circ} \mathrm{F} \text { ) }\end{array}$} & 6. Head off and coolant level raised to the steam lines \\
\hline & $\begin{array}{l}\text { 7. Head off, upper pool filled, and refueling transfer tube } \\
\text { open }\end{array}$ \\
\hline
\end{tabular}

RFOs were used in this study. Because a number of special tests were conducted during the first RFO, it was judged atypical. Therefore data from this outage were excluded from the analysis. On average, the plant enters POS 5 at 14 hours after shutdown and remains in POS 5 for 80 hours before entering POS 6 . During the return to power, the plant again enters POS 5 at 40 days after shutdown, remaining in POS 5 for 10.4 days. Based on this information, the three time windows were defined as follows:

Time Window 1: Starts 14 hours after shutdown and has a duration of 10 hours.

Time Window 2: Starts 24 hours after shutdown and has a duration of 70 hours.

Time Window 3: Starts 40 days after shutdown and has a duration of 10.4 days.

Although the plant can enter POS 5 during a refueling outage as early as 7 hours after shutdown, 7 hours was not used as the start time for Window 1 because the average value of 14 hours was judged to be more representative of the time required for the plant to enter POS 5 . However, to account for the possibility that the plant could enter POS 5 as soon as 7 hours after shutdown, the decay heat load used to represent Window 1 was the decay heat load 7 hours after shutdown. The decay heat used to represent Window 2 is the decay heat load 24 hours after shutdown. Similarly, the decay heat load used to represent Window 3 is the decay heat load 40 days after shutdown. The three time windows are depicted in Figure 4.2.
The configuration of the plant during POS 5 , as modeled in the Level $2 / 3$ analysis, was determined from requirements imposed by the technical specifications, plant procedures and practices during a refueling outage, critiques of refueling outages, and interviews with plant personnel. The technical specifications were used to define the minimum set of requirements. If a system was not required to be operable by the technical specifications, then the plant procedures and practices were reviewed. For example, the technical specifications do not require the HIS to be operable during POS 5; however, the habit at the plant is to keep at least one train operable. Thus, in this analysis, although the technical specifications do not require the HIS to be available, it was assumed that at least one train was always available. Even though the configuration of the plant continues to change during $\operatorname{POS} 5$, a plant configuration at the start of the accident was specified to model the plant in the Level $2 / 3$ analysis.

For example, the containment equipment hatch is removed during this POS. Thus, when the POS is initially entered, the hatch is attached (which is one plant configuration); then it is subsequently removed during the POS (creating a second configuration). However, it was judged that the time spent in the first configuration was so small that only the second configuration needed to be analyzed. The configuration of the plant at the start of the accident, as modeled in the Level $2 / 3$ analysis, is defined in the sections below. 


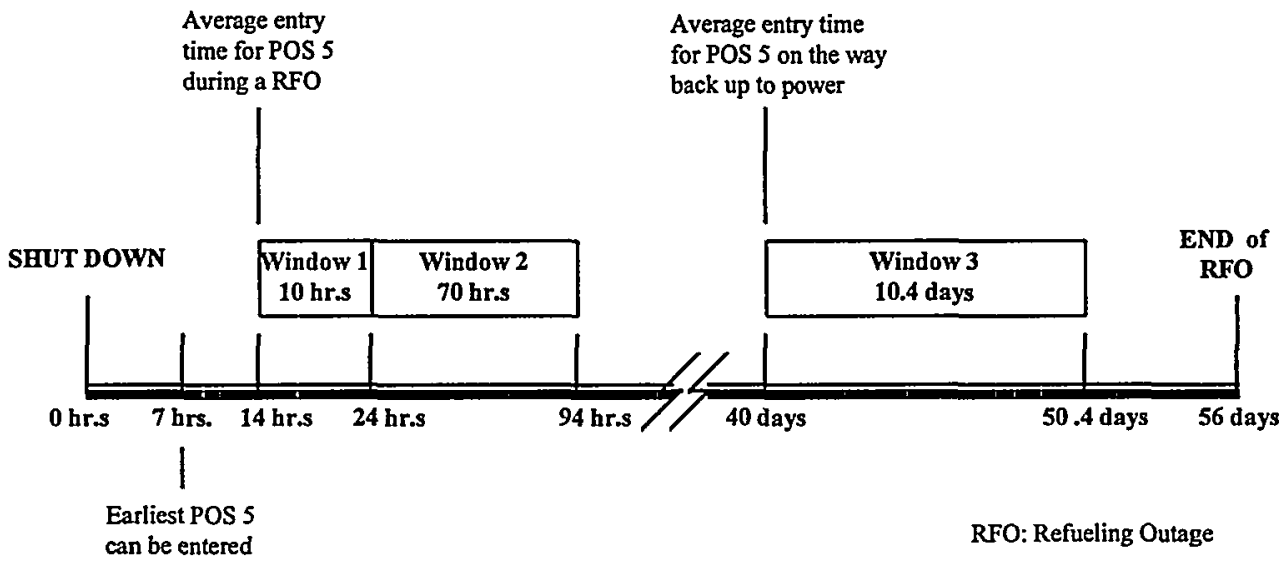

Figure 4.2 POS 5 time windows

Containment: The technical specifications do not require the integrity of the primary or the secondary containments to be maintained during POS 5. A review of the Grand Gulf refueling critiques indicated that the containment equipment hatch is typically removed shortly after entering POS 5. As modeled, the equipment hatch and both personnel locks are open when the accident is initiated. Given that the necessary support systems are available, the model allowed the containment to be closed prior to core damage and, if closed, the containment could be vented if necessary.

Drywell Integrity: The technical specifications do not require that the drywell integrity be maintained during POS 5. A review of the Grand Gulf refueling critiques indicated that the drywell personnel lock is open and the equipment hatch is typically removed early in POS 5. Furthermore, during POS 5 a portion of the upper reactor pool is drained and the drywell head is removed As modeled, either the drywell equipment hatch or the drywell personnel locks were open and remained open throughout the accident.

Reactor Pressure Vessel: In cold shutdown the reactor pressure vessel head is on. Although the technical specifications do not require any SRVs to be available, Grand Gulf administrative procedures dictate that at least two SRVs to be available. Therefore, in this analysis, the model allowed two available SRVs. The temperature of the vessel water is required by the technical specifications to be less than $200^{\circ} \mathrm{F}$. The water level can either be at the normal level or the natural circulation level. For the purposes of this analysis, it was assumed that at the start of the accident the reactor water was at the normal level and its temperature was $200^{\circ} \mathrm{F}$. The RPV head vent was assumed to be open at the start of the accident. The status of the MSIVs (i.e., open or closed) is accident specific.

Suppression Pool: The suppression pool inventory depends on the accident. Three levels were considered: (1) low water level (18 feet, 4.5 inches), (2) drained level (12 feet, 8 inches), and (3) empty, with 170,000 gallons available to HPCS from the condensate storage tank.

Hydrogen Ignition System: The technical specifications do not require the HIS to be available during POS 5 . However, because the usual practice at the plant is to perform train-based maintenance during a refueling outage, and because half of the igniters are on Train $A$ and half are on Train B, it was assumed in this analysis that at least one train of the HIS will always be available (note, however, that the HIS will not operate without ac power). 


\subsection{References}

[SERI, 1992]

[Drouin et al., 1989]

[Harper, 1994]

[USNRC, 1984]
System Energy Resources, Inc. "Grand Gulf Updated Final Safety Analysis Report," 1992."

M. T. Drouin, et al., "Analysis of Core Damage Frequency: Grand Gulf Unit 1 Internal Events," NUREG/CR-4550, SAND86-2084, Vol. 6, Rev. 1, Sandia National Laboratories, September 1989.

F. T. Harper, "Evaluation of Severe Accident Risks: Quantification of Major Input Parameters: Determination of Parameter Values Not Quantified Using Formal Expert Elicitation," NUREG/CR-4551, Vol. 2, Rev. 1, Part 6, Sandia National Laboratories, 1994."

USNRC, "Technical Specifications, Grand Gulf Nuclear Station Unit No. 1", Docket No. 50-416, Appendix "A" to License No. NPF-29, NUREG-0934, October, 1984.

- Copies are available for inspection or copying for a fee in the NRC Public Document room. 
Plant Description 


\section{Plant Damage State Analysis}

Plant damage states form the interface between the accident frequency analysis (i.e., Level 1 analysis) and the accident progression analysis (i.e., Level 2 analysis); as such they define the initial and boundary conditions for the Level 2 analysis. In the Level 1 analysis the sequence of events that will lead to core damage is identified. The minimum set of events that will result in core damage is called a cut set. In the plant damage state analysis, cut sets with similar characteristics that are important to the progression of the accident following core damage are grouped together; each group constitutes a plant damage state (PDS). The Level 1 analysis is documented in Volume 2 of this report.

\subsection{Development of Plant Damage States}

A four-step approach was used to develop the PDSs. Each step is discussed below.

In the first step, general features of the accidents that will define the initial and boundary conditions for the Level 2 analysis are identified. These general features define the configuration of the plant at the start of core damage and the status of systems than can be used to mitigate the accident.

- In the second step, specific systems and plant features are identified that address each of these general features. Each specific feature is call a characteristic; each possible configuration of each system or characteristic is called an attribute. More than one characteristic may be used to define a general feature. For example, the following four systems (i.e., characteristics) could be used to define the general features that addresses the status of core cooling : high pressure core spray (HPCS), low pressure coolant injection ( LPCI), standby service water crosstie (SSW), and condensate (CDS). That is, HPCS is one of four characteristics that defines the status of core cooling. The possible configurations, or attributes, of the HPCS system during the accident are: (a) HPCS available but not being used, (b) HPCS not available and not recoverable, and (c) HPCS not available but recoverable with the regaining of offsite power. In this example, the HPCS characteristic has three attributes. The list of characteristics and their associated attributes defines the possible plant/ system configuration for a particular accident, which is displayed as a string of alphanumeric characters. The first position corresponds to the first characteristic, the second position to the second characteristic, and so on. The alphanumeric character assigned to each position is the attribute for the appropriate characteristic.

- In the third step, the cut sets are reviewed and the appropriate attributes for each characteristic are assigned to each cut set. Because the list of characteristics generally describes the accident in less detail than the cut set, groups of cut sets will have the same string of letters. A unique string of letters is called an End State (ES). ESs are similar to PDSs except that they define the accident in more detail than the PDS. Although the number of ESs can be significantly less than the number of cut sets, typically there are still too many ESs to analyze individually in the Level 2 analysis.

- In the fourth and final step, the many ESs are combined into a manageable number of PDSs. This step is possible because within the resolution of the Level 2 analysis many of the ESs will result in similar accident progressions and releases of radioactive material. To form the PDSs, the ES characteristics are combined so that only the information needed to define the initial and boundary conditions for the Level 2 analysis are defined by the PDS. For example, the individual ESs indicate the availability of many different coolant injection systems (e.g., LPCI, SSW, and CDS). However, if LPCI is recoverable and the vessel is at low pressure, then the status of the other systems is not important for the model used in the Level 2 analysis. Thus, assuming that the other characteristics of the ESs are the same, all those ESs with LPCI recoverable would be combined regardless of the status of SSW and CDS. Through this process the majority of the ESs can be combined into a dozen or so PDSs; however, the actual number of PDSs developed will depend on the diversity of the accident sequences and the resolution desired for the Level 2 analysis.

The general features of the accident that were used in this study to develop the PDSs are: (1) the status of electric power, (2) the status of core cooling, (3) the status of containment heat removal, (4) the status of reactor pressure vessel (RPV) integrity, (5) the status of containment integrity, and (6) accident timing characteristics. Each of these general accident features is discussed below. 
Status of Electric Power: Some systems and components that can influence the progression of the accident following core damage were not modeled in the Level 1 analyses. Many of these systems depend primarily on electric power; therefore, in many cases this feature of the accident can be used to determine the availability of these systems following the onset of core damage. For example, offsite ac power is required to close the containment. Similarly, emergency ac power is required to operate the hydrogen ignition system.

Status of Core Cooling: This feature is used to identify systems that can be used to restore core coolant during the core damage process. Restoration of core cooling offers the potential to arrest the core damage process and prevent vessel failure. Preventing vessel failure can substantially reduce the consequences of the accident.

Status of Containment Heat Removal: This feature addresses the status of systems that can be used to remove decay heat from the containment, such as containment sprays and the suppression pool cooling systems. In cases where the containment is closed, the energy released to the containment atmosphere during core damage will pressurize the containment. These systems are used to attenuate this pressurization and thereby reduce the load on the containment structure. Containment heat removal is generally necessary to prevent containment failure. Containment sprays are also useful because they remove aerosols from the containment atmosphere and thereby reduce any potential release of radioactive material. Because the suppression pool is an integral part of containment heat removal, this feature also addresses the status of the suppression pool at the time of core damage (i.e., amount of water in the pool and temperature of the pool) and is used to identify situations where its performance may be impaired. The suppression pool serves as a heat sink for the reactor, supplies water to emergency core cooling systems (ECCS), and is an effective device for removing radioactive material released from the vessel.

Status of Reactor Pressure Vessel: This feature defines the integrity of the RPV and the pressure in the vessel at the time of core damage. The integrity of the vessel is important because it will determine the path by which steam and radioactive material will escape from the vessel. If the vessel integrity is maintained, the releases will pass from the vessel to the suppression pool via the safety relief valve (SRV) tailpipes. As mentioned previously, the suppression pool is an effective device for mitigating the release. For a loss-of-coolant accident (LOCA), the vessel releases will enter the drywell. For interfacing systems
LOCA, the release will bypass the containment altogether and enter the auxiliary building. If the vessel head vent is open, a portion of the release will enter the drywell while the remaining portion enters the suppression pool via the SRV tailpipes. When the vessel integrity is maintained, the pressure in the vessel will affect the timing of the accident, the amount of radioactive material released during core damage, and the pressure in the containment following vessel failure. The vessel pressure will also determine which systems can be used to provide makeup (i.e., high- or low-pressure systems).

Status of Containment Integrity: This feature defines the integrity of the containment boundary at the time of core damage. The integrity of the containment boundary is one of the most important factors for determining the severity of the accident. For severe core damage accidents in which the containment boundary remains intact, the offsite consequences are generally small. On the other hand, when the containment boundary is not maintained the consequences can be quite severe. Since in POS 5 the containment equipment hatch and personnel locks can be open, it is important to know the status of these penetrations at the time of core damage. This feature also addresses the status of the containment vent system, which can be used to relieve pressure in the containment when containment heat removal systems are not available or are inadequate. Opening the containment vent, however, will allow radioactive material in the containment atmosphere to enter the environment.

Accident Timing Characteristics: This feature defines the time-window status of the plant when the initiating event occurs and the amount of time that elapses between the occurrence of the initiating event and the onset of core damage. The time window will directly affect the amount of decay heat and the radionuclide inventory that is present at the start of the accident. The time window combined with the amount of time that elapses between the start of the accident and the onset of core damage will determine the amount of decay heat that is available at the onset of core damage, which in turn will affect the timing of key events following the onset of core damage (e.g., vessel failure and containment failure). The speed at which the accident proceeds can affect the amount of time available to restore core cooling and will also affect the relative timing between the release of radioactive material and the start of public evacuation. This last item can have a major impact on the magnitude of early health effects.

The characteristics that are used to define the ESs are provided in Appendix A.1. Similarly, the characteristics used to define the PDSs and the rationale used to collapse 
the ES characteristics into PDS characteristics are provided in Appendix A.2.

\subsection{Description of Plant Damage States}

The Level 1 analysis generated 28 core damage sequences, which contained a total of 38 cut sets. These cut sets were grouped into $22 \mathrm{ESs}$, which were then collapsed into twelve PDSs. The sequences contained in each ES and the ESs contained in each PDS are presented Appendix A.1. A brief description of each of the twelve PDSs is provided below. The first number in the PDS name identifies the time window in which the accident occurs; the second number identifies the PDS number. For example, PDS1-3 is the third PDS in Time Window 1.

\section{Plant Damage State PDS1-1:}

The accidents in PDS1-1 are initiated by a LOCA (A or S1) while the plant is in Time Window 1. The break drains the vessel to the top of the jet pumps (i.e., $2 / 3$ core height). The operators attempt to establish water solid operation (i.e., form a continuous loop of water between the RPV and the suppression pool) with low pressure ECCS. HPCS is unavailable because of maintenance or random hardware failures. In a LOCA, the water will drain from the vessel via the break into the drywell. To form a continuous loop of water, enough water must be pumped into the drywell (via the break) to flood the drywell up to the weir wall so that the volume of water in the drywell is connected with the volume of water in the suppression pool. To establish this connection between the drywell and the wetwell it is also necessary for the operators to dump the suppression pool makeup (SPMU) into the suppression pool. In this scenario, however, the operators fail to dump the SPMU in sufficient time to prevent ECCS from failing on inadequate suction head. Once the ECCS pumps fail, they are assumed to be lost for the entire accident. Unable to perform a water solid operation, the operators attempt to flood the containment with the SSW. To successfully flood the containment, the lower personnel lock must be closed. In this plant damage state, the operators do not close the lower personnel lock. Thus the containment is flooded up to the lower personnel lock at which point the water then enters the auxiliary building. It was assumed that the flooding operation would flood the auxiliary building and result in the loss of all core and containment cooling one hour after the start of the accident. Core damage is estimated to occur approximately one hour after the loss of core cooling (i.e., core damage is estimated to occur two hours after the initiating event).
At the onset of core damage, the vessel integrity is breached (via the break), the vessel is at low pressure, and the containment is open. Although offsite power is available during the accident, both the core cooling and containment cooling functions are lost for the entire accident.

\section{Plant Damage State PDS1-2:}

The accidents in PDS1-2 are initiated by a loss of offsite power (T1) followed by a failure of the Train B emergency diesel generator either to start or to run for sufficient time to prevent core damage. The initiating event occurs while the plant is in Time Window 1. The Train A emergency diesel generator is unavailable because of maintenance. HPCS is also unavailable because of either maintenance or random hardware failures. These events result in a station blackout (i.e., loss of all onsite and offsite ac power), leading to a loss of all emergency core cooling. Additionally, the station batteries deplete within 2 hours from the start of the accident. Without dc power, the SRVs cannot be opened to keep the vessel at low pressure. To complicate matters, the valves that isolate the low pressure piping and components on the shutdown cooling system (SDC) from the high pressure piping associated with the RPV require ac power to change position. Thus, without ac power, these valves remain open. Without core cooling the vessel inventory begins to boil, and the resulting steam pressurizes the RPV. The pressurization of the RPV fails the low pressure components associated with the SDC, resulting in a break outside the containment. The break drains the vessel to the top of the jet pumps (i.e., $2 / 3$ core height). Without a means to supply coolant to the core, the accident proceeds to core damage, which is estimated to occur 3.5 hours after initiation of the accident.

At the time of core damage the vessel integrity has been breached and the primary system is at low pressure. It is assumed that the loss of dc power precludes the recovery of offsite power. Without electric power, the containment cannot be closed and the core and containment cooling systems cannot be restored.

\section{Plant Damage State PDS1-3:}

This PDS is similar to PDS1-2 except that the station batteries continue to provide dc power for 12 hours. With dc power available, the operators are able to open two SRVs to keep the vessel depressurized. The operators then align the firewater system to provide coolant to the core. When the station batteries fail 12 hours after the initiating event, the SRVs close, the vessel pressurizes, and the firewater system is lost because of high vessel pressure. In this PDS, the operators manually isolate the low pressure 
components of the SDC from the high pressure primary system. (Note: In this PDS, as opposed to PDS1-2, a considerable amount of time passes between the loss of offsite power and the pressurization of the vessel, which provides the operators sufficient time to manually isolate the SDC.) Without core cooling the accident proceeds to core damage in approximately 12 hours.

At the time of core damage, the vessel is at system pressure with pressure relief provided by the SRVs cycling at their setpoints. The reactor vessel head vent is also open. It is assumed that the loss of dc power precludes the recovery of offsite power. Without electric power, the containment cannot be closed and the core and containment cooling systems cannot be restored.

\section{Plant Damage State PDS1-4:}

This PDS is similar to PDS1-2 except that the station batteries continue to provide dc power for at least 3.5 hours. In this PDS the operators fail to open two SRVs and align the firewater system for core injection. Furthermore, insufficient time exists for the operators to manually isolate the SDC from the primary system. Without core cooling, the vessel inventory begins to boil and the resulting steam pressurizes the RPV. The pressurization of the RPV fails the low pressure components associated with the SDC, resulting in a break outside the containment. The break drains the vessel to the top of the jet pumps (i.e., $2 / 3$ core height). Without a means to supply coolant to the core, the accident proceeds to core damage, which is estimated to occur 3.5 hours after the initiation of the accident.

At the time of core damage the vessel integrity has been breached, the primary system is at low pressure, and the containment equipment hatch is open. Because dc power is available, it is possible to restore offsite power after the onset of core damage. Following recovery of ac power, low pressure ECCS can be used to provide coolant to the core.

\section{Plant Damage State PDS1-5:}

The accidents in PDS1-5 are initiated by a valve misalignment that diverts vessel water to the suppression pool via the residual heat removal (RHR) system (H1) while the plant is in Time Window 1 . The diversion of water is automatically isolated when the vessel water level reaches Level 3. The operators recognize the diversion and attempt to restore core cooling using the water solid operation (i.e., form a water solid loop between the vessel and the suppression pool); however, the suppression pool is empty. Next the operators attempt to flood the containment by injecting water into the vessel using SSW. When the vessel is full, the water passes through the SRVs and enters the suppression pool. In this PDS, however, the operator fails to close the lower containment personnel lock. With the lower personnel lock open, the water being used to flood the containment will pass through the lock and flood the auxiliary building. When the auxiliary building is flooded, it is assumed that all core and containment cooling systems are lost. At this point in the accident, the water level in the vessel is at the main steam lines. Without core cooling, the temperature of the core coolant will increase until it reaches the saturation temperature, at which point it will begin to boil. The steam generated during the boiling process passes through the SRVs and is condensed in the suppression pool. Core damage is estimated to occur 7 hours after the initiating event.

At the onset of core damage, two SRVs are open, the reactor vessel head vent is closed, and the primary system is at low pressure. Even though offsite power is available, the containment is open and all core and containment cooling systems are lost for the entire accident.

\section{Plant Damage State PDS2-1:}

The accidents in PDS2-1 are initiated by a LOCA (A or S1). This PDS is the same as PDS1-1 except that the accident is initiated while the plant is in Time Window 2.

\section{Plant Damage State PDS2-2:}

The accidents in PDS2-2 are initiated by a loss of offsite power (T1). This PDS is the same as PDS1-2 except that the initiating event occurs while the plant is in Time Window 2.

\section{Plant Damage State PDS2-3:}

The accidents in PDS2-3 are initiated by a loss of offsite power (T1). This PDS is the same as PDS1-4 except that the initiating event occurs while the plant is in Time Window 2.

\section{Plant Damage State PDS2-4:}

The accidents in PDS2-4 are initiated by a diversion of vessel water to the suppression pool via the RHR system because of a misalignment of valves. This PDS is the same as PDS1-5 except that the initiating event occurs while the plant is in Time Window 2.

\section{Plant Damage State PDS2-5:}

The accidents in this PDS are initiated by a loss of all SSW (T5A), which leads to loss of both Train A and Train B ECCS. HPCS is unavailable because of maintenance or random hardware failures. The operators recognize the loss 
of SDC but are unable to establish water solid operation because all the ECCS injection systems are unavailable. Without core cooling, the core coolant inventory temperature increases until it reaches saturation, at which point the vessel coolant begins to boil and the vessel begins to pressurize. In this PDS, the operators do not open the SRVs to keep the vessel at low pressure, and therefore the vessel will pressurize to system pressure and will be maintained at system pressure with the SRVs providing pressure relief at their pressure setpoints. The steam generated in the vessel is directed to the suppression pool, via the SRV tailpipes, where it is condensed. Core damage is estimated to occur 12 hours after the initiating event.

At the onset of core damage, the primary system is at system pressure and the vessel head vent is open. All core and containment cooling systems are lost for the entire accident. The containment can be either open or closed. In the case where the containment is closed prior to core damage, the containment vent system is available to relieve the pressure in the containment.

\section{Plant Damage State PDS2-6:}

The accidents in PDS2- 6 are initiated by a valve misalignment that diverts vessel water to the suppression pool via the RHR system ( $\mathrm{Hl}$ ) while the plant is in Time Window 2. The diversion of water is automatically isolated when the vessel water level reaches Level 3 . The operators recognize the diversion and attempt to restore core cooling using the water solid operation (i.e., form a water solid loop between the vessel and the suppression pool). The operators turn on the ECCS pumps and pump water from the suppression pool into the vessel. In this PDS, the main steam isolation valves (MSIVs) are open at the start of the accident and the operators fail to close them during the accident. Also the operators do not turn ECCS off when the vessel is full. Instead the water fills the vessel and flows out through the steam lines to the turbine. This situation is allowed to continue until the ECCS suction strainers in the suppression pool are uncovered. At this time ECCS will fail. Furthermore, it is assumed that the resulting flood in the turbine building will fail any remaining core and containment cooling systems. With the water level now at the main steam lines, the coolant temperature will increase until it reaches saturation, at which point the water will begin to boil. The steam generated during the boiloff process will be transported to the condenser via the main stream lines. Without core cooling, the accident will proceed to core damage in 6.75 hours.

At the time of core damage the MSIVs are open, which establishes a direct path from the RPV to the turbine building, which is outside the containment. Because the MSIVs are open, the primary system is at low pressure. Offsite power is available and the containment can be either open or closed. All core and containment cooling systems are lost for the entire accident.

\section{Plant Damage State PDS3-1}

The accidents in PDS2-1 are initiated by a LOCA (A or S1). This PDS is similar to PDS1-1 except that the accident is initiated while the plant is in Time Window 3 and HPCS was initially available. In this PDS, the failure by the operators to dump SPMU results in a loss of all ECCS (both high and low pressure).

\subsection{Plant Damage State Results}

The core damage frequencies for the 12 PDSs are presented in Table 5.1. The mean total core damage frequency (i.e., the frequency that results from the combination of all 12 PDSs) is $2.1 \mathrm{E}-06 / \mathrm{yr}$; the frequencies at the $5^{\text {th }}$ and $95^{\text {th }}$ percentiles are $4.1 \mathrm{E}-07 / \mathrm{yr}$ and $5.6 \mathrm{E}-06 / \mathrm{yr}$, respectively.

The fractional contributions of individual PDS frequencies to the total core damage frequency are presented in Table 5.2. Two fractional contribution measures were calculated: fractional contribution to core damage frequency and to the mean core damage frequency. The first measure is calculated by dividing the PDS frequency for a single observation by the total core damage frequency for the same observation (an observation is one particular run in the many runs made during a Monte Carlo analysis). This process is repeated for all the observations, and descriptive statistics are then calculated for the resulting distributions. In the second calculation, the mean of each PDS is divided by the total mean core damage frequency. Because the first measure is calculated for each observation, it provides information on the fractional contribution across the entire distribution. The second measure, on the other hand, only provides information on the fractional contribution to the mean values, which may not be representative of the entire distribution. Hence, when fractional contributions are displayed in the remaining sections of this report, the first measure (calculated on an observation-by-observation basis) is used. The second measure is provided in this section of the report because it is the measure that was used to display the contribution of groups of initiating events to the core damage frequency in the Level 1 analysis reported in Volume 2 of this report.

The Level 1 analysis used a Latin hypercube sampling (LHS) sample size of 1000; the Level $2 / 3$ analysis used an 
LHS sample size of 200. Although a sample size of 1000 can be used in the Level 1 analysis, the large computational requirements of accident progression and consequence analyses precluded the use of such a large sample size in the Level $2 / 3$ analysis. When selecting the LHS sample size for the Level $2 / 3$ analyses, two objectives had to be considered: (1) the sample size had to be sufficiently large so that the Level 2/3 PDS results were reasonably similar to the Level 1 results, and (2) the sample size had to be small enough for the calculations to be performed in a timely manner. A sample size of 200 satisfied these two objectives. The PDS frequencies from these two samples are compared in Appendix A.3.

Table 5.1 Plant damage state frequencies

\begin{tabular}{|c|c|c|c|c|c||}
\hline \multirow{2}{*}{ Plant Damage States } & \multicolumn{3}{|c|}{ Descriptive Statistics* Plant Damage State Frequency (1/yr) } \\
\cline { 2 - 6 } & $5 \%$ & $50 \%$ & $95 \%$ & Mean & $\begin{array}{c}\text { Standard } \\
\text { Deviation }\end{array}$ \\
\hline PDS1-1 & $1.6 \mathrm{E}-09$ & $1.4 \mathrm{E}-08$ & $1.9 \mathrm{E}-07$ & $4.1 \mathrm{E}-08$ & $8.3 \mathrm{E}-08$ \\
\hline PDS1-2 & $1.4 \mathrm{E}-10$ & $4.3 \mathrm{E}-09$ & $1.3 \mathrm{E}-07$ & $2.3 \mathrm{E}-08$ & $4.8 \mathrm{E}-08$ \\
\hline PDS1-3 & $2.9 \mathrm{E}-09$ & $1.7 \mathrm{E}-08$ & $1.6 \mathrm{E}-07$ & $4.4 \mathrm{E}-08$ & $1.2 \mathrm{E}-07$ \\
\hline PDS1-4 & $6.0 \mathrm{E}-11$ & $2.0 \mathrm{E}-09$ & $3.5 \mathrm{E}-08$ & $9.2 \mathrm{E}-09$ & $2.9 \mathrm{E}-08$ \\
\hline PDS1-5 & $4.9 \mathrm{E}-10$ & $6.9 \mathrm{E}-09$ & $4.8 \mathrm{E}-08$ & $1.4 \mathrm{E}-08$ & $2.4 \mathrm{E}-08$ \\
\hline PDS2-1 & $1.3 \mathrm{E}-08$ & $1.4 \mathrm{E}-07$ & $1.5 \mathrm{E}-06$ & $3.5 \mathrm{E}-07$ & $6.6 \mathrm{E}-07$ \\
\hline PDS2-2 & $2.2 \mathrm{E}-08$ & $1.5 \mathrm{E}-07$ & $1.6 \mathrm{E}-06$ & $5.5 \mathrm{E}-07$ & $1.9 \mathrm{E}-06$ \\
\hline PDS2-3 & $2.7 \mathrm{E}-09$ & $2.9 \mathrm{E}-08$ & $4.5 \mathrm{E}-07$ & $1.1 \mathrm{E}-07$ & $3.7 \mathrm{E}-07$ \\
\hline PDS2-4 & $7.7 \mathrm{E}-09$ & $8.8 \mathrm{E}-08$ & $7.5 \mathrm{E}-07$ & $2.0 \mathrm{E}-07$ & $3.0 \mathrm{E}-07$ \\
\hline PDS2-5 & $8.6 \mathrm{E}-11$ & $2.7 \mathrm{E}-09$ & $5.3 \mathrm{E}-08$ & $1.3 \mathrm{E}-08$ & $3.4 \mathrm{E}-08$ \\
\hline PDS2-6 & $2.6 \mathrm{E}-11$ & $1.1 \mathrm{E}-09$ & $2.8 \mathrm{E}-08$ & $7.4 \mathrm{E}-09$ & $2.2 \mathrm{E}-08$ \\
\hline PDS3-1 & $6.3 \mathrm{E}-08$ & $3.8 \mathrm{E}-07$ & $2.4 \mathrm{E}-06$ & $7.3 \mathrm{E}-07$ & $1.2 \mathrm{E}-06$ \\
\hline Total & $4.1 \mathrm{E}-07$ & $1.4 \mathrm{E}-06$ & $5.6 \mathrm{E}-06$ & $2.1 \mathrm{E}-06$ & $2.7 \mathrm{E}-06$ \\
\hline
\end{tabular}

* Statistics based on an LHS sample size of 200 . 
Table 5.2 Plant damage state fractional contributions

\begin{tabular}{||c|c|c|c|c|c|c||}
\hline \multirow{2}{*}{$\begin{array}{c}\text { Plant } \\
\text { Damage } \\
\text { States }\end{array}$} & \multicolumn{5}{|c|}{ Descriptive Statistics*: Fraction Contribution (1/yr) } & Fractional \\
\cline { 2 - 7 } & $5 \%$ & $50 \%$ & $95 \%$ & Mean & STD Dev. & $\begin{array}{c}\text { Contribution } \\
\text { To Mean** }\end{array}$ \\
\hline PDS1-1 & $9.9 \mathrm{E}-04$ & $1.3 \mathrm{E}-02$ & $5.4 \mathrm{E}-02$ & $1.8 \mathrm{E}-02$ & $1.6 \mathrm{E}-02$ & 0.020 \\
\hline PDS1-2 & $1.8 \mathrm{E}-04$ & $3.7 \mathrm{E}-03$ & $7.1 \mathrm{E}-02$ & $1.5 \mathrm{E}-02$ & $3.0 \mathrm{E}-02$ & 0.011 \\
\hline PDS1-3 & $1.2 \mathrm{E}-03$ & $1.4 \mathrm{E}-02$ & $1.1 \mathrm{E}-01$ & $3.0 \mathrm{E}-02$ & $5.7 \mathrm{E}-02$ & 0.021 \\
\hline PDS1-4 & $5.4 \mathrm{E}-05$ & $1.9 \mathrm{E}-03$ & $2.2 \mathrm{E}-02$ & $5.7 \mathrm{E}-03$ & $1.4 \mathrm{E}-02$ & 0.004 \\
\hline PDS1-5 & $3.1 \mathrm{E}-04$ & $5.1 \mathrm{E}-03$ & $4.3 \mathrm{E}-02$ & $9.9 \mathrm{E}-03$ & $1.4 \mathrm{E}-02$ & 0.007 \\
\hline PDS2-1 & $9.2 \mathrm{E}-03$ & $1.2 \mathrm{E}-01$ & $4.1 \mathrm{E}-01$ & $1.5 \mathrm{E}-01$ & $1.3 \mathrm{E}-01$ & 0.17 \\
\hline PDS2-2 & $1.2 \mathrm{E}-02$ & $1.4 \mathrm{E}-01$ & $6.4 \mathrm{E}-01$ & $2.2 \mathrm{E}-01$ & $2.1 \mathrm{E}-01$ & 0.26 \\
\hline PDS2-3 & $1.3 \mathrm{E}-03$ & $2.4 \mathrm{E}-02$ & $2.5 \mathrm{E}-01$ & $5.9 \mathrm{E}-02$ & $9.6 \mathrm{E}-02$ & 0.53 \\
\hline PDS2-4 & $5.7 \mathrm{E}-03$ & $6.3 \mathrm{E}-02$ & $5.6 \mathrm{E}-01$ & $1.4 \mathrm{E}-01$ & $1.7 \mathrm{E}-01$ & 0.097 \\
\hline PDS2-5 & $4.4 \mathrm{E}-05$ & $1.8 \mathrm{E}-03$ & $4.3 \mathrm{E}-02$ & $1.0 \mathrm{E}-02$ & $2.3 \mathrm{E}-02$ & 0.006 \\
\hline PDS2-6 & $1.2 \mathrm{E}-05$ & $6.9 \mathrm{E}-04$ & $3.6 \mathrm{E}-02$ & $5.8 \mathrm{E}-03$ & $1.3 \mathrm{E}-02$ & 0.004 \\
\hline PDS3-1 & $6.7 \mathrm{E}-02$ & $3.0 \mathrm{E}-01$ & $7.6 \mathrm{E}-01$ & $2.0 \mathrm{E}-01$ & $9.5 \mathrm{E}-01$ & 0.35 \\
\hline \hline
\end{tabular}

* Statistics based on an LHS sample size of 200 .

** Fractional contribution to mean core damage frequency is the ratio of the mean individual PDS frequency to mean total core damage frequency. 
Plant Damage State Analysis 


\section{Accident Progression Analysis}

In the accident progression analysis, event tree techniques are used to delineate the many possible paths (i.e., accident progressions) that the accident can follow after the onset of core damage. The event tree used to model this portion of the accident is called an Accident Progression Event Tree (APET). Many different paths are possible because there is uncertainty about equipment operation, operator performance, and the outcomes of complex phenomena. Of primary concern is the identification of events that can affect the release of radioactive material from the core and the subsequent transport of this material through the engineered environment (e.g., primary system, containment, and auxiliary building) because this information will be used to estimate source terms in the subsequent analysis (see Section 7). The inputs to the APET are the PDSs described in Section 5. The products of the accident progression analysis are the delineations of the possible paths the accident may follow after the onset of core damage and the probability of each path. Because a typical APET used to model severe accidents can delineate thousands or even hundreds of thousands of paths, it is not practical to estimate a source term for each path individually. Instead, groups of progressions, called Accident Progression Bins (APBs), are formed; these groups have similar characteristics that affect the formation of the source term. In the subsequent analysis, the amount of radioactive material released to the environment is estimated for each APB. The following subsections will describe the APET model, identify the sources of information used to quantify the APET, discuss the characteristics of the accident used to develop the APBs, and provide a summary of results.

\subsection{Accident Progression Model}

The APET developed for this analysis is similar in concept and structure to the APETs used in the NUREG-1150 study; however, it is not as detailed. Compared to the NUREG-1150 APETs, the POS 5 APET includes fewer questions (i.e., top events). Issues were also addressed in less detail (e.g., hydrogen combustion phenomena), and formal expert judgement procedures were not used to quantify the APET. While there are substantially fewer questions included in the POS 5 APET, compared to the NUREG-1150 APETs, the POS 5 APET included a sufficient number of questions so that important interactions between phenomena, systems, and operator actions were captured. Experience and insights gained from other PRAs and the abridged analysis of POS 6 were used to focus the development of the trees and thereby limit the number of questions. In particular, the following factors allowed the size of the tree to be reduced (relative to the APET used in NUREG-1150): (1) In many cases, several related issues were combined and addressed as a single issue. (2) The plant and system configuration during shutdown minimized and/or eliminated the need to address many of the issues considered in the full-power PRA (e.g., after the drywell equipment hatch is removed, assessing the structural response of the drywell tc loads that occur during the accident is no longer necessary). (3) Where necessary, simplifying assumptions were used to limit the size of the analysis (e.g., the impact that the standby gas treatment system has on the release of radioactive material is not included in this analysis). The selection of appropriate top events was based on PRAs of full-power operation, characteristics of the PDSs, results from relevant deterministic calculations that were generated using state-of-the-art severe accident codes such as MELCOR [Summers et al., 1991], and knowledge of plant operation based on plant procedures, discussions with plant personnel, and relevant technical descriptions (e.g., technical specifications, safety analysis reports, and system descriptions).

\subsubsection{Major Assumptions}

The major assumptions made during the development and quantification of the APET are presented below. Additional assumptions are discussed in Appendix B.1, which provides a detailed discussion of the questions in the APET. The major assumptions include:

- During POS 5 the plant is in the cold shutdown mode of operation with the vessel head attached. Two SRVs are available to control the pressure in the vessel.

- Core damage is defined as the start of fuel heatup. The time to core damage and other timing characteristics of the accident were determined from MELCOR calculations performed for this study. MELCOR calculations were performed for various PDSs and are documented in Volume 6, Part 2 of this report.

- The containment and drywell are both open at the start of the accident. The containment can only be closed if offsite ac power is available; containment closure must be completed prior to the onset of core damage. The drywell is assumed to remain open throughout the accident. Also, for those accidents in which the containment was unsuccessfully flooded (i.e., the lower personnel 
lock was inadvertently left open resulting in a flood in the auxiliary building), it was assumed in the Level 1 analysis that the lock remained open prior to core damage. Because no credit is given for closing the containment after the onset of core damage, it is assumed that the containment remains open for the duration of the accident.

- To restore offsite power to the plant in the event that power is lost before or during the accident, dc power from the station batteries is required. If the station batteries deplete prior to resumption of offsite power, it is assumed that offsite power cannot be restored during the accident. steam and noncondensibles or from pressurization that accompanies a hydrogen burn. The auxiliary building is assumed to fail on a 5-psi overpressure. Prior to failure, it is assumed that no radioactive material enters the environment. Although the auxiliary building is not a leak-tight structure, it is assumed that the radioactive material is released into the building so slowly that negligible amounts of radiation escape into the environment prior to building failure. Neither the standby gas treatment system nor the ventilation system is modeled. Thus it is assumed that no engineered features are available to attenuate the release in the auxiliary building, and the only attenuation that the release will experience in the building is that caused by natural processes (e.g., natural deposition).

If the containment is closed prior to core damage, containment heat removal must be available to prevent subsequent containment failure from longterm overpressurization. This assumption is supported by MELCOR calculations, which show that the containment will ultimately fail from overpressure if the decay heat is not removed from the containment. For the PDSs developed for this analysis, containment heat removal is never available; therefore the containment will never remain intact throughout the accident. If the containment (1) is closed prior to core damage, (2) does not fail from loads accompanying hydrogen combustion or vessel failure, and (3) is not vented, it is assumed to fail late in the accident from the accumulation of steam and noncondensibles.
If the containment is closed prior to the onset of core damage and subsequently vented after the onset of core damage, it is assumed that the vent stays open throughout the accident. While the emergency operating procedures (EOPs) direct the operators to close the containment when its pressure drops below a certain pressure, without containment heat removal the containment must be vented again later in the accident when the pressure again increases above the vent pressure. No attempt was made in this analysis to model the opening and closing of the containment vent. Furthermore, because the availability of the containment purge system during POS 5 is not being modeled (and it is not required by the technical specification), it is assumed that the containment will be vented directly to the environment and will not pass through the containment purge system with its associated filters and charcoal beds.
If the containment fails, it is assumed to fail above the auxiliary building roof, thereby allowing radioactive releases to bypass the auxiliary building and enter the environment directly. The enclosure building that surrounds the portion of the containment above the auxiliary building roof is estimated to offer essentially no attenuation to the release. This assumption is consistent with that used in the Grand Gulf plant analysis performed as part of the NUREG-1150 study [Brown et al., 1990].

- Because the assessed containment failure pressure at the $99^{\text {th }}$ percentile is only $97 \mathrm{psig}$ and the drywell pressure required to prevent the SRVs from opening must exceed $100 \mathrm{psi}$, failure of the SRVs from high containment pressure is not considered in this study.

The Grand Gulf Emergency Operating Procedures are applicable after the onset of core damage, and the operators will continue to follow them.

- No operator actions were modeled that would require the operators to enter the containment or auxiliary building following the onset of core damage.

Recovery of coolant injection after the onset of core damage is only considered if (1) injection systems were not available prior to core damage but become available following core damage, or 
(2) conditions occur following the onset of core damage that would cause the operators to use a system that was available but not used prior to core damage.

If the reactor head vent is open and the vessel is pressurized, it is assumed that all the in-vessel releases escape though the head vent and bypass the suppression pool. This assumption is based on results from MELCOR calculations performed for this study. If the vessel is depressurized prior to core damage or the vessel is breached by a LOCA, the in-vessel release will either pass through the SRVs and enter the suppression pool or escape through the break, whichever the case may be.

-

It is assumed that the core cannot be cooled and vessel failure cannot be prevented by flooding the lower portion of the containment, which submerges in water the lower portion of the lower vessel head.

Although the containment is flooded in the LOCA PDSs, it is assumed that the break occurs above the water, and therefore any releases that occur before vessel failure will not be scrubbed by water.

\subsubsection{Overview of the APET}

The APET for POS 5 considers the progression of the accident from the onset of core damage-defined in this analysis as the start of fuel heatup as predicted by the MELCOR code-through the completion of the interactions between the core debris and the concrete structure below the vessel. The interactions that occur between the core debris released from the vessel and the concrete structures below the vessel are termed coreconcrete interactions (CCIs). To model these accidents, the APET addresses the 59 events or questions listed in Table 6.1. The first 17 questions are used to define the characteristics of the PDSs that form the initial conditions for the analysis. Following definition of the PDSs, the questions in the APET are divided into four general time regimes: (1) before core damage, (2) during the in-vessel phase of the core damage process, (3) from vessel failure to the start of significant CCIs, and (4) from the start of significant CCIs to the end of the accident. Events considered before core damage include events that are important to the accident progression but that were not included in the Level 1 analysis. These events include operator actions associated with containment closure and the initiation the of hydrogen ignition system (HIS). Questions in the second time regime address the core degradation and relocation processes in the vessel and the status of plant features that can be used to mitigate the release. Included during the in-vessel phase of the accident are events that address the recovery of core coolant, the status of the reactor vessel integrity and pressure, and the status of the containment and auxiliary building. To assess the status of containment integrity events associated with hydrogen combustion, containment heat removal and containment venting are also considered. Because many of the systems that can be used to mitigate the accident depend on ac power, the recovery of ac power during a station blackout is also addressed in this section of the APET. Events included in the vessel failure time regime include those that determine the likelihood that the core debris is cooled in the vessel, resulting in termination of the accident with the core in a safe stable condition. For accidents in which the core debris is not cooled and the vessel fails, events are included that address the phenomena associated with vessel failure (e.g., vessel melt-through, high pressure melt ejection, and steam explosions) and the accompanying loads. The responses of the containment or auxiliary building to these loads are also assessed in this section of the APET. Events that are included in the time regime after vessel failure include events associated with CCIs, the longterm pressurization of the containment from the steam and noncondensibles generated during the $\mathrm{CCI}$ process, and the status of the containment's integrity late in the accident. Because many of the systems that can be used to control the pressure in the containment depend on electric power (e.g., containment sprays and containment venting) the status of electric power is also addressed during this phase of the accident. By the end of the tree, the extent of core damage (i.e., only in-vessel releases versus both in-vessel and exvessel releases), the release path, and the status of the systems that can be used to mitigate the release have been identified. These features of the accident are then used as a basis for estimating the magnitude of the release in the radioactive release and transport analysis.

Each question included in the APET is discussed in detail in Appendix B, Section B.1. Because of the large number of questions included in the APET, it is not practical to represent the tree graphically. Instead, Boolean statements are used to represent the tree, which is then evaluated with the EVNTRE code [Griesmeyer et al., 1989]. The Boolean representation of the POS 5 APET is provided in Appendix B, Section B.2. 
Table 6.1 POS 5 accident progression event tree questions

\begin{tabular}{|c|c|}
\hline No. & APET Questions \\
\hline & Plant Damáge State Definitions \\
\hline 1 & What is the Plant Damage State? \\
\hline 2 & What is the status of electric power at core damage (PDS Char. 1)? \\
\hline 3 & What is the status of dc power at core damage (PDS Char. 1)? \\
\hline 4 & What is the status of high pressure injection at core damage (PDS Char. 2)? \\
\hline 5 & What is the status of low pressure injection at core damage (PDS Char. 2)? \\
\hline 6 & What is the status of containment sprays and SPC at core damage (PDS Char. 3)? \\
\hline 7 & What is the suppression pool level at the onset of core damage (PDS Char. 4)? \\
\hline 8 & What is the suppression pool temperature at the onset of core damage (PDS Char. 5)? \\
\hline 9 & What is the status of the reactor head vent at the onset of core damage (PDS Char. 6)? \\
\hline 10 & What is the status of the RPV integrity at the onset of core damage (PDS Char. 7)? \\
\hline 11 & What is the status of the containment access penetrations at the onset of core damage (PDS Char. 8)? \\
\hline 12 & What is the status of the containment vents system at the onset of core damage (PDS Char. 9)? \\
\hline 13 & When does core damage occur (PDS Char. 10)? \\
\hline 14 & While in POS 5, when does the initiating event occur (PDS Char. 11)? \\
\hline 15 & What type of event initiates the accident? \\
\hline 16 & What is the pressure in the RPV at the time of core damage? \\
\hline 17 & How much water is in the reactor pedestal cavity at the time of core damage? \\
\hline & Events that Occur Before Core Damage \\
\hline 18 & Is the containment equipment hatch opened at the start of the accident? \\
\hline 19 & Do the operators close the containment before core damage? \\
\hline 20 & Does the auxiliary building fail before core damage? \\
\hline 21 & What is the status of the drywell before core damage? \\
\hline 22 & Do the operators turn on the HIS before core damage? \\
\hline & Events that Occur During Core Damage \\
\hline 23 & Are the station batteries depleted during core damage? \\
\hline 24 & Is offsite power restored during core damage? \\
\hline 25 & Is the RPV isolated during core damage? \\
\hline 26 & Do the operators initiate containment sprays during core damage? \\
\hline 27 & Do the operators depressurize the RPV during core damage? \\
\hline 28 & What is the status of the SRV vacuum breakers during core damage? \\
\hline 29 & Is core cooling restored during core damage? \\
\hline 30 & What is the peak hydrogen concentration in the containment during CD? \\
\hline 31 & What is the fraction of zirconium that is oxidized in the vessel during core damage? \\
\hline 32 & Do the operators turn on the HIS during $\mathrm{CD}$ ? \\
\hline 33 & Does an uncontrolled hydrogen combustion event occur during CD? \\
\hline
\end{tabular}


Table 6.1 POS 5 accident progression event tree questions (continued)

\begin{tabular}{|l|l}
\hline \hline No. & \multicolumn{1}{c}{ APET Questions } \\
\hline 34 & What is the pressure in the containment during CD (no uncontrolled burn)? \\
35 & Does the containment fail from quasi-static loads during core damage? \\
36 & Do the operators vent the containment during core damage? \\
38 & What is the status of the containment during core damage? \\
\hline 39 & What is the size of the containment opening during core damage? \\
\hline 40 & Does the auxiliary building fail during core damage? \\
\hline 41 & Is there water in the RPV pedestal cavity just prior to VB? \\
42 & Is the core damage process arrested in the vessel? \\
43 & What fraction of the core debris would be mobil at VB? \\
44 & Does a large in-vessel steam explosion occur? \\
45 & Does an Alpha mode event occur? \\
46 & Does a large in-vessel steam explosion fail the vessel? \\
47 & What is the mode of VB? \\
48 & Does high pressure melt ejection occur? \\
49 & Does a large ex-vessel steam explosion accompany VB? \\
50 & Does the containment fail from pressure loads accompanying VB? \\
51 & What is the status of containment integrity just after VB? \\
52 & What is the size of the containment opening just after VB? \\
\hline 53 & Does the auxiliary building fail just after VB? \\
\hline 54 & Is ac power recovery late in the accident? \\
\hline 59 & Whe the core debris in the cavity coolable? \\
\hline
\end{tabular}

\subsection{Quantification of the Accident Progression Model}

The quantification of the APET consists primarily of assigning probabilities to the branches in the APET. These branches represent the possible outcomes for the various events included in the tree (e.g., the occurrence of human actions, the system responses, the occurrence of phenomenological events). The probabilities in the POS 5 APET were quantified using information from the following sources:
- Level 1 Analysis: The frequencies for the PDSs were obtained from the Level 1 analysis described in Volume 2 of this report.

- HRA analysis: A Human Reliability Analysis (HRA) was performed to determine the human error probability for operator actions during the core damage process (e.g., containment closure and the recovery of core cooling and containment cooling functions). For the sake of consistency, wherever possible, the same HRA models and techniques used in the Level I 
analysis were also used in this study. The results of the HRA analysis are provided in Appendix B.3.

- MELCOR calculations: A series of MELCOR calculations was performed specifically for this study. Results from these calculations helped guide the development and quantification of the APET. Specifically, the MELCOR calculations were used to determine the timing of key events (e.g., onset of core damage, vessel failure, containment failure) and the pressure, temperature, and composition histories of the containment and auxiliary building. The MELCOR calculations are documented in Volume 6, Part 2 of this report.

- Data from the NUREG-1150 PRAs: Where appropriate, data used in the NUREG-1150 full power PRA of Grand Gulf were also used in this study (e.g., structural capacity of the containment to static loads).

For those events judged to be important to risk, and for which there existed a large amount of uncertainty concerning the value to assign to the branch probability, an uncertainty distribution was assigned to the probability. Twenty-three variables in the APET were included in the uncertainty analysis. For the remaining events, those either judged to be less important or for which the branch probability was not believed to be uncertain, a single value was used. The primary sources of information used to quantify the questions in the APET are listed in Appendix B.3.1. Also, if the question was included in the uncertainty analysis, Appendix B.3.1 identifies the distribution that was used to characterize the uncertainty and provides the variable name.

\subsection{Accident Progression Bins}

As each path through the APET is evaluated, the result of that evaluation is stored by assigning it to an Accident Progression Bin (APB). The APBs are the means by which information is passed from the accident progression analysis to the source term analysis (see Section 7); as such the bin describes the evaluation in adequate detail for a source term (the release of radioactive material) to be estimated for it. The binning scheme for the POS 5 analysis utilizes 14 characteristics or quantities, each of which defines a certain feature of the accident progression (the definition of the APBs is analogous to the definition of PDSs). For each characteristic, the possible states are termed attributes. The selection of the characteristics and attributes is based on the information needed in the radionuclide release and transport analysis to estimate the source term. The fourteen characteristics used in this analysis are identified and described in Table 6.2. The attributes for each characteristic are identified and described in Appendix B.4.

\subsection{Evaluation of the Accident Progression Event Tree}

The Grand Gulf POS 5 APET was evaluated using the EVNTRE code [Griesmeyer and Smith, 1989]; the results from EVNTRE were post-processed with the PSTEVNT code [Higgins, 1989]. All the plant damage states are evaluated in a single APET and EVNTRE run; hence the probabilities for the APBs generated in this analysis are conditional on the occurrence of core damage, not a particular PDS. A path in the APET was dropped from the analysis when its probability dropped below 1.0E-07. Thus the APBs consist of groups of individual progressions, each having a conditional probability of at least $1.0 \mathrm{E}-07$. The logic used to form the APBs is included in the APET logic model described in Appendix B.2. The uncertainties associated with important events that affect the accident progression analysis were propagated through the APET using a stratified form of simple random sampling called Latin Hypercube Sampling (LHS) (see Section 2 for more discussion). In this technique, the APET is evaluated many times using different sets of inputs for each evaluation. The entire set of inputs is called the sample, whereas the set of inputs used for a single evaluation is called an observation. A sample consisting of 200 observations was used in this analysis. The evaluation of the APET resulted in the generation of 242 unique APBs.

\subsection{Results from Accident Progression Analysis}

Because there are far too many APBs to present and discuss each one individually, only aspects of the accident progression that have a major effect on the source term and risk will be discussed in this subsection. Features of the accident that can have a major impact on the amount of radioactive material released to the environment include the containment's integrity during the accident, the recovery of core cooling and arrest of the core damage process in the vessel, the coolability of the core debris that is released from the vessel, and the availability of plant features that can be used to attenuate the release (e.g., the suppression pool and the containment sprays). In none of these 
Table 6.2 Accident progression bin characteristics

\begin{tabular}{|c|c|}
\hline $\begin{array}{l}\text { Characteristic } \\
\text { No. }\end{array}$ & Description \\
\hline 1 & Identifies the plant damage state \\
\hline 2 & $\begin{array}{l}\text { Identifies the status of containment integrity during the various stages of } \\
\text { the accident. }\end{array}$ \\
\hline 3 & $\begin{array}{l}\text { Identifies the status of auxiliary building integrity during the various } \\
\text { stages of the accident }\end{array}$ \\
\hline 4 & Identifies the status of drywell integrity at the start of the accident \\
\hline 5 & Identifies the status of the reactor vessel integrity prior to core damage \\
\hline 6 & Identifies the status of the reactor head vent before core damage \\
\hline 7 & $\begin{array}{l}\text { Identifies the status of the SRV tailpipe vacuum breaker during core } \\
\text { damage }\end{array}$ \\
\hline 8 & $\begin{array}{l}\text { Identifies both the pressure in the reactor and the status of core coolant at } \\
\text { the time of vessel failure }\end{array}$ \\
\hline 9 & Identifies the status of containment sprays during core damage \\
\hline 10 & Identifies the amount of zirconium oxidized during core damage \\
\hline 11 & $\begin{array}{l}\text { Identifies the occurrence of high pressure melt ejection and steam } \\
\text { explosion events }\end{array}$ \\
\hline 12 & $\begin{array}{l}\text { Identifies the coolability of the core debris in the reactor pedestal cavity } \\
\text { following vessel failure }\end{array}$ \\
\hline 13 & Identifies the time window during POS 5 in which the accident occurs \\
\hline 14 & $\begin{array}{l}\text { Identifies the temperature of the suppression pool at the onset of core } \\
\text { damage }\end{array}$ \\
\hline
\end{tabular}

accidents are the containment sprays available. Simplified representations of the APET that address these aspects of the accident for all PDSs considered together, the LOCA PDS considered as a group, the Station Blackout (SBO) PDSs considered as a group, and the Other PDSs considered as a group are shown in Figures 6.1, 6.2, 6.3, and 6.4, respectively. The Other PDS group consists of those PDSs that are not LOCAs and not SBO; it includes the following: PDS1-5, PDS2-4, PDS2-5, and PDS2-6. Figure 6.1 is conditional on the occurrence of core damage, whereas the other figures are conditional on the occurrence of the PDS under consideration. The values displayed in the figures are mean conditional probabilities.
From the simplified tree presented in Figure 6.1, it can be seen that the most likely accidents in POS 5 have an open containment, the suppression pool is bypassed, and the vessel fails. The uncertainty in the probability that the containment is closed prior to core damage, and the uncertainty in the probability that core cooling is restored and vessel failure is prevented, are displayed in Figure 6.5. The probabilities displayed in Figure 6.5 are conditional on the occurrence of core damage. For the cases where the vessel fails, there exists a significant probability that the core debris will either be quenched in a flooded cavity or CCI will occur in a flooded cavity. For the former, the releases associated with $\mathrm{CCI}$ are prevented. For the latter, the releases are scrubbed by the water in the flooded cavity. 
Accident Progression Analysis

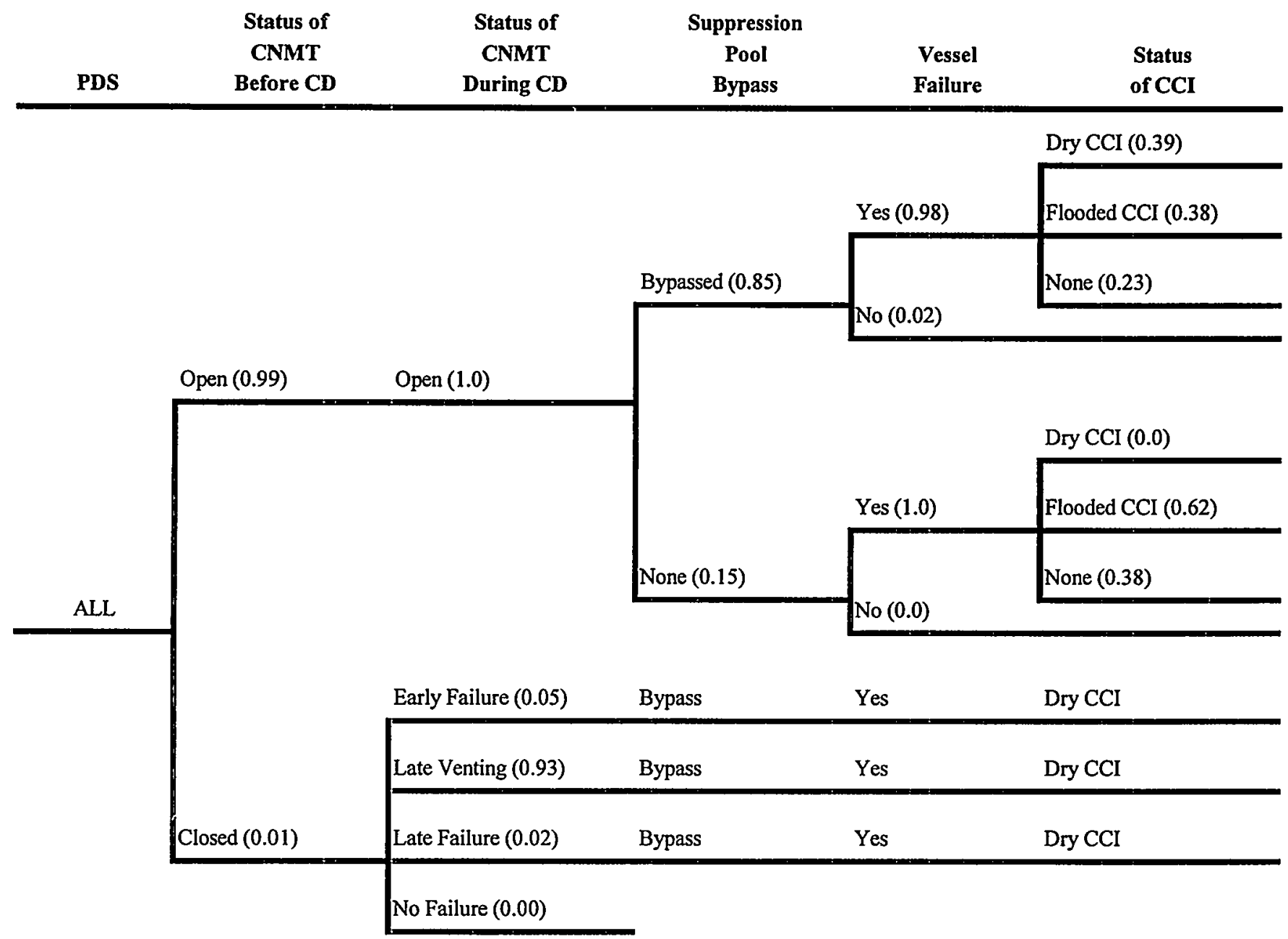

Figure 6.1 Simplified representation of POS 5 accident progressions

If the containment is closed prior to core damage, it is always predicted to either fail or to be vented after core damage because containment heat removal is not available in these accidents. Venting the containment late in the accident is the most likely scenario. Given that the containment is closed prior to core damage, the uncertainties in the probabilities that the containment fails early in the accident (early is defined as during core damage or at the time of vessel failure), that the containment is vented late in the accident, and that the containment fails late in the accident are displayed in Figure 6.6. From this figure it is clear that when the containment is closed, venting the containment late in the accident is the most likely event.
For the LOCA PDS group the containment is always open, the suppression pool is always bypassed, and core cooling never restored. Without core cooling the vessel is always predicted to fail. Since the containment was flooded prior to core damage in these PDSs, the core debris ejected from the vessel is released into a pool of water. The mean probability that the core debris is quenched and $\mathrm{CCI}$ is avoided is 0.38 . For those cases where $\mathrm{CCl}$ does occur, the releases will always be scrubbed by the water in the flooded cavity. Thus, while the vessel is always predicted to fail, in half of these cases the core debris is quenched. The primary difference in the source term between cases with core damage arrest and cases with no $\mathrm{CCl}$ is that, for the latter cases, it is still possible for ex-vessel steam explo- 


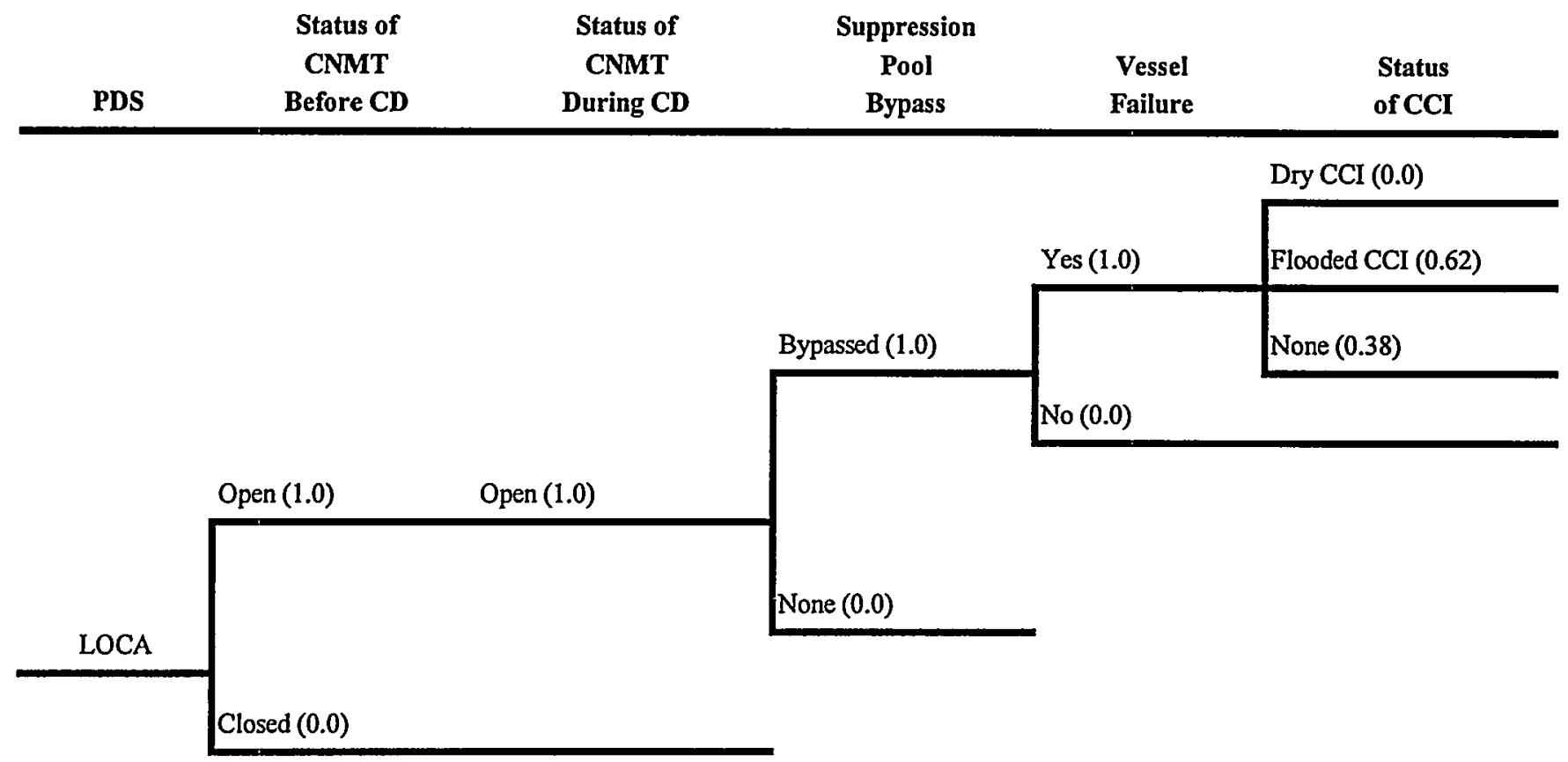

Figure 6.2 Simplified representation of LOCA PDS group accident progression

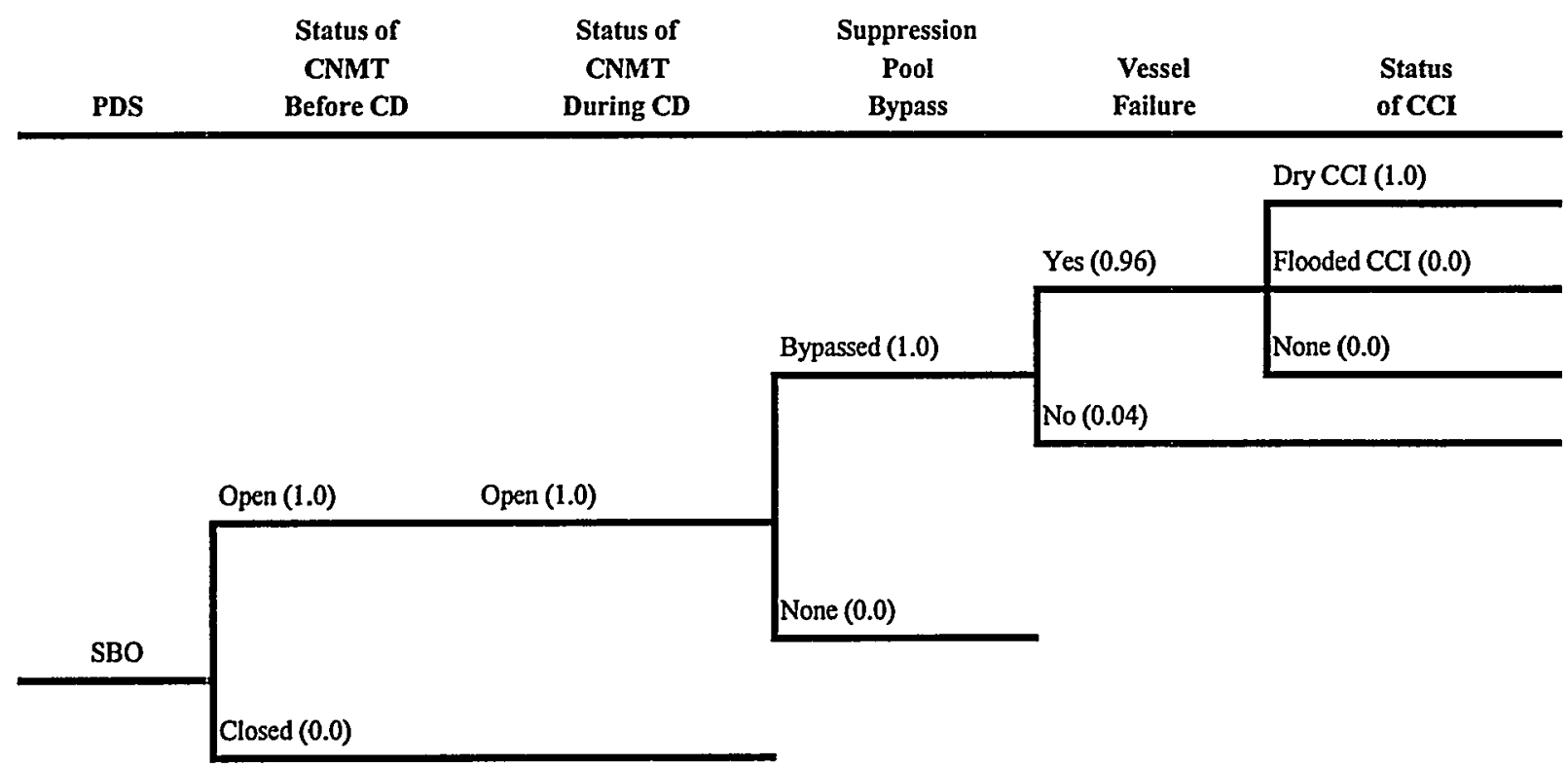

Figure 6.3 Simplified representation of Station Blackout PDS group accident progression (values for flooded $\mathrm{CCI}$ and no $\mathrm{CCI}$ are approximately 0.0 : i.e., $<1 \times 10^{-4}$ ) 


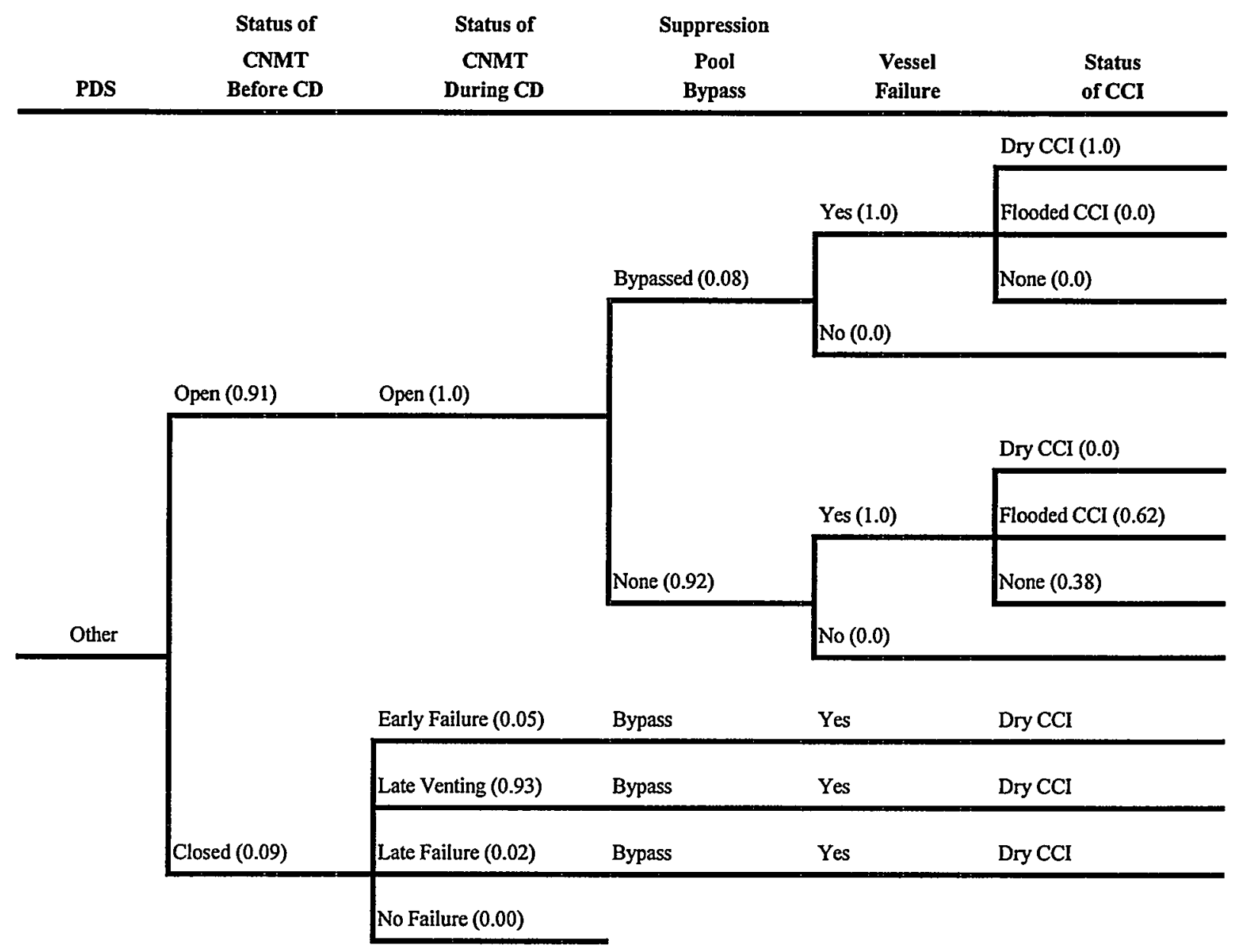

Figure 6.4 Simplified representation of Other PDS group accident progression

sions or debris ejected at high pressure to contribute to the release of radioactive material (in the source term model used for this analysis a "puff" release is also associated with vessel failure; however, this release is small compared to the other sources). Hence the source term associated with the latter cases tends to be larger. Thus for the LOCA accidents the release associated with the core damage process can be large because the suppression pool is bypassed and the containment sprays are unavailable. The ex-vessel releases, however, may be limited because there is a substantial probability that the core debris will be quenched, and for those cases where CCI occurs the releases will be scrubbed by a pool of water. For the LOCA accidents, the primary features that can attenuate the release are the auxiliary building and the pool of water formed when the containment was flooded.
For the SBO PDS group, the containment is always open and the suppression pool is bypassed. Although there are accidents in this PDS group in which ac power can be recovered and core cooling restored, the mean probability that the core damage process is arrested and vessel failure prevented is only 0.04 . For those accidents that involve vessel failure, the core debris is nearly always released into a dry cavity. Hence for this PDS group the most likely

1 For station blackout accidents that involve a break in the SDC system, which is subsequently isolated when ac power is recovered, it is conservatively assumed that all the in-vessel releases escape out the break and bypass the suppression pool before the break is isolated. This assumption is made because it is not known at what time during core damage that ac power is restored and the SDC system isolated. 


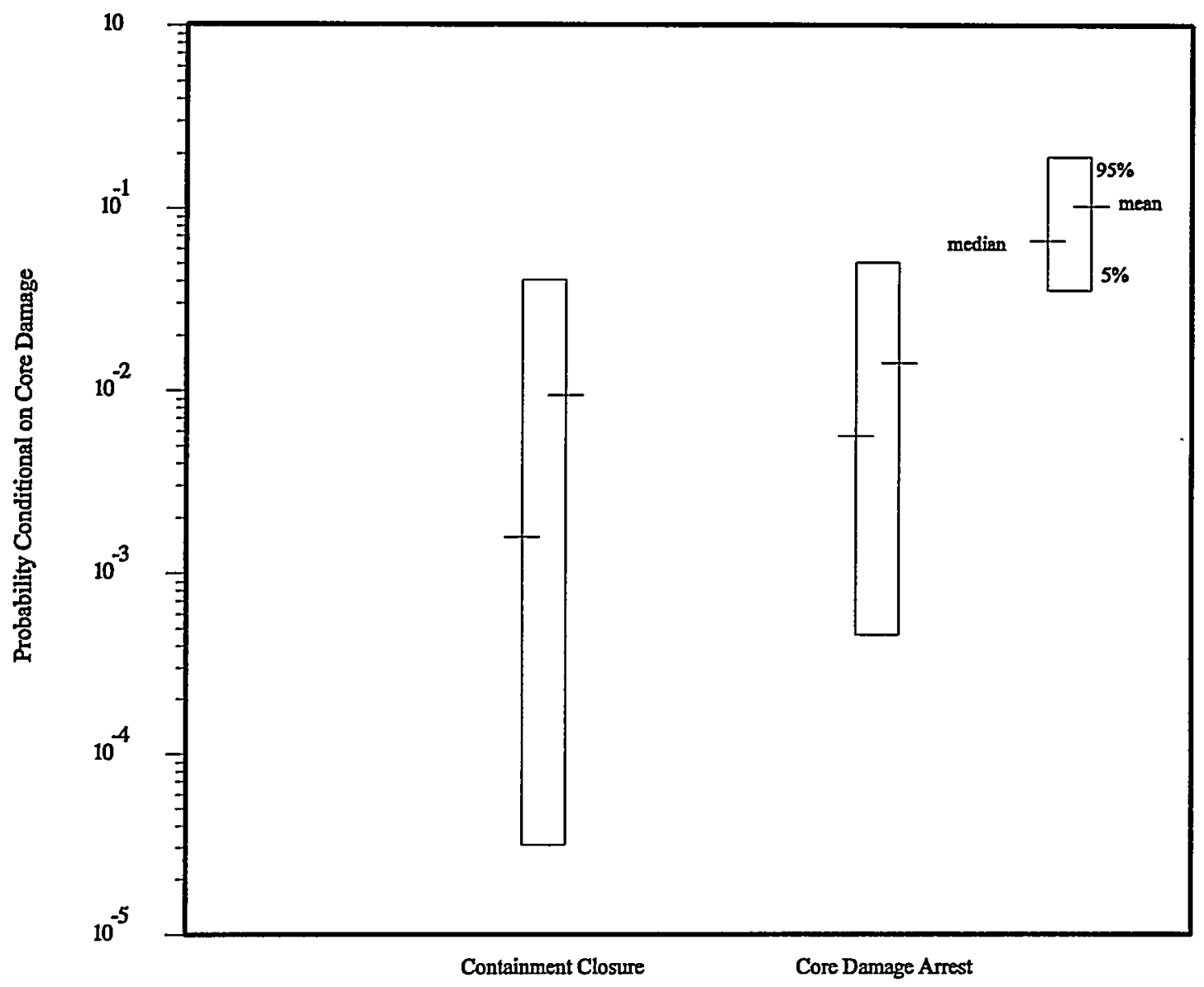

Figure 6.5 Probability of containment closure and probability of core damage arrest

scenario is that the vessel will fail and $\mathrm{CCI}$ will occur in a dry cavity. Because the in-vessel releases bypass the vessel and CCI nearly always occurs in a dry cavity, the releases associated with these accidents can be large.

As can be seen from Figure 6.4, for the Other PDS group many different accident progressions are possible. Of the three PDS groups discussed, this is the only group in which the containment can be closed prior to core damage. The mean probability that the containment is closed, however, is only 0.09 . If the containment is closed prior to core damage, it is always predicted to either fail or to be vented after core damage. Venting the containment late in the accident is the most likely scenario. The progressions in which the containment is closed prior to core damage stem from PDS2-5. In this PDS, the suppression pool is always bypassed, ${ }^{2}$ core cooling is never restored, and $\mathrm{CCI}$ always occurs in a dry cavity. The accidents in which the containment remains open during the entire accident consist of cases where the suppression pool is bypassed and cases where the radioactive material released during the core damage process passes through the suppression pool. For

2

In PDS2-5 the reactor head vent is open and the vessel is pressurized at the onset of core damage. If the reactor remains at high pressure, all of the releases will escape out the vent and bypass the suppression pool. If, however, the vessel is at low pressure the release will pass through the SRVs and enter the suppression pool. Although it is likely that the operators will depressurize the vessel during core damage, it is not known when during core damage the operators will perform the action. Hence it is conservatively assumed that the release escapes through the head vent while the vessel is pressurized. 


\section{Accident Progression Analysis}

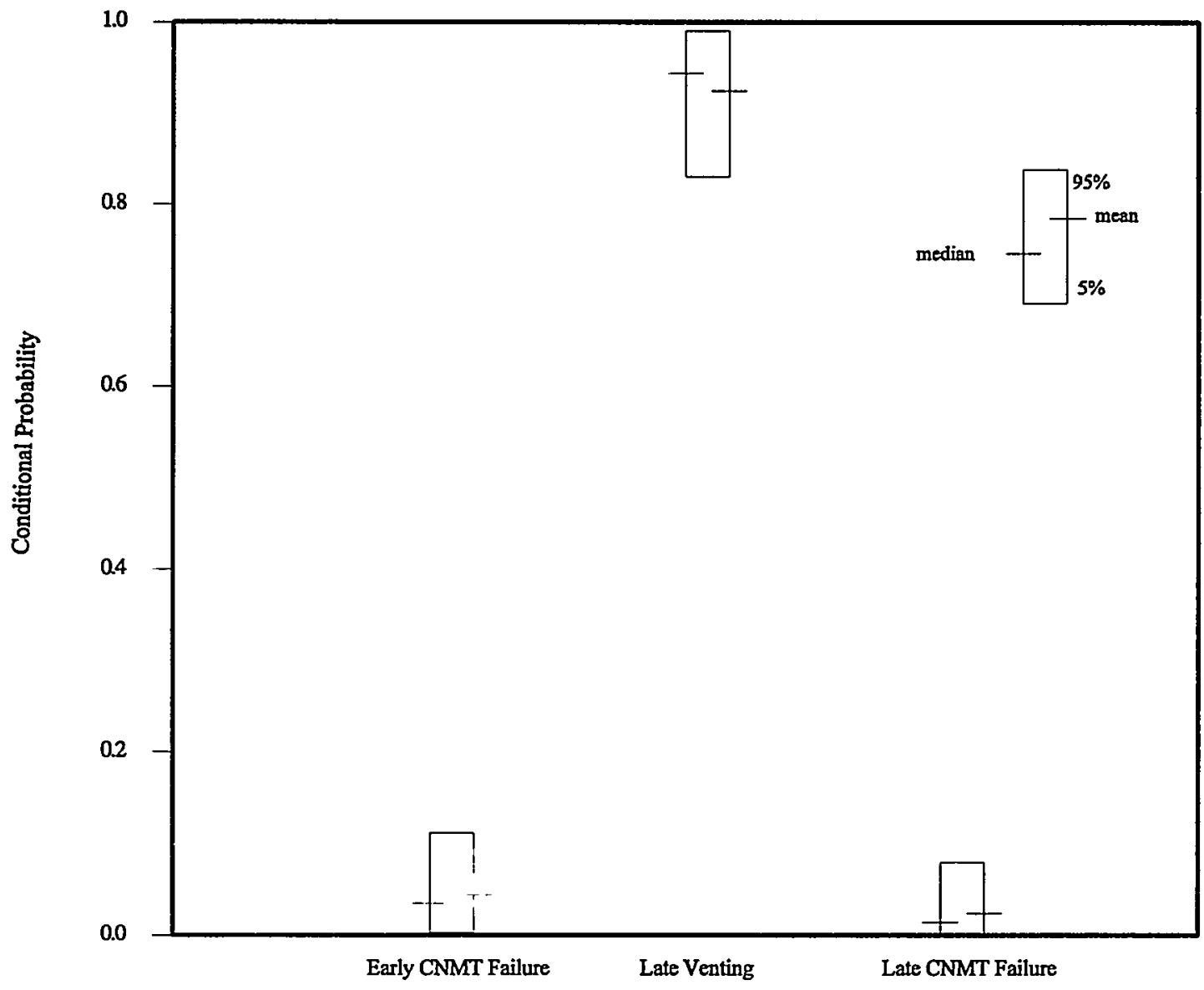

Figure 6.6 Probability of containment failure given that containment is closed before core damage

the cases where the suppression pool is bypassed, the vessel is always predicted to fail and the resulting $\mathrm{CCI}$ occurs in a dry cavity. For these accidents, there is very little attenuation of the release. The accidents that allow the invessel releases to pass through the suppression pool also have a flooded cavity. In these cases the vessel is always predicted to fail; however, there is a substantial probability that the core debris released from the vessel will be quenched. If the debris is not quenched, the $\mathrm{CCI}$ will occur under a pool of water, and any radioactive releases will be scrubbed by the water. For this event, both the in-vessel and a portion of the ex-vessel release will be scrubbed by a pool of water, and therefore it is likely that the release will be less than the previous case.
The configuration of the plant in POS 5 is such that very few plant features are available to attenuate the release when core damage occurs. Contrary to full power, in many of the accidents that could occur during POS 5, the containment is open at the start of the accident, the suppression pool is bypassed, and the containment sprays are not available. Also in these POS 5 accidents, there are very few PDSs where it is possible to recover core cooling and arrest the core damage process in the vessel. Without these features to attenuate the release, a large release to the environment is possible. 


\subsection{References}

[Summers et al., 1991]

[Griesmeyer et al., 1989]

[Higgins, 1989]

[Wheeler et al., 1989]

[Brown et al., 1990]

[Harper et al., 1991]
R. M. Summers et al., "MELCOR 1.8.0: A Computer Code for Nuclear Reactor Severe Accident Source Term and Risk Assessment Analyses," NUREG/CR-5531, SAND900364, Sandia National Laboratories, January 1991.

J. M. Griesmeyer et al., "A Reference Manual for the Event Progression Analysis code (EVNTRE)," NUREG/CR-5174, SAND88-1607, Sandia National Laboratories, September 1989.

S. J. Higgins, "A User's Manual for the Postprocessing Program PSTEVNT," NUREG/CR-5380, SAND88-2988, Sandia National Laboratories, November 1989.

T. A. Wheeler et al., "Analysis of Core Damage Frequency From Internal Events: Expert Judgement Elicitations," NUREG/CR-4550, SAND86-2084, Vol. 2, Part 2, Sandia National Laboratories, April 1989.

T. D. Brown et al., "Evaluation of Severe Accident Risks: Grand Gulf Unit 1," NUREG/CR-4551, SAND86-1309, Vol. 6, Rev. 1, Sandia National Laboratories, December 1990.

F. T. Harper et al., "Evaluation of Severe Accident Risks: Quantification of Major Input Parameters: Experts' Determination of Containment Loads and Molten Core Containment Interaction Issues," NUREG/CR-4551, Vol. 2, Rev. 1, Part 2, Sandia National Laboratories, April 1991. 
Accident Progression Analysis 


\section{Radionuclide Release and Transport Analysis}

The Radionuclide Release and Transport Analysis, commonly referred to as the source term analysis, addresses the release of radioactive material from the fuel and core debris and its subsequent transport and deposition in the primary system and containment. The inputs to the source term analysis are the accident progression bins (APBs) defined in the Accident Progression Analysis. The APBs describe the configuration of the plant, the status of systems that can be used to mitigate the release, and the occurrence of phenomena that can impact the source term. The product of the analysis is a collection of parameters, referred to as the source term, that characterizes the release of radioactive material from the containment ${ }^{1}$ to the environment. The source term is then used in the consequence analysis to estimate health effects attributable to the release of radioactive material for each APB.

\subsection{Definition of the Source Term}

The source term, as defined in this analysis, consists of the following information: the amount and type of radioactive material released from the containment, the timing characteristics of the release, the energy of the release, the elevation of the release, and the time when a general emergency is declared and evacuation plans are initiated (referred to as the warning time). The amount of material released is expressed as a fraction of the radionuclide ${ }^{2}$ inventory present in the core at the start of the accident for the radionuclides considered in this analysis. Although many different radionuclides would be released from the damaged fuel during an accident, health effects from only 60 radionuclides are considered in the consequence analysis. Furthermore, because in the source term analysis the release and transport of these radionuclides are of concern (not the health effects from the radionuclides), it is possible to form groups of radionuclides that are expected to have similar release and transport characteristics. The 60 radionuclides considered in the consequence analysis are combined into the nine release classes shown in Table 7.1. The definition for each release class is based on the definitions used in the NUREG-1150 study as discussed in Volume 1 of NUREG/CR-4551 [Gorham et al., 1993]. Similar to NUREG-1150, the timing of the release is

1 In this context, containment is generalized to include the region in which engineered features are available to attenuate a release before it enters the environment (e.g., the auxiliary building that surrounds the containment building).

2 The terms radionuclide, isotope, and fission product are used interchangeably in this report. characterized by two release segments: the first or early release segment and the second or late release segment. For each release segment, the start time of the release segment (relative to the start of the accident), the duration of the segment, the release fractions for the nine release classes, and the energy release rate are provided.

\subsection{Description of the Source Term Model}

The source term is estimated using a modified version of the parametric code GGSOR that was developed for the NUREG-1150 Grand Gulf plant study [Brown et al., 1990]. The concept of the parametric approach for estimating source terms is discussed in Section 2.0, and the code is described in detail in the XSOR Users Manual [Jow et al., 1993], so they will not be described in detail in this report. Instead, the basic parametric equation will be presented and aspects of the model that were modified will be identified. The version of GGSOR that was used in this analysis, GGSOR-P5, is listed in Appendix C.

GGSOR was modified to reflect plant configuration during plant operating state (POS) 5 and the types of accidents that are possible during this mode of operation. Specifically, GGSOR was modified to account for: (1) loss of coolant accidents (LOCAs) in the containment, (2) interfacing system LOCAs in the auxiliary building, (3) the passage of releases through the auxiliary building, (4) the passage of releases through the reactor pressure vessel head vent, and (5) the timing characteristics of the accidents initiated while the plant is in POS 5.

GGSOR accounts for two releases from the containment. The first release occurs roughly at the time of containment failure. The second begins after the first has finished. When the containment is open prior to core damage, or fails before vessel breach, the first release results from fission products that escape from the fuel while the core is still in the reactor pressure vessel (RPV) (i.e., in-vessel releases) and releases that occur at the time of vessel failure. For this case, the second release includes fission products that are released after vessel breach, which are referred to as the late releases. They include fission products from core-concrete interactions (CCI), material revolatilized from the RPV after vessel breach, and iodine released from the suppression pool (and in some cases the RPV cavity water). For situations where the containment fails many hours after vessel breach, both release segments consist of in-vessel releases, fission products released at vessel breach, and the 
Radionuclide Release and Transport

Table 7.1 Isotopes in each radionuclide release class

\begin{tabular}{|c|c|}
\hline Release Class & Isotopes Included \\
\hline $\begin{array}{l}\text { 1. Inert Gases } \\
\text { 2. Iodine }\end{array}$ & $\begin{array}{l}\mathrm{Kr}-85, \mathrm{Kr}-85 \mathrm{M}, \mathrm{Kr}-87, \mathrm{Kr}-88, \mathrm{Xe}-133, \mathrm{Xe}-135 \\
\mathrm{I}-131, \mathrm{I}-132, \mathrm{I}-133, \mathrm{I}-134, \mathrm{I}-135\end{array}$ \\
\hline 3. Cesium & $\mathrm{Rb}-86, \mathrm{Cs}-134, \mathrm{Cs}-136, \mathrm{Cs}-137$ \\
\hline 4. Tellurium & Sb-127, Sb-129, Te-127, Te-127M, Te-129, Te-129M, Te-131M, Te-132 \\
\hline 5. Strontium & Sr-89, Sr-90, Sr-91, Sr-92 \\
\hline 6. Ruthenium & Co-58, Co-60, Mo-99, Tc-99M, Ru-103, Ru-105, Ru-106, Rh-105 \\
\hline 7. Lanthanum & $\begin{array}{l}\text { Y-90, Y-91, Y-92, Y-93, Zr-95, Zr-97, Nb-95, La-140, La-141, La-142, } \\
\text { Pr-143, Nd-147, Am-241, Cm-242, Cm-244 }\end{array}$ \\
\hline 8. Cerium & Ce-141, Ce-143, Ce-144, Np-239, Pu-238, Pu-239, Pu-240, Pu-241 \\
\hline 9. Barium & $\mathrm{Ba}-139, \mathrm{Ba}-140$ \\
\hline
\end{tabular}

late releases. The timing and duration of these releases depend primarily on the plant damage state (PDS) and the time and mode of containment failure.

For radionuclide class $i$, the basic parametric equation for GGSOR has the following form:

$$
\begin{aligned}
& S_{t}=F_{C O R, t} F_{V E S, t}\left[\left(1-X_{H V S P L T}\right)\left(R_{1}+R_{2}+R_{3}\right)+\frac{X}{D F_{C A V, l}}\right] \frac{F_{C O N V, i}}{D F_{R B, i}} \\
& +F_{\mathrm{VBPUF}, i}\left(R_{4}+R_{\mathrm{S}}\right) \frac{F_{\text {CONC }, i}}{D F_{R B, i}} \\
& +\left(1-F_{C O R, t}-F_{V B P U F, l}\right) X_{L V} X_{E V S E} F_{E V S E, l}\left(R_{6}+R_{7}\right) \frac{F_{C O N C, l}}{D F_{R B, l}} \\
& +\left(1-F_{C O R, t}-F_{V B P U F, i}\right) X_{L V} X_{H P E} F_{D C H, i}\left(R_{6}+R_{7}\right) \frac{F_{C O N C, i}}{D F_{R B, i}} \\
& +\left(1-F_{C O R, t}-F_{V B P U F, l}\right) X_{L V} X_{C C I} F_{C C I, t}\left(R_{8}+R_{9}\right) \frac{F_{C O N C, l}}{D F_{R B, l}} \\
& +F_{C O R, i}\left(1-F_{V E S, l}\right) F_{R E V O, l}\left(R_{10}+R_{11}\right) \frac{F_{C O N C, i}}{D F_{R B, i}} \\
& +\left[F_{L T I I} F_{P O O L I}+F_{L T I Z} F_{C A V W I}\left(R_{12}+R_{13}\right)\right] R_{14}
\end{aligned}
$$

where

$$
\begin{aligned}
& R_{1}=\frac{X_{T L P} X_{P L B Y E}}{D F_{S P R V, i}}, \\
& R_{2}=\frac{X_{T L P}\left(1-X_{P L B Y E}\right)}{M A X\left(D F_{C P A, l}, D F_{S P R V, i}\right)}, \\
& R_{3}=\frac{\left(1-X_{\text {TLP }}\right)}{M A X\left(D F_{V P A, i}, D F_{S P R Y, l}\right)}, \\
& R_{4}=\frac{X_{P L B Y P}}{D F_{S P R C, i}}, \\
& R_{5}=\frac{\left(1-X_{P L B Y P}\right)}{M A X\left(D F_{C P A, i}, D F_{S P R C, i}\right)}, \\
& R_{6}=\frac{X_{P L B Y D}}{D F_{S P R C, i}}, \\
& R_{7}=\frac{\left(1-X_{P L B P D}\right)}{M A X\left(D F_{C P A, i} i D F_{S P R C, i}\right)}, \\
& R_{8}=\frac{X_{P L B Y C}}{M A X\left(D F_{C A V, i}, D F_{S P R C, l}\right)}, \\
& R_{9}=\frac{\left(1-X_{P L B Y C}\right)}{M A X\left(D F_{C A V, i}, D F_{C P A, l}, D F_{S P R C, i}\right)}, \\
& R_{P L B Y C},
\end{aligned}
$$




$$
\begin{aligned}
R_{11} & =\frac{\left(1-X_{P L B P C}\right)}{M A X\left(D F_{C P A, 1}, D F_{S P R C, l}\right)}, \\
R_{12} & =\frac{X_{P L P Y C}}{D F_{C P A, l}}, \\
R_{13} & =\frac{\left(1-X_{P L B Y C}\right)}{D F_{C P A, l}}, \\
R_{14} & =F_{C O N C, 1} \quad \text { (if no containment failure, use } F_{\text {CoNC }} \text { for Noble gases), } \\
& =1.0 \quad \text { (if containment failure), } \\
X_{C C 1} & =1-X_{K P B} \quad \text { (if DCH occurs), } \\
& =1-X_{E Y S B} \quad \text { (if an ex-vessel steam explosion occurs), } \\
& =1.0 \quad \text { (if neither DCH nor ex-vessel steam explosion occurs). }
\end{aligned}
$$

The first term on the right side of Equation 7.1 represents the in-vessel releases. The second term describes the puff release at vessel breach. The third term represents the ex-vessel steam explosion release. The fourth term represents the direct containment heating (DCH) release and is mutually exclusive with the third term. The fifth term represents the core-concrete interaction (CCI) release. The sixth term is the revolatilization release from the reactor coolant system after vessel breach and is for I, Cs, and $\mathrm{Te}$ classes only. The last term represents the evolution of iodine from the suppression pool and reactor cavity water late in the accident. The definitions of the various parameters in Equation 7.1 are as follows:

\section{$\mathrm{F}_{\mathrm{CAVWI}}=\quad$ fraction of initial iodine core inventory scrubbed by the cavity water during the $\mathrm{CCI}$ release.}

$$
\begin{array}{ll}
\mathrm{DF}_{\mathrm{SPRC}, \mathrm{i}}= & \begin{array}{l}
\text { scrubbing decontamination factor for } \\
\text { sprays acting on species i released into } \\
\text { containment after vessel breach. }
\end{array} \\
\mathrm{DF}_{\mathrm{SPRV}, \mathrm{i}}=\quad & \begin{array}{l}
\text { scrubbing decontamination factor for } \\
\text { sprays acting on species i released into } \\
\text { containment before vessel breach. }
\end{array}
\end{array}
$$

$$
\mathrm{DF}_{\mathrm{CAv}, \mathrm{i}}=
$$$$
\text { scrubbing decontamination factor for }
$$
aerosol species i released into cavity water during $\mathrm{CCI}$ release.

$$
\begin{array}{ll}
\mathrm{DF}_{\mathrm{CPA}, \mathrm{i}}= & \begin{array}{l}
\text { scrubbing decontamination factor for } \\
\text { aerosol species i flowing from drywell to } \\
\text { the suppression pool. }
\end{array} \\
\mathrm{DF}_{\mathrm{VPA}, \mathrm{i}}= & \begin{array}{l}
\text { scrubbing decontamination factor for } \\
\text { aerosol species i flowing from the vessel } \\
\text { to the suppression pool. }
\end{array}
\end{array}
$$

$$
\begin{aligned}
& \mathrm{F}_{\mathrm{CCI}, \mathrm{i}}=\quad \begin{array}{l}
\text { fraction of material released from the } \\
\text { melt during molten CCI. }
\end{array} \\
& \mathrm{F}_{\mathrm{CONC}, \mathrm{i}}=\quad \begin{array}{l}
\text { fraction of species i released from } \\
\text { containment for CCI and other releases } \\
\text { after vessel breach, not including the } \\
\text { effects of scrubbing by pools and sprays. }
\end{array} \\
& \mathrm{F}_{\mathrm{CONV}, \mathrm{i}}=\quad \begin{array}{l}
\text { fraction of species i released from } \\
\text { containment for material released into } \\
\text { containment before vessel breach, not } \\
\text { including the effects of scrubbing by } \\
\text { pools and sprays. }
\end{array}
\end{aligned}
$$$$
\mathrm{F}_{\mathrm{COR}, \mathrm{i}}=
$$$$
\text { fraction of initial inventory of species } i
$$
released from the fuel prior to vessel failure.

$\mathrm{F}_{\mathrm{DCH}, \mathrm{i}}=$

$\mathrm{X}_{\mathrm{EVSE}}=$

$\mathrm{F}_{\mathrm{EVSE}, \mathrm{i}}=$

$\mathrm{X}_{\mathrm{HPE}}=$

$\mathrm{X}_{\mathrm{LV}} \quad=$

$\mathrm{F}_{\mathrm{REVO}, \mathrm{i}}=$

$\mathrm{X}_{\mathrm{PLBYC}}=$

$\mathrm{X}_{\mathrm{PLBYD}}=$

fraction of species $i$ in the portion of the core involved in direct containment heating that is released to the drywell at vessel breach.

fraction of core material leaving the vessel that participates in an ex-vessel steam explosion and therefore is not available to participate in $\mathrm{CCI}$.

fraction of species $i$ in the portion of the core involved in an ex-vessel steam explosion that is released to the drywell at vessel breach.

fraction of core material leaving the vessel that participates in direct containment heating and therefore is not available to participate in CCI.

fraction of the core material that leaves the vessel after the vessel breach.

fraction of species i deposited on the surfaces of the reactor vessel and structural materials that is revaporized and released in the drywell after vessel breach.

fraction of $\mathrm{CCI}$ releases that bypass the suppression pool.

fraction of DCH releases or ex-vessel steam explosion releases that bypass the suppression pool. 


\begin{tabular}{|c|c|c|c|c|c|}
\hline $\mathrm{X}_{\mathrm{PLBYE}}$ & $=$ & $\begin{array}{l}\text { fraction of in-vessel releases that bypass } \\
\text { the suppression pool. }\end{array}$ & & & $\begin{array}{l}\text { initiated (evacuation starts a short time } \\
\text { later, however, because of the time it } \\
\text { takes to implement evacuation). }\end{array}$ \\
\hline $\mathrm{X}_{\mathrm{PLBYP}}$ & $=$ & $\begin{array}{l}\text { fraction of puff releases at vessel breach } \\
\text { that bypass the suppression pool. }\end{array}$ & $\mathrm{T} 1$ & $=$ & start of release segment 1 . \\
\hline$X_{\mathrm{TLP}}$ & $=$ & $\begin{array}{l}\text { fraction of the in-vessel releases that are } \\
\text { released into the drywell through } \\
\text { stuck-open safety relief valve (SRV) } \\
\text { tailpipe vacuum breakers. }\end{array}$ & $\begin{array}{l}\text { D1 } \\
\text { E1 }\end{array}$ & $=$ & $\begin{array}{l}\text { duration of release segment } 1 . \\
\text { energy release rate associated with release } \\
\text { segment } 1 .\end{array}$ \\
\hline$F_{V E S, i}$ & $=$ & $\begin{array}{l}\text { fraction of species i released from the fuel } \\
\text { that is released from the vessel. }\end{array}$ & $\mathrm{T} 2$ & $=$ & start of release segment 2 . \\
\hline$F_{\text {LTII }}$ & $=$ & $\begin{array}{l}\text { fraction of iodine in the suppression pool } \\
\text { that is volatilized and released after vessel } \\
\text { breach. }\end{array}$ & $\mathrm{E} 2$ & $=$ & $\begin{array}{l}\text { aurauon of release segment } L \text {. } \\
\text { energy release rate associated with release } \\
\text { segment } 2 \text {. }\end{array}$ \\
\hline
\end{tabular}

$\mathrm{F}_{\mathrm{LT12}}=$ fraction of iodine in the cavity water that is volatilized and released after vessel breach.
$\mathrm{X}_{\text {HVSPLT }}=\quad$ fraction of in-vessel release that passes through the reactor head vent.
$\mathrm{F}_{\mathrm{POOLI}}=$ fraction of initial core inventory for iodine scrubbed by the pool.
$\mathrm{DF}_{\mathrm{RB}, \mathrm{i}}=$ decontamination factor for aerosol species i flowing from the containment to the auxiliary building.

$\mathrm{S}_{\mathrm{i}} \quad=\quad$ fraction of the initial core inventory of species $i$ that is ultimately released to the environment.

$F_{\text {VBPUF, } i}=\quad$ fraction of initial core inventory of species $i$ that is released to the drywell as puff at the time of vessel breach.

$\mathrm{X}_{\mathrm{CCI}}=$ fraction of core material that leaves the vessel and participates in CCI.

In addition to the parameters that appear in Equation 7.1, a series of parameters are used to define (1) the timing of the release relative to the start of the accident and (2) other characteristics of the release that are important for the determination of the consequences. These additional parameters include:

TW = warning time, which is taken to correspond to when a general emergency is declared and evacuation procedures are
A detailed discussion of this equation is presented in NUREG/CR-5360 [Jow et al., 1993].

\subsection{Quantification of Source Term Model}

Information from NUREG-1150 was used extensively throughout this study; the source term analysis is no exception. It was assumed that the distributions for the parameters in Equation 7.1 developed in the NUREG-1150 study could, in most cases, also be used in this study. ${ }^{3}$ This assumption was based on guidance provided by an internal Source Term Advisory Group, which was convened for an earlier portion of the study (see Appendix F) to provide guidance on the use of existing methods and data, to review the source term issues being treated, and to identify any new issues that may be important to shutdown accidents. Guidance provided by this group was factored into this study of POS 5. As stated earlier (see Chapter 3), the methods used in the NUREG-1150 study were also used in this study. The rationale for selecting the parametric approach is discussed in Chapter 3. Guidance provided by the review group did not indicate anything fundamentally

3 Here the discussion concerns the parameters that are used to accident that is released to the environment. By using the same parameters it is implied that for a similar accident, the release fractions for a full power accident and a shutdown accident will be the same. Although the release fractions may be the same, the amount of radioactive material release to the environment will not be the same because of the differences in the radioactive inventories at the start of the accident. determine the fraction of the inventory at the start of the 
different with these shutdown accidents, compared to full power accidents, that would make the parametric approach unacceptable for its intended use. Based on a review of the expected accidents and their associated phenomena as well as results from MELCOR calculations performed for these shutdown accidents, the review group guidance also suggested that it would be acceptable to use the source term parameters developed in the NUREG-1150 as a starting point for this study, considering the large uncertainties already reflected by the distributions and the objectives of the study. This guidance is supported by a comparison of selected results from a series of MELCOR calculations of shutdown accidents with the NUREG-1150 full power source term distributions, which suggested that the two sets of information were in qualitative agreement. In most cases the results from the MELCOR calculations fell within the large uncertainty bans of the NUREG-1150 source term distributions. Although it was suggested that the NUREG1150 distributions could be used as a starting point, the guidance also warned that the distributions should be modified or that new distributions should be added when the plant configuration or other characteristics of the accident were sufficiently different from the full power conditions to introduce different initial conditions or phenomena. The release fractions and source terms predicted by MELCOR were generally not used directly in this analysis because the models and data used in MELCOR present one view of a severe accident; alternative and conflicting views also exist. The distributions from NUREG-1150 are based on results from formal expert judgment elicitations that consider and account for these alternative views. Selected results from MELCOR were used in this analysis, however, when the quantification of the parameter was very accident-specific (e.g., the timing of the release) or when a new parameter was added for this analysis and data from other sources were not available.

A review by the project staff of the POS 5 accidents indicated that in most cases the plant configuration and accident conditions associated with these accidents were sufficiently similar to the full power accidents to render modifications to the NUREG-1150 distributions unnecessary. Many of the source term parameters defined in the NUREG-1150 study depended on conditions in the containment and/or core and were not necessarily tied to specific accident sequences (e.g., $\mathrm{F}_{\mathrm{COR}}$ depends on the amount of zirconium oxidized in the core). In these cases, shutdown accidents with similar containment/core conditions were associated with the relevant parameter values. In other cases, however, the parameter values used in NUREG-1150 were tied to a specific accident sequence (e.g., short-term station blackout). In these cases, the rationale behind the quantification of the parameter was reviewed and the shutdown accident sequence that most closely matched the relevant attributes of the full power accident were associated with that parameter value. For some scenarios it was necessary to add additional parameters and distributions to reflect the configuration of the plant as it operates in POS 5. When new parameters were added for this analysis, data were obtained from relevant NUREG-1150 cases or from relevant MELCOR calculations that were performed for this analysis. Information from MELCOR calculations performed for this study was also used to quantify the source term parameters that define the timing and energy of the release because these parameters tend to be accident-specific, so the use of full power data would not be appropriate in this instance (the MELCOR calculations are documented in Volume 6, Part 2 of this report). For many accidents, the timing parameters are tightly coupled with the decay heat load available during the accident. The values assigned to the various parameters are listed in Appendix C.

Two of the parameters that appear in Equation 7.1 were not used in the NUREG-1150 Grand Gulf analysis: the fraction of radionuclides that passes through the reactor head vent $\left(\mathrm{X}_{\mathrm{HVSPLT}}\right)$ and the decontamination factor (DF) for the reactor building $\left(\mathrm{DF}_{\mathrm{RB}}\right)$. During full power operation, the reactor vessel head vent is closed; therefore $\mathrm{X}_{\mathrm{HVSPLT}}$ is not an issue. During shutdown, the reactor head vent is open at the start of the accident, allowing for the possibility that some of the in-vessel release will leave the vessel via the head vent. The value used for the fraction of the in-vessel release that passes through the head vent, $X_{\mathrm{HVSPLT}}$, was obtained from MELCOR calculations performed for this study. In the NUREG-1150 Grand Gulf analysis, the containment never failed into the auxiliary building, and therefore a DF for the auxiliary building was not required. In this analysis, however, the containment equipment hatch is often open, allowing radioactive material in the containment atmosphere to pass through the auxiliary building before escaping to the environment. While a reactor building DF was not developed for the Grand Gulf plant in NUREG-1150, reactor building DFs were developed for the Peach Bottom plant by the NUREG-1150 Source Term Expert Panel. Based on a review of the experts' rationale for the Peach Bottom DFs, it was determined that many factors (i.e., specification of initial and boundary conditions) present in the Grand Gulf analysis would make the calculation of the DF uncertain; thus the use of a single value as calculated from MELCOR would not be appropriate. Instead, it was decided by the project staff that the DF uncertainty distribution developed in NUREG-1150 for the Peach Bottom reactor building would be an acceptable surrogate for the Grand Gulf DF. In the Peach Bottom probabilistic risk assessment (PRA), 
different DFs were developed for different types of accidents. Because in these shutdown accidents the flow rate of steam and noncondensibles in the auxiliary building is fairly low and a considerable amount of condensation of steam exists on the structures in the building, the Peach Bottom DF selected for this study corresponded to accidents where there was little blow-down from the containment into the auxiliary building following containment failure (i.e., low flow rate of gases from containment to auxiliary building) and where a considerable amount of steam was available to condense. An assessment of the impact that the standby gas treatment system or the auxiliary building ventilation system would have on the reactor building DF was beyond the scope of this analysis; hence these systems were not modeled in this analysis.

The sources of information used to quantify the parameters in Equation 7.1 are listed in Table 7.2; the parameters included in the uncertainty analysis are also identified in this table. Sixteen of the parameters in Equation 7.1 were sampled in the uncertainty analysis; distributions for these parameters were based on information from the NUREG1150 study. Ten of these sixteen parameters were generated by the NUREG-1150 Source Term Expert Panel. For each parameter that was assessed by the panel, the distribution for the parameter, the reasoning that led each expert to his conclusions, and the aggregation of the individual distributions are fully described in NUREG/CR-4551, Volume 2, Part 4 [Harper et al., 1992]. The remaining parameters included in the uncertainty analysis were quantified by the NUREG- 1150 project staff and are discussed in NUREG-5360 [Jow et al., 1993].

Unless specifically identified, there is no correlation between any of the source term variables, but there is complete correlation within a variable. $\mathrm{F}_{\mathrm{COR}}$ is not correlated with $F_{\mathrm{VES}}, \mathrm{F}_{\mathrm{CONV}}$, or any other variable, but the values for the different cases for a given parameter and for the different radionuclide classes are completely correlated. That is, if the 0.05 quantile value is chosen for iodine for low zirconium oxidation, the 0.05 quantile value is also chosen for all the other radionuclide classes and for all values for high zirconium oxidation.

\subsection{Partitioning of Source Terms}

As discussed above, a source term is estimated for each APB. Furthermore, because the uncertainty analysis includes variables from the source term analysis, there will be many source term estimates for a single APB. In this analysis, approximately 35,000 source terms were generated. Because of the large computational requirements of the consequence analysis, consequences cannot be estimated for each individual source term. Instead, the individual source terms that are expected to result in similar early and chronic health effects are combined into source term groups (STGs); the PARTITION program [Iman et al., 1990] as modified for the LaSalle PRA in the PRUEP program [Brown et al., 1992] is used to form the STGs. The source term for each STG is a frequency-weighted average of the source terms for each of the APBs that are contained in the STG. Because a different radionuclide inventory will be used for each time window in the consequence calculations, the source terms from each time window were partitioned separately to ensure that each STG only contained source terms from a single time window.

\subsection{Results}

The source term is a collection of 28 parameters that characterize the radioactive release to the environment, and the health effects that result from the release are a complicated function of these parameters and additional parameters that affect the transport and deposition of the material in the environment. Therefore it is neither convenient nor particularly useful to infer the impact of the accident based on the source term-particularly when a consequence analysis, which will take these factors into account, is being performed. Furthermore, because source terms were generated for several hundred APB, it is not practical to present the source term for individual APBs. Instead, to document the product of the source term analysis, the source terms for the STGs are presented. The partitioning process resulted in the generation of 54 STGs. The source term associated with each STG is provided in Appendix C. A discussion of the effect that different accident characteristics have on risk will be deferred to chapter 9 of this report. 
Table 7.2 Sources of information used to quantify the source term parameters

\begin{tabular}{|c|c|c|}
\hline $\begin{array}{l}\text { Source Term } \\
\text { Parameter }\end{array}$ & $\begin{array}{l}\text { Included in } \\
\text { Uncertainty Analysis }\end{array}$ & Quantification Source \\
\hline $\mathrm{F}_{\text {CAVWI }}$ & No & Determined by various combination of other parameters \\
\hline $\mathrm{DF}_{\mathrm{SPRC}}$ & Yes & $\begin{array}{l}\text { NUREG-1150 Project Staff (DF } \mathrm{SPRC}_{\mathrm{SR}} \text { and } \mathrm{DF}_{\mathrm{SPRV}} \text { are completely correlated, } \\
\text { i.e., same LHS variable used for both distributions) }\end{array}$ \\
\hline $\mathrm{DF}_{\mathrm{SPRV}}$ & Yes & $\begin{array}{l}\text { NUREG-1150 Project Staff (DF } \\
\text { i.e., same LHS variable used for both distributions) }\end{array}$ \\
\hline $\mathrm{DF}_{\mathrm{CAV}}$ & Yes & NUREG-1150 Project Staff \\
\hline $\mathrm{DF}_{\mathrm{CPA}}$ & Yes & $\begin{array}{l}\text { NUREG-1150 Project Staff (DF } \mathrm{CPA}_{\mathrm{CA}} \text { and } \mathrm{DF}_{\mathrm{VPA}} \text { are completely correlated, i.e., } \\
\text { same LHS variable used for both distributions) }\end{array}$ \\
\hline $\mathrm{DF}_{\mathrm{VPA}}$ & Yes & $\begin{array}{l}\text { NUREG-1 } 150 \text { Project Staff (DF } \mathrm{CPA}_{\mathrm{CA}} \text { and } \mathrm{DF}_{\mathrm{VPA}} \text { are completely correlated, i.e., } \\
\text { same LHS variable used for both distributions) }\end{array}$ \\
\hline $\mathrm{F}_{\mathrm{CCI}}$ & Yes & $\begin{array}{l}\text { NUREG-1150 Source Term Expert Panel } \\
\text { Case 1: Coolable Debris Bed }\left(\mathrm{F}_{\mathrm{ccl}} \text { set to } 0.0\right) \\
\text { Case 2: Dry cavity with high level of zirconium oxidized in the core } \\
\text { Case 3: Dry cavity with low level of zirconium oxidized in the core } \\
\text { Case 4: Flooded cavity } w / \text { high level of zirconium oxidized in the core } \\
\text { Case 5: Flooded cavity with low level of zirconium oxidized in the core }\end{array}$ \\
\hline $\mathrm{F}_{\text {CONC }}$ & Yes & $\begin{array}{l}\text { NUREG-1150 Source Term Expert Panel } \\
\text { Case 1: No containment failure } \\
\text { Case 2: Early containment leak, subcooled suppression pool } \\
\text { Case 3: Early containment leak, saturated suppression pool } \\
\text { Case 4: Early containment rupture (includes case where containment } \\
\text { equipment hatch is open), subcooled suppression pool } \\
\text { Case 5: Early containment rupture (includes case where containment } \\
\text { equipment hatch is open), subcooled suppression pool } \\
\text { Case 6: Late containment leak } \\
\text { Case 7: Late containment rupture }\end{array}$ \\
\hline $\mathrm{F}_{\text {CoNv }}$ & Yes & $\begin{array}{l}\text { NUREG-1150 Source Term Expert Panel } \\
\text { Same cases as } F_{\text {conc }} \text { with the following consideration: } \\
\text { Case 8: LOCA in auxiliary building or open MSIVs, } F_{\text {CoNv }}=1.0\end{array}$ \\
\hline $\mathrm{F}_{\mathrm{COR}}$ & Yes & $\begin{array}{l}\text { NUREG-1150 Source Term Expert Panel } \\
\text { Case 1: High level of zirconium oxidation in the core } \\
\text { Case 2: Low level of zirconium oxidation in the core }\end{array}$ \\
\hline $\mathrm{F}_{\mathrm{DCH}}$ & Yes & NUREG-1150 Source Term Expert Panel \\
\hline $\mathrm{X}_{\mathrm{EVSE}}$ & No & $\begin{array}{l}\text { NUREG-1150 Project Staff } \\
\text { Case 1: Large amount of core debris participates in steam explosion } \\
\text { Case 2: Small amount of core debris participates in steam explosion }\end{array}$ \\
\hline $\mathrm{F}_{\text {EVSE }}$ & Yes & NUREG-1150 Project Staff \\
\hline
\end{tabular}


Radionuclide Release and Transport

Table 7.2 Sources of information used to quantify the source term parameters (continued)

\begin{tabular}{|c|c|c|}
\hline $\begin{array}{l}\text { Source Term } \\
\text { Parameter }\end{array}$ & $\begin{array}{c}\text { Included in } \\
\text { Uncertainty Analysis }\end{array}$ & Quantification Source \\
\hline$X_{\mathrm{HPE}}$ & No & $\begin{array}{l}\text { NUREG-1150 Project Staff } \\
\text { Case 1: Large amount of core debris participates in HPME } \\
\text { Case 2: Small amount of core debris participates in HPME }\end{array}$ \\
\hline $\mathrm{F}_{\mathrm{REVO}}$ & Yes & $\begin{array}{l}\text { NUREG-1150 Source Term Expert Panel } \\
\text { Case 1: No vessel failure }\left(\mathrm{F}_{\mathrm{REV}}=0.0\right) \\
\text { Case 2: Injection available after vessel failure } \\
\text { Case 3: No injection available after vessel failure }\end{array}$ \\
\hline$X_{\text {PLBYC }}$ & No & Set to 1.0 ; drywell personnel lock and/or equipment hatch open \\
\hline $\mathrm{X}_{\mathrm{PLBYD}}$ & No & Set to 1.0 ; drywell personnel lock and/or equipment hatch open \\
\hline $\mathrm{X}_{\text {PLBYE }}$ & No & NUREG-1150 Project Staff \\
\hline$X_{\text {PLBYP }}$ & No & Set to 1.0 ; drywell personnel lock and/or equipment hatch open \\
\hline $\mathrm{X}_{\mathrm{TLP}}$ & No & $\begin{array}{l}\text { NUREG-1150 Project Staff } \\
\text { Case 1: Reactor vessel is at low pressure during core damage } \\
\text { Case 2: Reactor vessel is at high pressure during core damage }\end{array}$ \\
\hline $\mathrm{F}_{\mathrm{VES}}$ & Yes & $\begin{array}{l}\text { NUREG-1150 Source Term Expert Panel } \\
\text { Case 1: Reactor vessel at high pressure during core damage } \\
\text { Case 2: Reactor vessel at low pressure during core damage }\end{array}$ \\
\hline$F_{L T 11}$ & Yes & $\begin{array}{l}\text { NUREG-1150 Source Term Expert Panel }\left(\mathrm{F}_{\mathrm{LT11}} \text { and } \mathrm{F}_{\mathrm{LTT2}} \text { are completely }\right. \\
\text { correlated, i.e., same LHS variable used for both distributions) } \\
\text { Case 1: Subcooled suppression pool } \\
\text { Case 2: Saturated suppression pool }\end{array}$ \\
\hline $\mathrm{F}_{\mathrm{LT12}}$ & Yes & $\begin{array}{l}\text { NUREG-1150 Source Term Expert Panel }\left(F_{L T 1} \text { and } F_{L T 12} \text { are completely }\right. \\
\text { correlated, i.e., same LHS variable used for both distributions) } \\
\text { Case 1: Dry cavity } \\
\text { Case 2: Flooded cavity } \\
\text { Case 3: No core-concrete interactions in cavity }\end{array}$ \\
\hline $\mathrm{X}_{\text {HVSPLT }}$ & No & $\begin{array}{l}\text { MELCOR calculations for POS } 5 \\
\text { Case 1: Head vent open } \\
\text { Case 2: Head vent closed }\left(\mathrm{X}_{\text {HVSPLT }}=0.0\right)\end{array}$ \\
\hline $\mathrm{F}_{\mathrm{POOLI}}$ & No & Determined by various combination of other parameters \\
\hline $\mathrm{DF}_{\mathrm{RB}}$ & Yes & $\begin{array}{l}\text { NUREG-1150 Source Term Expert Panel } \\
\text { (Distribution from NUREG-1150 Peach Bottom Analysis: drywell shell } \\
\text { melt-through with saturated suppression pool) }\end{array}$ \\
\hline $\mathrm{F}_{\mathrm{VBPUF}}$ & Yes & NUREG-1150 Project Staff \\
\hline $\mathrm{X}_{\mathrm{cCI}}$ & No & Determined by various combination of other parameters \\
\hline
\end{tabular}


Table 7.2 Sources of information used to quantify the source term parameters (concluded)

\begin{tabular}{|c|c|c|}
\hline $\begin{array}{c}\text { Source Term } \\
\text { Parameter }\end{array}$ & $\begin{array}{c}\text { Included in } \\
\text { Uncertainty Analysis }\end{array}$ & Quantification Source \\
\hline TW & No & $\begin{array}{l}\text { MELCOR calculations for POS } 5 \\
\text { Case 1: PDS1-1 } \\
\text { Case 2: PDS1-2 and PDS1-4 } \\
\text { Case 3: PDS1-3 } \\
\text { Case 4: PDS1-5 } \\
\text { Case 5: PDS2-1 } \\
\text { Case 6: PDS2-2 and PDS2-3 } \\
\text { Case 7: PDS2-4 } \\
\text { Case 8: PDS2-5: Containment equipment hatch is open } \\
\text { Case 9: PDS2-5: Containment fails during core damage } \\
\text { Case 10: PDS2-5: Containment fails at vessel breach } \\
\text { Case 11: PDS2-5: Containment vented or fails late in the accident } \\
\text { Case 12: PDS2-6 } \\
\text { Case 13: PDS3-1 }\end{array}$ \\
\hline $\mathrm{T} 1$ & No & $\begin{array}{l}\text { MELCOR calculations for POS } 5 \\
\text { Same cases as TW except that Cases } 1,4,5,7 \text {, and } 13 \text { are divided to } \\
\text { distinguish between cases where the auxiliary building fails during core } \\
\text { damage from those that fail at the time of vessel failure }\end{array}$ \\
\hline D1 & No & $\begin{array}{l}\text { MELCOR calculations for POS } 5 \\
\text { Case 1: Containment rupture or venting } \\
\text { Case 2: Auxiliary building failure at the time of vessel failure } \\
\text { Case 3: Short duration } \\
\text { Case 4: Medium duration } \\
\text { Case 5: Long duration }\end{array}$ \\
\hline E1 & No & $\begin{array}{l}\text { MELCOR calculations for POS } 5 \\
\text { Case 1: Flooded CNMT \& Aux. Bldg failure at VB (no } \mathrm{H}_{2} \text { burn) } \\
\text { Case 2: Aux. Bldg fails during } \mathrm{CD}\left(\mathrm{H}_{2} \text { burn) or } \mathrm{CNMT} \text { fails via rupture }\right. \\
\text { Case 3: } \mathrm{SBO} \text { and the Aux. Bldg fails at beginning of } \mathrm{CD} \text {, or } \\
\text { CNMT fails via a leak, or CNMT open before core damage } \\
\text { Case 4: } \mathrm{PDS} 2-6 \text { (Aux. Bldg fails prior to core damage) }\end{array}$ \\
\hline $\mathrm{T} 2$ & No & Combination of previously defined parameters $(\mathrm{T} 2=\mathrm{T} 1+\mathrm{D} 1)$ \\
\hline $\mathrm{D} 2$ & No & $\begin{array}{l}\text { MELCOR calculations for POS } 5 \\
\text { Case 1: No vessel breach } \\
\text { Case 2: All other cases }\end{array}$ \\
\hline $\mathrm{E} 2$ & No & $\begin{array}{l}\text { MELCOR calculations for POS } 5 \\
\text { Case 1: No vessel breach } \\
\text { Case 2: CNMT not flooded } \\
\text { Case 3: Flooded CNMT }\end{array}$ \\
\hline
\end{tabular}




\subsection{References}

[Gorham et al., 1993]

[Brown et al., 1990]

[Jow et al., 1993]

[Cybulskis et al., 1989]

[Harper et al., 1992]

[Iman et al., 1990]

[Brown et al., 1992]
E. D. Gorham et al., "Evaluation of Severe Accident Risks: Methodology for the Containment Source Term, Consequence, and Risk Integration Analysis," NUREG/CR-4551, SAND86-1309, Vol. 1, Rev. 1, Sandia National Laboratories, December 1993.

T. D. Brown et al., "Evaluation of Severe Accident Risks: Grand Gulf Unit 1," NUREG/CR-4551, SAND86-1309, Vol. 6, Rev. 1, Sandia National Laboratories, December 1990.

H.-N. Jow et al., "XSOR Code User's Manual," NUREG/CR-5360, SAND890943, Sandia National Laboratories, November 1993.

P. Cybulskis et al., "Assessment of the XSOR Codes," NUREG/CR-5346, BMI2171, Battelle Columbus Division, November 1989.

F. T. Harper, "Evaluation of Severe Accident Risks: Quantification of Major Input Parameters: Experts' Determination of Source Term Issues," NUREG/CR4551, Vol. 2, Rev. 1, Part 4, Sandia National Laboratories, June 1992.

R. L. Iman et al., "PARTITION: A Program for Defining the Source Term/Consequence Interface in the NUREG-1150 Probabilistic Risk Assessments," NUREG/CR-5262, SAND88-2940, Sandia National Laboratories, May 1990.

T. D. Brown et al., "Integrated Risk Assessment for the LaSalle Unit 2 Nuclear Power Plant," NUREG/CR-5305, SAND90-2765, Vols. 1-2, Sandia National Laboratories, August 1992. 


\section{Consequence Analysis}

As is typically done in probabilistic risk assessments (PRAs) of nuclear power plants, the consequences to the general public that result from a release of radioactive material were estimated. In this study, onsite doses were also estimated, something not typically estimated in full power PRAs of nuclear power plants. Another important difference between this analysis and those previously performed for full power accidents is that the radionuclides in the fuel have had, in some cases, a significant amount of time to decay, resulting in a different inventory than was present at shutdown. The ORIGEN2 code [Croff, et al., 1989] was used to calculate the inventories associated with the shutdown accidents analyzed in this study; a unique inventory was defined for each of the three time windows. The resulting inventories were reduced to include only the 60 radionuclides currently available in the MACCS code [Chanin et al., 1990; Jow et al., 1990; Rollstin et al., 1990]. These 60 radionuclide inventories were then used as the basis for both the onsite and offsite consequence calculations. These inventories, which do not include short-lived radionuclides, are appropriate for both the onsite and offsite analyses because the reactor has been in shutdown for at least seven hours at the beginning of the accident, thus allowing decay of the short-lived radionuclides. The radionuclide inventories are provided in Appendix D.1. The following subsections provide an overview of the methodology and list the pertinent results for both the onsite and offsite consequences.

\subsection{Onsite Consequences}

During shutdown the plant is in a configuration in which many of the systems designed to contain and mitigate a release are unavailable (e.g., during POS 5 the containment is open to the auxiliary building). Without these mitigative features, a release of radioactive material can pose an immediate hazard to personnel at the plant. For this reason, a method for estimating the potential doses to onsite personnel was developed as part of this study. Because onsite consequences have seldom been considered in the analysis of severe accidents at nuclear power plants, an accepted methodology for performing onsite consequence assessments does not exist; therefore, a simple scoping analysis was performed in this study. In this onsite consequence assessment, only the doses and dose rates are estimated for the surrounding region near the plant (referred to as the parking lot region); health effects to the onsite population are not estimated in this study. Because many simplifying assumptions are used in this assessment, the calculations should be viewed as scoping in nature. The intent of these calculations is to provide some insight into the potential magnitude of the onsite doses and dose rates. The method and results are discussed in the following two sections.

\subsubsection{Method}

Doses and dose rates were estimated for a range of distances from the reactor. For comparative purposes, two different wake-effect models were used to estimate the relative concentrations downwind of the reactor. The first model was developed by Ramsdell [1990]. The second model actually consists of two models: a model developed by Wilson, which was used to estimate doses within 100 meters of the plant, and a model utilized by the NRC, which was used to estimate doses beyond 100 meters [Wilson, 1984; USNRC, 1982]. The Ramsdell model was developed by using multiple linear regression to fit experimental results to a statistical model that included the following four variables: (1) wind speed, (2) distance, (3) building area, and (4) stability. The result was that the exponent on each of these variables was determined by the experimental results. The Wilson and NRC models are based on Gaussian plume theory. The NRC model allows for plume meander during low speed and stable atmospheric conditions for distances less than $800 \mathrm{~m}$. The Wilson models uses experimental data to fit the Gaussian model to the relative concentration for distances very close to the release point. Relative concentration marks an interesting difference between the two sets of models: In the Ramsdell model, the relative concentration is somewhat proportional to the wind speed and the stability class; in the Wilson and NRC models, the relative concentration is predicted to be inversely proportional to the wind speed. Using the integrated air concentrations for each building wake-effect model, the dose and dose rate were estimated for each partitioned source term group (see Section 7).

The major simplifying assumptions used in this analysis were that radioactive decay was neglected during the exposure time, the directional dependence of the weather was ignored, and a single radioactive release location and building area was assumed. Two different weather scenarios were used for each set of models. The first scenario assumes stable conditions (stability class $F$ ) with a wind speed of $1 \mathrm{~m} / \mathrm{s}$. The second scenario assumes unstable conditions (stability class A) with a wind speed of $5 \mathrm{~m} / \mathrm{s}$. The dose calculation considered exposure from both the immersion and inhalation pathways and is a 50-year committed dose. The dose rate only considered exposure from the immersion pathway. Doses were calculated assuming both exposure to the entire release and exposure 
to only the first 15 minutes of the release. A dose rate was calculated for each of the release segments defined in the source term analysis. The code that implements these models is listed in Appendix D.2.

\subsubsection{Results}

Table 8.1-1 contains the mean total dose, 15-minute dose, and dose rates for each segment of the release based on the Wilson/Reg. Guide building wake effect model for several distances from the containment and for the two weather conditions. Similar estimates of mean doses and dose rates based on the Ramsdell model are shown in Table 8.1-2. Note that one cannot multiply the dose rate in Table 8.1-1 and 8.1-2 by the exposure time to determine the corresponding total dose because the dose rate is only based on the immersion pathway, while the total dose includes exposure from both the immersion and the inhalation pathways and is a 50-year committed dose. Results from these calculations for each of the source term groups are provided in Appendix D.3. The only uncertainties included in the onsite analysis were the uncertainties in the magnitude of the source term and the likelihood of the source term; an assessment of the uncertainties in the onsite consequence models and the inputs to these models was beyond the scope of this project. Because these calculations are scoping in nature and because they are intended only to provide some insight into the potential magnitude of the onsite doses and dose rates, only mean values are provided, with the understanding that the uncertainty associated with these estimates could be large.

Because the relative air concentration predicted by the Wilson/Reg. Guide model is inversely proportional to the wind speed, the dose and dose rates that correspond to a wind speed of $1 \mathrm{~m} / \mathrm{s}$ are larger than the doses that correspond to a wind speed of $5 \mathrm{~m} / \mathrm{s}$. The opposite relationship holds for the Ramsdell model. For the weather condition with F stability and a wind speed of $1 \mathrm{~m} / \mathrm{s}$, the doses predicted by the Wilson/Reg. Guide model are considerably greater than the corresponding doses predicted by the Ramsdell model. For the weather condition with $\mathrm{A}$ stability and a wind speed of $5 \mathrm{~m} / \mathrm{s}$, the doses predicted by the Wilson/Reg. Guide model are greater than the corresponding doses predicted by the Ramsdell model when the receptor is within 100 meters of the plant. Beyond 100 meters, the doses predicted by the Ramsdell model are the greatest. A comparison of the results presented in Tables 8.1-1 and 8.1-2 shows that the two different models produce considerably different results. This difference highlights the fact that there exists a considerable amount of uncertainty regarding the modeling of the transport of radioactive material in the vicinity near the plant (i.e., onsite region).

For comparison to the doses reported in Tables 8.1-1 and $8.1-2$, the lethal dose in $50 \%$ of the population (LD50) is approximately 300 rem acute gamma-ray total body exposure [Evans et al., 1993]. Tables 8.1-1 and 8.1-2 show that the possible onsite consequences of an accident during shutdown could be significant.

\subsection{Offsite Consequences}

Offsite consequences were calculated with version 1.5.11.1 of the MACCS code [Chanin et al., 1993] for each of the source term groups defined in the partitioning process. This code has been in use for some time and will not be

Table 8.1-1 Mean parking lot doses and dose rates predicted by Wilson model

\begin{tabular}{|c|c|c|c|c|c|c|c|c|}
\hline \multirow{3}{*}{$\begin{array}{l}\text { Distance } \\
\text { (m) }\end{array}$} & \multicolumn{4}{|c|}{ A Stability, Wind Speed $=5 \mathrm{~m} / \mathrm{s}$} & \multicolumn{4}{|c|}{ F Stability, Wind Speed $=1 \mathrm{~m} / \mathrm{s}$} \\
\hline & \multicolumn{2}{|c|}{ Dose Rate $(\mathrm{rem} / \mathrm{hr})^{\mathrm{a}}$} & \multicolumn{2}{|c|}{ Dose $(\mathrm{rem})^{b}$} & \multicolumn{2}{|c|}{ Dose Rate $(\mathrm{rem} / \mathrm{hr})^{\mathrm{a}}$} & \multicolumn{2}{|c|}{ Dose (rem) ${ }^{b}$} \\
\hline & $\begin{array}{c}\text { First } \\
\text { Release }\end{array}$ & $\begin{array}{l}\text { Second } \\
\text { Release }\end{array}$ & $\begin{array}{c}15 \mathrm{~min} \\
\text { Exposure }\end{array}$ & $\begin{array}{l}\text { Exposure to } \\
\text { Entire Release }\end{array}$ & $\begin{array}{c}\text { First } \\
\text { Release }\end{array}$ & $\begin{array}{l}\text { Second } \\
\text { Release }\end{array}$ & $\begin{array}{c}15 \mathrm{~min} \\
\text { Exposure }\end{array}$ & $\begin{array}{l}\text { Exposure to } \\
\text { Entire Release }\end{array}$ \\
\hline 10 & 45,000 & 17,000 & 550,000 & $41,000,000$ & 220,000 & 85,000 & $2,700,000$ & $200,000,000$ \\
\hline 50 & 1,800 & 680 & 22,000 & $1,600,000$ & 8,900 & 3,400 & 110,000 & $8,100,000$ \\
\hline 100 & 75 & 29 & 920 & 68,000 & 2,600 & 1,000 & 32,000 & $2,400,000$ \\
\hline 250 & 5 & 2 & 57 & 4,300 & 670 & 260 & 8,200 & 610,000 \\
\hline 500 & 1 & 0.2 & 7 & 520 & 240 & 90 & 2,900 & 210,000 \\
\hline
\end{tabular}

a: The dose rate calculation only considered exposure from the immersion pathway.

$b$ : The dose calculation considered exposure from both the immersion and inhalation pathways and is a 50 year committed dose. 
Table 8.1-2 Mean parking lot doses and dose rates predicted by Ramsdell model

\begin{tabular}{|c|c|c|c|c|c|c|c|c|}
\hline \multirow{3}{*}{$\begin{array}{l}\text { Distance } \\
\text { (m) }\end{array}$} & \multicolumn{4}{|c|}{ A Stability, Wind Speed $=5 \mathrm{~m} / \mathrm{s}$} & \multicolumn{4}{|c|}{ F Stability, Wind Speed $=1 \mathrm{~m} / \mathrm{s}$} \\
\hline & \multicolumn{2}{|c|}{ Dose Rate $(\mathrm{rem} / \mathrm{hr})^{3}$} & \multicolumn{2}{|c|}{ Dose (rem) ${ }^{b}$} & \multicolumn{2}{|c|}{ Dose Rate $(\mathrm{rem} / \mathrm{hr})^{\mathrm{a}}$} & \multicolumn{2}{|c|}{ Dose (rem) } \\
\hline & $\begin{array}{c}\text { First } \\
\text { Release }\end{array}$ & $\begin{array}{l}\text { Second } \\
\text { Release }\end{array}$ & $\begin{array}{c}15 \mathrm{~min} \\
\text { Exposure }\end{array}$ & $\begin{array}{l}\text { Exposure to } \\
\text { Entire Release }\end{array}$ & $\begin{array}{c}\text { First } \\
\text { Release }\end{array}$ & $\begin{array}{l}\text { Second } \\
\text { Release }\end{array}$ & $\begin{array}{c}15 \mathrm{~min} \\
\text { Exposure }\end{array}$ & $\begin{array}{c}\text { Exposure to } \\
\text { Entire } \\
\text { Release }\end{array}$ \\
\hline 10 & 460 & 180 & 5,600 & 420,000 & 340 & 130 & 4,100 & 310,000 \\
\hline 50 & 75 & 29 & 920 & 68,000 & 55 & 21 & 670 & 50,000 \\
\hline 100 & 34 & 13 & 420 & 31,000 & 25 & 10 & 310 & 23,000 \\
\hline 250 & 12 & 5 & 150 & 11,000 & 9 & 3 & 110 & 8,100 \\
\hline 500 & 6 & 2 & 68 & 5,000 & 4 & 2 & 50 & 3,700 \\
\hline
\end{tabular}

a: The dose rate calculation only considered exposure from the immersion pathway.

b: The dose calculation considered exposure from both the immersion and inhalation pathways and is a 50 year committed dose.

described in any detail here. Although the variables thought to be the largest contributors to the uncertainty in risk were sampled from distributions in the accident frequency analysis, the accident progression analysis, and the source term analysis, no analogous treatment of uncertainties was performed in the consequence analysis. Variability in the weather was fully accounted for, but the uncertainty in other parameters such as dry deposition speed or evacuation rate was not considered.

\subsubsection{Description of the Offsite Consequence Analysis}

MACCS tracks the dispersion of the radioactive material in the atmosphere from the power plant and computes its deposition on the ground. MACCS then calculates the effects of this radioactivity on the population and the environment. Doses and the ensuing health effects from 60 radionuclides are computed for the following pathways: immersion or cloudshine, inhalation from the plume, groundshine, deposition on the skin, inhalation of resuspended ground contamination, ingestion of contaminated water, and ingestion of contaminated food.

MACCS treats atmospheric dispersion by the use of multiple, straight-line Gaussian plumes. Each plume can have a different direction, duration, and initial radionuclide concentration. Cross-wind dispersion is treated by a multi-step function. Both dry and wet deposition are treated as independent processes. The weather variability is treated by means of a stratified sampling process.

For early exposure, the following pathways are considered: immersion or cloudshine, inhalation from the plume, groundshine, deposition on the skin, and inhalation of resuspended ground contamination. For long-term exposure, MACCS considers the following four pathways: groundshine, inhalation of resuspended ground contamination, ingestion of contaminated water, and ingestion of contaminated food. The direct exposure pathways (groundshine and inhalation of resuspended ground contamination) produce doses in the population living in the area surrounding the plant. The indirect exposure pathways (ingestion of contaminated water and food) produce doses in those who ingest food or water emanating from the area around the accident site. The contamination of water bodies is estimated for the washoff of material deposited on the land as well as direct deposition. The food pathway model includes direct deposition onto crops and uptake from the soil.

Both short-term and long-term mitigative measures are modeled in MACCS. Short-term actions include evacuation, sheltering, and emergency relocation out of the emergency planning zone. Population relocation is defined in the MACCS emergency response model as temporary relocation of nonevacuating people following deposition. The model provides two alternatives for temporary relocation (hot-spot relocation and normal relocation) at a user-specified time after plume arrival, each conditional on a projected dose from cloudshine, groundshine, cloud inhalation, and resuspension inhalation that exceeds a userspecified limit. Long-term actions include later relocation and restrictions on land use and crop disposition.

Relocation and land decontamination, interdiction, and condemnation are based on projected long-term doses from groundshine and inhalation of resuspended radioactivity. The disposal of agricultural products is based on the products' contamination levels, and the removal of 
Table 8.2-1 Offsite consequence measures calculated for POS 5

\begin{tabular}{|c|c|}
\hline Consequence Measure & Description \\
\hline Early fatalities & $\begin{array}{l}\text { Number of fatalities occurring within } 1 \text { year of the accident because of } \\
\text { early exposure (i.e., exposure incurred within seven days of the accident). }\end{array}$ \\
\hline $\begin{array}{l}\text { Total latent cancer } \\
\text { fatalities }\end{array}$ & $\begin{array}{l}\text { Number of latent cancer fatalities caused by both early and chronic } \\
\text { exposure (i.e., chronic exposure is that incurred more than seven days after } \\
\text { the accident). }\end{array}$ \\
\hline $\begin{array}{l}\text { Population dose within } 50 \\
\text { miles }\end{array}$ & $\begin{array}{l}\text { Population dose, expressed in effective dose equivalents for whole body } \\
\text { exposure (rem), resulting from early and chronic exposure pathways within } \\
50 \text { miles of the reactor. Because of the nature of the chronic pathways } \\
\text { models, the actual exposure from food and water consumption may take } \\
\text { place beyond } 50 \text { miles (e.g., food and water originating within } 50 \text { miles of } \\
\text { the plant may be consumed by people located beyond } 50 \text { miles). }\end{array}$ \\
\hline $\begin{array}{l}\text { Population dose within } \\
\text { entire region }\end{array}$ & $\begin{array}{l}\text { Population dose, expressed in effective dose equivalents for whole body } \\
\text { exposure (rem), resulting from early and chronic exposure pathways within } \\
\text { the surrounding region. }\end{array}$ \\
\hline $\begin{array}{l}\text { Individual early fatality } \\
\text { probability within one mile }\end{array}$ & $\begin{array}{l}\text { Probability of dying within one year for an individual within one mile of } \\
\text { the site exclusion boundary (i.e., ef/pop, where ef is the number of early } \\
\text { fatalities within one mile of the exclusion boundary, and pop is the } \\
\text { population within one mile of the exclusion boundary). }\end{array}$ \\
\hline $\begin{array}{l}\text { Individual latent cancer } \\
\text { fatality probability within } \\
10 \text { miles }\end{array}$ & $\begin{array}{l}\text { Probability of dying from cancer for an individual within ten miles of the } \\
\text { plant (i.e., cf/pop, where cf is the number of cancer fatalities resulting from } \\
\text { direct exposure in the resident population within ten miles of the plant, and } \\
\text { pop is the population size within ten miles of the plant). The calculation } \\
\text { does not include ingestion but does include integrated groundshine and } \\
\text { inhalation exposure. }\end{array}$ \\
\hline
\end{tabular}

farmland from crop production is based on ground contamination criteria. The health effects models link the dose received by an organ to predicted morbidity or mortality rates. The models used in MACCS calculate both the short-term and long-term effects for a number of organs.

The MACCS consequence model calculates a large number of different consequence measures. Results for the following six consequence measures are given in this report: early fatalities, total latent cancer fatalities, population dose within 50 miles, population dose for the entire region, early fatality risk within 1 mile, and latent cancer fatality risk within 10 miles. These consequence measures are described in Table 8.2-1.

\subsubsection{MACCS Input for Grand Gulf}

The input used in this study is identical to that used for Grand Gulf in the NUREG-1150 study, with the exception of the core inventories (see Appendix D.1) and the source terms, which resulted from GGSOR-P5. Because the methods used to calculate the MACCS parameters and the parameter values developed using those methods are documented Volume 2, Part 7 of NUREG/CR-4451 [Sprung et al., 1990], only a small portion of the MACCS input is presented here.

The emergency response assumptions used in the NUREG1150 study were also used in this study. It is assumed that $99.5 \%$ of the population within 10 miles of the plant 
evacuates, and the remaining $0.5 \%$ of the population does not evacuate and continues normal activity. In addition to evacuation, two criteria were used to relocate the population during the emergency phase of the accident. The first criterion is termed hot spot relocation and calls for relocation of the population 12 hours after plume passage if the projected effective dose equivalent from one week of exposure exceeds 50 rem. The second criterion is termed normal relocation and calls for relocation of the population 24 hours after plume passage if the projected effective dose equivalent from one week of exposure exceeds $25 \mathrm{rem}$.

Table 8.2-2 lists the MACCS input parameters that have strong site dependencies, and it presents the values of these parameters used in the MACCS calculations for the Grand Gulf site. The evacuation delay period begins when general emergency conditions occur, and it ends when the general public starts to evacuate. Non-farm wealth includes personal, business, and public property; the farmland fractions do not add to one because not all farmland is under cultivation. In addition to the site-specific data presented in Table 8.2-2, the Grand Gulf MACCS calculations used one year of meteorological data from the Grand Gulf site and regional population data developed from the 1980 census tapes. ${ }^{1}$ Table 8.2-3 gives the population within certain distances of the plant as summarized from the MACCS demographic input. Table 8.2-4 lists the shielding parameters used in this analysis.

\subsubsection{Results from the Offsite Consequence Analysis}

Offsite consequences were estimated for each of the 54 source term groups defined in the source term analysis. The mean (over weather variation) consequences for these source term groups are presented in Table D.4-1 in Appendix D.4. These mean consequences are the values used in the aggregate risk calculations. Aggregate risk was defined in Chapter 3; results from the risk calculation are presented in Chapter 9.

Table 8.2-2 Site-specific input data for Grand Gulf MACCS calculations

\begin{tabular}{lc}
\hline \hline Parameter & Value \\
\hline Reactor Power Level (MWt) & 3833 \\
Containment Height (m) & 32 \\
Containment Width (m) & 32 \\
Exclusion Zone Distance (m) & 696 \\
Evacuation Delay (h) & 1.25 \\
Evacuation Speed (m/s) & 3.7 \\
Farmland Fractions by Crop Categories & \\
$\quad$ Pasture & 0.7 \\
Stored Forage & 0.05 \\
Grains & 0.18 \\
Green Leafy Vegetables & 0.0005 \\
Legumes and Seeds & 0.13 \\
Roots and Tubers & 0.0008 \\
Other Food Crops & 0.004 \\
Non-Farm Wealth (\$/person) & 53,000 \\
Farm Wealth & 1824 \\
Value (\$/hectare) & 0.30 \\
Fraction in Improvements & \\
\hline \hline
\end{tabular}
Gulf was based on the 1980 census data and an objective of this study is to compare the risk from full power with the risk from shutdown, these data were also used in this study instead of the more recent 1990 census data. 
Consequence Analysis

Table 8.2-3 Population surrounding plant

\begin{tabular}{cc|l}
\hline \hline \multicolumn{2}{c|}{$\begin{array}{l}\text { Distance from Plant } \\
(\mathrm{km})\end{array}$} & Population \\
\hline 1.6 & 1.0 & 34 \\
4.8 & 3.0 & 879 \\
16.1 & 10.0 & 10,255 \\
48.3 & 30.0 & 97,395 \\
160.9 & 100.0 & $1,614,883$ \\
563.3 & 350.0 & $22,259,422$ \\
1609.3 & 1000.0 & $142,024,448$ \\
\hline \hline
\end{tabular}

Table 8.2-4 Shielding factors used for Grand Gulf MACCS calculations

\begin{tabular}{ccc}
\hline \multirow{2}{*}{ Radiation Pathway } & \multicolumn{2}{c}{ Population Response } \\
\cline { 2 - 3 } & Evacuate & $\begin{array}{l}\text { Normal } \\
\text { Activity }\end{array}$ \\
\hline Cloudshine & 1.0 & 0.75 \\
Groundshine & 0.5 & 0.33 \\
Inhalation & 1.0 & 0.41 \\
Skin & 1.0 & 0.41 \\
\hline \hline
\end{tabular}




\subsection{References}

[Croff et al., 1989] A. G. Croff, et al., "ORIGEN2: Isotope Generation and Depletion Code," RSIC, Oak Ridge National Laboratory, Oak Ridge, TN, CCC-371, September 1989.

[Chanin et al., 1990] D. I. Chanin et. al., "MELCOR Accident Analysis Consequence Code System," NUREG/CR4691, SAND86-1562, Sandia National Laboratories, Vol 1, February 1990.

[Jow et al., 1990] H-N Jow et. al., "MELCOR Accident Analysis Consequence Code System," NUREG/CR-4691, SAND86-1562, Sandia National Laboratories, Vol 2, February 1990.

[Rollstin et al., 1990] J. A. Rollstin et. al., "MELCOR Accident Analysis Consequence Code System," NUREG/CR4691, SAND86-1562, Sandia National Laboratories, Vol 3, February 1990.

[Ramsdell, 1990] J. V. Ramsdell, Jr., "Diffusion in Building Wakes for Ground-Level Releases," Atmospheric Environment, Vol. 24B, No. 3, 377-388, (1990).

[Wilson, 1984]

Wilson in Atmospheric Science and Power Production, Ed. Randerson, D., DOE/TIC-27601, 299, (1984).

[USNRC, 1982]

U.S. Nuclear Regulatory Commission, Regulatory Guide 1.145, Revision 1, November 1982.

[Chanin et al., 1993] D. Chanin, et al., "MACCS Version 1.5.11.1: A Maintenance Release of the Code," NUREG/CR6059, SAND92-2146, Sandia National Laboratories, October 1993.

[Sprung et al., 1990] J. L. Sprung et al., "Evaluation of Severe Accident Risks: Quantification of Major Input Parameters, MACCS Input," NUREG/CR-4551, SAND86-1309, Vol. 2, Rev. 1, Part 7, Sandia National Laboratories, December 1990.

[Evans et al., 1993] J. S. Evans, et al., "Health Effects Model for Nuclear Power Plant Consequence Analysis, Part I: Introduction, Integration, and Summary," NUREG/CR-4214, Rev. 2, Part 1, ITRI-141, Inhalation Toxicology Research Institute, Lovelace Biomedical and Environment Research Institute, October 1993. 
Consequence Analysis 


\section{Risk Analysis}

As discussed in Section 2, the risk calculation combines the results from the previous four constituent analyses. Results from the accident frequency and accident progression analyses determine the frequency of a release, while results from the source term and consequence analyses determine the magnitude of the release and the resulting consequences to the offsite population. Aggregate risk is the sum, over all accidents, of the accident frequency multiplied by the consequence of the accident. In the following subsections, the aggregate risk results are presented and the contributors to risk are discussed.

\subsection{Risk Results}

The aggregate risk results for plant operating state (POS) 5 are presented in Table 9.1; it provides statistics that describe the risk distributions for each of the consequence measures defined in Table 8.2-1. The descriptive statistics include the following measures: $5^{\text {th }}$ percentile, median value, $95^{\text {th }}$ percentile, mean value, and standard deviation. The descriptive statistics for the aggregate risk distributions for each of the plant damage states (PDSs) are provided in Appendix E.1. The aggregate risk results calculated in this study account for the length of time, on average, that the plant is in POS 5 during a calendar year. (The time the plant is in POS 5 is factored into the core damage frequency and is discussed in Volume 2 of this report.) These risk results are not conditional on being in POS 5 .

As stated previously, aggregate risk is a function of both the frequency of the source term and the consequences that result from the source term for each of the accidents delineated in the study. The frequency of the source term is the frequency of the initiating event, coupled with the probability that the initiating event leads to core damage, coupled with the probability that the core damage accident leads to a certain source term. Because in these accidents the containment is nearly always open or bypassed, the frequency of a release occurring is essentially the frequency of core damage. To determine the magnitudes of the various possible releases, each with its own probability conditional on core damage, the accident progression and source term analyses considered factors such as (1) the probability that the core damage process is arrested, (2) the probability that the suppression pool is bypassed, and (3) the probability that core debris released from the vessel is quenched. The core damage frequency can have a strong impact on the frequency of the source term and thus on risk. In general, for a given set of accidents, an increase in the core damage frequency will result in an increase in risk. The extent to which an increase in the core damage frequency results in an increase in risk will depend on which accidents are affected and the severity of the consequences associated with these accidents. The factors that lead to the core damage frequencies used in this study are discussed in Volume 2 of this report.

Many factors can affect the magnitude and severity of the release, which in turn will affect risk. Factors associated with the POS 5 accidents and that tend to increase risk include the following:

- In many accidents the containment equipment hatch was open during the entire accident. An open hatch provides a path for radionuclides to escape from the containment to the auxiliary building and then out into the environment.

Table 9.1 Distributions for aggregated risk for POS 5 (all values are per calendar year; population doses are in person-rem)

\begin{tabular}{|c|c|c|c|c|c|}
\hline \multirow{3}{*}{$\begin{array}{c}\text { Consequence } \\
\text { Measure }\end{array}$} & \multicolumn{5}{|c|}{ Descriptive Statistics* } \\
\hline & \multicolumn{3}{|c|}{ Percentiles } & \multirow[b]{2}{*}{ Mean } & \multirow{2}{*}{$\begin{array}{l}\text { Standard } \\
\text { Deviation }\end{array}$} \\
\hline & 5 th & 50th & 95th & & \\
\hline Core Damage Frequency & 4.1E-07 & $1.4 \mathrm{E}-06$ & $5.6 \mathrm{E}-06$ & 2.1E-06 & 2.7E-06 \\
\hline Early Fatality Risk & 3.7E-11 & $2.8 \mathrm{E}-09$ & $3.9 \mathrm{E}-08$ & $1.4 \mathrm{E}-08$ & $5.4 \mathrm{E}-08$ \\
\hline Total Latent Cancer Risk & 4.3E-04 & $1.9 \mathrm{E}-03$ & $1.2 \mathrm{E}-02$ & $3.8 \mathrm{E}-03$ & 7.7E-03 \\
\hline Population Dose within 50 miles of the plant & $1.3 \mathrm{E}-01$ & 5.3E-01 & $3.1 \mathrm{E}+00$ & $9.9 \mathrm{E}-01$ & $1.9 \mathrm{E}+00$ \\
\hline Population Dose within 1000 miles of the plant & $9.9 \mathrm{E}-01$ & $4.4 \mathrm{E}+00$ & $2.8 \mathrm{E}+01$ & $8.7 \mathrm{E}+00$ & $1.8 \mathrm{E}+01$ \\
\hline Individual Early Fatality Risk -0 to 1 mile & $4.2 \mathrm{E}-13$ & 2.7E-11 & $3.0 \mathrm{E}-10$ & $9.6 \mathrm{E}-11$ & $3.4 \mathrm{E}-10$ \\
\hline Individual Latent Cancer Risk- 0 to 10 miles & $2.5 \mathrm{E}-10$ & $9.4 \mathrm{E}-10$ & 4.9E-09 & $1.6 \mathrm{E}-09$ & $2.4 \mathrm{E}-09$ \\
\hline
\end{tabular}

* Statistics based on a Latin hypercube sampling (LHS) of 200 observations. 
- Two plant features that can be used to attenuate the release of radioactive aerosols are the suppression pool and the containment sprays. In both the loss of coolant accident (LOCA) and the station blackout (SBO) PDSs, the radioactive material released from the damaged fuel bypassed the suppression pool. The containment sprays were not available in any of the POS 5 accidents.

- In many of the accidents core cooling was not restored early in the accident, precluding any possibility of arresting the core damage process before the vessel failed. Once the vessel fails, the core debris in the vessel is released into the reactor cavity, allowing for the possibility of coreconcrete interactions. A significant amount of radioactive material can be released during this exvessel phase of the accident.

A number of factors associated with these POS 5 accidents also tend to decrease risk. These factors include the following:

- For those accidents in which the containment equipment hatch is open, the releases pass through the auxiliary building before escaping into the environment. Because of its large volume and surface area, the auxiliary building provides a location for the radionuclides to be attenuated by deposition and thereby reduce the source term to the environment.

- The accidents delineated for these shutdown conditions progress sufficiently slowly that there is typically a considerable amount of time available for the public to respond to the accident and evacuate before they are exposed to the release. This is primarily important for the early health effects consequence measures which are more strongly affected by the time available to evacuate.

- Radioactive decay has reduced the radioactive potential of these shutdown accidents relative to the inventory that is present immediately after the reactor is shut down. This factor is primarily important for early health effects, which are more strongly affected by the shorter lived radionuclides. This affect is much less noticeable for latent health effects, which are more strongly affected by the long lived isotopes.

- The population density around the Grand Gulf plant is relatively low. Although many factors influence the magnitude of the consequences, in general, for a given release, a smaller population denotes a smaller number of fatalities. Of the four Mark III plants in the United States, Grand Gulf has the fewest number of people living within 50 miles of the plant, according to the 1990 census data. The Mark III plant with the greatest 50-mile population has a population more than an order of magnitude greater than the Grand Gulf 50-mile population.

\subsection{Contributors to Risk}

This subsection provides the fractional contributions to risk for various groups of accidents. The fractional contribution, as used in this study, is defined as the ratio of the aggregate risk for a group of accidents (e.g., a PDS) to the total aggregate risk. This calculation is performed for each observation included in the uncertainty analysis (an observation is one particular run in the many runs made in a Monte Carlo type analysis) and results in a distribution of fractional contribution values. The mean of this distribution is called the mean fractional contribution to risk (MFCR). The MFCR is not equivalent to the simple ratio of the mean risk for a group of accidents to the total mean risk, which is referred to as fractional contribution to mean risk (FCMR); the FCMR is not used in this report.

Although the MFCR and FCMR have often been used to characterize the contributors that on average are important to risk, these mean measures do not reflect the uncertainty in the fractional contributions. As is evident from Table 9.2, the distributions of fractional contributions to risk are all highly uncertain. This conclusion follows from the wide spread between the $5^{\text {th }}$ and $95^{\text {th }}$ percentiles. Because the MFCR does not reflect the uncertainty in the distribution of fractional contribution to risk, additional descriptive statistics are provided that reflect this uncertainty and should be considered when assessing these results.

Table 9.2 provides the fractional contributions to the core damage frequency, the early fatality risk, and the total latent cancer risk for the following three PDS groups: LOCAs, SBOs, and Other Transients. The fractional contributions to the population dose risk measures (not shown in Table 9.2 for the sake of brevity) are similar to the fractional contributions to the total latent cancer risk measure. From Table 9.2 it can be seen that, on average, the SBO PDS group is the dominant contributor to the total early fatality risk. Because of the large amount of overlap among the three distributions, as is evident from the descriptive statistics provided in Table 9.2, on any given 
Risk Analysis

Table 9.2 Fractional contributions to aggregate risk for the LOCA, SBO, and Other Transients PDS groups

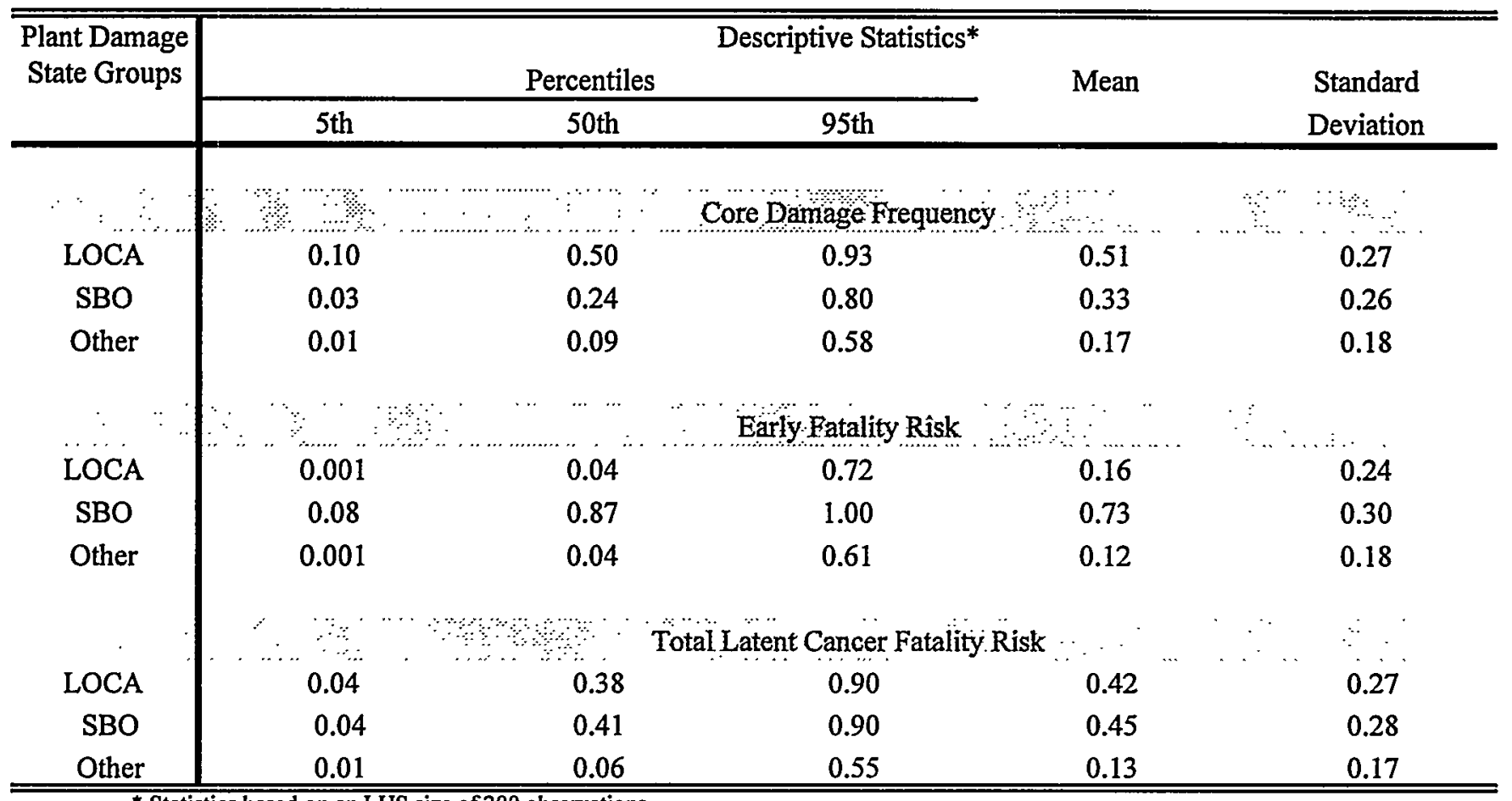

* Statistics based on an LHS size of 200 observations.

observation the contribution from the three groups can vary. That is, for one observation the SBO group may be dominant, whereas for another observation the LOCA group may be the dominant group. On average, however, the SBO is the dominant contributor. The large contribution of the SBO PDS group to early fatality risk can be attributed to its relatively high contribution to the core damage frequency coupled with the fact that the containment equipment hatch is open, the suppression pool is bypassed, and the auxiliary building fails early in these accidents. Combined, these factors cause the SBOs to have relatively high risk values. The LOCA PDS group, however, is not a dominant contributor to early fatality risk even though it is a dominant contributor to the core damage frequency. This condition results primarily because the dominant contributors to the LOCA core damage frequency are LOCA accidents that are initiated while the plant is in Time Window 3 (i.e., PDS3-1). A number of factors can potentially reduce the number of early fatalities that occur when the accident is initiated in Time Window 3 relative to the other time windows. These factors include the following: (1) radioactive decay has reduced the inventory of short-lived radionuclides that are important to early health effects, (2) because of lower decay heat the accidents progress more slowly, allowing more time for the population to evacuate, and (3) the release is spread out over a longer time, which helps reduce the concentration of radionuclides in the environment. For these reasons, Time window 3 is a negligible contributor to early fatality risk. The early fatality risk distributions for the LOCA, SBO, and Other PDS groups are provided in Figure 9.1.

For latent cancer health effects, the LOCA and SBO PDS groups are on average the dominant contributors to risk. Because the radionuclides important to the latent health effects tend to have long half lives, these risk measures are not particularly sensitive to the time of accident occurrence relative to shutdown. Latent cancers primarily depend on the total amount of radioactive material released, not on the time of release (i.e., early in the accident versus late in the accident). Because latent cancers are not strongly dependent on the timing characteristics of the accident (i.e., start of release or release duration), the latent cancer risk will depend on the likelihood of the accident and on the total amount of radioactive material released. In all of the core damage accidents delineated in this study, the 
Risk Analysis

Table 9.3 Fractional contribution to aggregate risk for Time Windows 1, 2, and 3

\begin{tabular}{|c|c|c|c|c|c|}
\hline \multirow{3}{*}{$\begin{array}{c}\text { Plant Damage } \\
\text { State Groups }\end{array}$} & \multicolumn{5}{|c|}{ Descriptive Statistics* } \\
\hline & \multicolumn{3}{|c|}{ Percentiles } & \multirow[t]{2}{*}{ Mean } & \multirow{2}{*}{$\begin{array}{c}\text { Standard } \\
\text { Deviation }\end{array}$} \\
\hline & 5 th & 50 th & 95th & & \\
\hline & & & age $F_{I}$ & & \\
\hline TW1 & 0.01 & 0.06 & 0.19 & 0.08 & 0.08 \\
\hline TW2 & 0.21 & 0.62 & 0.89 & 0.58 & 0.20 \\
\hline \multirow[t]{2}{*}{ TW3 } & 0.07 & 0.30 & 0.76 & 0.34 & 0.20 \\
\hline & \multicolumn{5}{|c|}{ Early Fatality Risk } \\
\hline TW1 & 0.010 & 0.12 & 0.94 & 0.22 & 0.25 \\
\hline TW2 & 0.06 & 0.88 & 0.99 & 0.78 & 0.25 \\
\hline \multirow[t]{2}{*}{ TW3 } & 0.000 & 0.000 & 0.005 & 0.003 & 0.02 \\
\hline & \multicolumn{5}{|c|}{ Total Latent Cancer Fatality Risk } \\
\hline TW1 & 0.01 & 0.07 & 0.24 & 0.09 & 0.10 \\
\hline TW2 & 0.26 & 0.71 & 0.94 & 0.67 & 0.20 \\
\hline TW3 & 0.02 & 0.19 & 0.60 & 0.24 & 0.19 \\
\hline
\end{tabular}

containment is either open at the start of the accident or fails during the accident, and in most of the accidents the core damage process is not arrested in the vessel. Thus, while the timing of the accident may vary, when the uncertainty in the source term is considered, all the accidents will result in roughly similar releases of radioactive material to the environment. Thus, as can be seen in Table 9.2, the mean fractional contribution to latent cancer risks tends to be roughly similar to the mean fractional contribution to the core damage frequency for each of the PDS groups. The fractional contributions from the LOCA and Other Transients tend to be less than their fractional contribution to the core damage frequency because portions of the release for these PDSs are scrubbed by either the suppression pool or the pool formed by flooding the containment. The fractional contribution from the SBO PDS group tends to be greater than the fractional contribution to the core damage frequency because for these accidents the containment is open at the start of the accident, the auxiliary building fails early in the accident, the vessel nearly always fails, CCI nearly always occurs, and the releases are rarely scrubbed by water. Therefore the releases associated with the SBO tend to be large relative to the other accidents analyzed in this study. The latent cancer risk distributions for the LOCA, SBO, and Other PDS groups are displayed in Figure 9.2.

The mean fractional contributions to aggregate risk for Time Windows 1,2, and 3 are presented in Table 9.3. On average, Time Window 2 is the dominant contributor to early health effects, followed by Time Window 1 . As explained above, Time Window 3 is a negligible contributor to the early health effects. For latent cancers, Time Window 2 is again the dominant contributor, on average, followed this time by Time Window 3 and then Time Window 1. As discussed above, for the latent health effects, the fractional contributions to risk are roughly similar to the contribution to the core damage frequency. 
Risk Analysis

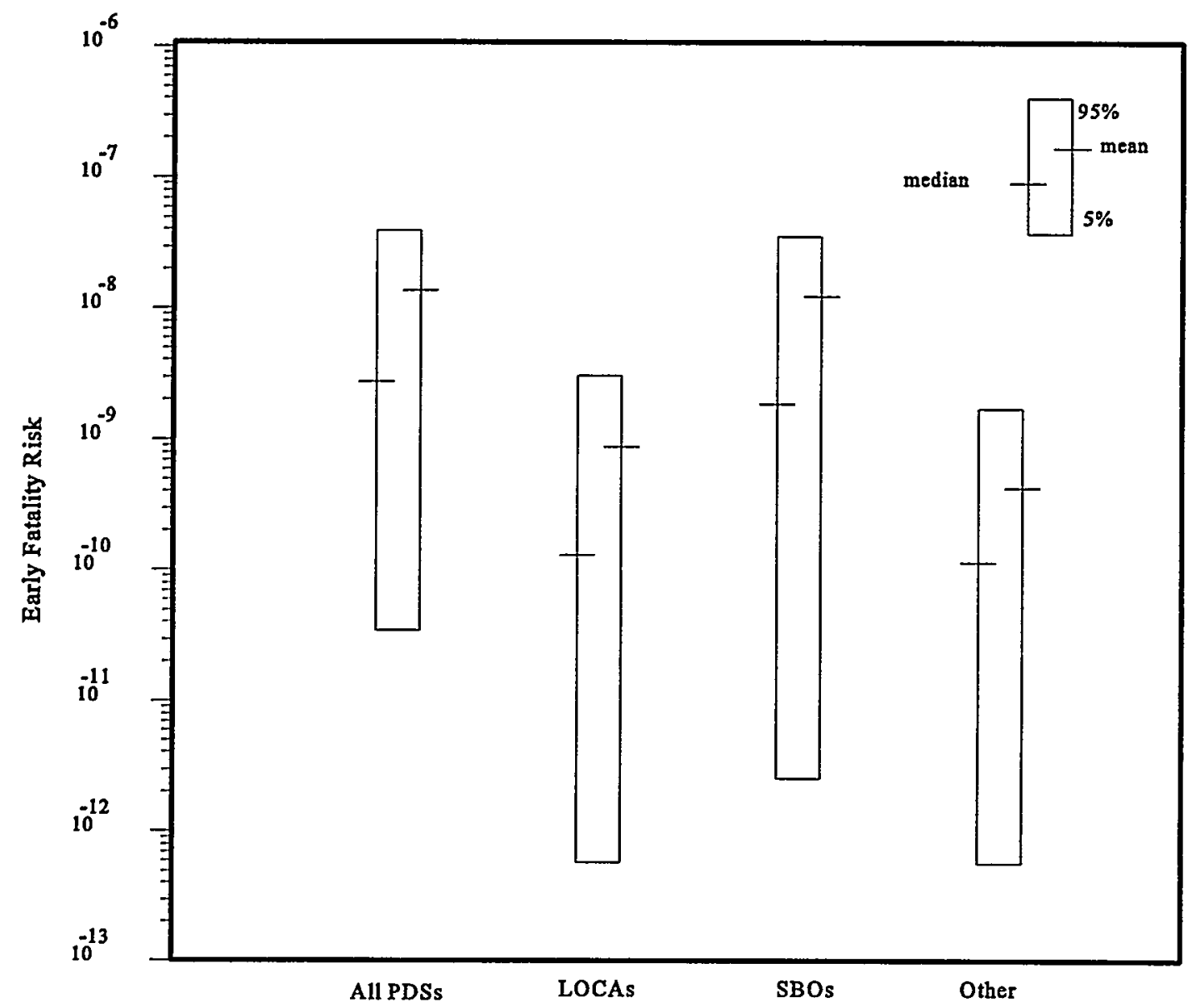

Figure 9.1 Early fatality risks for PDS groups 
Risk Analysis

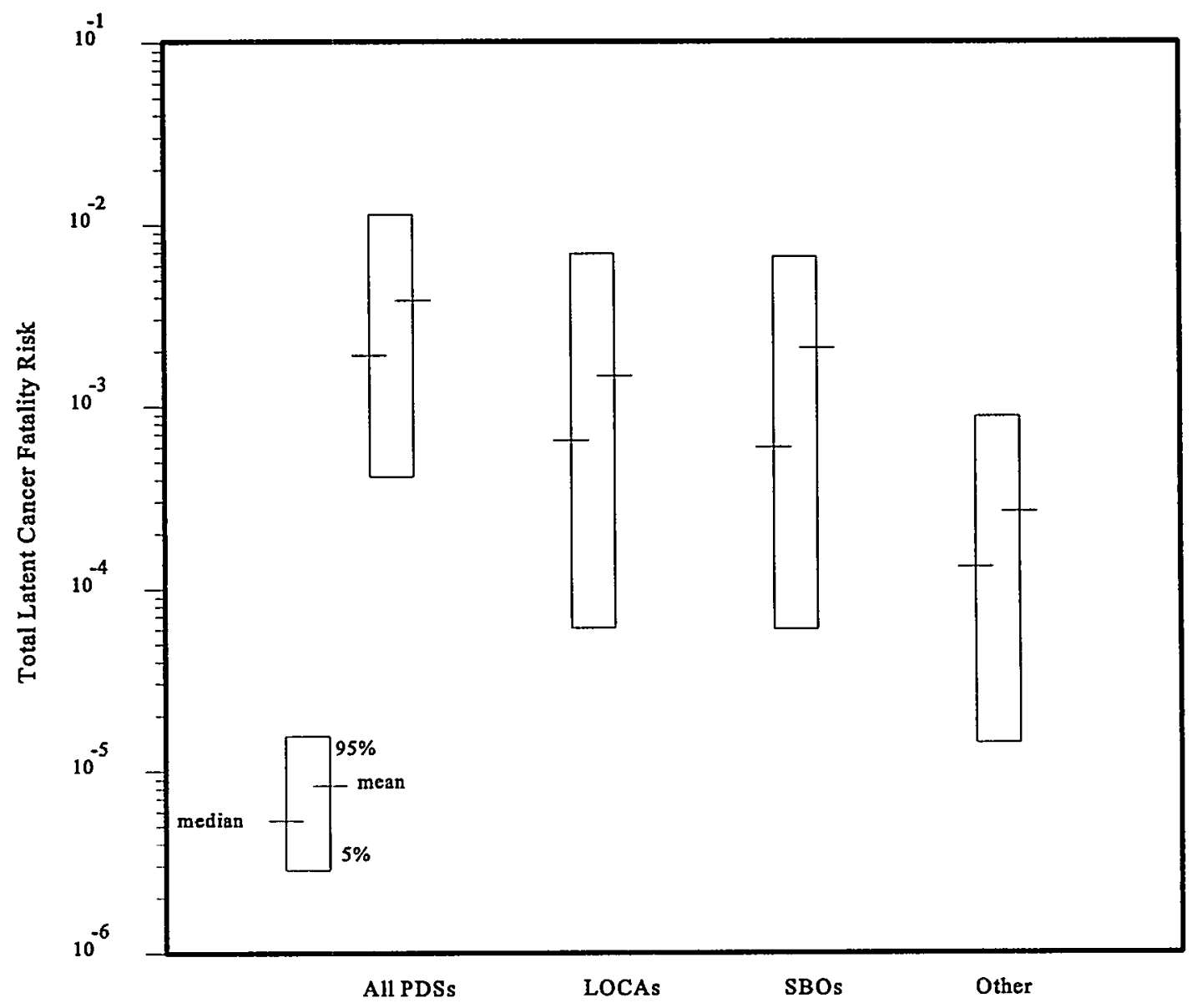

Figure 9.2 Total latent cancer risk for PDS groups 


\section{Comparison to Full Power Results}

An objective of this study is to assess the risk significance of plant operating state (POS) 5 . In the previous section the risk results were presented. In this section these risk results will be compared to the risks of full power operation. From this comparison the risk significance of POS 5 relative to full power can be determined. The comparison will help place the results from this study in context. The risk to the general public from the operation of the Grand Gulf nuclear plant during full power operation was analyzed in NUREG1150 [USNRC, 1990; Brown et. al., 1990]. That analysis showed that the full power risks at Grand Gulf were small relative to other plants analyzed in the NUREG-1150 study.

\subsection{Comparison Between POS 5 and Full Power Risk}

The POS 5 and full power distributions for early fatality risk, total latent cancer risk, population dose within 50 miles risk, and population dose within 1000 miles risk are displayed in Figures 10.1, 10.2, 10.3, and 10.4 respectively. Aggregate risk is a function of both the frequency and the consequences of accidents. Because the core damage frequency is a major contributor to the frequency of the accidents, the POS 5 and full-power core damage frequency distributions are displayed in Figure 10.5. Descriptive statistics for the aggregate risk measures and the total core damage frequency from the two analyses are listed in Table 10.1 .

The aggregate risk measures calculated in the NUREG1150 full power analysis are displayed in Table 10.1 along with the risk results calculated in this study of POS 5 . The risks from POS 5 compare to the full power risks as follows: The mean values from each study do not differ by more than a factor of 5 , and the risk values at the $50^{\text {th }}$ and $95^{\text {th }}$ percentiles from the two studies do not differ by more than an order of magnitude. The mean, median, $5^{\text {th }}$ percentile, and $95^{\text {th }}$ percentile values from the POS 5 risk distributions are all greater than the corresponding values from the full power distributions. The full power risk at the 5 th percentile is approximately an order of magnitude less than the corresponding POS 5 risk value.

The mean early fatality risk of POS 5 is only a factor of 1.7 greater than the full power risk. For POS 5 , the risk at the $95^{\text {th }}$ percentile is $4 \mathrm{E}-08 / \mathrm{yr}$ and drops by three orders of magnitude to $4 \mathrm{E}-11 / \mathrm{yr}$ at the $5^{\text {th }}$ percentile. For full power, the risk at the $95^{\text {th }}$ percentile is $3 \mathrm{E}-08 / \mathrm{yr}$ and drops by four orders of magnitude to $3 \mathrm{E}-12 / \mathrm{yr}$ at the $5^{\text {th }}$ percentile.

The mean total latent cancer fatality risk of POS 5 is a factor of four greater than the corresponding full power risk. For POS 5, the risk at the $95^{\text {th }}$ percentile is $1 \mathrm{E}-02 / \mathrm{yr}$ and drops by slightly more than one order of magnitude to $4 \mathrm{E}-04 / \mathrm{yr}$ at the $5^{\text {th }}$ percentile. For full power, the risk at the $95^{\text {th }}$ percentile is $2 \mathrm{E}-03 / \mathrm{yr}$ and drops by slightly more than two orders of magnitude to $1 \mathrm{E}-05 / \mathrm{yr}$ at the $5^{\text {th }}$ percentile.

The smallest difference between the POS 5 and full power mean risk values is in the early fatality risk measure. The greatest difference is for the mean total latent cancer fatality risk and the individual latent cancer fatality risk. Some of the difference in the latent cancer fatality risk measures results from the different models used in the MACCS calculations that were performed for the two studies. MACCS version 1.5.11.1 [Chanin et al., 1993] was used to estimate offsite consequences in the POS 5 probabilistic risk assessment (PRA); an earlier version was used in the NUREG-1150 plant studies. Cancer risk coefficients implemented in MACCS version 1.5.11.1 are two to three times greater than those utilized in earlier versions of the MACCS code. The total latent cancer and the individual latent cancer fatality risk measures are directly affected by these cancer risk coefficients.

The POS 5 mean core damage frequency is a factor of two lower than the full power value. The $95^{\text {th }}$ percentile value from the POS 5 distribution is less than the corresponding values from the full power distribution, while the median and $5^{\text {th }}$ percentile values from the POS 5 distribution are greater than the full power distribution.

This comparison shows that the risk from POS 5 is not insignificant compared to the risk from full power operation. In fact, although the mean risk values from the two studies are similar (i.e., do not differ by more than a factor of 5), the mean risk values from POS 5 are actually greater than the full power risk values. Hence the full power risk distributions by themselves do not completely characterize the risks associated with the operation of this plant. To accurately characterize the plant's risks, results from this study suggest that it may be necessary to include other modes of operation in addition to the full power mode of operation. This can have important implications for assessments that rely on the total risk from a plant, such as when comparisons are made to the safety goals. 
Comparison to Full Power Results

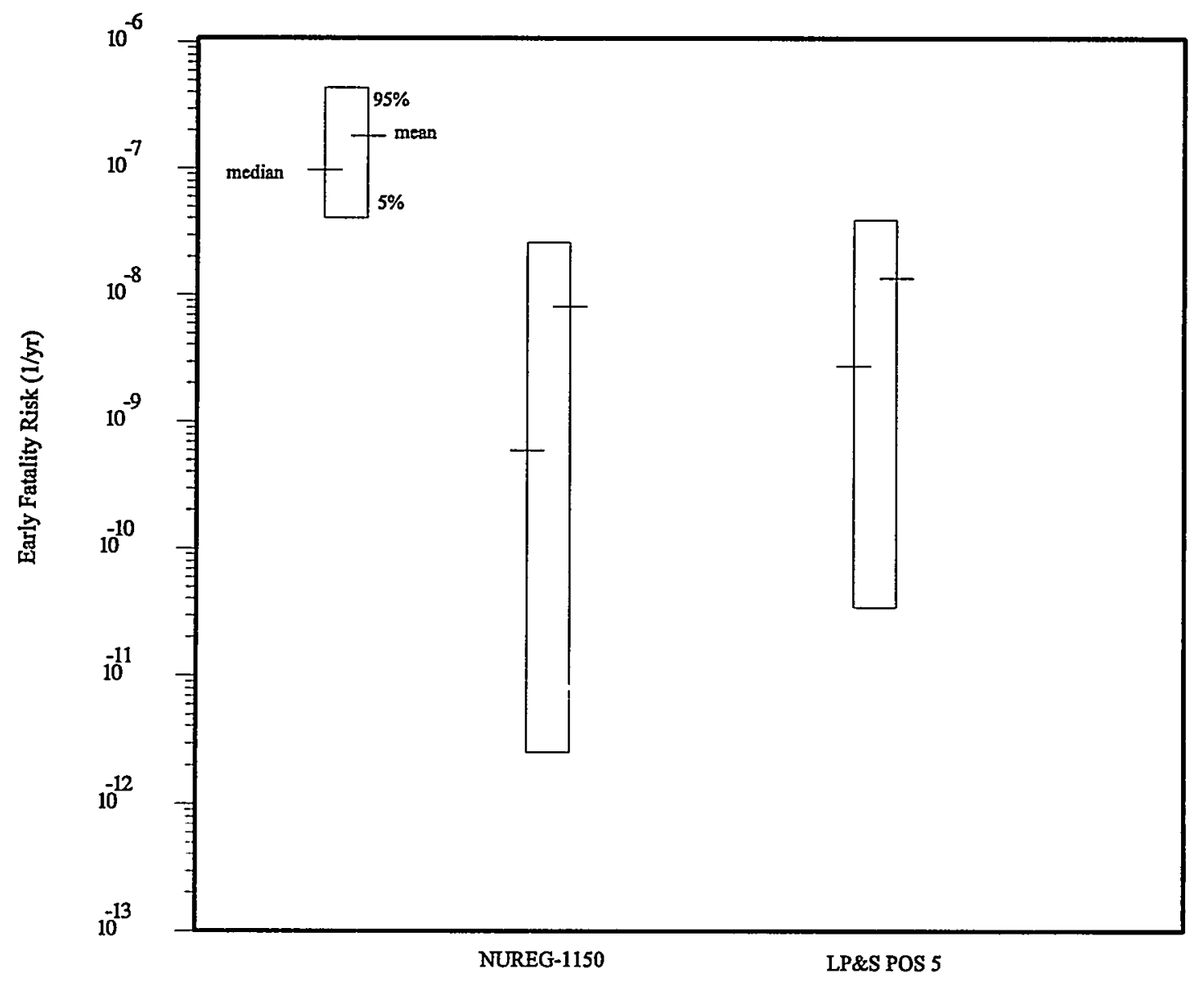

Figure 10.1 Comparison of Grand Gulf full power and POS 5 early fatality risk 


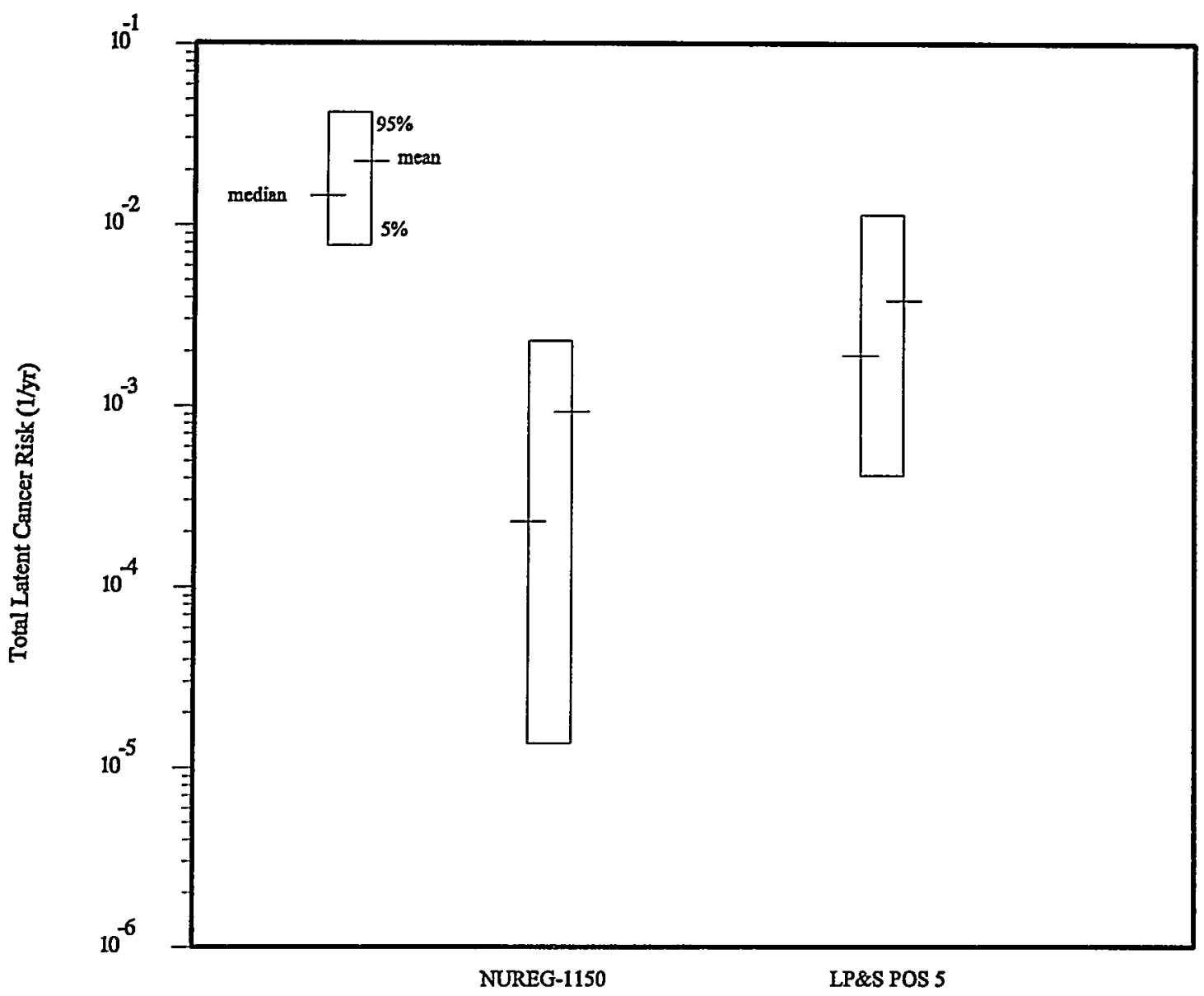

Figure 10.2 Comparison of Grand Gulf full power and POS 5 total latent cancer risk 
Comparison to Full Power Results

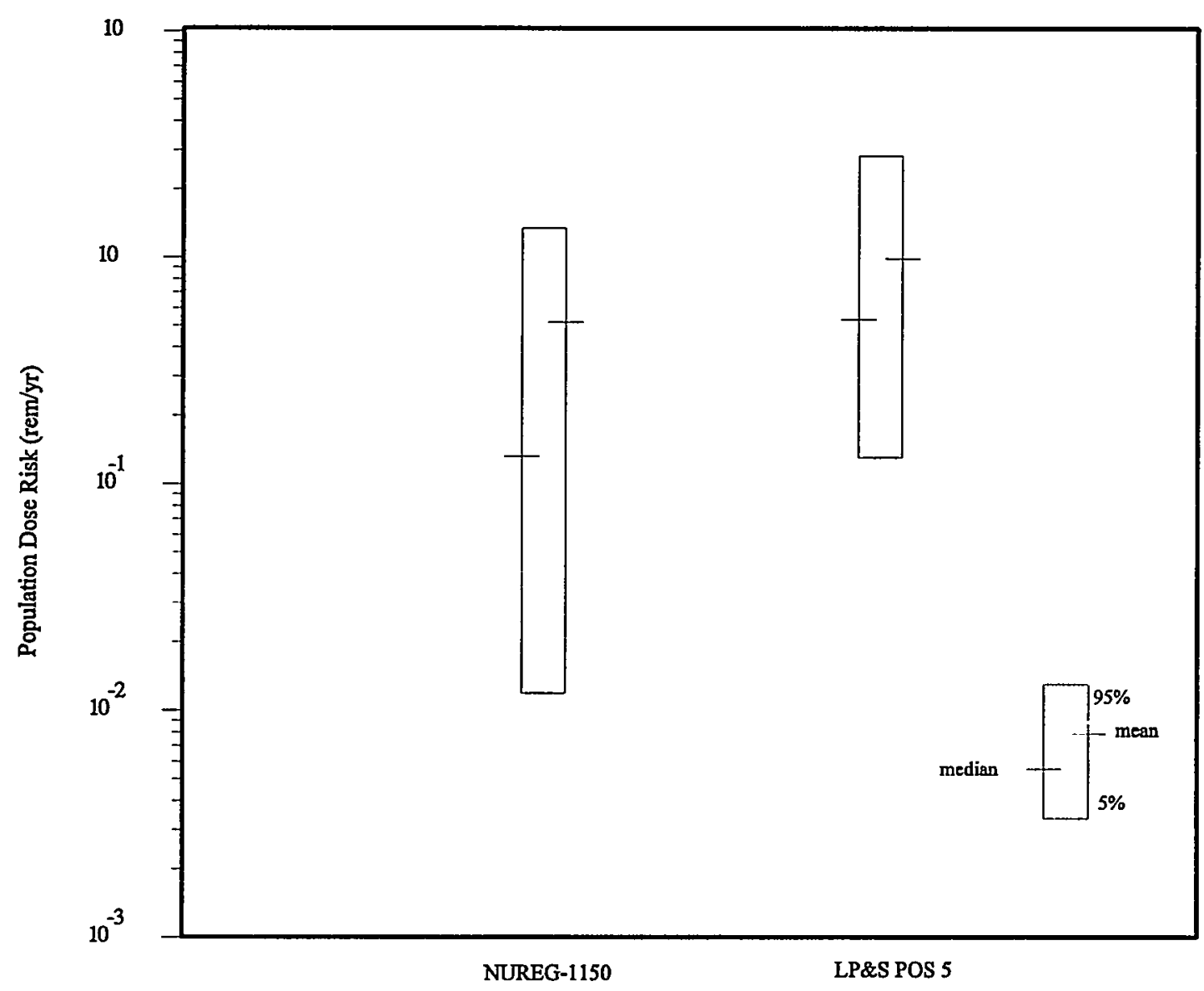

Figure 10.3 Comparison of Grand Gulf full power and POS 5 population dose within 50 miles of the site risk 


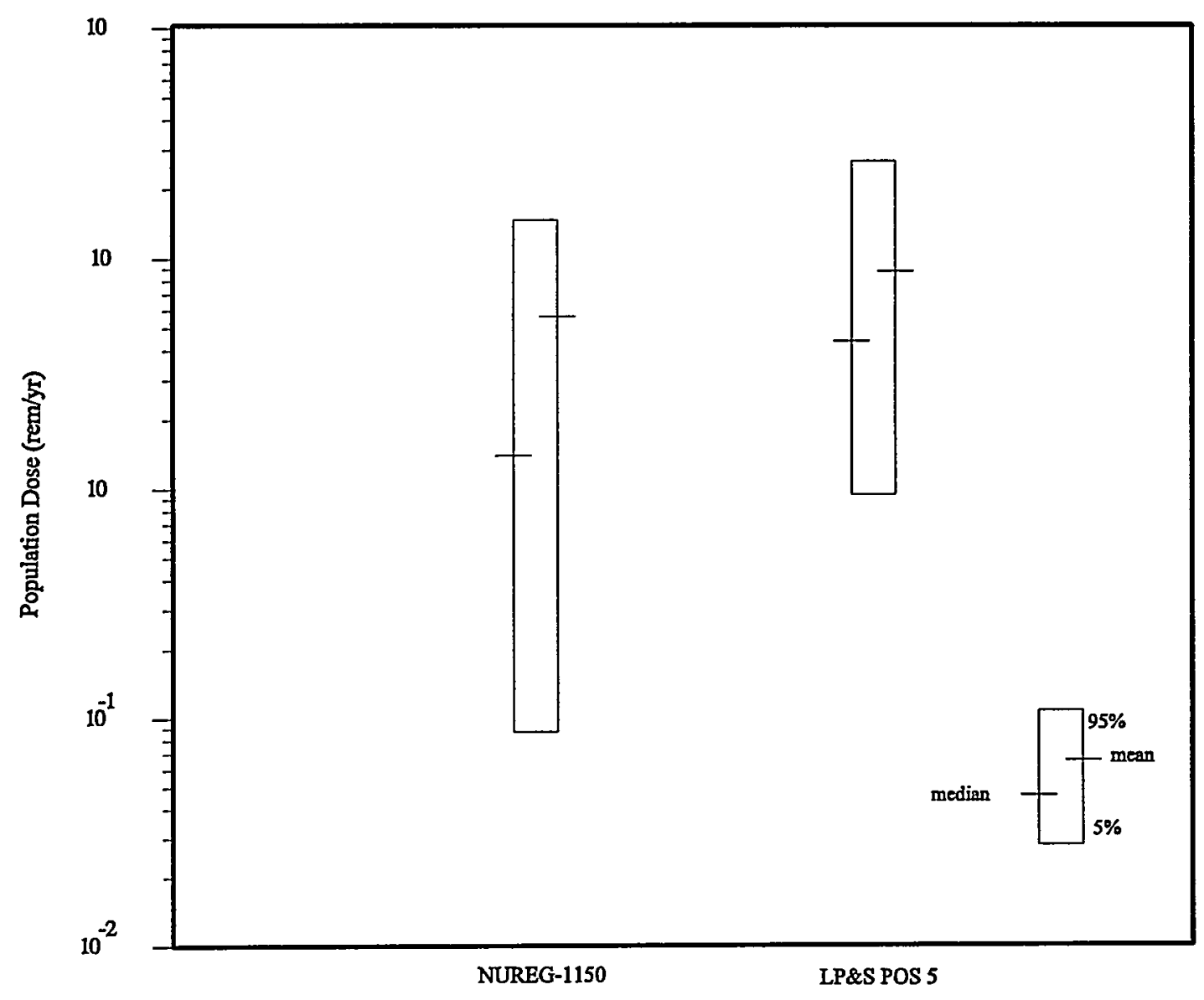

Figure 10.4 Comparison of Grand Gulf full power and POS 5 population dose within 1000 miles of the site risk 


\section{Comparison to Full Power Results}

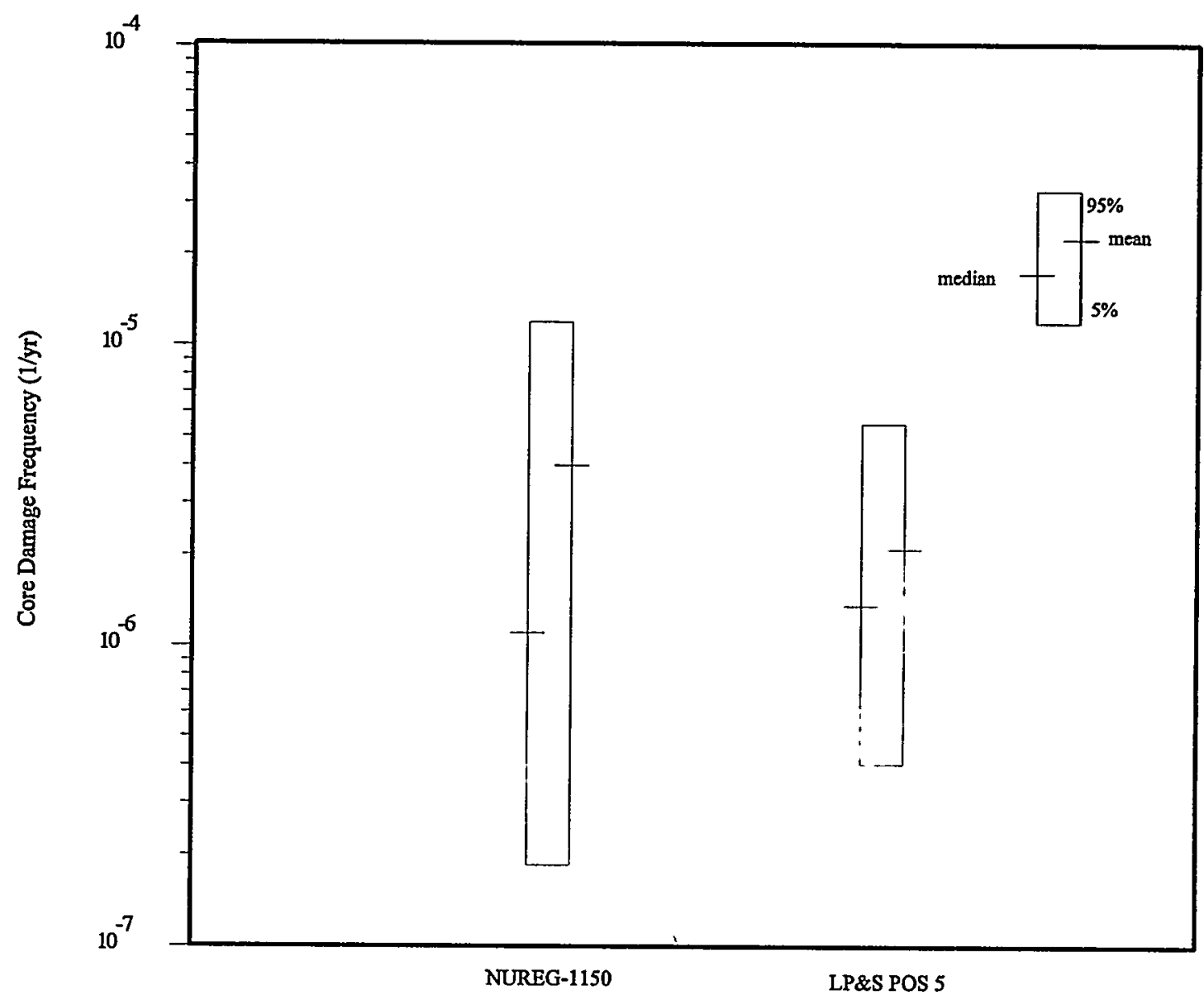

Figure 10.5 Comparison of Grand Gulf full power and POS 5 core damage frequencies 
Table 10.1 Distributions for aggregate risk for POS 5 and for full power (all values are per year; population doses are in person-rem)

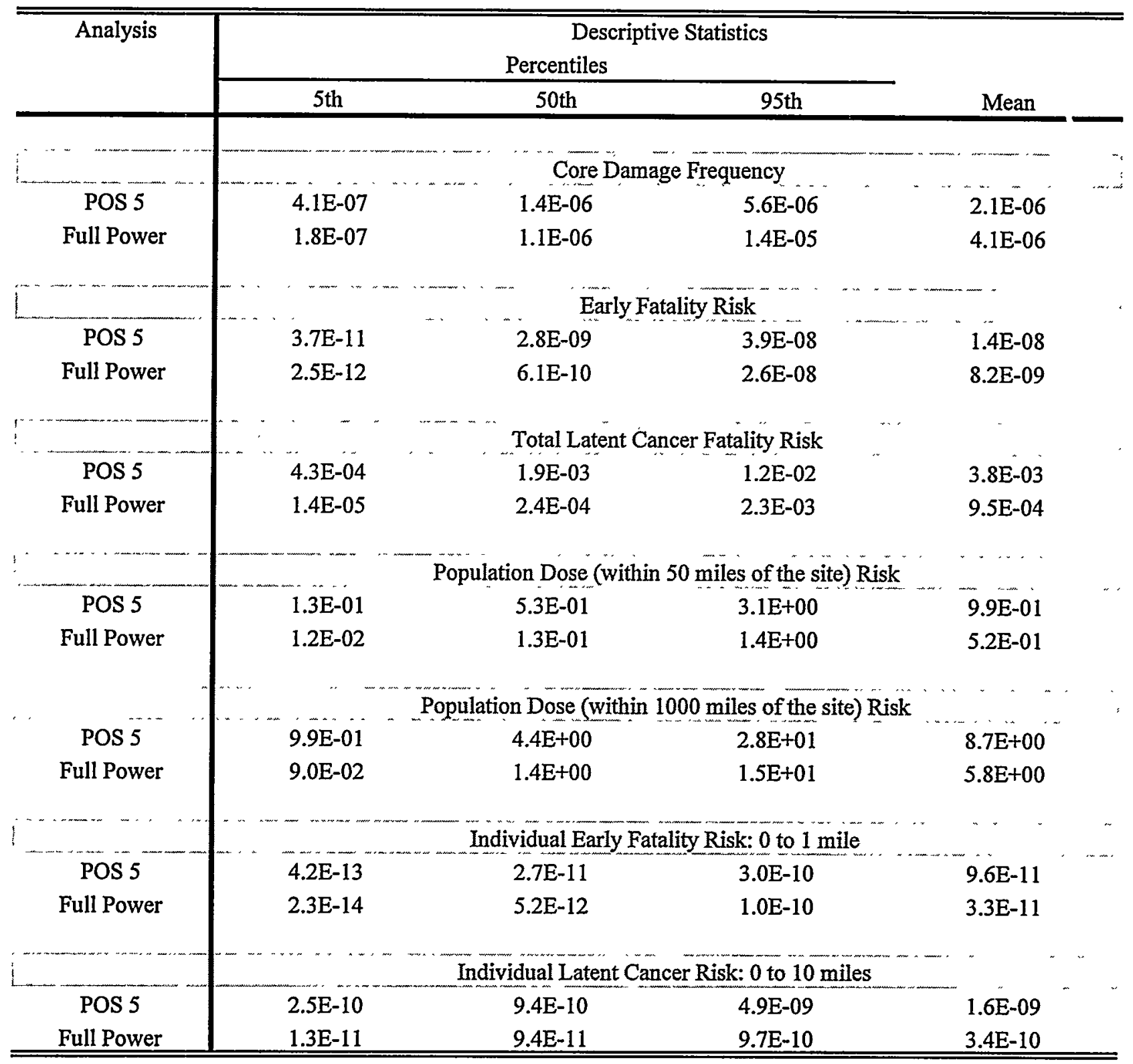

" Full power results were extracted from Volume 6 of NUREG/CR-4551 [Brown et al, 1990] 
Comparison to Full Power Results

\subsection{References}

[USNRC, 1990]

U.S. Nuclear Regulatory Commission, "Severe Accident Risks: An Assessment for Five U. S. Nuclear Power Plants," NUREG-1150, Vols. 1-3, December 1990-January 1991.

[Brown et al., 1990]

T. D. Brown et al., "Evaluation of Severe Accident Risks: Grand Gulf Unit 1," NUREG/CR-4551, SAND86-1309, Vol. 6, Rev. 1, Sandia National Laboratories, December 1990.

[Chanin et al., 1993]

D. Chanin et al., "MACCS Version 1.5.11.1: A Maintenance Release of the Code," NUREG/CR6059, SAND92-2146, Sandia National Laboratories, October 1993. 


\section{Open Issues}

The study presented in this volume is for a single plant operating state (POS). The study assesses the risk associated only with POS 5 . The study does not attempt to assess the risk with the entire low power and shutdown (LP\&S) regime of operation. Although the Level 1 screening study and other qualitative insights suggest that POS 5 is the risk-dominant mode of shutdown, no detailed study has been performed on the other POSs to confirm this supposition. Furthermore, only accidents initiated from traditional internal events were analyzed in this study. Hence the risk calculated for POS 5 is not complete in the sense that it does not include accidents initiated by internal fires or floods and also does not include accidents initiated by seismic events.

Note that reducing the risk in one POS by changing when equipment is available and unavailable can shift the risk to another POS. Because this study only addresses the risk associated with one POS, the effect of these changes on overall risk (i.e., risk across all the POSs) cannot be quantitatively assessed.

The risk results and the risk perspectives presented in this report account for the amount of time, on average, that the plant is in POS 5 in any given year. Additional perspectives could be obtained by expressing the risk results conditional on being in POS 5 (i.e., the results given that the plant is in POS 5). These additional perspectives were beyond the scope and resources allocated for this project and hence were not generated in this study.

For cases where the containment equipment hatch is open during the accident, the auxiliary building could play a major role in mitigating the release. The auxiliary building acts as a large holdup volume, allowing time for natural processes to remove radionuclides from the building atmosphere before being released into the environment. Thus the building is an important plant feature that mitigates the release to the environment. Although the auxiliary building was included in the MELCOR calculations performed for this study, no detailed analyses of the auxiliary building's structural capacity or its capability to retain radionuclides were performed. Instead, assumptions were used in the modeling of this issue, any of which, if changed, could have a significant impact on the results because the most likely accident scenarios consist of an open containment where the radioactive releases pass through the auxiliary building. The modeling assumptions include the following:

- $\quad$ Although the auxiliary building is not a leak-tight structure, it was assumed that none of the radioactive material escapes the building prior to its assumed failure pressure of $5 \mathrm{psig}$. This is only an issue for the accidents in which the operators flood the containment. In these accidents the auxiliary building pressurizes very slowly and is predicted to stay intact until vessel failure. In all of the other accidents in which the containment is open, the building is predicted to fail prior to core damage.

- $\quad$ Neither the ventilation system nor the standby gas treatment system were modeled, and therefore the effect (either beneficial or detrimental) that these systems would have on the building's ability to retain radioactive material was not considered.

- The decontamination factor used in this analysis could be approximated by the decontamination factor distribution of the auxiliary building developed in the NUREG-1150 analysis of the Peach Bottom plant.

In addition to the issues discussed above, a more general issue can affect the risk results generated in this study: the use of NUREG-1150 data, which were developed for accidents initiated at power conditions for these shutdown accidents. The NUREG-1150 data were used extensively to quantify the parameters in the accident progression event tree and the parametric source term expression.

(Information about the timing of the accident, e.g., start of core damage, auxiliary building failure time, and temperature and pressure histories, was obtained from MELCOR calculation performed specifically for this analysis.) 
Open Issues 


\section{Appendix A Supporting Information for the Plant Damage State Analysis}

This appendix contains information that was used to support the plant damage state (PDS) analysis. The approach used to develop PDSs is described in Section 5.1 of the main report.

\section{A.1 Development of End States}

Sixteen characteristics were used to define the End States (ESs). These 16 characteristics and their associated attributes are defined in Table A.1 (definitions for the initiating event types listed in Table A.1 are provided in Chapter 4 of Volume 2 of this report). In the Level 1 analysis, 28 sequences that contained a total of 38 cut sets were delineated. These cut sets were reviewed and the appropriate attribute for each characteristic was assigned to each cut set. The result was a 16-position alphanumeric string for each cut set. A unique string defines an ES. Through this process, 22 ESs were defined. A list of these ESs is provided in Table A.2.

\section{A.2 Development of Plant Damage States}

Review of the ESs suggested that 11 characteristics would adequately define the PDSs. The characteristics and attributes for the PDSs are presented in Table A.3. ES characteristic 1 was dropped because there were too many initiating events to treat each one separately, and in most cases the Level 2 analysis does not depend on the initiating event. This characteristic was included in the ES analysis for the sake of completeness. For cases where it is important to know the initiating event (e.g., loss of coolant accident [LOCA] or loss of offsite power), this information was included in a characteristic that addressed the event. For example, PDS Characteristic 1, Status of Electric Power, is used to identify accidents initiated by a loss of offsite power. Similarly, PDS Characteristic 7 is used to identify accidents initiated by a LOCA. Because in the Level 2 analysis large LOCAs (i.e., A) and intermediate size LOCAs (i.e., S1) are treated the same, this distinction does not have to be maintained in the PDS definition. ES Characteristics 3-6, Status of Core Cooling, were combined under one PDS characteristic. ES Characteristic 9, Status of Suppression Pool Makeup System, was incorporated into PDS Characteristic 3, Status of Containment Sprays and Suppression Pool Cooling, and Characteristic 4, Status of Suppression Pool Level. In going from ESs to PDSs, for a given characteristic the attributes were often changed to eliminate redundant or unnecessary information and to incorporate information from other characteristics that were eliminated.

In the development of PDSs from ESs, a series of assumptions was made with regard to the station blackout ESs. These assumptions include the following:

- PDS1-2 consists of two sequences. In the first case the station batteries deplete within two hours of the initiating event, resulting in the closure of the safety relief valves (SRVs) and the subsequent pressurization of the vessel. With the vessel pressurized, the firewater system cannot be used as an alternate source of injection. In this scenario, the vessel pressurizes to $440 \mathrm{psig}$ and fails the shutdown cooling system, resulting in an interfacing system LOCA. Core damage is estimated to occur 3.5 hours after the initiating event. In the second sequence the station batteries supply emergency dc power for at least 3.5 hours. In this scenario, two hours after the initiating event the operators open the SRVs and use the firewater system as an alternate source of coolant makeup. Injection is continued until the batteries deplete, resulting in the closure of the SRVs and the pressurization of the vessel. The firewater system cannot provide makeup once the vessel pressurizes. The batteries deplete sometime between 3.5 and 12 hours after the initiating event. In this analysis it is conservatively assumed that the batteries fail 3.5 hours after the initiating event, and therefore the firewater system injects water for only 1.5 hours. Following the loss of the firewater system, the accident progresses in a manner similar to the first sequence. Thus the major difference between the two sequences is that core damage is delayed by approximately 1.5 hours in the second sequence. This delay of 1.5 hours is not sufficient to warrant a separate PDS; therefore the second sequence is modeled as though the firewater system was never used.

- In PDS1-3 it is assumed that the operators manually isolate the shutdown cooling (SDC) system before the vessel begins to pressurize following the loss of core cooling, thereby preventing an interfacing systems LOCA in the SDC 
Appendix A

system. This action is possible in these accidents because there is a significant amount of time between the initiating event and the pressurization of the vessel (i.e., 12 hours). A.lthough this action was included in the Level 1 model for Time Window 2, it was not included in the model for Time Window 1 because isolation of the SDC system in Time Window 1 does not prevent core damage. However, had this action been included in the Level 1 model, the cut sets that would have survived truncation would have the SDC system isolated. Thus, while the ES indicates there is a LOCA in the SDC system (Attribute F for Characteristic 14), the PDS indicates the vessel is at high pressure with pressure relief being provided by the SRVs.

- PDS2-2 consists of five similar sequences. In the first two sequences the station batteries deplete within 5.5 hours of the initiating event, resulting in the closure of the SRVs and the subsequent pressurization of the vessel. With the vessel pressurized, the firewater system cannot be used as an alternate source of injection. In this scenario, the vessel pressurizes to $440 \mathrm{psig}$ and fails the shutdown cooling system resulting in an interfacing system LOCA. Core damage is estimated to occur 5.5 hours after the initiating event. In the remaining three sequences the station batteries supply emergency dc power long enough for the operators to open the SRVs and align the firewater system to provide coolant to the core. The firewater system provides coolant makeup until the batteries deplete, at which point the accident progress in a manner similar to the first two sequences except that core damage is delayed by several hours. Each of these last three sequences has a different battery depletion tim. (i.e., battery depletion times of 3, 5.5, and 12 hours). To keep the analysis manageable by reducing the number of PDS that needed to be analyzed, the last three sequences were combined with the first two sequences and were modeled as though the firewater system was not used.

The consolidation of ES characteristics and the simplifying assumptions regarding the use of the firewater system in the station blackout ESs resulted in the generation of twelve PDSs. A list of the ESs that are contained in each PDS is presented in Table A.2. The PDS definitions and their contribution to the point estimate core damage frequency are presented in Table A.4.

\section{A.3 Comparison Between Level 1 and Level 2/3 PDS Frequencies}

The Level 1 analysis documented in Volume 2 of this report used a Latin hypercube sampling (LHS) size of 1000, whereas the Level $2 / 3$ analysis used a sample size of 200 . While a sample size of 1000 can be used in the Level 1 analysis, the large computational requirements of accident progression and consequence analyses precluded the use of such a large sample size in the Level $2 / 3$ analysis. When selecting the LHS size for the Level $2 / 3$ analyses, two objectives had to be considered: (1) the sample size had to be sufficiently large so that the Level $2 / 3$ PDS results were reasonably similar to the Level 1 results, and (2) the sample size had to be small enough that the calculations could be performed in a timely manner. A sample size of 200 satisfied both objectives. The PDS frequencies from these two samples are compared in Table A.5. 


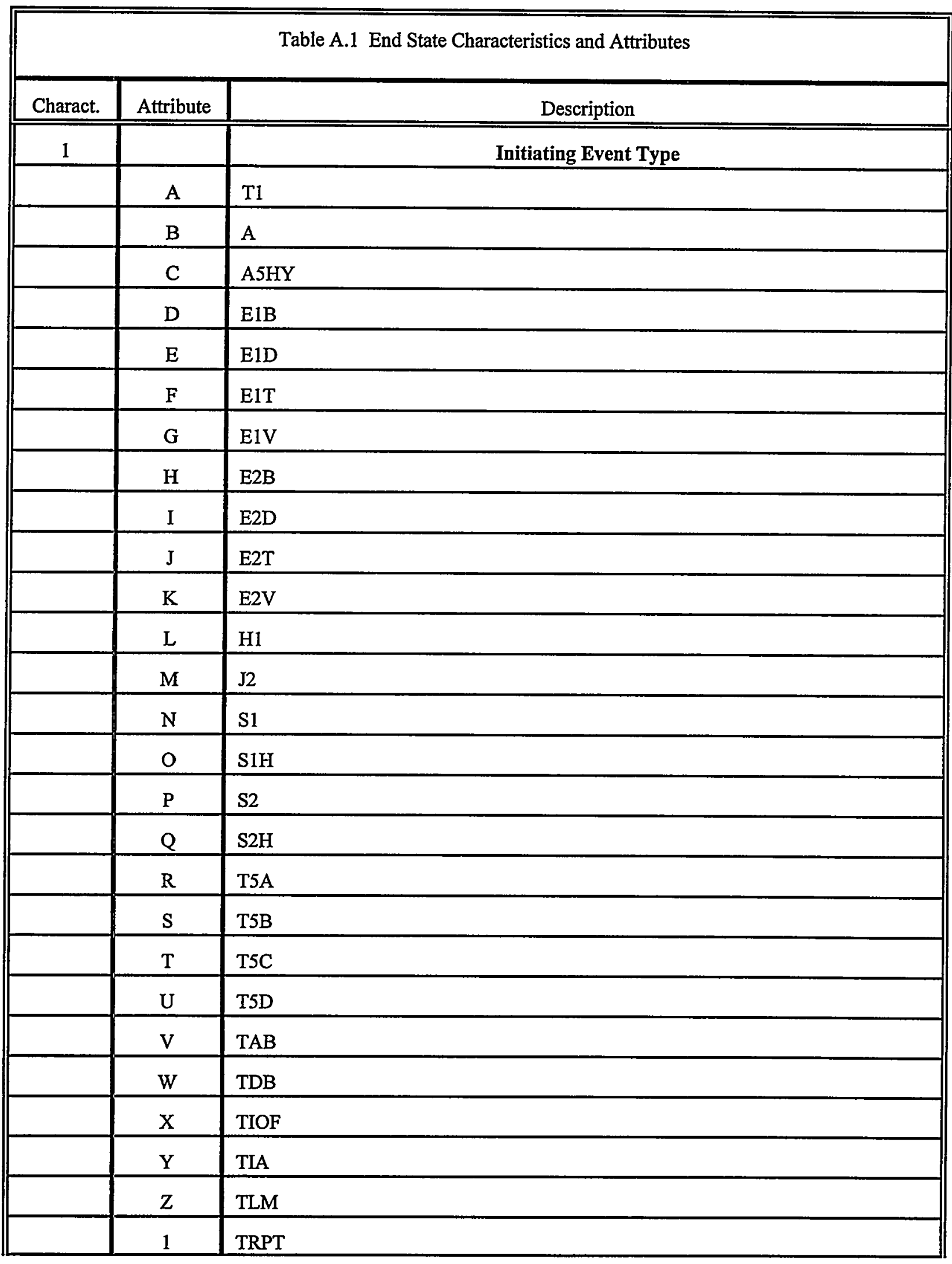


Appendix A

\begin{tabular}{|c|c|c|}
\hline Charact. & Attribute & Description \\
\hline & & AVAILABILITY OF ELECTRICAL POWER \\
\hline \multirow[t]{7}{*}{2} & & Availability of Electrical Power \\
\hline & A & Offsite power (OSP) available, $\mathrm{AC}$ and $\mathrm{DC} \mathrm{B}$ power available \\
\hline & B & OSP not available - but recoverable \\
\hline & $\mathrm{C}$ & OSP not available - not recoverable, delayed failure of core cooling \\
\hline & $\mathrm{D}$ & OSP not available - not recoverable, prompt failure of core cooling \\
\hline & E & OSP available - Emergency $A C$ and $D C$ power not available and not recoverable \\
\hline & & STATUS OF CORE COOLING \\
\hline \multirow[t]{4}{*}{3} & & Status of HPCS \\
\hline & A & HPCS available \\
\hline & B & HPCS not available - not recoverable \\
\hline & $\mathrm{C}$ & HPCS not available - but recoverable \\
\hline \multirow[t]{5}{*}{4} & & Status of LPCI \\
\hline & A & LPCI Train B available \\
\hline & $\mathrm{B}$ & LPCI not available - not recoverable \\
\hline & $\mathrm{C}$ & LPCI not available - but recoverable \\
\hline & $\mathrm{D}$ & LPCI not available - but recoverable with recovery of OSP \\
\hline \multirow[t]{5}{*}{5} & & Status of Service Water Crosstie \\
\hline & A & SSW Crosstie available \\
\hline & $\mathrm{B}$ & SSW Crosstie not available - not recoverable \\
\hline & $\mathrm{C}$ & SSW Crosstie not available - but recoverable \\
\hline & $\mathrm{D}$ & SSW Crosstie not available - but recoverable with recovery of OSP \\
\hline & & \\
\hline
\end{tabular}




\begin{tabular}{|c|c|c|}
\hline Charact. & Attribute & Description \\
\hline \multirow[t]{6}{*}{6} & & Status of Condensate \\
\hline & A & CDS available \\
\hline & B & CDS not available - not recoverable \\
\hline & $\mathrm{C}$ & CDS not available - but recoverable \\
\hline & $\mathrm{D}$ & CDS not available - but recoverable with recovery of OSP \\
\hline & & STATUS OF CONTAINMENT HEAT REMOVAL \\
\hline \multirow[t]{4}{*}{7} & & Status of Suppression Pool Level \\
\hline & A & Water at "Low Level" or "Drained Level" \\
\hline & B & Suppression pool is empty \\
\hline & $\mathrm{C}$ & Suppression pool level is at the ECCS suction strainers \\
\hline \multirow[t]{4}{*}{8} & & Status of Suppression Pool Temperature \\
\hline & A & Suppression Pool is sub-cooled \\
\hline & B & Suppression Pool is saturated \\
\hline & $\mathrm{C}$ & Suppression Pool temperature is not applicable \\
\hline \multirow[t]{6}{*}{9} & & Status of Suppression Pool Makeup \\
\hline & A & SPMU has been used \\
\hline & B & SPMU is available but not used because it was not previously needed \\
\hline & $\mathrm{C}$ & SPMU is available but not used because of operator error \\
\hline & $\mathrm{D}$ & SPMU is not available but can be recovered with recovery of OSP \\
\hline & E & SPMU is not available and cannot be recovered \\
\hline \multirow[t]{4}{*}{10} & & Status of Containment Sprays and Suppression Pool Cooling \\
\hline & A & CS/SPC available with heat exchangers \\
\hline & B & CS/SPC not available - not recoverable \\
\hline & $\mathrm{C}$ & CS/SPC not available - but recoverable with recovery of OSP \\
\hline
\end{tabular}


Appendix A

\begin{tabular}{|c|c|c|}
\hline Charact. & Attribute & Description \\
\hline & & STATUS OF REACTOR VESSEL AND CONTAINMENT INTEGRITY \\
\hline \multirow[t]{3}{*}{11} & & Status of the Reactor Vessel Head Vent \\
\hline & A & Head vent is open during the accident \\
\hline & B & Operators close the head vent prior to core damage \\
\hline \multirow[t]{3}{*}{12} & & Status of Containment Lower Personnel Lock \\
\hline & A & Containment lower personnel lock is open \\
\hline & B & Containment status is unknown \\
\hline \multirow[t]{4}{*}{13} & & Status of Containment Vent System \\
\hline & A & CVS not required - but available \\
\hline & B & CVS not required - not available \\
\hline & $\mathrm{C}$ & CVS not required - not available - but recoverable with recovery of OSP \\
\hline \multirow[t]{8}{*}{14} & & Status of RPV Pressure \\
\hline & A & Vessel at high pressure - SRVs available but not used \\
\hline & $\mathrm{B}$ & Vessel at high pressure - SRVs available but operator failed to use them \\
\hline & C & Vessel at high pressure - SRVs not available \\
\hline & D & Vessel at low pressure - SRVs are open by operator \\
\hline & $\mathrm{E}$ & Vessel at low pressure - SRVs available and the vessel is open by LOCA \\
\hline & $\mathrm{F}$ & Vessel at low pressure - SRVs available and the vessel is open by SDC break \\
\hline & G & Vessel at low pressure - SRVs available and the vessel is open by open MSIVs \\
\hline & & \\
\hline & & \\
\hline & & \\
\hline & & \\
\hline
\end{tabular}




\begin{tabular}{|c|c|c|}
\hline \multicolumn{3}{|r|}{ Table A.1 End State Characteristics and Attributes } \\
\hline \multirow[t]{2}{*}{ Charact. } & Attribute & Description \\
\hline & & TIMING CHARACTERISTICS \\
\hline \multirow[t]{11}{*}{15} & & Time to Core Damage \\
\hline & A & Core damage occurs in 2 hour \\
\hline & B & Core damage occurs in 2.35 hours \\
\hline & $\mathrm{C}$ & Core damage occurs in 3 hours \\
\hline & $\underline{\mathrm{D}}$ & Core damage occurs in 3.5 hours \\
\hline & $\mathrm{E}$ & Core damage occurs in 5.5 hours \\
\hline & $\mathrm{F}$ & Core damage occurs in 6.75 hours \\
\hline & G & Core damage occurs in 7 hours \\
\hline & $\mathrm{H}$ & Core damage occurs in 7.35 hours \\
\hline & I & Core damage occurs in 9.75 hours \\
\hline & $\mathbf{J}$ & Core damage occurs in 12 hours \\
\hline \multirow[t]{4}{*}{16} & & Time Window \\
\hline & 1 & Time window 1: Ranges from 14 to 24 hours after shutdown \\
\hline & 2 & Time window 2: Ranges from 24 to 94 hours after shutdown \\
\hline & 3 & Time window 3: Ranges from 40 to 50.4 days after shutdown \\
\hline
\end{tabular}


Appendix A

Table A.2 End State and Plant Damage State Definitions

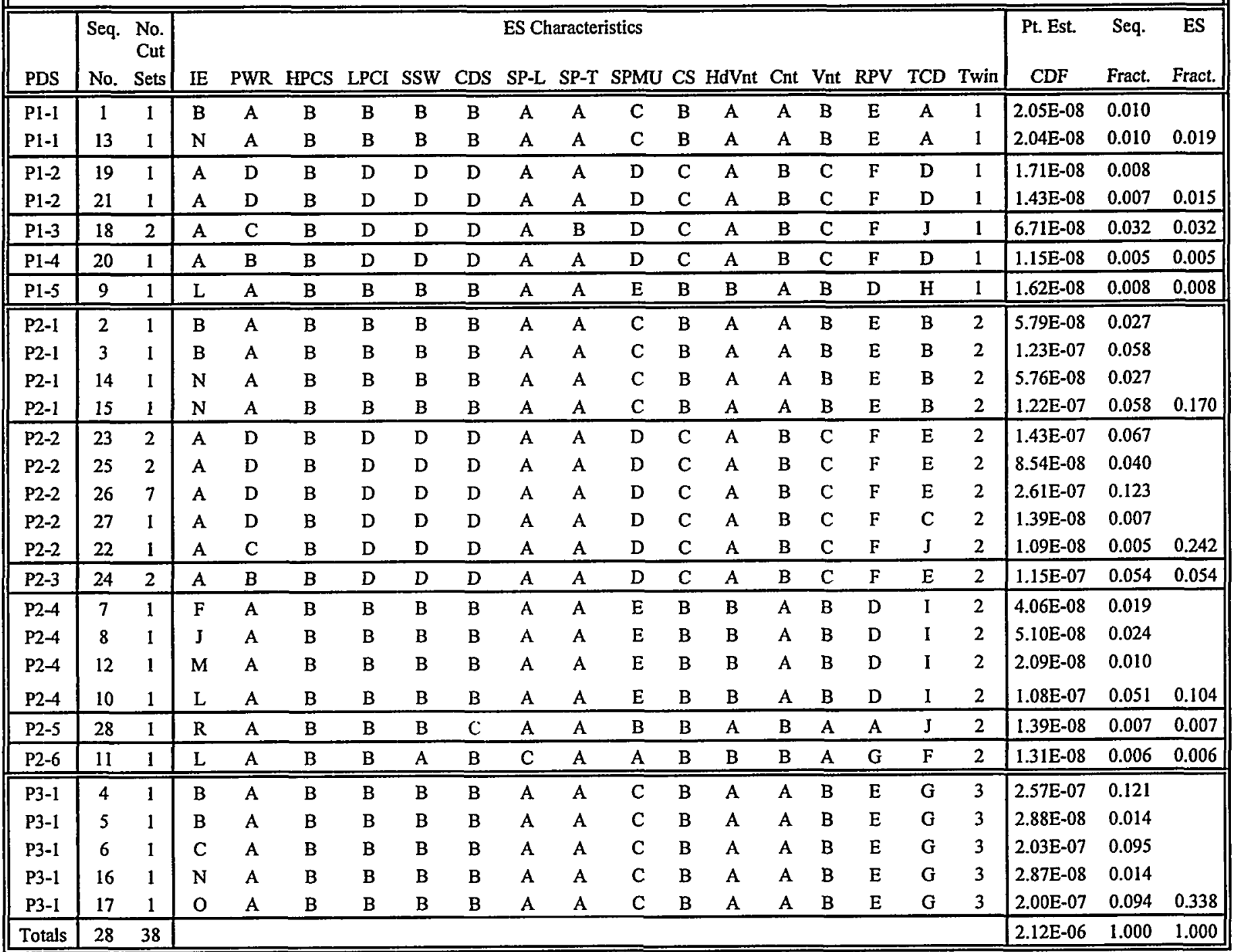


Table A.3 Plant Damage State Characteristics and Attributes

\begin{tabular}{|c|c|c|}
\hline Charact. & Attribute & Description \\
\hline & & STATUS OF ELECTRICAL POWER \\
\hline \multirow[t]{7}{*}{1} & & Status of Electrical Power \\
\hline & A & Offsite power (OSP) available \\
\hline & B & OSP not available - but recoverable \\
\hline & $\mathrm{C}$ & OSP not available - not recoverable, delayed failure of core cooling \\
\hline & $\mathrm{D}$ & OSP not available - not recoverable, prompt failure of core cooling \\
\hline & $\mathrm{E}$ & OSP available - Emergency $\mathrm{AC}$ and $\mathrm{DC}$ power not available and not recoverable \\
\hline & & STATUS OF CORE COOLING \\
\hline \multirow[t]{5}{*}{2} & & Status of Core Coolant Injection \\
\hline & A & Core injection is not available and cannot be recovered \\
\hline & B & LPCI and/or SSW crosstie are unavailable due to operator error \\
\hline & $\mathrm{C}$ & LPCI and/or SSW crosstie are unavailable but recoverable with recovery of OSP \\
\hline & & STATUS OF CONTAINMENT HEAT REMOVAL \\
\hline \multirow[t]{4}{*}{3} & & Status of Containment Sprays and Suppression Pool Cooling \\
\hline & A & $\mathrm{CS} / \mathrm{SPC}$ is not available and cannot be recovered \\
\hline & B & $\mathrm{CS} / \mathrm{SPC}$ is not available but can be recovered with recovery of OSP \\
\hline & $\mathrm{C}$ & $\mathrm{CS} / \mathrm{SPC}$ is available \\
\hline \multirow[t]{3}{*}{4} & & Status of Suppression Pool Level \\
\hline & A & Water at "Low Level" or "Drained Level" \\
\hline & B & Suppression pool level is at the ECCS suction strainers \\
\hline \multirow[t]{3}{*}{5} & & Status of Suppression Pool Temperature \\
\hline & A & Suppression Pool is sub-cooled \\
\hline & B & Suppression Pool is saturated \\
\hline
\end{tabular}




\section{Appendix A}

\begin{tabular}{|c|c|c|}
\hline & & Table A.3 Plant Damage State Characteristics and Attributes \\
\hline \multirow[t]{2}{*}{ Charact. } & Attribute & Description \\
\hline & & STATUS OF REACTOR VESSEL INTEGRITY \\
\hline \multirow[t]{3}{*}{6} & & Status of RPV Head Vent \\
\hline & A & Head vent is open during the accident \\
\hline & B & Operators close the head vent prior to core damage \\
\hline \multirow[t]{7}{*}{7} & & Status of RPV Pressure and Integrity \\
\hline & A & Primary system is at system pressure \\
\hline & $\mathrm{B}$ & Primary system is at low pressure ( $>400 \mathrm{psia})$ \\
\hline & $\mathrm{C}$ & Primary system is at low pressure; RPV is breached by a LOCA inside containment \\
\hline & $\mathrm{D}$ & Primary system is at low pressure; RPV is breached by a LOCA in SDC system \\
\hline & $\mathrm{E}$ & Primary system is at low pressure; RPV is breached by open MSIVs \\
\hline & & STATUS OF CONTAINMENT INTEGRITY \\
\hline \multirow[t]{3}{*}{8} & & Status of Containment Lower Personnel Lock \\
\hline & A & Containment lower personnel lock is open \\
\hline & B & Containment status is unknown \\
\hline \multirow[t]{4}{*}{9} & & Status of Containment Vent System \\
\hline & A & CVS is unavailable and cannot be recovered \\
\hline & B & CVS is unavailable but can be recovered with recovery of OSP \\
\hline & $\mathrm{C}$ & CVS is available but has not been used because is has not been needed \\
\hline & & \\
\hline & & \\
\hline & & \\
\hline & & \\
\hline & & \\
\hline & & \\
\hline
\end{tabular}


Appendix A

\begin{tabular}{|c|c|c|}
\hline & & Table A.3 Plant Damage State Characteristics and Attributes \\
\hline \multirow[t]{2}{*}{ Charact. } & Attribute & Description \\
\hline & & TIMING CHARACTERISTICS \\
\hline \multirow[t]{9}{*}{10} & & Time to Core Damage \\
\hline & A & Core damage occurs in 2 hour \\
\hline & B & Core damage occurs in 2.35 hours \\
\hline & $\mathrm{C}$ & Core damage occurs in 3.5 hours \\
\hline & $\mathrm{D}$ & Core damage occurs in 5.5 hours \\
\hline & $\mathrm{E}$ & Core damage occurs in 6.75 hours \\
\hline & $\mathrm{F}$ & Core damage occurs in 7 hours \\
\hline & $\mathrm{G}$ & Core damage occurs in 9.75 hours \\
\hline & $\mathrm{H}$ & Core damage occurs in 12 hours \\
\hline \multirow[t]{4}{*}{11} & & Time Windows \\
\hline & $\mathrm{A}$ & Time Window 1: Ranges from 14 to 24 hours after shutdown \\
\hline & $\mathrm{B}$ & Time Window 2: Ranges from 24 to 94 hours after shutdown \\
\hline & $\mathrm{C}$ & Time Window 3: Ranges from 40 to 50.4 days after shutdown \\
\hline
\end{tabular}


Appendix A

\begin{tabular}{||c|c|c||}
\hline \multicolumn{3}{|c||}{ Table A.4 Plant Damage State Definitions } \\
\hline \hline Plant Damage States & Alpha-numeric Description & $\begin{array}{c}\text { Fractional Contribution } \\
\text { to PE CD Frequency }\end{array}$ \\
\hline PDS1-1 & A-A-AAA-AC-AA-A1 & 0.019 \\
\hline PDS1-2 & D-C-BAA-AD-AB-C1 & 0.015 \\
\hline PDS1-3 & C-C-BAB-AA-AB-H1 & 0.032 \\
\hline PDS1-4 & B-C-BAA-AD-AB-C1 & 0.005 \\
\hline PDS1-5 & A-A-AAA-BB-AA-F1 & 0.008 \\
\hline PDS2-1 & A-A-AAA-AC-AA-B2 & 0.170 \\
\hline PDS2-2 & D-C-BAA-AD-AB-D2 & 0.242 \\
\hline PDS2-3 & B-C-BAA-AD-AB-D2 & 0.054 \\
\hline PDS2-4 & A-A-AAA-BB-AA-G2 & 0.104 \\
\hline PDS2-5 & A-B-AAA-AA-BC-H2 & 0.007 \\
\hline PDS2-6 & A-B-ABA-BE-BC-E2 & 0.006 \\
\hline PDS3-1 & A-A-AAA-AC-AA-F3 & 0.338 \\
\hline & & 1.00 \\
\hline Total & & \\
\hline
\end{tabular}


Appendix A

\begin{tabular}{|c|c|c|c|c|c|c|c|}
\hline \multirow[t]{2}{*}{$\begin{array}{c}\text { Plant Damage } \\
\text { States }\end{array}$} & \multirow{2}{*}{$\begin{array}{l}\text { LHS } \\
\text { Sample } \\
\text { Size }\end{array}$} & \multicolumn{4}{|c|}{$\begin{array}{l}\text { Descriptive Statistics': Core Damage Frequency } \\
(1 / \mathrm{yr})\end{array}$} & \multicolumn{2}{|c|}{ Fractional Contribution ${ }^{2}$} \\
\hline & & $5 \%$ & $50 \%$ & $95 \%$ & Mean & FCM-CDF & MFC-CDF \\
\hline \multirow[t]{2}{*}{ PDS1-1 } & 200 & $1.59 \mathrm{E}-09$ & $1.41 \mathrm{E}-08$ & $1.92 \mathrm{E}-07$ & $4.13 \mathrm{E}-08$ & 0.020 & 0.018 \\
\hline & 1000 & $1.31 \mathrm{E}-09$ & $1.51 \mathrm{E}-08$ & $1.56 \mathrm{E}-07$ & $4.05 \mathrm{E}-08$ & 0.020 & 0.017 \\
\hline \multirow[t]{2}{*}{ PDS1-2 } & 200 & $1.44 \mathrm{E}-10$ & $4.27 \mathrm{E}-09$ & $1.29 \mathrm{E}-07$ & $2.29 \mathrm{E}-08$ & 0.011 & 0.015 \\
\hline & 1000 & $2.15 \mathrm{E}-10$ & $4.93 \mathrm{E}-09$ & 1.11E-07 & $2.48 \mathrm{E}-08$ & 0.012 & 0.016 \\
\hline \multirow[t]{2}{*}{ PDS1-3 } & 200 & $2.94 \mathrm{E}-09$ & $1.73 \mathrm{E}-08$ & $1.62 \mathrm{E}-07$ & $4.39 \mathrm{E}-08$ & 0.021 & 0.030 \\
\hline & 1000 & $2.82 \mathrm{E}-09$ & $1.75 \mathrm{E}-08$ & $1.22 \mathrm{E}-07$ & $3.59 \mathrm{E}-08$ & 0.018 & 0.027 \\
\hline \multirow[t]{2}{*}{ PDS1-4 } & 200 & $5.98 \mathrm{E}-11$ & $1.99 \mathrm{E}-09$ & $3.49 \mathrm{E}-08$ & $9.19 \mathrm{E}-09$ & 0.004 & 0.006 \\
\hline & 1000 & 9.33E-11 & $1.97 \mathrm{E}-09$ & $3.79 \mathrm{E}-08$ & 9.34E-09 & 0.005 & 0.007 \\
\hline \multirow[t]{2}{*}{ PDS1-5 } & 200 & $4.89 \mathrm{E}-10$ & $6.85 \mathrm{E}-09$ & $4.78 \mathrm{E}-08$ & $1.36 \mathrm{E}-08$ & 0.007 & 0.010 \\
\hline & 1000 & $4.78 \mathrm{E}-10$ & $6.03 \mathrm{E}-09$ & $6.68 \mathrm{E}-08$ & $1.59 \mathrm{E}-08$ & 0.008 & 0.011 \\
\hline \multirow[t]{2}{*}{ PDS2-1 } & 200 & $1.28 \mathrm{E}-08$ & $1.35 \mathrm{E}-07$ & $1.45 \mathrm{E}-06$ & $3.52 \mathrm{E}-07$ & 0.168 & 0.153 \\
\hline & 1000 & 1.13E-08 & $1.35 \mathrm{E}-07$ & $1.41 \mathrm{E}-06$ & 3.67E-07 & 0.184 & 0.154 \\
\hline \multirow[t]{2}{*}{ PDS2-2 } & 200 & $2.22 \mathrm{E}-08$ & $1.45 \mathrm{E}-07$ & $1.60 \mathrm{E}-06$ & 5.53E-07 & 0.264 & 0.217 \\
\hline & 1000 & $2.12 \mathrm{E}-08$ & $1.72 \mathrm{E}-07$ & $1.63 \mathrm{E}-06$ & 4.47E-07 & 0.224 & 0.210 \\
\hline \multirow[t]{2}{*}{ PDS2-3 } & 200 & $2.74 \mathrm{E}-09$ & $2.93 \mathrm{E}-08$ & $4.46 \mathrm{E}-07$ & $1.11 \mathrm{E}-07$ & 0.053 & 0.059 \\
\hline & 1000 & $3.05 \mathrm{E}-09$ & $2.87 \mathrm{E}-08$ & $3.33 \mathrm{E}-07$ & $9.29 \mathrm{E}-08$ & 0.046 & 0.055 \\
\hline \multirow[t]{2}{*}{ PDS2-4 } & 200 & $7.67 \mathrm{E}-09$ & $8.80 \mathrm{E}-08$ & $7.52 \mathrm{E}-07$ & $2.03 \mathrm{E}-07$ & 0.097 & 0.140 \\
\hline & 1000 & $6.04 \mathrm{E}-09$ & $9.93 \mathrm{E}-08$ & $8.19 \mathrm{E}-07$ & $2.14 \mathrm{E}-07$ & 0.107 & 0.140 \\
\hline \multirow[t]{2}{*}{ PDS2-5 } & 200 & $8.55 \mathrm{E}-11$ & $2.74 \mathrm{E}-09$ & $5.32 \mathrm{E}-08$ & $1.26 \mathrm{E}-08$ & 0.006 & 0.010 \\
\hline & 1000 & $1.06 \mathrm{E}-10$ & $2.59 \mathrm{E}-09$ & $6.01 \mathrm{E}-08$ & $1.47 \mathrm{E}-08$ & 0.007 & 0.010 \\
\hline \multirow[t]{2}{*}{ PDS2-6 } & 200 & $2.56 \mathrm{E}-11$ & $1.11 \mathrm{E}-09$ & 2.83E-08 & $7.43 \mathrm{E}-09$ & 0.004 & 0.006 \\
\hline & 1000 & $1.69 \mathrm{E}-11$ & $1.07 \mathrm{E}-09$ & $5.22 \mathrm{E}-08$ & $1.17 \mathrm{E}-08$ & 0.006 & 0.009 \\
\hline \multirow[t]{2}{*}{ PDS3-1 } & 200 & $6.25 \mathrm{E}-08$ & $3.75 \mathrm{E}-07$ & $2.40 \mathrm{E}-06$ & $7.27 \mathrm{E}-07$ & 0.347 & 0.338 \\
\hline & 1000 & $6.50 \mathrm{E}-08$ & $3.75 \mathrm{E}-07$ & $2.29 \mathrm{E}-06$ & $7.26 \mathrm{E}-07$ & 0.363 & 0.343 \\
\hline \multirow[t]{2}{*}{ Total } & 200 & $4.07 \mathrm{E}-07$ & $1.37 \mathrm{E}-06$ & $5.56 \mathrm{E}-06$ & $2.10 \mathrm{E}-06$ & & \\
\hline & 1000 & 4.13E-07 & $1.34 \mathrm{E}-06$ & $5.38 \mathrm{E}-06$ & $2.00 \mathrm{E}-06$ & & \\
\hline
\end{tabular}

Note 1: A LHS sample size of 200 was used in the Level 2/3 and integrated analyses whereas a LHS sample size of 1000 was used in the Level 1 analysis

Note 2: $\quad F C M-C D F=$ Fractional contribution to mean core damage frequency

MFC-CDF $=$ Mean fractional contribution to core damage frequency 
Appendix A 


\section{Appendix B Supporting Information for the Accident Progression Analysis}

This appendix contains information that was used to support the accident progression analysis. The many possible progressions of the accident following the onset of core damage are delineated and evaluated using event tree techniques. The event tree developed to model the Level 2 portion of the accident is called an accident progression event tree (APET). The APET is evaluated using the EVNTRE code [Griesmeyer et al., 1989] as implemented in Version 0.95 Alpha of the SETAC event tree processing code suite. EVNTRE calculates the probability of each path through the APET and combines the paths into groups, specified by the user, called accident progression bins (APBs). These APBs can then be further grouped or rebinned and sorted using the PSTEVNT code. Section B.1 provides a discussion of each of the questions in the APET; the APET that forms the input to the EVNTRE code is listed in Section B.2. The input format for EVNTRE is described in ETPRE user's manual [Roginski, 1994]; the concept of a case structure, as described in the ETPRE user's manual, is vital for understanding Section B.2. The quantification of the APET is discussed in Section B.3. A description of the characteristics used to form the APBs is provided in Section B.4.

\section{B.1 Description of the Grand Gulf POS 5 APET}

In the following section, the purpose of each question in the APET is discussed, the branches are defined, and the sources of information used to quantify the branches are presented. The probabilities assigned to each branch are also presented; for cases where a distribution of probabilities is used, the mean of the distribution is displayed. The Latin hypercube sampling (LHS) code was used to generate the inputs for the branches that are quantified with a distribution. The distribution types listed in the APET correspond to distributions supported by the LHS code. A single APET is used to evaluate all the PDSs.

Question 1. What is the Plant Damage State?

Number of Branches: 12

Number of Cases: 1

Number of Cases Sampled: 1

The branches for this question are:

1. PDS1-1 PDS1-1: Loss of coolant accident (LOCA) in Time Window 1

2. PDS1-2 PDS1-2: Station Blackout accident in Time Window 1

3. PDS1-3 PDS1-3: Station Blackout accident in Time Window 1

4. PDS1-4 PDS1-4: Station Blackout accident in Time Window 1

5. PDS1-5 PDS1-5: Flooded containment accident in Time Window 1

6. PDS2-1 PDS2-1: LOCA in Time Window 2

7. PDS2-2 PDS2-2: Station Blackout accident in Time Window 2

8. PDS2-3 PDS2-3: Station Blackout accident in Time Window 2

9. PDS2-4 PDS2-4: Flooded containment accident in Time Window 2

10. PDS2-5 PDS2-5: High pressure core damage accident in Time Window 2

11. PDS2-6 PDS2-6: Open main steam isolation valve accident in Time Window 2

12. PDS3-1 PDS3-1: LOCA in Time Window 3

This question defines the probability of each plant damage state (PDS), conditional on the occurrence of core damage. Twelve PDSs were defined in this analysis. The branch probabilities are sampled. The probability distribution for each PDS is based on the frequency of each PDS obtained from the IRRAS code.

The quantification for this question is:

$\begin{array}{lll}\text { Branch 1: } & \text { PDS1-1 } & 0.019 \\ \text { Branch 2: } & \text { PDS1-2 } & 0.015 \\ \text { Branch 3: } & \text { PDS1-3 } & 0.032 \\ \text { Branch 4: } & \text { PDS1-4 } & 0.005 \\ \text { Branch 5: } & \text { PDS1-5 } & 0.008\end{array}$


Appendix B

$\begin{array}{lll}\text { Branch 6: } & \text { PDS2-1 } & 0.17 \\ \text { Branch 7: } & \text { PDS2-2 } & 0.242 \\ \text { Branch 8: } & \text { PDS2-3 } & 0.054 \\ \text { Branch 9: } & \text { PDS2-4 } & 0.104 \\ \text { Branch 10: PDS2-5 } & 0.007 \\ \text { Branch 11: PDS2-6 } & 0.006 \\ \text { Branch 12: PDS3-1 } & 0.338\end{array}$

Question 2. What is the status of electric power at core damage (PDS Char. 1)?

Number of Branches: 3

Number of Cases: 3

Number of Cases Sampled: None

The branches for this question are:

1. aOSP Offsite and onsite electric power are available.

2. OSP-nDIVAC Offsite power is available; however, the onsite ac buses are failed.

3. nOSP Neither offsite nor onsite ac power is available.

This question is used to define the PDSs. This question addresses PDS characteristic 1 , which defines the availability of electric power at the onset of core damage. For PDSs initiated by a loss of offsite power (PDS1-2, PDS1-3, PDS1-4, PDS22, and PDS2-3), both offsite and onsite power are unavailable, and therefore the probability of Branch 3 is set to 1.0. For all the other PDSs, the probability of Branch 1 is set to 1.0. Branch 2 is not used in this analysis but was included for the sake of completeness.

Question 3. What is the status of de power at core damage (PDS Char. 1)?

Number of Branches: 2

Number of Cases: 2

Number of Cases Sampled: None

The branches for this question are:

1. nDC-BCD The station batteries have depleted and dc power cannot be recovered.

2. $\mathrm{aDC}-\mathrm{BCD} \quad \mathrm{DC}$ power is available at the onset of core damage.

This question is used to define the PDSs. This question addresses PDS characteristic 1, which defines the availability of electric power at the onset of core damage. If ac power is not available and the station batteries deplete, it is assumed that neither ac nor dc power can be recovered. For PDS1-2, PDS1-3, and PDS2-2, the probability of Branch 1 is set to 1.0. For all the other PDSs, the probability of Branch 2 is set to 1.0.

Question 4. What is the status of high pressure injection at core damage (PDS Char. 2)?

Number of Branches: 3

Number of Cases: 1

Number of Cases Sampled: None

The branches for this question are:

1. nHPInj High pressure injection is not available and cannot be recovered.

2. rHPInj High pressure injection is not available but can be recovered with the recovery of OSP.

3. HPInj High pressure injection is available but not being used.

This question is used to define the PDSs. This question addresses PDS characteristic 2, which defines the availability of core coolant injection at onset of core damage. For all the PDSs defined in this analysis, the probability of Branch 1 is set to 1.0. 
Question 5. What is the status of low pressure injection at core damage (PDS Char. 2)?

Number of Branches: 4

Number of Cases: 4

Number of Cases Sampled: None

The branches for this question are:

1. nLPInj

Low pressure injection is not available and cannot be recovered.

2. nLPInj-op

3. rLPInj

Low pressure injection is not available because of operator errors.

4. aLPInj

Low pressure injection is not available but can be recovered with the recovery of OSP.

Low pressure injection is available but not being used.

This question is used to define the PDSs. This question addresses PDS characteristic 2, which defines the availability of core coolant injection at the onset of core damage. For PDSs initiated by a loss of offsite power (PDS1-2, PDS1-3, PDS1-4, PDS2-2, and PDS2-3), low pressure injection is not available but can be recovered once offsite power is restored to the plant; therefore, the probability of Branch 3 is set to 1.0. For PDS2-6, the service water cross-tie system was available; however, the operators fail to use this system, and therefore the probability of Branch 2 is set to 1.0. For all the other PDSs, the probability of Branch 1 is set to 1.0. There are no PDSs in this analysis in which low pressure injection is available at the onset of core damage, and therefore Branch 4 is never used in this analysis.

Question 6. What is the status of the containment sprays and suppression pool cooling at core damage (PDS Char. 3)?

\section{Number of Branches: 4 \\ Number of Cases: 3 \\ Number of Cases Sampled: None}

The branches for this question are:

1. nCS

2. $\mathrm{rCS}$

3. alignCS

The containment sprays (CS) are not available and cannot be recovered.

4. autoCS

The CS are not available but can be recovered with the recovery of OSP. The CS are available but not aligned to provide containment cooling. The CS are available and aligned to provide containment cooling.

This question is used to define the PDSs. This question addresses PDS characteristic 3, which defines the status of CS at the onset of core damage. For the PDSs initiated by a loss of offsite power (PDS1-2, PDS1-3, PDS1-4, PDS2-2 and PDS2-3) CS are not available but can be recovered if offsite power is restored; therefore, the probability of Branch 2 is set to 1.0. For all the other PDSs the CS are not available and cannot be recovered, and therefore the probability of Branch 1 is set to 1.0.

\section{Question 7. What is the suppression pool level at core damage (PDS Char. 4)? \\ Number of Branches: 2 \\ Number of Cases: 2 \\ Number of Cases Sampled: None}

The branches for this question are:

1. SPL-Lo The suppression pool level is no lower than the low water level (LWL).

2. SPL-Strain The suppression pool has been drained to the ECCS suction strainers.

This question is used to define the PDSs. This question addresses PDS characteristic 4 , which defines the level of the suppression pool at the onset of core damage. For PDS2-6, the probability of Branch 2 is set to 1.0. For all the other PDSs, the suppression pool is no lower than the LWL, and therefore the probability of Branch 1 is set to 1.0. 
Appendix B

Question 8. What is the suppression pool temperature at core damage (PDS Char. 5)?

Number of Branches: 2

Number of Cases: 3

Number of Cases Sampled: None

The branches for this question are:

1. SPT-Sub The suppression pool is subcooled at the onset of core damage.

2. SPT-Sat The suppression pool is saturated at the onset of core damage.

This question is used to define the PDSs. This question addresses PDS characteristic 5, which defines the temperature of the suppression pool at the onset of core damage. For PDS1-3, the suppression pool is saturated at the time of core damage; therefore, the probability of Branch 2 is set to 1.0. For all of the other PDSs, the suppression pool is subcooled, and therefore the probability of Branch 1 is set to 1.0.

Question 9. What is the status of the reactor head vent at core damage (PDS Char. 6)?

Number of Branches: 2

Number of Cases: 2

Number of Cases Sampled: None

The branches for this question are:

1. RPV-nVnt The reactor head vent is not open during core damage.

2. RPV-OVnt The reactor head vent is open during core damage.

This question is used to define the PDSs. This question addresses PDS characteristic 6, which defines the status of the reactor head vent at the time of core damage. The reactor head vent is a two-inch pipe that vents the reactor to the sump located in the reactor cavity directly below the vessel. Although this line is closed and is not used during normal operation, it is open during cold shutdown, and therefore will be open at the start of the accident. The plant's inadequate heat removal procedures, however, direct the operators to close motor-operated valves on the head vent line in the event that core cooling cannot be maintained. The valves cannot be closed during a station blackout. Furthermore, if the operators have failed to follow procedures in a particular accident, it is assumed that the operators will also fail to close the head vent. The head vent is only closed in PDSs PDS1-5, PDS2-4, and PDS2-6; therefore, the probability of Branch 1 is set to 1.0. For all the other PDSs the head vent is open, in which case the probability of Branch 2 is set to 1.0 .

Question 10. What is the status of the reactor pressure vessel integrity at core damage (PDS Char. 7)?

Number of Branches: 5

Number of Cases: 6

Number of Cases Sampled: None

The branches for this question are:

1. RPV-HiP

2. RPV-LoP

3. RPV-LOCA

4. RPV-ILOCA

5. RPV-oMSIV
The reactor vessel has not been breached. The pressure in the vessel is at system pressure with pressure relief being provided by the SRVs at their setpoints.

The reactor vessel has not been breached. At least two SRVs are open and the pressure in the vessel is low ( $<400 \mathrm{psia})$.

The reactor vessel has been breached by a LOCA located inside the containment. The vessel is at low pressure at the onset of core damage.

The reactor vessel has been breached by a LOCA located outside the containment. The vessel is at low pressure at the onset of core damage.

The main steam isolation valves (MSIVs) are open, and therefore the vessel is at low pressure at the onset of core damage. 
This question is used to define the PDSs. This question addresses PDS characteristic 7, which defines the status of the reactor integrity and the pressure of the reactor vessel at the onset of core damage. For PDSs initiated by a LOCA (PDS1-1, PDS2-1, PDS3-1), the probability for Branch 3 is set to 1.0. For PDSs initiated by a loss of offsite power followed by a break outside the containment in the shutdown cooling system (PDS1-2, PDS1-4, PDS2-2, and PDS2-3), the probability for Branch 4 is set to 1.0. For PDS2-6, in which the operators fail to close the MSIVs, the probability for Branch 5 is set to 1.0. Two SRVs are open in PDS1-5 and PDS2-4; therefore, the vessel is at low pressure and the probability for Branch 2 is set to 1.0. For the remaining PDSs, the reactor vessel is pressurized to system pressure, and therefore for these PDSs the probability of Branch 1 is set 1.0 .

Question 11. What is the status of the containment access penetrations at core damage (PDS Char. 8)?

Number of Branches: 2

Number of Cases: 2

Number of Cases Sampled: None

The branches for this question are:

1. o-LPersLk The lower containment personnel lock is open at the onset of core damage.

2. LPersLk-Unk The status of the lower personnel lock is unknown at the onset of core damage.

This question is used to define the PDSs. This question addresses PDS characteristic 8, which defines the status of the lower containment personnel lock. The lower personnel lock will be open if the operators flooded the containment and failed to close the lower personnel lock, or if the accident is initiated by a loss of offsite power (the closure of the containment equipment hatch requires offsite power). There are only two PDSs for which the status of the containment penetrations is not known: PDS2-5 and PDS2-6. For these two PDSs, the probability for Branch 2 is set to 1.0. For all the other PDSs, the lower containment personnel lock and/or the containment equipment hatch are open; therefore, the probability of Branch 1 is set to 1.0. It should be noted, however, that even if the operators close the equipment hatch in PDS2-6, the MSIVs are still open and any releases will escape into the turbine building.

Question 12. What is the status of the containment vent system at core damage (PDS Char. 9)?

Number of Branches: 3

Number of Cases: 4

Number of Cases Sampled: None

The branches for this question are:

1. nCVS The containment vent system (CVS) is unavailable at the onset of core damage and cannot be recovered during the accident.

2. rCVS The CVS is unavailable at the onset of core damage but can be recovered following the recovery of offsite power.

3. aCVS The CVS is available at the onset of core damage.

This question is used to define the PDSs. This question addresses PDS characteristic 9 , which defines the status of the containment vent system. To open the containment vent requires emergency ac power. For PDSs initiated by a loss of offsite power (PDS1-2, PDS1-3, PDS1-4, PDS2-2, and PDS2-3), the CVS is unavailable but recoverable when offsite power is restored to the plant. For these PDSs, the probability for Branch 2 is set to 1.0. For PDS2-5 and PDS2-6, the CVS is available; therefore, the probability for Branch 3 is set to 1.0. For all the other PDSs, the CVS is not available and cannot be recovered, and therefore the probability of Branch 1 is set to 1.0.

Question 13. When does core damage occur (PDS Char. 10)?

Number of Branches: 8

Number of Cases: 9

Number of Cases Sampled: None 
Appendix B

The branches for this question are:

1. TCD2

2. TCD2p3

3. TCD3

4. TCD5

5. TCD6

6. TCD7

7. TCD9

8. TCD12
Core damage occurs approximately 2 hours after the initiation of the accident. Core damage occurs approximately 2.35 hours after the initiation of the accident. Core damage occurs approximately 3.5 hours after the initiation of the accident. Core damage occurs approximately 5.5 hours after the initiation of the accident. Core damage occurs approximately 6.75 hours after the initiation of the accident. Core damage occurs approximately 7 hours after the initiation of the accident. Core damage occurs approximately 9.75 hours after the initiation of the accident. Core damage occurs approximately 12 hours after the initiation of the accident.

This question is used to define the PDSs. This question addresses PDS characteristic 10, which defines the time at which core damage occurs. In this analysis, core damage is defined at the start of significant fuel heatup. The times to core damage were obtained from a series of MELCOR calculations that were performed specifically for this analysis. These calculations are documented in Part 2 of this Volume. For PDS1-1, the probability for Branch 1 is set to 1.0. For PDS2-1, the probability for Branch 2 is set to 1.0. For PDS1-2 and PDS1-4, the probability for Branch 3 is set to 1.0. For PDS2-2 and PDS2-3, the probability for Branch 4 is set to 1.0. For PDS2-6, the probability for Branch 5 is set to 1.0. For PDS1-5 and PDS3-1, the probability for Branch 6 is set to 1.0. For PDS2-4, the probability for Branch 7 is set to 1.0. For PDS1-3 and PDS2-5, the probability for Branch 8 is set to 1.0 .

Question 14. While in POS 5, when does the initiating event occur (PDS Char. 11)?

Number of Branches: 3

Number of Cases: 5

Number of Cases Sampled: None

The branches for this question are:

1. IE-Win 1 The initiating event occurs while the plant is in Time Window 1 . Time Window 1 ranges from 14 hours after shutdown to 24 hours after shutdown.

2. IE-Win2 The initiating event occurs while the plant is in Time Window 2. Time Window 2 ranges from 24 hours after shutdown to 94 hours after shutdown.

3. IE-Win3 The initiating event occurs while the plant is in Time Window 3 . Time Window 3 ranges from 40 days after shutdown to 50.4 days after shutdown.

This question is used to define the PDSs. This question addresses PDS characteristic 11 , which defines the Time Window that the plant is in when the initiating event occurs. The Time Window defines the radionuclide inventory and the decay heat load at the start of the accident. Time Windows 1 and 2 occur during POS 5 prior to the plant entering Operating Condition 5 (Refueling). Time Window 3 occurs during POS 5 on the way back up to power after refueling. PDS1-1, PDS1-2, PDS13, PDS1-4, and PDS1-5 occur while the plant is in Time Window 1; therefore, for these PDSs the probability for Branch 1 is set to 1.0. PDS2-1, PDS2-2, PDS2-3, PDS2-4, PDS2-5 and PDS2-6 occur while the plant is in Time Window 2, and therefore for these PDSs the probability for Branch 2 is set to 1.0. PDS3-1 occurs while the plant is in Time Window 3 , and therefore for this PDS the probability for Branch 3 is set to 1.0 .

Question 15. What type of event initiates the accident?

Number of Branches: 3

Number of Cases: 4

Number of Cases Sampled: None

The branches for this question are:

1. IE-LOCA

2. IE-SBO
The accident is initiated by a loss of coolant accident (LOCA).

The accident is initiated by a loss of offsite power followed by loss of emergency Division $A$ and $B$ power that results in a station blackout (SBO). 
3. IE-Other The accident is not initiated by a LOCA and is not initiated by a loss of offsite power.

This is a summary question and is used to summarize information from previous questions. This question partitions the accidents into three classes: (1) those initiated by a LOCA, (2) those initiated by a loss of offsite power that result in a station blackout, and (3) those initiated by all other types of events.

Case 1: This case includes those accidents initiated by a LOCA (i.e., PDS1-1, PDS2-1, and PDS3-1). The quantification for this case is:

$\begin{array}{lll}\text { Branch 1: } & \text { IE-LOCA } & 1.0 \\ \text { Branch 2: } & \text { IE-SBO } & 0.0 \\ \text { Branch 2: } & \text { IE-Other } & 0.0\end{array}$

Case 2: This case includes those accidents initiated by a loss of offsite power while the plant is Time Window 1 followed by a loss of all emergency ac power resulting in a station blackout scenario. The quantification for this case is:

$\begin{array}{lll}\text { Branch 1: } & \text { IE-LOCA } & 0.0 \\ \text { Branch 2: } & \text { IE-SBO } & 1.0 \\ \text { Branch 2: } & \text { IE-Other } & 0.0\end{array}$

Case 3: This case includes those accidents initiated by a loss of offsite power while the plant is Time Window 2 followed by a loss of all emergency ac power resulting in a station blackout scenario. The quantification for this case is:

$\begin{array}{lll}\text { Branch 1: } & \text { IE-LOCA } & 0.0 \\ \text { Branch 2: } & \text { IE-SBO } & 1.0 \\ \text { Branch 2: } & \text { IE-Other } & 0.0\end{array}$

Case 4: This case includes all the remaining accidents that are not captured by Cases 1 through 3 . The quantification for this case is:

$\begin{array}{lll}\text { Branch 1: } & \text { IE-LOCA } & 0.0 \\ \text { Branch 2: } & \text { IE-SBO } & 0.0 \\ \text { Branch 3: } & \text { IE-Other } & 1.0\end{array}$

Question 16. What is the pressure in the reactor pressure vessel at the time of core damage?

Number of Branches: 2

Number of Cases: 2

Number of Cases Sampled: None

The branches for this question are:

1. RPV-HiP-BCD The reactor vessel is at system pressure (i.e., $1000 \mathrm{psia}$ ) at the onset of core damage.

2. RPV-LoP-BCD The reactor vessel is at low pressure (i.e., $<400 \mathrm{psia}$ ) at the onset of core damage.

This is a summary question and is used to summarize information from previous questions. This question partitions the accidents into two categories: (1) those accidents in which the vessel is pressurized and pressure relief is being provided by the SRVs cycling at their setpoints, (2) those accidents in which the vessel is at low pressure.

Case 1: This case includes those accidents identified as being at high pressure in Question 10. The quantification for this case is: 
Appendix B

$\begin{array}{lll}\text { Branch 1: } & \text { RPV-HiP-BCD } & 1.0 \\ \text { Branch 2: } & \text { RPV-LOP-BCD } & 0.0\end{array}$

Case 2: This case includes all the remaining the accidents in which the reactor vessel is not at system pressure; therefore, they are at low pressure ( $<400$ psia). The reactor vessel will be at low pressure if: (1) the accident is initiated by a LOCA, (2) there is an unisolated LOCA outside the containment, (3) the MSIVs are open, or (4) the operators open two SRVs. The reactor head vent does not have the capacity to keep the reactor vessel depressurized in the event that core cooling is unavailable. Thus an open head vent will not by itself maintain the vessel at low pressure. The quantification for this case is:

Branch 1: RPV-HiP-BCD 0.0

Branch 2: RPV-LoP-BCD 1.0

Question 17. How much water is in the reactor pedestal cavity at the time of core damage?

Number of Branches: 2

Number of Cases: 3

Number of Cases Sampled: None

The branches for this question are:

1. Cav-Dry-BCD The reactor cavity is flooded at the onset of core damage.

2. Cav-Fld-BCD The reactor cavity is essentially dry at the onset of core damage.

This is a summary question and is used to summarize information from previous questions. This question partitions the accidents into two categories: (1) those accidents in which the reactor cavity is flooded with water at the onset of core damage and (2) those accidents in which the reactor cavity is essentially dry at the onset of core damage. The reactor cavity is located directly below the reactor and is partly recessed in the drywell floor. Water on the drywell floor will drain into the reactor cavity where it is collected in sumps. These sumps are then periodically drained. Water can enter the drywell from several sources: (1) normal equipment leakage, (2) LOCA, (3) overflow from the suppression pool (e.g., during containment flooding operations).

Case 1: This case includes those accidents initiated by a LOCA. In these accidents the operators flood the containment to a level that corresponds to the bottom of the lower containment personnel lock. The lower personnel lock is open in these accidents, and therefore the containment is only flooded to this level. The quantification for this case is:

\section{Branch 1: Cav-Dry-BCD $\quad 0.0$ \\ Branch 2: Cav-Fld-BCD 1.0}

Case 2: This case includes those accidents that were not initiated by a LOCA but in which the operators flood the containment to a level that corresponds to the bottom of the lower containment personnel lock in an attempt to prevent core damage. In these accidents, the operators fail to close the lower personnel lock, and therefore the containment is only flooded to this level. The quantification for this case is:

\section{Branch 1: Cav-Dry-BCD 0.0}

Branch 2: Cav-Fld-BCD 1.0

Case 3: This case includes all the remaining accidents that were not initiated by a LOCA and in which the operators did not flood the containment. In these accidents the reactor cavity was essentially dry. Normal leakage from the equipment would not flood the cavity during the time-frame of interest. The quantification for this case is:

Branch 1: Cav-Dry-BCD 1.0

Branch 2: Cav-Fld-BCD $\quad 0.0$ 
Question 18. Is the containment equipment hatch open at the start of the accident?

Number of Branches: 2

Number of Cases: 1

Number of Cases Sampled: None

The branches for this question are:

1. nOCnt-S The containment equipment hatch is not open at the start of the accident.

2. OCnt-S The containment equipment hatch is open at the start of the accident.

This question addresses the status of the containment equipment at the start of the accident. The equipment hatch can be removed as soon as the plant enters POS 5. A review of the Grand Gulf refueling outage critiques indicated that the removal process typically begins soon after the plant enters POS 5; similarly, the closure process is typically performed near the end of POS 5. Thus the amount of time that the plant is in POS 5 with the equipment hatch in place is relatively small compared to the total amount of time that the plant is in POS 5. Therefore, while there will be a small portion of time during Time Window 1 and Time Window 3 in which the equipment hatch is in place, for this analysis it is assumed that the equipment hatch is always removed at the start of the accident. The quantification for this question is:

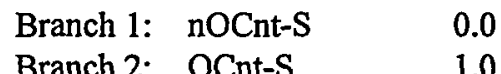

Question 19. Do the operators close the containment before core damage?

Number of Branches: 2

Number of Cases: 5

Number of Cases Sampled: 1

The branches for this question are:

1. nOCnt-BCD The containment access penetrations are not open at the onset of core damage.

2. OCnt-BCD The containment access penetrations are open at the onset of core damage.

This question determines whether the operators successfully replace the containment equipment hatch and close the containment personnel locks prior to the onset of core damage. The information used to assess this issue was obtained from the Grand Gulf plant. The Grand Gulf plant utilizes a 19-ft-diameter, steel pressure seating equipment hatch. The hatch is attached from inside the containment via 20 bolts. The hatch uses two compression seals (gasket concept) around its periphery to maintain tightness along the mating surfaces. The hatch is stored inside the containment in a storage bin above the opening. Offsite ac power is required to move and position the hatch. Each personnel airlock consists of a cylindrical steel shell with steel bulkheads at each end and two steel doors in the bulkheads, which open toward the reactor. Sealing of each door is accomplished by two continuous inflatable seals, which surround the door edge. In this analysis, successful closure of the access penetrations requires the following two conditions: (1) offsite power must be available to move the equipment hatch into place, (2) there must be sufficient time before the onset of core damage to position and attach the equipment hatch. In this analysis it is estimated that at least 5 hours is needed to perform the required action to close the containment. Because of the severe environment that will be present in the containment following the onset of core damage, no credit is given for closure of the containment after the onset of core damage. This issue was addressed in the Human Reliability Analysis (HRA) that was performed for the Level 2 analysis and is discussed further in Appendix B.3.

Case 1: This case includes those accidents in which the containment access penetrations were not open at the start of the accident ; therefore, there are no penetrations to close. The quantification for this case is:

Branch 1: nOCnt-BCD $\quad 1.0$

Branch 2: OCnt-BCD $\quad 0.0$ 
Case 2: This case includes those accidents in which the operators failed to close the lower personnel lock and therefore were unable to successfully flood the containment and prevent core damage. Because of these prior operator errors, and because the lower personnel lock is already open at the onset of core damage, it is also assumed that the equipment hatch is open. The quantification for this case is:

$\begin{array}{lll}\text { Branch 1: } & \text { nOCnt-BCD } & 0.0 \\ \text { Branch 2: } & \text { OCnt-BCD } & 1.0\end{array}$

Case 3: This case includes those accidents initiated by a loss of offsite power. Without offsite power the containment cannot be closed. The quantification for this case is:

$$
\begin{array}{lll}
\text { Branch 1: } & \text { nOCnt-BCD } & 0.0 \\
\text { Branch 2: } & \text { OCnt-BCD } & 1.0
\end{array}
$$

Case 3: This case includes those accidents that were estimated to progress to core damage in less than 5 hours, which therefore precluded successful closure of the containment. The quantification for this case is:

$$
\begin{array}{lll}
\text { Branch 1: } & \text { nOCnt-BCD } & 0.0 \\
\text { Branch 2: } & \text { OCnt-BCD } & 1.0
\end{array}
$$

Case 4: This case includes those accidents in which the containment is open at the start of the accident, offsite power is available, and core damage does not occur for at least 5 hours. Furthermore, the operators have not committed previous errors that would preclude closure of the containment. In these accidents it is possible that the operators will close the containment. This case is sampled; the distribution for the probability that the operators fail to close the containment was developed in the HRA and is discussed in Appendix B.3. The quantification (mean values) for this case is:

Branch 1: nOCnt-BCD 0.898

Branch 2: OCnt-BCD $\quad 0.102$
Lognormal distribution with a mean of 0.102 and an error factor of 5 .

Question 20. Does the auxiliary building fail before core damage?

Number of Branches: 2

Number of Cases: 4

Number of Cases Sampled: None

The branches for this question are:

1. nOAux-BCD The auxiliary building fails before the onset of core damage.

2. OAux-BCD The auxiliary building does not fail before the onset of core damage.

This question determines the status of the auxiliary building integrity at the onset of core damage. The Grand Gulf plant utilizes a secondary containment that completely encloses the primary containment. The secondary containment consists of the auxiliary building and the enclosure building. The auxiliary building, which contains safety systems, fuel storage and shipping equipment and necessary auxiliary support systems, surrounds the lower portions of the containment. The containment personnel locks and the containment equipment hatch allow direct access from the containment to the auxiliary building. Thus, if the containment access penetrations are open, steam and radioactive releases from the containment will pass directly into the auxiliary building. It is assumed that the pressure-retaining capability of the auxiliary building is 5 psig (assumed strength of doors for industrial buildings). The enclosure building encases the upper portion of the containment above the auxiliary building roof and provides a boundary for the standby gas treatment system, which maintains a negative pressure in the volume between the containment and enclosure building to ensure that leakage of radioactive materials from the containment is filtered prior to release to the environment. The enclosure building has virtually no pressure retaining capability and is essentially isolated from the auxiliary building. 
Case 1: This case includes those accidents in which there is an interfacing systems loss of coolant accident (LOCA) in the auxiliary building. In these accidents, the low pressure components of the shutdown cooling system (or ADHRS) are not isolated from the primary system. Following the loss of core cooling, the reactor vessel pressurizes, resulting in a LOCA in the shutdown cooling system in the auxiliary building. The steam released from the vessel immediately following the LOCA pressurizes and fails the auxiliary building. The quantification for this case is:

$\begin{array}{lll}\text { Branch 1: } & \text { nOAux-BCD } & 0.0 \\ \text { Branch 2: } & \text { OAux-BCD } & 1.0\end{array}$

Case 2: This case includes those accidents in which the operators fail to close the main steam isloation valves (MSIVs). Steam generated in the vessel following the loss of core cooling is transported to the condenser via the main steam lines and released into the turbine building. The steam released from the reactor vessel pressurizes and fails the turbine building. Because the turbine building and the auxiliary building are similar (they are large volumes with little pressure retaining capability), releases into the turbine building are treated as though they were released into the auxiliary building and a separate analysis of the turbine building is not performed. The quantification for this case is:

$\begin{array}{lll}\text { Branch 1: } & \text { nOAux-BCD } & 0.0 \\ \text { Branch 2: } & \text { OAux-BCD } & 1.0\end{array}$

Case 3: This case includes those accidents in which the containment access penetrations were not open at the start of the accident, the MSIVs were not opened, and there was not an interfacing systems LOCA in the auxiliary building. Thus the auxiliary building is effectively isolated from the containment and does not pressurize. The quantification for this case is:

$\begin{array}{lll}\text { Branch 1: } & \text { nOAux-BCD } & 1.0 \\ \text { Branch 2: } & \text { OAux-BCD } & 0.0\end{array}$

Case 4: This case includes those accidents in which the containment access penetrations were open during core damage; however, the MSIVs were closed and an interfacing systems LOCA did not occur in the auxiliary building. The LOCAs that are included in this case occur when the reactor vessel is depressurized; therefore, there is not a large release of steam that would threaten the auxiliary building (i.e., most of the reactor vessel inventory drains out of the vessel and is not available to be converted to steam). In the remaining accidents the steam that is generated in the vessel is released to the suppression pool where it is condensed. For the cases with an open reactor head vent, there is an insufficient amount of steam released via the vent to threaten the auxiliary building before the onset of core damage.

Branch 1: nOAux-BCD 1.0

Branch 2: OAux-BCD $\quad 0.0$

Question 21. What is the status of the drywell before core damage?

Number of Branches: 2

Number of Cases: 1

Number of Cases Sampled: None

The branches for this question are:

1. Cls-DW-BCD Both the drywell personnel lock and the containment equipment hatch are closed before the onset of core damage.

2. Op-DW-BCD Either the drywell personnel lock and/or the drywell equipment hatch are open before the onset of core damage. 


\section{Appendix B}

This question determines the status of the drywell integrity at the onset of core damage. The status of drywell integrity is an important feature of the accident because as long as the drywell's integrity is maintained, releases (i.e., steam and radioactive material) in the drywell are forced through the suppression pool. The suppression pool condenses the steam and thereby reduces the pressure load in the containment. Also, the suppression pool is an effective device for removing radioactive material from the drywell atmosphere. There are, however, no Technical Specification requirements for drywell integrity while the plant is in POS 5. A review of the Grand Gulf refueling outage critiques indicated that the drywell personnel lock is generally open during POS 5 and that the drywell equipment hatch is also removed during POS 5 . The drywell head is also detached from the drywell during POS 5. Discussion with plant personnel indicated that during an accident the operators will be more concerned with closure of the containment instead of closure of the drywell. Because reattachment of the drywell equipment hatch and the drywell head are time-consuming tasks and because closure of the drywell will not be the operators' primary concern, in this analysis it is assumed that the drywell will be open for the duration of the accident. The quantification for this question is:

$\begin{array}{lll}\text { Branch 1: } & \text { Cls-DW-BCD } & 0.0 \\ \text { Branch 2: } & \text { Op-DW-BCD } & 1.0\end{array}$

Question 22. Do the operators turn on the Hydrogen Ignition System before core damage?

Number of Branches: 2

Number of Cases: 2

Number of Cases Sampled: 1

The branches for this question are:

1. nHIS-BCD The operators do not turn on the Hydrogen Ignition System (HIS) before core damage.

2. HIS-BCD The operators turn on the HIS before the onset of core damage.

This question determines the status of the HIS at the onset of core damage. The Grand Gulf containment utilizes an HIS to control the accumulation of hydrogen during accident conditions. In the core region there is an abundant supply of zirconium (i.e., fuel cladding, channel boxes), which, at the elevated temperatures typical of core damage accidents, readily reacts with steam to produce hydrogen. The function of the HIS is to prevent the buildup of large quantities of hydrogen inside the containment during accident conditions. This function is accomplished by igniting, via a spark, small amounts of hydrogen before they have a chance to accumulate. The HIS consists of 90 General Motors ac division glow plugs (Model 7G), 45 powered by each ac power division. The HIS is manually actuated. Igniters are located throughout the containment and drywell volumes. The Grand Gulf Emergency Procedures direct the operators to enter the Hydrogen Control section of the Containment Control Procedure (GGNS EP-3) if the water level drops below the top of the active fuel (TAF) or if the water level in the reactor vessel cannot be determined. The Hydrogen Control Procedures direct the operators to activate the HIS if the drywell hydrogen concentration is less than $9 \%$ and the containment hydrogen concentration is below the Hydrogen Deflagration Overpressure Limit (HDOL). This issue was addressed in the Human Reliability Analysis (HRA) that was performed for the Level 2 analysis and is discussed further in Appendix B.3.

Case 1: This case includes those accidents initiated by a loss of offsite power that result in a station blackout. In station blackout scenarios where the reactor vessel water level has reached the top of the active fuel (TAF), the operators would be unable to determine hydrogen concentrations and therefore could not determine whether or not the hydrogen concentration could be maintained below the HDOL. Given this situation, the emergency procedure guides the operators to "secure and prevent" operation of the igniters. Thus it is assumed that the operators would not turn the HIS to the on position until they had recovered power and could determine the hydrogen levels (see Question 31). The quantification for this case is:

$\begin{array}{lll}\text { Branch 1: } & \text { nHIS-BCD } & 1.0 \\ \text { Branch 2: } & \text { HIS-BCD } & 0.0\end{array}$

Case 2: This case includes those accidents in which emergency ac power is available. The water level in the reactor vessel has dropped below the TAF and very little if any hydrogen has been produced. In this situation, the 
Hydrogen Control section of the Containment Control procedures would direct the operators to turn the HIS to the on position. This case is sampled. The distribution for the probability that the operators fail to turn on the HIS was developed in the HRA and is discussed further in Appendix B.3. The quantification (mean values) for this case is:

Branch 1: nHIS-BCD $\quad 0.054$ Lognormal distribution with a mean of 0.054 and an

Branch 2: HIS-BCD error factor of 5 .

\section{Question 23. Do the station batteries deplete during core damage? \\ Number of Branches: 3 \\ Number of Cases: 5 \\ Number of Cases Sampled: None}

The branches for this question are:

1. nDC-CD $\mathrm{DC}$ power is not available during core damage.

2. DC-ECD DC power is available during the early portion (i.e., first 1.5 hours) of core damage.

3. DC-LCD DC power is available during core damage.

This question determines the availability of dc power during core damage. The availability of dc power is important for the following two reasons: (1) dc power is required to keep the safety relief valves open and maintain the vessel at low pressure, and (2) dc power is required to restore offsite power to the plant (see Question 24). Restoration of offsite power is considered during two different portions of the core damage time regime: (1) during the first 1.5 hours of core damage and (2) from 1.5 hours after the onset of core damage to vessel failure (i.e., vessel failure is defined as the end of the core damage time regime). Because the restoration of offsite power depends of the availability of dc power, these two time regimes were also used to determine the availability of dc power. The failure probabilities for the time windows are based on a distribution developed for the NUREG-1150 Grand Gulf plant analysis that models the failure probability of the station batteries versus time for station blackout sequences [Wheeler et al., 1989]. The failure probabilities used in this analysis are conditional on dc power being available at the onset of core damage.

Case 1: This case includes those accidents in which offsite and onsite power are available. With ac power available, the battery chargers supply the necessary dc power and battery depletion is not an issue. For the PDSs analyzed in this study, there were no failures of the dc bus, and therefore, with ac power available, dc power is also assured. The quantification for this case is:

$\begin{array}{lll}\text { Branch 1: } & \text { nDC-CD } & 0.0 \\ \text { Branch 2: } & \text { DC-ECD } & 0.0 \\ \text { Branch 3: } & \text { DC-LCD } & 1.0\end{array}$

Case 2: This case includes those accidents in which dc power was not available before core damage and therefore is not available during core damage (no credit is given for recovery of the station batteries). The quantification for this case is:

$\begin{array}{lll}\text { Branch 1: } & \text { nDC-CD } & 1.0 \\ \text { Branch 2: } & \text { DC-ECD } & 0.0 \\ \text { Branch 3: } & \text { DC-LCD } & 0.0\end{array}$

Case 3: This case includes those accidents initiated by a loss of offsite power that result in a station blackout during Time Window 1. In these accidents, dc power is available at the onset of core damage. For this case the following times are used for core damage and vessel breach, respectively: 3.5 hours and 14.4 hours. With these times, the first time period starts at 3.5 hours and ends at 5.5 hours; the second time period starts at 5.5 hours and ends at 14.4 hours. Therefore, the value for Branch 2 is the probability that dc power is available at 5.5 hours given that it was 
Appendix B

available at 3.5 hours. Similarly, the value for Branch 3 is the probability that dc power is available between 5.5 hours and 14.4 hours given that it was available at 3.5 hours. The quantification for this case is:

$\begin{array}{lll}\text { Branch 1: } & \text { nDC-CD } & 0.011 \\ \text { Branch 2: } & \text { DC-ECD } & 0.242 \\ \text { Branch 3: } & \text { DC-LCD } & 0.747\end{array}$

Case 4: This case includes those accidents initiated by a loss of offsite power that result in a station blackout during Time Window 2. In these accidents, dc power is available at the onset of core damage. For this case, the following times are used for core damage and vessel breach, respectively: 5.5 hours and 12.6 hours. With these times, the first time period starts at 5.5 hours and ends at 7 hours; the second time period starts at 7 hours and ends at 12.6 hours. Therefore, the value for Branch 2 is the probability that dc power is available at 7 hours given that it was available at 5.5 hours. Similarly, the value for Branch 3 is the probability that dc power is available between 7 hours and 12.6 hours given that it was available at 3.5 hours. The quantification for this case is:

$\begin{array}{lll}\text { Branch 1: } & \text { nDC-CD } & 0.015 \\ \text { Branch 2: } & \text { DC-ECD } & 0.103 \\ \text { Branch 3: } & \text { DC-LCD } & 0.882\end{array}$

Case 5: This case is not used.

Question 24. Is offsite power restored during core damage?

Number of Branches: 4

Number of Cases: 7

Number of Cases Sampled: 4

The branches for this question are:

1. nAC-CD Neither offsite nor onsite power is available during core damage.

2. OSP-CD Only offsite power is available during core damage (ac emergency bus failed-note this situation does not occur in this analysis).

3. EAC-CD $\quad A C$ power is available during the early portion (i.e., first 1.5 hours) of core damage.

4. LAC-CD AC power is available during all of the core damage process.

This question determines the availability of ac power during core damage. The availability of ac power is important because it will determine which systems can be used to mitigate the accident (e.g., core cooling systems, containment heat removal systems, Hydrogen Ignition System, Containment Vent System). Restoration of offsite power is considered during two different portions of the core damage time regime: (1) during the first 1.5 hours of core damage and (2) from 1.5 hours after the onset of core damage to vessel failure (i.e., vessel failure is defined as the end of the core damage time regime). The first time regime corresponds to the time available to restore coolant to the core and arrest the core damage process. The duration of this first time regime is 1.5 hours, which corresponds to the point in the core damage process where approximately $10 \%$ of the core is damaged. It was estimated that restoration of coolant to the core with only $10 \%$ of the core damaged will prevent any further damage. After this point in the core damage progression (i.e., the second time regime) there is a rapid escalation in the amount of core damage, and therefore it is assumed that beyond this point the likelihood of core damage arrest is very small (see Question 41). It is important to also consider the second time regime because the availability of ac power is also important after the failure of the vessel (i.e., containment venting and containment heat removal).

The probability of recovering offsite power during a given time period is determined by sampling from a set of distributions for power recovery [Iman and Hora, 1988] (also see Volume 2 of this report). These distributions reflect the type of electrical switchyard at Grand Gulf, as explained in NUREG-1032 [Baranowsky, 1985]. To supply ac power to the safety systems, not only does ac power have to be restored to the site, but de power must be available as well; dc power is required for circuit breaker control power. After the station batteries have been depleted, it is very difficult to return ac power to the safety systems. Although the circuit breakers can be moved manually, this procedure is very complicated and slow. Thus, 
for the time frame considered in this analysis, it is assumed that once dc power is lost, ac power cannot be recovered. The generation of the power recovery curves used in this analysis is discussed in Appendix G of Volume 2, Part 2 of this report.

Case 1: This case includes those accidents in which offsite and onsite power are available at the start of the accident, and therefore ac power is still available. The quantification for this case is:

$\begin{array}{lll}\text { Branch 1: } & \text { nAC-CD } & 0.0 \\ \text { Branch 2: } & \text { OSP-CD } & 0.0 \\ \text { Branch 3: } & \text { EAC-CD } & 1.0 \\ \text { Branch 4: } & \text { LAC-CD } & 0.0\end{array}$

Case 2: This case includes those accidents in which dc power is not available. The lack of dc power implies that both offsite an onsite ac power are unavailable. Without dc power, offsite power cannot be recovered. Furthermore, in this analysis no credit is given for recovery of the emergency diesel generator during the core damage process. Therefore, in this case all ac power is unavailable. The quantification for this case is:

$\begin{array}{lll}\text { Branch 1: } & \text { nAC-CD } & 1.0 \\ \text { Branch 2: } & \text { OSP-CD } & 0.0 \\ \text { Branch 3: } & \text { EAC-CD } & 0.0 \\ \text { Branch 4: } & \text { LAC-CD } & 0.0\end{array}$

Case 3: This case includes those accidents initiated by a loss of offsite power that result in a station blackout during Time Window 1 . Because dc power is only available during the early time period, ac power can only be restored during this time period (i.e., in the late core damage time period dc power is not available; therefore, ac power cannot be restored during the late time period). For this case the following times are used for core damage and vessel breach, respectively: 3.5 hours and 14.4 hours. With these times, the early core damage time period starts at 3.5 hours and ends at 5.5 hours; the late core damage time period starts at 5.5 hours and ends at 14.4 hours. The value for Branch 3 is the probability of recovering ac power during the early core damage time period, given that offsite power was not available at the onset of core damage. The quantification for this case is:

$\begin{array}{lll}\text { Branch 1: } & \text { nAC-CD } & 0.69 \\ \text { Branch 2: } & \text { OSP-CD } & 0.0 \\ \text { Branch 3: } & \text { EAC-CD } & 0.31 \\ \text { Branch 4: } & \text { LAC-CD } & 0.0\end{array}$

Power recovery distribution.

Case 4: This case includes those accidents initiated by a loss of offsite power that result in a station blackout during Time Window 1. Because dc power is available during the entire core damage process, ac power can be restored during either the early or the late core damage time period. For this case the following times are used for core damage and vessel breach, respectively: 3.5 hours and 14.4 hours. With these times, the early core damage time period starts at 3.5 hours and ends at 5.5 hours; the late core damage time period starts at 5.5 hours and ends at 14.4 hours. The value for Branch 3 is the probability of recovering ac power during the early core damage time period, given that offsite power was not available at the onset of core damage. The value for Branch 4 is the probability of recovering ac power during the late core damage time period, given that offsite power was not available at the onset of core damage. The quantification for this case is:

$\begin{array}{lll}\text { Branch 1: } & \text { nAC-CD } & 0.16 \\ \text { Branch 2: } & \text { OSP-CD } & 0.0 \\ \text { Branch 3: } & \text { EAC-CD } & 0.31 \\ \text { Branch 4: } & \text { LAC-CD } & 0.53\end{array}$

Power recovery distribution. Power recovery distribution.

Case 5: This case includes those accidents initiated by a loss of offsite power that result in a station blackout during Time Window 2. Because dc power is only available during the early time period, ac power can only be restored during this time period (i.e., in the late core damage time period dc power is not available; therefore, ac power 


\section{Appendix B}

cannot be restored during the late time period). For this case the following times are used for core damage and vessel breach, respectively: 5.5 hours and 12.6 hours. With these times, the early core damage time period starts at 5.5 hours and ends at 7 hours; the late core damage time period starts at 7 hours and ends at 12.6 hours. The value for Branch 3 is the probability of recovering ac power during the early core damage time period, given that offsite power was not available at the onset of core damage. The quantification for this case is:

$\begin{array}{lll}\text { Branch 1: } & \text { nAC-CD } & 0.74 \\ \text { Branch 2: } & \text { OSP-CD } & 0.0 \\ \text { Branch 3: } & \text { EAC-CD } & 0.26 \\ \text { Branch 4: } & \text { LAC-CD } & 0.0\end{array}$

Power recovery distribution.

Case 6: This case includes those accidents initiated by a loss of offsite power that result in a station blackout during Time Window 2. Because dc power is available during the entire core damage process, ac power can be restored during either the early or the late core damage time period. For this case the following times are used for core damage and vessel breach, respectively: 5.5 hours and 12.6 hours. With these times, the early core damage time period starts at 5.5 hours and ends at 7 hours; the late core damage time period starts at 7 hours and ends at 12.6 hours. The value for Branch 3 is the probability of recovering ac power during the early core damage time period, given that offsite power was not available at the onset of core damage. The value for Branch 4 is the probability of recovering ac power during the late core damage time period, given that offsite power was not available at the onset of core damage. The quantification for this case is:
Branch 1: nAC-CD
0.11
Branch 2: OSP-CD
0.0
Branch 3: EAC-CD 0.26
Branch 4: LAC-CD $\quad 0.43$
Power recovery distribution.
Power recovery distribution.

Case 7: This case is not used.

Question 25. Is the reactor pressure vessel isolated during core damage?

Number of Branches: 2

Number of Cases: 5

Number of Cases Sampled: 1

The branches for this question are:

1. Op-RPV-CD A breach in the reactor vessel integrity is not isolated during core damage.

2. Cls-RPV-CD The reactor vessel integrity is maintained during core damage.

This question determines the status of the reactor pressure vessel (RPV) integrity during core damage. The status of vessel integrity is important because it impacts (1) the pressure in the vessel, (2) the release path of steam and radionuclides during core damage, and (3) the possibility of arresting the core damage process. This question is primarily concerned with station blackout accidents that result in a break in the shutdown cooling (SDC) system, and whether the isolation valves (FOO8 and FOO9) in the SDC system isolate when offsite power is recovered. If the break is isolated and core coolant is restored to the vessel, it is possible that the core damage process will be arrested and vessel failure avoided. This question does not address the status of the safety relief valves or the reactor head vent; these issues are addressed in other questions.

Case 1: This case includes those accidents that are initiated by a loss of coolant accident (LOCA). It is assumed that the LOCA cannot be isolated, and therefore the RPV remains breached. The quantification for this case is:

Branch 1: Op-RPV-CD $\quad 1.0$

Branch 2: Cls-RPV-CD $\quad 0.0$ 
Case 2: This case includes those accidents that have an open main steam isolation valve (MSIV) before core damage. In these accidents the operators failed to recognize that an MSIV is open or were unable for some reason to close the MSIV. Because there was ample time to close the MSIV before core damage, yet it wasn't closed, no credit is given for closing the MSIV during core damage. Thus the vessel remains breached. The quantification for this case is:

\section{Branch 1: Op-RPV-CD $\quad 1.0$ \\ Branch 2: Cls-RPV-CD $\quad 0.0$}

Case 3: This case includes those accidents that were initiated by a loss of offsite power that resulted in a station blackout. In these accidents, the isolation valves on the SDC system were open when power was lost, and because these are ac power valves, they remain open following the loss of ac power. Following the loss of core cooling, the vessel pressurizes and ruptures the SDC system outside the containment, resulting in an interfacing systems LOCA. This case includes those accidents in which ac power is recovered during the early time regime of core damage. The valves should automatically close following the recovery of ac power. If the valves do not automatically close, the operators can close the valves. The issue being addressed is whether the valves were sufficiently damaged during the early phases of core damage so that the resulting damage would preclude closure of the valves. Because ac power is recovered early in the core damage process, it is expected that the environment experienced by the valves would would not be severe; therefore, it is expected that the valves would close. However, there is uncertainty regarding the performance of the valves (and the associated control logic) in this environment. Therefore, a maximum entropy distribution was used to characterize the uncertainty in the probability that the valve will close. The quantification for this case is:

\section{Branch 1: Op-RPV-CD $\quad 0.1$ Branch 2: Cls-RPV-CD $\quad 0.9$}

Maximum Entropy with an Lower Bound of 0.5, an Upper Bound of 1.0 and a Mean of 0.9 ,

Case 4: This case includes accidents that are similar to the accidents addressed by the previous case, except in this case offsite power is not recovered. Without ac power the break cannot be isolated, and therefore the vessel remains breached. This case also includes those accidents in which ac power is recovered late during the core damage process. In these accidents, compared to accidents in which ac power is restored early during core damage, the isolation valves will experience a more severe environment; therefore, their performance is even less certain. Furthermore, the recovery of power can occur any time during the late time period of core damage. The closer in time to vessel failure that power is recovered, the less important the closure of the valves becomes, since most of the in-vessel releases will have already escaped the vessel via the break. Following vessel failure, the isolation of the break is not particularly important because the containment is open in these accidents and the releases will escape into the auxiliary building in either case. The quantification for this case is:

$\begin{array}{lll}\text { Branch 1: } & \text { Op-RPV-CD } & 1.0 \\ \text { Branch 2: } & \text { Cls-RPV-CD } & 0.0\end{array}$

Case 5: This case includes those accidents in which the vessel was not breached prior to core damage. Nothing has happened to this point in the accident that would change that condition. The quantification for this case is:

Branch 1: Op-RPV-CD $\quad 0.0$

Branch 2: Cls-RPV-CD $\quad 1.0$

Question 26. Do the operators initiate the containment sprays during core damage?

Number of Branches: 2

Number of Cases: 7

Number of Cases Sampled: 0

The branches for this question are: 


\section{Appendix B}

1. $\mathrm{nCS}-\mathrm{CD}$

The containment sprays (CS) are not used during core damage.

2. $C S-C D$

The CS are used during core damage.

This question determines whether the CS were used during core damage. The use of the CS is important because it: (1) can reduce the pressure in the containment and (2) reduce the amount of airborne radioactive material that is present in the containment atmosphere. The Grand Gulf Emergency Procedures (GGNS EP-3) direct the operators to initiate the CS if the containment pressure is above 2.2 psig.

Case 1: This case includes those accidents in which the CS are not available. The quantification for this case is:

$\begin{array}{lll}\text { Branch 1: } & \text { nCS-CD } & 1.0 \\ \text { Branch 2: } & \text { CS-CD } & 0.0\end{array}$

Case 2: This case includes those accidents in which the CS are available or recoverable, yet the containment is open and the CS system is not aligned to automatically start. In this analysis, it is assumed that if the containment is open and the CS are not aligned to automatically start, the CS will not be used because containment pressure control is not an issue. In this situation, it is assumed that the operators' primary concern will be to restore core cooling and that they will use any available systems to provide coolant to the core. The quantification for this case is:

$\begin{array}{lll}\text { Branch 1: } & \text { nCS-CD } & 1.0 \\ \text { Branch 2: } & \text { CS-CD } & 0.0\end{array}$

Case 3: This case is similar to the previous case except that the containment is closed; however, the MSIVs are open or there is an interfacing systems LOCA. Again, the pressure in the containment is not an issue in these accidents because all of the releases (both steam and radioactive material) during core damage will bypass the containment. The quantification for this case is:

$\begin{array}{lll}\text { Branch 1: } & \text { nCS-CD } & 1.0 \\ \text { Branch 2: } & \text { CS-CD } & 0.0\end{array}$

Case 4: This case includes those accidents in which low pressure coolant injection is aligned to the shutdown cooling (SDC) system, yet was not used to provide makeup to the core. These previous operator errors preclude its used in the CS mode of operation. The quantification for this case is:

$\begin{array}{lll}\text { Branch 1: } & \text { nCS-CD } & 1.0 \\ \text { Branch 2: } & \text { CS-CD } & 0.0\end{array}$

Case 5: This case includes those accidents in which CS are recoverable; however, emergency ac power is not available (i.e., either station blackout or the ac buses have failed), and therefore the CS system cannot be used. The quantification for this case is:

$\begin{array}{lll}\text { Branch 1: } & \text { nCS-CD } & 1.0 \\ \text { Branch 2: } & \text { CS-CD } & 0.0\end{array}$

Case 6: This case includes those accidents in which CS are recoverable and ac power is restored during core damage. In this case the containment is closed and the reactor vessel integrity is maintained. The Grand Gulf Emergency Procedures will direct the operators to initiate the CS. It is likely the operators will follow the procedures and initiate the CS; a small probability is assigned to failure of the CS to account for failure of the system and/or failure of the operators to follow the procedures. The quantification for this case is:

$\begin{array}{lll}\text { Branch 1: } & \text { nCS-CD } & 0.01 \\ \text { Branch 2: } & \text { CS-CD } & 0.99\end{array}$


Case 7: This case includes those accidents in which CS are available. In this case the containment is closed and the reactor vessel integrity is maintained. The Grand Gulf Emergency Procedures will direct the operators to initiate the CS. It is likely the operators will follow the procedures and initiate CS; a small probability is assigned to the failure of the CS to account for failure of the system and/or failure of the operators to follow the procedures. The quantification for this case is:

Branch 1: nCS-CD 0.01

Branch 2: CS-CD 0.99

Question 27. Do the operators depressurize the reactor pressure vessel during core damage?

Number of Branches: 2

Number of Cases: 5

Number of Cases Sampled: 2

The branches for this question are:

1. RPV-HiP-CD The pressure in the reactor vessel is at system pressure (i.e., approx. 1000 psia) during core damage.

2. RPV-LoP-CD The reactor vessel remains at low pressure $(<400 \mathrm{psia})$ during core damage.

This question determines the pressure in the reactor pressure vessel (RPV) during core damage. The pressure in the vessel is important because it (1) determines which systems can be used to restore coolant to the core, (2) affects the amount of hydrogen produced during core damage, (3) affects the amount of radioactive material that is retained in the vessel, (4) affects the probability of in-vessel steam explosions, (5) affects the dispersal core debris during core damage, which in turn affects the magnitude of the loads that accompany vessel breach and also affects the coolability of the ejected core debris (i.e., assuming water is available to cool the core debris).

Case 1: This case includes those accidents in which the reactor vessel is breached by either a LOCA, an unisolated interfacing systems LOCA, or an open main steam isloation valve. Since the reactor vessel is breached, it is at low pressure. The quantification for this case is:

Branch 1: RPV-HiP-CD $\quad 0.0$

Branch 2: RPV-LoP-CD 1.0

Case 2: This case includes those accidents in which the reactor vessel integrity is maintained (i.e., no breach) and dc power is not available. Since the safety relief valves (SRVs) require dc power to remain open, without dc power the SRVs will close and the vessel will pressurize. An open reactor head vent will not prevent the vessel from pressurizing. The quantification for this case is:

Branch 1: RPV-HiP-CD $\quad 1.0$

Branch 2: RPV-LoP-CD $\quad 0.0$

Case 3: This case includes those accidents that were initiated by a loss of offsite power that resulted in a station blackout followed by a break in the shutdown cooling (SDC) systems outside the containment. In these accidents, ac power is restored during core damage and the break is isolated. With the break isolated, the vessel will pressurize. This case determines whether the operators open at least two SRVs so that low pressure injection systems, if available, can be used to cool the core. The quantification for this case is the same as Case 5. Although this case is somewhat different from Case 5 in that an interfacing systems LOCA occurred and was subsequently isolated, the vessel will still pressurize and conditions are still available that would lead the operators to depressurize the vessel. Thus it is judged that within the resolution of this analysis the use of Case 5 quantification for this case is appropriate. The quantification for this case is: 
Appendix B

$\begin{array}{llll}\text { Branch 1: } & \text { RPV-HiP-CD } & 0.054 & \begin{array}{l}\text { Bounded Lognormal distribution with a mean of } \\ \text { Branch 2: }\end{array} \\ \text { RPV-LoP-CD } & 0.946 & 0.054 \text { and an error factor of } 5 .\end{array}$

Case 4: This case includes those accidents in which at least two SRVs were open before core damage. Because dc power is available in these accidents and there is no reason for the operators to close the SRVs, the SRVs remain open during core damage. With the SRVs open, the reactor vessel remains at low pressure. The quantification for this case is:

$\begin{array}{lll}\text { Branch 1: } & \text { RPV-HiP-CD } & 0.0 \\ \text { Branch 2: } & \text { RPV-LoP-CD } & 1.0\end{array}$

Case 5: This case includes those accidents in which the SRVs were not opened before core damage, resulting in the reactor vessel being at high pressure at the onset of core damage. In some of these scenarios, the Level 1 model did not address the issue of vessel depressurization because depressurization of the vessel would not preclude core damage (e.g., if no injection systems were available). In any case, based on the conditions present in the plant during the core damage process, the Grand Gulf Emergency Procedures would indicate that emergency depressurization of the reactor vessel is required. This case is sampled; the distribution for the probability that the operators fail to depressurize the vessel was developed in the Human Reliability Analysis and is discussed in Appendix B.3. The quantification (mean values) for this case is:

$\begin{array}{lll}\text { Branch 1: RPV-HiP-CD } & 0.054 & \begin{array}{l}\text { Bounded Lognormal distribution with a mean of } \\ 0.054 \text { and an error factor of } 5 .\end{array}\end{array}$

Branch 2: RPV-LoP-CD $\quad 0.946$

\section{Question 28. What is the status of the safety relief valve vacuum breakers during core damage? Number of Branches: 2 \\ Number of Cases: 3 Number of Cases Sampled: 1}

The branches for this question are:
1. Op-SRV-Bkr
A vacuum breaker on a safety relief valve (SRV) tailpipe sticks open and remains open during core damage.
2. Cls-SRV-Bkr The vacuum breakers on the SRV tailpipes remain closed during core damage.

This question determines whether any of the vacuum breakers on the SRV tailpipes stick open and remain open during core damage. The status of the SRV tailpipe vacuum breakers is important because if a vacuum breaker is open a portion of the release from the reactor vessel will enter the drywell and thus bypass the suppression pool. The suppression pool is an effective device for condensing steam and trapping radioactive material that is released from the vessel. Tailpipe vacuum breakers will open after the associated SRV discharges steam through the tailpipe into the suppression pool. When the steam in the tailpipe condenses on the pipe walls, a vacuum is formed in the tailpipe. The vacuum breaker is designed to relieve this vacuum and thereby prevent suppression pool water from being drawn up into the tailpipe. In this analysis, a stuck open tailpipe vacuum breaker is significant only if it is the vacuum breaker on the tailpipe for an SRV that is expected to be open after core damage occurs. Thus the cases below consider which vacuum breakers are challenged by the sequence during the boil-down phase of the accident. This question reflects only significant vacuum breakers sticking open (i.e., one that will result in fission product releases bypassing the suppression pool).

Case 1: This case includes those accidents in which the reactor vessel was depressurized before core damage. In this situation the SRVs are not cycled repeatedly. Because the SRVs and their associated vacuum breakers are not cycled, it is very unlikely that a vacuum breaker will stick open. The quantification for this case is: 


\section{Branch 1: Op-SRV-Bkr $\quad 0.00$ \\ Branch 2: Cls-SRV-Bkr 1.00}

Case 2: This case includes those accidents in which the reactor is at high pressure before core damage and the reactor vessel head vent is open. Although the head vent will not prevent the vessel from pressurizing, it will significantly limit the number of cycles of the SRVs. Because the SRVs and their associated vacuum breakers are not cycled repeatedly, it is very unlikely that a vacuum breaker will stick open. The quantification for this case is:

$$
\begin{array}{lll}
\text { Branch 1: } & \text { Op-SRV-Bkr } & 0.00 \\
\text { Branch 2: } & \text { Cls-SRV-Bkr } & 1.00
\end{array}
$$

Case 3: This case includes those accidents in which the reactor is at high pressure before core damage and the operators closed the reactor vessel head vent. The SRVs are cycled to maintain the reactor pressure vessel (RPV) at the SRV setpoints (i.e., near system pressure). Since the SRVs are repeatedly opened and closed, the SRV tailpipe vacuum breakers are also required to open and close a number of times. In this case the probability that the vacuum breakers stick open is not negligible. The probability that a vacuum breaker sticks open is based on the distribution used in the NUREG-1150 Grand Gulf plant analysis [Brown et al., 1990]. The quantification for this case is:

\section{Branch 1: Op-SRV-Bkr $\quad 0.25$ \\ Branch 2: Cls-SRV-Bkr 0.75 \\ Number of Branches: 3 \\ Number of Cases: 8 \\ Number of Cases Sampled: 0}

Uniform Distribution between 0.01 and 0.5

Question 29. Is core cooling restored during core damage?

The branches for this question are:

1. E-CorCool

2. L-CorCool

3. n-CorCool
Core cooling is restored early during the core damage process.

Core cooling is restored late during the core damage process.

Core cooling is not restored during the core damage process.

This question determines whether core cooling is restored to the reactor vessel during the core damage process. The time that core cooling is restored is important because it affects (1) whether the core damage process can be arrested before the vessel fails, (2) the amount of hydrogen produced, and (3) the amount of core debris produced. The core damage time regime is divided into two segments or time regimes: (1) during the first 1.5 hours of core damage and (2) from 1.5 hours after the onset of core damage to vessel failure (i.e., vessel failure is defined as the end of the core damage time regime). The first time regime corresponds the time available to restore coolant to the core and arrest the core damage process. The duration of this first time regime is 1.5 hours, which corresponds to the point in the core damage process where approximately $10 \%$ of the core is damaged. It was estimated that the restoration of coolant to the core with only $10 \%$ of the core damage will prevent any further damage. After this point in the core damage progression (i.e., the second time regime) there is a rapid escalation in the amount of core damage, and therefore it is assumed that beyond this point the likelihood of core damage arrest is very small (see Question 41).

Case 1: This case includes those accidents in which high pressure injection is available (i.e., HPCS). Although this case never occurs in this analysis, it is included for the sake of completeness. The quantification for this case is:

$$
\begin{array}{lll}
\text { Branch 1: } & \text { E-CorCool } & 1.00 \\
\text { Branch 2: } & \text { L-CorCool } & 0.00 \\
\text { Branch 3: } & \text { n-CorCool } & 0.00
\end{array}
$$

Case 2: This case includes those accidents in which high pressure injection is recoverable (i.e., HPCS) and ac power is restored in the early time segment of the core damage process. It is assumed that HPCS will automatically 
inject into the reactor vessel. This case never occurs in this analysis; however, it is included for the sake of completeness. The quantification for this case is:

$\begin{array}{lll}\text { Branch 1: } & \text { E-CorCool } & 1.00 \\ \text { Branch 2: } & \text { L-CorCool } & 0.00 \\ \text { Branch 3: } & \text { n-CorCool } & 0.00\end{array}$

Case 3: This case includes those accidents in which high pressure injection is recoverable (i.e., HPCS) and ac power is restored in the late time segment of the core damage process. It is assumed that HPCS will automatically inject into the reactor vessel. This case never occurs in this analysis; however, it is included for the sake of completeness. The quantification for this case is:

$\begin{array}{lll}\text { Branch 1: } & \text { E-CorCool } & 0.00 \\ \text { Branch 2: } & \text { L-CorCool } & 1.00 \\ \text { Branch 3: } & \text { n-CorCool } & 0.00\end{array}$

Case 4: This case includes those accidents in which the vessel is at high pressure during the core damage process and no high pressure injection systems are available or recoverable (i.e., HPCS). The quantification for this case is:
Branch 1: E-CorCool
0.00
Branch 2: L-CorCool
0.00
Branch 3: n-CorCool
1.00

Case 5: This case includes those accidents in which low pressure injection systems are available, though they are not aligned to automatically inject into the reactor vessel, and the operators fail to use these systems to cool the core. Because credit was given for using these systems in the Level 1 analysis and nothing happened during core damage that would make additional systems available, it is assumed that the operators are unable to provide coolant to the core. The quantification for this case is:
Branch 1: E-CorCool
0.00
Branch 2: L-CorCool
0.00
Branch 3: n-CorCool
1.00

Case 6: This case includes those accidents in which at least one low pressure injection system is recoverable and ac power is restored in the early time segment of the core damage process. If the accident involves a loss of coolant accident (LOCA) in an interfacing system, the break has been isolated. It is assumed that the low pressure injection system will automatically inject into the reactor vessel following the recovery of ac power. The quantification for this case is:
Branch 1: E-CorCool
1.00
Branch 2: L-CorCool
0.00
Branch 3: n-CorCool
0.00

Case 7: This case includes those accidents in which at least one low pressure injection system is recoverable and ac power is restored in the late time segment of the core damage process. If the accident involves a LOCA in an interfacing system, the break has been isolated. It is assumed that the low pressure injection system will automatically inject into the reactor vessel following the recovery of ac power. The quantification for this case is:

$\begin{array}{lll}\text { Branch 1: } & \text { E-CorCool } & 0.00 \\ \text { Branch 2: } & \text { L-CorCool } & 1.00 \\ \text { Branch 3: } & \text { n-CorCool } & 0.00\end{array}$


Case 8: This case includes those accidents in which either no injection systems are available, ac power is not available, or the accident involves a LOCA in an interfacing system (i.e., shutdown cooling). In the latter case it is assumed that if injection was provided to the reactor vessel, all the suppression pool water would be pumped out the break into the auxiliary building. The resulting flood in the auxiliary building would then fail all core cooling and containment cooling systems and the accident would proceed as though injection was never available. The quantification for this case is:
Branch 1: E-CorCool
0.00
Branch 2: L-CorCool
0.00
Branch 3: $\mathrm{n}$-CorCool $\quad 1.00$

\title{
Question 30. What is the peak hydrogen concentration in the containment during core damage? Number of Branches: 5 \\ Number of Cases: 5 \\ Number of Cases Sampled: 0
}

The branches for this question are:
1. $\mathrm{H} 2<4$
2. $\mathrm{H} 2<8$
3. $\mathrm{H} 2<12$
4. $\mathrm{H} 2<16$
5. $\mathrm{H} 2>16$

\begin{abstract}
The concentration of hydrogen in the containment is less than $4 \%$ by volume. The concentration of $\mathrm{H}_{2}$ in the containment is between $4 \%$ and $8 \%$ by volume. The concentration of $\mathrm{H}_{2}$ in the containment is between $8 \%$ and $12 \%$ by volume. The concentration of $\mathrm{H}_{2}$ in the containment is between $12 \%$ and $16 \%$ by volume. The concentration of hydrogen in the containment is greater than $16 \%$ by volume.
\end{abstract}

This question determines the concentration of hydrogen in the containment during the core damage process. The concentration of hydrogen is important because it will determine the likelihood of a hydrogen combustion event and also the magnitude of the resulting load on the containment. This question is also used to determine the amount of zirconium that was oxidized in the vessel (see Question 31). The ranges for the concentrations were selected to correspond to the ranges used for the load distributions that were developed during the NUREG-1150 project as documented in NUREG/CR-4551 [Harper et al., 1991].

Case 1: This case includes those accidents in which core cooling is restored early during the core damage process. In this case, core cooling is restored before a significant fraction of the core is damaged, which thereby limits the amount of hydrogen produced. The quantification for this case is:

$\begin{array}{lll}\text { Branch 1: } & \mathrm{H} 2<4 & 1.00 \\ \text { Branch 2: } & \mathrm{H} 2<8 & 0.00 \\ \text { Branch 3: } & \mathrm{H} 2<12 & 0.00 \\ \text { Branch 4: } & \mathrm{H} 2<16 & 0.00 \\ \text { Branch 5: } & \mathrm{H} 2>16 & 0.00\end{array}$

Case 2: This case includes those accidents in which the core damage process occurs with the vessel at high pressure and core cooling is either not restored or restored during the late time regime of the core damage process. The containment is closed, there are no open main steam isolation valves (MSIVs), and a LOCA in an interfacing system does not occur. The amount of hydrogen produced is based on the zirconium oxidation curves developed in the project and documented in NUREG/CR-4551 [Harper et al., 1990]. The concentrations were determined based on the assumption that the containment is closed and the drywell and the containment volumes are well mixed. The quantification for this case is:

$\begin{array}{lll}\text { Branch 1: } & \mathrm{H} 2<4 & 0.014 \\ \text { Branch 2: } & \mathrm{H} 2<8 & 0.095 \\ \text { Branch 3: } & \mathrm{H} 2<12 & 0.127\end{array}$




$\begin{array}{lll}\text { Branch 4: } & \mathrm{H} 2<16 & 0.207 \\ \text { Branch 5: } & \mathrm{H} 2>16 & 0.557\end{array}$

Case 3: This case includes those accidents in which the core damage process occurs with the vessel at low pressure, and core cooling is either not restored or restored during the late time regime of the core damage process. The containment is closed, there are no open MSIVs, and a LOCA in an interfacing system does not occur. The amount of hydrogen produced is based on the zirconium oxidation curves developed in the NUREG-1150 project [Harper et al., 1990]. The concentrations were determined based on the assumption that the containment is closed and the drywell and the containment volumes are well mixed. The quantification for this case is:

$\begin{array}{lll}\text { Branch 1: } & \mathrm{H} 2<4 & 0.010 \\ \text { Branch 2: } & \mathrm{H} 2<8 & 0.060 \\ \text { Branch 3: } & \mathrm{H} 2<12 & 0.140 \\ \text { Branch 4: } & \mathrm{H} 2<16 & 0.205 \\ \text { Branch 5: } & \mathrm{H} 2>16 & 0.585\end{array}$

Case 4: This case includes those accidents in which the core damage process occurs with the vessel at high pressure and core cooling is either not restored or restored during the late time regime of the core damage process. The containment is effectively open to the auxiliary building (i.e., either the equipment hatch is open, the MSIVs are open, or there is a LOCA in the auxiliary building). (Note, the cases with the open MSIVs or the LOCA in the auxiliary building are not particularly important because in these cases the auxiliary building will fail early in the accident as a result of the steam release into the auxiliary building). The amount of hydrogen produced is based on the zirconium oxidation curves developed in the NUREG-1150 project [Harper et al., 1990]. The concentrations were determined based on the assumption that the containment, drywell, and auxiliary building atmospheres are well mixed. Results from MELCOR calculations indicated that the atmosphere of the bottom floor of the auxiliary building does not readily mix with the other volumes. Thus the bottom floor of the auxiliary building is not included in these calculations. The quantification for this case is:

$\begin{array}{lll}\text { Branch 1: } & \mathrm{H} 2<4 & 0.066 \\ \text { Branch 2: } & \mathrm{H} 2<8 & 0.213 \\ \text { Branch 3: } & \mathrm{H} 2<12 & 0.303 \\ \text { Branch 4: } & \mathrm{H} 2<16 & 0.243 \\ \text { Branch 5: } & \mathrm{H} 2>16 & 0.175\end{array}$

Case 5: This case includes those accidents in which the core damage process occurs with the vessel at low pressure and core cooling is either not restored or restored during the late time regime of the core damage process. The containment is effectively open to the auxiliary building (i.e., either the equipment hatch is open, the MSIVs are open, or there is a LOCA in the auxiliary building). The amount of hydrogen produced is based on the zirconium oxidation curves developed in the NUREG-1150 project [Harper et al., 1990]. The concentrations were determined based on the assumption that the containment, drywell, and auxiliary building atmospheres are well mixed. Results from MELCOR calculations indicated that the atmosphere of the bottom floor of the auxiliary does not readily mix with the other volumes. Thus the bottom floor of the auxiliary building is not included in these calculations. The quantification for this case is:

$\begin{array}{lll}\text { Branch 1: } & \mathrm{H} 2<4 & 0.040 \\ \text { Branch 2: } & \mathrm{H} 2<8 & 0.205 \\ \text { Branch 3: } & \mathrm{H} 2<12 & 0.325 \\ \text { Branch 4: } & \mathrm{H} 2<16 & 0.250 \\ \text { Branch 5: } & \mathrm{H} 2>16 & 0.180\end{array}$


Question 31. What is fraction of zirconium that is oxidized in the vessel during core damage?

Number of Branches: 2

Number of Cases: 2

Number of Cases Sampled: 0

The branches for this question are:

1. ZrOxid $<21$

Less than 21 percent of the zirconium in the vessel is oxidized during core damage.

2. $\quad$ ZrOxid $>21$

More than 21 percent of the zirconium is the vessel is oxidized during core damage.

This question determines the fraction of zirconium in the vessel that is oxidized during the core damage process. The fraction of zirconium oxidized is important because it will affect the amount of radioactive material released during the core damage process. The amount of zirconium oxidized during core damage will also affect the magnitude of the loads accompanying vessel failure because unoxidized zirconium can react with steam during vessel failure and enhance the resulting load. The ranges for the fraction of zirconium oxidized were selected to correspond to the ranges used for the load distributions and the source term parameter distributions that were developed during the NUREG-1150 project [Harper et al., 1991; Harper et al., 1992]. Because the hydrogen concentrations in the containment are based on a certain fraction of zirconium being oxidized, this information is used to determine the fraction of zirconium oxidized.

Case 1: This case includes two types of accidents. The first type consists of accidents in which the containment is closed and the hydrogen concentration is greater than 16 percent. A hydrogen concentration of $16 \%$ corresponds to slightly more than $21 \%$ zirconium oxidation. The second type consists of accidents in which the containment is open to the auxiliary building and the hydrogen concentration is greater than $8 \%$. In this case, a hydrogen concentration of $9 \%$ corresponds to approximately $21 \%$ zirconium oxidation. The quantification for this case is:

Branch 1: $\quad$ ZrOxid $<21 \quad 0.00$

Branch 2: $\quad$ ZrOxid $>21 \quad 1.00$

Case 2: This case includes two types of accidents. The first type consists of accidents in which the containment is closed and the hydrogen concentration is less than 16 percent. A hydrogen concentration of $16 \%$ corresponds to slightly more than $21 \%$ zirconium oxidation. The second type consists of accidents in which the containment is open to the auxiliary building and the hydrogen concentration is less than $8 \%$. In this case, a hydrogen concentration of $9 \%$ corresponds to approximately $21 \%$ zirconium oxidation. The quantification for this case is:

Branch 1: $\quad$ ZrOxid $<21 \quad 1.00$

Branch 2: $\quad$ ZrOxid $>21 \quad 0.00$

Question 32. Do the operators turn on the hydrogen igniter system during core damage?

Number of Branches: 2

Number of Cases: 4

Number of Cases Sampled: 1

The branches for this question are:

1. nHIS-CD The hydrogen igniters are on during core damage.

2. HIS-CD The hydrogen igniters are not on during core damage.

This question determines the status of the hydrogen ignition system (HIS) during the core damage process. The status of the HIS is important because if it is on and the containment is closed, the hydrogen generated during the core damage process will be burned with a minimal pressure load on the containment. This question is primarily concerned with the situation where the HIS could not be turned on prior to core damage because ac power was not available; however, ac power is recovered during core damage. 
Appendix B

Case 1: This case includes those accidents in which the operators turned on the igniters before the onset of core damage. There is no reason for the operators to turn the HIS off, and therefore the HIS remains on during core damage. The quantification for this case is:

$$
\begin{aligned}
& \text { Branch 1: nHIS-CD } 0.00 \\
& \text { Branch 2: } \quad \text { HISS-CD } \quad 1.00
\end{aligned}
$$

Case 2: This case includes those accidents in which ac power was available before core damage and there was sufficient time for the operators to recognize the need to turn on the HIS. In these accidents, however, the operators failed to turn on the HIS. If the operators failed to turn on the HIS before core damage, there is no reason to believe they will turn on the HIS during core damage. The quantification for this case is:

$$
\begin{array}{lll}
\text { Branch 1: } & \text { nHIS-CD } & 1.00 \\
\text { Branch 2: } & \text { HIS-CD } & 0.00
\end{array}
$$

Case 3: This case includes those accidents in which ac power was not available before core damage but is recovered during core damage before the hydrogen concentration reaches $8 \%$. In these accidents, the lack of ac power before core damage precluded the use of the HIS before core damage. This question assesses the probability that the operators will fail to turn on the HIS following the recovery of ac power. This case is sampled; the distribution for the probability that the operators fail to turn on the HIS is the same distribution that was developed for Case 2 of Question 22. The rationale for this distribution is discussed in Appendix B.3. The quantification for this case is:

$$
\begin{array}{lll}
\text { Branch 1: } & \text { nHIS-CD } & 0.054 \\
\text { Branch 2: } & \text { HIS-CD } & 0.946
\end{array}
$$

Lognormal distribution with a mean of 0.054 and an error factor of 5.

Case 4: This case includes those accidents in which either ac power was not recovered during core damage or was recovered late during the core damage process and the hydrogen concentration was above $8 \%$.

$\begin{array}{lll}\text { Branch 1: } & \text { nHIS-CD } & 1.00 \\ \text { Branch 2: } & \text { HIS-CD } & 0.00\end{array}$

\section{Question 33. Does an uncontrolled hydrogen combustion event occur during core damage? Number of Branches: 3 \\ Number of Cases: 6 Number of Cases Sampled: 2}

The branches for this question are:
1. $\mathrm{nBrn}-\mathrm{H} 2$
The hydrogen does not burn.
2. $\mathrm{Brn}-\mathrm{H} 2$
A deflagration occurs in the containment during core damage.
3. Brn-Dif
The hydrogen burns as a diffusion flame.

This question determines whether the hydrogen in the containment is ignited and, if it is ignited, whether it burns as a large deflagration or as a relatively benign diffusion flame. The type of burn that occurs is important because is will affect the magnitude of the loads on the containment.

Case 1: This case includes those accidents in which the HIS is on and the hydrogen is initially released into the containment (i.e., MSIVs are not open and there is not a LOCA in the auxiliary building). The HIS will burn the hydrogen as a diffusion flame. The quantification for this case is:

$$
\text { Branch 1: } \quad \mathrm{nBrn}-\mathrm{H} 2 \quad 0.00
$$


Appendix B

$\begin{array}{lll}\text { Branch 2: } & \text { Brn-H2 } & 0.00 \\ \text { Branch 3: } & \text { Brn-Dif } & 1.00\end{array}$

Case 2: This case includes those accidents in which the HIS is not on and the hydrogen concentration is less than $4 \%$. With this concentration, the hydrogen will not burn as a deflagration The quantification for this case is:

$\begin{array}{lll}\text { Branch 1: } & \text { nBrn-H2 } & 1.00 \\ \text { Branch 2: } & \text { Brn-H2 } & 0.00 \\ \text { Branch 3: } & \text { Brn-Dif } & 0.00\end{array}$

Case 3: This case includes those accidents in which ac power is available before core damage or was recovered early during the core damage process; however, the HIS was not turned on. Because ac power is available, there will be plenty of ignition sources; the same assumption is used in this analysis as was used in the NUREG-1150 Grand Gulf plant analysis: that the hydrogen will burn. There is uncertainty, however, as to whether it will burn as a deflagration or as a diffusion flame. If there are ample ignition sources and the hydrogen burns before it has had a chance to accumulate, diffusion flames or small relatively benign burns are possible. If the hydrogen ignites after it has had a chance to accumulate, it is likely that it will burn as a deflagration. The case is sampled; the distribution is based on the distribution used in the NUREG-1150 Grand Gulf plant analysis [Brown et al., 1990]. The quantification (mean values) for this case is:

$\begin{array}{lll}\text { Branch 1: } & \text { nBrn-H2 } & 0.00 \\ \text { Branch 2: } & \text { Brn-H2 } & 0.25 \\ \text { Branch 3: } & \text { Brn-Dif } & 0.75\end{array}$

UNIFORM distribution between 0.5 and 1.0

Case 4: This case includes those accidents that are initiated by a loss of offsite power and result in a station blackout. In these accidents ac power is recovered late during the core damage process. Furthermore, it is assumed that the hydrogen burns as a deflagration because: (1) it is not known when ac power is recovered, so it is assumed that it is recovered after the accumulation of a significant amount of hydrogen, and (2) ac power is available, so there are plenty of ignition sources to ignite the hydrogen. The quantification for this case is:

$\begin{array}{lll}\text { Branch 1: } & \text { nBrn-H2 } & 0.00 \\ \text { Branch 2: } & \text { Brn-H2 } & 1.00 \\ \text { Branch 3: } & \text { Brn-Dif } & 0.00\end{array}$

Case 5: This case includes those accidents that are initiated by a loss of offsite power and result in a station blackout. In these accidents ac power is not recovered during the core damage process. Without identifiable ignition sources, there is uncertainty as to whether the hydrogen will ignite. If it does ignite, it is conservatively assumed that ignition occurs after a significant amount of hydrogen has accumulated in the containment. This case is sampled; the distribution is based on information from the NUREG-1150 Grand Gulf plant analysis. Although the NUREG-1150 analysis used a complicated distribution developed by a panel of experts, in this analysis, for the sake of simplicity, a uniform distribution between 0.0 and 0.75 is used to characterize the uncertainty in the likelihood that the hydrogen will ignite. The lower and upper bounds of the uniform distribution correspond to the upper and lower bounds on the expert distribution from NUREG-1150. The quantification (mean values) for this case is:

\begin{tabular}{|c|c|c|c|}
\hline Branch 1: & $\mathrm{nBrn}-\mathrm{H} 2$ & 0.50 & \\
\hline Branch 2: & $\mathrm{Brn}-\mathrm{H} 2$ & 0.50 & Uniform distribution between 0.0 and 0.75 \\
\hline Branch 3: & Brn-Dif & 0.00 & \\
\hline
\end{tabular}

Case 6: This case is not used. 
Appendix B

Question 34. What is the pressure in the containment during core damage (no uncontrolled burn)?

Number of Branches: 2

Number of Cases: 3

Number of Cases Sampled: 0

The branches for this question are:

1. P-Lo The pressure is less than the vent pressure threshold $(\mathrm{P}<20 \mathrm{psig})$.

2. P-Vnt The pressure in the containment exceeds the vent threshold.

This question determines whether the base pressure (i.e., not including pressure spikes from hydrogen combustion) in the containment exceeds the vent threshold. The Grand Gulf Emergency Procedures (EP-3) direct the operators to vent the containment once the pressure exceeds 20 psig and cannot be maintained below 22 psig. During the accident, the containment will pressurize because of the accumulation of steam and non-condensibles (e.g., hydrogen). If the containment is open, it will only pressurize to the failure pressure of the auxiliary building, which is well below the vent threshold.

Case 1: This case includes those accidents in which the containment is open to the auxiliary building (i.e., the containment equipment hatch has been removed). In this situation, the containment pressure will not exceed the auxiliary building failure pressure, which is well below the vent threshold. The quantification for this case is:

$\begin{array}{lll}\text { Branch 1: } & \text { P-Lo } & 1.00 \\ \text { Branch 2: } & \text { P-Vnt } & 0.00\end{array}$

Case 2: This case includes those accidents in which the reactor vessel is open to the auxiliary building (i.e., open MSIVs or a LOCA in the auxiliary building). In this situation, the containment will not pressurize significantly because the steam and non-condensibles released from the vessel bypass the containment and enter the auxiliary building directly. The quantification for this case is:

$\begin{array}{lll}\text { Branch 1: } & \text { P-Lo } & 1.00 \\ \text { Branch 2: } & \text { P-Vnt } & 0.00\end{array}$

Case 3: This case includes those accidents in which the containment is closed and the releases from the reactor vessel either enter the containment directly (i.e., a LOCA or stuck open head vent or SRV vacuum breaker) or enter the containment after first passing through the suppression pool. For the accidents analyzed in this study, all of the LOCAs occur with the vessel depressurized and the containment open. For the cases with an open reactor pressure vessel (RPV) head vent, MELCOR calculations indicated that there is no rapid or significant pressurization. Thus there are no cases where the containment will pressurize to the vent threshold. The quantification for this case is:

$\begin{array}{lll}\text { Branch 1: } & \text { P-Lo } & 1.00 \\ \text { Branch 2: } & \text { P-Vnt } & 0.00\end{array}$

Question 35. Does the containment fail from quasi-static loads during core damage?

Number of Branches: 3

Number of Cases: 6

Number of Cases Sampled: 0

The branches for this question are:

1. nCF-CD The containment does not fail from a hydrogen burn during core damage.

2. CF-Rpt-CD The containment fails in the rupture mode (nominal hole size is $1 \mathrm{ft}^{2}$ ) from a hydrogen burn during core damage.

3. CF-Lk-CD The containment fails in the leak mode (nominal hole size is $0.1 \mathrm{ft}^{2}$ ) from a hydrogen burn during core damage. 
This question determines whether the containment fails from a hydrogen burn during core damage. The status of the containment integrity is important because failure of the containment will result in path for radionuclides to enter the environment. Similar to the NUREG-1150 Grand Gulf plant analysis [Brown et al., 1990], two failure sizes are defined: a leak and a rupture. A leak is defined as a failure that will not result in rapid depressurization of the containment; a nominal hole size of $0.1 \mathrm{ft}^{2}$ is assigned to this mode of failure. A rupture is defined as a failure that will result in rapid depressurization of the containment; a nominal hole size of $1 \mathrm{ft}^{2}$ is assigned to this mode of failure. Based on previous structural analysis of the Grand Gulf containment, it was concluded that the most likely location for failure is the region near the junction of the dome and the cylindrical wall [Brown et al., 1990]. A failure in this location will most likely result in a release to the enclosure building that surrounds the containment dome. Because the enclosure building has virtually no pressure retaining capability and is essentially isolated from the auxiliary building, it is assumed that following containment failure the release goes directly from the containment into the environment.

To determine if the containment fails from quasi-static loads that accompany the combustion of hydrogen, a distribution that characterizes the uncertainty in the load that results from the combustion of a specific concentration of hydrogen was convolved with the Grand Gulf containment failure pressure distribution. The result of this convolution is the probability that the containment fails, given a specified concentration of hydrogen is ignited, and burns. The hydrogen load distributions generated by the expert panels in the NUREG-1150 project [Harper et al., 1991] were used in this study. Similarly, the containment failure pressure distribution developed for the NUREG-1150 Grand Gulf plant analysis [Harper et al., 1994] was also used in this analysis.

Case 1: This case includes those accidents in which either the containment is open to the auxiliary building (i.e., the containment equipment hatch has been removed), the reactor vessel is open to the auxiliary building, the hydrogen does not burn, or (if the hydrogen burns) it burns as a diffusion flame. In this situation, the containment does not fail from a hydrogen combustion event. The quantification for this case is:

$\begin{array}{lll}\text { Branch 1: } & \text { nCF-CD } & 1.00 \\ \text { Branch 2: } & \text { CF-Rpt-CD } & 0.00 \\ \text { Branch 3: } & \text { CF-Lk-CD } & 0.00\end{array}$

Case 2: This case includes those accidents in which the containment is closed and the concentration of hydrogen in the containment is less than $8 \%$. While the hydrogen burns as a deflagration, the resulting load is not sufficient to fail the containment. In this situation, the containment does not fail from a hydrogen combustion event. The quantification for this case is:

$\begin{array}{lll}\text { Branch 1: } & \text { nCF-CD } & 1.00 \\ \text { Branch 2: } & \text { CF-Rpt-CD } & 0.00 \\ \text { Branch 3: } & \text { CF-Lk-CD } & 0.00\end{array}$

Case 3: This case includes those accidents in which the containment is closed, the concentration of hydrogen in the containment is between $8 \%$ and $12 \%$, and the hydrogen burns as a deflagration. The probability of containment failure was obtained from the convolution of the hydrogen load distribution with the containment failure pressure distribution. The quantification for this case is:

$\begin{array}{lll}\text { Branch 1: } & \text { nCF-CD } & 0.79 \\ \text { Branch 2: } & \text { CF-Rpt-CD } & 0.19 \\ \text { Branch 3: } & \text { CF-Lk-CD } & 0.02\end{array}$

Case 4: This case includes those accidents in which the containment is closed, the concentration of hydrogen in the containment is between $12 \%$ and $16 \%$, and the hydrogen burns as a deflagration. The probability of containment failure was obtained from the convolution of the hydrogen load distribution with the containment failure pressure distribution. The quantification for this case is:

Branch 1: $\quad$ nCF-CD $\quad 0.13$ 
Appendix B

$\begin{array}{lll}\text { Branch 2: } & \text { CF-Rpt-CD } & 0.49 \\ \text { Branch 3: } & \text { CF-Lk-CD } & 0.38\end{array}$

Case 5: This case includes those accidents in which the containment is closed, the concentration of hydrogen in the containment is greater than $16 \%$, and the hydrogen burns as a deflagration. The probability of containment failure was obtained from the convolution of the hydrogen load distribution with the containment failure pressure distribution. The quantification for this case is:

$\begin{array}{lll}\text { Branch 1: } & \text { nCF-CD } & 0.04 \\ \text { Branch 2: } & \text { CF-Rpt-CD } & 0.50 \\ \text { Branch 3: } & \text { CF-Lk-CD } & 0.46\end{array}$

Case 6 This case is not used.

Question 36. Do the operators vent the containment during core damage?

Number of Branches: 2

Number of Cases: 5

Number of Cases Sampled: 2

The branches for this question are:

1. nVnt-CD The containment is not vented during core damage.

2. Vnt-CD The operators vent the containment during core damage.

This question determines whether the operators vent the containment during core damage. The status of containment venting is important because opening the containment vent establishes a path from the containment to the environment that bypasses the auxiliary building, which allows airborne radioactive material in the containment to escape directly to the environment. The size of the vent path is equivalent to a rupture in the containment. The Grand Gulf Emergency Procedures (EP-3) direct the operators to vent the containment if the containment pressure is greater than $20 \mathrm{psig}$ and cannot be maintained below 22 psig. Although the procedures allow the operators to close the vent once the pressure drops below 20 psig, the operators would have to open the vent again later in the accident because containment cooling is not available and the steam and non-condensibles generated during the accident would again cause the containment pressure to exceed 22 psig. This analysis makes no attempt to model the opening and the closing of the vent to maintain the pressure below 20 psig. Instead, it is assumed that once the vent is opened, it remains open for the duration of the accident.

Case 1: This case includes those accidents in which either the containment vent system is not available or the containment pressure is below the vent threshold pressure. The quantification for this case is:

$\begin{array}{lll}\text { Branch 1: } & \text { nVnt-CD } & 1.00 \\ \text { Branch 2: } & \text { Vnt-CD } & 0.00\end{array}$

Case 2: This case includes those accidents in which the pressure in the containment is above the vent threshold. In these accidents, the reactor vessel was at high pressure before core damage, and the operators followed the procedures and depressurized the reactor vessel during core damage. The containment vent system is available (i.e., either it was available from the start of the accident or was recoverable and ac power was recovered during core damage). Because the operators followed procedures and depressurized the reactor vessel, it is likely that they will follow procedures and vent the containment. This case is sampled; the distribution that characterizes the uncertainty in the probability that the operators will fail to vent the containment was developed in the Human Reliability Analysis (HRA) and is discussed in Appendix B.2. The quantification (mean value) for this case is:

$\begin{array}{llll}\text { Branch 1: } & \text { nVnt-CD } & 0.031 & \begin{array}{l}\text { Lognormal distribution with a mean of } 0.031 \text { and an } \\ \text { error factor of 5. }\end{array} \\ \text { Branch 2: } & \text { Vnt-CD } & 0.969 & \end{array}$


Case 3: This case is the same as the previous case except that the operators failed to follow procedures and depressurize the reactor vessel. Because the operators failed to follow procedures, it is assumed that they will not follow procedures and vent the containment. The quantification for this case is:

$\begin{array}{lll}\text { Branch 1: } & \text { nVnt-CD } & 1.0 \\ \text { Branch 2: } & \text { Vnt-CD } & 0.0\end{array}$

Case 4: This case includes those accidents in which the pressure in the containment is above the vent threshold. In these accidents, the reactor vessel was at low pressure before core damage and remains at low pressure during core damage. The containment vent system is available (i.e., either it was available from the start of the accident or was recoverable and ac power was recovered during core damage). In these accidents the operators have not failed to follow procedures during core damage; therefore, it is likely that they will follow procedures and vent the containment. This case is sampled; the distribution that characterizes the uncertainty in the probability that the operators will fail to vent the containment was developed in the HRA and is discussed in Appendix B.2. The quantification (mean value) for this case is:

$$
\begin{array}{lll}
\text { Branch 1: } & \text { nVnt-CD } & 0.031 \\
\text { Branch 2: } & \text { Vnt-CD } & 0.969
\end{array}
$$

Lognormal distribution with a mean of 0.031 and an error factor of 5 .

Case 5: This case includes those accidents in which ac power was not available before core damage and the pressure in the containment rises above the vent threshold during core damage. In these accidents, the containment vent system was recoverable; however, ac power was not restored during core damage. Without ac power the containment vent cannot be opened. The quantification for this case is:

$\begin{array}{lll}\text { Branch 1: } & \text { nVnt-CD } & 1.0 \\ \text { Branch 2: } & \text { Vnt-CD } & 0.0\end{array}$

\section{Question 37. What is the status of the containment during core damage? \\ Number of Branches: 2 \\ Number of Cases: 2 \\ Number of Cases Sampled: 0}

The branches for this question are:

1. nOCnt-CD The containment is breached during core damage.

2. OCnt-CD The containment remains intact during core damage.

This question summarizes the status of containment integrity at the end of core damage. It includes both situations where the containment was breached prior to core damage and situations where it was breached during core damage. The question does not address the size of the breach.

Case 1: This case includes those accidents in which either the containment access penetrations were open before core damage, the operators vented the containment, or the containment failed from a hydrogen combustion event during core damage. The quantification for this case is:

$$
\begin{array}{lll}
\text { Branch 1: } & \text { nOCnt-CD } & 0.00 \\
\text { Branch 2: } & \text { OCnt-CD } & 1.00
\end{array}
$$

Case 2: This case includes all other accidents in which the containment remains intact during core damage. The quantification for this case is: 
Appendix B

Branch 1: nOCnt-CD 1.00

Branch 2: $\quad$ OCnt-CD $\quad 0.00$

Question 38. What is the size of the containment opening during core damage?

Number of Branches: 3

Number of Cases: 4

Number of Cases Sampled: 0

The branches for this question are:

1. Cnt-Rpt-CD The containment opening is the size of a rupture (nominal size is $1.0 \mathrm{ft}^{2}$ ).

2. Cnt-Lk-CD The containment opening is the size of a leak (nominal size is $0.1 \mathrm{ft}^{2}$ ).

3. Cnt-NF-CD The containment remains intact during core damage.

This question summarizes the size of the containment opening during core damage.

Case 1: This case includes those accidents in which the containment remains intact during core damage. The quantification for this case is:

$\begin{array}{ll}\text { Branch 1: } & \text { Cnt-Rpt-CD } 0.00 \\ \text { Branch 2: } & \text { Cnt-Lk-CD } 0.00 \\ \text { Branch 3: } & \text { Cnt-NF-CD } 1.00\end{array}$

Case 2: This case includes those accidents in which either the containment access penetrations were open prior to core damage, the containment was vented, or a hydrogen combustion event ruptured the containment during core damage. The quantification for this case is:

$\begin{array}{ll}\text { Branch 1: } & \text { Cnt-Rpt-CD } 1.00 \\ \text { Branch 2: } & \text { Cnt-Lk-CD } 0.00 \\ \text { Branch 3: } & \text { Cnt-NF-CD } 0.00\end{array}$

Case 3: This case includes those accidents in which a hydrogen combustion event caused a leak in the containment during core damage. The quantification for this case is:
Branch 1:
Cnt-Rpt-CD 0.00
Branch 2: $\quad$ Cnt-Lk-CD 1.00
Branch 3: Cnt-NF-CD 0.00

Case 4: This case is not used.

Question 39. Does the auxiliary building fail during core damage?

Number of Branches: 2

Number of Cases: 8

Number of Cases Sampled: 0

The branches for this question are:

1. nOAux-CD The auxiliary building remains intact during core damage.

2. OAux-CD The auxiliary building either fails prior to or during core damage.

This question determines the status of the auxiliary building integrity during core damage. The status of the auxiliary building integrity is important because, for cases with either the reactor vessel or the containment open to the auxiliary building, it defines when the radioactive material is released to the environment. If the containment is closed prior to core 
damage and the reactor vessel is not open to the auxiliary building, a subsequent containment failure will bypass the auxiliary building (i.e., containment fails above auxiliary building roof).

Case 1: This case includes those accidents in which the auxiliary building failed prior to core damage. The quantification for this case is:

Branch 1: $\quad$ nOAux-CD 0.00

Branch 2: $\quad$ OAux-CD 1.00

Case 2: This case includes those accidents in which the reactor vessel is open to the auxiliary building (i.e., open MSIVs or a LOCA in auxiliary building) during core damage. Results for MELCOR indicate that the auxiliary building will fail prior to core damage; therefore, it is open during core damage. The quantification for this case is:

$$
\begin{aligned}
& \text { Branch 1: nOAux-CD } 0.00 \\
& \text { Branch 2: } \quad \text { OAux-CD } 1.00
\end{aligned}
$$

Case 3: This case includes those accidents in which the auxiliary building did not fail before core damage, the reactor is not open to the auxiliary building, and the containment was closed prior to the onset of core damage. In this situation, any releases of steam and/or non-condensibles will bypass the auxiliary building. The quantification for this case is:

$$
\begin{array}{lll}
\text { Branch 1: } & \text { nOAux-CD } & 1.00 \\
\text { Branch 2: } & \text { OAux-CD } & 0.00
\end{array}
$$

Case 4: This case includes those accidents in which the containment is open to the auxiliary building and there is a hydrogen deflagration in the containment. The pressure load from the containment will fail the relatively weak auxiliary building structure. The quantification for this case is:

$$
\begin{array}{ll}
\text { Branch 1: } & \text { nOAux-CD } 0.00 \\
\text { Branch 2: } & \text { OAux-CD } 1.00
\end{array}
$$

Case 5: This case includes those accidents in which the containment is open to the auxiliary building and the hydrogen generated during core damage burns as a diffusion flame. Based on sensitivity calculations using MELCOR, it is expected that the auxiliary building will fail. The quantification for this case is:

$$
\begin{array}{lll}
\text { Branch 1: } & \text { nOAux-CD } & 0.01 \\
\text { Branch 2: } & \text { OAux-CD } & 0.99
\end{array}
$$

Case 6: This case includes those accidents initiated by a LOCA. The containment is open to the auxiliary building and it is flooded with water. In this case, the hydrogen generated during core damage does not burn. Based on results from MELCOR, the pressure in the auxiliary building is sufficiently low that failure of the auxiliary building is not expected before vessel failure. The quantification for this case is:

$$
\begin{array}{ll}
\text { Branch 1: } & \text { nOAux-CD } 1.00 \\
\text { Branch 2: } & \text { OAux-CD } 0.00
\end{array}
$$

Case 7: This case includes those accidents in which the reactor vessel is not breached prior to vessel failure; however, either the reactor head vent or a vacuum breaker on a SRV tailpipe sticks open. The containment is open and the auxiliary building is intact before the onset of core damage. The portion of the steam that leaves the reactor vessel via the open head vent or vacuum break will bypass the suppression pool. Based on results from MELCOR, the steam that bypasses the suppression pool is sufficient to pressurize the auxiliary building to its failure point. The quantification for this case is: 
Appendix B

$$
\begin{array}{lll}
\text { Branch 1: } & \text { nOAux-CD } & 0.00 \\
\text { Branch 2: } & \text { OAux-CD } & 1.00
\end{array}
$$

Case 8: This case includes those accidents in which the reactor vessel is not breached prior to vessel failure and the reactor head vent remains closed. There are no stuck open SRV tailpipe vacuum breakers. The containment is open and the auxiliary building is intact before the onset of core damage. The steam generated in the vessel is directed to the suppression pool where it is condensed. In this case, the hydrogen generated during core damage does not burn. The pressure in the auxiliary building remains below failure pressure. The quantification for this case is:

Branch 1: $\quad$ nOAux-CD 1.00

Branch 2: $\quad$ OAux-CD $\quad 0.00$

Question 40. Is there water in the reactor pressure vessel pedestal cavity just prior to vessel breach?

Number of Branches: 2

Number of Cases: 2

Number of Cases Sampled: 0

The branches for this question are:

1. Cav-Dry The cavity below the reactor vessel is essentially dry.

2. Cav-Fld The reactor cavity is flooded with water.

This question determines the amount of water that is in the reactor cavity at the time of vessel failure. The amount of water in the cavity (i.e., dry or flooded) is important because it will determine if ex-vessel steam explosions are possible, it can impact the loads at vessel breach, and it will affect the coolability of debris released from the vessel following vessel failure. For this analysis, the only sources of water in the drywell before vessel failure are from either a LOCA or for cases where the operators deliberately try to flood the containment. The containment sprays (CS) are in the containment; therefore use of the sprays will not flood the cavity. Also, because in these accidents the drywell personnel lock and/or equipment hatch is open, significant pressure differences between the drywell and containment will not exist, and therefore the possibility of pushing water over the weir wall is not a concern.

Case 1: This case includes those accidents in which the operators deliberately attempt to flood the containment (the LOCAs are included in these accidents). The cavity is flooded up to the bottom the of reactor vessel. The quantification for this case is:

$\begin{array}{lll}\text { Branch 1: } & \text { Cav-Dry } & 0.00 \\ \text { Branch 2: } & \text { Cav-Fld } & 1.00\end{array}$

Case 2: This case includes all the other accidents in which the operators do not flood the containment. The quantification for this case is:

$\begin{array}{lll}\text { Branch 1: } & \text { Cav-Dry } & 1.00 \\ \text { Branch 2: } & \text { Cav-Fld } & 0.00\end{array}$

Question 41. Is the core damage process arrested in the vessel?

Number of Branches: 2

Number of Cases: 4

Number of Cases Sampled: 1

The branches for this question are:

1. nCDArrest The core damage process is arrested in the vessel.

2. CDArrest The core damage process is not arrested. 
This question determines whether the core damage process is arrested in the vessel. The state of the core in the vessel is important because if the core damage process is arrested, vessel failure is precluded and the release of radioactive material from the core debris is terminated. The heat generated by the core (and core debris) will fail the vessel if coolant is not supplied to the core. The probability of vessel failure depends on when the coolant is restored to the vessel during the core damage process. The in-vessel phase of the core damage process was divided into two time regimes. The first time regime corresponds to the time available to restore coolant to the core and arrest the core damage process. The duration of this first time regime is 1.5 hours, which corresponds to the point in the core damage process where approximately $10 \%$ of the core is damaged. It is estimated that restoration of coolant to the core with only $10 \%$ of the core damaged will prevent any further damage. After this point in the core damage progression (i.e., the second time regime) there is a rapid escalation in the amount of core damage, and therefore it is assumed that beyond this point the likelihood of core damage arrest is very small.

Case 1: This case includes those accidents in which the vessel integrity is breached via a LOCA (either inside the containment or in an interfacing system) and the LOCA is not isolated during core damage. In these accidents, the use of injection will result in a flood in the auxiliary building (i.e., for LOCAs inside the containment the lower personnel lock is open), which will in turn fail any remaining injection systems. Thus, without coolant injection the core cannot be continuously cooled. The quantification for this case is:

Branch 1: . nCDArrest 1.00

Branch 2: $\quad$ CDArrest $\quad 0.00$

Case 2: This case includes those accidents in which core cooling is restored during the early time regime of the core damage process before a significant fraction of the core has been damaged. In this case, most of the fuel is still in its original geometry (although the cladding may have failed); therefore, a significant debris bed has not formed. The restoration of core cooling in this situation will arrest the core damage process. The quantification for this case is:

$\begin{array}{lll}\text { Branch 1: } & \text { nCDArrest } & 0.00 \\ \text { Branch 2: } & \text { CDArrest } & 1.00\end{array}$

Case 3: This case includes those accidents in which core cooling is restored during the late time regime of the core damage process. Because in this case most of the fuel has been damaged by the time coolant is restored, it is unlikely that the core damage process will be arrested. The probability of vessel failure will depend on whether the core debris forms a coolable debris bed or instead forms a dense layer on the bottom head of the vessel. This case is sampled; the distribution used to characterize the uncertainty in the probability that the core debris is not coolable is a Maximum Entropy Distribution with a lower value, a mean value, and a upper value of $0.0,0.01$, and 0.5 respectively. The quantification for this case is:

$\begin{array}{lll}\text { Branch 1: } & \text { nCDArrest } & 0.99 \\ \text { Branch 2: } & \text { CDArrest } & 0.01\end{array}$

Maximum Entropy Distribution: Lower value $=0.00$, Mean $=0.01$, Upper value $=0.5$

Case 4: This case includes those accidents in which core cooling is not restored, and therefore vessel failure is assured. The quantification for this case is:

$\begin{array}{lll}\text { Branch 1: } & \text { nCDArrest } & 1.00 \\ \text { Branch 2: } & \text { CDArrest } & 0.00\end{array}$

Question 42. What fraction of the core debris would be mobile at vessel breach?

Number of Branches: 2

Number of Cases: 2

Number of Cases Sampled: 0

The branches for this question are: 
Appendix B

1. HiLiqVB A large amount of core debris (nominally $40 \%$ ) is mobile when vessel breach occurs.

2. LoLiqVB A small amount of core debris (nominally $10 \%$ ) is mobile when vessel breach occurs.

This question determines the amount of core debris that is mobile at the time of vessel breach (VB). The amount of core debris that is mobile is important because it affects (1) the mode of vessel failure, (2) the magnitude of the loads that accompany vessel failure, and (3) the probability that the core debris released from the vessel is coolable for cases with water in the reactor cavity. Nominal values are used to characterize the amount of core debris that would be mobile at VB. A nominal value of $10 \%$ represents low mobility, whereas a nominal value of $40 \%$ represents high mobility. The $10 \%$ value represents the range from $0 \%$ to $20 \%$ molten, and the $40 \%$ value represents any larger quantities. The nominal values used for this question and the probabilities associated with the various levels of mobility are based on the NUREG-1150 Grand Gulf plant analysis [Brown et al., 1990].

In the NUREG-1150 analyses, it was felt that the amount of material molten at VB was tightly coupled to the mode of vessel failure [Harper et al., 1994]. If the vessel fails early, then the mobility will be low. In boiling water reactors, early vessel failure would be caused by melt flowing through an instrument tube and failing the tube outside the vessel. If the melt were to freeze and plug the tube, then vessel failure would be delayed until a massive creep rupture occurs. Hence the major uncertainty is whether the melt flowing in the instrument tube will freeze. If the vessel fails by a massive creep rupture and water is being injected into the vessel, it is likely that it will fail with a low mobility. On the other hand, if there is no water injection and a massive creep rupture occurs, it is uncertain as to how much core debris will be molten.

Case 1: This case includes those accidents in which injection is restored during core damage. The quantification for this case is:

$\begin{array}{lll}\text { Branch 1: } & \text { HiLiqVB } & 0.025 \\ \text { Branch 2: } & \text { CDArrest } & 0.975\end{array}$

Case 2: This case includes those accidents in which injection is not restored during core damage. The quantification for this case is:

$\begin{array}{lll}\text { Branch 1: } & \text { HiLiqVB } & 0.100 \\ \text { Branch 2: } & \text { CDArrest } & 0.900\end{array}$

Question 43. Does a large in-vessel steam explosion occur?

Number of Branches: 2

Number of Cases: 3

Number of Cases Sampled: 2

The branches for this question are:

1. nVStmExp No large in-vessel steam explosions occur prior to VB.

2. VStmExp A large in-vessel steam explosion occurs prior to VB.

This question determines whether a large in-vessel steam explosion occurs prior to VB. The occurrence of a large in-vessel steam explosion is important because a steam explosion can fail the vessel. The quantification of the question is based on information from NUREG-1150 as documented in NUREG/CR-4551 [Harper et al., 1994]. In the NUREG-1150 analysis the likelihood of a steam explosion depended on the reactor vessel pressure.

Case 1: This case includes those accidents in which injection is restored early during the core damage process, before a significant fraction of the core has been damaged. Because only a small fraction of the core is damaged, there is not enough molten core debris to cause a large in-vessel steam explosion. The quantification for this case is:

$\begin{array}{lll}\text { Branch 1: } & \text { nVStmExp } & 1.00 \\ \text { Branch 2: } & \text { VStmExp } & 0.00\end{array}$ 
Case 2: This case includes those accidents in which the reactor vessel is depressurized and coolant injection is either restored late during the core damage process or is not restored. In either case, a significant fraction of the core will be damaged, which allows for the possibility of a large in-vessel steam explosion. The likelihood of a steam explosion depends on the pressure in the reactor vessel. Based on information used in NUREG-1150, steam explosions are more likely when the pressure is low than when the pressure is near system pressure (i.e., 1000 psia). Results from experimental programs and experience in the metal industry suggest that the occurrence of a steam explosion can be treated as a stochastic event. The likelihood of a steam explosion is, however, very uncertain. In this analysis, a maximum entropy distribution is used to characterize the uncertainty in the probability that a steam explosion occurs. The mean of the maximum entropy distribution corresponds to the value used in the NUREG1150 analysis for the probability of a steam explosion when the vessel is at low pressure. The quantification for this case is:

$\begin{array}{lll}\text { Branch 1: } & \text { nVStmExp } & 0.14 \\ \text { Branch 2: } & \text { VStmExp } & 0.86\end{array}$

$$
\begin{aligned}
& \text { Maximum Entropy: Lower Bound }=0.001, \text { Mean }= \\
& 0.86 \text {, Upper } \text { Bound }=1.0
\end{aligned}
$$

Case 3: This case is the same as the previous case except that the pressure in the reactor vessel is at the system pressure (i.e., approximately 1000 psia). Based on information used in NUREG-1150, steam explosions are less likely when the pressure is high than when the pressure is low. Similar to the previous case, a maximum entropy distribution is used to characterize the uncertainty in the probability that a steam explosion occurs. The mean of the maximum entropy distribution corresponds to the value used in the NUREG-1150 analysis for the probability of a steam explosion when the vessel is at high pressure. The quantification for this case is:
Branch 1:
Branch 2:
Branch 1:
Branch 2:
nVStmExp
VStmExp
0.10

Maximum Entropy: Lower Bound $=0.001$, Mean $=$ 0.10, Upper Bound $=1.0$

Question 44. Does an Alpha Mode event occur?

Number of Branches: 2

Number of Cases: 3

Number of Cases Sampled: 2

The branches for this question are:

1. nAlpha

2. Alpha
An Alpha Mode event occurs.

An Alpha Mode event does not occur.

This question determines whether a Alpha Mode event occurs. An Alpha Mode event is an energetic fuel-coolant interaction (i.e., steam explosion) that fails the vessel and generates a missile that then fails the containment. The occurrence of an Alpha Mode event is important because it provides a path for radioactive material to escape both the reactor vessel and the containment. This event was postulated and analyzed during the Reactor Safety Study [USNRC, 1975]. The quantification for this question is based on distributions used in the NUREG-1150 study as documented in NUREG/CR-4551[Harper et al., 1994]. In the NUREG-1150 analysis the likelihood of an Alpha Mode event depended on the pressure in the reactor vessel.

Case 1: This case includes those accidents in which a large in-vessel steam explosion occurs with the reactor vessel at low pressure. The quantification for this case is:

$$
\begin{array}{llll}
\text { Branch 1: } & \text { nVStmExp } & 0.990 & \\
\text { Branch 2: } & \text { VStmExp } & 0.010 & \text { User Distribution with a Mean of } 0.01
\end{array}
$$

Case 2: This case includes those accidents in which a large in-vessel steam explosion occurs with the reactor vessel at system pressure. The probability of an Alpha Mode event at high pressure was estimated in NUREG-1150 to be 
Appendix B

an order of magnitude lower than the probability of an Alpha Mode event at low pressure. The quantification for this case is:

Branch 1: $\quad$ nVStmExp 0.999

Branch 2: VStmExp $\quad 0.001$

User Distribution with a Mean of 0.001

Question 45. Does a large in-vessel steam explosion fail the vessel?

Number of Branches: 5

Number of Cases: 3

Number of Cases Sampled: 0

The branches for this question are:

1. SE-Alpha An Alpha Mode fails the reactor vessel.

2. SE-BtHd An in-vessel steam explosion causes the bottom head of the reactor vessel to fail (nominal failure size of $340 \mathrm{ft}^{2}$ ).

3. SE-LgBrch An in-vessel steam explosion causes a large breach $\left(22 \mathrm{ft}^{2}\right)$ in the reactor vessel.

4. SE-SmBrch An in-vessel steam explosion causes a small breach $\left(1 \mathrm{ft}^{2}\right)$ in the reactor vessel.

5. SE-nFail The reactor vessel does not fail from an in-vessel steam explosion

This question determines whether an in-vessel steam explosion fails the reactor vessel and, if it does fail the reactor vessel, it determines the size of the failure. Failure of the reactor vessel is important because it establishes a path for radioactive material to escape the vessel and bypass the suppression pool, and it releases the core debris into the reactor cavity below the reactor vessel. If the cavity is dry, the core debris will interact with the concrete cavity and will continue to release radioactive material. If the cavity is flooded, the potential exists for the core debris to be cooled, in which case the release of radioactive material from the debris is terminated. The quantification of this question is based on the probabilities developed in the NUREG-1 150 project as documented in NUREG/CR-4551 [Harper et al., 1994].

Case 1: This case includes those accidents in which an Alpha Mode event occurs. By definition, an Alpha Mode event results in a large failure to the reactor vessel. The quantification for this case is:

$\begin{array}{lll}\text { Branch 1: } & \text { SE-Alpha } & 1.00 \\ \text { Branch 2: } & \text { SE-BtHd } & 0.00 \\ \text { Branch 3: } & \text { SE-LgBrch } & 0.00 \\ \text { Branch 4: } & \text { SE-SmBrch } & 0.00 \\ \text { Branch 5: } & \text { SE-nFail } & 0.00\end{array}$

Case 2: This case includes those accidents in which a large in-vessel steam explosion occurs. The quantification of this case is based on probabilities developed in the NUREG-1150 project. The quantification for this case is:

$\begin{array}{lll}\text { Branch 1: } & \text { SE-Alpha } & 0.00 \\ \text { Branch 2: } & \text { SE-BtHd } & 0.20 \\ \text { Branch 3: } & \text { SE-LgBrch } & 0.10 \\ \text { Branch 4: } & \text { SE-SmBrch } & 0.10 \\ \text { Branch 5: } & \text { SE-nFail } & 0.60\end{array}$

Case 3: This case includes those accidents in which a large in-vessel steam explosion does not occur, and therefore the reactor vessel does not fail from a steam explosion. The quantification for this case is:

$\begin{array}{lll}\text { Branch 1: } & \text { SE-Alpha } & 0.00 \\ \text { Branch 2: } & \text { SE-BtHd } & 0.00\end{array}$




\section{Branch 3: $\quad$ SE-LgBrch 0.00 \\ Branch 4: $\quad$ SE-SmBrch 0.00 \\ Branch 5: SE-nFail $\quad 1.00$}

\section{Question 46. What is the mode of vessel failure? \\ Number of Branches: 5 \\ Number of Cases: 7 \\ Number of Cases Sampled: 0}

The branches for this question are:

1. VB-Alpha

2. VB-BtHd

3. VB-LgBrch

4. VB-SmBrch

5. nVB
An Alpha Mode fails the reactor vessel.

The bottom head of the reactor vessel fails (nominal failure size of $340 \mathrm{ft}^{2}$ ).

The mode of vessel failure is a large breach $\left(22 \mathrm{ft}^{2}\right)$.

The mode of vessel failure is a small breach $\left(1 \mathrm{ft}^{2}\right)$.

The reactor vessel does not fail.

This question determines the mode of vessel failure. This question (1) summarizes previous reactor vessel failure modes caused by large in-vessel steam explosions and (2) considers reactor vessel failure modes caused by core debris attack. Failure of the reactor vessel is important because it establishes a path for radioactive material to escape the vessel and bypass the suppression pool, and it releases the core debris into the reactor cavity below the reactor vessel. If the cavity is dry, the core debris will interact with the concrete cavity and will continue to release radioactive material. If the cavity is flooded, the potential exists for the core debris to be cooled, in which case the release of radioactive material from the debris is terminated.

The vessel is predicted to fail if the core damage process is not arrested (See Question 41). The quantification of this question is based on the probabilities developed in the NUREG-1150 project as documented in NUREG/CR-4551 [Harper et al., 1994]. Given that the reactor vessel fails from core debris attack, the following modes of reactor vessel failure were considered:

1. global thermally induced fracture/creep-rupture of the lower head;

2. ejection of an in-core instrument guide tube or control rod drive (CRD); and

3. flow of molten core materials through a guide tube, $C R D$, or drain line leading to their thermally induced rupture below the bottom head.

The most likely failure mode is flow-induced thermal failure of a guide tube or drain. Molten material can enter the tube and flow beyond the reactor vessel wall. Thermal weakening followed by rupture of the wall can occur if the melt gives up its latent and sensible heat to the guide tube or drain walls. It is uncertain whether the presence of water in these tubes will prevent tube failure. This uncertainty has been included in the probability assigned to this mode of failure. This failure mode will result in a small hole. It was assessed that there is a very small probability of multiple tube failures resulting in a large hole. It was also estimated that thermally induced binding between the guide tube and the vessel will prevent pressure ejection of the in-core instrument guide tube.

Case 1: This case summarizes previous failures caused by in-vessel steam explosions; it includes those accidents in which the reactor vessel fails as a result of an Alpha Mode event. The quantification for this case is:

$\begin{array}{lll}\text { Branch 1: } & \text { VB-Alpha } & 1.00 \\ \text { Branch 2: } & \text { VB-BtHd } & 0.00 \\ \text { Branch 3: } & \text { VB-LgBrch } & 0.00 \\ \text { Branch 4: } & \text { VB-SmBrch } & 0.00 \\ \text { Branch 5: } & \text { nVB } & 0.00\end{array}$


Case 2: This case summarizes previous failures caused by in-vessel steam explosions; it includes those accidents in which the reactor vessel bottom head fails. The quantification for this case is:

$\begin{array}{lll}\text { Branch 1: } & \text { VB-Alpha } & 0.00 \\ \text { Branch 2: } & \text { VB-BtHd } & 1.00 \\ \text { Branch 3: } & \text { VB-LgBrch } & 0.00 \\ \text { Branch 4: } & \text { VB-SmBrch } & 0.00 \\ \text { Branch 5: } & \text { nVB } & 0.00\end{array}$

Case 3: This case summarizes previous failures caused by in-vessel steam explosions; it includes those accidents in which an in-vessel steam explosion results in a large breach to the reactor vessel. The quantification for this case is:

$\begin{array}{lll}\text { Branch 1: } & \text { VB-Alpha } & \mathbf{0 . 0 0} \\ \text { Branch 2: } & \text { VB-BtHd } & 0.00 \\ \text { Branch 3: } & \text { VB-LgBrch } & 1.00 \\ \text { Branch 4: } & \text { VB-SmBrch } & 0.00 \\ \text { Branch 5: } & \text { nVB } & 0.00\end{array}$

Case 4: This case summarizes previous failures caused by in-vessel steam explosions; it includes those accidents in which an in-vessel steam explosion results in a small breach to the reactor vessel. The quantification for this case is:

$\begin{array}{lll}\text { Branch 1: } & \text { VB-Alpha } & 0.00 \\ \text { Branch 2: } & \text { VB-BtHd } & 0.00 \\ \text { Branch 3: } & \text { VB-LgBrch } & 0.00 \\ \text { Branch 4: } & \text { VB-SmBrch } 1.00 \\ \text { Branch 5: } & \text { nVB } & 0.00\end{array}$

Case 5: This case includes those accidents in which the core damage process is arrested in the vessel and a large invessel steam explosion either does not occur or does not fail the vessel. Thus in this situation the reactor vessel does not fail. The quantification for this case is:

$\begin{array}{lll}\text { Branch 1: } & \text { VB-Alpha } & 0.00 \\ \text { Branch 2: } & \text { VB-BtHd } & 0.00 \\ \text { Branch 3: } & \text { VB-LgBrch } & 0.00 \\ \text { Branch 4: } & \text { VB-SmBrch } 0.00 \\ \text { Branch 5: } & \text { nVB } & 1.00\end{array}$

Case 6: This case includes those accidents in which the core damage process is not arrested in the vessel and a large in-vessel steam explosion either does not occur or does not fail the vessel. Thus in this situation the core debris causes thermally induced reactor vessel failure. The probabilities assigned to the branches for this case are based on probabilities developed in the NUREG-1150 study as documented in NUREG/CR-4551 [Harper et al., 1994]. The quantification for this case is:

$\begin{array}{lll}\text { Branch 1: } & \text { VB-Alpha } & 0.000 \\ \text { Branch 2: } & \text { VB-BtHd } & 0.249 \\ \text { Branch 3: } & \text { VB-LgBrch } & 0.005 \\ \text { Branch 4: } & \text { VB-SmBrch } & 0.746 \\ \text { Branch 5: } & \text { nVB } & 0.000\end{array}$

Case 7: This case is not used. 


\section{Question 47. Does high pressure melt ejection accompany vessel failure? \\ Number of Branches: 2 \\ Number of Cases: 2 \\ Number of Cases Sampled: 0}

The branches for this question are:

1. nHPME A high pressure melt ejection event does not accompany reactor vessel failure.

2. HPME A high pressure melt ejection event accompanies reactor vessel failure.

This question determines whether the core debris is released from the reactor vessel as a high pressure melt ejection (HPME). This question is referenced in the source term analysis to determine the source term associated with vessel breach. If HPME occurs, the fraction of radionuclides released during direct containment heating $(\mathrm{DCH}), \mathrm{F}_{\mathrm{DCH}}$ is applied to the core debris ejected at vessel breach. If the reactor vessel is at high pressure when the vessel fails, the core debris will likely be ejected at a high velocity. Because of its high velocity, it is expected that the ejected material will undergo extensive fragmentation, and the result will be an HPME event. Thus if the reactor vessel fails at high pressure, HPME is likely. The quantification of the issue is based on probabilities developed in the NUREG-1150 project as documented in NUREG/CR-4551 [Brown et al, 1990].

Case 1: This case includes those accidents in which either the reactor vessel does not fail or the reactor vessel is depressurized when it fails. Thus in this situation a HMPE event does not occur. The quantification for this case is:

$\begin{array}{lll}\text { Branch 1: } & \text { nHPME } & 1.00 \\ \text { Branch 2: } & \text { HPME } & 0.00\end{array}$

Case 2: This case includes those accidents in which the reactor vessel is at high pressure when it fails. The probability that an HPME event occurs is based on the probability used in the NUREG-1150 analysis as documented in NUREG/CR-4551 [Brown et al., 1990]. The quantification for this case is:

$\begin{array}{lll}\text { Branch 1: } & \text { nHPME } & 0.20 \\ \text { Branch 2: } & \text { HPME } & 0.80\end{array}$

Question 48. Does a large ex-vessel steam explosion accompany vessel failure?

Number of Branches: 2

Number of Cases: 3

Number of Cases Sampled: 1

The branches for this question are:

1. nExStmE A large ex-vessel steam explosion accompanies reactor vessel failure.

2. ExStmE A large ex-vessel steam explosion does not occur.

This question determines whether a large ex-vessel steam explosion accompanies reactor vessel failure. This question is referenced in the source term analysis to determine the source term associated with vessel breach. This question is not, however, used to address the quasi-static loads associated with vessel breach. The probability of containment failure at the time of reactor vessel failure (see Question 49) is based on the expected loads from all sources and does not attempt to distinguish between loads from individual events (e.g., steam explosions, direct containment heating, hydrogen combustion). Thus, while the loads from steam explosions are not explicitly represented as a separate source, they were considered when the loads associated with vessel failure where addressed. The quantification of the issue is based on probabilities developed in the NUREG-1150 project as documented in NUREG/CR-4551 [Brown et al., 1990].

The dropping of hot metal into water has been observed to cause energetic and violent reactions commonly known as fuelcoolant interaction (FCIs) or steam explosions. They appear to be more likely when the water is considerably below its 
Appendix B

saturation temperature. At Sandia National Laboratories, steam explosions were observed in $86 \%$ of the tests ( 32 of 37 tests) in which hot metal was dropped into water at ambient pressure [Harper et al., 1994]. Some of these explosions were extremely energetic; others were not. For an ex-vessel steam explosion to occur in a severe reactor accident, there must be water in the reactor cavity prior to vessel failure, or water must enter the cavity coincident with the debris, and the vessel must fail allowing the core debris to enter the reactor cavity.

Case 1: This case includes those accidents in which either the reactor vessel does not fail or an Alpha Mode event occurs. The effect that an Alpha Mode event has on the source term is addressed in the question that addresses HPME events (see Question 47). The quantification for this case is:

\begin{tabular}{|c|c|}
\hline Drail & $\mathrm{nEx}$ \\
\hline Branch 2: & ExStmE \\
\hline
\end{tabular}

Case 2: This case includes those accidents in which core debris is released from the reactor vessel into either a flooded reactor cavity or a dry cavity coincident with water from the reactor vessel that is supplied by an injection source. In this situation, steam explosions are possible. Results from experimental programs and experience in the metal industry suggest that the occurrence of a steam explosion can be treated as a stochastic event. The likelihood of a steam explosion is, however, very uncertain. In this analysis, a maximum entropy distribution is used to characterize the uncertainty in the probability that a steam explosion occurs. The mean of the maximum entropy distribution corresponds to the value used in the NUREG-1150 analysis for the probability of an ex-vessel steam explosion. The quantification for this case is:

\begin{tabular}{|c|c|}
\hline . & $\mathrm{nExStmE}$ \\
\hline Branch 2: & ExStmE \\
\hline
\end{tabular}

Maximum Entropy: Lower Bound $=0.001$, Mean $=$ 0.86, Upper Bound $=1.0$

Case 3: This case includes those accidents in which the reactor cavity is dry, and therefore an ex-vessel steam explosion is not possible. The quantification for this case is:

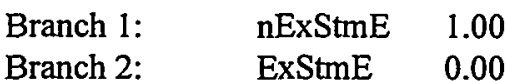

Question 49. Does the containment fail from pressure loads accompanying vessel failure?

Number of Branches: 3

Number of Cases: 11

Number of Cases Sampled: 0

The branches for this question are:

1. nCF-VB The containment does not fail from pressure loads accompanying vessel failure.

2. CF-Rpt-VB The containment fails in the rupture mode (nominal hole size is $1.0 \mathrm{ft}^{2}$ ) from pressure loads accompanying vessel failure.

3. CF-Lk-VB The containment fails in the leak mode (nominal hole size is $0.1 \mathrm{ft}^{2}$ ) from pressure loads accompanying vessel failure.

This question determines whether the containment fails from pressure loads accompanying vessel failure. The status of the containment integrity is important because failure of the containment will result in a path for radionuclides to enter the environment. Similar to the NUREG-1150 Grand Gulf plant analysis [Brown et al., 1990], two failure sizes are defined: a leak and a rupture. The containment failure issue is discussed in Question 35.

To determine if the containment fails from pressure loads that accompany vessel failure, a distribution that characterizes the uncertainty in load that results from vessel failure was convolved with the Grand Gulf containment failure pressure distribution. The result of this convolution is the probability that the containment fails given a specified pressure load. The 
distributions for the pressure loads accompanying vessel failure generated by the expert panels in the NUREG-1150 project [Harper et al., 1991] were used in this study. These distributions consider loads that result from steam explosions, direct containment heating, hydrogen combustion, and reactor vessel blowdown. The containment failure pressure distribution developed for the NUREG-1150 Grand Gulf plant analysis [Harper et al., 1994] was also used in this analysis.

Case 1: This case includes those accidents in which the vessel does not fail and, therefore, there are no pressure loads to cause containment failure. The quantification for this case is:

$\begin{array}{lll}\text { Branch 1: } & \text { nCF-VB } & 1.00 \\ \text { Branch 2: } & \text { CF-Rpt-VB } & 0.00 \\ \text { Branch 3: } & \text { CF-Lk-VB } & 0.00\end{array}$

Case 2: This case includes those accidents in which the containment was already ruptured. This case also includes those accidents in which the containment fails as a result of an Alpha Mode event. In either case, the containment is already ruptured; therefore, further failure of the containment is not considered. The quantification for this case is:

$\begin{array}{lll}\text { Branch 1: } & \text { nCF-VB } & 0.00 \\ \text { Branch 2: } & \text { CF-Rpt-VB } & 1.00 \\ \text { Branch 3: } & \text { CF-Lk-VB } & 0.00\end{array}$

Case 3: This case includes those accidents in which the reactor vessel is pressurized when it fails; a large amount of molten core debris is released at the time of vessel failure. The probability of containment failure was obtained from the convolution of the pressure load distribution with the containment failure pressure distribution. The quantification for this case is:

$\begin{array}{lll}\text { Branch 1: } & \text { nCF-VB } & 0.22 \\ \text { Branch 2: } & \text { CF-Rpt-VB } & 0.35 \\ \text { Branch 3: } & \text { CF-Lk-VB } & 0.43\end{array}$

Case 4: This case includes those accidents in which the reactor vessel is pressurized when it fails; a small amount of molten core debris is released at the time of vessel failure. The probability of containment failure was obtained from the convolution of the pressure load distribution with the containment failure pressure distribution. The quantification for this case is:

$\begin{array}{lll}\text { Branch 1: } & \text { nCF-VB } & 0.41 \\ \text { Branch 2: } & \text { CF-Rpt-VB } & 0.27 \\ \text { Branch 3: } & \text { CF-Lk-VB } & 0.32\end{array}$

Case 5: This case includes those accidents in which the reactor vessel is at low pressure when it fails; more than $21 \%$ of the zirconium in the core debris is oxidized, and it is released from the vessel into a flooded reactor cavity. The probability of containment failure was obtained from the convolution of the pressure load distribution with the containment failure pressure distribution. The quantification for this case is:

$\begin{array}{lll}\text { Branch 1: } & \text { nCF-VB } & 0.90 \\ \text { Branch 2: } & \text { CF-Rpt-VB } & 0.04 \\ \text { Branch 3: } & \text { CF-Lk-VB } & 0.06\end{array}$

Case 6: This case includes those accidents in which the reactor vessel is at low pressure when it fails; less than $21 \%$ of the zirconium in the core debris is oxidized, and it is released from the vessel into a flooded reactor cavity. The probability of containment failure was obtained from the convolution of the pressure load distribution with the containment failure pressure distribution. The quantification for this case is:

Branch 1: $\quad$ nCF-VB $\quad 0.82$ 


$$
\begin{array}{lll}
\text { Branch 2: } & \text { CF-Rpt-VB } & 0.10 \\
\text { Branch 3: } & \text { CF-Lk-VB } & 0.08
\end{array}
$$

Case 7: This case includes those accidents in which the hydrogen igniters have been on during core damage; therefore, the hydrogen generated during core damage has been consumed. Because the vessel fails at low pressure into a dry cavity, it is unlikely that there will be a rapid production of hydrogen at the time of vessel failure. Hence it is likely that the igniters will burn the hydrogen with minimal pressurization of the containment. A small probability is assigned to containment failure to account for the possibility of the rapid generation of hydrogen when the core debris and water are released at the time of vessel failure. The quantification for this case is:

$$
\begin{aligned}
& \text { Branch 1: } \quad \text { nCF-VB } \quad 0.99 \\
& \text { Branch 2: CF-Rpt-VB } 0.005 \\
& \text { Branch 3: CF-Lk-VB } 0.005
\end{aligned}
$$

Case 8: This case includes those accidents in which the reactor vessel fails at low pressure and releases the core debris into a dry cavity. During core damage, hydrogen accumulated in the containment; however, it did not ignite because of a lack of ignition sources. The peak hydrogen concentration in the containment before vessel failure was between 8 and $12 \%$. Because the vessel fails at low pressure into a dry cavity, it is unlikely that there will be a rapid production of hydrogen at the time of vessel failure; however, the hot debris released into the containment will provide ample ignition sources for the preexisting hydrogen. In this situation, ignition is certain. The containment failure probability used for this case is the same probability used for the corresponding case for hydrogen burns during core damage (see Question 35). The quantification for this case is:

$$
\begin{array}{lll}
\text { Branch 1: } & \text { nCF-VB } & 0.79 \\
\text { Branch 2: } & \text { CF-Rpt-VB } & 0.19 \\
\text { Branch 3: } & \text { CF-Lk-VB } & 0.02
\end{array}
$$

Case 9: This case includes those accidents in which the reactor vessel fails at low pressure and releases the core debris into a dry cavity. During core damage, hydrogen accumulated in the containment; however, it did not ignite because of a lack of ignition sources. The peak hydrogen concentration in the containment before vessel failure was between 12 and $16 \%$. Because the vessel fails at low pressure into a dry cavity, it is unlikely that there will be a rapid production of hydrogen at the time of vessel failure; however, the hot debris released into the containment will provide ample ignition sources for the preexisting hydrogen. In this situation, ignition is certain. The containment failure probability used for this case is the same probability used for the corresponding case for hydrogen burns during core damage (see Question 35). The quantification for this case is:

$$
\begin{aligned}
& \text { Branch 1: } \quad \text { nCF-VB } \quad 0.13 \\
& \text { Branch 2: } \quad \text { CF-Rpt-VB } 0.49 \\
& \text { Branch 3: } \quad \text { CF-Lk-VB } 0.38
\end{aligned}
$$

Case 10: This case includes those accidents in which the reactor vessel fails at low pressure and releases the core debris into a dry cavity. During core damage, hydrogen accumulated in the containment, however, it did not ignite because of a lack of ignition sources. The peak hydrogen concentration in the containment before vessel failure was greater than $16 \%$. Since the vessel fails at low pressure into a dry cavity, it is unlikely that there will be a rapid production of hydrogen at the time of vessel failure, however, the hot debris released into the containment will provide ample ignition sources for the preexisting hydrogen. In this situation, ignition is certain. The containment failure probability used for this case is the same probability used for the corresponding case for hydrogen burns during core damage (See Question 35). The quantification for this case is:

$\begin{array}{lll}\text { Branch 1: } & \text { nCF-VB } & 0.04 \\ \text { Branch 2: } & \text { CF-Rpt-VB } & 0.50 \\ \text { Branch 3: } & \text { CF-Lk-VB } & 0.46\end{array}$


Case 11: This case includes those accidents in which the reactor vessel fails at low pressure and releases the core debris into a dry cavity. In these accidents, either the hydrogen generated during core damage ignited, or the peak hydrogen concentration was less than $8 \%$. In either case, there is an insufficient amount of hydrogen to cause containment failure. The quantification for this case is:

$\begin{array}{lll}\text { Branch 1: } & \text { nCF-VB } & 1.00 \\ \text { Branch 2: } & \text { CF-Rpt-VB } & 0.00 \\ \text { Branch 3: } & \text { CF-Lk-VB } & 0.00\end{array}$

Question 50. What is the status of containment integrity just after vessel failure?

Number of Branches: 2

Number of Cases: 2

Number of Cases Sampled: 0

The branches for this question are:

1. nOCnt-VB The containment is breached at the time of vessel failure.

2. OCnt-VB The containment is still intact just after vessel failure.

This question summarizes the status of containment integrity at the end of vessel failure. This question summarizes failures that occurred before vessel failure and also includes failures that occurred at the time of vessel failure. This question does not address the size of the breach.

Case 1: This case includes those accidents in which either the containment was breached during or prior to core damage, the containment failed from an Alpha Mode event, or the containment failed from pressure loads that accompanied vessel breach. The quantification for this case is:

Branch 1: nOCnt-VB $\quad 0.00$

Branch 2: $\quad$ OCnt-VB 1.00

Case 2: This case includes all other accidents in which the containment is still intact after vessel failure. The quantification for this case is:

Branch 1: nOCnt-VB 1.00

Branch 2: $\quad$ OCnt-VB $\quad 0.00$

Question 51. What is the size of the containment opening just after vessel failure?

Number of Branches: 3

Number of Cases: 4

Number of Cases Sampled: 0

The branches for this question are:

1. Cnt-Rpt-VB The containment opening is the size of a rupture (nominal size is $1.0 \mathrm{ft}^{2}$ ).

2. Cnt-Lk-VB The containment opening is the size of a leak (nominal size is $0.1 \mathrm{ft}^{2}$ ).

3. Cnt-NF-VB The containment is still intact after vessel failure.

This question summarizes the size of the containment opening at the end of the vessel breach time regime.

Case 1: This case includes those accidents in which the containment is still intact at the end of the vessel breach time regime. The quantification for this case is:

Branch 1: $\quad$ Cnt-Rpt-VB 0.00 
Appendix B

$\begin{array}{lll}\text { Branch 2: } & \text { Cnt-Lk-VB } & 0.00 \\ \text { Branch 3: } & \text { Cnt-NF-VB } & 1.00\end{array}$

Case 2: This case includes those accidents in which either the containment was ruptured prior to or during core damage, the containment failed as a result of an Alpha Mode event, or pressure loads accompanying vessel failure caused a rupture in the containment. The quantification for this case is:

$\begin{array}{ll}\text { Branch 1: } & \text { Cnt-Rpt-VB } 1.00 \\ \text { Branch 2: } & \text { Cnt-Lk-VB } 0.00 \\ \text { Branch 3: } & \text { Cnt-NF-VB } 0.00\end{array}$

Case 3: This case includes those accidents in which a hydrogen combustion event during core damage or pressure loads accompanying vessel breach caused a leak in the containment. The quantification for this case is:

$\begin{array}{ll}\text { Branch 1: } & \text { Cnt-Rpt-VB } 0.00 \\ \text { Branch 2: } & \text { Cnt-Lk-VB } 1.00 \\ \text { Branch 3: } & \text { Cnt-NF-VB } 0.00\end{array}$

Case 4: This case is not used.

Question 52. Does the auxiliary building fail due to loads accompanying vessel failure?

Number of Branches: 2

Number of Cases: 3

Number of Cases Sampled: 0

The branches for this question are:

1. nOAux-VB The auxiliary building is intact at the end of the vessel breach time regime.

2. OAux-VB The auxiliary building has failed by the end of the vessel breach time regime.

This question determines the status of the auxiliary building integrity at the end of the vessel breach time regime. The status of the auxiliary building integrity is important because, for cases with either the reactor vessel or the containment open to the auxiliary building, it defines when the radioactive material is released to the environment. As discussed in Question 35, for accidents in which the containment is closed prior to core damage and the reactor vessel is not open to the auxiliary building (i.e., via open MSIVs or a LOCA in the auxiliary building), any subsequent failure of the containment will occur above the auxiliary building roof, and therefore all of the containment releases will bypass the auxiliary building.

Case 1: This case includes those accidents in which the auxiliary building failed prior to vessel failure. The quantification for this case is:

Branch 1: $\quad$ nOAux-VB $\quad 0.00$

Branch 2: $\quad$ OAux-VB 1.00

Case 2: This case includes those accidents in which the auxiliary building did not fail prior to vessel failure, the reactor is not open to the auxiliary building, and the containment was closed prior to the onset of core damage. In this situation, any releases of steam and/or non-condensibles will bypass the auxiliary building. The quantification for this case is:

Branch 1: $\quad$ nOAux-VB 1.00

Branch 2: $\quad$ OAux-VB $\quad 0.00$

Case 4: This case includes those accidents in which either the containment or the reactor vessel (i.e., via open MSIVs or a LOCA in the auxiliary building) is open to the auxiliary building. Results from MELCOR indicated 
that in all the relevant scenarios, the auxiliary building will fail at the time of vessel failure from pressure loads that accompany vessel failure or will fail shortly after vessel failure from the accumulation of steam and noncondensibles. The quantification for this case is:

Branch 1: $\quad$ nOAux-VB $\quad 0.00$

Branch 2: $\quad$ OAux-VB 1.00

Question 53. What is the status of de power late in the accident?

Number of Branches: 2

Number of Cases: 5

Number of Cases Sampled: None

The branches for this question are:

1. nDC-Late DC power is not available during the late time regime (i.e., after vessel failure).

2. DC-Late DC power is available during the late time regime (i.e., after vessel failure).

This question determines the availability of dc power during the late time regime. The availability of dc power is important because dc power is required to restore offsite power to the plant (see Question 24). Restoration of offsite power during the late time regime is considered from the time of vessel failure until two hours after vessel failure. Because the restoration of offsite power depends of the availability of dc power, the time regime used for restoration of offsite power was also used for $\mathrm{dc}$ power. The probability that dc power is not available during the late time regime is based on a distribution that was developed for the NUREG-1150 Grand Gulf plant analysis, which models the failure probability of the station batteries versus time for station blackout sequences [Wheeler et al., 1989]. The failure probabilities used in this question are conditional on dc power being available at the time of vessel failure.

Case 1: This case includes those accidents in which ac power was available before vessel failure. With ac power available, the battery chargers supply the necessary dc power and battery depletion is not an issue. For the PDSs analyzed in this study, there were no failures of the dc bus; therefore, with ac power available, dc power is also assured. The quantification for this case is:

Branch 1: nDC-Late $\quad 0.0$

Branch 2: DC-Late $\quad 1.0$

Case 2: This case includes those accidents in which dc power was not available at the time of vessel failure and therefore is not available after vessel failure (no credit is given for recovery of the station batteries). The quantification for this case is:
Branch 1: nDC-Late
1.0
Branch 2: DC-Late
0.0

Case 3: This case includes those accidents initiated by a loss of offsite power that result in a station blackout during Time Window 1. In these accidents, dc power is available at the time of vessel failure. For this case the following times are used for the start and end of the time period considered for restoration of ac power during the late time period: 14.4 hours and 16.4 hours. Therefore, the value for Branch 2 is the probability that de power is available at 16.4 hours given that it was available at 14.4 hours. The quantification for this case is:
Branch 1: nDC-Late
0.18
Branch 2: DC-Late
0.82

Case 4: This case includes those accidents initiated by a loss of offsite power that result in a station blackout during Time Window 2. In these accidents, dc power is available at the time of vessel failure. For chis case the following times are used for the start and end of the time period considered for restoration of ac power during the late time 


\section{Appendix B}

period: 12.6 hours and 14.6 hours. Therefore, the value for Branch 2 is the probability that de power is available at 14.6 hours given that it was available at 12.6 hours. The quantification for this case is:

$$
\begin{array}{lll}
\text { Branch 1: } & \text { nDC-Late } & 0.16 \\
\text { Branch 2: } & \text { DC-Late } & 0.84
\end{array}
$$

Case 5: This case is not used.

\section{Question 54. Is offsite power restored after vessel breach core damage? Number of Branches: 2 Number of Cases: 5 Number of Cases Sampled: 2}

The branches for this question are:

1. nAC-Late Neither offsite nor onsite power is available after vessel breach.

2. OSP-Late Only offsite power is available after vessel breach (note this situation does not occur in this analysis).

3. AC-Late AC power is available after vessel breach.

This question determines the availability of ac power during the late time period after vessel failure. The availability of ac power is important because it will determine which systems can be used to mitigate the accident (e.g., containment heat removal systems and Containment Vent System). Restoration of offsite power during the late time regime is considered from the time of vessel failure until two hours after vessel failure. Two hours was selected because containment venting will typically be required within two hours of vessel breach; also, for situations where the containment is open, the containment sprays must be initiated shortly after vessel breach if they are to be effective as a means of trapping radioactive material released during the interactions between the core debris and the concrete cavity.

The probability of recovering offsite power during a given time period is determined by sampling from a set of distributions for power recovery [Iman and Hora, 1988] (see also Volume 2 of this report). These distributions reflect the type of electrical switchyard at Grand Gulf, as explained in NUREG-1032 [Baranowsky, 1985]. To get ac power to the safety systems, not only does ac power have to be restored to the site, but dc power must be available as well. DC power is required for circuit breaker control power; once the station batteries have been depleted, it is very difficult to get ac power back to the safety systems. Although the circuit breakers can be moved manually, this procedure is very complicated and slow. Thus, for the time frame considered in this analysis, it is assumed that once dc power is lost, ac power cannot be recovered. The generation of the power recovery curves used in this analysis is discussed in Appendix G of Volume 2, Part 2 of this report.

Case 1: This case includes those accidents in which offsite and onsite power are available at the start of the accident; therefore, ac power is still available. The quantification for this case is:

$\begin{array}{lll}\text { Branch 1: } & \text { nAC-Late } & 0.0 \\ \text { Branch 2: } & \text { OSP-Late } & 0.0 \\ \text { Branch 3: } & \text { AC-Late } & 1.0\end{array}$

Case 2: This case includes those accidents in which dc power is not available. The lack of dc power implies that both offsite and onsite ac power are unavailable. Without dc power, offsite power cannot be recovered. Furthermore, in this analysis no credit is given for recovery of the emergency diesel generator during the core damage process. Therefore, in this case all ac power is unavailable. The quantification for this case is:

$\begin{array}{lll}\text { Branch 1: } & \text { nAC-Late } & 1.0 \\ \text { Branch 2: } & \text { OSP-Late } & 0.0 \\ \text { Branch 3: } & \text { AC-Late } & 0.0\end{array}$


Case 3: This case includes those accidents initiated by a loss of offsite power that result in a station blackout during Time Window 1. DC power is available at the time of vessel failure. For this case the following times are used for the start and end of the time period considered for restoration of ac power during the late time period: 14.4 hours and 16.4 hours. Therefore, the value for Branch 3 is the probability that ac power is recovered during the late time regime given that ac power was not available at the time of vessel failure. The quantification for this case is:

$$
\begin{array}{llll}
\text { Branch 1: } & \text { nAC-Late } & 0.78 & \\
\text { Branch 2: } & \text { OSP-Late } & 0.0 & \\
\text { Branch 3: } & \text { EAC-Late } & 0.22 & \text { Power recovery distribution. }
\end{array}
$$

Case 4: This case includes those accidents initiated by a loss of offsite power that result in a station blackout during Time Window 2. DC power is available at the time of vessel failure. For this case the following times are used for the start and end of the time period considered for restoration of ac power during the late time period: 12.6 hours and 14.6 hours. Therefore, the value for Branch 3 is the probability that ac power is recovered during the late time regime given that ac power was not available at the time of vessel failure. The quantification for this case is:

$$
\begin{array}{llll}
\text { Branch 1: } & \text { nAC-Late } & 0.73 & \\
\text { Branch 2: } & \text { OSP-Late } & 0.0 & \\
\text { Branch 3: } & \text { EAC-Late } & 0.27 & \text { Power recovery distribution. }
\end{array}
$$

Case 5: This case is not used.

\section{Question 55. Is the core debris in the cavity coolable? \\ Number of Branches: 3 \\ Number of Cases: 8 \\ Number of Cases Sampled: 0}

The branches for this question are:
1. nCCI
2. FldCCI
3. DryCCI
The core debris is cooled in the cavity; there are no core-concrete interactions (CCI).
The core debris is not coolable; CCI occurs in a flooded cavity.
The core debris is not coolable; $\mathrm{CCI}$ occurs in a dry cavity.

This question determines whether the core debris released from the reactor vessel is cooled by water in the cavity below the reactor vessel. If the debris is not coolable, this question also determines whether the CCI take place in a dry or flooded cavity. The coolability of the core debris is important because it will affect the amount of radioactive material released from the core debris. If the core debris is coolable, it is assumed in the source term analysis that no radioactive material is released from the core debris in the cavity. If the core debris is not in a coolable configuration but the CCI occurs in a flooded cavity, some of the radioactive material released from the core debris will be trapped in the overlying pool of water.

The quantification of this question is based on probabilities developed in the NUREG-1150 study as documented in NUREG/CR-4551 [Harper et al., 1994]. The core debris bed will not be coolable if it is finely fragmented or if the debris reagglomerates after vessel failure. The coolability of the debris that is released at vessel breach, as well as that of the debris slowly released following vessel breach, is considered in this question. The core debris must be coolable in both cases if the $\mathrm{CCI}$ is not to occur. If any of the core debris released to the cavity, either at the time of vessel failure or after vessel failure, is not coolable, $\mathrm{CCI}$ will be initiated. Once $\mathrm{CCI}$ has been established, it is assumed that all the material in the reactor cavity participates in CCI. Thus the coolability of the debris released after vessel breach is important only if the debris released at vessel breach is coolable.

The likelihood that the debris released after vessel breach is coolable is the same for all the cases that have water in the cavity. The debris released after vessel breach was most likely solid at vessel breach. As the decay heat melts this remaining debris, it is released from the vessel. Thus it is likely that this debris will be released with a low amount of superheat. It is 


\section{Appendix B}

expected that the debris bed that forms from this material will consist of large particles that may not be entirely molten. Assuming there is water in the cavity, it is likely that the debris bed will be coolable.

If the reactor pressure vessel fails at high pressure, most of the debris will be ejected from the cavity. Although this material will be finely fragmented, it will be coolable because it is spread throughout the drywell in a thin layer. In this case, the coolablility of the debris in the cavity is based on the material that is released after vessel breach.

Case 1: This case includes those accidents in which the vessel does not fail, and therefore no CCI occurs. The quantification for this case is:

$\begin{array}{lll}\text { Branch 1: } & \text { nCCI } & 1.0 \\ \text { Branch 2: } & \text { FldCCI } & 0.0 \\ \text { Branch 3: } & \text { DryCCI } & 0.0\end{array}$

Case 2: This case includes those accidents in which the vessel fails and the core debris is released into a dry cavity. Core cooling was not restored to the vessel during core damage. Furthermore, if the vessel was pressurized during core damage, none of the low pressure injection systems are aligned to automatically inject into the vessel when the vessel depressurizes at the time of vessel failure. Because there is no water in the cavity, $\mathrm{CCI}$ in a dry cavity is assured. The quantification for this case is:

$\begin{array}{lll}\text { Branch 1: } & \text { nCCI } & 0.0 \\ \text { Branch 2: } & \text { FldCCI } & 0.0 \\ \text { Branch 3: } & \text { DryCCI } & 1.0\end{array}$

Case 3: This case includes those accidents in which the vessel, which is at system pressure, fails and the core debris is released into a dry cavity. Core coolant is being provided to the vessel and will enter the cavity coincident with the core debris. Because the vessel fails at high pressure, most of the debris released at vessel breach is ejected from the cavity. Thus the coolability of the debris in the cavity is based on the material released after vessel failure. As explained above, it is likely that the debris released after vessel failure is coolable. If the debris bed is not cooled, $\mathrm{CCI}$ will occur in a flooded cavity. The quantification for this case is:

$\begin{array}{lll}\text { Branch 1: } & \text { nCCI } & 0.80 \\ \text { Branch 2: } & \text { FldCCI } & 0.20 \\ \text { Branch 3: } & \text { DryCCI } & 0.00\end{array}$

Case 4: This case includes those accidents in which the debris is released from the vessel at low pressure into a dry cavity. Core coolant is being provided to the vessel and will enter the cavity coincident with the core debris.

Because the vessel fails at low pressure, most of the debris released at vessel breach will remain in the cavity. Even though water is released from the vessel at the time of vessel failure, the debris will contact essentially a dry floor, and $\mathrm{CCI}$ is likely to initiate. Once CCI is established, gases and steam flow upward through the debris and create a resistance to water that would penetrate and cool the debris. If the debris bed is not cooled, which is likely, CCI will occur in a flooded cavity. The quantification for this case is:

$\begin{array}{lll}\text { Branch 1: } & \text { nCCI } & 0.16 \\ \text { Branch 2: } & \text { FldCCI } & 0.84 \\ \text { Branch 3: } & \text { DryCCI } & 0.00\end{array}$

Case 5: This case includes those accidents in which the debris is released from the vessel at high pressure into a flooded cavity. Because the vessel fails at high pressure, most of the debris released at vessel breach is ejected from the cavity. Thus the coolability of the debris in the cavity is based on the material released after vessel failure. As explained above, it is likely that the debris released after vessel failure is coolable. If the debris bed is not cooled, CCI will occur in a flooded cavity. The quantification for this case is: 
$\begin{array}{lll}\text { Branch 1: } & \text { nCCI } & 0.80 \\ \text { Branch 2: } & \text { FldCCI } & 0.20 \\ \text { Branch 3: } & \text { DryCCI } & 0.00\end{array}$

Case 6: This case includes those accidents in which the debris is released from the vessel at low pressure into a flooded cavity. In this case, the debris also has a high amount of super heat. Because the vessel fails at low pressure, most of the debris released at vessel breach will remain in the cavity. Even though there is water in the cavity, it is likely that the core debris will agglomerate because of its high superheat. Thus it is likely that the core debris released at the time of vessel failure will not be coolable. Therefore, even though it is likely that the debris release after vessel breach is coolable, it is likely that $\mathrm{CCl}$ will be initiated by the debris released at the time of vessel breach. If the debris is not cooled, $\mathrm{CCI}$ will occur in a flooded cavity. The quantification for this case is:

$\begin{array}{lll}\text { Branch 1: } & \text { nCCI } & 0.16 \\ \text { Branch 2: } & \text { FldCCI } & 0.84 \\ \text { Branch 3: } & \text { DryCCI } & 0.00\end{array}$

Case 7: This case includes those accidents in which the debris is released from the vessel at low pressure into a flooded cavity. In this case, the debris has a small amount of super heat. Because the vessel fails at low pressure, most of the debris released at vessel breach will remain in the cavity. Even though there is water in the cavity and the debris has a small amount of superheat, it is uncertain whether the debris released at vessel breach will be coolable. If the debris is not cooled, CCI will occur in a flooded cavity. The quantification for this case is:

$\begin{array}{lll}\text { Branch 1: } & \text { nCCI } & 0.40 \\ \text { Branch 2: } & \text { FldCCI } & 0.60 \\ \text { Branch 3: } & \text { DryCCI } & 0.00\end{array}$

Case 8: This case is not used.

Question 56. Do the operators vent the containment after vessel breach?

Number of Branches: 2

Number of Cases: 3

Number of Cases Sampled: 1

The branches for this question are:

1. nVnt-Late The containment is not vented after vessel breach.

2. Vnt-Late The operators vent the containment after vessel breach.

This question determines whether the operators vent the containment after vessel breach. The status of containment venting is important because opening the containment vent establishes a path from the containment to the environment that bypasses the auxiliary building, which allows airborne radioactive material in the containment to escape directly to the environment. The size of the vent path is equivalent to a rupture in the containment. The Grand Gulf Emergency Procedures (EP-3) direct the operators to vent the containment if the containment pressure is greater than $20 \mathrm{psig}$ and cannot be maintained below 22 psig. Although the procedures allow the operators to close the vent once the pressure drops below $20 \mathrm{psig}$, the operators would have to open the vent again later in the accident because containment cooling is not available and the steam and noncondensibles generated during the accident would again cause the containment pressure to exceed $22 \mathrm{psig}$. This analysis makes no attempt to model the opening and the closing of the vent to maintain the pressure below $20 \mathrm{psig}$. Instead, it is assumed that once the vent is opened, it remains open for the duration of the accident.

Case 1: This case includes those accidents in which either the containment vent system is not available or the containment pressure is below the vent threshold pressure (i.e., containment already ruptured or reactor vessel open to auxiliary building). The quantification for this case is: 


\section{Appendix B}

$\begin{array}{lll}\text { Branch 1: } & \text { nVnt-Late } & 1.00 \\ \text { Branch 2: } & \text { Vnt-Late } & 0.00\end{array}$

Case 2: This case includes those accidents in which the pressure in the containment is above the vent threshold. MELCOR calculations for the relevant scenarios indicate that the containment pressure will exceed the vent threshold at the time of vessel failure or shortly after vessel failure. The containment vent system is either available or was recoverable and ac power is recovered (i.e., either during core damage or after vessel breach). The Grand Gulf Emergency Procedures will direct the operators to vent the containment in this situation. This case is sampled; the distribution that characterizes the uncertainty in the probability that the operators will fail to vent the containment was developed in the Human Reliability Analysis and is discussed in Appendix B.3. The quantification (mean value) for this case is:

$\begin{array}{lll}\text { Branch 1: } & \text { nVnt-Late } & 0.031 \\ \text { Branch 2: } & \text { Vnt-Late } & 0.969\end{array}$

Lognormal distribution with a mean of 0.031 and an error factor of 5 .

Case 3: This case includes those accidents in which the pressure in the containment is above the vent threshold; however, ac power is not available. Without ac power the containment vent cannot be opened. The quantification for this case is:

Branch 1: $\quad$ nVnt-Late 1.0

Question 57. Does the containment fail late in the accident?

Number of Branches: 3

Number of Cases: 2

Number of Cases Sampled: 0

The branches for this question are:

1. nCF-Late The containment does not fail late in the accident.

2. CF-Rpt-Late The containment fails in the rupture mode (nominal hole size is $1.0 \mathrm{ft}^{2}$ ) late in the accident.

3. CF-Lk-Late The containment fails in the leak mode (nominal hole size is $0.1 \mathrm{ft}^{2}$ ) late in the accident.

This question determines whether the containment fails late in the accident. The status of the containment integrity is important because failure of the containment will result in a path for radionuclides to enter the environment. Similar to the NUREG-1150 Grand Gulf plant analysis [Brown et al., 1990], two failure sizes are defined: a leak and a rupture. The containment failure issue is discussed in Question 35.

For the PDSs considered in this analysis, the accidents in which the containment could be closed prior to the onset of core damage also had the characteristic that containment cooling was not available and could not be recovered. Therefore, in all the accidents addressed in this study, the containment will be breached at some point during the accident.

Case 1: This case includes those accidents in which the containment was ruptured either before vessel failure or at the time of vessel failure or the containment was vented after vessel failure. In any case, the containment boundary is already breached. The quantification for this case is:

Branch 1: nCF-Late $\quad 1.00$

Branch 2: CF-Rpt-Late $\quad 0.00$

Branch 3: CF-Lk-Late $\quad 0.00$ 
Case 2: This case includes those accidents in which the containment was intact at the end of the vessel breach time regime and was not vented during the late time regime. Without containment cooling, the containment will fail from the accumulation of steam and non-condensibles generated during the accident. The quantification for this case is:

Branch 1: nCF-Late $\quad 0.00$

Branch 2: CF-Rpt-Late $\quad 0.50$

Branch 3: CF-Lk-Late $\quad 0.50$

Question 58. What is the status of containment integrity late in the accident?

Number of Branches: 2

Number of Cases: 2

Number of Cases Sampled: 0

The branches for this question are:

1. nOCnt-Late The containment does not remain intact throughout the accident.

2. OCnt-Late The containment is still intact at the end of the accident.

This question summarizes the status of containment integrity at the end of the accident. This question summarizes failures that occurred before vessel failure, at the time of vessel failure, and after vessel failure. The question does not address the size of the breach.

Case 1: This case includes those accidents in which the containment was breached during the accident. The quantification for this case is:

Branch 1: $\quad$ nOCnt-Late 0.00

Branch 2: $\quad$ OCnt-late $\quad 1.00$

Case 2: This case includes all other accidents in which the containment is still intact at the end of the accident. The quantification for this case is:

$\begin{array}{lll}\text { Branch 1: } & \text { nOCnt-Late } 1.00 \\ \text { Branch 2: } & \text { OCnt-Late } \quad 0.00\end{array}$

Question 59. What is the size of the containment opening at the end of the accident?

Number of Branches: 3

Number of Cases: 4

Number of Cases Sampled: 0

The branches for this question are:

1. Cnt-Rpt-Late The containment opening is the size of a rupture (nominal size is $1.0 \mathrm{ft}^{2}$ ).

2. Cnt-Lk-Late The containment opening is the size of a leak ( nominal size is $0.1 \mathrm{ft}^{2}$ ).

3. Cnt-NF-Late The containment is still intact after vessel failure.

This question summarizes the size of the containment opening at the end of the accident.

Case 1: This case includes those accidents in which the containment is still intact at the end of the accident. The quantification for this case is:

Branch 1: Cnt-Rpt-Late $\quad 0.00$ 
Appendix B

$\begin{array}{lll}\text { Branch 2: } & \text { Cnt-Lk-Late } & 0.00 \\ \text { Branch 3: } & \text { Cnt-NF-Late } & 1.00\end{array}$

Case 2: This case includes those accidents in which either the containment was ruptured prior to or during vessel failure, vented after vessel failure, or was ruptured late in the accident from the accumulation of steam and noncondensibles. The quantification for this case is:

$\begin{array}{lll}\text { Branch 1: } & \text { Cnt-Rpt-Late } & 1.00 \\ \text { Branch 2: } & \text { Cnt-Lk-Late } & 0.00 \\ \text { Branch 3: } & \text { Cnt-NF-Late } & 0.00\end{array}$

Case 3: This case includes those accidents in which loads caused a leak containment at some point during the accident. The quantification for this case is:

$\begin{array}{lll}\text { Branch 1: } & \text { Cnt-Rpt-VB } 0.00 \\ \text { Branch 2: } & \text { Cnt-Lk-VB } 1.00 \\ \text { Branch 3: } & \text { Cnt-NF-VB } 0.00\end{array}$

Case 4: This case is not used. 


\section{B.2 Listing of POS 5 APET}

A listing of the accident progression event tree (APET) is provided in this section of the appendix. The APET in this format is provided as input to the EVNTRE code preprocessor ETPRE [Roginski, 1994], which is used to evaluate the event tree. The logic that is used to form the accident progression bins is provided near the end of the APET listing and is identified by the keyword "Binning". 
APET for POS 5, Ver. 2

One-Eval 1.0

$\stackrel{2}{a}$

PDS Definition

What is the Plant Damage State?

PDS1-1 PDS1-2 PDS1-3 PDS1-4 PDS1-5 PDS2-1 PDS2-2 PDS2-3 PDS2-4 PDS2-5 PDS2-6 PDS3-1

(SFP1-1) (SFP1-2) (SFP1-3) (SFP1-4) (SFP1-5) (SFP2-1) (SFP2-2) (SFP2-3) (SFP2-4) (SFP2-5) (SFP2-6) (Excess)

$\begin{array}{lllllllllllll}\$ & 0.019 & 0.015 & 0.032 & 0.005 & 0.008 & 0.17 & 0.242 & 0.054 & 0.104 & 0.007 & 0.006 & 0.338\end{array}$

What is the status of electric power at core damage (PDS Char. 1)?

PDS1-2 + PDS1-3 + PDS1-4

PDS2-2 + PDS2-3

$\begin{array}{lll}\text { aOSP } & \text { OSP-nDIVAC } & \text { nOSP } \\ 0.0 & 0.0 & 1.0 \\ 0.0 & 0.0 & 1.0 \\ 1.0 & 0.0 & 0.0\end{array}$

What is the status of dc power at core damage (PDS Char. 1)?

PDS1-2 + PDS1-3 + PDS2-2

$\mathrm{NDC}-\mathrm{BCD} \quad \mathrm{aDC}-\mathrm{BCD}$

Otherwise

$1.0 \quad 0.0$

$\square$
$\dot{1}$
$\ddot{\sigma}$

What is the status of high pressure injection at core damage (PDS Char. 2)?

$$
\text { nHPInj }
$$

nHPInj

rHPInj

HPInj

What is the status of low pressure injection at core damage (PDS Char. 2)?

$\begin{array}{lllll} & \text { nLPInj } & \text { nLPInj-op } & \text { rLPInj } & \text { aLPInj } \\ \text { PDS1-2 + PDS1-3 + PDS1-4 } & 0.0 & 0.0 & 1.0 & 0.0 \\ \text { PDS2-2 + PDS2-3 } & 0.0 & 0.0 & 1.0 & 0.0 \\ \text { PDS2-6 } & 0.0 & 1.0 & 0.0 & 0.0 \\ \text { Otherwise } & 1.0 & 0.0 & 0.0 & 0.0\end{array}$

What is the status of containment sprays and SPC at core damage (PDS Char. 3)?

PDS1-2 + PDS1-3 + PDS1-4

PDS2-2 + PDS2-3

Otherwise

$\begin{array}{ll}\text { nCS } & \text { rCS } \\ 0.0 & 1.0 \\ 0.0 & 1.0 \\ 1.0 & 0.0\end{array}$

$\mathrm{rCS}$
1.0
1.0

0.0

alignCS

0.0

0.0

0.0

SPL-Lo SPL-Strain

?ִ

What is the suppression pool level at the onset of core damage (PDS Char. 4)?

PDS2-6

Otherwise

0.0

1.0
0.0 


$\begin{array}{lll} & \text { SPT-Sub } & \text { SPT-Sat } \\ \text { PDS1-3 } & 0.0 & 1.0 \\ \text { Otherwise } & 1.0 & 0.0\end{array}$

What is the status of the reactor head vent at the onset of core damage (PDS Char. 6)?

$\begin{array}{lll} & \text { RPV-nVnt } & \text { RPV-OVnt } \\ \text { PDS1-5 + PDS2-4 + PDS2-6 } & 1.0 & 0.0 \\ \text { Otherwise } & 0.0 & 1.0\end{array}$

What is the status of the RPV integrity at the onset of core damage (PDS Char. 7)?

$\begin{array}{llllll} & \text { RPV-HiP } & \text { RPV-LoP } & \text { RPV-LOCA } & \text { RPV-ILOCA } & \text { RPV-oMSIV } \\ \text { PDS1-1 + PDS2-1 + PDS3-1 } & 0.0 & 0.0 & 1.0 & 0.0 & 0.0 \\ \text { PDS1-2+ PDS1-4 } & 0.0 & 0.0 & 0.0 & 1.0 & 0.0 \\ \text { PDS2-2 + PDS2-3 } & 0.0 & 0.0 & 0.0 & 1.0 & 0.0 \\ \text { PDS2-6 } & 0.0 & 0.0 & 0.0 & 0.0 & 1.0 \\ \text { PDS1-5 + PDS2-4 } & 0.0 & 1.0 & 0.0 & 0.0 & 0.0 \\ \text { Otherwise } & 1.0 & 0.0 & 0.0 & 0.0 & 0.0\end{array}$

What is the status of the containment access penetrations at the onset of core damage (PDS Char. 8)?

$\begin{array}{lll}\text { PDS2-5 + PDS2-6 } & \text { o-LPersLk } & \text { LPersLk-Unk } \\ \text { Otherwise } & 0.0 & 1.0 \\ & 1.0 & 0.0\end{array}$

What is the status of the containment vents system at the onset of core damage (PDS Char. 9)?

$\begin{array}{llll} & \text { nCVS } & \text { rCVS } & \text { aCVS } \\ \text { PDS1-2 + PDS1-3 + PDS1-4 } & 0.0 & 1.0 & 0.0 \\ \text { PDS2-2 + PDS2-3 } & 0.0 & 1.0 & 0.0 \\ \text { PDS2-5 + PDS2-6 } & 0.0 & 0.0 & 1.0 \\ \text { Otherwise } & 1.0 & 0.0 & 0.0\end{array}$

When does core damage occur (PDS Char. 10)?

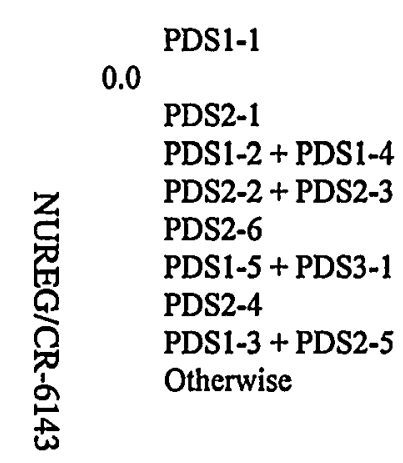

$\begin{array}{llll}\text { TCD2 } & \text { TCD2p3 } & \text { TCD3 } & \text { TCD5 } \\ 1.0 & 0.0 & 0.0 & 0.0 \\ 0.0 & & & \\ 0.0 & 1.0 & 0.0 & 0.0 \\ 0.0 & 0.0 & 1.0 & 0.0 \\ 0.0 & 0.0 & 0.0 & 1.0 \\ 0.0 & 0.0 & 0.0 & 0.0 \\ 0.0 & 0.0 & 0.0 & 0.0 \\ 0.0 & 0.0 & 0.0 & 0.0 \\ 0.0 & 0.0 & 0.0 & 0.0 \\ & 0.0 & 0.0 & 0.0\end{array}$

$\begin{array}{llll}\text { TCD6 } & \text { TCD7 } & \text { TCD9 } & \text { TCD12 } \\ 0.0 & 0.0 & 0.0 & \\ & & & \\ 0.0 & 0.0 & 0.0 & 0.0 \\ 0.0 & 0.0 & 0.0 & 0.0 \\ 0.0 & 0.0 & 0.0 & 0.0 \\ 1.0 & 0.0 & 0.0 & 0.0 \\ 0.0 & 1.0 & 0.0 & 0.0 \\ 0.0 & 0.0 & 1.0 & 0.0 \\ 0.0 & 0.0 & 0.0 & 1.0 \\ 0.0 & 0.0 & 0.0 & 1.0 \text { SNA }\end{array}$


While in POS 5, when does the initiating event occur (PDS Char. 11)?

PDS1-1 + PDS1-2 + PDS1-3 + PDS1-4 + PDS1-5 1.0

PDS2-1 + PDS2-2 + PDS2-3 + PDS2-4

IE-Win1 IE-Win2 IE-Win3

PDS2-5 + PDS2-6

PDS3-1

$0.0-1.0$

0.0

0.0

1.0

Otherwise

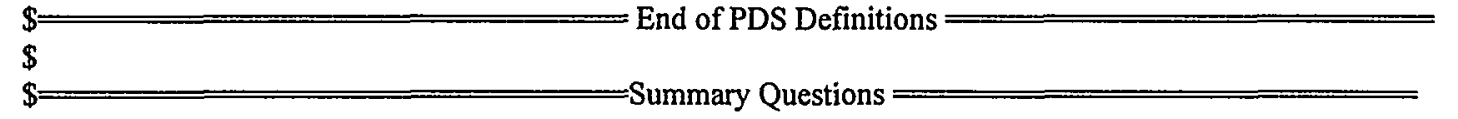

What type of event initiates the accident?

$\begin{array}{llll} & \text { IE-LOCA } & \text { IE-SBO } & \text { IE-Other } \\ \text { PDS1-1 + PDS2-1 + PDS3-1 } & 1.0 & 0.0 & 0.0 \\ \text { PDS1-2 + PDS1-3 + PDS1-4 } & 0.0 & 1.0 & 0.0 \\ \text { PDS2-2 + PDS2-3 } & 0.0 & 1.0 & 0.0 \\ \text { Otherwise } & 0.0 & 0.0 & 1.0\end{array}$

$\$$ This question summarizes the type of IE

at is the pressure in the RPV at the time of core damage?

$\begin{array}{llll}0 & & \text { RPV-HiP-BCD } & \text { RPV-LoP-BCD } \\ \dot{d} & \text { RPV-HiP } & 1.0 & 0.0 \\ \infty & \text { Otherwise } & 0.0 & 1.0\end{array}$

How much water is in the reactor pedestal cavity at the time of core damage?

$\begin{array}{llll} & \text { Cav-Dry-BCD } & \text { Cav-Fld-BCD } & \\ \text { IE-LOCA } & 0.00 & 1.00 & \text { \$(1): Containment flooded in LOCAs } \\ \text { PDS1-5 + PDS2-4 } & 0.00 & 1.00 & \text { \$ (2): CNMT flooded in these PDSs } \\ \text { Otherwise } & 1.00 & 0.00 & \text { \$ (3): Cavity esscentially dry. }\end{array}$

$\$=$ Events before $C D$

Is the containment equipment hatch opened at the start of the accident?

$\begin{array}{ll}\text { nOCnt-S } & \text { OCnt-S } \\ 0.0 & 1.0\end{array}$

Do the operators close the containment before core damage?

$\begin{array}{lll} & \text { nOCnt-BCD } & \text { OCnt-BCD } \\ \text { nOCnt-S } & 1.0 & 0.0 \\ \text { o-LPersLk } & 0.0 & 1.0 \\ \text { IE-SBO } & 0.0 & 1.0 \\ \text { TCD2 + TCD2p3 + TCD3 } & 0.0 & 1.0 \\ \text { Otherwise } & \text { (Excess) } & \text { (HEP5-CNT) }\end{array}$

$\$$ This question addresses the status of the equipment hatch before CD.

$\$$ In this analysis it is assumed that the containment equipment hatch

$\$$ is always open at the start of the accident.

$\$(1)$ : The equip. hatch was closed at the start of the accident are remains closed.

$\$(2)$ : Low personnel lock was open at CD by definition of the PDS

$\$$ (3): The hatch was open prior to IE - SBO precludes closure

$\$(4)$ : CD occurs within 3 hours of the IE - not enough time to close containment

$\$(5)$ : CD occurs $>5$ hours of IE - Cnt closure possible. 
$\Xi \quad$ Does the auxiliary building fail before core damage?

$\begin{array}{lll} & \text { nOAux-BCD } \\ \text { RPV-ILOCA } & 0.0 & 1.0 \\ \text { RPV-oMSIV } & 0.0 & 1.0 \\ \text { nOCnt-S } & & 1.0 \\ \text { Otherwise } & & 1.0\end{array}$

\section{OAux-BCD}

\$ (1): ILOCA in Aux Bldg, steam from LOCA fails Aux Bldg

$\$(2)$ : RPV Level at steam lines; boiloff of coolant will fail Aux Bldg

1.0

What is the status of the drywell before core damage? Cls-DW-BCD

0.0
0.0 \$ (3): The CNMT is closed at the start of the accident, thus, no early Aux Bldg failure
$0.0 \quad \$(4)$ : Cnt is open BCD and SP subcooled

$\$$ In this analysis it is assumed that the drywell remains open for the duration

Op-DW-BCD \$ of the accident.

1.0

Do the operators turn on the HIS before core damage?

\begin{tabular}{|c|c|c|c|}
\hline & nHIS-BCD & HIS-BCD & $\$$ This question determines whether the HIS was actuated prior to $\mathrm{CD}$ \\
\hline E-SBO & 1.0 & 0.00 & $\begin{array}{l}\text { Il not operate without Div } 1 \text { or } 2 \text { power. It is assumed } \\
\text { I not actuate a system that won't work. }\end{array}$ \\
\hline se & (HEP-HIS-nSBO) & (Excess) & ilable. EOP call for the HIS to be turned on \\
\hline
\end{tabular}

幽

Data: HEP-HIS-nSBO 0.054 LOGNORMAL 0.0545

$\$=$ Events During Core Damage

Do the station batteries depleted during core damage?

$\begin{array}{lllll} & \text { nDC-CD } & \text { DC-ECD } & \text { DC-LCD } & \\ \text { /IE-SBO } & 0.000 & 0.000 & 1.000 & \text { \$(1): Not a SBO, thus dc power available } \\ \text { nDC-BCD } & 1.000 & 0.000 & 0.000 & \text { \$(2): SBO \& dc power failed before CD } \\ \text { IE-Win1 } & 0.011 & 0.242 & 0.747 & \text { \$(3): SBO during TW-1 (dc avail. @ 3.5 hr) } \\ \text { IE-Win2 } & 0.015 & 0.103 & 0.882 & \text { \$(4): SBO during TW-2 (dc avail. @ 5.5 hr) } \\ \text { Otherwise } & 0.000 & 1.000 & 0.000 & \text { \$(5): This case shouldn't be used. }\end{array}$

Is offsite power restored during core damage?

\begin{tabular}{|c|c|c|c|c|c|}
\hline & $\mathrm{nAC}-\mathrm{CD}$ & OSP-CD & EAC-CD & LAC-CD & \\
\hline /IE-SBO & 0.0 & 0.0 & 1.0 & 0.0 & \$ (1): Not a SBO, OSP available \\
\hline $\mathrm{nDC}-\mathrm{CD}$ & 1.0 & 0.0 & 0.0 & 0.0 & $\$(2)$ : No dc power, thus, no ac \\
\hline IE-Winl \& DC-ECD & (Excess) & 0.0 & (AC-ECD-TW1) & 0.0 & \$ (3): SBO in TW-1, dc early, OSP recoverable \\
\hline IE-Win1 & (Excess) & 0.0 & (AC-ECD-TW1) & (AC-LCD-TW1) & $\$$ (4): SBO in TW-1, dc available all of CD, OSP recvr \\
\hline IE-Win2 \& DC-ECD & (Excess) & 0.0 & (AC-ECD-TW2) & 0.0 & \$ (5): SBO in TW-2, dc early, OSP recoverbable \\
\hline IE-Win2 & (Excess) & 0.0 & (AC-ECD-TW2) & (AC-LCD-TW2) & $\$(6)$ : SBO in TW-2, dc available all of CD, OSP recvr \\
\hline Otherwise & 1.0 & 0.0 & 0.0 & 0.0 & $\$(7)$ : This case shouldn't be used. \\
\hline
\end{tabular}

Data: AC-ECD-TW1 0.5 File C:LLPSIPOS5ICALCLLHS200LAPETAACRECBE.LSP \$ Distributions from PWRRECBE.FOR; uses Power recovery curves 
Data: AC-LCD-TW1 0.5 File C:LLPSIPOS5ICALCLLHS200IAPETIACRECBE.LSP \$ generated by MODEI.FOR

Data: AC-ECD-TW2 0.5 File C:LLPSPOS5ICALCLHS200LAPETACRECBE.LSP

Data: AC-LCD-TW2 0.5 File C:LLPSTPOS5ICALCLLHS200\APETACRECBE.LSP

Is the RPV isolated during core damage?

$\begin{array}{lc}\text { IE-LOCA } & \\ \text { RPV-OMSIV } & 1.0 \\ \text { RPV-ILOCA \& IE-SBO \& EAC-CD } & \\ \text { RPV-ILOCA } & 1.0 \\ \text { Otherwise } & \end{array}$

Op-RPV-CD
1.0
0.0
(Excess)
0.0
0.0

Cls-RPV-CD

$0.0 \quad \$(1)$ : IE was a LOCA

$\$(2)$ : MSIVs are open - no credit for closing during CD

(ISO-SDC) \$(3): Failure of SDC F008 and F009 to close due to severe invironment \$ (4): SDC F008 and F009 open - no ac power

$1.0 \$(5):$ RPV integrity maintained.

Data: ISO-SDC 0.9 MAXIMUM ENTROPY 0.50 .91 .0

Do the operators initiate containment sprays during core damage?

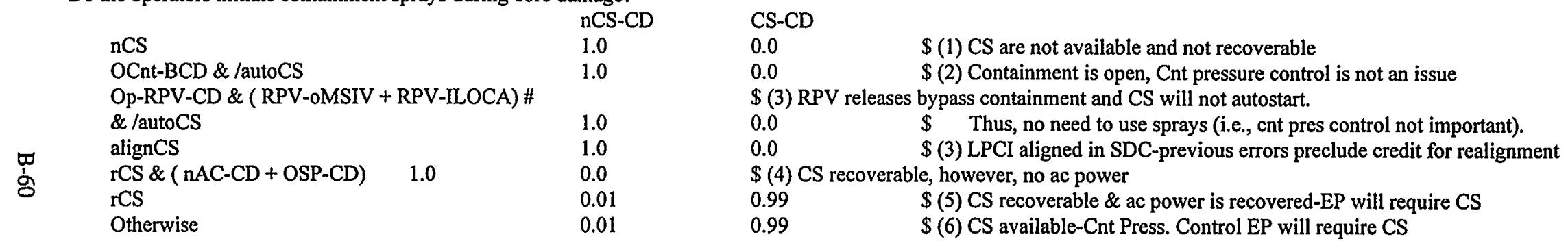

Do the operators depressurize the RPV during core damage?

\begin{tabular}{|c|c|c|}
\hline & RPV-HiP-CD & RPV-LoP-CD \\
\hline Op-RPV-CD $\quad 0.0$ & 1.0 & $V$ open via a breach (i.e., $I$ \\
\hline $\mathrm{DC}-\mathrm{CD}$ & 1.0 & dc power, \\
\hline RPV-ILOCA (HEP-PRPV-HiP) & (Excess) & \$ (3): HRA Quantifica \\
\hline $\begin{array}{l}\text { RPV-LoP-BC } \\
\text { Otherwise }\end{array}$ & $\begin{array}{l}0.0 \\
\text { (HEP-PRPV-HiP) }\end{array}$ & $\begin{array}{l}\text { \$ the recovery subsequent isolation of the SDC F008 and F009 } \\
\$(4): \text { RPV at low pressure before CD and still at low pressure } \\
\$(5): R P V \text { at high pressure. EP call for depressurization. }\end{array}$ \\
\hline
\end{tabular}

Data: HEP-PRPV-HiP 0.054 BOUNDED LOGNORMAL 0.054 50.0010 .999

What is the status of the SRV vacuum breakers during core damage?

$\begin{array}{lllll} & & \text { Op-SRV-Bkr } & \text { Cls-SRV-Bkr } & \\ \vdots & \text { RPV-LoP-BCD } & 0.0 & 1.00 & \$(1): \text { SRVs open, very few SRV cycles, if any, thus, unlikely to fail } \\ \vdots & \text { RPV-OVnt } & 0.0 & 1.00 & \$(2): \text { The open vent will limit the number of SRV cycles, thus, failure unlikely } \\ 0 & \text { Otherwise } & \text { (SRV-VBkr) } & \text { (Excess) } & \$(3): \text { RPV vent closed and RPV is pressurized - NUREG-1150 Quantification }\end{array}$


Is core cooling restored during core damage?

\begin{tabular}{|c|c|c|c|c|c|}
\hline & E-CorCool & L-CorCool & n-CorCool & \multirow{2}{*}{\multicolumn{2}{|c|}{$\$(1)$ : This case will never be used. }} \\
\hline HPInj & 1.0 & 0.0 & 0.0 & & \\
\hline rHPInj \& EAC-CD & 1.0 & 0.0 & 0.0 & \multicolumn{2}{|c|}{$\$(2)$ : This case will never be used. } \\
\hline rHPInj \& LAC-CD & 0.0 & 1.0 & 0.0 & \multicolumn{2}{|c|}{ \$ (2): This case will never be used. } \\
\hline RPV-HiP-CD 0.0 & 0.0 & 1.0 & \multicolumn{3}{|c|}{ \$ (3): RPV at high pressure and no high pressure makeup } \\
\hline nLPInj-op & 0.0 & 0.0 & 1.0 & \multicolumn{2}{|c|}{$\$(4)$ : Low pressure makeup is unavailable due to operator error. } \\
\hline rLPInj \& EAC-CD & $C D+R P V-0$ & + RPV-LOCA)\# & \multicolumn{3}{|c|}{ \$ (5): LPInj recoverable early, ac power avail. \& not unisolated ILOCA } \\
\hline & 1.0 & 0.0 & 0.0 & \multirow{2}{*}{\multicolumn{2}{|c|}{$\begin{array}{l}\text { \$Recvry of LPInj w/ an unisol. ILOCA would drain SP \& fld Aux Bldg } \\
\text { erable late, ac power avail. \& not unisolated ILOCA }\end{array}$}} \\
\hline rLPInj \& LAC-CD & $-C D+R P V-0$ & + RPV-LOCA) \# & \$ (6): LPInj recoverable late, ac power avail. \& not unisolated ILOCA & & \\
\hline & 0.0 & 1.0 & 0.0 & \$ Recvr & w/ an unisol. ILOCA would drain SP \& fld Aux Bldg \\
\hline Otherwise & 0.0 & 0.0 & 1.0 & \multicolumn{2}{|c|}{$\$(7)$ : Either no injection, no ac power, or RPV not isolated. } \\
\hline \multicolumn{6}{|c|}{ at is the peak hydrogen concentration in the containment during $\mathrm{CD}$ ? } \\
\hline & $\mathrm{H} 2<4$ & $\mathrm{H} 2<8$ & $\mathrm{H} 2<12$ & $\mathrm{H} 2<16$ & $\mathrm{H} 2>16$ \\
\hline E-CorCool & 1.000 & 0.000 & 0.000 & 0.000 & $0.000 \$(1)$ : Injection before significant $\mathrm{H} 2$ production \\
\hline \multicolumn{6}{|c|}{ RPV-HiP-CD \& nOCnt-BCD \& /RPV-oMSIV \& /RPV-ILOCA \# } \\
\hline nOCNT-BCD \& $/ R$ & $\begin{array}{l}0.014 \\
\text { /RPV-ILOC }\end{array}$ & 0.095 & 0.127 & 0.207 & 0.557 \$ (2): RPV at HiP and CNMT Closed, \\
\hline & 0.010 & 0.060 & 0.140 & 0.205 & $0.585 \$(3):$ RPV at LoP and CNMT Closed, \\
\hline RPV-HiP & 0.066 & 0.213 & 0.303 & 0.243 & 0.175 \$ (4): RPV at HiP, CNMT Open to Aux. Bldg \\
\hline Otherwise & 0.040 & 0.205 & 0.325 & 0.250 & 0.180 \$ (5): RPV at LoP, CNMT Open to Aux. Bldg \\
\hline
\end{tabular}

What is the fraction of zirconium that is oxidized in the vessel during core damage?

$$
\text { ZrOxid<21 ZrOxid }>21
$$

$\mathrm{H} 2>16+(\mathrm{OCnt}-\mathrm{BCD}+\mathrm{RPV}-\mathrm{oMSIV}+\mathrm{RPV}-\mathrm{ILOCA}) \& / \mathrm{H} 2<4 \& / \mathrm{H} 2<8 \# \$(1)$ CNMT closed and H2 concentration greater than $16 \%$ or CNMT open

$\begin{array}{llll} & 0.00 & 1.00 & \$ \text { and H2 concentration is greater than } 8 \% \\ \text { Otherwise } & 1.00 & 0.00 & \$(2) \text { CNMT closed and } \mathrm{H} 2<16 \% \text { or CNMT open and } \mathrm{H} 2<8 \%\end{array}$

Do the operators turn on the HIS during $\mathrm{CD}$ ?

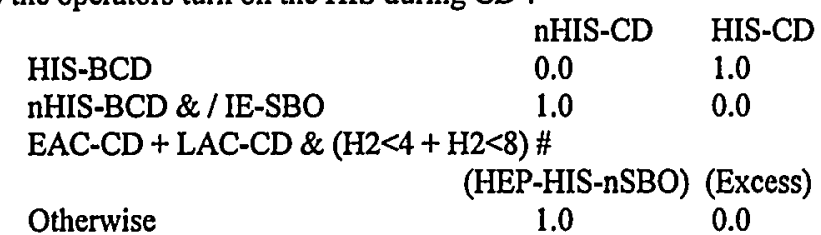

Does an uncontrolled hydrogen combustion event occur during $\mathrm{CD}$ ?
HIS-CD \& /RPV-ILOCA \& /RPV-oMSIV $\mathrm{H} 2<4$
$\$$ This question is primarially concerned with whether the operators turn HIS on following $\$$ the recovery of ac power but before the $\mathrm{H}_{2}$ exceeds the safe zone.

$\$(1)$ : HIS prior to core damage, thus, still on

$\$(2)$ : Not a SBO, operators fail to turn on HIS, thus, still off.

$\$(3)$ : $\mathrm{SBO}$, ac recovered early during $\mathrm{CD}$ or ac recovered late but

$\$ \mathrm{H}_{2}$ concentration is less than $8 \%$.

$\$(4)$ : Either ac power recovered late during $C D$ or wasn't recovered.

$\begin{array}{ll}\text { Brn-H2 } & \text { Brn-Dif } \\ 0.0 & 1.0\end{array}$

$\begin{array}{ll}0.0 & 1.0 \\ 0.0 & 0.0\end{array}$

$\$(1)$ : Igniter are on with release of $\mathrm{H} 2$ to CNMT $\$(2)$ : The $H 2$ concentration is $<4 \%$, will not burn 


\author{
$/ \mathrm{IE}-\mathrm{SBO}+\mathrm{IE}-\mathrm{SBO} \& \mathrm{EAC}-\mathrm{CD} \quad 0.0$ \\ $\mathrm{IE}-\mathrm{SBO} \& / \mathrm{nAC}-\mathrm{CD}$ \\ IE-SBO \\ Otherwise
}

Data: Brn-nSBO 0.75 UNIFORM 0.51 .0

Data: Brn-SBO 0.5 UNIFORM 0.0 0.75 \$as a deflagration
$\$(3)$ : ACpower available (plenty of ignition sources)

$\begin{array}{ll}\text { (Excess) } & (\mathrm{Brn}-\mathrm{nSBO}) \\ 0.0 & 1.0 \\ & \\ \text { (Excess) } & (\mathrm{Brn}-\mathrm{SBO}) \\ 1.0 & 0.0\end{array}$

0.0

$\$(4)$ : SBO with late recovery of $A C$, ignition assured,

$\$$ however, concentration not known, assume high

$\$(5)$ : $\mathrm{SBO}$, ac not recovered, few ignition sources

0.0

$\$$ This case shouldn't be used.

\$ Based on NUREG/CR-4551, Vol 6 (Appendix A)

\$ Min \& Max values correspond to ignition freq. in NURE/CR-4551, Vol. 6, (Appendix A)

What is the pressure in the containment during $\mathrm{CD}$ (no uncontrolled burn)? \$ This question addresses the pressure in the containment during

$\begin{array}{llll} & \text { P-Lo } & \text { P-Vnt } & \text { \$ core damage. } \\ \text { OCnt-BCD } & 1.0 & 0.0 & \text { \$(1): Containment is open, thus, pressure is low } \\ \text { Op-RPV-CD \& / RPV-LOCA } & 1.0 & 0.0 & \text { \$ (2): RPV is open to containment or turbine building } \\ \text { Otherwise } & 1.0 & 0.0 & \text { \$(3): Cnt closed, MELCOR calc. indicate P<20 psig. }\end{array}$

Does the containment fail from quasi-static loads during core damage?

$\begin{array}{lll}\text { OCnt-BCD + Brn-Dif + nBrn-H2 } & \text { nCF-CD } \\ \text { + RPV-oMSIV } & & \\ & \mathrm{H} 2<4+\mathrm{H} 2<8 & 1.0 \\ \mathrm{H} 2<12 & 1.0 & 0.00 \\ \mathrm{H} \quad & & 0.79 \\ \mathrm{H} 2<16 & 0.13 \\ \mathrm{H} 2>16 & 0.04 \\ \text { Otherwise } & & 1.0\end{array}$

\section{CF-Rpt-CD CF-Lk-CD}

0.00

0.00

0.19

0.49

0.50

0.00

0.00

\$ HiS, or no deflag.

\$(1) Either Cnt open or Open MSIV, H2 burned with $\$(2)$ Peak $\mathrm{H} 2$ concen. is $<8 \%$
0.02
0.38
0.46
\$ (3) Peak H2 concen. between 8 \& $12 \%$
$\$$ (4) Peak H2 concen. between $12 \& 16 \%$
0.46
0.00
$\$(5)$ Peak $\mathrm{H} 2$ concen. is $>16 \%$
$\$$ (6) This case shouldn't be used.

Do the operators vent the containment during core damage?

\begin{tabular}{|c|c|}
\hline \multicolumn{2}{|r|}{ nVnt-CD } \\
\hline nCVS + P-Lo & 0.0 \\
\hline RPV-HiP \& nOCnt-BCD \& nCS-CD \& RPV & \\
\hline$\&(\mathrm{aCVS}+\mathrm{rCVS} \&(\mathrm{EAC}-\mathrm{CD}+\mathrm{LAC}-\mathrm{CD})$ & (HEP-EVnt) \\
\hline RPV-HiP \& nOCnt-BCD \& nCS-CD \& RPV & \\
\hline$\&(\mathrm{aCVS}+\mathrm{rCVS} \&(\mathrm{EAC}-\mathrm{CD}+\mathrm{LAC}-\mathrm{CD}))$ & \\
\hline $\mathrm{VS}+\mathrm{rCVS} \&(\mathrm{EAC}-\mathrm{CD}+\mathrm{LAC}-\mathrm{CD})$ & (HEP. \\
\hline herwise & 1.0 \\
\hline
\end{tabular}

Vnt-CD

$\$(1)$ : Either CVS not available, or CNMT pressure below vent threshold

$\$(2)$ : Cnt Pres $>20$ psig and CVS availabile and operators previously

(Excess)

$\$$ followed CPC EP (i.e., depressurized the RPV)

0.00

$\$(3)$ : Cnt Pres $>20$ psig and CVS availabile and operators previousiy

$\begin{array}{ll}\text { (Excess) } & \$(4): \text { Cnt Pres }>20 \text { psig and CVS is available, EP requ } \\ 0.0 & \$(5): \text { Cnt Pres }>20 \text { psig, however CVS not available. }\end{array}$

$\$$ failed to followed CPC EP (i.e., depressurized the RPV)

Data: HEP-EVnt 0.031 LOGNORMAL 0.0315

What is the status of the containment during core damage?

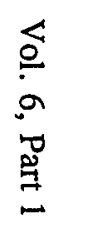

OCnt-CD

$\$(1)$ : CNMT open before $C D$, vented or fails during $C D$

$0.0 \quad \$(2):$ CNMT intact. 
What is the size of the containment opening during core damage?

$\begin{array}{lllll} & \text { Cnt-Rpt-CD } & \text { Cnt-Lk-CD } & \text { Cnt-NF-CD } & \\ \text { nOCnt-CD } & 0.0 & 0.0 & 1.0 & \text { \$ CNMT intact. } \\ \text { OCnt-BCD + CF-Rpt-CD + Vnt-CD } & 1.0 & 0.0 & 0.0 & \text { \$ CNMT hatches open, vented or failed by ruptured } \\ \text { CF-Lk-CD } & 0.0 & 1.0 & 0.0 & \text { \$ CNMT failure mode is a leak } \\ \text { Otherwise } & 0.0 & 0.0 & 1.0 & \text { \$ This case shouldn't be used. }\end{array}$

Does the auxiliary building fail during core damage?

$\begin{array}{llll} & \text { nOAux-CD } & \text { OAux-CD } & \\ \text { OAux-BCD } & 0.0 & 1.0 & \$(1): \text { Aux. Bldg open BCD } \\ \text { (RPV-ILOCA + RPV-oMSIV ) \& Op-RPV-CD } & 0.0 & 1.0 & \$(2): \text { RPV open to aux. bldg during CD } \\ \text { nOCnt-BCD } & 1.0 & 0.0 & \text { \$(3): Cnt closed BCD; any Cnt failure will be above Aux. Bldg } \\ \text { Brn-H2 } & 0.0 & 1.0 & \text { \$(4): } \mathrm{H}_{2} \text { in the Cnt or Aux Bldg without HIS, possible severe burn } \\ \text { Brn-Dif } & 0.01 & 0.99 & \text { \$(5): } \mathrm{H}_{2} \text { burns via the HIS, thus, less severe burn } \\ \text { RPV-LOCA } & 1.0 & 0.0 & \text { \$(6): LOCA with Open Cnt, MELCOR shows no Early Aux. Bldg failure } \\ \text { RPV-OVnt + Op-SRV-Bkr } & 0.0 & 1.0 & \text { \$(7): RPV head vnt or SRV vacuum breaker open (Based on MELCOR) } \\ \text { Otherwise } & 1.0 & 0.0 & \text { \$(8): Intact RPV, steam condensed in suppression pool }\end{array}$

$\$=$ Events Accompanying Vessel Breach

菌

Is there water in the RPV pedestal cavity just prior to VB? \$ This question addressed the amount of water in the pedestal cavity below the RPV

Cav-FId-BCD 0.0
Otherwise

$$
\text { Otherwise }
$$

1.0

1.0

Is the core damage process arrested in the vessel?

(RPV-ILOCA + RPV-LOCA)
$\&$ Op-RPV-CD
E-CorCool
L-CorCool
Otherwise

Cav-Fld

$\$(1)$ : Cavity flooded before $\mathrm{CD}$, thus, still flooded

0.0

$\$(2)$ : No other souces of water to cavity, thus, dry
1.0

0.01

(Excess)

1.0

\section{nCDArrest CDArrest}

0.0

0.99

(CDArst-L)

0.0
$\$$ This question addresses the coolability of the core debris in the vessel
\$ (1): I-LOCA not isolated, thus, insufficient core coverage

$\$(2)$ : Coolant restored early in the accident $\Rightarrow$ likely that debris is coolable

$\$(3)$ : Coolant restored late in the accident $\Rightarrow$ likely debris is not coolable

$\$(4)$ : Coolant is not restored $\Rightarrow$ debris is not cooled.

Data: CDArst-L 0.01 MAXIMUM ENTROPY 0.000 .010 .5

What fraction of the core debris would be mobil at VB?

$\begin{array}{ll}\text { HiLiqVB } & \text { LoLiqVB } \\ 0.025 & 0.975\end{array}$

$0.100 \quad 0.900$

Does a large in-vessel steam explosion occur?

E-CorCool
nVStmExp

1.0

\section{VStmExp}

0.0

(2)

\$Quantification is based on NUREG-1150.

$\$(1)$ : Coolant restored during core damage

$\$(2)$ : Coolant either not restored or restored late in the accident

$\$$ This question addresses the likelihood that a large steam explosion occurs in the vessel $\$$ Quantification: Mean value is based on NUREG-1150.

$\$(1)$ : Coolant restored early $\Rightarrow$ not enough core debris to result in a large steam explosion 
Data: StmExp-LoP 0.86 MAXIMUM ENTROPY 0.0010 .861 .0

Data: StmExp-HiP 0.10 MAXIMUM ENTROPY 0.001 0.10 1.0

Does an Alpha mode event occur?

VStmExp \& RPV-LoP
VStmExp

VStmExp

Data: A-LoP 0.01 = Alpha-Dist $/$ StmExp-LoP

Data: A-HiP $0.001=$ Alpha-Dist $/ 10.0 /$ StmExp-H

Data: Correlate Alpha-Dist StmExp-LoP 0.999

nAlpha
(Excess)
(Excess)
1.0
StmExp-LoP
10.0 / StmExp-

xp-LoP 0.999
xp-HiP 0.999

Does a large in-vessel steam explosion fail the vessel?

\begin{tabular}{lllllll}
\multicolumn{1}{c}{} & SE-Alpha & SE-BtHd & SE-LgBrch & SE-SmBrch & SE-nFail \\
$\square$ & Alpha & 1.0 & 0.0 & 0.0 & 0.0 & 0.0 \\
$\vdots$ & VStmExp & 0.0 & 0.20 & 0.10 & 0.10 & 0.60 \\
$\vdots$ & Otherwise & 0.0 & 0.0 & 0.0 & 0.0 & 1.0
\end{tabular}

\$ This question addresses the likelihood that a large stm expls. fails the RPV and Cnt. Alpha \$Quantification is based on NUREG-1150.

(A-LoP) $\quad \$(1)$ : Steam explosion occurs when the vessel is at low pressure

(A-HiP) $\quad \$(2)$ : Steam explosion occurs when the vessel is at system pressure

$0.0 \$(3)$ : There is not in-vessel steam explosion.

ditional on a Stm Expl, the Alpha distrib. is divided by the Stm

$\$$ Stm Expl distrib. Note: Prob. of Alpha mode at high $P$ is 0.1 the prob. at low $P$.

What is the mode of VB?

$\begin{array}{lll} & \text { VB-Alpha } & \text { VB-BtHd } \\ \text { SE-Alpha } & 1.0 & 0.0 \\ \text { SE-BtHd } & 0.0 & 1.0 \\ \text { SE-LgBrch } & 0.0 & 0.0 \\ \text { SE-SmBrch } & 0.0 & 0.0 \\ \text { CDArrest } & 0.0 & 0.0 \\ \text { HiLiqVB + LoLiqVB } & 0.0 & 0.249 \\ \text { Otherwise } & 0.0 & 0.0\end{array}$

$\begin{array}{lll}\text { VB-LgBrch } & \text { VB-SmBrch } & \mathrm{nVB} \\ 0.0 & 0.0 & 0.0 \\ 0.0 & 0.0 & 0.0 \\ 1.0 & 0.0 & 0.0 \\ 0.0 & 1.0 & 0.0 \\ 0.0 & 0.0 & 1.0 \\ 0.005 & 0.746 & 0.0 \\ 0.0 & 0.0 & 1.0\end{array}$
\$ Quant. based on adjusted NUREG-1150 values $\$$ (1): Alpha mode occurs
$\$$ (2): In-vessel Stm.Exp. occurs: Used M.Berman values
$\$(3)$ : No Stm. Exp.

Does high pressure melt ejection occur?

$\begin{array}{lll} & \text { nHPME } & \text { HPME } \\ \text { nVB + RPV-LoP-CD } & 1.0 & 0.0 \\ \text { Otherwise } & 0.20 & 0.80\end{array}$

\$ This event is only used in the source term analysis

$\$(1)$ : Either no VB, or RPV depressurized

$\$(2)$ : RPV fails at high pressure

\author{
$\$(1)$ : Alpha mode event \\ $\$$ (2) StmExp causes bottom head failure \\ $\$(3)$ StmExp causes large breach \\ $\$$ (4) StmExp causes small breach \\ $\$(5)$ Core damae arrested invessel \\ $\$(6)$ No StmExp, No core cooling \\ $\$$ This case shouldn't be used
}

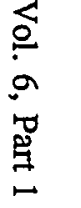

Does a large ex-vessel steam explosion accompany VB?

$\begin{array}{ll} & \mathrm{nExStmE} \\ \text { nVB + Alpha } 1.0 & 0.0 \\ \text { Cav-Fld + E-CorCool + L-CorCool \# }\end{array}$

ExStmE

$\$(1)$ : Either no vessel failure or alpha mode event, thus, no ex-vessel steam explosion

$\$(2)$ : Vessel fails into a flooded cavity or water enters cavity coincident with debris 

Otherwise
(Excess)
(ExStmE)
$\$$

Does the containment fail from pressure loads accompanying VB?

\begin{tabular}{|c|c|c|c|c|c|}
\hline & & nCF-VB & CF-Rpt-VB & CF-Lk-VB & \\
\hline $\mathrm{nVB}$ & & 1.0 & 0.0 & 0.00 & $\$(1)$ : No VB, thus, no loads to cause CNMT failure \\
\hline Cnt-Rpt-CD + Alpha & & 1.0 & 0.0 & 0.00 & $\$(2)$ : CNMT already fail or is open \\
\hline RPV-HiP-CD \& HiLiqVB & & 0.22 & 0.35 & 0.43 & \$ (3) VB @ HiP with large amount of core debris ejected \\
\hline RPV-HiP-CD & 0.41 & 0.27 & 0.32 & \$(4) VB@ & ith small amount of core debris ejected \\
\hline Cav-Fld \& ZrOxid $>21$ & & 0.90 & 0.04 & 0.06 & $\begin{array}{l}\$(5) \text { : VB @ LoP with flooded cavity and a large fraction } \\
\$ \text { of Zr oxidized }\end{array}$ \\
\hline Cav-Fld & & 0.82 & 0.10 & 0.08 & $\$(6)$ Same as above except small fract of inves $\mathrm{Zr}$ oxid. \\
\hline HIS-CD & & 0.99 & 0.005 & 0.005 & $\$(7)$ : Slow release of $\mathrm{H} 2$ with igniters on. \\
\hline $\mathrm{nBm}-\mathrm{H} 2 \& \mathrm{H} 2<12$ & & 0.79 & 0.190 & 0.02 & $\$(8): 8$ to $12 \% \mathrm{H} 2$ accumuled $\mathrm{BCD}$; no burn $\mathrm{BCD}$ \\
\hline $\mathrm{nBm}-\mathrm{H} 2 \& \mathrm{H} 2<16$ & & 0.13 & 0.49 & 0.38 & $\$(9): 12$ to $16 \% \mathrm{H} 2$ accumuled $\mathrm{BCD}$; no burn $\mathrm{BCD}$ \\
\hline $\mathrm{nBm}-\mathrm{H} 2 \&$ H2 $>16$ & & 0.04 & 0.50 & 0.46 & $\$(10):>16 \% \mathrm{H} 2$ accumuled $B C D ;$ no burn $B C D$ \\
\hline Otherwise & & 1.0 & 0.0 & 0.0 & $\$(11):<8 \% \mathrm{H} 2$ accumuled $\mathrm{BCD} ;$ no burn $\mathrm{BCD}$ \\
\hline
\end{tabular}

ఝ্

What is the status of containment integrity just after VB?

\section{nOCnt-VB}

1.0

1.0

Otherwise

0.0

What is the size of the containment opening just after VB?

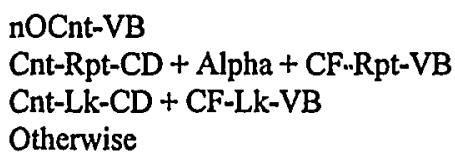

Cnt-Rpt-VB
0.00
1.00
0.00
0.00

\section{$\$$ Mean based on value used in NUREG-1150}

\$ Note: This does not include ILOCAs

OCnt-VB

\$ (1): CNMT hatches open or fails during CD or at VB

0.0

$\$(2)$ : CNMT is intact

\$ Note: This does not include ILOCAs

Cnt-Lk-VB Cnt-NF-VB

$\begin{array}{lll}0.00 & 1.00 & \$(1): \text { The CNMT is intact } \\ 0.00 & 0.00 & \text { \$(2): CNMT rupture during CD or VB or Alpha failure } \\ 1.00 & 0.00 & \text { \$(3): CNMT leak @ CD (and no rupt @ VB) or leak at VB } \\ 0.00 & 1.00 & \text { \$(4): No CNMT failure }\end{array}$

Does the auxiliary building fail just after VB?

$\begin{array}{llll} & \text { nOAux-VB } & \text { OAux-VB } & \\ \text { OAux-CD } & 0.0 & 1.0 & \text { \$(1): Aux BIdg has already failed } \\ \text { nOCnt-BCD \& /RPV-ILOCA \& /RPV-oMSIV } & 1.0 & 0.0 & \text { \$ (2):CNMT was closed and no ILOCA or no open MSIV } \\ \text { Otherwise } & 0.0 & 1.0 & \$(3) \text { : MELCOR calc. show Aux. Bldg will fail shortly after VB }\end{array}$

党

Event Associated with Ex-Vessel Phase of the Accident

What is the status of dc power late in the accident?

$\begin{array}{llll} & \text { nDC-Late } & \text { DC-Late } & \\ \text { InAC-CD } & 0.00 & 1.00 & \$(1): \text { ac power avail, thus, de available }\end{array}$




$\begin{array}{llll}\text { nDC-CD } & 1.00 & 0.00 & \$(2) \text { : dc power not available before CD, thus, not avail. late } \\ \text { IE-Win1 } & 0.18 & 0.82 & \$(3): \text { no ac or dc during CD for TW-1 } \\ \text { IE-Win2 } & 0.16 & 0.84 & \$(4) \text { : no ac or dc during CD for TW-2 } \\ \text { Otherwise } & 1.00 & 0.00 & \$(5) \text { : This case shouldn't be used }\end{array}$

Is ac power recovery late in the accident?

$\begin{array}{lll} & \text { nAC-Late } & \text { OSP-Late } \\ \text { InAC-CD } & 0.0 & 0.0 \\ \text { nDC-Late } & 1.0 & 0.0 \\ \text { IE-Win1 } & \text { (Excess) } & 0.0 \\ \text { IE-Win2 } & \text { (Excess) } & 0.0 \\ \text { Otherwise } & 0.0 & 0.0\end{array}$

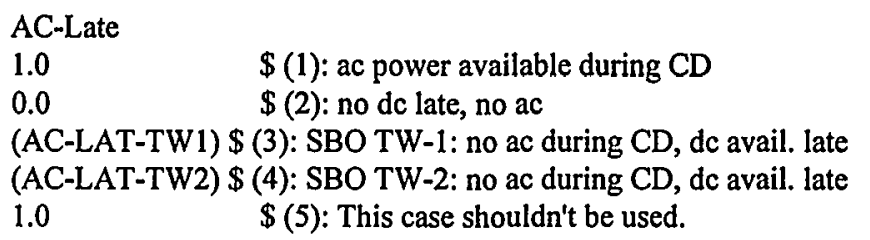

Data: AC-LAT-TW1 0.5 File C:ILPSIPOS5ICALCILHS200\APETACRECBE.LSP Data: AC-LAT-TW2 0.5 File C:LPSIPOS5ICALCLLHS200LAPETACRECBE.LSP

Is the core debris in the cavity coolable?

nVB

Cav-Dry \& n-CorCool \& /aLPInj 0.00

$\mathrm{nCCl}$

1.00

0.00

0.80

0.16

0.80

0.16

0.40

Cav-Fid \&

Cav-Fld

Otherwise

1.00

FldCCI
0.00
1.00
0.20
0.84
0.20
0.84
0.60
0.00

DryCCI
0.00

$\$(2)$ : LP inject. will not start at VB

$0.00 \$$ (3): Cav. Dry and vessel fails at high pressure

$0.00 \$(4)$ : Cav. Dry and vessel fails at low pressure

$0.00 \$(5)$ : Cav. Flooded and vessel fails at high pressure

$0.00 \$ \$(6)$ : Cav. Fld, vessel fails at LP with lots of debris

$0.00 \$(7)$ : Cav. Fld, vessel fails at LP with little debris

$0.00 \quad \$$ This case shouldn't be used.

Do the operators vent the containment after VB?

\section{nVnt-Late Vnt-Late}

nCVS + Cnt-Rpt-VB + Op-RPV-CD \& \#

(RPV-ILOCA + RPV-oMSIV) 1.0

aCVS + rCVS \& AC-Late

Otherwise

\section{0}

(HEP-EVnt)

1.0

\section{$\$$ is open to the Cnt or tubine bulding}

(Excess) $\quad \$(2)$ : Cnt Pres $>20$ psig and CVS is available, EP requires venting

$0.0 \$(3)$ : Cnt Pres $>20$ psig, however CVS not available.

Does the containment fail late in the accident?

$\begin{array}{lll}\text { nCF-Late } & \text { CF-Rpt-Late } & \text { CF-Lk-Late } \\ 1.0 & 0.0 & 0.00 \\ 0.0 & 0.5 & 0.50\end{array}$

$\$(1)$ : Containment already breached.

$\$(2)$ : CNMT fails from late overpressuriation by steam $\$(3)$ : Mean of failure distribution

OCnt-Late

$0.0 \quad 1.0 \quad \$(1)$ : CNMT already open or failed

1.0

$\$(2)$ : CNMT intact 
What is the size of the containment opening late in the accident?

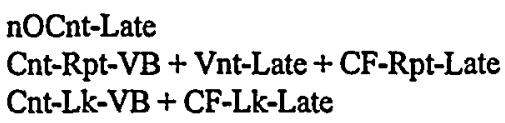

Otherwise

Cnt-Rpt-Late
0.0
1.0
0.0
0.0

Cnt-Lk-J.ate

\section{0}

0.0

1.0

0.0
Cnt-NF-Late

1.0

0.0

0.0

1.0
$\$(1)$ : CNMT intact

$\$$ (2): CNMT rupture or open

$\$(3)$ : CNMT fail as a leak (no ruptures)

$\$$ This case shouldn't be used.

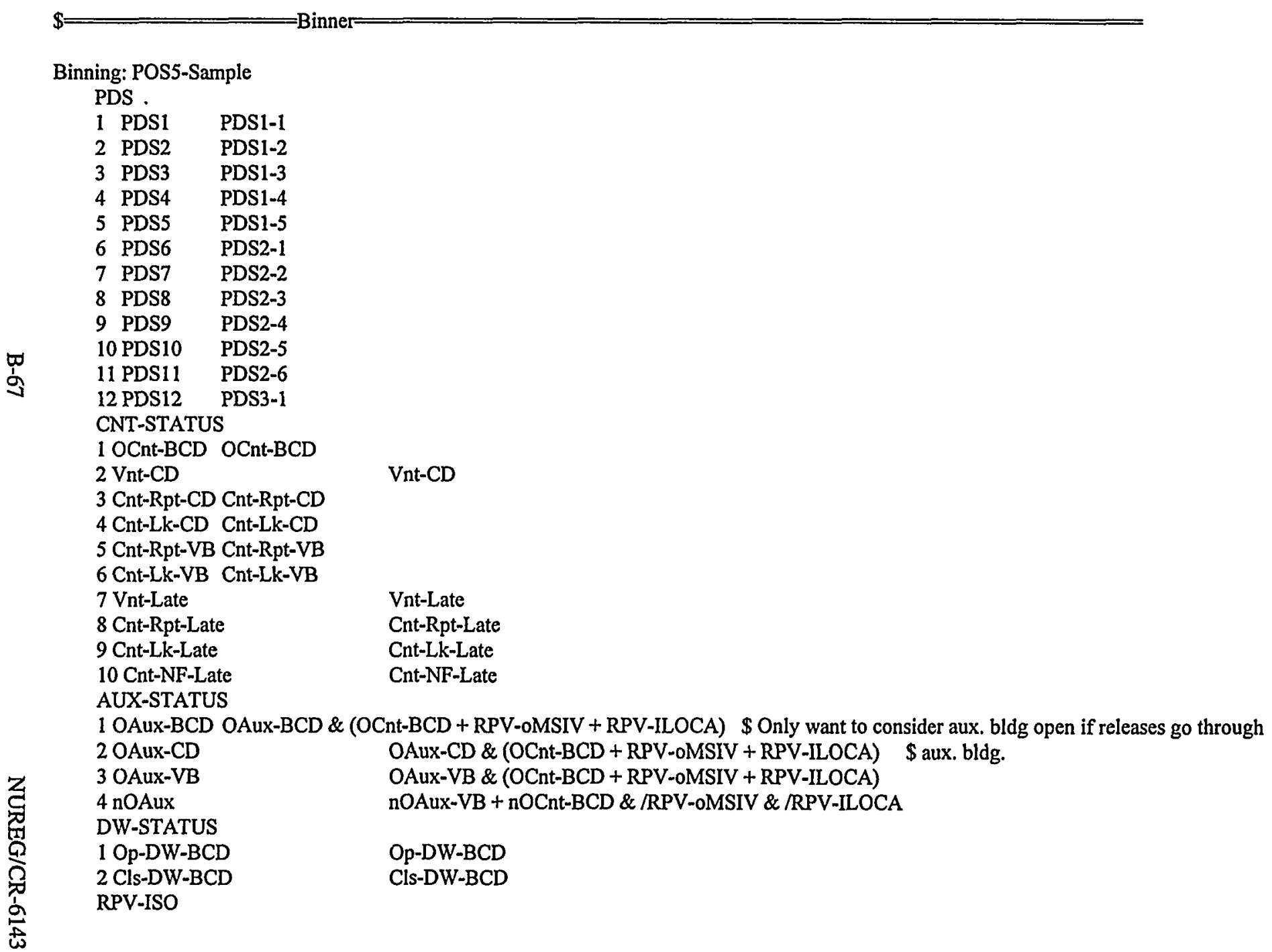




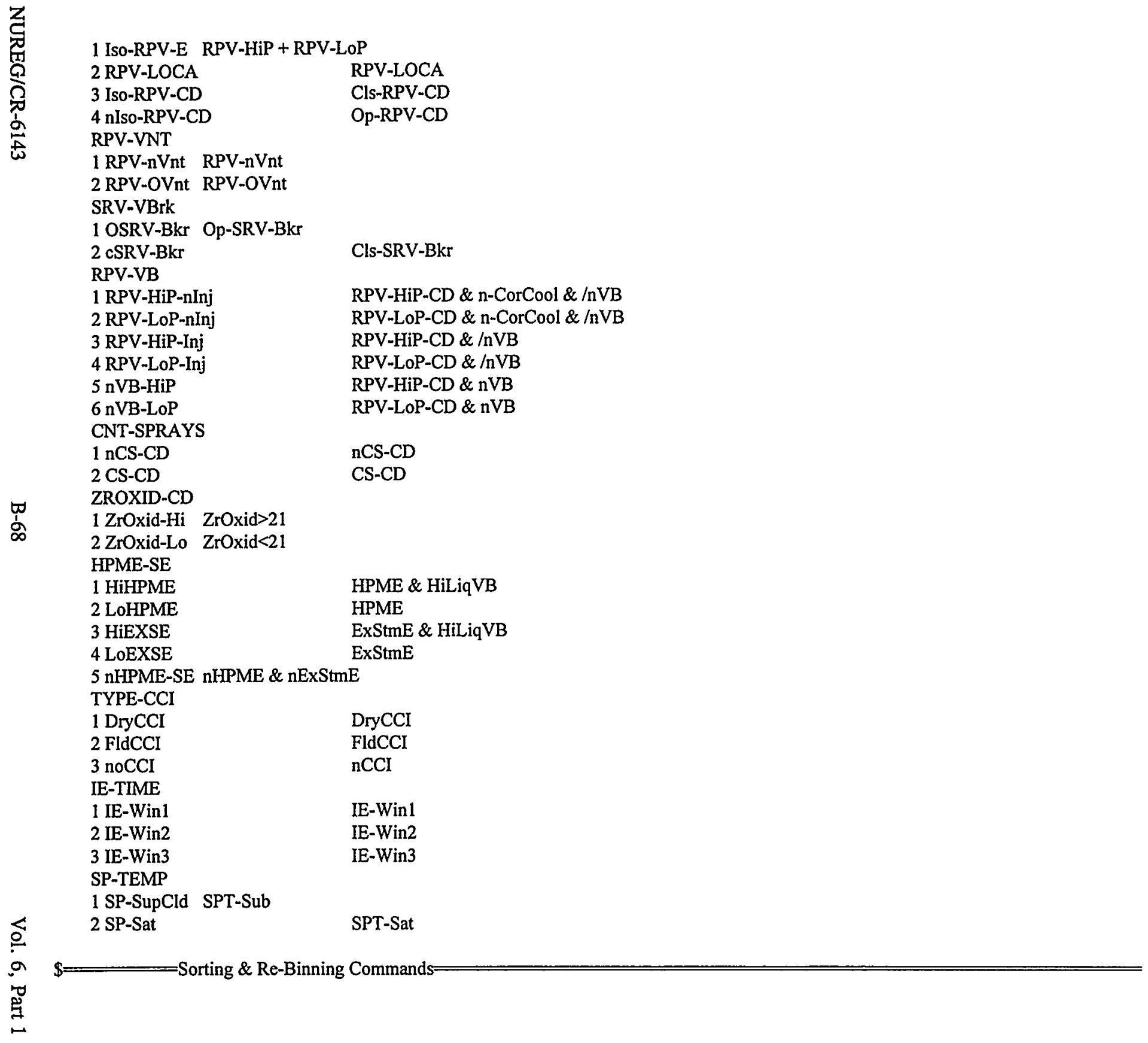


Sorting:

CNT-STATUS

\pm Rebinning:

$\$=$ Distributions=

Dataset:

RAN-REC-LOSP 250 UNIFORM 1.0500 .999

SFP1-1 0.092 File C:LLPSIPOS5ICALCLLHS200LAPETGGP5PDS.LHS

SFP1-2 0.042 File C:ILPSIPOS5ICALCLLHS200\APETIGGPSPDS.LHS SFP1-3 0.158 File C:LLPSIPOS5ICALCLLHS200LAPET GGPSPDS.LHS SFP1-4 0.065 File C:LLPSTPOS5|CALCLLHS200\APETIGGP5PDS.LHS SFP1-5 0.012 File C:LLPSIPOS5TCALCLLHS200LAPETGGP5PDS.LHS SFP2-1 0.014 File C:LLPSTPOS5/CALCLLHS200LAPETGGP5PDS.LHS SFP2-2 0.214 File C:LLPSIPOS5ICALCLLHS200\APETIGGP5PDS.LHS SFP2-3 0.020 File C:LLPSIPOS5ICALCLLHS200\APET TGP5PDS.LHS SFP2-4 0.043 File C:LLPSTPOS5ICALCLLHS200LAPETGGP5PDS.LHS SFP2-5 0.091 File C:LLPSIPOS5ICALCLLHS200\APETIGGP5PDS.LHS SFP2-6 0.021 File C:LPSSPOS5ICALCLLHS200\APETIGGP5PDS.LHS

to SFP3-1 0.102 File C:LLPSIPOS5ICALCLLHS200\APETGGP5PDS.LHS

Alpha-Dist 0.001 CONTINUOUS LOGARITHMIC 14 \#

$\begin{array}{ll}9.99 \mathrm{E}-8 & 0.0000 \# \\ 1.00 \mathrm{E}-7 & 0.1818 \# \\ 1.00 \mathrm{E}-6 & 0.2696 \# \\ 1.01 \mathrm{E}-5 & 0.3552 \# \\ 1.00 \mathrm{E}-4 & 0.5611 \# \\ 1.00 \mathrm{E}-3 & 0.7162 \# \\ 5.03 \mathrm{E}-3 & 0.8200 \# \\ 1.00 \mathrm{E}-2 & 0.911 \# \\ 3.71 \mathrm{E}-2 & 0.9670 \# \\ 5.42 \mathrm{E}-2 & 0.9760 \# \\ 1.01 \mathrm{E}-1 & 0.9844 \# \\ 2.81 \mathrm{E}-1 & 0.9920 \# \\ 5.70 \mathrm{E}-1 & 0.9960 \# \\ 9.97 \mathrm{E}-1 & 1.0000\end{array}$

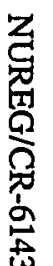

STVAR2 0.5 UNIFORM 0.010

STVAR3 0.5 UNIFORM 0.01 .0

STVAR4 0.5 UNIFORM 0.010

STVAR5 0.5 UNIFORM 0.01 .0

STVAR6 0.5 UNIFORM 0.01 .0

STVAR7 0.5 UNIFORM 0.01 .0 
Appendix B

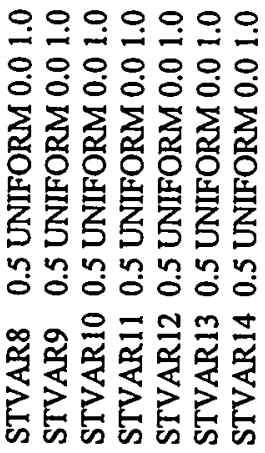




\section{B.3 Quantification of the POS 5 APET}

This section of the appendix provides supporting information for the quantification of Accident Progression Analysis. Appendix subsection B.3.1 lists the sources of information that were used to quantify the APET. Appendix subsection B.3.2 provides the rationale that was used to determine the human error probabilities that were used in the Human Reliability Analysis.

\section{B.3.1 Sources of Information Used to Quantify the APET}

Table B.3.1-1 lists each question in the APET, indicates whether or not it was included in the uncertainty analysis (i.e., sampled), and lists the primary sources of information that were used to quantify the question. Also, if the question was included in the uncertainty analysis, Table B.3.1-1 identifies the distribution that was used to characterize the uncertainty and the variable name. A more detailed discussion of the information sources used to quantify each question is provided in the discussion of each question in section B.1. 
Table B.3.1-1

APET Quantification

\begin{tabular}{|c|c|c|c|c|}
\hline APET Question & Sampled & Distribution Type & Variable Name & Quantification Source \\
\hline What is the Plant Damage State? & Yes & Computed from IRRAS & SFP1-1...SFP3-1 & Based on IRRAS Output \\
\hline What is the status of electric power at core damage (CD) (PDS Char. 1)? & No & None & & PDS Description \\
\hline What is the status of dc power at $\mathrm{CD}$ (PDS Char. 1)? & No & None & & PDS Description \\
\hline What is the status of high pressure injection at $\mathrm{CD}$ (PDS Char. 2)? & No & None & & PDS Description \\
\hline What is the status of low pressure injection at $\mathrm{CD}$ (PDS Char. 2)? & No & None & & PDS Description \\
\hline What is the status of containment sprays and SPC at CD (PDS Char. 3)? & No & None & & PDS Description \\
\hline What is the suppression pool level at the onset of $\mathrm{CD}$ (PDS Char. 4)? & No & None & & PDS Description \\
\hline What is the suppression pool temperature at the onset of CD (PDS Char. 5)? & No & None & & PDS Description \\
\hline What is the status of the reactor head vent at the onset of $\mathrm{CD}$ (PDS Char. 6)? & No & None & & PDS Description \\
\hline What is the status of the RPV integrity at the onset of CD (PDS Char. 7)? & No & None & & PDS Description \\
\hline What is the status of the CNMT access penetrations at CD (PDS Char. 8)? & No & None & & PDS Description \\
\hline What is the status of the CNMT vent system at the onset of CD (PDS Char. 9)? & No & None & & PDS Description \\
\hline When does CD occur (PDS Char. 10)? & No & None & & PDS Description \\
\hline While in POS 5 , when does the initiating event occur (PDS Char. 11)? & No & None & & PDS Description \\
\hline What type of event initiates the accident? & No & None & & Summary \\
\hline What is the pressure in the RPV at the time of CD? & No & None & & Summary \\
\hline How much water is in the reactor pedestal cavity at the time of $\mathrm{CD}$ ? & No & None & & Summary \\
\hline Is the containment equipment hatch opened at the start of the accident? & No & None & & Initial Conditions \\
\hline Do the operators close the containment before $\mathrm{CD}$ ? & Yes & $\begin{array}{l}\text { BOUNDED LOGNORMAL } \\
\text { Mean }=0.102, E F=5\end{array}$ & HEP5-CNT & $\begin{array}{l}\text { HRA Analysis } \\
\text { (Appendix B.3) }\end{array}$ \\
\hline Does the auxiliary building fail before $\mathrm{CD}$ ? & No & None & & $\begin{array}{l}\text { MELCOR Calculations } \\
\text { (Vol. 6, Part } 2 \text { of this report) }\end{array}$ \\
\hline What is the status of the drywell before $\mathrm{CD}$ ? & No & None & & Initial Conditions \\
\hline
\end{tabular}


Table B.3.1-1 (continued) APET Quantification

\begin{tabular}{|c|c|c|c|c|}
\hline APET Question & Sampled & Distribution Type & Variable Name & Quantification Source \\
\hline Do the operators tum on the HIS before CD? & Yes & $\begin{array}{l}\text { LOGNORMAL: } \\
\mathrm{MN}=0.054 \\
\mathrm{EF}=5\end{array}$ & HEP-HIS-nSBO & $\begin{array}{l}\text { HRA Analysis } \\
\text { (Appendix B.3) }\end{array}$ \\
\hline Do the station batteries depleted during CD? & No & None & & $\begin{array}{l}\text { Station Battery Failure Curve } \\
\text { [Wheeler et al., 1989] }\end{array}$ \\
\hline Is offsite power restored during $\mathrm{CD}$ ? & Yes & LOSP Non-recovery curves & $\begin{array}{l}\text { AC-ECD-TW1 } \\
\text { AC-LCD-TW1 } \\
\text { AC-ECD-TW2 } \\
\text { AC-LCD-TW2 }\end{array}$ & $\begin{array}{l}\text { See Volume } 2 \text { of this report for } \\
\text { Curves. Probabilities calculated using } \\
\text { ACRECBE.FOR }\end{array}$ \\
\hline Is the RPV isolated during $C D$ ? & Yes & $\begin{array}{l}\text { MAXIMUM ENTROPY } \\
\text { Lower End: } 0.5 \\
\text { Mean: } 0.9 \\
\text { Upper End: } 1.0\end{array}$ & ISO-SDC & Project Staff \\
\hline Do the operators initiate containment sprays during $C D$ ? & No & None & & PDS \\
\hline Do the operators depressurize the RPV during CD? & Yes & $\begin{array}{l}\text { BOUNDED LOGNORMAL } \\
\text { Mean }=0.54, E F=5\end{array}$ & HEP-PRPV-HiP & $\begin{array}{l}\text { HRA Analysis } \\
\text { (Appendix B.3) }\end{array}$ \\
\hline What is the status of the SRV vacuum breakers during CD? & Yes & $\begin{array}{l}\text { UNIFORM } \\
\text { Min. }=0.01 \\
\text { Max. }=0.5\end{array}$ & SRV-VBkr & $\begin{array}{l}\text { NUREG-1150 } \\
\text { [Brown et al., 1990] }\end{array}$ \\
\hline Is core cooling restored during $\mathrm{CD}$ ? & No & None & & \\
\hline What is the peak hydrogen concentration in the containment during CD? & No & None & & $\begin{array}{l}\text { NUREG-1150 } \\
\text { [Harper et al., 1991] }\end{array}$ \\
\hline What is the fraction of zirconium that is oxidized in the vessel during CD? & No & None & & Summary \\
\hline Do the operators turn on the HIS during CD? & Yes & $\begin{array}{l}\text { LOGNORMAL } \\
\mathrm{Mn}=0.054, \mathrm{EF}=5\end{array}$ & HEP-HIS-CD & $\begin{array}{l}\text { HRA Analysis } \\
\text { (Appendix B.3) }\end{array}$ \\
\hline Does an uncontrolled hydrogen combustion event occur during $C D$ ? & Yes & $\begin{array}{l}\text { UNIFORM: } \\
\text { Min. }=0.5, \text { Max. }=1.0 \\
\text { UNIFORM: } \\
\text { Min. }=0.0, \text { Max. } 0.75\end{array}$ & $\begin{array}{l}\text { Bm-nSBO } \\
\text { Bm-SBO }\end{array}$ & $\begin{array}{l}\text { Based on NUREG-1 } 150 \\
\text { [Brown et al., 1990] }\end{array}$ \\
\hline
\end{tabular}


Table B.3.1-1 (continued)

APET Quantification

\begin{tabular}{|c|c|c|c|c|}
\hline APET Question & Sampled & Distribution Type & Variable Name & Quantification Source \\
\hline What is the pressure in the containment during $\mathrm{CD}$ (no uncontrolled burn)? & No & None & & Project Staff \\
\hline Does the containment fail from quasi-static loads during $\mathrm{CD}$ ? & No & None & & $\begin{array}{l}\text { Based on NUREG-1 } 150 \\
\text { [Brown et al., 1990] }\end{array}$ \\
\hline Do the operators vent the containment during CD? & Yes & $\begin{array}{l}\text { LOGNORMAL } \\
\mathrm{Mn}=0.031, \mathrm{EF}=5\end{array}$ & HEP-EVnt & $\begin{array}{l}\text { HRA Analysis } \\
\text { (Appendix B.3) }\end{array}$ \\
\hline What is the status of the containment during CD? & No & None & & Summary \\
\hline What is the size of the containment opening during CD? & No & None & & Summary \\
\hline Does the auxiliary building fail during $C D$ ? & No & None & & Project Staff \\
\hline Is there water in the RPV pedestal cavity just prior to VB? & No & None & & Summary \\
\hline Is the core damage process arrested in the vessel? & Yes & $\begin{array}{l}\text { MAXIMUM ENTROPY } \\
\text { Lower Bound }=0.0 \\
\text { Mean }=0.01 \\
\text { Upper Bound }=0.5\end{array}$ & CDArst-L & Project Staff \\
\hline What fraction of the core debris would be mobil at VB? & No & None & & $\begin{array}{l}\text { NUREG-1150 } \\
\text { [Brown et al., 1990] }\end{array}$ \\
\hline Does a large in-vessel steam explosion occur? & Yes & $\begin{array}{c}\text { MAXIMUM ENTROPY } \\
\text { Lower Bound }=0.001 \\
\text { Mean }=0.86 \\
\text { Upper Bound }=1.0 \\
\text { MAXIMUM ENTROPY } \\
\text { Lower Bound }=0.001 \\
\text { Mean }=0.10 \\
\text { Upper Bound }=1.0\end{array}$ & $\begin{array}{l}\text { StmExp-LoP } \\
\text { StmExp-HiP }\end{array}$ & $\begin{array}{l}\text { Project Staff: Mean value based on } \\
\text { NUREG-1150 } \\
\text { [Brown et al., 1990] }\end{array}$ \\
\hline Does an Alpha mode event occur? & Yes & $\begin{array}{l}\text { Aggregated Distribution } \\
\text { Aggregated Distribution }\end{array}$ & $\begin{array}{l}\text { A-LoP } \\
\text { A-HiP }\end{array}$ & $\begin{array}{l}\text { NUREG-1150 } \\
\text { NUREG-1150 }\end{array}$ \\
\hline Does a large in-vessel steam explosion fail the vessel? & No & None & & NUREG-1150 \\
\hline What is the mode of VB? & No & None & & NUREG-1150 \\
\hline Does high pressure melt ejection occur? & No & None & & NUREG-1150 \\
\hline
\end{tabular}

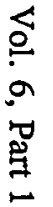




\begin{tabular}{|c|c|c|c|c|}
\hline \multicolumn{5}{|c|}{$\begin{array}{l}\text { Table B.3.1-1 (concluded) } \\
\text { APET Quantification }\end{array}$} \\
\hline APET Question & Sampled & Distribution Type & Variable Name & Quantification Source \\
\hline Does a large ex-vessel steam explosion accompany VB? & Yes & $\begin{array}{l}\text { MAXIMUM ENTROPY } \\
\text { Lower Bound }=0.001 \\
\text { Mean }=0.86 \\
\text { Upper Bound }=1.0\end{array}$ & ExStmE & $\begin{array}{l}\text { Project Staff: Mean values based on } \\
\text { NUREG-1150 } \\
\text { [Brown et al., 1990] }\end{array}$ \\
\hline Does the containment fail from pressure loads accompanying VB? & No & None & & $\begin{array}{l}\text { Based on NUREG-1150 } \\
\text { [Brown et al., 1990] }\end{array}$ \\
\hline What is the status of containment integrity just after VB? & No & None & & Summary \\
\hline What is the size of the containment opening just after VB? & No & None & & Summary \\
\hline Does the auxiliary building fail just after VB? & No & None & & Project Staff \\
\hline What is the status of dc power late in the accident? & No & None & & $\begin{array}{l}\text { Station Battery Failure Curve } \\
\text { [Wheeler et al., 1989] }\end{array}$ \\
\hline Is ac power recovery late in the accident? & Yes & LOSP Non-recovery Curves & $\begin{array}{l}\text { AC-LAT-TW1 } \\
\text { AC-LAT-TW2 }\end{array}$ & $\begin{array}{l}\text { See Volume } 2 \text { of this report for } \\
\text { Curves. Probabilities calculated with } \\
\text { ACRECBE.FOR }\end{array}$ \\
\hline Is the core debris in the cavity coolable? & No & None & & $\begin{array}{l}\text { NUREG-1150 } \\
\text { [Brown et al., 1990] }\end{array}$ \\
\hline Do the operators vent the containment after VB? & Yes & $\begin{array}{l}\text { Same distribution that was } \\
\text { used for venting during core } \\
\text { damage }\end{array}$ & & \\
\hline Does the containment fail late in the accident? & No & None & & Project Staff \\
\hline What is the status of the containment late in the accident? & No & None & & Summary \\
\hline What is the size of the containment opening late in the accident? & No & None & & Summary \\
\hline
\end{tabular}




\section{B.3.2 Level 2 Human Reliability Analysis}

This subsection describes the Human Reliability Analysis (HRA) that was performed for the Level 2 analysis. The approach used in this analysis is the same approach that was used in the Level 1 analysis. The general methodology used for conducting the HRA and determining the Human Error Probabilities (HEPs) for the identified human actions was the Accident Sequence Evaluation Program Human Reliability Analysis Procedure (ASEP HRAP) [Swain, 1987]. The details of the HRA methodology used in this study are described in Chapter 10 of Volume 2 of this report.

The following four HEPs were developed for the Accident Progression Analysis:

HEP1: Closure of the containment before the onset of core damage,

HEP2: Initiation of the Hydrogen Igniter System,

HEP3: Initiation of containment venting, and

HEP4: Depressurization of the reactor vessel during core damage.

A simple listing of each of the Level 2 human actions includes its mean HEP, and its associated error factor is presented in Table B.3.2-1. The calculation and supporting rationale for each of the individual HEPs using the ASEP HRAP procedure is presented in Tables B.3.2-2 through B.3.2-41. According to the ASEP HRAP, the HEPs obtained with the ASEP HRAP procedure are assumed to be median values from a lognormal distribution. The median values were converted to means for use in the analysis using the following formula:

$$
\text { Mean }=\text { Median } * \exp \left\{[\ln (\text { error factor })]^{2} / 5.412\right\} \text {. }
$$

Table B.3.2-1

Summary of HEPs for Level 2 Analysis

\begin{tabular}{llll}
\hline HEP & Distribution & Mean Value & Error Factor \\
\hline HEP1 & Lognormal & 0.102 & 5 \\
HEP2 & Lognormal & 0.054 & 5 \\
HEP3 & Lognormal & 0.031 & 5 \\
HEP4 & Lognormal & 0.054 & 5 \\
\hline
\end{tabular}


Table B.3.2-2

HEP 1 Calculation

\begin{tabular}{||l|l||}
\hline Human Action Event (1) & Containment Closure \\
\hline Event Description (2) & $\begin{array}{l}\text { Containment Closure is the operator action to diagnose the need and accomplish the actions leading to containment } \\
\text { closure. }\end{array}$ \\
\hline Event Context (3) & $\begin{array}{l}\text { An initiating event has occurred and vessel temperature is increasing due to inadequate decay heat removal. If the } \\
\text { operators initiate containment closure early enough, the negative impacts of accident scenarios resulting in core } \\
\text { damage can be lessened. }\end{array}$ \\
\hline Applicable Procedures (4) & $\begin{array}{l}\text { No specific procedures indicate when containment should be closed. However, the GGNS Shutdown Protection Plan } \\
\text { used during refueling outages specifies that containment not be open during Operating Condition 3. If vessel water } \\
\text { temperature exceeds 200 degrees F with the vessel head on, Operating Condition 3 is entered. GGNS does have } \\
\text { procedures describing the process of containment closure. }\end{array}$ \\
\hline
\end{tabular}

Table B.3.2-3

HEP 1: Sequence Timing and Indications

\begin{tabular}{|c|c|c|c|}
\hline $\begin{array}{l}\text { Event/Occurrence } \\
\text { (of most interest) } \\
\text { (1) }\end{array}$ & $\begin{array}{c}\text { Time }\left(\mathrm{T}_{0}\right) \\
\text { Operator } \\
\text { Alerted } \\
(2)\end{array}$ & $\begin{array}{l}\text { Annunciator/Indication } \\
\text { (3) }\end{array}$ & $\begin{array}{l}\text { Comments/ } \\
\text { Source of } \\
\text { Information } \\
\text { (4) }\end{array}$ \\
\hline $\begin{array}{l}\text { Operators need to initiate } \\
\text { containment closure early } \\
\text { in accident scenarios in } \\
\text { order to lessen the impact } \\
\text { of core damage. }\end{array}$ & 0 & $\begin{array}{l}\text { Vessel water temperature has increased to } 200 \text { degrees } \\
\text { F. due to inadequate decay heat removal. With the } \\
\text { vessel head on and vessel temperature at } 200 \text { degrees F, } \\
\text { Operating Condition (OC) } 4 \text { is left and OC } 3 \text { is entered. } \\
\text { In OC } 3 \text {, containment is supposed to be closed per the } \\
\text { GGNS Shutdown Protection Plan. Interviews with } \\
\text { operators indicated that they would begin to close } \\
\text { containment when vessel temperature reached } 200 \\
\text { degrees. Interviews also indicated that an inability to } \\
\text { establish a normal means of shutdown cooling within } 1 \\
\text { hour of an accident would also be a cue to initiate } \\
\text { containment closure. }\end{array}$ & $\begin{array}{l}\text { The assumption that the cue for containment } \\
\text { closure occurs at time "0" is appropriate because } \\
\text { the thermal-hydraulic calculations for the time } \\
\text { available to close containment before core damage } \\
\text { etc., assumed vessel temperature was initially at } \\
200 \text { degrees } F \text {. While vessel temperature could, in } \\
\text { fact, be }<=140 \text { degrees when the initiating event } \\
\text { occurs, the time available for the crew to close } \\
\text { containment would functionally be the same } \\
\text { because their is no reason to assume that they } \\
\text { would initiate containment closure prior to vessel } \\
\text { temperature reaching } 200 \text { degrees } F \text {. }\end{array}$ \\
\hline
\end{tabular}


Table B.3.2-4

HEP 1: Potential Operator Action

\begin{tabular}{||l|l|l|l||}
\hline \multicolumn{1}{|c|}{$\begin{array}{c}\text { Description } \\
\text { of Event } \\
\text { (1) }\end{array}$} & $\begin{array}{c}\text { Number of } \\
\text { Abnormal Events } \\
\text { (2) }\end{array}$ & $\begin{array}{c}\text { Activities (Tasks) } \\
\text { Required to Perform } \\
\text { Action and Procedures } \\
\text { (3) }\end{array}$ & $\begin{array}{c}\text { Comments/ } \\
\text { Source of Information } \\
\text { (4) }\end{array}$ \\
\hline $\begin{array}{l}\text { For any number of possible reasons, } \\
\text { a condition of inadequate decay heat } \\
\text { removal exists and normal means of } \\
\text { SDC are not available. Vessel } \\
\text { temperature is approaching boiling } \\
\begin{array}{l}>200 \text { degrees F) and the operators } \\
\text { could decide to close containment to } \\
\text { avoid problems later if they are } \\
\text { unable to restore adequate cooling. }\end{array}\end{array}$ & $\begin{array}{l}\text { One } \\
\text { 1. Close containment equipment } \\
\text { hatch, including using crane to move } \\
19 \text { high hatch into place and } \\
\text { torquing down nuts to seal } \\
\text { containment. } \\
\text { 2. Close airlock at 119 foot elevation } \\
\text { level, including removal of the } \\
\text { plywood frame, cables, hoses, etc. } \\
\text { required to accomplish the } \\
\text { necessary actions were obtained } \\
\text { through telephone discussions with } \\
\text { plant personnel and through } \\
\text { responses to written questions } \\
\text { submitted to GGNS. }\end{array}$ & $\begin{array}{l}\text { 3. Close airlock at 208' level. } \\
\text { Significantly less removal of } \\
\text { material required at this airlock than } \\
\text { at 119'. }\end{array}$ \\
\hline
\end{tabular}


Table B.3.2-5

HEP 1: Time Available to Diagnose and Perform the Task

\begin{tabular}{|c|c|c|c|c|}
\hline $\begin{array}{l}\text { Action } \\
\text { (1) }\end{array}$ & $\begin{array}{c}\text { Time by Which } \\
\text { Operator Must } \\
\text { Act }\left(T_{c d}\right) \\
\text { (2) }\end{array}$ & $\begin{array}{c}\text { Time at Which } \\
\text { Operator } \\
\text { is Alerted } \\
\text { that Symptom } \\
\text { has Occurred }\left(T_{0}\right) \\
\text { (3) }\end{array}$ & $\begin{array}{c}\text { Maximum Time } \\
\text { Available } \\
\text { to Perform the } \\
\text { Identified } \\
\text { Operator Activities } \\
\left(T_{\mathrm{m}}\right) \\
(4) \\
\end{array}$ & $\begin{array}{l}\text { Comments/ } \\
\text { Source of Information } \\
\text { (5) }\end{array}$ \\
\hline $\begin{array}{l}\text { Diagnose the } \\
\text { need and carry- } \\
\text { out the actions } \\
\text { required to } \\
\text { close primary } \\
\text { containment. }\end{array}$ & 5 hours & 0 & 5 hours & $\begin{array}{l}\text { MELCOR calculations indicate that for cases } \\
\text { where the containment can be closed, TAF would } \\
\text { not be reached for } 8 \text { to } 10 \text { hours. However, with } \\
\text { the head vent open, the temperature in containment } \\
\text { (in the very worst and unlikely case) could reach } \\
123 \text { degrees in } 5 \text { hours and up to } 145 \text { degrees in } 8 \\
\text { hours. It was judged that temperatures greater than } \\
123 \text { degrees might restrict the crews ability to work } \\
\text { in containment. Thus, it was conservatively } \\
\text { assumed that they would only have } 5 \text { hours. It } \\
\text { should be noted, however, that the containment } \\
\text { cooling system would have to fail and the initial } \\
\text { containment temperature be at } 100 \text { degrees at the } \\
\text { start of the accident (both of which are unlikely) } \\
\text { for containment to reach } 123 \text { degrees in } 5 \text { hours. }\end{array}$ \\
\hline
\end{tabular}


Table B.3.2-6

HEP 1: Operator Action Performance Time

\begin{tabular}{|c|c|c|c|c|c|}
\hline $\begin{array}{l}\text { Activities } \\
\text { (1) }\end{array}$ & $\begin{array}{l}\text { Location } \\
\text { (2) }\end{array}$ & $\begin{array}{c}\text { Travel } \\
\text { Time }\left(T_{L}\right) \\
(3)\end{array}$ & $\begin{array}{l}\text { Performance } \\
\text { Time }\left(T_{p}\right) \\
\text { (4) }\end{array}$ & $\begin{array}{l}\text { Total } \\
\text { Action } \\
\text { Time }\left(T_{\mathfrak{z}}\right) \\
\quad(5)\end{array}$ & $\begin{array}{c}\text { Comments/ } \\
\text { Source of Information } \\
(6)\end{array}$ \\
\hline $\begin{array}{l}\text { 1. Close containment } \\
\text { equipment hatch, } \\
\text { including using crane to } \\
\text { move } 19^{\prime} \text { high hatch } \\
\text { into place and torquing } \\
\text { down nuts to seal } \\
\text { containment. }\end{array}$ & $\begin{array}{l}\text { Inside wall of } \\
\text { primary } \\
\text { containment }\end{array}$ & -- & $\begin{array}{l}\text { Estimated travel } \\
\text { and performance } \\
\text { time for closing } \\
\text { the equipment } \\
\text { hatch was } 4 \text { hours. }\end{array}$ & 4 hours & $\begin{array}{l}\text { Specific estimates regarding performance time were obtained } \\
\text { from GGNS personnel. Per the GGNS Shutdown Protection } \\
\text { Plan, different groups of individuals are assigned } \\
\text { responsibility for closing the hatch, and the two airlocks } \\
\text { during an outage. Thus, closing of the airlocks could be done } \\
\text { in parallel with the closing of the hatch. GGNS personnel } \\
\text { claim that the hatch could be in place with the nuts on in about } \\
2 \text { hours. An additional hour would be required to torque down } \\
\text { the nuts to ensure an air tight seal. This estimate assumes no } \\
\text { problems occur, e.g., problems with the crane. Thus, to } \\
\text { provide some accounting for potential delays, it was assumed } \\
4 \text { hours would be necessary to seal containment. This estimate } \\
\text { also assumes a crew of four would be available to close the } \\
\text { hatch. }\end{array}$ \\
\hline $\begin{array}{l}\text { 2. Close airlock at } 119^{\prime} \\
\text { level, including removal } \\
\text { of the plywood frame, } \\
\text { cables, hoses, etc. }\end{array}$ & $\begin{array}{l}119^{\prime} \text { level in } \\
\text { containment }\end{array}$ & -- & $\begin{array}{l}\text { Estimated travel } \\
\text { and performance } \\
\text { time for closing } \\
\text { the airlock was } 1 \\
\text { hour. }\end{array}$ & $\begin{array}{l}0 \text { minutes. } \\
\text { Assumed to } \\
\text { be done in } \\
\text { parallel } \\
\text { with hatch } \\
\text { closure }\end{array}$ & $\begin{array}{l}\text { GGNS estimated that the plywood frame (which is built to } \\
\text { help reduce contamination) could be dismantled, the quick } \\
\text { release hoses detached and thrown-out, the electrical wires } \\
\text { disconnected, and the } 119 \text { ' level airlock shut within a hour } \\
\text { "easily" (it takes about } 10 \text { min. to close the airlocks } \\
\text { themselves. The inner door alone will seal containment. }\end{array}$ \\
\hline $\begin{array}{l}\text { 3. Close airlock at } 208^{\prime} \\
\text { level. Significantly less } \\
\text { removal of material } \\
\text { required at this airlock } \\
\text { than at } 119^{\prime} \text {. }\end{array}$ & $\begin{array}{l}208 \text { ' level in } \\
\text { containment }\end{array}$ & -- & $\begin{array}{l}\text { Estimated travel } \\
\text { and performance } \\
\text { time for closing } \\
\text { the airlock was } 30 \\
\text { minutes }\end{array}$ & $\begin{array}{l}\text { 0 minutes } \\
\text { Assumed to } \\
\text { be done in } \\
\text { parallel } \\
\text { with hatch } \\
\text { closure } \\
4 \text { hours } \\
\text { Total }\end{array}$ & $\begin{array}{l}\text { With less material to be removed, GGNS estimated it would } \\
\text { take } 30 \text { minutes to close the } 208^{\prime} \text { level air lock. } \\
\text { Regardless, both airlocks could be closed in the time required } \\
\text { to close the hatch. }\end{array}$ \\
\hline
\end{tabular}


Table B.3.2-7

HEP 1: Diagnosis Time for Operator Action

\begin{tabular}{|c|c|c|c|c|}
\hline $\begin{array}{l}\text { Action } \\
\text { (1) }\end{array}$ & $\begin{array}{l}\text { Maximum Time } \\
\text { Available }\left(T_{m}\right) \\
\text { (2) }\end{array}$ & $\begin{array}{c}\text { Total Action } \\
\text { Time }\left(T_{2}\right) \\
\text { (3) }\end{array}$ & $\begin{array}{l}\text { Time Available } \\
\text { to Diagnosis }\left(T_{d}\right) \\
\text { (4) }\end{array}$ & $\begin{array}{c}\text { Comments/ } \\
\text { Source of } \\
\text { Information } \\
\text { (5) }\end{array}$ \\
\hline $\begin{array}{l}\text { Diagnose the need to initiate } \\
\text { containment closure }\end{array}$ & 5 hours & 4 hours & 60 minutes & \\
\hline
\end{tabular}

Table B.3.2-8

HEP 1: Diagnosis Analysis

\begin{tabular}{||l|l|l|l|l||}
\hline \multicolumn{1}{|c|}{$\begin{array}{c}\text { Action } \\
\text { (1) }\end{array}$} & \multicolumn{1}{|c|}{$\begin{array}{c}\text { Failure to } \\
\text { Diagnose } \\
\text { (2) }\end{array}$} & $\begin{array}{c}\text { Skill-Based } \\
\text { (3) }\end{array}$ & $\begin{array}{c}\text { Comments/ } \\
\text { Final HEP } \\
\text { (4) }\end{array}$ & $\begin{array}{c}\text { Source of Information } \\
\text { (5) }\end{array}$ \\
\hline $\begin{array}{l}\text { Diagnose the need to } \\
\text { initiate containment } \\
\text { closure. }\end{array}$ & $\begin{array}{l}\text { Per ASEP HRAP Table 8-3, the } \\
\text { median value from ASEP } \\
\text { Figure 8-1 for 60 minutes } \\
\text { diagnosis time was assigned. }\end{array}$ & Median = 1.0E-4 & $\begin{array}{l}\text { Discussions with plant personnel indicated that } \\
\text { the operators would be concerned about an } \\
\text { open containment and would initiate closure if } \\
\text { vessel temperature reached 200 degrees } F . \\
\text { However, they also acknowledged that they } \\
\text { would not want to unnecessarily close } \\
\text { containment. One operator indicated that even } \\
\text { if 200 degrees was not reached, if they were } \\
\text { unable to restore normal shutdown cooling in } \\
\text { an hour, then they would initiate containment } \\
\text { closure. }\end{array}$ \\
\hline
\end{tabular}


Table B.3.2-9

HEP 1: Post-Diagnosis Action Type Identification per Step 10, Table 8-1 of ASEP HRAP

\begin{tabular}{||c|l|l|l|l|l||}
\hline \multicolumn{1}{|c|}{$\begin{array}{c}\text { Action } \\
\text { (1) }\end{array}$} & \multicolumn{1}{|c|}{$\begin{array}{c}\text { Safety Systems } \\
\text { Failed } \\
\text { (2) }\end{array}$} & $\begin{array}{c}\text { EOPs, Training, } \\
\text { Use EOPs Well } \\
\text { Designed EOPs } \\
\text { (3) }\end{array}$ & $\begin{array}{c}\text { Individual } \\
\text { Operator Must } \\
\text { Perform Concurrent } \\
\text { Tasks } \\
\text { (4) }\end{array}$ & $\begin{array}{c}\text { Dynamic or } \\
\text { Step-by-Step } \\
\text { (5) }\end{array}$ & $\begin{array}{c}\text { Comments/ } \\
\text { Source of } \\
\text { Information } \\
\text { (6) }\end{array}$ \\
\hline $\begin{array}{l}\text { Close } \\
\text { containment } \\
\text { equipment } \\
\text { hatch and both } \\
\text { airlocks. }\end{array}$ & $\begin{array}{l}\text { N/A - Operators may or } \\
\text { may not have entered } \\
\text { EOPs at this point. } \\
\text { Regardless, closing } \\
\text { containment is carried-out } \\
\text { by individuals other than } \\
\text { operators and is clearly a } \\
\text { step x step task. }\end{array}$ & $\begin{array}{l}\text { Discussions with } \\
\text { plant personnel } \\
\text { indicated that they } \\
\text { were knowledgeable } \\
\text { about the need for the } \\
\text { actions and the } \\
\text { requirements. }\end{array}$ & No & Step-by-Step & \\
\hline
\end{tabular}


Table B.3.2-10

HEP 1: Post-Diagnosis Stress-Level Identification per Step 10, Table 8-1 of ASEP HRAP

\begin{tabular}{|c|c|c|c|c|c|c|}
\hline $\begin{array}{l}\text { Action } \\
\text { (1) }\end{array}$ & $\begin{array}{c}\mathrm{T}_{\mathrm{m}}<\mathbf{h} \\
\text { After } \\
\mathbf{1 E} \\
\text { (2) }\end{array}$ & $\begin{array}{l}\text { Recirc. } \\
\text { Phase } \\
\text { in } \\
\text { Large } \\
\text { LOCA } \\
\text { (3) }\end{array}$ & $\begin{array}{l}\text { More } \\
\text { Than Two } \\
\text { Safety } \\
\text { Systems } \\
\text { Fail } \\
\text { (4) }\end{array}$ & $\begin{array}{c}\text { Operator } \\
\text { Familiar } \\
\text { W/Sequence } \\
\text { (5) }\end{array}$ & $\begin{array}{c}\text { Stress Level } \\
\text { (ด) }\end{array}$ & $\begin{array}{c}\text { Comments/ } \\
\text { Source of Information } \\
(7)\end{array}$ \\
\hline $\begin{array}{l}\text { 1. Close } \\
\text { equipment } \\
\text { hatch }\end{array}$ & N/A ${ }^{1}$ & N/A & Maybe & Maybe & $\begin{array}{l}\text { Extremely } \\
\text { High }\end{array}$ & $\begin{array}{l}\text { With the crew being instructed to close } \\
\text { containment in an emergency (accident) } \\
\text { situation in which containment temperature } \\
\text { is likely to be increasing, extremely high } \\
\text { stress must be assumed. }\end{array}$ \\
\hline $\begin{array}{l}\text { 2. Close } \\
\text { airlocks at } \\
119^{\prime} \text { and } 208^{\prime} \\
\text { levels }\end{array}$ & $\mathrm{N} / \mathrm{A}^{1}$ & N/A & Maybe & Maybe & Moderately High & $\begin{array}{l}\text { Since closing the airlocks can be done } \\
\text { essentially in parallel with closing the hatch, } \\
\text { the time available to accomplish these tasks } \\
\text { is much greater than that for closing the } \\
\text { hatch. In addition, the work for closing the } \\
\text { airlocks could be done before containment } \\
\text { temperature has increased much and, in any } \\
\text { case, the work is mainly done in the door } \\
\text { area. Thus, only moderately high stress was } \\
\text { assumed for these actions. }\end{array}$ \\
\hline
\end{tabular}

At least moderately high stress was assumed for all events. 
Table B.3.2-11

HEP 1: Total HEP

\begin{tabular}{|c|c|c|c|c|c|}
\hline $\begin{array}{l}\text { Action } \\
\text { (1) }\end{array}$ & $\begin{array}{c}\text { Original } \\
\text { Operator HEP } \\
\left.\text { (HEP }_{\mathrm{op}}\right) \\
\text { (2) }\end{array}$ & $\begin{array}{c}\text { Independent } \\
\text { Check/Correction } \\
\text { HEP } \\
\left(\text { HEP }_{\mathrm{r} 2}\right) \\
\text { (3) } \\
\end{array}$ & $\begin{array}{l}\text { Total HEP } \\
\text { (4) }\end{array}$ & $\begin{array}{l}\text { EF } \\
(5)\end{array}$ & $\begin{array}{l}\text { Comments/ } \\
\text { Source of } \\
\text { Information } \\
(6)\end{array}$ \\
\hline $\begin{array}{l}\text { 1. Diagnose need to } \\
\text { close primary } \\
\text { containment }\end{array}$ & $\begin{array}{l}\text { Median }=1.0 \mathrm{E}-4 \\
\mathrm{EF}=30 \\
\text { Mean }=8.5 \mathrm{E}-4\end{array}$ & & $\begin{array}{ll}\text { Med. } & \text { Mean } \\
1.0 \mathrm{E}-4 & 8.5 \mathrm{E}-4\end{array}$ & $(30)$ & \\
\hline $\begin{array}{l}\text { 2. Close containment } \\
\text { equipment hatch, } \\
\text { including using crane } \\
\text { to move } 19 \text { ' high hatch } \\
\text { into place and torquing } \\
\text { down nuts to seal } \\
\text { containment. }\end{array}$ & $\begin{array}{l}\text { Median }=0.05 \\
\text { Mean }=0.081\end{array}$ & $\begin{array}{l}\text { Does not really make sense to give credit for a } \\
\text { second check on closing the equipment hatch. } \\
\text { There would very likely be insufficient time to } \\
\text { get it closed if the crew failed to start the task } \\
\text { when directed. Similarly, assuming some sort of } \\
\text { unnoticed error in sealing the hatch was possible } \\
\text { (it is difficult to think of one), the time available } \\
\text { would most likely be insufficient. }\end{array}$ & $\begin{array}{lc}\text { Med. } & \text { Mean } \\
0.05 & 0.081\end{array}$ & $(5)$ & \\
\hline $\begin{array}{l}\text { 3. Close airlock at } \\
119 \text { 'level, including } \\
\text { removal of plywood } \\
\text { frame, cables, hoses, } \\
\text { etc. }\end{array}$ & $\begin{array}{l}\text { Med. }=0.02 \\
\text { Mean }=0.032\end{array}$ & $\begin{array}{l}\text { Credit for a second check was given in this } \\
\text { instance. Given the number of people around } \\
\text { during shutdown, the fact that a specific team is } \\
\text { responsible, and the ample time available, a } \\
\text { failure to start this task could be recovered. } \\
\text { Second check values for action } 2 \text { were: } \\
\text { Median }=0.2 \\
\text { Mean }=0.323\end{array}$ & $0.004 \quad 0.01$ & $(5)$ & $\begin{array}{l}\text { Second check } \\
\text { HEPs are } \\
\text { multiplied by the } \\
\text { original HEP for } \\
\text { each action. }\end{array}$ \\
\hline $\begin{array}{l}\text { 4. Close airlock at } 208^{\prime} \\
\text { level. Significantly less } \\
\text { removal of material } \\
\text { required at this airlock } \\
\text { than at } 119^{\prime} \text {. }\end{array}$ & $\begin{array}{l}\text { Med. }=0.02 \\
\text { Mean }=0.032\end{array}$ & $\begin{array}{l}\text { Yes, rationale the same as for airlock at } 119^{\prime} \\
\text { level. }\end{array}$ & $\begin{array}{l}\frac{0.004}{0.058} \frac{0.01}{0.102} \\
\text { Total Median HEP } \\
=0.058 \\
\text { Total Mean } \\
\text { HEP }=0.102\end{array}$ & $\frac{(5)}{(5)}$ & $\begin{array}{l}\text { The error factor } \\
\text { associated with } \\
\text { the dominant } \\
\text { HEPs was } \\
\text { assigned. }\end{array}$ \\
\hline
\end{tabular}


Table B.3.2-12

HEP 2 Calculation

\begin{tabular}{|c|c|}
\hline Human Action Event (1) & Initiation of Hydrogen Igniter System \\
\hline Event Description (2) & $\begin{array}{l}\text { Initiation of the Hydrogen Igniter System (HIS) is the operator action to diagnose the need and accomplish the actions } \\
\text { leading to the hydrogen igniters being turned on per procedure. }\end{array}$ \\
\hline Event Context (3) & $\begin{array}{l}\text { An initiating event has occurred and the reactor vessel level has dropped to Top of Active Fuel (TAF). Vessel level } \\
\text { reaching TAF ( }-167 \text { in.) is an entry condition for the Hydrogen Control section of GGNS EP-3 (Containment Control). } \\
\text { If the operators initiate the HIS before hydrogen concentration levels reach } 9 \% \text {, potential explosions related to } \\
\text { hydrogen release in the drywell and primary containment may be prevented. It is assumed that the containment } \mathrm{H}_{2} \\
\text { concentration is in the safe zone of the Hydrogen Deflagration Overpressure Limit and presumably can be maintained } \\
\text { there upon entering EP-3. (Note. In Station Blackout Scenarios where level has reached TAF, the operators would be } \\
\text { unable to determine Hydrogen concentration levels and therefore could not determine whether or not } \mathrm{H}_{2} \text { concentration } \\
\text { could be maintained in the safe zone of the Hydrogen Deflagration Overpressure Limit (HDOL). Given this situation, } \\
\text { the EP guides the operators to "secure and prevent operation" of the igniters. Thus, it is assumed that the operators } \\
\text { would not turn the hydrogen igniters to the on position until they had recovered power and could determine the } \mathrm{H}_{2} \\
\text { levels.) }\end{array}$ \\
\hline Applicable Procedures (4) & Hydrogen Control Section of Emergency Procedure 3 (EP-3, Containment Control, GGNS, Rev. 21) \\
\hline
\end{tabular}


Table B.3.2-13

HEP 2: Sequence Timing and Indications

\begin{tabular}{|c|c|c|c|}
\hline $\begin{array}{c}\text { Event/Occurrence } \\
\text { (of most interest) } \\
\text { (1) }\end{array}$ & $\begin{array}{c}\text { Time }\left(\mathrm{T}_{0}\right) \\
\text { Operator } \\
\text { Alerted } \\
(2)\end{array}$ & $\begin{array}{l}\text { Annunciator/Indication } \\
\text { (3) }\end{array}$ & $\begin{array}{l}\text { Comments/ } \\
\text { Source of } \\
\text { Information } \\
\quad(4) \\
\end{array}$ \\
\hline $\begin{array}{l}\text { Operators need to initiate } \\
\text { the HIS to prevent the } \\
\text { build-up of large pockets } \\
\text { of hydrogen and the } \\
\text { associated potential } \\
\text { explosions which could } \\
\text { damage containment. }\end{array}$ & O & $\begin{array}{l}\text { The primary indicator is vessel level reaching TAF. } \\
\text { Numerous alarms will have sounded prior to or in } \\
\text { conjunction with vessel level reaching TAF. Thus, the } \\
\text { operators will be tracking a significant drop in water } \\
\text { level. Another indicator would be containment or } \\
\text { drywell } \mathrm{H}_{2} \text { concentration above } 0.5 \% \text {, which also is an } \\
\text { entry condition for the hydrogen control section of EP-3 } \\
\text {. In all Initiating Event scenarios except for a LOCA, } \\
\text { vessel level dropping to TAF would occur over a several } \\
\text { hour period ( } 3 \text { to } 13 \text { hours depending on the event). For } \\
\text { the LOCA scenario, TAF would be reached more or less } \\
\text { immediately, but core damage would not be reached for } \\
\text { approximately an hour after TAF is reached. At least } 1 \\
\text { hour would be available between TAF and core damage } \\
\text { for all relevant scenarios. }\end{array}$ & $\begin{array}{l}\text { MELCOR calculations determined both the time } \\
\text { before TAF would be reached for the various } \\
\text { initiators and the time between TAF and Core } \\
\text { Damage. It should be noted that hydrogen build- } \\
\text { up after core damage would not reach a level that } \\
\text { would mandate not using the hydrogen igniters for } \\
\text { at least another } 30 \text { to } 60 \text { minutes, depending on } \\
\text { the accident scenario. }\end{array}$ \\
\hline
\end{tabular}


Table B.3.2-14

HEP 2: Potential Operator Action

\begin{tabular}{||l|l|l|l||}
\hline \multicolumn{1}{|c|}{$\begin{array}{c}\text { Description } \\
\text { of Event } \\
\text { (1) }\end{array}$} & $\begin{array}{c}\text { Number of } \\
\text { Abnormal Events } \\
\text { (2) }\end{array}$ & $\begin{array}{c}\text { Activities (Tasks) } \\
\text { Required to Perform } \\
\text { Action and Procedures } \\
\text { (3) }\end{array}$ & $\begin{array}{c}\text { Comments/ } \\
\text { Source of Information } \\
\text { (4) }\end{array}$ \\
\hline $\begin{array}{l}\text { For any number of possible reasons, } \\
\text { vessel level has dropped to TAF. }\end{array}$ & $\begin{array}{l}\text { One abnormal event is assumed per } \\
\text { Table 8-1, Step 9.b.1. While failures } \\
\text { of several systems may occur in the } \\
\text { relevant scenarios, } \\
\text { the entry condition for the EP-3 is } \\
\text { clear and essentially only one } \\
\text { diagnosis is required (level below - } \\
\text { 167"). The procedure should guide } \\
\text { the operators to make the } \\
\text { appropriate response. }\end{array}$ & $\begin{array}{l}\text { Operate the igniters from the control } \\
\text { room. }\end{array}$ & \\
\hline
\end{tabular}

ఝ্ 
Table B.3.2-15

HEP 2: Time Available to Diagnose and Perform the Task

\begin{tabular}{|c|c|c|c|c|}
\hline $\begin{array}{l}\text { Action } \\
\text { (1) }\end{array}$ & $\begin{array}{c}\text { Time by Which } \\
\text { Operator Must } \\
\text { Act }\left(\mathrm{T}_{e d}\right) \\
\text { (2) }\end{array}$ & $\begin{array}{c}\text { Time at Which } \\
\text { Operator } \\
\text { is Alerted } \\
\text { that Symptom } \\
\text { has Occurred }\left(\mathrm{T}_{0}\right) \\
\text { (3) }\end{array}$ & $\begin{array}{l}\text { Maximum Time } \\
\text { Available } \\
\text { to Perform the } \\
\text { Identified } \\
\text { Operator Activities } \\
\left(T_{\mathrm{m}}\right) \\
(4)\end{array}$ & $\begin{array}{c}\text { Comments/ } \\
\text { Source of Information } \\
(5)\end{array}$ \\
\hline $\begin{array}{l}\text { Diagnose the } \\
\text { need and carry- } \\
\text { out the actions } \\
\text { required to } \\
\text { initiate the } \\
\text { hydrogen } \\
\text { ignition system. }\end{array}$ & 1 hour & 0 & 1 hour & $\begin{array}{l}\text { Results from MELCOR calculations indicated that } \\
\text { core damage would not occur for at least an hour } \\
\text { after reaching TAF. } \mathrm{H}_{2} \text { would not be released until } \\
\text { core damage occurred. Thus, at least } 1 \text { hour would } \\
\text { be available to diagnose the need and initiate the } \\
\text { hydrogen igniters. In fact, as noted above, } \\
\text { hydrogen build-up after core damage would not } \\
\text { reach a level that would mandate not using the } \\
\text { hydrogen igniters for at least another } 30 \text { to } 60 \\
\text { minutes, depending on the accident scenario. }\end{array}$ \\
\hline
\end{tabular}

Table B.3.2-16

HEP 2: Operator Action Performance Time

\begin{tabular}{|c|c|c|c|c|c|}
\hline $\begin{array}{l}\text { Activities } \\
\text { (1) }\end{array}$ & $\begin{array}{l}\text { Location } \\
\text { (2) }\end{array}$ & $\begin{array}{c}\text { Travel } \\
\text { Time }\left(\mathbf{T}_{\mathbf{L}}\right) \\
(\mathbf{3}) \\
\end{array}$ & $\begin{array}{l}\text { Performance } \\
\text { Time }\left(T_{p}\right) \\
(4)\end{array}$ & $\begin{array}{c}\text { Total Action } \\
\text { Time }\left(T_{a}\right) \\
(5) \\
\end{array}$ & $\begin{array}{c}\text { Comments/ } \\
\text { Source of Information } \\
(6) \\
\end{array}$ \\
\hline $\begin{array}{l}\text { Operate Hydrogen } \\
\text { Igniter System }\end{array}$ & Control Room & -- & 1 minute & 1 minute & $\begin{array}{l}\text { Per ASEP Table } 8-1 \text {, Step } 5 \mathrm{~b} \text {, a } 1 \text { min. travel and } \\
\text { manipulation time was assumed for actions in the } \\
\text { control room. }\end{array}$ \\
\hline
\end{tabular}


Table B.3.2-17

HEP 2: Diagnosis Time for Operator Action

\begin{tabular}{|c|c|c|c|c|}
\hline $\begin{array}{l}\text { Action } \\
\text { (1) }\end{array}$ & $\begin{array}{l}\text { Maximum Time } \\
\text { Available }\left(T_{m}\right) \\
\text { (2) }\end{array}$ & $\begin{array}{c}\text { Total Action } \\
\text { Time }\left(T_{2}\right) \\
\text { (3) }\end{array}$ & $\begin{array}{l}\text { Time Available } \\
\text { to Diagnosis }\left(T_{d}\right) \\
\text { (4) }\end{array}$ & $\begin{array}{c}\text { Comments/ } \\
\text { Source of } \\
\text { Information } \\
(5) \\
\end{array}$ \\
\hline $\begin{array}{l}\text { Diagnose the need to initiate } \\
\text { Hydrogen Ignition System }\end{array}$ & 1 hour & 1 minute & Approx. 59 minutes & \\
\hline
\end{tabular}

Table B.3.2-18

HEP 2: Diagnosis Analysis

\begin{tabular}{||c|l|c|l|l||}
\hline \multicolumn{1}{|c|}{$\begin{array}{c}\text { Action } \\
\text { (1) }\end{array}$} & \multicolumn{1}{|c|}{$\begin{array}{c}\text { Failure to } \\
\text { Diagnose } \\
\text { (2) }\end{array}$} & $\begin{array}{c}\text { Skill-Based } \\
\text { (3) }\end{array}$ & \multicolumn{1}{c|}{$\begin{array}{c}\text { Adjusted/ } \\
\text { Final HEP } \\
\text { (4) }\end{array}$} & $\begin{array}{c}\text { Comments/ } \\
\text { Source of Information } \\
\text { (5) }\end{array}$ \\
\hline $\begin{array}{l}\text { Diagnose the need to } \\
\text { initiate the Hydrogen } \\
\text { Igniter System. }\end{array}$ & $\begin{array}{l}\text { Per ASEP HRAP Table 8-3, the } \\
\text { median value from ASEP } \\
\text { Figure 8-1 for approx. 59 } \\
\text { minutes diagnosis time was } \\
\text { assigned. }\end{array}$ & & Median = 1.0E-4 \\
$\mathrm{EF}=30$ & Mean = 8.5E-4 & \\
\hline
\end{tabular}


Table B.3.2-19

HEP 2: Post-Diagnosis Action Type Identification per Step 10, Table 8-1 of ASEP HRAP

\begin{tabular}{|l|l|l|l|l|l|}
\hline \multicolumn{1}{|c|}{$\begin{array}{c}\text { Action } \\
\text { (1) }\end{array}$} & \multicolumn{1}{|c|}{$\begin{array}{c}\text { Safety Systems } \\
\text { Failed } \\
\text { (2) }\end{array}$} & $\begin{array}{l}\text { EOPs, Training, } \\
\text { Use EOPs, Well } \\
\text { Designed EOPs } \\
\text { (3) }\end{array}$ & $\begin{array}{c}\text { Individual } \\
\text { Operator Must } \\
\text { Perform Concurrent } \\
\text { Tasks } \\
\text { (4) }\end{array}$ & $\begin{array}{c}\text { Dynamic or } \\
\text { Step-by-Step } \\
\text { (5) }\end{array}$ & $\begin{array}{c}\text { Comments/ } \\
\text { Source of } \\
\text { Information } \\
\text { (6) }\end{array}$ \\
\hline $\begin{array}{l}\text { Operate } \\
\text { Hydrogen } \\
\text { Ignition System }\end{array}$ & $\begin{array}{l}\text { N/A - No additional safety } \\
\text { systems are assumed to fail } \\
\text { at this point. }\end{array}$ & $\begin{array}{l}\text { Although the lower } \\
\text { bound value from the } \\
\text { diagnosis model was } \\
\text { not used, the EOPs } \\
\text { are clear and the } \\
\text { operators need only } \\
\text { initiate the system } \\
\text { from the control } \\
\text { room. Thus, the } \\
\text { actions were not } \\
\text { assumed to be } \\
\text { dynamic. }\end{array}$ & Step-by-Step & & \\
\hline
\end{tabular}


Table B.3.2-20

HEP 2: Post-Diagnosis Stress-Level Identification per Step 10, Table 8-1 of ASEP HRAP

\begin{tabular}{|c|c|c|c|c|c|c|}
\hline $\begin{array}{l}\text { Action } \\
\text { (1) }\end{array}$ & $\begin{array}{c}\mathbf{T}_{\mathrm{m}}<2 \mathrm{~h} \\
\text { After } \\
\mathbf{1 E} \\
(2)\end{array}$ & $\begin{array}{c}\text { Recirc. } \\
\text { Phase } \\
\text { in } \\
\text { Large } \\
\text { LOCA } \\
\text { (3) } \\
\end{array}$ & $\begin{array}{c}\text { More } \\
\text { Than Two } \\
\text { Safety } \\
\text { Systems } \\
\text { Fail } \\
\text { (4) }\end{array}$ & $\begin{array}{c}\text { Operator } \\
\text { Familiar } \\
\text { W/Sequence } \\
\text { (5) }\end{array}$ & $\begin{array}{c}\text { Stress Level } \\
\text { (6) }\end{array}$ & $\begin{array}{l}\text { Comments/ } \\
\text { Source of Information } \\
\text { (7) }\end{array}$ \\
\hline $\begin{array}{l}\text { Initiate } \\
\text { Hydrogen } \\
\text { Ignition } \\
\text { System }\end{array}$ & $\mathrm{N} / \mathrm{A}^{1}$ & Maybe & Maybe & Maybe & $\begin{array}{l}\text { Extremely } \\
\text { High }\end{array}$ & $\begin{array}{l}\text { With vessel level dropping to TAF in } \\
\text { scenarios where safety systems have failed } \\
\text { and possibly in the context of a LOCA, } \\
\text { extremely high stress was assumed. }\end{array}$ \\
\hline
\end{tabular}

It least moderately high stress was assumed for all events.

崠

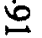


Table B.3.2-21

HEP 2: Total HEP

\begin{tabular}{|c|c|c|c|c|c|}
\hline $\begin{array}{l}\text { Action } \\
\text { (1) }\end{array}$ & $\begin{array}{c}\text { Original } \\
\text { Operator HEP } \\
\left(\text { HEP }_{\text {op }}\right) \\
\text { (2) }\end{array}$ & $\begin{array}{c}\text { Independent } \\
\text { Check/Correction } \\
\text { HEP } \\
\left(\text { HEP }_{r 2}\right) \\
\text { (3) }\end{array}$ & $\begin{array}{l}\text { Total HEP } \\
\text { (4) }\end{array}$ & $\begin{array}{l}\mathbf{E F} \\
(5)\end{array}$ & $\begin{array}{l}\text { Comments/ } \\
\text { Source of } \\
\text { Information } \\
(6)\end{array}$ \\
\hline $\begin{array}{l}\text { 1. Diagnose } \\
\text { need to } \\
\text { initiate } \\
\text { hydrogen } \\
\text { igniter } \\
\text { system. } \\
\end{array}$ & $\begin{array}{l}\text { Median }=1.0 \mathrm{E}-4 \\
E F=30 \\
\text { Mean }=8.5 \mathrm{E}-4\end{array}$ & & $\begin{array}{ll}\text { Med. } & \text { Mean } \\
1.0 \mathrm{E}-4 & 8.5 \mathrm{E}-4\end{array}$ & & \\
\hline $\begin{array}{l}2 . \text { Initiate } \\
\text { hydrogen } \\
\text { igniter } \\
\text { system. }\end{array}$ & $\begin{array}{l}\text { Med. }=0.05 \\
\text { Mean }=0.081\end{array}$ & $\begin{array}{l}\text { Credit for a second check and third was given in this } \\
\text { instance. Given the initial time available for the task, the } \\
\text { fact that additional time would be available even after core } \\
\text { damage (CD) was reached (at least } 30 \text { minutes and in most } \\
\text { cases an hour), the importance of the action, the clarity of } \\
\text { the EP, and the simplicity of the action, an initial failure to } \\
\text { operate the system would have some probability of being } \\
\text { recovered. Second and third check HEPs for the action } \\
\text { were: } \\
\text { Median }=0.5 \\
\text { Mean }=0.81\end{array}$ & $\frac{0.013}{0.013} \quad \frac{0.053}{0.054}$ & (5) & $\begin{array}{l}\text { The second } \\
\text { check HEP is } \\
\text { multiplied by the } \\
\text { original HEP for } \\
\text { a given action. } \\
\text { The resulting } \\
\text { product is } \\
\text { multiplied by the } \\
\text { HEP for the } \\
\text { third check. }\end{array}$ \\
\hline $\begin{array}{l}\text { Total HEP } \\
\text { and Error } \\
\text { Factor }\end{array}$ & & & $\begin{array}{l}\text { Total Median HEP } \\
=0.013 \\
\text { Total Mean } \\
\text { HEP }=0.054\end{array}$ & (5) & $\begin{array}{l}\text { The error factor } \\
\text { associated with } \\
\text { the dominant } \\
\text { HEP was } \\
\text { assigned. }\end{array}$ \\
\hline
\end{tabular}


Table B.3.2-22

HEP 3 Calculation

\begin{tabular}{||l|l||}
\hline Human Action Event (1) & Initiation of Containment Venting \\
\hline Event Description (2) & $\begin{array}{l}\text { Initiation of containment venting is the operator action to diagnose the need and accomplish the actions leading to the } \\
\text { venting of containment. The goal is to reduce extreme pressure build-up in containment and avoid a potential loss of } \\
\text { primary containment during an accident. An emergency procedure (EP-3) clearly addresses the situation. }\end{array}$ \\
\hline Event Context (3) & $\begin{array}{l}\text { An initiating event has occurred and for a number of possible reasons has resulted in core damage and eventually in } \\
\text { vessel breach. The relevant indicators for venting are that drywell pressure is greater than 1.23 PSIG and containment } \\
\text { pressure has reached 20 PSIG. Drywell pressure above 1.23 PSIG is the entry condition for the containment pressure } \\
\text { control section of GGNS EP-3 (Containment Control). Many hours have elapsed since the initiating event } \\
\text { (approximately 16 hours). At this point in any of the relevant scenarios, the plant conditions would not be changing } \\
\text { rapidly and an Emergency Response Team would be in place. Containment venting is clearly indicated in EP-3 and } \\
\text { given that the relevant parameters have been reached, the Emergency Director would have to override EP-3 to prevent } \\
\text { venting. The present analysis assumed that the Emergency Director would not have any basis for overriding EP-3 and } \\
\text { that the crew would be attempting to follow the emergency procedures to prevent any damage to primary containment. }\end{array}$ \\
\hline Applicable Procedures (4) & $\begin{array}{l}\text { Containment Pressure Control Section of Emergency Procedure 3 (EP-3, Containment Control, GGNS, Rev. 21) and } \\
\text { Attachment 13 of the EPs (05-S-01-EP-2, Attachment 13, Rev. 19). }\end{array}$ \\
\hline
\end{tabular}


Table B.3.2-23

HEP 3: Sequence Timing and Indications

\begin{tabular}{|c|c|c|c|}
\hline $\begin{array}{l}\text { Event/Occurrence } \\
\text { (of most interest) } \\
\text { (1) }\end{array}$ & $\begin{array}{c}\text { Time }\left(\mathbf{T}_{0}\right) \\
\text { Operator } \\
\text { Alerted } \\
(2)\end{array}$ & $\begin{array}{l}\text { Annunciator/Indication } \\
\text { (3) }\end{array}$ & $\begin{array}{l}\text { Comments/ } \\
\text { Source of } \\
\text { Information } \\
(4)\end{array}$ \\
\hline $\begin{array}{l}\text { Operators need to initiate } \\
\text { containment venting to } \\
\text { avoid overpressurization } \\
\text { and possible loss of } \\
\text { primary containment. }\end{array}$ & 0 & $\begin{array}{l}\text { The primary indicators are drywell pressure greater than } \\
1.23 \text { PSIG and containment pressure at or above } 20 \text { PSIG. } \\
\text { Core damage and a breach of the vessel has occurred. } \\
\text { Numerous alarms will have sounded prior to reaching this } \\
\text { point and drywell and containment pressure will be } \\
\text { paramount to the crews in their attempts to minimize the } \\
\text { impact of the accident. }\end{array}$ & $\begin{array}{l}\text { MELCOR calculations determined the time at which } \\
\text { the relevant parameters would be reached and the } \\
\text { time available for the operators to respond to the } \\
\text { cues in order to prevent a loss of primary } \\
\text { containment. In regards to any hesitancy on the part } \\
\text { of the operators to vent containment after core } \\
\text { damage, GGNS indicated that at this point in time } \\
\text { EP-3 would be followed. GGNS did acknowledge } \\
\text { that the issue is being discussed in the ongoing } \\
\text { Severe Accident work. }\end{array}$ \\
\hline
\end{tabular}

Table B.3.2-24

HEP 3: Potential Operator Action

\begin{tabular}{|c|c|c|c|}
\hline $\begin{array}{l}\text { Description } \\
\text { of Event } \\
\text { (1) }\end{array}$ & $\begin{array}{c}\text { Number of } \\
\text { Abnormal Events } \\
\text { (2) }\end{array}$ & $\begin{array}{c}\text { Activities (Tasks) } \\
\text { Required to Perform } \\
\text { Action and Procedures } \\
\text { (3) }\end{array}$ & $\begin{array}{l}\text { Comments/ } \\
\text { Source of Information } \\
\text { (4) }\end{array}$ \\
\hline $\begin{array}{l}\text { For any of several possible reasons, } \\
\text { core damage has occurred and has } \\
\text { been followed by vessel breach. The } \\
\text { parameters for entry into the venting } \\
\text { procedure have been met. Substantial } \\
\text { time is available for diagnosing the } \\
\text { need and performing the relevant } \\
\text { actions }\end{array}$ & $\begin{array}{l}\text { One abnormal event is assumed per } \\
\text { Table } 8-1, \text { Step } 9 \text {. While failures of } \\
\text { several systems may occur in the } \\
\text { relevant scenarios, } \\
\text { the entry condition for the relevant leg } \\
\text { of EP-3 is clear. At least two hours are } \\
\text { available for making the diagnosis and } \\
\text { the procedure should guide the } \\
\text { operators to make the appropriate } \\
\text { response. }\end{array}$ & $\begin{array}{l}\text { Operators will be required to override } \\
\text { four containment vent path isolation } \\
\text { interlocks per EP Attachment } 13 \text { and } \\
\text { open six valves from the control room. } \\
\text { Jumpering through the interlocks will } \\
\text { require use of Jumper Kit No. } 13 \text {. Two } \\
\text { relays in the main control room and } \\
\text { two in the upper control room will } \\
\text { require "jumpering." }\end{array}$ & $\begin{array}{l}\text { Per Attachment } 13 \text {, Jumper Kit No. } \\
13 \text { is stored in the control room } \\
\text { emergency locker. Vent procedure is } \\
\text { clear. Relays were not inspected for } \\
\text { adequacy of labeling. }\end{array}$ \\
\hline
\end{tabular}


Table B.3.2-25

HEP 3: Time Available to Diagnose and Perform the Task

\begin{tabular}{|c|c|c|c|c|}
\hline $\begin{array}{l}\text { Action } \\
\text { (1) }\end{array}$ & $\begin{array}{l}\text { Time by } \\
\text { Which } \\
\text { Operator } \\
\text { Must } \\
\text { Act }\left(T_{\text {cd }}\right) \\
\text { (2) }\end{array}$ & $\begin{array}{l}\text { Time at Which } \\
\text { Operator } \\
\text { is Alerted } \\
\text { that Symptom } \\
\text { has Occurred } \\
\left(T_{0}\right) \\
(3)\end{array}$ & $\begin{array}{l}\text { Maximum Time } \\
\text { Available } \\
\text { to Perform the } \\
\text { Identified } \\
\text { Operator Activities } \\
\left(T_{m}\right) \\
(4)\end{array}$ & $\begin{array}{c}\text { Comments/ } \\
\text { Source of Information } \\
\text { (5) }\end{array}$ \\
\hline $\begin{array}{l}\text { Diagnose the } \\
\text { need and carry- } \\
\text { out the actions } \\
\text { required to vent } \\
\text { containment. }\end{array}$ & 3 hours & 0 & $\begin{array}{l}3 \text { hours } \\
\text { Results from MELCOR } \\
\text { calculations indicated that the } \\
\text { indicators for containment } \\
\text { venting would occur more or less } \\
\text { in conjunction with the } \\
\text { occurrence of a vessel breach } \\
\text { related to core damage. Regarding } \\
\text { the time available for the } \\
\text { operators to respond, the } \\
\text { emergency procedure directs that } \\
\text { the operators vent containment } \\
\text { when pressure reaches } 20 \text { PSIG. } \\
\text { (continued in next column). }\end{array}$ & $\begin{array}{l}\text { (continued from previous column) However, if containment } \\
\text { pressure increases to } 22 \text { PSIG, the emergency procedure } \\
\text { essentially instructs the operators to "abandon" the core and use } \\
\text { whatever injection systems are available for containment sprays, } \\
\text { i.e., save containment. It seems extremely unlikely that the } \\
\text { operators would delay venting and put themselves in this } \\
\text { position. } \\
\text { T-H calculations indicated that it would take about three hours } \\
\text { for pressure to increase from } 20 \text { to } 22 \text { PSIG. Thus, it seemed } \\
\text { reasonable to assume that if the operators were going to follow } \\
\text { procedure and vent, they would do so within the three hours after } \\
\text { the relevant parameters were reached. In actuality, } 10 \text { to } 14 \text { hours } \\
\text { would have to elapse before containment pressure would become } \\
\text { great enough to fail containment and the operators could vent at } \\
\text { any point prior to that time. }\end{array}$ \\
\hline
\end{tabular}


Table B.3.2-26

HEP 3: Operator Action Performance Time

\begin{tabular}{|c|c|c|c|c|c|}
\hline $\begin{array}{l}\text { Activities } \\
\text { (1) }\end{array}$ & $\begin{array}{l}\text { Location } \\
\text { (2) }\end{array}$ & $\begin{array}{c}\text { Travel } \\
\text { Time }\left(T_{L}\right) \\
(3)\end{array}$ & $\begin{array}{l}\text { Performance } \\
\text { Time }\left(T_{p}\right) \\
(4)\end{array}$ & $\begin{array}{l}\text { Total Action } \\
\text { Time }\left(\mathbf{T}_{2}\right) \\
(5)\end{array}$ & $\begin{array}{l}\text { Comments/ } \\
\text { Source of Information } \\
\text { (6) }\end{array}$ \\
\hline $\begin{array}{l}\text { 1. Jumper two different } \\
\text { sets of relays in the } \\
\text { main control room. }\end{array}$ & $\begin{array}{l}\text { Main control } \\
\text { room }\end{array}$ & $\begin{array}{l}\text { Per ASEP } \\
\text { HRAP, Table } \\
8-1, \text { Step 5c, } \\
\underline{2 \text { minutes }} \\
\text { travel time } \\
\text { was } \\
\text { assumed. }\end{array}$ & $\begin{array}{l}\text { Per ASEP HRAP, } \\
\text { Table } 8-1, \text { Step 5a, } \\
\text { five minutes were } \\
\text { assumed necessary } \\
\text { to retrieve and } \\
\text { read Attachment } \\
13 \text {. It was } \\
\text { conservatively } \\
\text { assumed that the } \\
\text { two relays could } \\
\text { be jumpered } \\
\text { within } 15 \text { minutes. }\end{array}$ & $\begin{array}{l}\text { Per columns } 3 \text { and } \\
4 \text { of this Table, } \\
\text { Total action time } \\
\text { would be } 22 \\
\text { minutes. }\end{array}$ & $\begin{array}{l}\text { Retrieving and reading Attachment } 13 \text { and jumpering } \\
\text { the two sets of relays in the main control room were } \\
\text { assumed to be completely dependent actions. }\end{array}$ \\
\hline $\begin{array}{l}\text { 2. Jumper two different } \\
\text { sets of relays in the } \\
\text { upper control room. }\end{array}$ & $\begin{array}{l}\text { Upper control } \\
\text { room }\end{array}$ & $\begin{array}{l}\text { Travel time } \\
\text { to upper } \\
\text { control (up } \\
\text { one flight of } \\
\text { stairs) was } \\
\text { conservativel } \\
\text { y assumed to } \\
\text { be } \underline{15} \\
\underline{\text { minutes. }}\end{array}$ & $\begin{array}{l}\text { As noted above, } \\
\text { performance time } \\
\text { for jumpering two } \\
\text { relays could be } \\
\text { easily } \\
\text { accomplished } \\
\text { within } 15 \text { minutes. }\end{array}$ & $\begin{array}{l}\text { Travel plus } \\
\text { performance time } \\
\text { equals about } \underline{30} \\
\text { minutes. }\end{array}$ & $\begin{array}{l}\text { Except for retrieving and reading Attachment } 13 \text {, this } \\
\text { set of actions could be accomplished in parallel with } \\
\text { the set of actions in activity } \# 1 \text { discussed above. } \\
\text { However, given the procedural demands for a second } \\
\text { check on each step of the task and the time available, } \\
\text { it was assumed that the actions would occur serially. } \\
\text { Jumpering the two sets of relays in the upper control } \\
\text { room were assumed to be completely dependent } \\
\text { actions. }\end{array}$ \\
\hline $\begin{array}{l}\text { 3. Open six valves from } \\
\text { the control room to vent } \\
\text { containment (per } \\
\text { Attachment } 13 \text { ). }\end{array}$ & $\begin{array}{l}\text { Main Control } \\
\text { Room }\end{array}$ & -. & 6 minutes & $\begin{array}{l}6 \text { minutes } \\
\text { Total Action Time } \\
\text { for the three sets of } \\
\text { actions would be } \\
\text { equal to: } 22+30+ \\
6 \text { minutes, which } \\
\text { is equal to } 58 \\
\text { minutes (or about } \\
1 \text { hour). }\end{array}$ & $\begin{array}{l}\text { Per ASEP Table } 8-1 \text {, Step } 5 b \text {, a } 1 \text { min. travel and } \\
\text { manipulation time was assumed for actions in the } \\
\text { control room. Opening the relevant valves was } \\
\text { assumed to be a completely dependent set of actions. }\end{array}$ \\
\hline
\end{tabular}


Table B.3.2-27

HEP 3: Diagnosis Time for Operator Action

\begin{tabular}{|c|c|c|c|c|}
\hline $\begin{array}{l}\text { Action } \\
\text { (1) }\end{array}$ & $\begin{array}{l}\text { Maximum Time } \\
\text { Available }\left(T_{m}\right) \\
\text { (2) }\end{array}$ & $\begin{array}{c}\text { Total Action } \\
\text { Time }\left(T_{2}\right) \\
(3)\end{array}$ & $\begin{array}{l}\text { Time Available } \\
\text { to Diagnosis }\left(T_{d}\right) \\
\text { (4) }\end{array}$ & $\begin{array}{c}\text { Comments/ } \\
\text { Source of } \\
\text { Information } \\
(5) \\
\end{array}$ \\
\hline $\begin{array}{l}\text { Diagnose the need to vent } \\
\text { containment. }\end{array}$ & 3 hours & 1 hours & 2 hours & \\
\hline
\end{tabular}

Table B.3.2-28

HEP 3: Diagnosis Analysis

\begin{tabular}{|c|c|c|c|c|}
\hline $\begin{array}{l}\text { Action } \\
\text { (1) }\end{array}$ & $\begin{array}{l}\text { Failure to } \\
\text { Diagnose } \\
\text { (2) }\end{array}$ & $\begin{array}{l}\text { Skill-Based } \\
\text { (3) }\end{array}$ & $\begin{array}{c}\text { Adjusted/ } \\
\text { Final HEP } \\
\text { (4) }\end{array}$ & $\begin{array}{c}\text { Comments/ } \\
\text { Source of Information } \\
(5)\end{array}$ \\
\hline $\begin{array}{l}\text { Diagnose the need to } \\
\text { vent containment. }\end{array}$ & $\begin{array}{l}\text { Per ASEP HRAP Table } 8-3 \text {, the } \\
\text { median value from ASEP } \\
\text { Figure } 8-1 \text { for } 2 \text { hours } \\
\text { diagnosis time was assigned. }\end{array}$ & N/A & $\begin{array}{l}\text { Median }=6.0 \mathrm{E}-5 \\
\mathrm{EF}=30 \\
\text { Mean }=5.1 \mathrm{E}-4\end{array}$ & \\
\hline
\end{tabular}


Table B.3.2-29

HEP 3: Post-Diagnosis Action Type Identification per Step 10, Table 8-1 of ASEP HRAP

\begin{tabular}{||l|l|l|l|l|l||}
\hline \multicolumn{1}{|c|}{\begin{tabular}{|c|c|c|} 
Action \\
(1)
\end{tabular}} & \multicolumn{1}{|c|}{$\begin{array}{c}\text { Safety Systems } \\
\text { Failed } \\
\text { (2) }\end{array}$} & $\begin{array}{l}\text { EOPs, Training, } \\
\text { Use EOPs, Well } \\
\text { Designed EOPs } \\
\text { (3) }\end{array}$ & $\begin{array}{c}\text { Individual } \\
\text { Operator Must } \\
\text { Perform Concurrent } \\
\text { Tasks } \\
\text { (4) }\end{array}$ & $\begin{array}{c}\text { Dynamic or } \\
\text { Step-by-Step } \\
\text { (5) }\end{array}$ & $\begin{array}{c}\text { Comments/ } \\
\text { Source of } \\
\text { Information } \\
\text { (6) }\end{array}$ \\
\hline $\begin{array}{l}\text { Carry-out the } \\
\text { three sets of } \\
\text { actions } \\
\text { necessary to } \\
\text { vent } \\
\text { containment. }\end{array}$ & $\begin{array}{l}\text { N/A - No additional safety } \\
\text { systems are assumed to fail } \\
\text { at this point. }\end{array}$ & $\begin{array}{l}\text { Although the lower } \\
\text { bound value from the } \\
\text { diagnosis model was } \\
\text { not used, the EOPs } \\
\text { and Attachment 13 } \\
\text { are clear and the } \\
\text { tasks are } \\
\text { straightforward. } \\
\text { Thus, the actions } \\
\text { were not assumed to } \\
\text { be dynamic. }\end{array}$ & So & & \\
\hline
\end{tabular}


Table B.3.2-30

HEP 3: Post-Diagnosis Stress-Level Identification per Step 10, Table 8-1 of ASEP HRAP

\begin{tabular}{|c|c|c|c|c|c|c|}
\hline $\begin{array}{l}\text { Action } \\
\text { (1) }\end{array}$ & $\begin{array}{c}\mathbf{T}_{\mathrm{m}}<\mathbf{2 h} \\
\text { After } \\
\mathbf{1 E} \\
(\mathbf{2})\end{array}$ & $\begin{array}{c}\text { Recirc. } \\
\text { Phase } \\
\text { in } \\
\text { Large } \\
\text { LOCA } \\
(3) \\
\end{array}$ & $\begin{array}{c}\text { More } \\
\text { Than Two } \\
\text { Safety } \\
\text { Systems } \\
\text { Fail } \\
(4) \\
\end{array}$ & $\begin{array}{l}\text { Operator } \\
\text { Familiar } \\
\text { W/Sequence } \\
\quad(5)\end{array}$ & $\begin{array}{l}\text { Stress Level } \\
\text { (6) }\end{array}$ & $\begin{array}{c}\text { Comments/ } \\
\text { Source of Information } \\
\text { (7) }\end{array}$ \\
\hline $\begin{array}{l}\text { Carry-out the } \\
\text { three sets of } \\
\text { actions } \\
\text { necessary to } \\
\text { vent } \\
\text { containmen } \\
t\end{array}$ & $\mathrm{~N} / \mathrm{A}^{1}$ & See comments & $\begin{array}{l}\text { See } \\
\text { comments }\end{array}$ & $\begin{array}{l}\text { Yes - see } \\
\text { comments }\end{array}$ & $\begin{array}{l}\text { Moderately } \\
\text { High }\end{array}$ & $\begin{array}{l}\text { Given the substantial time available to } \\
\text { accomplish the task, the fact that an } \\
\text { Emergency Response Team would be in } \\
\text { place, and that a verifier would accompany } \\
\text { the individual performing the jumpering of } \\
\text { the relays, moderate as opposed to extremely } \\
\text { high stress was assigned. }\end{array}$ \\
\hline
\end{tabular}


Table B.3.2-31

HEP 3: Total HEP

\begin{tabular}{|c|c|c|c|c|c|}
\hline $\begin{array}{l}\text { Action } \\
\text { (1) }\end{array}$ & $\begin{array}{c}\text { Original } \\
\text { Operator HEP } \\
\left(\mathrm{HEP}_{\mathrm{op}}\right) \\
(2)\end{array}$ & $\begin{array}{c}\text { Independent } \\
\text { Check/Correction } \\
\text { HEP } \\
\left(\mathrm{HEP}_{\mathrm{r} 2}\right) \\
\text { (3) }\end{array}$ & $\begin{array}{c}\text { Total HEP } \\
\text { (4) }\end{array}$ & $\begin{array}{l}\text { EF } \\
(5)\end{array}$ & $\begin{array}{l}\text { Comments/ } \\
\text { Source of } \\
\text { Information } \\
\text { (6) }\end{array}$ \\
\hline $\begin{array}{l}\text { 1. Diagnose need } \\
\text { to vent } \\
\text { containment }\end{array}$ & $\begin{array}{l}\text { Median }=6.0 \mathrm{E}-5 \\
\mathrm{EF}=30 \\
\text { Mean }=5.1 \mathrm{E}-4\end{array}$ & N/A & $\begin{array}{ll}\text { Med. } & \text { Mean } \\
6.0 \mathrm{E}-5 & 5.1 \mathrm{E}-4\end{array}$ & (30) & \\
\hline $\begin{array}{l}\text { 2. Jumper two } \\
\text { different sets of } \\
\text { relays in the main } \\
\text { control room. }\end{array}$ & $\begin{array}{l}\text { Med. }=0.02 \\
\text { Mean }=0.032\end{array}$ & $\begin{array}{l}\text { Credit for a second check was given in this instance. Given the } \\
\text { initial time available for the tasks, the fact that a verifier would } \\
\text { accompany the individual assigned to perform the task and } \\
\text { written verification is required, the fact that feedback would be } \\
\text { immediate (decrease in pressure), and the seriousness of the } \\
\text { action, any initial failures in performing the actions would have } \\
\text { some probability of being recovered. Second check HEPs for } \\
\text { the action were: } \\
\text { Median }=0.2 \\
\text { Mean }=0.323\end{array}$ & $0.004 \quad 0.01$ & $(5)$ & $\begin{array}{l}\text { The second check } \\
\text { HEP is multiplied } \\
\text { by the original HEP } \\
\text { for a given action. }\end{array}$ \\
\hline $\begin{array}{l}\text { 3. Jumper two } \\
\text { different sets of } \\
\text { relays in the upper } \\
\text { control room. }\end{array}$ & $\begin{array}{l}\text { Med. }=0.02 \\
\text { Mean }=0.032\end{array}$ & Same as for action 2 above & $0.004 \quad 0.01$ & (5) & \\
\hline $\begin{array}{l}\text { 4. Open six valves } \\
\text { from the control } \\
\text { room to vent } \\
\text { containment (per } \\
\text { Attachment } 13 \text { ). }\end{array}$ & $\begin{array}{l}\text { Med. }=0.02 \\
\text { Mean }=0.032\end{array}$ & Same as for action 2 above & $0.004 \quad 0.01$ & (5) & \\
\hline $\begin{array}{l}\text { Total HEP and } \\
\text { Error Factor }\end{array}$ & & & $\begin{array}{l}\text { Total Median HEP }= \\
0.012 \\
\text { Total Mean } \\
\text { HEP }=0.031\end{array}$ & (5) & $\begin{array}{l}\text { The error factor } \\
\text { associated with the } \\
\text { dominant HEPs } \\
\text { was assigned. }\end{array}$ \\
\hline
\end{tabular}


Table B.3.2-32

HEP 4 Calculation

\begin{tabular}{||l|l||}
\hline \hline Human Action Event (1) & Depressurize Reactor Pressure Vessel (RPV) \\
\hline Event Description (2) & Operators must depressurize the vessel per emergency operating procedures. \\
\hline Event Context (3) & $\begin{array}{l}\text { An initiating event has occurred, vessel makeup has not been provided for any of several reasons, and the reactor } \\
\text { vessel level has dropped to Top of Active Fuel (TAF). As level continues to drop, if vessel pressure is high, emergency } \\
\text { depressurization is indicated in several places in EP-2, e.g., vessel level reaching - 210 in. In addition, depressurization } \\
\text { is indicated in EP-3 under several different sections related to containment control, e.g., suppression pool, drywell, and } \\
\text { containment temperature control and containment pressure control. Given the circumstances, several of the parameters } \\
\text { indicating the need to depressurize will be reached before core damage. HEP 4 assesses the probability that the } \\
\text { operators and Emergency Response Team would fail to depressurize given that the EPs direct them to do so. }\end{array}$ \\
\hline Applicable Procedures (4) & EP-2 (RPV Control, GGNS, Rev. 19), EP-3 (Containment Control, GGNS, Rev. 21) \\
\hline \hline
\end{tabular}

Table B.3.2-33

菅

\begin{tabular}{|c|c|c|c|}
\hline $\begin{array}{l}\text { Event/Occurrence } \\
\text { (of most interest) } \\
\text { (1) }\end{array}$ & $\begin{array}{c}\text { Time }\left(\mathrm{T}_{0}\right) \\
\text { Operator } \\
\text { Alerted } \\
(2)\end{array}$ & $\begin{array}{l}\text { Annunciator/Indication } \\
\text { (3) }\end{array}$ & $\begin{array}{l}\text { Comments/ } \\
\text { Source of } \\
\text { Information } \\
\text { (4) }\end{array}$ \\
\hline $\begin{array}{l}\text { Vessel level is dropping } \\
\text { and parameters are } \\
\text { reached which indicate } \\
\text { that the operators should } \\
\text { depressurize the vessel }\end{array}$ & 0 & $\begin{array}{l}\text { Several indicators are likely, including vessel level } \\
\text { reaching - } 210 \text { in., SP temperature and RPV pressure } \\
\text { outside the safe zone of the Heat Capacity Temperature } \\
\text { Limit, drywell temperature above } 330 \text { degrees F, } \\
\text { containment temperature below } 180 \text { degrees F, and/or } \\
\text { containment pressure outside the safe zone of the } \\
\text { Pressure Suppression Pressure (PSP). }\end{array}$ & $\begin{array}{l}\text { Because of the several ways in which the situation } \\
\text { of interest could be reached, it was not precisely } \\
\text { determined when the relevant parameters would } \\
\text { be reached. The question is whether or the not the } \\
\text { operators would depressurize in a "reasonable" } \\
\text { amount of time given that the relevant parameters } \\
\text { were reached. }\end{array}$ \\
\hline
\end{tabular}


Table B.3.2-34

HEP 4: Potential Operator Action

\begin{tabular}{|l|l|l|l|}
\hline \multicolumn{1}{|c|}{$\begin{array}{c}\text { Description } \\
\text { of Event } \\
\text { (1) }\end{array}$} & $\begin{array}{c}\text { Number of } \\
\text { Abnormal Events } \\
\text { (2) }\end{array}$ & $\begin{array}{c}\text { Activities (Tasks) } \\
\text { Required to Perform } \\
\text { Action and Procedures } \\
\text { (3) }\end{array}$ & $\begin{array}{c}\text { Comments/ } \\
\text { Source of Information } \\
\text { (4) }\end{array}$ \\
\hline $\begin{array}{l}\text { For any number of possible reasons, } \\
\text { vessel level has dropped to TAF and } \\
\text { makeup is not being provided. } \\
\begin{array}{l}\text { Vessel temperature and pressure is } \\
\text { increasing. }\end{array}\end{array}$ & $\begin{array}{l}\text { One abnormal event is assumed per } \\
\text { Table 8-1, Step 9.b. While failures } \\
\text { of several systems may occur in the } \\
\text { relevant scenarios, } \\
\text { the entry conditions for EP-2 and the } \\
\text { EP-3 are clear and any of several } \\
\text { different conditions will indicate } \\
\text { depressurization (see above). } \\
\text { Essentially the original abnormal } \\
\text { event is continuing. The EPs should } \\
\text { guide the operators to make the } \\
\text { appropriate response. }\end{array}$ & $\begin{array}{l}\text { Depressurize the vessel from the } \\
\text { control room using SRVs, MSIVs, } \\
\text { or any available means. }\end{array}$ & \\
\hline
\end{tabular}




\begin{tabular}{|c|c|c|c|c|}
\hline \multicolumn{5}{|c|}{$\begin{array}{l}\text { Table B.3.2-35 } \\
\text { HEP 4: Time Available to Diagnose and Perform the Task }\end{array}$} \\
\hline $\begin{array}{l}\text { Action } \\
\text { (1) }\end{array}$ & $\begin{array}{l}\text { Time by Which } \\
\text { Operator Must } \\
\text { Act }\left(T_{c d}\right) \\
\text { (2) }\end{array}$ & $\begin{array}{c}\text { Time at Which } \\
\text { Operator } \\
\text { is Alerted } \\
\text { that Symptom } \\
\text { has Occurred }\left(\mathrm{T}_{\mathrm{o}}\right) \\
\text { (3) }\end{array}$ & $\begin{array}{l}\text { Maximum Time } \\
\text { Available } \\
\text { to Perform the } \\
\text { Identified } \\
\text { Operator Activities } \\
\left(T_{m}\right) \\
(4)\end{array}$ & $\begin{array}{c}\text { Comments/ } \\
\text { Source of Information } \\
\text { (5) }\end{array}$ \\
\hline $\begin{array}{l}\text { Diagnose the } \\
\text { need and carry- } \\
\text { out the actions } \\
\text { required to } \\
\text { depressurize } \\
\text { the RPV. }\end{array}$ & 1 hour & 0 & 1 hour & $\begin{array}{l}\text { As noted above, the time available for the action } \\
\text { was not precisely determined because of the } \\
\text { different ways in which the need to depressurize } \\
\text { could be reached. The relevant constants are that } \\
\text { TAF has been reached, adequate vessel makeup is } \\
\text { not being provided, and several different indicators } \\
\text { should signal the need to depressurize. Obviously } \\
\text { the situation is not good and the concern is whether } \\
\text { or the not the operators will follow procedure and } \\
\text { depressurize within an hour after the indicators are } \\
\text { reached. If the operators follow procedure and } \\
\text { depressurize as instructed by the EPs, then the } \\
\text { consequences of the accident might be reduced. }\end{array}$ \\
\hline
\end{tabular}


Table B.3.2-36

HEP 4: Operator Action Performance Time

\begin{tabular}{|c|c|c|c|c|c|}
\hline $\begin{array}{l}\text { Activities } \\
\text { (1) }\end{array}$ & $\begin{array}{l}\text { Location } \\
\text { (2) }\end{array}$ & $\begin{array}{c}\text { Travel } \\
\text { Time }\left(\mathrm{T}_{\mathrm{L}}\right) \\
(3)\end{array}$ & $\begin{array}{l}\text { Performance } \\
\text { Time }\left(T_{P}\right) \\
(4)\end{array}$ & $\begin{array}{l}\text { Total Action } \\
\text { Time }\left(\mathrm{T}_{2}\right) \\
(5)\end{array}$ & $\begin{array}{l}\text { Comments/ } \\
\text { Source of Information } \\
(6)\end{array}$ \\
\hline $\begin{array}{l}\text { Open SRVs, MSIVs, } \\
\text { etc. to depressurize } \\
\text { vessel }\end{array}$ & Control Room & -. & 1 minute & 1 minute & $\begin{array}{l}\text { Per ASEP Table } 8-1 \text {, Step } 5 b \text {, a } 1 \mathrm{~min} \text {. travel and } \\
\text { manipulation time was assumed for actions in the } \\
\text { control room. }\end{array}$ \\
\hline
\end{tabular}

Table B.3.2-37

HEP 4: Diagnosis Time for Operator Action

\begin{tabular}{|c|c|c|c|c|}
\hline $\begin{array}{l}\text { Diagnose the need to } \\
\text { depressurize the RPV }\end{array}$ & 1 hour & 1 minute & Approx. 59 minutes & \\
\hline
\end{tabular}

Table B.3.2-38

HEP 4: Diagnosis Analysis

\begin{tabular}{|c|c|c|c|c|}
\hline $\begin{array}{l}\text { Action } \\
\text { (1) }\end{array}$ & $\begin{array}{l}\text { Failure to } \\
\text { Diagnose } \\
\text { (2) }\end{array}$ & $\begin{array}{c}\text { Skill-Based } \\
\text { (3) }\end{array}$ & $\begin{array}{c}\text { Adjusted/ } \\
\text { Final HEP } \\
\text { (4) }\end{array}$ & $\begin{array}{c}\text { Comments/ } \\
\text { Source of Information } \\
(\mathbf{5})\end{array}$ \\
\hline $\begin{array}{l}\text { Diagnose the need to } \\
\text { depressurize the } \\
\text { vessel. }\end{array}$ & $\begin{array}{l}\text { Per ASEP HRAP Table } 8-3 \text {, the } \\
\text { median value from ASEP } \\
\text { Figure } 8-1 \text { for approx. } 59 \\
\text { minutes diagnosis time was } \\
\text { assigned. }\end{array}$ & & $\begin{array}{l}\text { Median }=1.0 \mathrm{E}-4 \\
\mathrm{EF}=30 \\
\text { Mean }=8.5 \mathrm{E}-4\end{array}$ & \\
\hline
\end{tabular}


Table B.3.2-39

HEP 4: Post-Diagnosis Action Type Identification per Step 10, Table 8-1 of ASEP HRAP

\begin{tabular}{|c|c|c|c|c|c|}
\hline $\begin{array}{c}\text { Action } \\
\text { (1) }\end{array}$ & $\begin{array}{l}\text { Safety Systems } \\
\text { Failed } \\
\text { (2) }\end{array}$ & $\begin{array}{l}\text { EOPs, Training, } \\
\text { Use EOPs, Well } \\
\text { Designed EOPs } \\
\text { (3) }\end{array}$ & $\begin{array}{c}\text { Individual } \\
\text { Operator Must } \\
\text { Perform Concurrent } \\
\text { Tasks } \\
\text { (4) }\end{array}$ & $\begin{array}{c}\text { Dynamic or } \\
\text { Step-by-Step } \\
\text { (5) }\end{array}$ & $\begin{array}{l}\text { Comments/ } \\
\text { Source of } \\
\text { Information } \\
\text { (6) }\end{array}$ \\
\hline $\begin{array}{l}\text { Depressurize } \\
\text { the vessel }\end{array}$ & $\begin{array}{l}\text { N/A - No additional safety } \\
\text { systems are assumed to fail } \\
\text { at this point. }\end{array}$ & $\begin{array}{l}\text { Although the lower } \\
\text { bound value from the } \\
\text { diagnosis model was } \\
\text { not used, the EOPs } \\
\text { are clear and the } \\
\text { operators need only } \\
\text { open SRVs from the } \\
\text { control room. Thus, } \\
\text { the actions were not } \\
\text { assumed to be } \\
\text { dynamic. }\end{array}$ & No & Step-by-Step & \\
\hline
\end{tabular}


Table B.3.2-40

HEP 4: Post-Diagnosis Stress-Level Identification

per Step 10, Table 8-1 of ASEP HRAP

\begin{tabular}{|c|c|c|c|c|c|c|}
\hline $\begin{array}{l}\text { Action } \\
\text { (1) }\end{array}$ & $\begin{array}{c}\mathrm{T}_{\mathrm{m}}<2 \mathrm{~h} \\
\text { After } \\
\mathbf{1 E} \\
(2)\end{array}$ & $\begin{array}{l}\text { Recirc. } \\
\text { Phase } \\
\text { in } \\
\text { Large } \\
\text { LOCA } \\
\text { (3) } \\
\end{array}$ & $\begin{array}{l}\text { More } \\
\text { Than Two } \\
\text { Safety } \\
\text { Systems } \\
\text { Fail } \\
\text { (4) }\end{array}$ & $\begin{array}{c}\text { Operator } \\
\text { Familiar } \\
\text { W/Sequence } \\
\text { (5) }\end{array}$ & $\begin{array}{l}\text { Stress Level } \\
\text { (6) }\end{array}$ & $\begin{array}{l}\text { Comments/ } \\
\text { Source of Information } \\
(7)\end{array}$ \\
\hline $\begin{array}{l}\text { Depressurize the } \\
\text { vessel }\end{array}$ & $\mathrm{N} / \mathrm{A}^{1}$ & Maybe & Maybe & Maybe & $\begin{array}{l}\text { Extremely } \\
\text { High }\end{array}$ & $\begin{array}{l}\text { With vessel level dropping to TAF in } \\
\text { scenarios where safety systems have failed } \\
\text { and possibly in the context of a LOCA, } \\
\text { extremely high stress was assumed during } \\
\text { the time period of interest. }\end{array}$ \\
\hline
\end{tabular}

1 At least moderately high stress was assumed for all events. 
Table B.3.2-41

HEP 4: Total HEP

\begin{tabular}{|c|c|c|c|c|c|}
\hline $\begin{array}{l}\text { Action } \\
\text { (1) }\end{array}$ & $\begin{array}{c}\text { Original } \\
\text { Operator HEP } \\
\left(\text { HEP }_{\text {op }}\right) \\
\text { (2) }\end{array}$ & $\begin{array}{c}\text { Independent } \\
\text { Check/Correction } \\
\text { HEP } \\
\left(\text { HEP }_{\mathrm{r} 2}\right) \\
\text { (3) }\end{array}$ & $\begin{array}{c}\text { Total HEP } \\
\text { (4) }\end{array}$ & $\begin{array}{l}\text { EF } \\
\text { (5) }\end{array}$ & $\begin{array}{l}\text { Comments/ } \\
\text { Source of } \\
\text { Information } \\
(0)\end{array}$ \\
\hline $\begin{array}{l}\text { 1. Diagnose } \\
\text { need to } \\
\text { depressurize the } \\
\text { vessel }\end{array}$ & $\begin{array}{l}\text { Median }=1.0 \mathrm{E}-4 \\
\mathrm{EF}=30 \\
\text { Mean }=8.5 \mathrm{E}-4\end{array}$ & & $\begin{array}{ll}\text { Med. } & \text { Mean } \\
1.0 \mathrm{E}-4 & 8.5 \mathrm{E}-4\end{array}$ & (30) & \\
\hline $\begin{array}{l}\text { 2. Open SRVs, } \\
\text { MSIVs, etc. to } \\
\text { depressurize } \\
\text { vessel. }\end{array}$ & $\begin{array}{l}\text { Med. }=0.05 \\
\text { Mean }=0.081\end{array}$ & $\begin{array}{l}\text { Credit for a second and third check was given in this } \\
\text { instance. The EPs are clear, the action is simple, and an } \\
\text { initial failure to carry-out the needed actions would be } \\
\text { likely to be detected by the crew, i.e., the operators } \\
\text { would be monitoring the relevant parameters. Second } \\
\text { and third check HEPs for the action were: } \\
\text { Median }=0.5 \\
\text { Mean }=0.81\end{array}$ & $\frac{0.013}{0.013} \quad \frac{0.053}{0.054}$ & (5) & $\begin{array}{l}\text { The second } \\
\text { check HEP is } \\
\text { multiplied by the } \\
\text { original HEP for } \\
\text { a given action. } \\
\text { The resulting } \\
\text { product is } \\
\text { multiplied by the } \\
\text { HEP for the } \\
\text { third check. }\end{array}$ \\
\hline $\begin{array}{l}\text { Total HEP and } \\
\text { Error Factor }\end{array}$ & & & $\begin{array}{l}\text { Total Median HEP } \\
=0.013 \\
\text { Total Mean } \\
\text { HEP }=0.054\end{array}$ & (5) & $\begin{array}{l}\text { The error factor } \\
\text { associated with } \\
\text { the dominant } \\
\text { HEP was } \\
\text { assigned. }\end{array}$ \\
\hline
\end{tabular}


Appendix B

\section{B.4 Accident Progression Bin Definitions}

As each path through the APET is evaluated, the result of that evaluation is stored by assigning it to an Accident Progression Bin (APB). The APBs are the means by which information is passed from the accident progression analysis to the source term analysis (see Section 7), and as such the bin describes the evaluation in enough detail that a source term (the release of radioactive material) can be estimated for it. The binning scheme for the POS 5 analysis utilizes fourteen characteristics or quantities that define a certain feature of the accident progression (the definition of the APBs is analogous to the definition of PDSs). A bin is defined by specifying a letter for each of the 14 characteristics, where each letter for each characteristic has a certain meaning. For each characteristic, the possible states are termed attributes. The selection of the characteristics and attributes is based on the information that is needed in the radionuclide release and transport analysis to estimate the source term. The fourteen characteristics used in this analysis and their associated attributes are identified and described in Table B.4-1. The logic used to assign individual accident progressions to APBs is included in the APET input listed in Appendix B, Section B.2. 
Table B.4-1

Accident Progression Bin Definitions

\begin{tabular}{|c|c|c|}
\hline Attribute & Mnemonic & Description \\
\hline \multicolumn{3}{|c|}{ Characteristic 1: Plant Damage State (PDS) } \\
\hline$A-P$ & $\begin{array}{l}\text { PDS1-1 - } \\
\text { PDS3-1 }\end{array}$ & Attributes A through P identify individually the 16 PDSs \\
\hline \multicolumn{3}{|c|}{ Characteristic 2: Containment Status (CNT-STATUS) } \\
\hline A & OCnt-BCD & $\begin{array}{l}\text { The containment equipment hatch is open before core damage and remains open } \\
\text { throughout the accident. }\end{array}$ \\
\hline $\mathrm{B}$ & Vnt-CD & The containment is vented during core damage. \\
\hline $\mathrm{C}$ & Cnt-Rpt-CD & $\begin{array}{l}\text { The containment fails, via a rupture in the containment wall above the auxiliary } \\
\text { building roof, during core damage. }\end{array}$ \\
\hline $\mathrm{D}$ & Cnt-Lk-CD & $\begin{array}{l}\text { The containment fails, via a leak in the containment wall above the auxiliary } \\
\text { building roof, during core damage. }\end{array}$ \\
\hline E & Cnt-Rpt-VB & $\begin{array}{l}\text { The containment fails, via a rupture in the containment wall, from loads } \\
\text { accompanying vessel failure. }\end{array}$ \\
\hline F & Cnt-Lk-VB & $\begin{array}{l}\text { The containment fails, via a leak in the containment wall, from loads } \\
\text { accompanying vessel failure. }\end{array}$ \\
\hline G & Vnt-Late & The containment is vented during the late time regime. \\
\hline $\mathrm{H}$ & Cnt-Rpt-Late & $\begin{array}{l}\text { The containment fails, via a rupture in the containment wall, from loads that } \\
\text { occur late in the accident. }\end{array}$ \\
\hline I & Cnt-Lk-Late & $\begin{array}{l}\text { The containment fails, via a leak in the containment wall, from loads that occur } \\
\text { late in the accident. }\end{array}$ \\
\hline $\mathrm{J}$ & Cnt-NF-Late & $\begin{array}{l}\text { The containment is closed prior to core damage and its pressure boundary is } \\
\text { maintained during the remaining portion of the accident. }\end{array}$ \\
\hline \multicolumn{3}{|c|}{ Characteristic 3: Auxiliary building pressure integrity status - AUX-STATUS } \\
\hline A & OAux-BCD & $\begin{array}{l}\text { The containment is open during the accident and the auxiliary building fails } \\
\text { from overpressurization prior to the onset of core damage. }\end{array}$ \\
\hline B & OAux-CD & $\begin{array}{l}\text { The containment is open during the accident and the auxiliary building fails } \\
\text { from overpressurization during core damage. }\end{array}$ \\
\hline $\mathrm{C}$ & OAux-VB & $\begin{array}{l}\text { The containment is open during the accident and the auxiliary building fails } \\
\text { from overpressurization after vessel failure. }\end{array}$ \\
\hline $\mathrm{D}$ & nOAux & The containment is closed prior to the onset of core damage. \\
\hline
\end{tabular}


Table B.4-1 (continued)

\begin{tabular}{|c|c|c|}
\hline Attribute & Mnemonic & Description \\
\hline \multicolumn{3}{|c|}{ Characteristic 4: Drywell pressure integrity status (DW-STATUS) } \\
\hline A & Op-DW-BCD & $\begin{array}{l}\text { The drywell equipment hatch and/or personnel lock are open at the start of the } \\
\text { accident. This is the case that is assumed in this analysis. }\end{array}$ \\
\hline B & Cls-DW-BCD & $\begin{array}{l}\text { The drywell equipment hatch and personnel lock are closed prior to the onset of } \\
\text { core damage. }\end{array}$ \\
\hline \multicolumn{3}{|c|}{ Characteristic 5: Status of the reactor vessel pressure boundary (RPV-ISO) } \\
\hline A & Iso-RPV-E & $\begin{array}{l}\text { The reactor vessel is isolated and its pressure boundary is intact prior to core } \\
\text { damage. }\end{array}$ \\
\hline B & RPV-LOCA & $\begin{array}{l}\text { The accident is initiated by a pipe break in the drywell (i.e., LOCA); thus, the } \\
\text { reactor vessel pressure boundary is breached prior to core damage. }\end{array}$ \\
\hline $\mathrm{C}$ & Iso-RPV-CD & $\begin{array}{l}\text { The reactor vessel was not isolated prior to core damage (either open MSIVs or } \\
\text { an unisolated interfacing systems LOCA); the vessel is, however, isolated during } \\
\text { core damage. }\end{array}$ \\
\hline $\mathrm{D}$ & nIso-RPV-CD & $\begin{array}{l}\text { The reactor vessel was not isolated prior to core damage (either open MSIVs or } \\
\text { an unisolated interfacing systems LOCA) and the vessel is not isolated during } \\
\text { core damage. It is assumed that any releases from the vessel, prior to vessel } \\
\text { failure, will bypass the containment and will pass directly into the auxiliary } \\
\text { building. }\end{array}$ \\
\hline
\end{tabular}

Characteristic 6: Status of the reactor head vent prior to core damage (RPV-VNT)
A RPV-nVnt
The reactor head vent is closed during core damage.
B RPV-OVnt
The reactor head vent is open throughout the accident.

Characteristic 7: Status of the SRV tailpipe vacuum breakers (SRV-VBkr)
A OSRV-VBkr
A vacuum breaker on the SRV tailpipe that is being used to relieve the pressure in the RPV sticks open either before or during core damage.
B $\quad$ cSRV-VBkr
None of the vacuum breakers on the SRV tailpipes stick open prior to vessel failure.

Characteristic 8: Status of the reactor vessel just prior to vessel failure (RPV-VB)
A RPV-HiP-nInj
The vessel is pressurized (i.e., near system pressure) just prior to vessel failure and core coolant is not being injected into the vessel.
B RPV-LoP-nInj The pressure in the vessel is less than 200 psig just prior to vessel failure and core coolant is not being injected into the vessel.
C RPV-HiP-Inj The vessel is pressurized (i.e., near system pressure) just prior to vessel failure and core coolant is being injected into the vessel.
D RPV-LoP-Inj The pressure in the vessel is less than 200 psig just prior to vessel failure and core coolant is being injected into the vessel. 
Table B.4-1 (continued)

\begin{tabular}{|c|c|c|}
\hline Attribute & Mnemonic & Description \\
\hline \multicolumn{3}{|c|}{ Characteristic 8: Status of the reactor vessel just prior to vessel failure (RPV-VB) (continued) } \\
\hline $\mathrm{E}$ & nVB-HiP & $\begin{array}{l}\text { Core cooling is restored with the vessel at high pressure and the core damage } \\
\text { process is arrested in the vessel and the accident is terminated with the core in a } \\
\text { safe stable state (e.g., TMI). }\end{array}$ \\
\hline $\mathrm{F}$ & nVB-LoP & $\begin{array}{l}\text { Core cooling is restored with the vessel at low pressure and the core damage } \\
\text { process is arrested in the vessel and the accident is terminated with the core in a } \\
\text { safe stable state (e.g., TMI). }\end{array}$ \\
\hline \multicolumn{3}{|c|}{ Characteristic 9: Status of containment sprays (CNT-SPRAYS) } \\
\hline A & $\mathrm{nCS}-\mathrm{CD}$ & Containment sprays are not used during the core damage process. \\
\hline B & CS-CD & Containment sprays are used during the core damage process. \\
\hline \multicolumn{3}{|c|}{ Characteristic 10: Fraction of zirconium oxidized in the vessel prior to vessel failure (ZROXID-CD) } \\
\hline A & ZrOxid-Hi & $\begin{array}{l}\text { The fraction of zirconium oxidized in the vessel prior to vessel failure is greater } \\
\text { than } 0.21 \text {. }\end{array}$ \\
\hline B & ZrOxid-Lo & $\begin{array}{l}\text { The fraction of zirconium oxidized in the vessel prior to vessel failure is less } \\
\text { than } 0.21 \text {. }\end{array}$ \\
\hline \multicolumn{3}{|c|}{ Characteristic 11: Fraction of core participating in HPME or steam explosions (HPME-SE) } \\
\hline A & HiHPME & Forty percent of the core participates in HPME \\
\hline B & LoHPME & Ten percent of the core participates in HPME \\
\hline $\mathrm{C}$ & HiEXSE & $\begin{array}{l}\text { An HPME event does not occur; however, } 40 \% \text { percent of the core participates } \\
\text { in an ex-vessel steam explosion. }\end{array}$ \\
\hline $\mathrm{D}$ & LoEXSE & $\begin{array}{l}\text { An HPME event does not occur; however, } 10 \% \text { of the core participates in an ex- } \\
\text { vessel steam explosion. }\end{array}$ \\
\hline $\mathrm{E}$ & nHPME-SE & Neither an HPME event nor an ex-vessel steam explosion occurs. \\
\hline \multicolumn{3}{|c|}{ Characteristic 12: Status of the core debris in the reactor pedestal cavity (TYPE-CCI) } \\
\hline A & DryCCI & Core-concrete interactions proceed in a dry cavity following vessel failure. \\
\hline B & FldCCI & Core-concrete interactions proceed in a flooded cavity following vessel failure. \\
\hline $\mathrm{C}$ & noCCI & $\begin{array}{l}\text { The core debris in the cavity is quenched and core-concrete interactions are } \\
\text { avoided. }\end{array}$ \\
\hline
\end{tabular}


Appendix B

Table B.4-1 (concluded)

\begin{tabular}{|c|c|c|}
\hline Attribute & Mnemonic & Description \\
\hline \multicolumn{3}{|c|}{ Characteristic 13: Initiating event time window (IE-TIME) } \\
\hline A & IE-Win 1 & $\begin{array}{l}\text { The initiating event occurs sometime between } 14 \text { hours after shutdown and } 24 \\
\text { hours after shutdown. }\end{array}$ \\
\hline B & IE-Win2 & $\begin{array}{l}\text { The initiating event occurs sometime between } 24 \text { hours after shutdown and } 94 \\
\text { hours after shutdown. }\end{array}$ \\
\hline B & IE-Win3 & $\begin{array}{l}\text { The initiating event occurs sometime between } 40 \text { days after shutdown and } 50 \\
\text { days after shutdown. }\end{array}$ \\
\hline \multicolumn{3}{|c|}{ Characteristic 14: Temperature of the Suppression Pool (SP-TEMP) } \\
\hline A & SP-SupCld & The suppression pool is subcooled at the onset of core damage. \\
\hline B & SP-Sat & The suppression pool is saturated at the onset of core damage. \\
\hline
\end{tabular}




\section{B.5 References}

[Griesmeyer et al., 1989]

J. M. Griesmeyer et al., "A Reference Manual for the Event Progression Analysis Code (EVNTRE)," NUREG/CR-5174, SAND88-1607, Sandia National Laboratories, September 1989.

[Roginski, 1994]

R. J. Roginski, "ETPRE User's Manual Version 3.00," SAND93-3834, Sandia National Laboratories, May 1994.

[Swain, 1987]

[Wheeler et al., 1989]

A. D. Swain, "Accident Sequence Evaluation Program Human Reliability Analysis Procedure," NUREG/CR-4772, February 1987.

T. A. Wheeler et al., "Analysis of Core Damage Frequency From Internal Events: Expert Judgement Elicitation," NUREG/CR-4550, SAND86-2084, Vol. 2, Sandia National Laboratories, April 1989.

[Iman and Hora, 1988]

R. L. Iman and S. C. Hora, "Modeling Time to Recovery and Initiating Event Frequency for Loss of Off-Site Power Incidents and Nuclear Power Plants," NUREG/CR-5032, SAND87-2428, Sandia National Laboratories, January 1988.

[Baranowsky, 1985]

P. W. Baranowsky, "Evaluation of Station Blackout Accidents at Nuclear Power Plants: Technical Findings Related to Unresolved Safety Issue A-44," NUREG/CR-1032, May 1985.

[Brown et al., 1990]

T. D. Brown et al., "Evaluation of Severe Accident Risks: Grand Gulf Unit 1," NUREG/CR-4551, SAND86-1309, Vol. 6, Rev. 1, Sandia National Laboratories, December 1990.

[Harper et al., 1991]

F. T. Harper, "Evaluation of Severe Accident Risks: Quantification of Major Input Parameters: Experts' Determination of Containment Loads and Molten Core Containment Interaction Issues," NUREG/CR-4551, Vol. 2, Rev. 1, Part 2, Sandia National Laboratories, April 1991.

[Harper et al., 1990]

F. T. Harper, "Evaluation of Severe Accident Risks: Quantification of Major Input Parameters: Expert Opinion Elicitation on In-Vessel Issues," NUREG/CR-4551, Vol. 2, Rev. 1, Part 1, Sandia National Laboratories, December 1990.

[Harper et al., 1992] F. T. Harper, "Evaluation of Severe Accident Risks: Quantification of Major Input Parameters: Experts' Determination of Structural Response Issues," NUREG/CR-4551, Vol. 2, Rev. 1, Part 3, Sandia National Laboratories, March 1992.

[Harper et al., 1994] F. T. Harper, "Evaluation of Severe Accident Risks: Quantification of Major Input Parameters: Determination of Parameter Values Not Quantified Using Formal Expert Elicitation," NUREG/CR-4551, Vol. 2, Rev. 1, Part 6, Sandia National Laboratories, 1994."

[USNRC, 1975]

U. S. Nuclear Regulatory Commission, "Reactor Safety Study - An Assessment of Accident Risks in U. S. Commercial Nuclear Power Plants," WASH-1400 (NUREG-75/014), October 1975.

* Copies are available for inspection or copying for a fee in the NRC Public Document room. 
Appendix B 


\section{Appendix C Supporting Information for the Source Term Analysis}

This appendix provides supporting information for the source term analysis. Section C.1 provides a list of the FORTRAN code GGSORP5.FOR that implements the parametric expressions used to estimate the source terms. Section C.2 provides a listing of the input file for GGSORP5 that contains the data for the parameters in the parametric expression. Section C.3 is a listing of the source term for each source term group defined using the PARTITION code (the version modified in the LaSalle probabilistic risk assessment performed in the PRUEP program).

\section{C.1 Listing of GGSORP5.FOR}

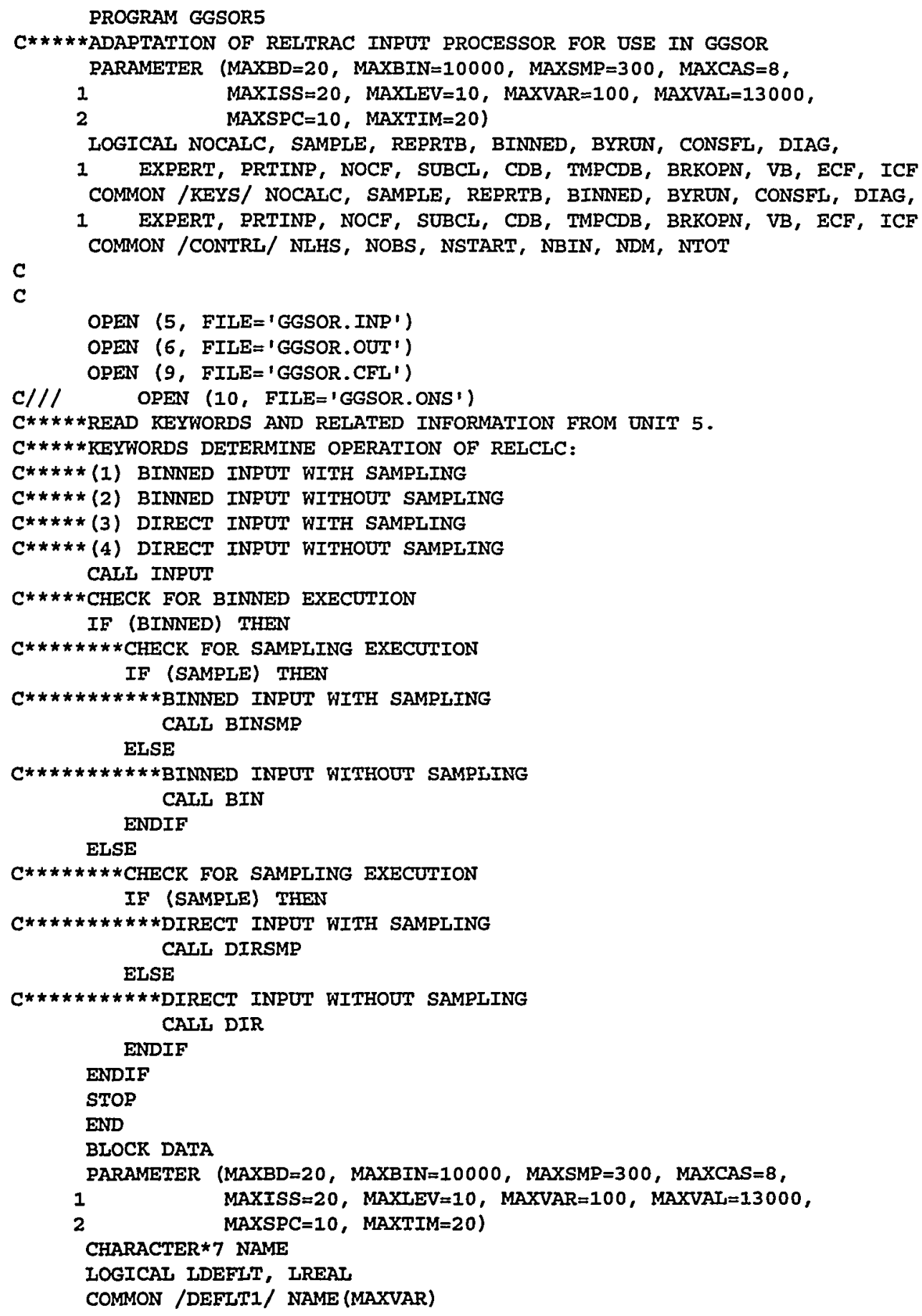


Appendix C

COMMON /DEFLT2/ NVAR, NVAL, NVCB1, NVCB2, NVCB3, NVCB4, 1

2

NVCB5, IDIMEN (3, MAXVAR), ISPOS (MAXVAR) ISMPPS (MAXVAL) , IPNT (MAXVAR) , IDEFLT (MAXVAL), LREAL (MAXVAL)

$C \star \star \star \star \star$ DEFINE VARIABLE NAMES AND CORRESPONDING DIMENSIONS TO BE SET $C * * \star * *$ THROUGH DEFAULT AND SAMPLE VECTOR SUBSTITUTION FOR BINNED $C * * * * *$ EXECUTION. VARIABLE NAMES AND DIMENSIONS CORRESPOND EXACTLY $C * * * * *$ TO ORDER OF VARIABLES IN COMMON BLOCKS:

$C * * * * *$ (1) BASVAL, (2) BINNED, AND (3) EXPERT

$C \star \star \star \star \star A S$ IF THESE COMMON BLOCKS ARE CONCATENTATED. DATA NAME /

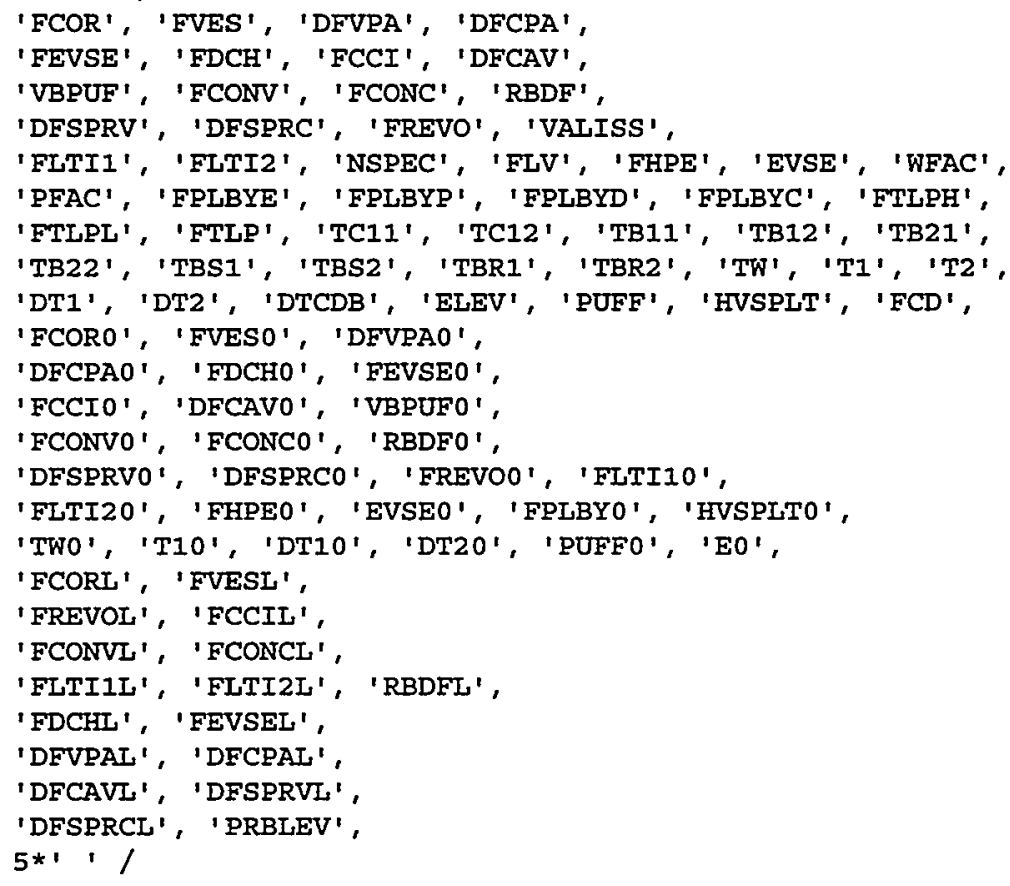

$C \star \star \star \star *$ DEFINE 3 DIMENSIONS FOR EACH OF THE VARIABLES

DATA IDIMEN /

MAXSPC $, 1,1, \operatorname{MAXSPC}, 1,1, \operatorname{MAXSPC}, 1,1, \operatorname{MAXSPC}, 1,1$, MAXSPC $1,1, \operatorname{MAXSPC}, 1,1, \operatorname{MAXSPC}, 1,1, \operatorname{MAXSPC}, 1,1$, MAXSPC , 1, 1, MAXSPC, 1, 1, MAXSPC, 1, 1, MAXSPC, 1, 1, MAXSPC $, 1,1$, MAXSPC, 1, 1, MAXSPC $, 1,1$, MAXISS , 1, 1, $1,1,1,1,1,1,1,1,1,1,1,1,1,1,1,1,1,1,1,1,1$, $1,1,1,1,1,1,1,1,1,1,1,1,1,1,1,1,1,1$, 
$C \star \star \star \star \star$ DEFINE NUMBERS OF VALUES IN COMMON BLOCRS:

$C \star \star \star \star \star$ (1) BASVAL, (2) BINNED, AND (3) EXPERT

DATA NVCB1 / 205 /, NVCB2 / $1356 /$, NVCB3 / 11370 /, NVCB4 / 0 /,

END

SUBROUTINE INPUT

$C * \star \star * *$ PROCESS KEYWORD INPUT ON UNIT 5

PARAMETER (MAXLEN $=101$ )

PARAMETER (MAXBD $=20, M A X B I N=10000, M A X S M P=300, M A X C A S=8$,

MAXISS $=20, M A X L E V=10, M A X V A R=100, M A X V A L=13000$,

2 MAXSPC $=10, \operatorname{MAXTIM}=20$ )

COMMON /CONTRI/ NLHS, NOBS, NSTART, NBIN, NDM, NTOT

LOGICAL NOCALC, SAMPLE, REPRTB, BINNED, BYRON, CONSFL, DIAG,

1 EXPERT, PRTINP, NOCF, SUBCL, CDB, TMPCDB, BRKOPN, VB, ECF, ICF COMMON /KEYS/ NOCALC, SAMPLE, REPRTB, BINNED, BYRUN, CONSFL, DIAG,

1 EXPERT, PRTINP, NOCF, SUBCL, CDB, TMPCDB, BRKOPN, VB, ECF, ICF CHARACTER BINARR* (MAXBD), BTITLE*80, TITLE*80 COMMON /BINS/ BINARR (MAXBIN), BTITLE, TITLE CHARACTER $* 80$ FILNAM CHARACTER 80 DEFFIL, SAMFIL, VECFIL COMMON /FILBLK/ DEFFIL, SAMFIL, VECEIL CHARACTER CARD* (MAXLEN), CVAL* (MAXLEN), KEYWRD*20

$C * \star * * \star$ SET LOGICAL TYPES FOR FREE FORMAT SUBROUTINE RDSTRG

C LOGICAL EOR, LVAI, TYPE(4)

C

C*****INITIALLIZE COLUMN POINTER FOR CURRENT RECORD

$I C=1$

$C \star \star \star \star \star$ READ RECORD READ $(5,1001)$ CARD

$C * \star * * *$ READ MODE SWITCH

CALI RDSTRG (CARD, IC, KEYWRD, LVAL, IVAL, RVAL, KLNGTH, TYPE, EOR)

$C \star \star \star \star \star$ CHECK FOR BINNED OR DIRECT EXECUTION

IF (KEYWRD ( $1:$ KLNGTH) .EQ. 'BINNED') THEN

$C \star \star \star \star \star \star \star \star$ SET BINNED EXECUTION TYPE

BINNED = . TRUE.

ELSE IF (KEYWRD ( $1:$ KLNGTH) .EQ. 'DIRECT') THEN

$C \star \star \star \star \star \star \star \star$ SET DIRECT EXECUTION TYPE

BINNED $=$.FALSE.

ELSE

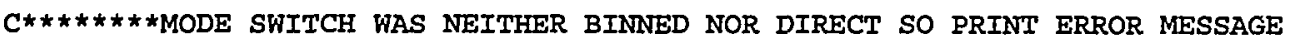
$\operatorname{WRITE}(6,5030)$ STOP

ENDIF

$C \star \star \star \star \star$ SET DEFAULT VAIUES

SAMPLE $=$. FAISE.

NOCALC $=$. FALSE .

PRTINP $=$.FAISE.

NOBS $=1$

REPRTB = . FAISE.

BYRUN = FALSE.

CONSFL $=$. FALSE

DIAG = . FALSE .

EXPERT $=$. FALSE .

$C * * * \star *$ INITIALLIZE NUMBER OF BINS

NBIN $=0$

$C \star \star \star \star \star$ READ TITLE

$\operatorname{READ}(5,1001)$ TITLE

C $\star \star \star \star *$ PRINT MESSAGE FOR EXECUTION TYPE AND TITLE

WRITE $(6,1003)$ KEYWRD (1:KLNGTH), TITLE

$\operatorname{WRITE}(6,1002)$ CARD

WRITE $(6,1002)$ TITLE

Vol. 6, Part 1 


\section{Appendix C}

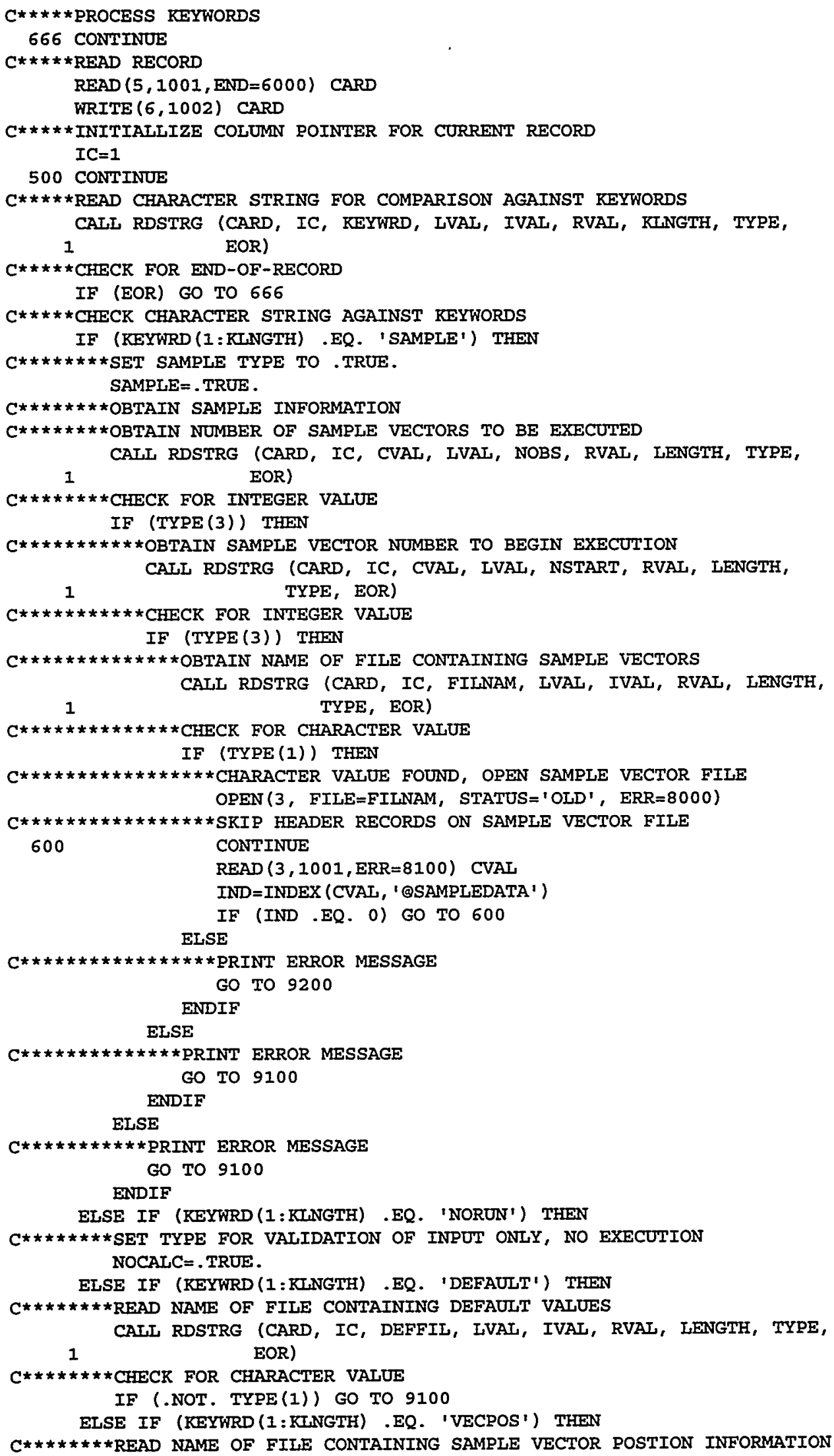


1 CALL RDSTRG (CARD

C $\star \star \star \star \star \star \star *$ CHECR FOR CHARACTER VAIUE

IF (.NOT. TYPE(1)) GO TO 9100

ELSE IF (KEYWRD ( $1:$ KINGTH) .EQ. 'BINFILE') THEN

C $\star \star \star \star \star \star \star \star$ READ A BIN ARRAY FILE

C $\star \star \star \star \star \star \star \star$ CHECK FOR BINNED EXECUTION

IF (BINNED) THEN

C $\star \star \star \star \star \star \star \star \star \star \star$ READ NAME OF FILE CONTAINING BIN INFORMATION

1

CALL RDSTRG (CARD, IC, FILNAM, LVAL, IVAL, RVAL, LENGTH,

IF (TYPE (1)) THEN

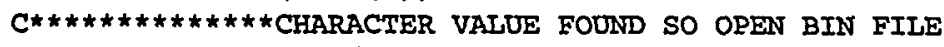

OPEN $(4$, FILE $=$ FILNAM， STATUS $=$ 'OLD'， ERR $=8000)$ ELSE

C $\star \star \star \star \star \star \star \star \star \star \star \star \star \star \star *$ PRINT ERROR MESSAGE

GO TO 9200

ENDIF

ELSE

C $\star * \star * \star \star \star \star \star * \star$ NO BINS USED FOR DIRECT EXECUTION, PRINT ERROR MESSAGE GO TO 9300

ENDIF

ELSE IF (KEYWRD ( $1:$ KL.NGTH) .EQ. 'PRTINP') THEN

C $\star \star \star \star \star \star \star \star$ SET CONTROL FLAG PRTINP

PRTINP=.TRUE.

ELSE IF (KEYWRD (1:KLNGTH) .EQ. 'REPORTB') THEN

C $* * \star \star * \star * \star$ SET CONTROL FLAG REPORTB REPRTB $=$.TRUE.

ELSE IF (KEYWRD ( $1:$ KLNGTH) .EQ. 'KPBYRUN') THEN

C $\star \star \star \star \star \star \star \star$ SET CONTROI FLAG KPBYRUN BYRUN $=$. TRUE.

ELSE IF (KEYWRD ( $1:$ KLINGTH) .EQ. ' CONSFL') THEN

C $\star \star \star \star \star \star \star$ SET CONTROL FLAG CONSFL CONSFL $=$. TRUE .

ELSE IF (KEYWRD ( 1 :KLNGTH) .EQ. 'DIAG') THEN

$\mathrm{C} \star \star \star \star \star \star \star \star$ SET DIAGNOSTIC PRINT CONTROL FLAG DIAG $D I A G=. T R U E$.

ELSE IF (KEYWRD ( 1 :KLNGTH) .EQ. 'EXPERT') THEN

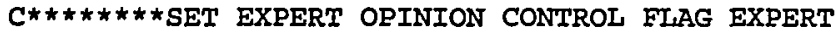
EXPERT $=$.TRUE.

ELSE

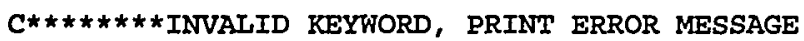
WRITE $(6,5020)$ KEYWRD ( 1 : KLNGTH) NOCALC $=$.TRUE.

ENDIF

GO TO 500

6000 CONTINUE

C*****VALIDATE COMBINATION OF FLAGS

IF (EXPERT .AND. ((.NOT. BINNED) .OR. (.NOT. SAMPLE))) THEN WRITE $(6,6001)$

STOP

ENDIF

IF (NOCALC) STOP

$C * \star \star \star \star$ PRINT CONTROL INFORMATION

IF (SAMPLE) WRITE $(6,5025)$ NOBS, NSTART

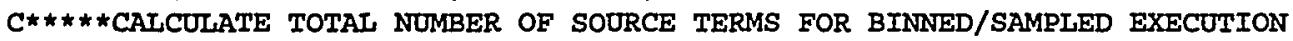

IF (BINNED .AND. BYRUN) THEN

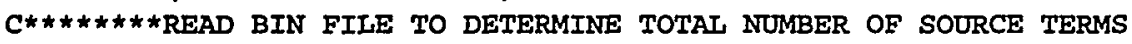
NTOT $=0$ NSAMPL=NSTART + NOBS - 1

C $\star \star \star \star \star \star \star \star$ READ TITIE RECORD READ $(4,1001)$ BTITLE 


\section{Appendix C}

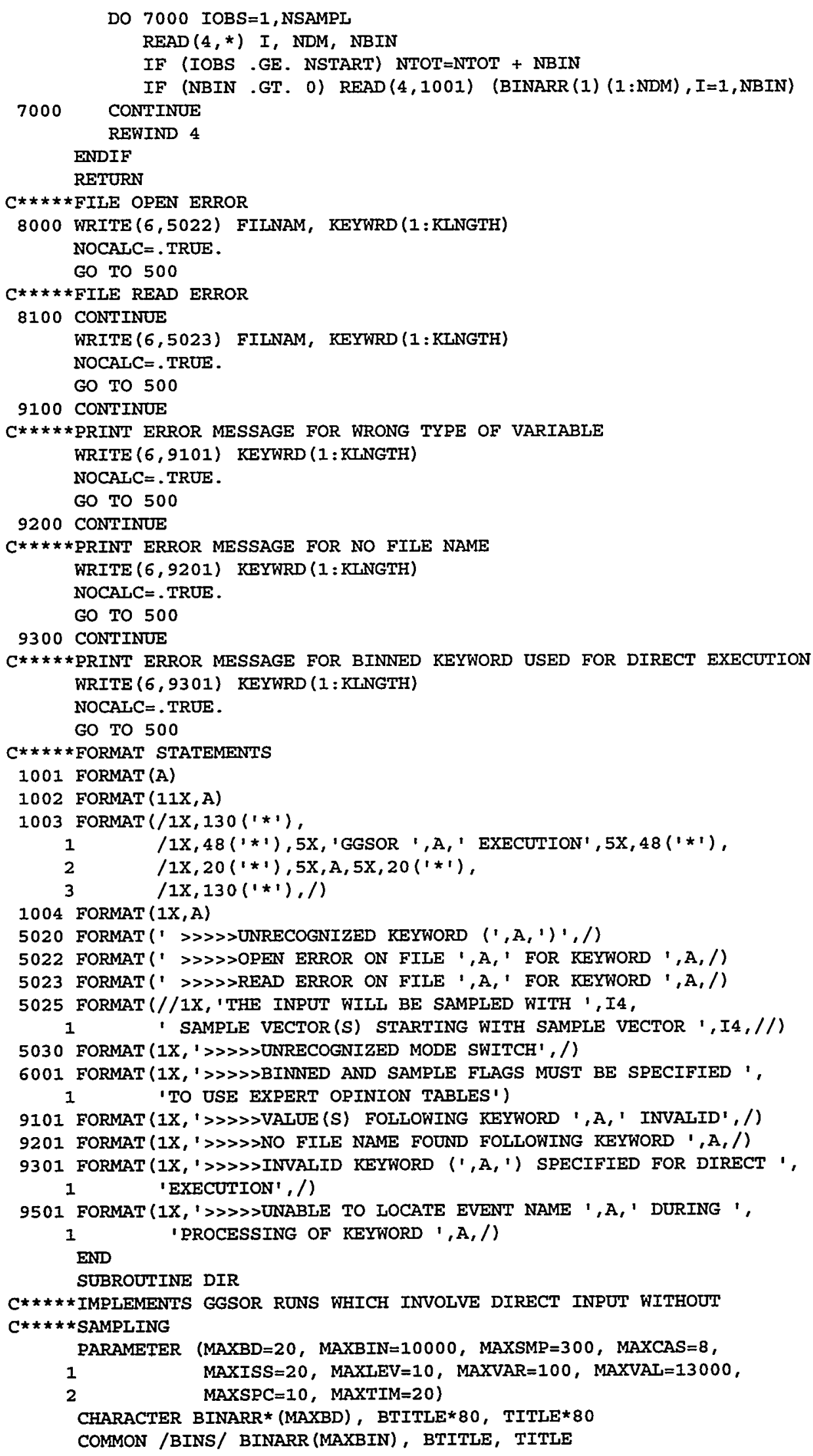


COMMON /CONTRL/ NLHS, NOBS, NSTART, NBIN, NDM, NTOT CHARACTER*7 NAME

LOGICAL LDEFLT, IREAL

COMMON /DEELT1/ NAME (MAXVAR)

COMMON /DEFLT2/ NVAR, NVAL, NVCB1, NVCB2, NVCB3, NVCB4,

1

2

3 NVCB5, IDIMEN (3,MAXVAR), ISPOS (MAXVAR) ISMPPS (MAXVAL) , IPNT (MAXVAR), LDEFLT (MAXVAL), IREAL (MAXVAL)

LOGICAL NOCALC, SAMPLE, REPRTB, BINNED, BYRUN, CONSFL, DIAG, 1 EXPERT, PRTINP, NOCF, SUBCL, CDB, TMPCDB, BRKOPN, VB, ECF, ICF COMMON /KEYS/ NOCALC, SAMPLE, REPRTB, BINNED, BYRUN, CONSFL, DIAG,

1 EXPERT, PRTINP, NOCF, SUBCL, CDB, TMPCDB, BRKOPN, VB, ECF, ICF COMMON /SRCTRM/ ST (MAXSPC), STE (MAXSPC), STCCI (MAXSPC),

1 STL (MAXSPC), STIL, STRVOL (MAXSPC), 2 ST1 (MAXSPC), ST2 (MAXSPC), RV (MAXSPC)

COMMON /BASVAL/ FCOR (MAXSPC), FVES (MAXSPC), DFVPA (MAXSPC), 1 DFCPA (MAXSPC), FEVSE (MAXSPC), FDCH (MAXSPC), 2 FCCI (MAXSPC), DFCAV (MAXSPC), VBPUF (MAXSPC), 3 FCONV (MAXSPC), FCONC (MAXSPC), RBDF (MAXSPC) 4 DFSPRV (MAXSPC)， DFSPRC (MAXSPC)， FREVO (MAXSPC), 5 VALISS (MAXISS), FLTI1, FLTI2, NSPEC, FLV, FHPE, 6 EVSE, WFAC, PFAC, FPLBYE, FPLBYP, FPLBYD, 7 FPLBYC, FTLPH, FTLPL, FTLP, TC11, TC12, TB11, 8 TB12, TB21, TB22, TBS1, TBS2, TBR1, TBR2, TW, 9 T1, T2, DT1, DT2, DTCDB, ELEV, PUFF, HVSPLT, FCD COMMON /BINNED/ FCORO (MAXSPC,MAXCAS), FVESO (MAXSPC,MAXCAS), DFVPAO (MAXSPC, MAXCAS), DFCPAO (MAXSPC, MAXCAS), FDCHO (MAXSPC, MAXCAS) , FEVSEO (MAXSPC, MAXCAS), FCCIO (MAXSPC, MAXCAS), DFCAVO (MAXSPC, MAXCAS), VBPUFO (MAXSPC, MAXCAS), FCONVO (MAXSPC, MAXCAS), FCONCO (MAXSPC, MAXCAS), RBDFO (MAXSPC, MAXCAS), DFSPRVO (MAXSPC, MAXCAS), DFSPRCO (MAXSPC, MAXCAS), FREVOO (MAXSPC, MAXCAS) , FLTI10 (MAXCAS), FLTI20 (MAXCAS) , FHPE0 (MAXCAS), EVSEO (MAXCAS), FPLBYO (3), HVSPLTO, TWO (MAXTIM), T10 (MAXTIM), DT10 (MAXTIM)， DT20 (MAXTIM)， PUFF0 (MAXTIM), EO (MAXTIM)

COMMON /EXPERT/ FCORL (MAXSPC,MAXLEV, MAXCAS), FVESL (MAXSPC, MAXLEV, MAXCAS), FREVOL (MAXSPC, MAXIEV, MAXCAS), FCCIL (MAXSPC, MAXIEV, MAXCAS), FCONVL (MAXSPC, MAXIEV, MAXCAS), FCONCL (MAXSPC, MAXIEV, MAXCAS), FLTIIL (MAXLEV, MAXCAS) , FLTI2L (MAXIEV, MAXCAS) , RBDFL (MAXSPC, MAXLEV, MAXCAS), FDCHU (MAXSPC, MAXLEV, MAXCAS), FEVSEL (MAXSPC, MAXLEV, MAXCAS), DFVPAI (MAXSPC, MAXIEV, MAXCAS), DFCPAL (MAXSPC, MAXLEV, MAXCAS), DFCAVL (MAXSPC, MAXLEV, MAXCAS), DFSPRVL (MAXSPC, MAXLEV, MAXCAS), DFSPRCL (MAXSPC, MAXLEV, MAXCAS), PRBLEV (MAXLEV)

COMMON /LHSBLK/ XLHS (MAXSMP) DATA IOBS / 1 /, IBIN / 1 /

C

C

$C * \star \star \star \star$ SET NUMBER OF SAMPLE VALUES $X I H S(1)=0.0$

$C \star \star \star \star \star D E F I N E$ VARIABLE NAMES FOR DEFAULT INPUT CALL DEEINE

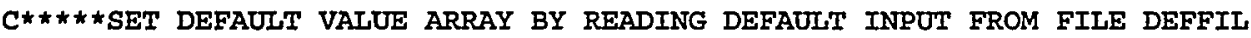
CALL SETDEF 


\section{Appendix C}

$C \star \star \star \star *$ PRINT DEFAULT VALUE INFORMATION IF (PRTINP) CALL WRTPAR

C*****TERMINATE EXECUTION IF ONLY VALIDATING INPUT OR ERROR ENCOUNTERED

$C \star \star \star \star \star$ DURING READING OF INPUT DATA IF (NOCALC) THEN

$C * \star \star * \star * \star *$ PRINT MESSAGE $\operatorname{WRITE}(6,1010)$ STOP ENDIF

C $\star \star \star \star \star$ TRANSFER DEFAULT VALUE ARRAY TO COMMON BLOCK VALUES CALI TRANS $(\operatorname{FCOR}(1), \operatorname{FCORO}(1,1), \operatorname{FCORL}(1,1,1))$

$C * * * * *$ PRINT CONTENTS OF COMMON BLOCKS IF (REPRTB) CALL WRREL

$C \star \star \star \star \star$ SET TOTAL NUMBER OF SOURCE TERMS NTOT $=1$

C*****WRITE HEADER TO CONSEQUENCE DATA FILE IF (CONSFL) WRITE $(9,1006)$ TITLE, NDM, NSPEC, NTOT, NOBS

$C * * * * *$ WRITE STANDARD HEADER TO SPECIALIZED CONSEQUENCE DATA FILE

C $/ / /$ IF (CONSFL) WRITE $(10,5001)$ TITLE, NDM, NSPEC, NTOT, NOBS

$C \star \star \star \star \star$ PERFORM SOURCE TERM CALCULATIONS

CALI GGSORC (IOBS, IBIN)

$C \star \star \star \star \star$ PRINT PROCESSING SUMMARY WRITE $(6,2003)$ RETURN

$C \star \star \star \star \star$ FORMAT STATEMENTS

1006 FORMAT $(1 X, A, / 1 X, 4 I 10)$

1010 FORMAT (/1X, 'EXECUTION TERMINATED FOLLOWING VALIDATION OF INPUT')

2003 FORMAT (/1X,' SINGLE DIRECT EXECUTION PROCESSED')

5001 FORMAT $\left(1 \mathrm{X}, \mathrm{A}, /, 1 \mathrm{X},{ }^{\prime} \mathrm{NDM}=1, I 5,1 \quad\right.$ NSPEC $=1, I 5,{ }^{\prime} \quad$ NTOT $=1, \mathrm{I} 5$,

1 NOBS $=1, I 5$ END SUBROUTINE DIRSMP

$C \star \star \star \star \star$ IMPLEMENTS GGSOR RUNS WHICH INVOLVE DIRECT INPUT WITH

$C * \star \star \star \star$ SAMPLING

PARAMETER (MAXBD $=20, \operatorname{MAXBIN}=10000, \operatorname{MAXSMP}=300, \operatorname{MAXCAS}=8$,

2 MAXISS $=20$, MAXLEV $=10$, MAXVAR $=100$, MAXVAI $=13000$, MAXSPC $=10$, MAXTIM $=20$ )

CHARACTER BINARR* (MAXBD)， BTITLE*80，TITLE*80

COMMON /BINS/ BINARR (MAXBIN), BTITLE, TITLE

COMMON /CONTRL/ NLHS, NOBS, NSTART, NBIN, NDM, NTOT

CHARACTER*7 NAME

LOGICAL LDEFLT, LREAL

COMMON /DEFLT1/ NAME (MAXVAR)

COMMON /DEFLT2/ NVAR, NVAL, NVCB1, NVCB2, NVCB3, NVCB4,

1

2

3

LOGICAI NOCAIC, SAMPLE, REPRTB, BINNED, BYRUN, CONSFL, DIAG,

1 EXPERT, PRTINP, NOCF, SUBCL, CDB, TMPCDB, BRKOPN, VB, ECF, ICF COMMON /KEYS/ NOCALC, SAMPLE, REPRTB, BINNED, BYRUN, CONSFL, DIAG, 1 EXPERT, PRTINP, NOCF, SUBCL, CDB, TMPCDB, BRKOPN, VB, ECF, ICF COMMON /SRCTRM/ ST (MAXSPC), STE (MAXSPC), STCCI (MAXSPC),

1 STL (MAXSPC)，STIL， STRVOL (MAXSPC), 2 ST1 (MAXSPC), ST2 (MAXSPC), RV (MAXSPC)

COMMON /BASVAL/ FCOR (MAXSPC), FVES (MAXSPC), DFVPA (MAXSPC), DFCPA (MAXSPC) , FEVSE (MAXSPC), FDCH (MAXSPC), FCCI (MAXSPC), DFCAV (MAXSPC), VBPUF (MAXSPC), FCONV (MAXSPC)， FCONC (MAXSPC)， RBDF (MAXSPC), DFSPRV (MAXSPC)， DFSPRC (MAXSPC), FREVO (MAXSPC), VALISS (MAXISS), FLTI1, FLTI2, NSPEC, FLV, FHPE, EVSE, WFAC, PFAC, FPLBYE, FPLBYP, FPLBYD, FPLBYC, FTLPH, FTLPL, FTLP, TC11, TC12, TB11, TB12, TB21, TB22, TBS1, TBS2, TBR1, TBR2, TW, 


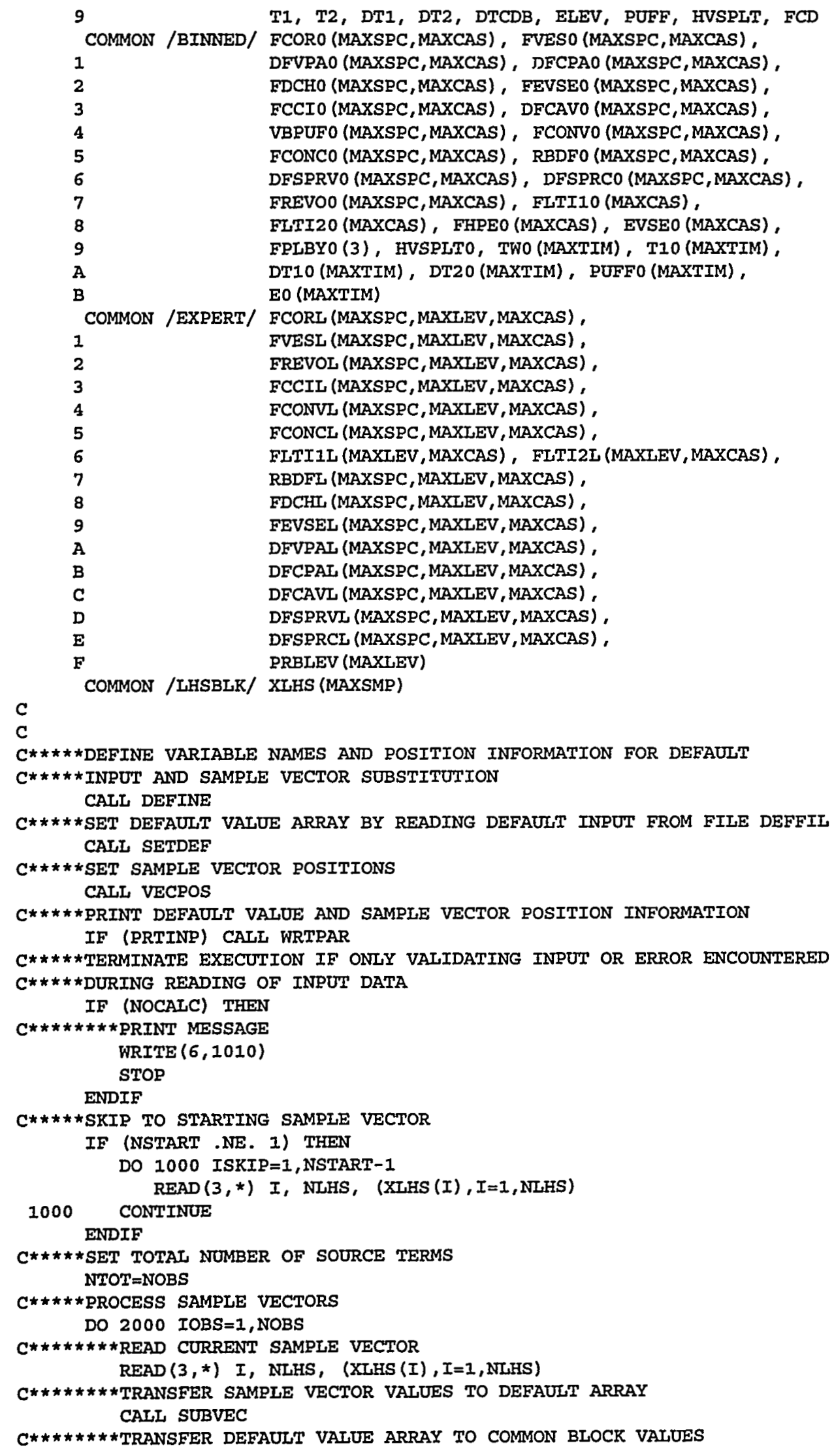




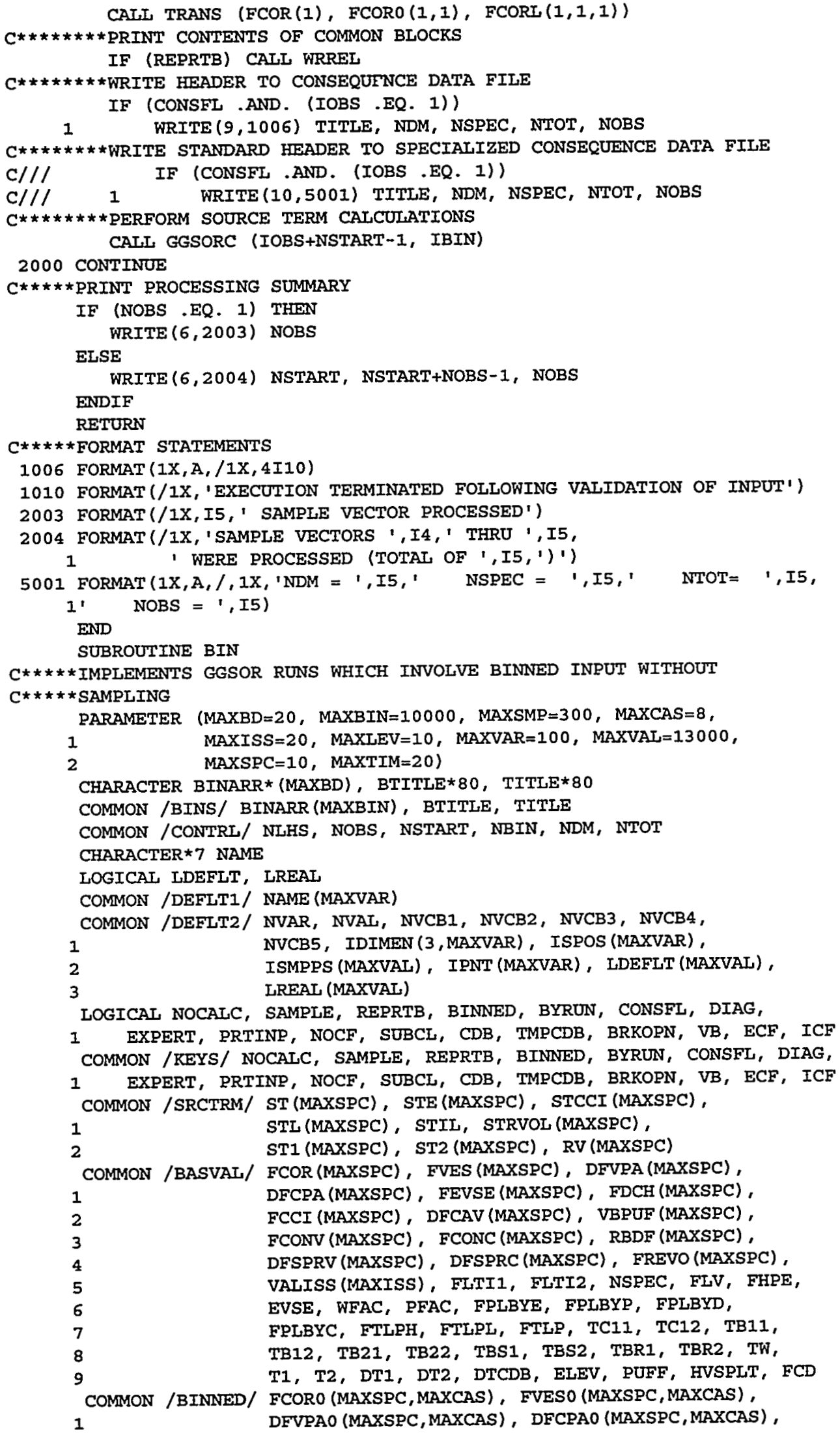




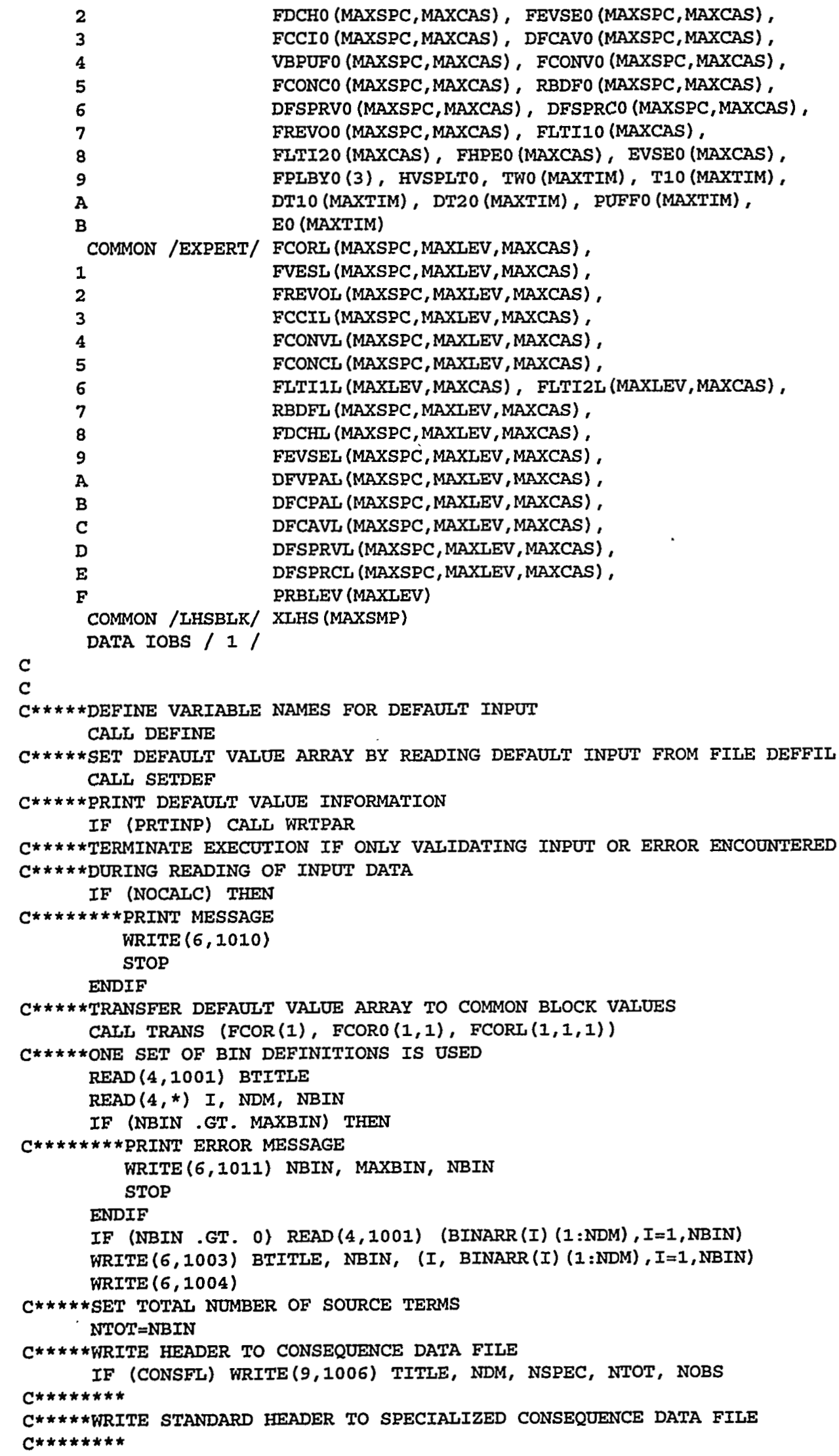


Appendix C

C/// IF (CONSFL) WRITE $(10,5001)$ TITLE, NDM, NSPEC, NTOT, NOBS

$C \star \star \star \star \star$ LOOP OVER INDIVIDUAL BINS

DO 1000 IBIN $=1$, NBIN

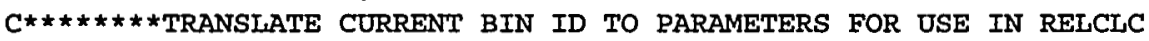
CALL BINTRN (IBIN)

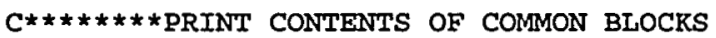
IF (REPRTB) CALL WRREL

C $\star \star \star \star \star \star \star \star \star$ PERFORM SOURCE TERM CALCULATIONS

1000 CONTINUE

CALL GGSORC (IOBS, IBIN)

$C * \star * \star *$ PRINT NUMBER OF BINS PROCESSED WRITE $(6,2003)$ NBIN RETURN

$C * \star \star * \star$ FORMAT STATEMENTS

1001 FORMAT (A)

1002 FORMAT (1X,A)

1003 FORMAT $(/ / 1 \mathrm{X}, 130$ ('='),

$1 / / 1 \mathrm{X}$, 'BINNING INFORMATION',

$2 \quad / I X, A$,

3 //IX, 'THE FOLLOWING ',I7,' BIN(S) ARE TO BE PROCESSED: ',

$4 \quad / /(1 X, I 7, '-1, A))$

1004 FORMAT $\left(/ 1 \mathrm{X}, 130\left({ }^{\prime}=1\right), / /\right)$

1006 FORMAT (1X,A, /1X,4I10)

1010 FORMAT (/IX, 'EXECUTION TERMINATED FOLLOWING VALIDATION OF INPUT')

1011 FORMAT (/IX,' $\gg>>$ NUMBER OF BINS (',I7,') READ FROM FILE IS ', ' LARGER THAN ALLOWED DIMENSION $(1, I 7,1) '$ ',

$2 \quad / 1 X, ' \gg>>$ INCREASE PARAMETER MAXBIN TO AT LEAST $1, I 7$,

3 / $3 \mathrm{X}, ' \gg \gg>>$ EXECUTION TERMINATED')

2003 FORMAT (/IX,I7,' BIN (S) PROCESSED')

5001 FORMAT (IX, A, //, 1X, 'NDM = ',I5,' NSPEC $=$ ', I5, ' NTOT= ', I5,

1 NOBS $=1, I 5, / 1$

ENDD

SUBROUTINE BINSMP

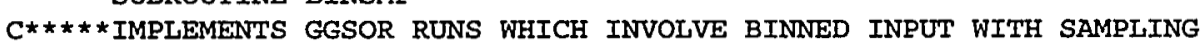

PARAMETER (MAXBD $=20$, MAXBIN $=10000$, MAXSMP $=300$, MAXCAS $=8$,

1

2 MAXISS $=20, M A X L E V=10, \operatorname{MAXVAR}=100, \operatorname{MAXVAL}=13000$, MAXSPC $=10$, MAXTIM $=20$ )

CHARACTER BINARR* (MAXBD)， BTITLE*80, TITLE*80

COMMON /BINS/ BINARR (MAXBIN); BTITLE, TITLE

COMMON /CONTRL/ NLHS, NOBS, NSTART, NBIN, NDM, NTOT

CHARACTER* 7 NAME

LOGICAI LDEFLT, LREAL,

COMMON /DEFLT1/ NAME (MAXVAR)

COMMON /DEFLT2! NVAR, NVAL, NVCB1, NVCB2, NVCB3, NVCB4,

1

2

3

LOGICAL NOCALC, SAMPLE, REPRTB, BINNED, BYRUN, CONSFL, DIAG,

1 EXPERT, PRTINP, NOCF, SUBCL, CDB, TMPCDB, BRROPN, VB, ECF, ICF COMMON /KEYS/ NOCALC, SAMPLE, REPRTB, BINNED, BYRUN, CONSFL, DIAG,

1 EXPERT, PRTINP, NOCF, SUBCL, CDB, TMPCDB, BRKOPN, VB, ECF, ICF COMMON /SRCTRM/ ST (MAXSPC), STE (MAXSPC), STCCI (MAXSPC),

1 STL (MAXSPC), STIL, STRVOL (MAXSPC),

2 ST1 (MAXSPC), ST2 (MAXSPC), RV (MAXSPC)

COMMON /BASVAL/ FCOR (MAXSPC), FVES (MAXSPC), DFVPA (MAXSPC),

1 DFCPA (MAXSPC), FEVSE (MAXSPC), FDCH (MAXSPC), FCCI (MAXSPC), DFCAV (MAXSPC), VBPUF (MAXSPC), FCONV (MAXSPC) , FCONC (MAXSPC), RBDF (MAXSPC), DFSPRV (MAXSPC), DFSPRC (MAXSPC), FREVO (MAXSPC), VAIISS (MAXISS), FLTI1, FLTI2, NSPEC, FLV, FHPE, EVSE, WFAC, PFAC, FPLBYE, FPLBYP, FPLBYD, FPLBYC, FTLPH, FTLPL, FTLP, TC11, TC12, TB11, TB12, TB21，TB22，TBS1, TBS2, TBR1, TBR2，TW， 
9 T1, T2, DT1, DT2, DTCDB, ELEV, PUFF, HVSPLT, FCD COMMON /BINNED/ FCORO (MAXSPC,MAXCAS), FVESO (MAXSPC,MAXCAS), 1 DFVPAO (MAXSPC, MAXCAS), DFCPAO (MAXSPC, MAXCAS), FDCHO (MAXSPC, MAXCAS), FEVSEO (MAXSPC,MAXCAS), FCCIO (MAXSPC, MAXCAS), DFCAVO (MAXSPC, MAXCAS), VBPUFO (MAXSPC,MAXCAS), FCONVO (MAXSPC, MAXCAS), FCONCO (MAXSPC, MAXCAS), RBDFO (MAXSPC, MAXCAS), DFSPRVO (MAXSPC, MAXCAS), DFSPRCO (MAXSPC, MAXCAS), FREVO0 (MAXSPC,MAXCAS), FLTI10 (MAXCAS), FLTI20 (MAXCAS), FHPEO (MAXCAS), EVSEO (MAXCAS), FPLBYO (3), HVSPLTO, TWO (MAXTIM), T10 (MAXTIM), DT10 (MAXTIM), DT20 (MAXTIM), PUFFO (MAXTIM),

COMMON /EXPERT/ FCORL (MAXSPC, MAXLEV, MAXCAS), FVESI (MAXSPC, MAXLEV, MAXCAS), FREVOL (MAXSPC, MAXIEV, MAXCAS), FCCIL (MAXSPC, MAXLEV, MAXCAS), FCONVL (MAXSPC, MAXIEV, MAXCAS), FCONCL (MAXSPC, MAXLEV, MAXCAS), FLTI1L (MAXIEV, MAXCAS), FLTI2L (MAXIEV, MAXCAS), RBDFL (MAXSPC, MAXLEV, MAXCAS), FDCHU (MAXSPC, MAXILEV, MAXCAS), FEVSEL (MAXSPC, MAXIEV, MAXCAS), DEVPAI (MAXSPC, MAXIEV, MAXCAS), DFCPAI (MAXSPC, MAXLEV, MAXCAS), DFCAVI (MAXSPC, MAXIEV, MAXCAS), DESPRVL (MAXSPC, MAXIEV, MAXCAS), DFSPRCL (MAXSPC, MAXIEV, MAXCAS). PRBLEV (MAXIEV)

$C$ XLHS (MAXSMP)

$C * * \star * *$ DEFINE VARIABLE NAMES AND POSITION INFORMATION FOR DEFAULT $C \star \star \star \star \star$ INPUT AND SAMPLE VECTOR SUBSTITUTION

CAII DEFINE

$C \star * \star \star \star$ SET DEFAULT VALUE ARRAY BY READING DEFAULT INPUT FROM FIIE DEFFIL CALL SETDEF

$C * * * * *$ SET SAMPLE VECTOR POSITIONS CALI VECPOS

$C \star \star \star \star \star$ PRINT DEFAULT VALUE AND SAMPLE VECTOR POSITION INFORMATION IF (PRTINP) CALL WRTPAR

$C * * * * *$ TERMINATE EXECUTION IF ONLY VALIDATING INPUT OR ERROR ENCOUNTERED

$C \star * \star * \star$ DURING READING OF INPUT DATA

IF (NOCALC) THEN

$C \star * \star \star * \star * *$ PRINT MESSAGE WRITE $(6,1010)$ STOP

ENDIF

$C \star \star \star \star \star$ READ TITLE RECORD

READ $(4,1001)$ BTITLE

IF (.NOT. BYRUN) THEN

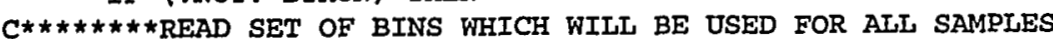
READ (4,*) I, NDM, NBIN IF (NBIN .GT. MAXBIN) THEN

C $\star * \star * \star \star \star \star \star * \star *$ PRINT ERROR MESSAGE WRITE $(6,1011)$ NBIN, MAXBIN, NBIN STOP

ENDIF

IF (NBIN .GT. 0) $\operatorname{READ}(4,1001)$ (BINARR (I) (I:NDM), I=1,NBIN)

WRITE $(6,1003)$ IOBS, BTITLE, NBIN,

1 $(I, \operatorname{BINARR}(I)(1:$ NDM $), I=1$, NBIN $)$

WRITE $(6,1004)$

C $\star * \star \star \star \star * \star$ SET TOTAL NUMBER OF SOURCE TERMS

Vol. 6, Part 1

C-13

NUREG/CR-6143 
Appendix C

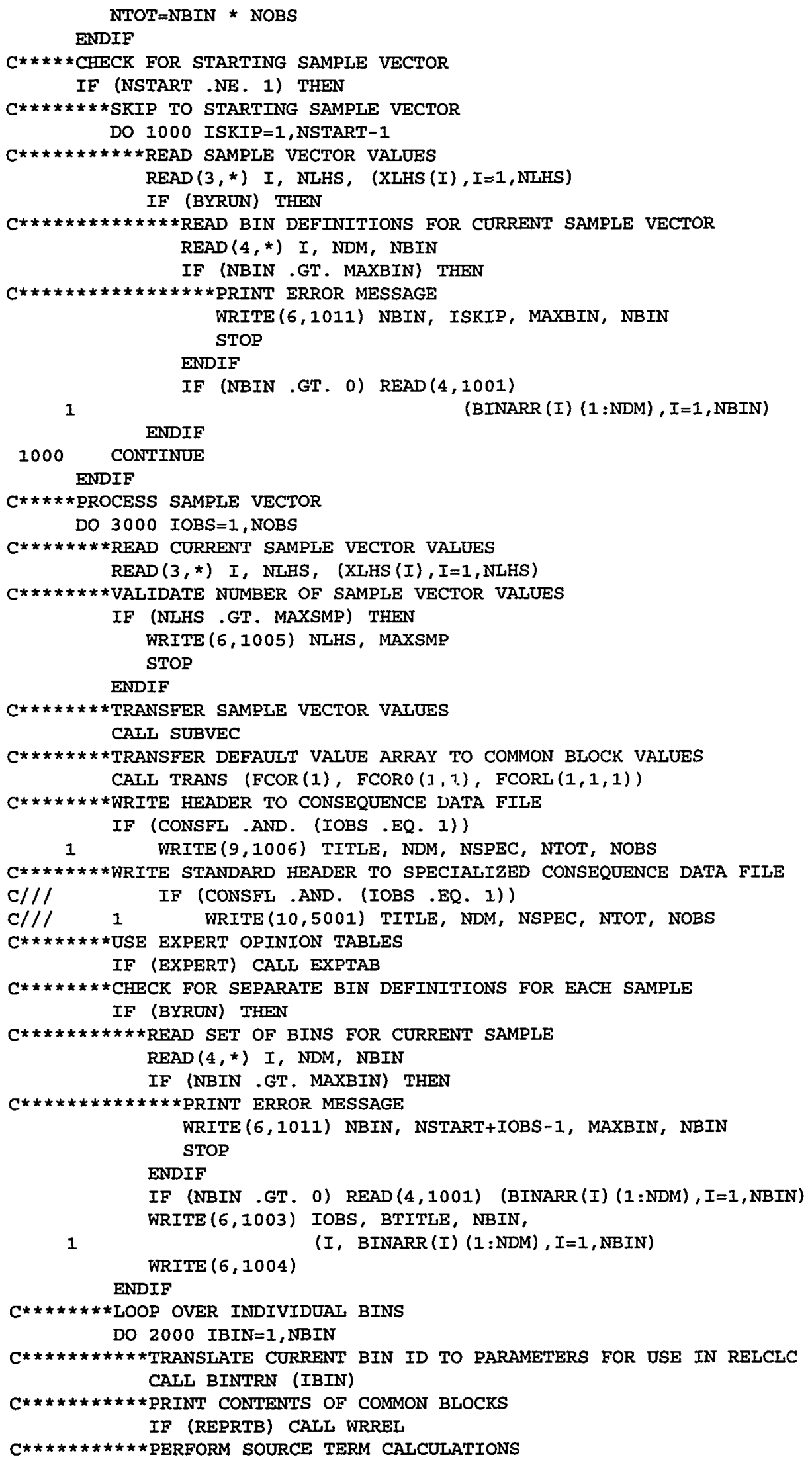


2000

CALL GGSORC (IOBS+NSTART-1, IBIN)

$C \star \star \star \star \star \star \star \star$ PRINT NUMBER OF BINS PROCESSED FOR CURRENT SAMPLE VECTOR

3000 CONTINUS WRITE $(6,2003)$ NBIN, IOBS+NSTART-I

\section{RETURN}

$C * * \star \star \star$ FORMAT STATEMENTS

1001 FORMAT (A)

1002 FORMAT $(1 \mathrm{X}, \mathrm{A})$

1003 FORMAT $\left(/ / 1 X, 130\left(^{\prime}={ }^{\prime}\right)\right.$,

$1 / / 1 X, ' B I N N I N G$ INFORMATION FOR SAMPLE VECTOR ',I4,

$2 \quad / 1 \mathrm{X}, \mathrm{A}$,

3 //1X, 'THE FOLLOWING ',I7,' BIN(S) ARE TO BE PROCESSED:',

$4 \quad / /(1 \mathrm{X}, I 7,1-1, \mathrm{~A}))$

1004 FORMAT $(/ 1 \mathrm{X}, 130(1=1), / /)$

1005 FORMAT (/1X, ' $\gg \gg>N U M B E R$ OF SAMPLE VECTOR VALUES (',I4,

1 i) READ FROM UNIT 3 EXCEEDS ',

$2 \quad / 1 X, ' \gg \gg \gg$ MAXIMUM NUMBER ALLOWED (MAXSMP=',I4, ')',

$3 \quad(1 \mathrm{X}, 1 \gg \gg>$ EXECUTION TERMINATED')

1006 FORMAT ( $1 \mathrm{X}, \mathrm{A}, / 1 \mathrm{X}, 4 \mathrm{I} 10)$

1010 FORMAT (/1X, 'EXECUTION TERMINATED FOLLOWING VALIDATION OF INPUT')

1011 FORMAT $(/ 1 \mathrm{X}, 1 \gg \gg$ MUMBER OF BINS $(1, I 7,1)$ READ FROM UNIT 4,1,

1 'SAMPLE VECTOR ',I4,

2 ' , IS LAARGER THAN AILOWED DIMENSION $(1, I 7,1) '$,

$3 \quad / I X, ' \gg \gg$ INCREASE PARAMETER MAXBIN TO AT LEAST ', I7,

$4 \quad / 1 X, ' \gg>>$ EXECUTION TERMINATED')

2003 FORMAT ( $/ 1 X, I 7, '$ BIN(S) PROCESSED FOR SAMPLE VECTOR ', I4)

5001 FORMAT (1X,A, //,1X, 'NDM = ',I5,' NSPEC $=$ ', I5,' NTOT= ', I5,

1' NOBS $=1, I 5, /)$

END

SUBROUTINE DEFINE

$C * \star \star \star \star$ DEFINE NAMES AND DIMENSIONS OF VARIABLES TO BE SET THROUGH

$C \star \star \star \star \star$ DEFAULT INPUT AND SAMPLE VECTOR SUBSTITUTION

PARAMETER (MAXBD $=20, \operatorname{MAXBIN}=10000, \operatorname{MAXSMP}=300$, MAXCAS $=8$,

1 MAXISS $=20, \operatorname{MAXIEV}=10, \operatorname{MAXVAR}=100$, MAXVAI $=13000$,

$2 \quad$ MAXSPC $=10, \operatorname{MAXTIM}=20$ )

CHARACTER*7 NAME

LOGICAL IDEFLT, LREAI

COMMON /DEFLT1/ NAME (MAXVAR)

COMMON /DEFLT2/ NVAR, NVAL, NVCB1, NVCB2, NVCB3, NVCB4,

1

3 LREAL (MAXVAL)

LOGICAL NOCAIC, SAMPLE, REPRTB, BINNED, BYRUN, CONSFL, DIAG,

1 EXPERT, PRTINP, NOCF, SUBCL, CDB, TMPCDB, BRKOPN, VB, ECF, ICF COMMON /KEYS/ NOCALC, SAMPLE, REPRTB, BINNED, BYRUN, CONSFL, DIAG, C

1 EXPERT, PRTINP, NOCF, SUBCL, CDB, TMPCDB, BRKOPN, VB, ECF, ICF

C

$C \star \star \star \star \star$ SET DEFAULT TYPES TO .FALSE.

DO $1000 I=1$, MAXVAI

LDEFLT $(I)=$. FAISE .

1000 CONTINUE

$C \star \star \star \star \star$ INITIALIIZE NOMBER OF VARIABLES NVAR $=0$

$C \star \star \star \star \star$ INITIALLIZE TOTAL NUMBER OF VAIUES

NVAL $=1$

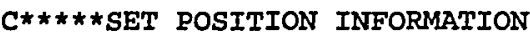

DO 2000 IVAR=1, MAXVAR

$C * \star * \star * \star * *$ CHECK FOR BLANR VARIABLE NAME

IF (NAME (IVAR) .EQ. ' 'I) GO TO 3000

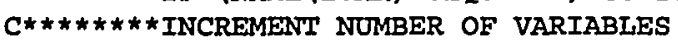

NVAR $=$ NVAR +1

Vol. 6, Part 1

C-15

NUREG/CR-6143 
Appendix C

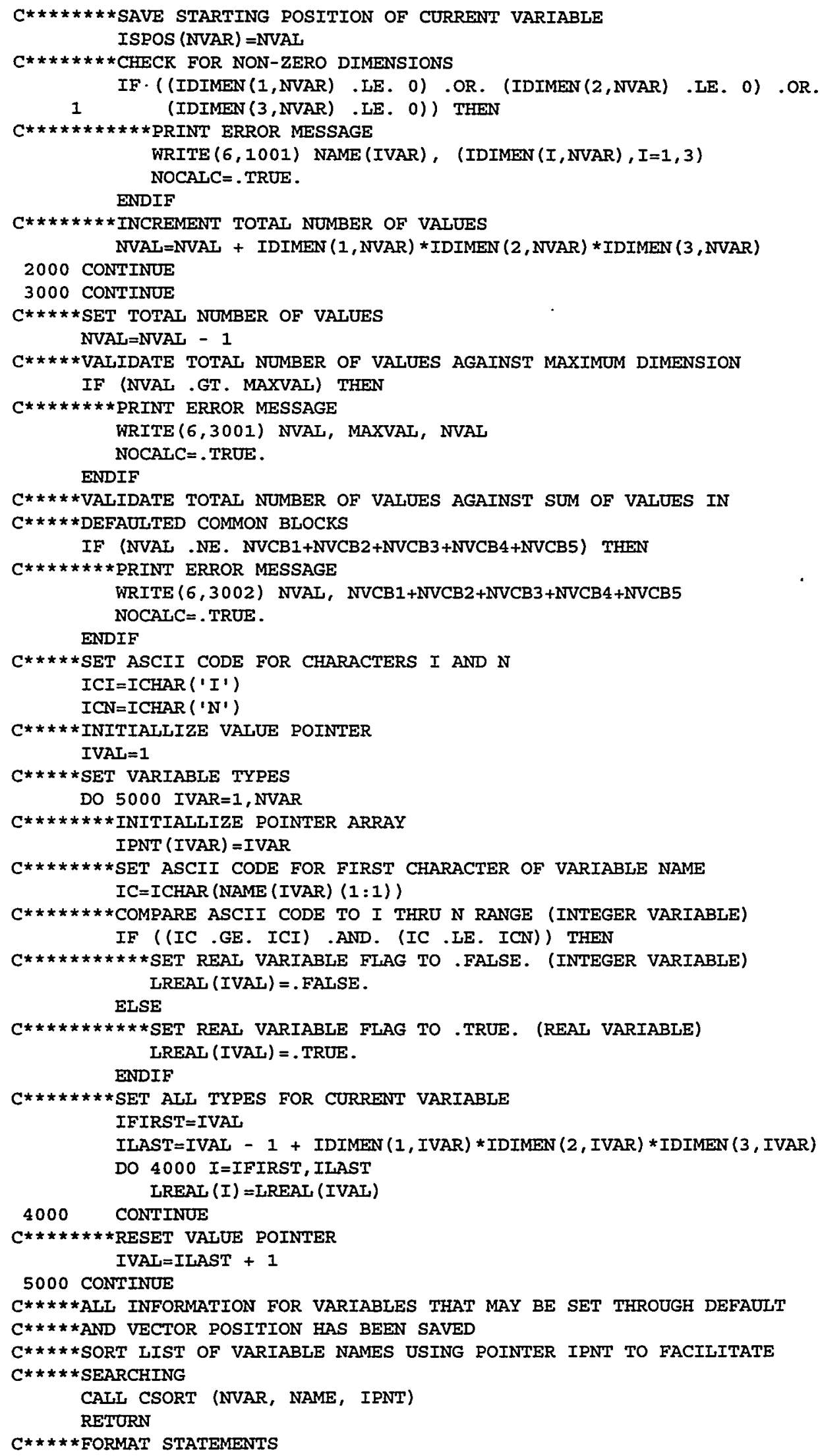


1001 FORMAT (1X, ' $\gg \gg>D I M E N S I O N S$ FOR VARIABLE ',A,' MUST BE GREATER ', 'THAN 0 ',

$/ 1 \mathrm{X}$, ' $\gg \gg>$ DIMENSION $1=1$, I5, ', DIMENSION $2=1$, I5,

1 , DIMENSION $3=1, I 5$,

/IX,' $\gg \gg \gg$ CHECK VARIABLE DEFINITIONS IN SUBROUTINE DEFINE', /)

3001 FORMAT ( $1 \mathrm{X}$, ' $\gg \gg \gg>$ NOMBER OF VARIABLES (NVAR=',I5,') EXCEEDS ',

1 'DIMENSION (MAXVAR=',I5,' ')',

$2 / 1 X, 1 \gg \gg>$ CHECK VARIABLE DEFINITIONS IN SUBROUTINE DEFINE ',

3 'AND/OR',

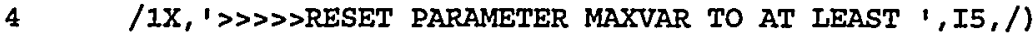

3002 FORMAT ( $1 \mathrm{X}, 1 \gg \gg \gg$ MUMBER OF VAIUES WHICH CAN BE SET THROUGH ',

1 'DEFAULT AND VECTOR SUBSTITUTION $(1, I 5, ')$ ',

$2 / I X, ' \gg \gg>$ SHOULD BE EQUAI TO THE TOTAL NUMBER OF VALUES ',

3 IIN THE COMMON BLOCRS TO BE SET ',

$4 \quad / 1 X,{ }^{\prime} \gg>(N V C B 1+N V C B 2+N V C B 3+N V C B 4+N V C B 5=1, I 5, ')$,

5 '-- SUBROUTINE DEFINE',//)

END

SUBROUTINE SETDEF

$C * * * *$ SET DEFAULT VALUES BY READING VARIABLE NAMES AND CORRESPONDING

C $* * * *$ VALUES FROM FILE DESIGNATED FOR DEFAULT VALUES (DEFFIL)

PARAMETER (MAXLEN $=101$, MAXVLN $=20)$

PARAMETER (MAXBD $=20, \operatorname{MAXBIN}=10000, \operatorname{MAXSMP}=300, \mathrm{MAXCAS}=8$,

1

MAXISS $=20, \operatorname{MAXLEV}=10, \mathrm{MAXVAR}=100, \mathrm{MAXVAL}=13000$,

$2 \quad M A X S P C=10, \operatorname{MAXTIM}=20$ )

CHARACTER* 7 NAME

LOGICAL LDEFLT, LREAL

COMMON /DEFLTI/ NAME (MAXVAR)

COMMON /DEFLT2/ NVAR, NVAL, NVCB1, NVCB2, NVCB3, NVCB4,

1

2 ISMPPS (MAXVAL), IDNT (MAXVAR), IDEFLT (MAXVAI),

3 LREAL (MAXVAL)

LOGICAL NOCALC, SAMPLE, REPRTB, BINNED, BYRUN, CONSFI, DIAG,

1 EXPERT, PRTINP, NOCF, SUBCL, CDB, TMPCDB, BRKOPN, VB, ECF, ICF COMMON /KEYS/ NOCALC, SAMPLE, REPRTB, BINNED, BYRUN, CONSFL, DIAG,

1 EXPERT, PRTINP, NOCF, SUBCL, CDB, TMPCDB, BRKOPN, VB, ECF, ICF CHARACTER 80 DEFFIL, SAMFIL, VECFIL

COMMON /FILBLK/ DEFFIL, SAMFIL, VECFII

CHARACTER* (MAXLEN) CARD

CHARACTER* (MAXVLN) CVAL, TMPVAL

DIMENSION INDX (3)

LOGICAL EOR, TYPE(4), LVAI

CHARACTER $* 10$ IFRMT

COMMON /VALUES/ RVH (MAXVAL)

DIMENSION IVL (MAXVAL)

c

EQUIVAIENCE (IVL, RVL)

C

$C \star \star \star \star \star$ PRINT HEADER MESSAGE

IF (PRTINP) WRITE $(6,1003)$ DEFFIL

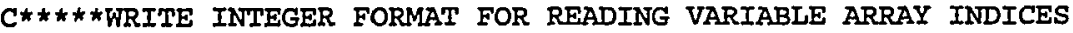

WRITE (IFRMT, 1004) MAXVLN

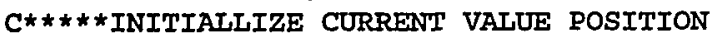

IVPOS $=-1$

$C \star \star \star \star \star$ OPEN DEFAULT FILE

OPEN (1, FILE=DEFFII, STATUS=' OLD', $E R R=9100)$

1000 CONTINOE

$C \star \star \star \star \star$ READ RECORD

$\operatorname{READ}(1,1001, \mathrm{END}=8000)$ CARD

$C \star \star \star \star \star$ PRINT RECORD

IF (PRTINR) WRITE $(6,1002)$ CARD

$C * \star * * *$ INITIALIIZE COLUMN POINTER FOR CURRENT RECORD

$I \mathrm{C}=1$

2000 CONTINUE

Vol. 6, Part 1 
Appendix C

$C * \star * \star *$ READ NEXT VALUE ON RECORD

CALI RDSTRG (CARD, IC, CVAL, IVAL, IVAL, RVAL, LENGTH, TYPE, EOR)

$C \star \star \star \star \star$ CHECK FOR END-OF-RECORD

IF (EOR) GO TO 1000

$C \star \star \star \star *$ CHECK FOR CHARACTER VALUE (VARIABLE NAME)

IF (TYPE (1)) THEN

$C \star \star \star \star \star \star \star \star$ INITIAIIIZE ARRAY SPECIFICATIONS

$\operatorname{INDX}(1)=1$

$\operatorname{INDX}(2)=1$

$\operatorname{INDX}(3)=1$

C $\star \star \star \star \star \star \star \star \star$ CHARACTER VALUE (VARIABLE NAME) FOUND

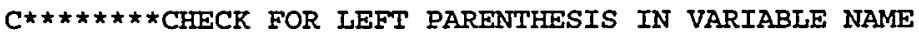

ILPAR=INDEX (CVAL, ' (')

IF (ILPAR . NE. 0) THEN

C $\star \star \star \star \star \star \star \star \star \star \star$ FOUND LEFT PARENTHESIS, CHECK FOR RIGHT PARENTHESIS

IRPAR=INDEX (CVAI, ')')

IF (IRPAR .NE. 0) THEN

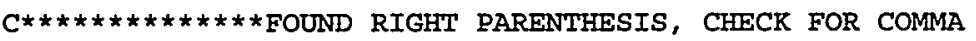

ICOMMA=INDEX (CVAL, 1,1$)$

IF (ICOMMA .NE. O) THEN

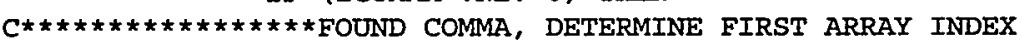

IS $=$ ILPAR + 1

$I E=I C O M M A$

$\begin{array}{ll}3000 & \text { IND }=1 \\ \text { CONTINUE }\end{array}$

$I E=I E-1$

IF (CVAI (IE:IE) .EQ. ' ') GO TO 3000

IF (IE .GE. IS) THEN

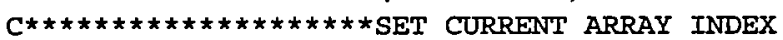

TMPVAL $=$ ' '

TMPVAL (MAXVIN+IS-IE : MAXVLN) =CVAL (IS : IE)

READ (TMPVAL, IFRMT, ERR $=9200$ ) INDX (IND)

ELSE

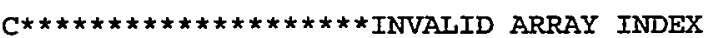

WRITE $(6,3001)$ IND, CVAL (1: LENGTH)

NOCALC $=$. TRUE.

ENDIF

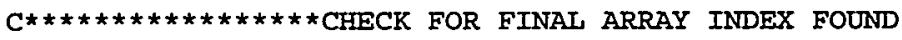

IF (ICOMMA .GT. O) THEN

$I S=I C O M M A+1$

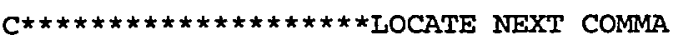

ICOMMA = INDEX (CVAI (IS : LENGTH)，', ')

IF (ICOMMA .GT. O) THEN

$I C O M M A=I S+I C O M M A-1$

IE $=$ ICOMMA

ELSE

IE $=$ IRPAR

ENDIF

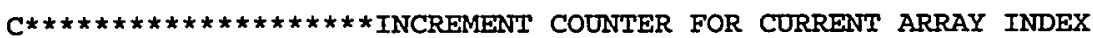

IND $=$ IND +1

IF (IND .GT. 3) THEN

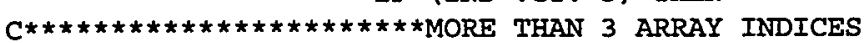

WRITE $(6,3002)$ CVAI ( 1 :LENGTH)

IVPOS $=-1$

NOCAIC $=$. TRUE.

GO TO 2000

ENDIF

GO TO 3000

ELSE

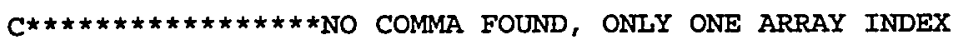

$I S=I L P A R+1$

IE $=$ IRPAR 


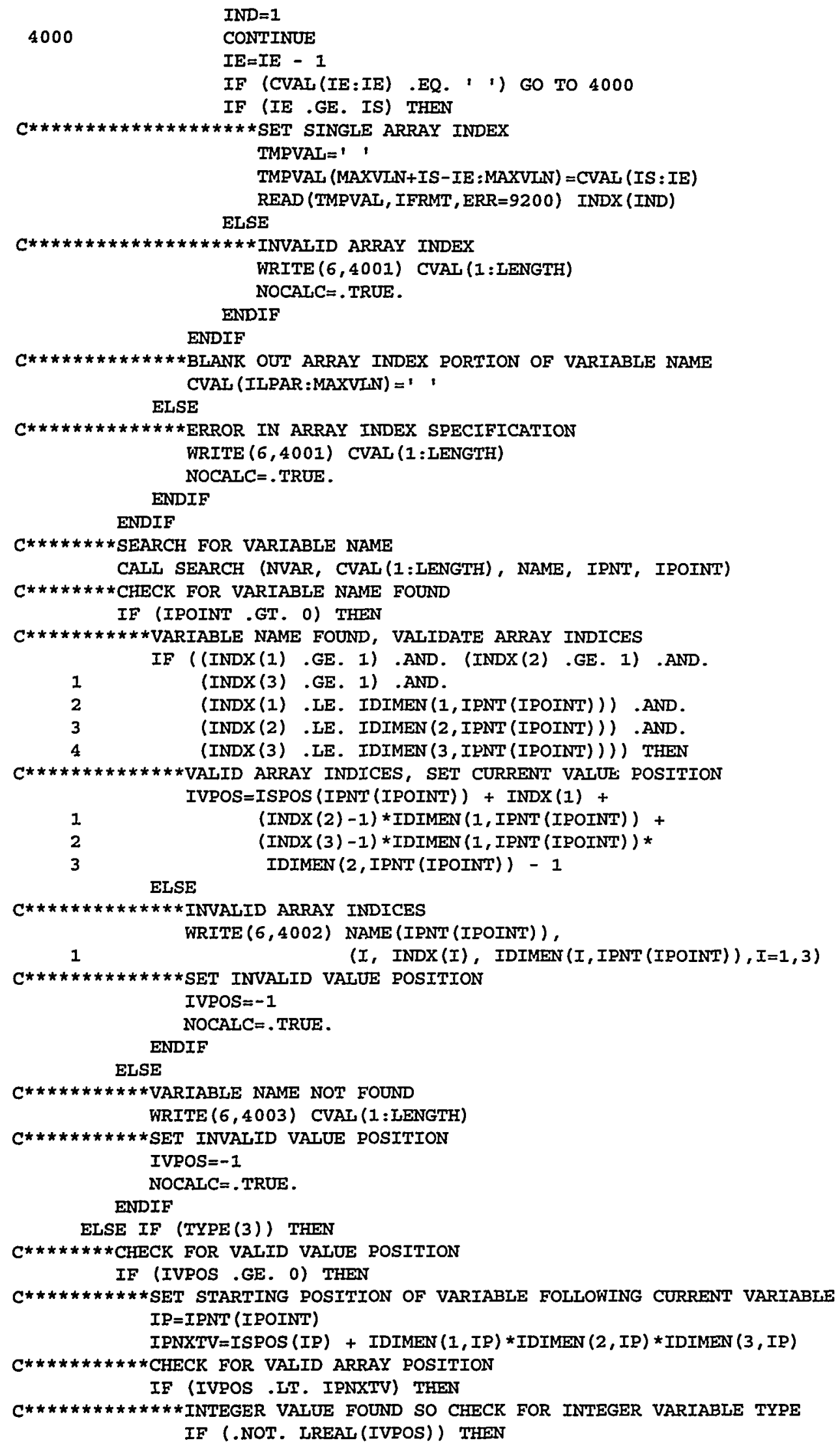


Appendix C

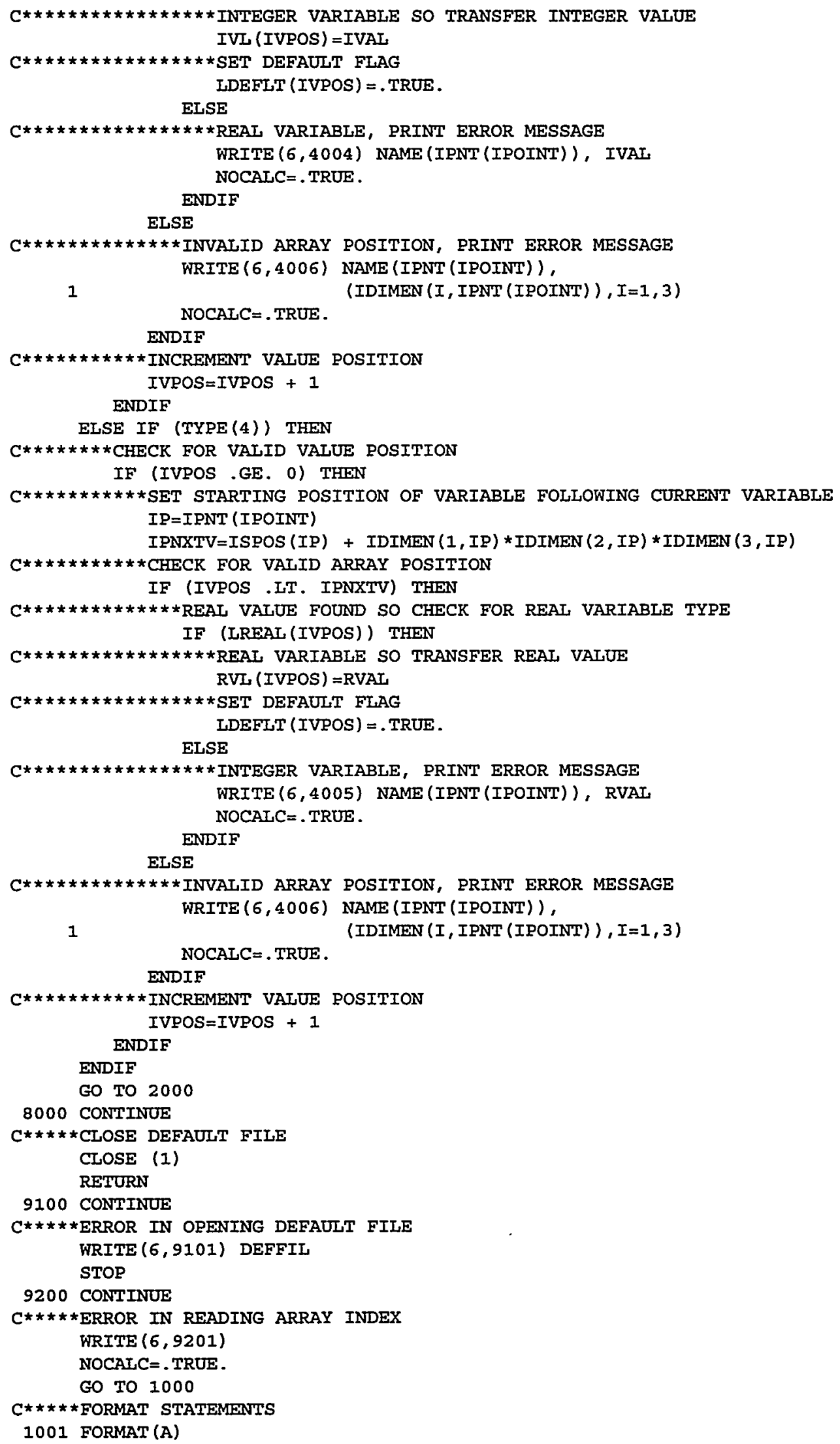


1002 FORMAT $(11 \mathrm{X}, \mathrm{A})$

1003 FORMAT (' 1 ', $/ 1 \mathrm{X}, 130(1 * 1)$,

$1 / 1 X, 53(1 * 1), 5 \mathrm{X}$, 'DEFAULT INPUT', $5 \mathrm{X}, 54\left(1 *^{\prime}\right)$,

$2 \quad / 1 X, 17(1 * 1), 5 X, '$ FILE $=', A, 5 X, 17(1 * 1)$,

$3 / 1 x, 130(1 * 1), / 1)$

1004 FORMAT (' (I', I2,' ')')

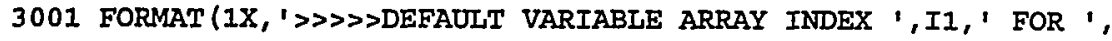

1 'VARIABLE ', A,' IS INVALID', /)

3002 FORMAT (1X,' $\gg \gg>M O R E$ THAN 3 ARRAY INDICES GIVEN FOR VARIABLE ',A,

1 'ON DEFAULT FILE')

4001 FORMAT(1X,' $\gg \gg \gg D E F A U L T$ VARIABLE ARRAY INDEX FOR ',

1 'VARIABLE ', $A$, ' IS INVALID',/)

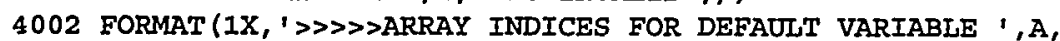

1 ' ARE OUT OF RANGE:'

$2 /(1 X, 1 \gg \gg \gg$ INDEX',I2,' =',I5,', VALID RANGE $=1$ TO 1, I5, /: $)$ )

4003 FORMAT $(1 X, 1 \gg \gg \gg$ DEFAULT VARIABLE NAME ', A,

1 ' NOT FOUND IN DEFAULT VARIABLE LIST',/)

4004 FORMAT (IX,' $\gg>>$ PATEMPT TO DEFAULT REAL VARIABLE (',A,

I ' ') TO INTEGER VALUE $(1, I 10,1) ', /)$

4005 FORMAT (1X,' $\gg \gg \gg$ PTTEMPT TO DEFAULT INTEGER VARIABLE (',A,

1 ' ') TO REAL VALUE (1,1PE10.3,1)',/)

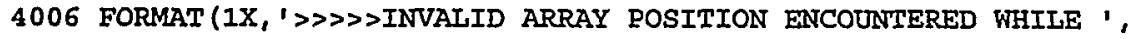

1 'SETTING DEFAULT VALUES FOR VARIABLE--',

8001 FORMAT (' 1 ')

$A, '(1, I 2,1,1, I 2,1,1, I 2,1) ')$

9101 FORMAT (1X,' $\gg \gg>$ ERROR ORENING DEFAULT FILE ', $A, /$ )

9201 FORMAT (1X,' $\gg \gg>$ ERROR IN READING PREVIOUS ARRAY INDEX')

END

SUBROUTINE VECPOS

$C \star * \star * \star$ SET SAMPLE VECTOR POSITIONS BY READING VARIABLE NAMES AND

$C \star \star \star \star \star$ CORRESPONDING SAMPLE VECTOR POSITIONS FROM FILE DESIGNATED

$C \star \star \star \star \star F O R$ SAMPLE VECTOR POSITIONS (SAMFIL)

PARAMETER (MAXBD $=20, \operatorname{MAXBIN}=10000, \operatorname{MAXSMP}=300, \operatorname{MAXCAS}=8$,

1

2

MAXISS $=20, M A X L E V=10, M A X V A R=100, M A X V A L=13000$,

PARAMETER (MAXIEN=101, MAXVLN=20)

CHARACTER*7 NAME

LOGICAL LDEFLT, LREAL

COMMON /DEFLTI/ NAME (MAXVAR)

COMMON /DEFLT2/ NVAR, NVAL, NVCB1, NVCB2, NVCB3, NVCB4,

1 NVCB5, IDIMEN (3,MAXVAR), ISPOS (MAXVAR),

2 ISMPPS (MAXVAL), IPNT (MAXVAR), LDEFLT (MAXVAI),

3 LREAL (MAXVAL)

LOGICAL NOCALC, SAMPLE, REPRTB, BINNED, BYRUN, CONSFL, DIAG,

1 EXPERT, PRTINP, NOCF, SUBCL, CDB, TMPCDB, BRKOPN, VB, ECF, ICF COMMON /KEYS/ NOCALC, SAMPLE, REPRTB, BINNED, BYRUN, CONSEL, DIAG,

1 EXPERT, PRTINP, NOCF, SUBCL, CDB, TMPCDB, BRKOPN, VB, ECF, ICF

CHARACTER $* 80$ DEFFIL, SAMFIL, VECFIL

COMMON /FILBLK/ DEFFIL, SAMFIL, VECFIL

CHARACTER* (MAXLEN) CARD

CHARACTER* (MAXVLN) CVAL, TMPVAL

CHARACTER* 10 IFRMT

DIMENSION INDX (3)

LOGICAI EOR, TYPE(4), LVAI

C

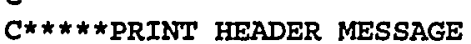

IF (PRTINP) WRITE $(6,1003)$ SAMFIL

$C \star \star \star \star \star$ WRITE INTEGER FORMAT FOR READING VARIABLE ARRAY INDICES

WRITE (IFRMT, 1004) MAXVIN

$C \star \star \star \star \star$ INITIAILIZE CURRENTI VALUE POSITION

IVPOS $=-1$

$C \star \star \star \star \star$ OPEN SAMPLE VECTOR POSITION FILE

Vol. 6, Part 1

C-21

NUREG/CR-6143 
Appendix C

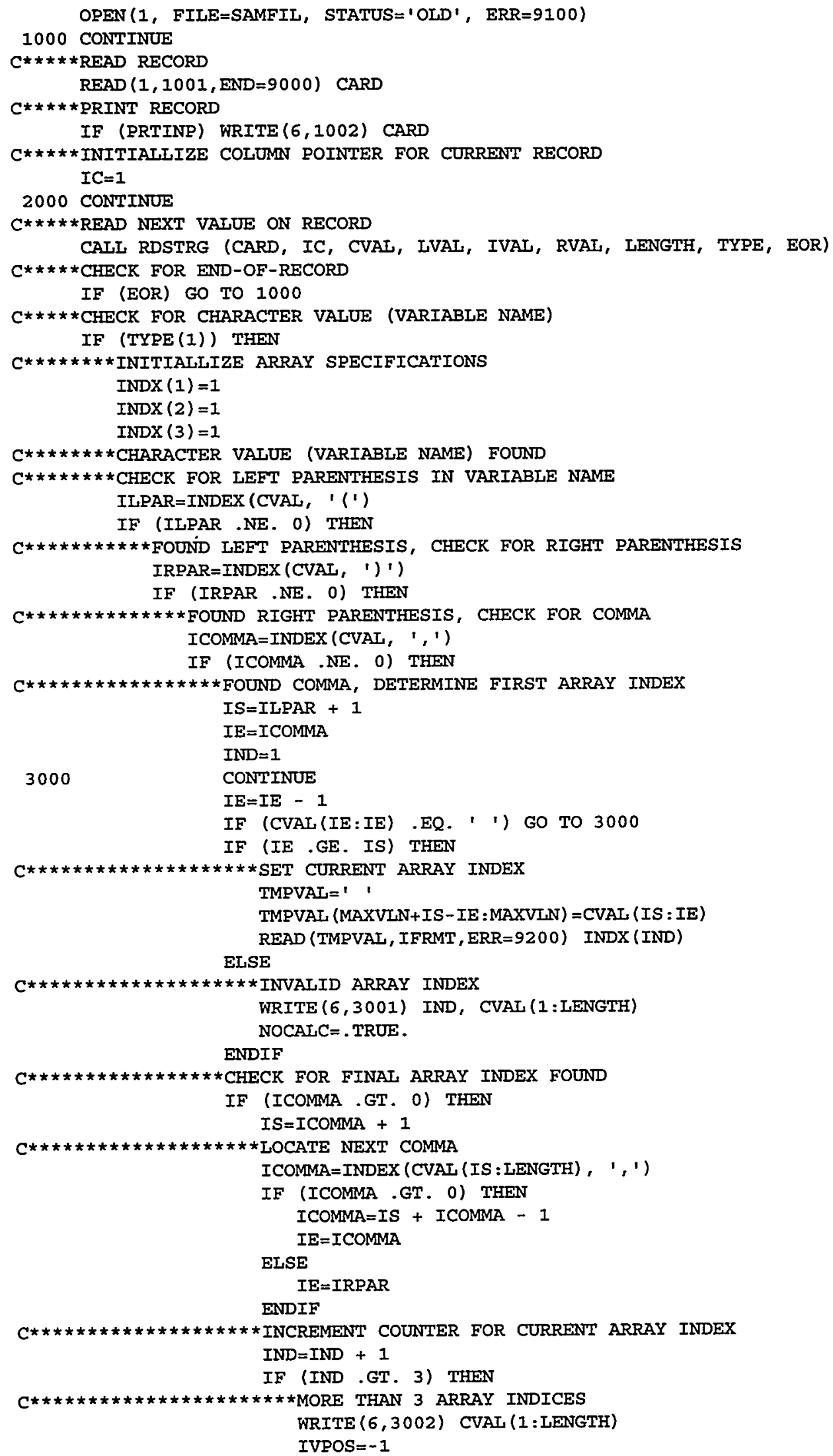




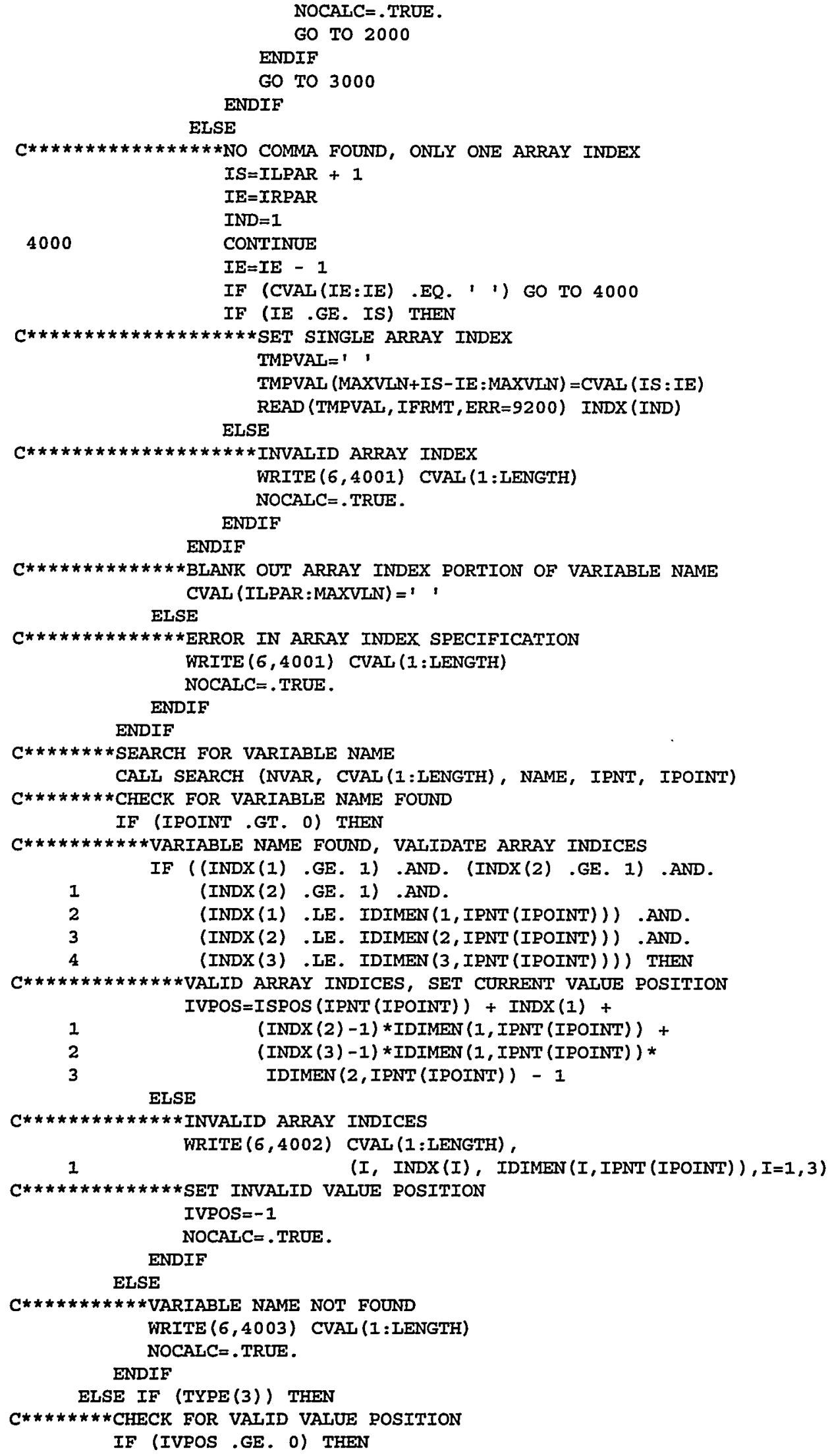


Appendix C

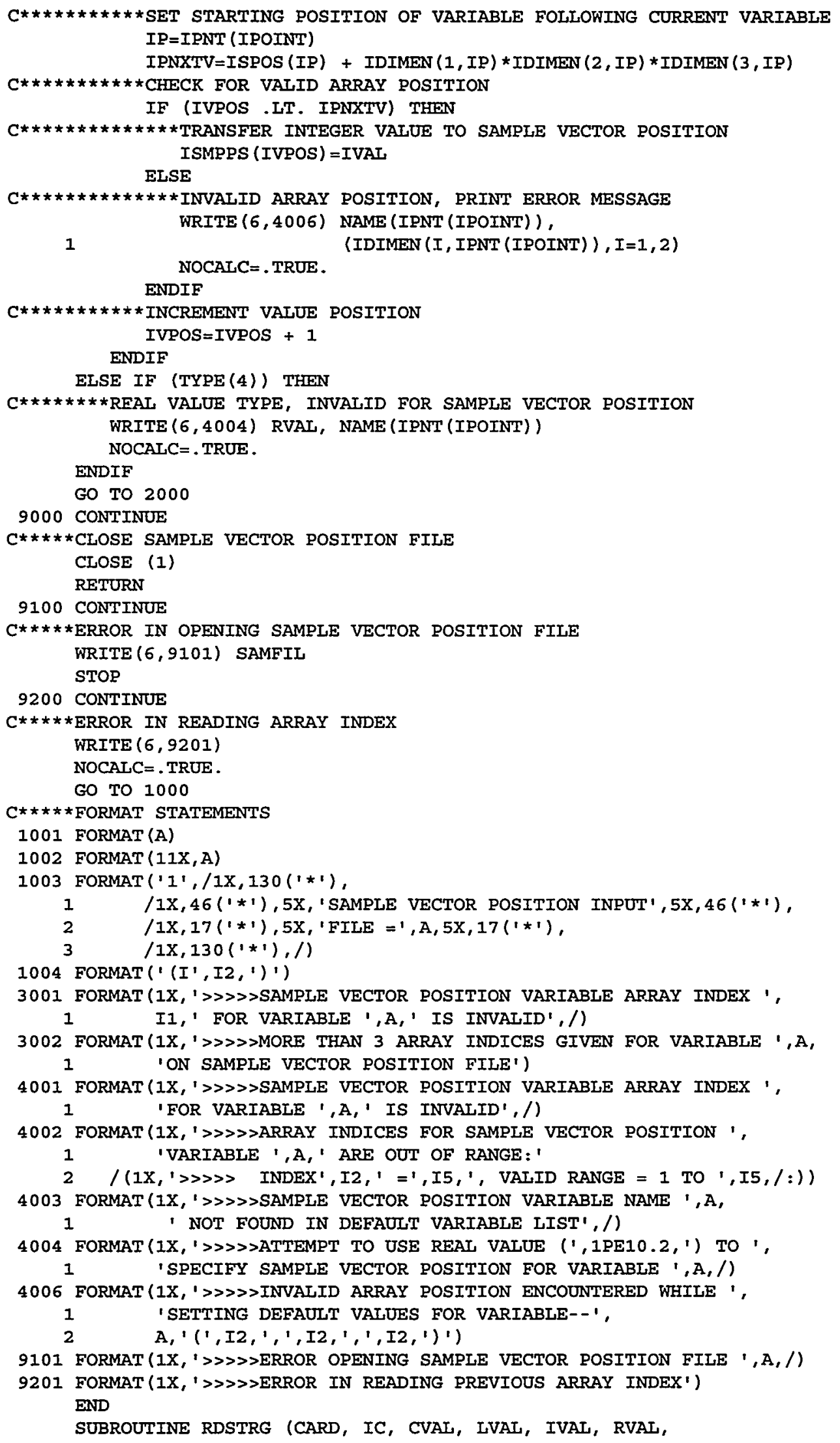


1 LENGTH, TYPE, EOR)

C*****CONVERTS A RECORD STRING TO A CHARACTER VALUE, A LOGICAL VALUE,

$C \star \star \star \star \star A$ REAL VALUE, AND AN INTEGER VALUE

PARAMETER ( IL $=100)$

CHARACTER* $(*)$ CARD, CVAL

CHARACTER* (IL) TMPCRD

CHARACTER*8 RFRMT

LOGICAI EOR, FIRST, LVAL, TYPE (4)

c

DATA FIRST / .TRUE. /

C

$C \star \star \star \star \star$ CHECK FOR FIRST TIME INTO ROUTINE

IF (FIRST) THEN

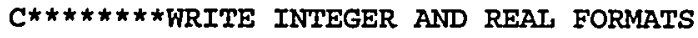

WRITE (RFRMT, 1002) IL

C $\star \star \star \star \star \star \star \star$ RESET INITIALLIZATION TYPE

FIRST $=$. FALSE.

ENDIF

LVAL $=$. FALSE .

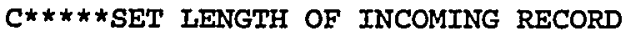

ILMAX =LEN (CARD)

$C * \star * \star \star$ SET IENGTH OF CHARACTER VARIABLE LENCVAL=LEN (CVAL)

$C \star \star \star \star \star$ INITIALLIZE VARIABLE FLAG TYPES ( $1=$ CHAR, 2=IOGIC, 3=INTEG, 4=REAL)

DO $1000 \quad I=1,4$

$\operatorname{TYPE}(I)=$. FALSE.

1000 CONTINUE

$C \star \star \star \star \star$ INTIALLIZE END-OF-RECORD TYPE

EOR= . FALSE.

$C \star \star \star \star \star$ RESET STARTING POSITION FOR CHARACTER POINTER

$I C=I C-1$

C $\star \star \star \star \star$ SEARCH FOR FIRST NON-BLANK CHARACTER

2000 CONTINUE

$C * * \star * \star$ INCREMENT CHARACTER POINTER

$I C=I C+1$

$C \star \star \star \star \star$ CHECK FOR END OF RECORD

IF (IC .GT. ILMAX) GO TO 9100

$C \star \star \star \star \star$ CHECK FOR BLANK CHARACTER (STRING DELIMITER)

IF (CARD (IC:IC) .EQ. ' I) GO TO 2000

$C \star \star \star \star \star$ CHECK FOR BEGINNING OF COMMENT

IF (CARD(IC:IC) .EQ. ' \$') GO TO 9100

$C \star * \star \star \star$ CHECK FOR COMMA CHARACTER (STRING DELIMITER)

IF (CARD(IC:IC) .EQ. ',') GO TO 2000

$C * * * * *$ CHECK FOR QUOTE CHARACTER (CHARACTER STRING DELIMITER)

IF (CARD (IC:IC) .EQ. ' 'I) THEN

$C * * * \star * * \star *$ SAVE STARTING POSITION OF CHARACTER STRING

$I S=I C+1$

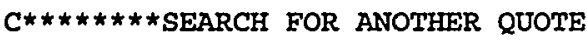
IC $=$ INDEX (CARD (IS : ILMAX),,$\cdots$,

IF (IC .EQ. O) THEN

$C \star \star \star \star \star \star \star \star \star \star \star \star$ QUOTE NOT FOUND SO CONTINUE SEARCH FOR BLANK TO TERMINATE

$C \star \star \star \star \star \star \star \star \star \star \star \star \star$ CHARACTER STRING

IC $=$ IS - 1

ELSE

C***********QUOTE FOUND

$I C=I S+I C-1$

GO TO 3100

ENDIF

C $\star \star \star \star \star \star \star \star$ SEARCH FOR END OF CHARACTER STRING (' SIGNIFIES BEGINNING AND

$C \star \star \star \star \star \star \star \star * E N D$ OF CHARACTER STRING)

3000 CONTINUE

$C \star \star \star \star \star \star \star \star$ INCREMENT CHARACTER POINTER

$I C=I C+I$

Vol. 6, Part 1 
Appendix C

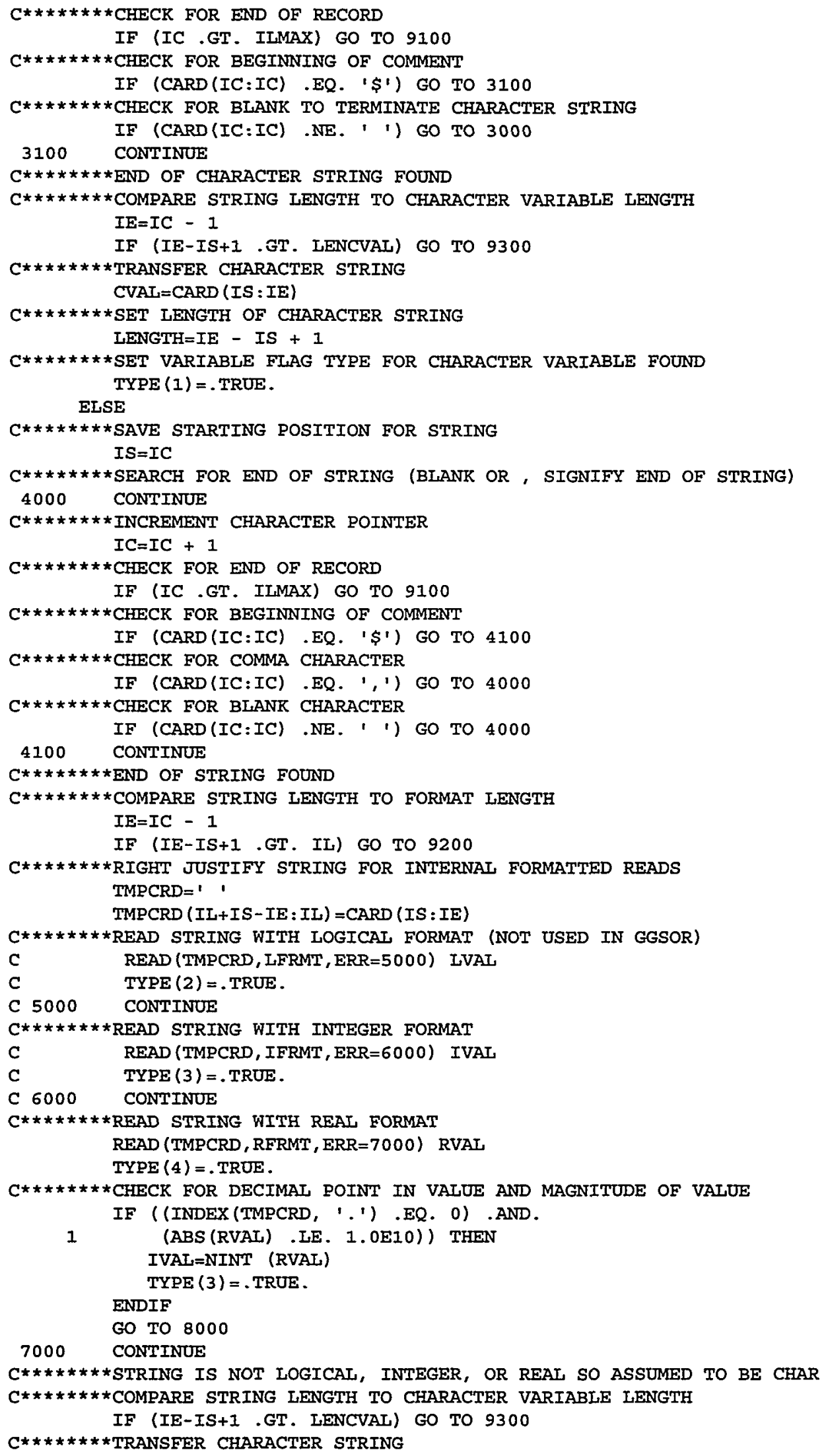




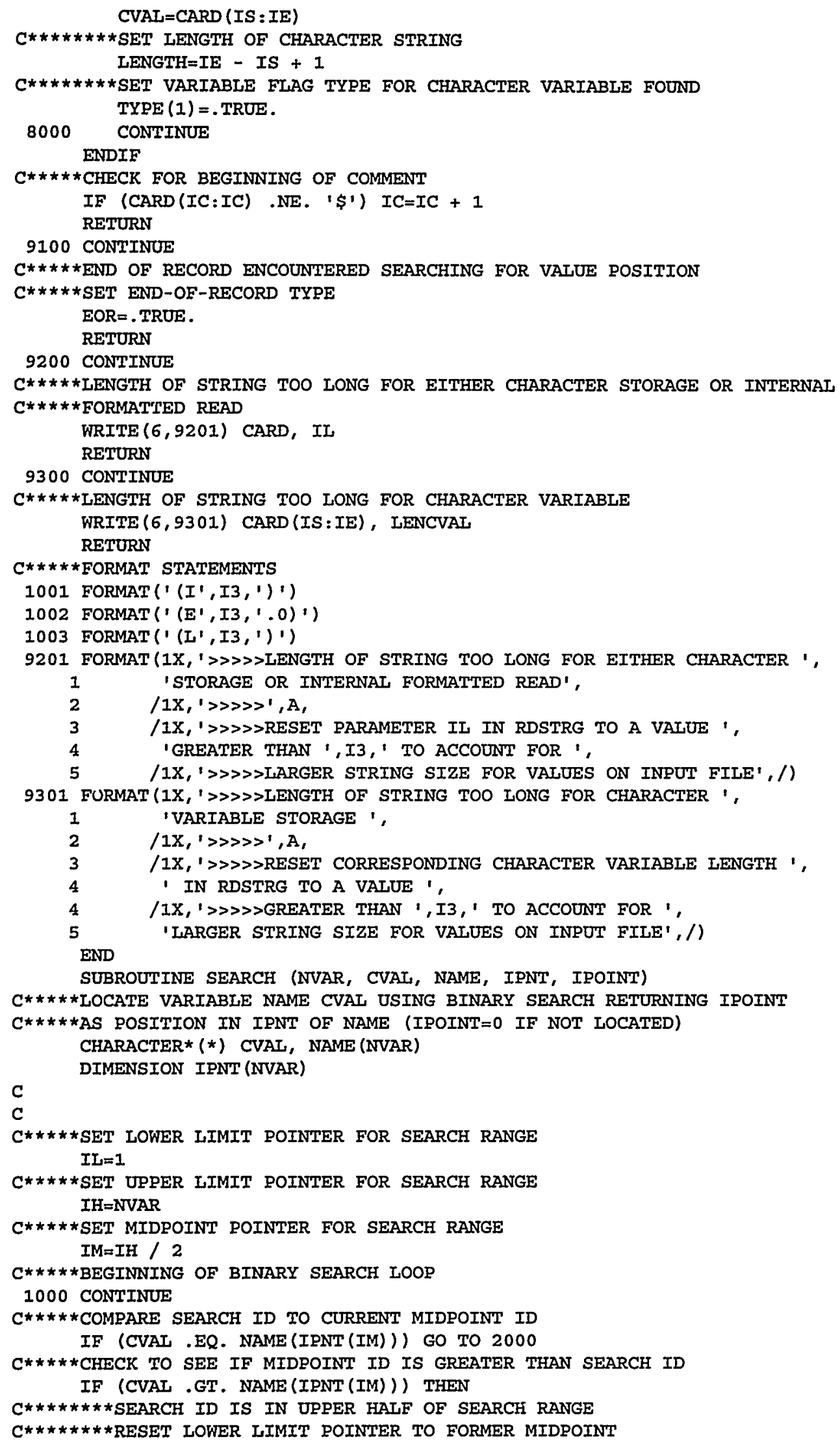


Appendix C

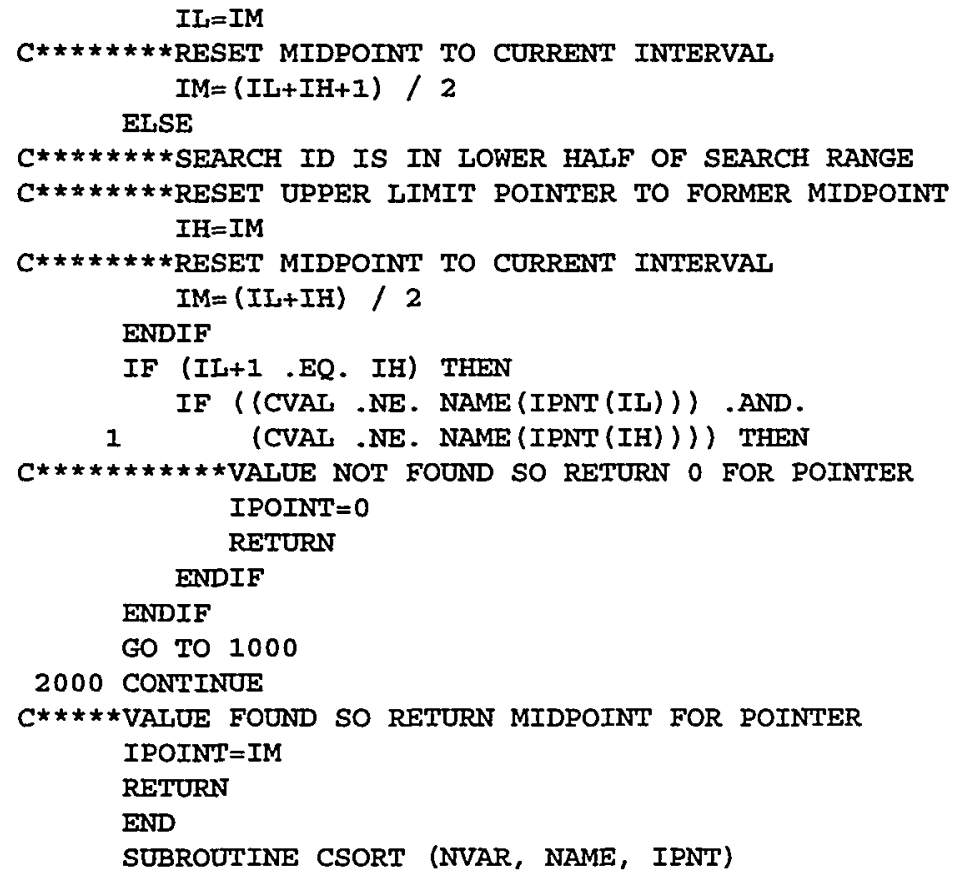




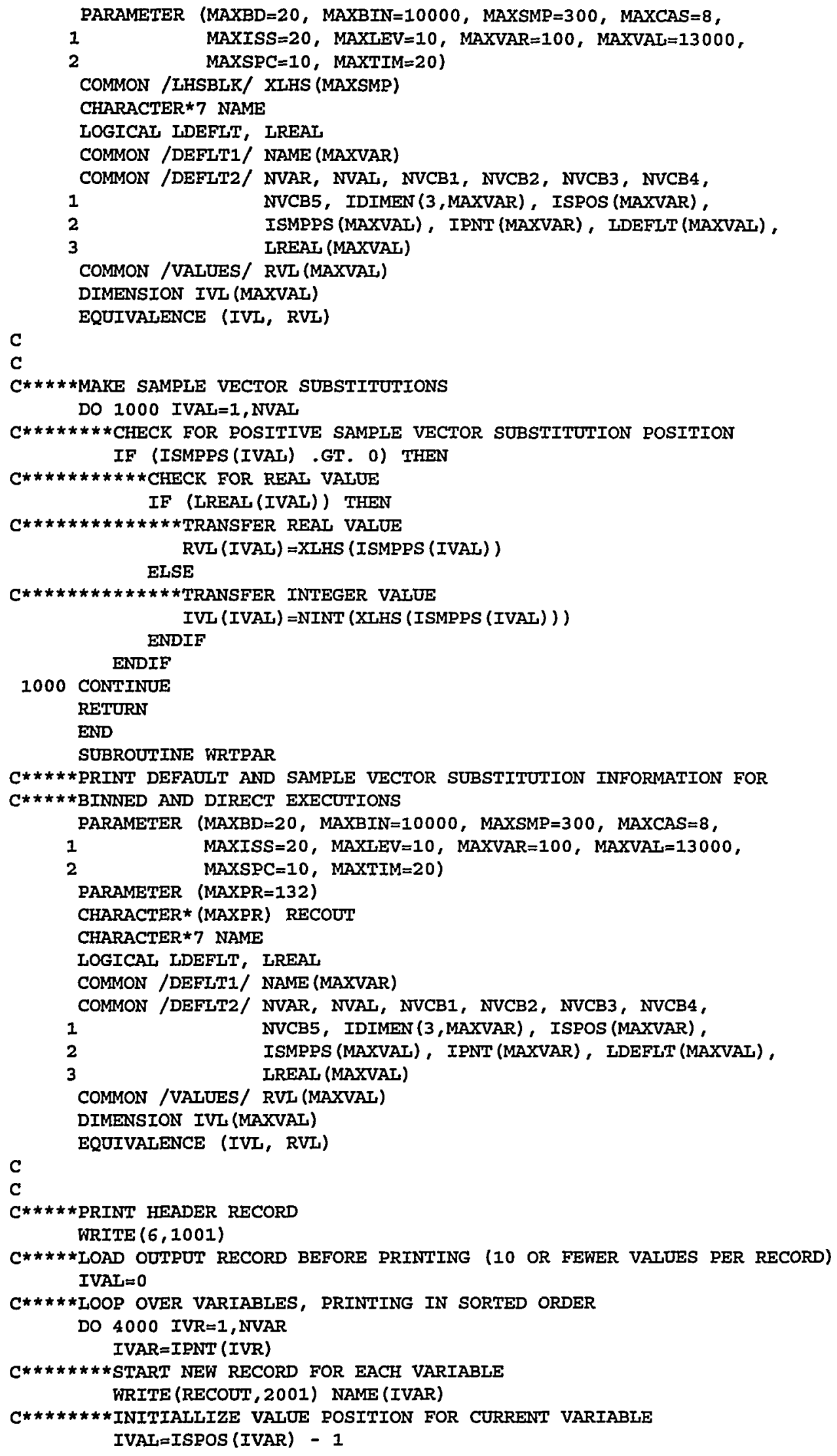

Vol. 6, Part 1 
Appendix C

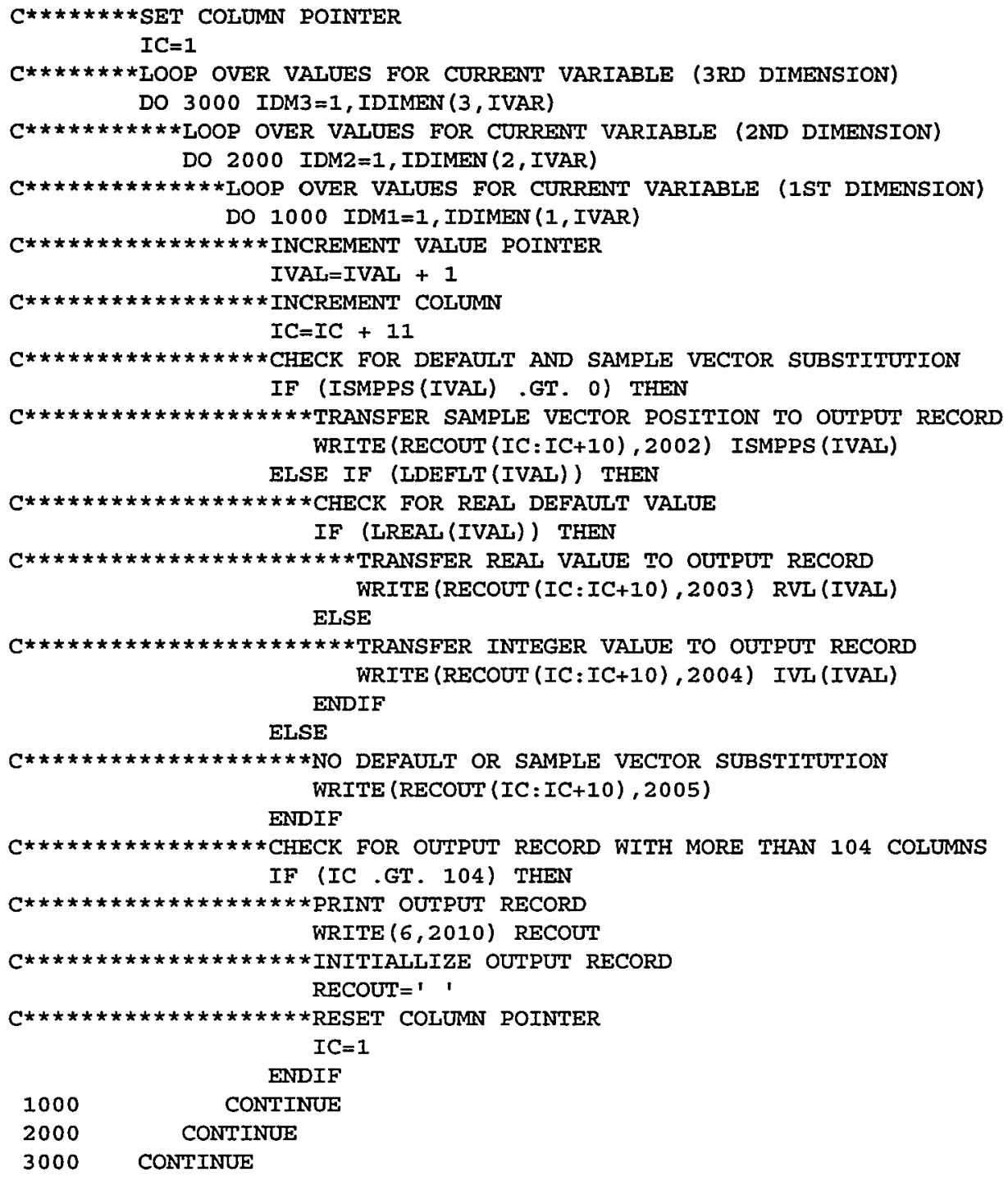




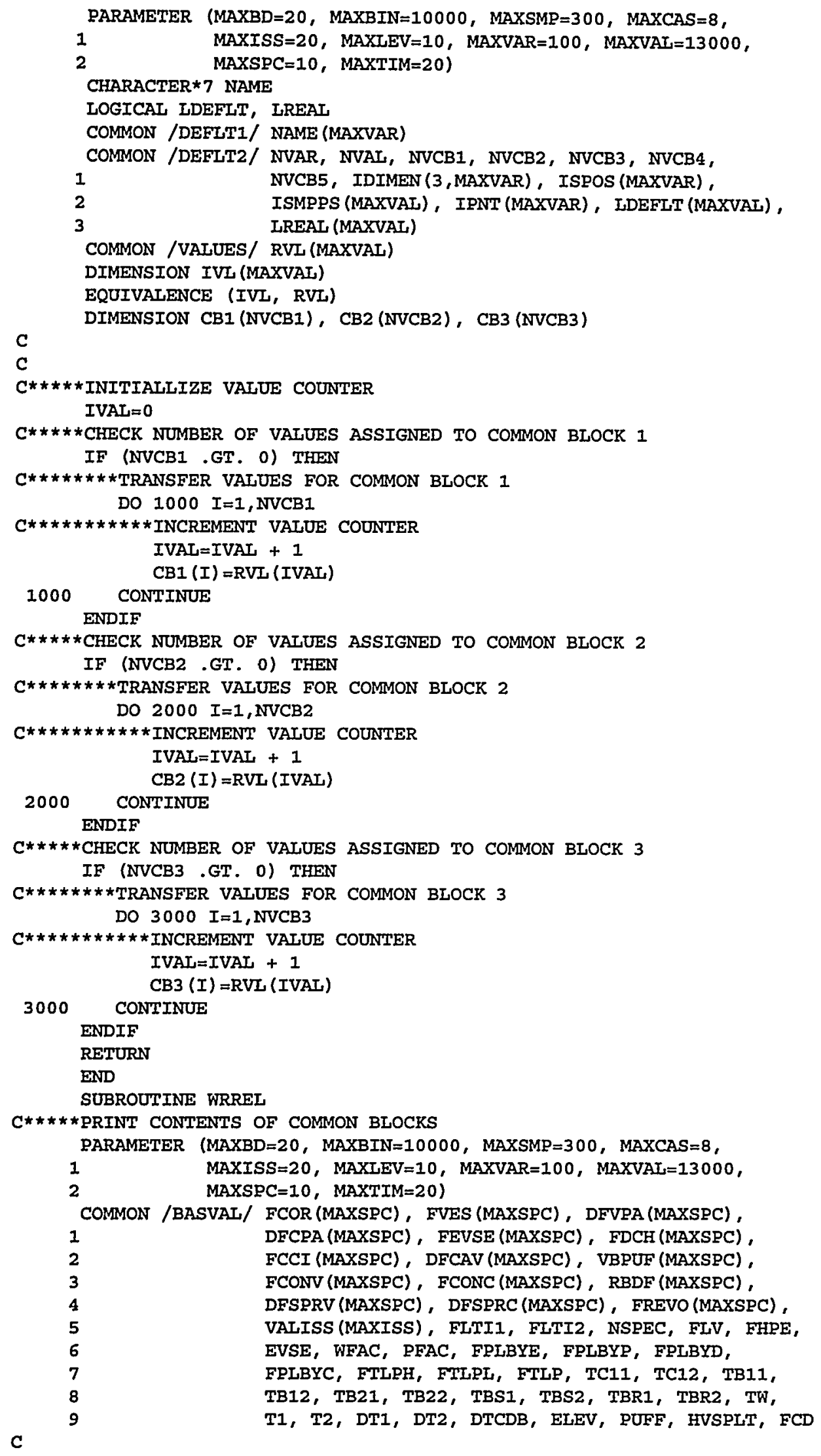




\section{Appendix C}

C

WRITE $(6,1001)$

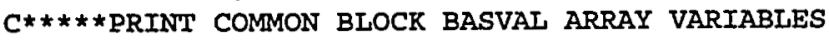

WRITE $(6,1002)$ 'BASVAI', 'BASE VALUES FOR GGSOR'

WRITE $(6,1003)$ 'FCOR ', 'FVES ', 'DFVPA ', 'DFCPA',

2

DO $1000 \mathrm{~K}=1$, NSPEC

'FEVSE ', 'FDCH ', 'FCCI ', 'DFCAV ',

IVBPUF ', 'FCONV '

WRITE $(6,1004)$ FCOR (K), FVES (K), DFVPA (K), DFCPA (K) ,

1 FEVSE $(K), F D C H(K), F C C I(K), \operatorname{DFCAV}(K)$, $\operatorname{VBPUF}(K), F C O N V(K)$

1000 CONTINUE

WRITE $(6,1003)$ 'FCONC ', 'RBDF ', 'DFSPRV ', 'DFSPRC ',

1

DO $2000 \quad K=1$, NSPEC

$\operatorname{WRITE}(6,1004) \operatorname{FCONC}(K), \operatorname{RBDF}(K), \operatorname{DFSPRV}(K), \operatorname{DFSPRC}(\mathrm{K})$, 1 FREVO (K)

2000 CONTINUE

WRITE $(6,1003)$ 'VALISS '

DO 3000 IISS $=1$, MAXISS

WRITE $(6,1004)$ VAIISS (IISS)

3000 CONTINUE

$C * * * * *$ PRINT COMMON BLOCK BASVAL SINGLE VARIABLES

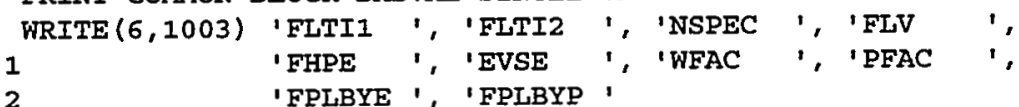

'FPLBYE ', 'FPLBYP '

WRITE $(6,1004)$ FLTI1, FITI2, FLOAT (NSPEC)， FLV,

1 FHPE, EVSE, WFAC, PFAC,

2 FPLBYE, FPLBYP

WRITE $(6,1003)$ 'FPLBYD ', 'FPLBYC ', 'FTLPH ', 'FTLPL ',

IFTLP ', 'TC11 ', 'TC12 ', 'TB11 ',

2

WRITE $(6,1004)$ FPLBYD, FPLBYC, FTLPH, FTLPL,

1 FTLP, TC11, TC12, TB11,

2 TB12, TB21

WRITE $(6,1003)$ 'TB22 $\quad$ 'TBS1 ', 'TBS2 ', 'TBR1 ',

$\begin{array}{llllll}1 & \text { 'TBR2 } & \text { ', ITW } & \text { 'T 'T1 ', 'T2 }\end{array}$

WRITE $(6,1004)$ TB22, TBS1, TBS2, TBR1,

1 TBR2, TW, T1, T2,

2 DT1, DT2

WRITE $(6,1003)$ 'DTCDB ', 'ELEV ', 'PUFF ।

WRITE $(6,1004)$ DTCDB, ELEV, PUFF

RETURN

$C \star \star \star \star \star$ FORMAT STATEMENTS

1001 FORMAT ('1')

1002 FORMAT $\left(/ / 1 \mathrm{X}, 130\left(^{\prime}={ }^{\prime}\right)\right.$,

$1 \quad / 1 \mathrm{X}, 5\left(1 *^{\prime}\right), 1^{\prime}$ CONTENTS OF COMMON BLOCK ', A, ', $5\left({ }^{\prime} *^{\prime}\right)$,

$2 \quad / 7 X, A$,

$3 \quad\left(1 \mathrm{X}, 130\left({ }^{\prime}={ }^{\prime}\right)\right)$

1003 FORMAT $(/ 3 \mathrm{X}, 10(\mathrm{~A} 7,4 \mathrm{X}))$

1004 FORMAT (1X, 1P, 10E11.3)

END

SUBROUTINE BINTRN (IBIN)

$C \star \star \star \star \star$ PERFORM BIN TRANSLATION

$\mathrm{C}$

$C=====$ BIN DIMENSIONS

$\mathrm{C}$

$C=====\operatorname{INDX}(1):$ PLANT DAMAGE STATES

C 1: PLANT DAMAGE STATE 1

C 2: PLANT DAMAGE STATE 2

C 3: PLANT DAMAGE STATE 3 
C

C

12: PIANT DAMAGE STATE 12

$C===:=\operatorname{INDX}(2):$ CONTAINMENT STATUS

C 1: CONTAINMENT EQUIPMENT HATCH IS OPEN

C 2: CONTAINMENT VENTED DURING CORE DAMAGE

C 3: CONTAIMMENT RUPTURE ABOVE AUX BLDG DURING CORE DAMAGE

C 4: CONTAINMENT LEAK ABOVE AUX BIDG DURING CORE DAMAGE

C 5: CONTAINMENT RUPTURE ABOVE AUX BLDG DURING VESSEL FAILURE

C 6: CONTAINMENT LEAK ABOVE AUX BLDG DURING VESSEL FAILURE

C 7: CONTAINMENT VENTED DURING ILATE TIME REGIME

C 8: CONTAINMENT RUPTURE LATE IN ACCIDENT

C 9: CONTAINMENT LEAK IATE IN ACCIDENT

C 10: CONTAINMENT CLOSED

$\mathrm{C}=====$ INDX (3): AUXILIARY BUILDING PRESSURE INTEGRITY STATUS

C 1: CONTAIMMENT OREN AND AUX BLDG FAILS PRIOR TO CORE DAMAGE

C 2: CONTAIMMENT OREN AND AUX BLDG FAILS DURING CORE DAMAGE

C 3: CONTAINMENT OPEN AND AUX BLDG FAILS AFTER VESSEL FAILURE

C 4 : CONTAINMENT CLOSED

$C==\approx=\approx$ INDX (4) : DRYWELI PRESSURE INTEGRITY STATUS

C 1: DRYWELL EQUIPMENT HATCH OREN

C 2: DRYWELL EQUIPMENT HATCH CLOSED

$C==2==$ INDX $(5)$ : REACTOR VESSEL PRESSURE BOUNDARY STATUS

C 1: ISOLATED

C 2: PIPE BREAK IN DRYWELL (LOCA) PRIOR TO CORE DAMAGE

C 3: OREN MSIV OR UNISOLATED INTERFACING SYSTEMS LOCA

C 4: UNISOLATED PRIOR TO CORE DAMAGE, ISOLATED DURING CORE DAMAGE

$C=====$ INDX $(6)$ : REACTOR HEAD VENT STATUS PRIOR TO CORE DAMAGE

C 1: CLOSED

C 2: OPEN

$C=====$ INDX (7): THE SRV TAILPIPE VACUUM BREAKERS STATUS

C $\quad$ 1: STICKS OPEN

C 2: DOES NOT STICK OPEN

$C====:$ INDX $(8)$ : REACTOR VESSEL STATUS PRIOR TO VESSEL FAILURE

C I: PRESSURIZED, NO COOLANT INJECTION

C 2: UNPRESSURIZED, NO COOLANT INJECTION

C 3: PRESSURIZED, COOLANT INJECTION

C 4 : UNRRESSURIZED, COOLANT INJECTION

C 5: PRESSURIZED, CORE COOLING RESTORED, CORE DAMAGE ARRESTED

C 6: UNPRESSURIZED, CORE COOLING RESTORED, CORE DAMAGE ARRESTED

$C=:==2$ INDX $(9):$ CONTAIMMENT SPRAYS STATUS

C 1 : NOT USED

C 2 : USED

$C=====$ INDX $(10)$ : ZIRCONIUM FRACTION OXIDIZED PRIOR TO VESSEL FAILURE

C I: GREATER THAN 0.21

C 2 : LESS THAN 0.21

C $== \pm=2$ INDX(11): CORE FRACTION PARTICIPATING IN HPME OR STEAM EXPLOSION

C $1: 40 \%$ OF CORE PARTICIPATES IN HPME

C 2: 10\% OF CORE PARTICIPATES IN HPME

C 3: 40\% OF CORE PARTICIPATES IN EX-VESSEL STEAM EXPLOSION

C 4: 10\% OF CORE PARTICIPATES IN EX-VESSEL STEAM EXPLOSION

C 5: NO HPME, NO EX-VESSEL STEAM EXPLOSION

$C=====$ INDX (12) : CORE DEBRIS STATUS IN THE REACTOR PEDESTAL CAVITY

C 1: CORE-CONCRETE INTERACTIONS IN DRY CAVITY

C 2: CORE-CONCRETE INTERACTIONS IN FLOODED CAVITY

C 3: NO CORE-CONCRETE INTERACTIONS, CAVITY CORE DEBRIS QUENCHED

C==E== INDX(13): INITIATING EVENT TIME WINDOW

C $\quad$ 1: 14 TO 24 HOURS AFTER SHUTDOWN

C $2: 24$ TO 94 HOURS AFTER SHUTDOWN

C 3: 40 TO 50 DAYS AFTER SHUTDOWN

$C== \pm= \pm$ INDX (14): SUPPRESSION POOL TEMPERATURE

C I: SUBCOOLED SUPPRESSION POOL

Vol. 6, Part 1 
Appendix C

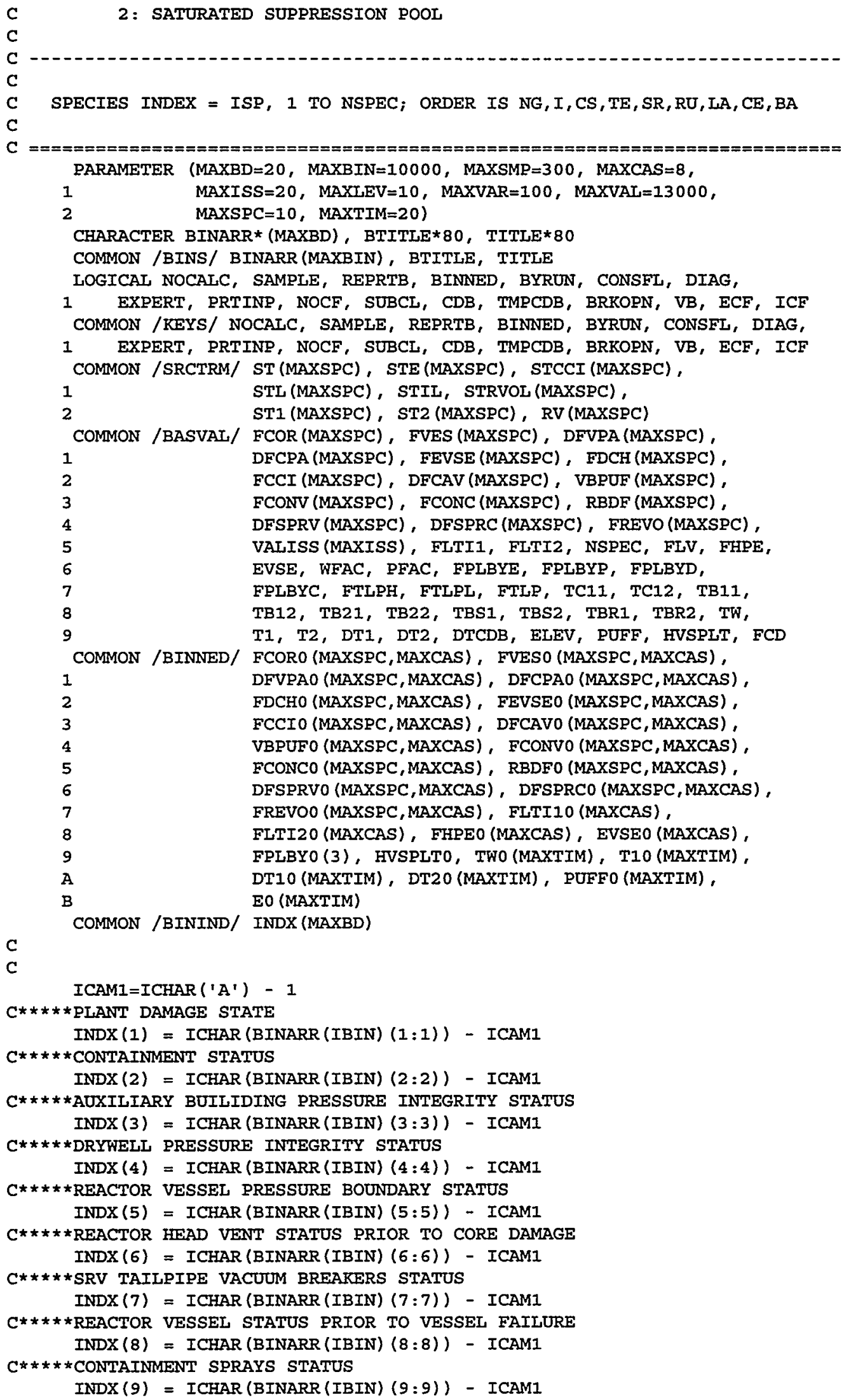


C*****ZIRCONIUM FRACTION OXIDIZED PRIOR TO VESSEL FAILURE $\operatorname{INDX}(10)=$ ICHAR (BINARR (IBIN) $(10: 10)$ ) - ICAMI

$C \star \star \star \star \star C O R E$ FRACTION PARTICIPATING IN HPME OR STEAM EXPLOSION $\operatorname{INDX}(11)=$ ICHAR (BINARR (IBIN) (11:11)) - ICAMI

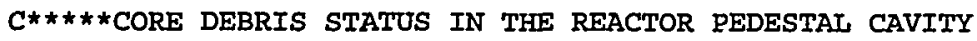
$\operatorname{INDX}(12)=$ ICHAR (BINARR (IBIN) (12:12)) - ICAMI

$C \star \star \star * \star$ INITIATING EVENT TIME WINDOW $\operatorname{INDX}(13)=\operatorname{ICHAR}($ BINARR (IBIN) $(13: 13))$ - ICAMI

$C * \star \star \star \star$ SUPPRESSION POOL TEMPERATURE $\operatorname{INDX}(14)=\operatorname{ICHAR}($ BINARR (IBIN) $(14: 14))$ - ICAM1

$C * \star \star \star *$ SET LOGICAL FLAGS TO BE PASSED TO GGSORC

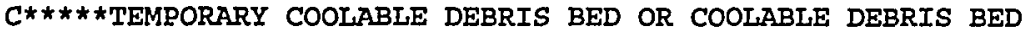
TMPCDB = . FALSE.

$\mathrm{CDB}=(\operatorname{INDX}(12) \quad . E Q \cdot 3)$

$C \star * \star * *$ VESSEL BREACH

$\mathrm{VB}=(\operatorname{INDX}(8)$.IE. 4)

$C \star \star \star \star \star$ SUPPRESSION POOL TEMPERATURE SUBCL $=(\operatorname{INDX}(14)$.EQ. 1)

$C * * * * *$ NO CONTAINMENT FAILURE FLAG NOCF $=($ INDX (2) .EQ. 10)

$C \star * \star * *$ EARLY CF BEFORE VB INCLUDES CASE WITH ILOCA OR OPEN MSIVS $E C F=((\operatorname{INDX}(2) . L E .4)$.OR. (INDX (5) .EQ. 3))

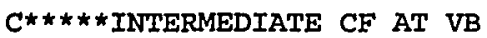
$I C F=(\operatorname{INDX}(2) \quad . E Q .5) \quad . O R .(\operatorname{INDX}(2) \quad . E Q .6)$

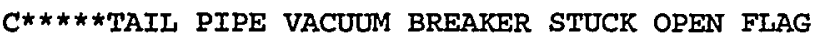
BRROPN $=($ INDX (7) .EQ. 1)

$C * \star \star \star *$ REACTOR HEAD VENT SPLIT FRACTION

IF ((INDX (6) .EQ. 2) .AND. (INDX (5) .EQ. 1)) THEN HVSPLT $=$ HVSPLTO

ELSE

HVSPLT $=0.0$

ENDIF

$C * * * * *$ LOOP OVER SPECIES

DO 500 ISP $=1$, NSPEC

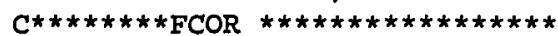

IF (INDX (8) .GE. 5) THEN

FCOR (ISP) $=$ FCD * FCORO (ISP, 2)

ELSE IF (INDX(10) .EQ. 1) THEN FCOR (ISP) $=$ FCORO (ISP, 1$)$

ELSE IF (INDX (10) .EQ. 2) THEN FCOR (ISP) $=$ FCORO $($ ISP, 2$)$ ENDIF

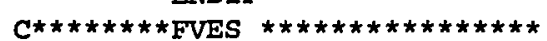

IF ((INDX (8) .EQ. 1) .OR. (INDX(8) .EQ. 3) .OR.

1 (INDX (8) .EQ. 5)) THEN

$C \star \star \star \star \star \star \star \star \star \star * *$ HIGH PRESSURE AT VB FVES (ISP) $=$ FVESO $($ ISP , I )

ELSE

$C * * \star * \star * \star * \star * \star$ LOW PRESSURE AT VB FVES (ISP) $=$ FVESO (ISP , 2)

ENDIF

$C \star \star * \star \star \star * *$ REVOLATILIZATION AFTER VESSEL BREACH

IF (INDX (8) . GE. 5) THEN

$C \star \star \star \star \star \star \star \star \star \star \star \star$ NO VESSEL BREACH, NO REVOLATIIIZATION FREVO (ISP) $=0.0$

ELSE IF ( $(\operatorname{INDX}(8)$.EQ. 3) .OR. (INDX(8) .EQ. 4)) THEN

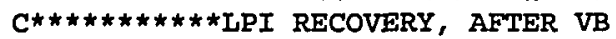
ELSE FREVO (ISP) $=$ FREVOO (ISP, 3 )

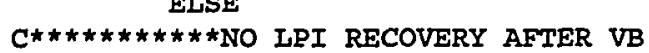
FREVO (ISP) $=$ FREVOO $($ ISP , I) ENDIF

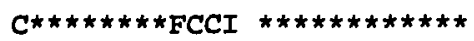

Vol. 6, Part 1 
Appendix C

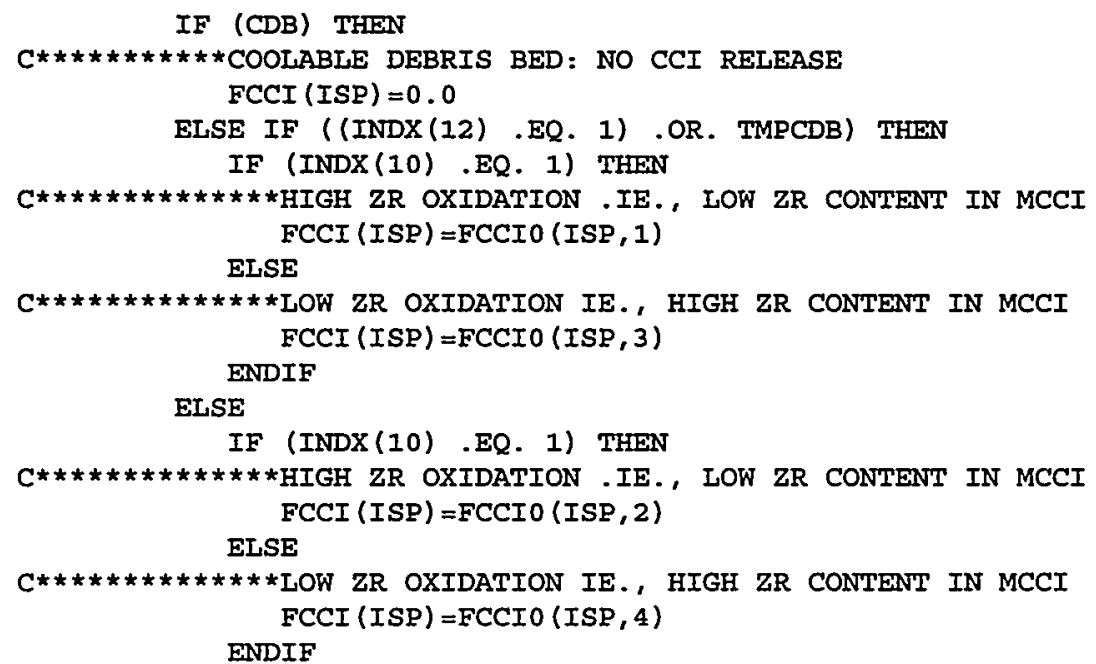


IF ((INDX (5) .EQ. 3) .OR. (INDX (5) .EQ. 4)) THEN

$F C O N V(I S P)=1.0$

ENDIF

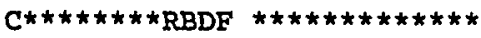

IF ((INDX (3) .GE. 1) .AND. (INDX (3) .IE. 3)) THEN

C $\star \star \star \star \star \star \star \star \star \star \star \star$ RELEASE GOES THROUGH REACTOR BUILDING $\operatorname{RBDF}($ ISP) $=\mathrm{RBDFO}($ ISP, 1 )

ELSE

C $\star \star \star \star \star \star \star \star \star \star \star \star$ REACTOR BUILDING BYPASSED

$\operatorname{RBDF}(I S P)=1.0$

ENDIF

C $\star \star \star \star \star \star \star * \star$ FDCH OR EX-VESSEL STEAM EXPLOSION

IF (INDX (11) .EQ. 5) THEN

$\mathrm{C} * \star * \star * \star * * * * *$ NO DCH, NO STEAM EXPLOSION

FHPE $=0.0$

EVSE $=0.0$

FDCH (ISP) $=0.0$

FEVSE (ISP) $=0.0$

ELSE IF (INDX(11) .IE. 2) THEN

$C * \star \star \star * * \star * \star * \star D C H$, NO STEAM EXPLOSION

EVSE $=0$.

FEVSE (ISP) $=0$.

FDCH (ISP) $=$ FDCHO (ISP, 1 )

IF (INDX (11) .EQ. 1) THEN

FHPE=FHPEO (1)

ELSE

FHPE $=$ FHPEO 0 (2)

ELSE

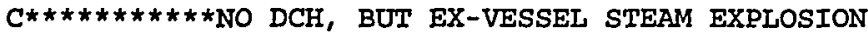

FHPE $=0.0$

FDCH (ISP) $=0.0$

FEVSE (ISP) $=$ FEVSE0 $($ ISP, 1 )

IF (INDX (11) .EQ. 3) THEN

EVSE $=$ EVSE 0 ( 1 )

ELSE

EVSE=EVSE0 (2)

ENDIF

ONDIF

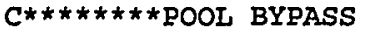

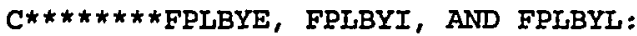

$C \star \star \star \star \star \star * \star$ FOR EARLY PHASE, ASSUME VACUUM BREAKER STICKS OREN FOR ASSIGNING

$C \star \star \star \star \star \star \star \star$ FPLBYE. IF BRROPEN IS FALSE, SET FPLBYE TO 0.0 I.ATER

IF (INDX (4) .EQ. 1) THEN

$C \star \star \star \star \star \star \star \star \star \star \star \star$ COMPLETE POOL BYPASS

FPLBYE=FPLBYO (3)

FPLBYI=FPLBYO (3)

FPLBYL $=$ FPLBYO (3)

ELSE

$C \star * \star \star \star \star \star * \star * * *$ NOMINAL POOL BYPASS

FPLBYE=FPLBYO (1)

FPLBYI $=$ FPLBYO (1)

FRLBYL=FPLBYO (1)

ENDIF

$C \star \star \star \star \star \star \star \star$ IF BRKORN IS FALSE, THEN FPLBYE=0.0 IRREGARDLESS OF DRYWELL

$C \star \star \star \star \star \star \star \star$ LEAKAGE SINCE EVERYTHING GOES THROUGH POOL

IF (.NOT. BRKOPN) FPLBYE $=0.0$

IF (BRROPN) THEN

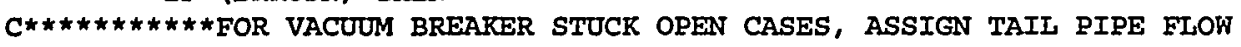

C $\star \star \star \star \star \star \star \star \star \star \star \star *$ FRACTION FOR HIGH PRESSURE VERSUS LOW PRESSURE SEQUENCES

IF ((INDX (8) .EQ. 1) .OR. (INDX (8) .EQ. 3) .OR.

1

$(\operatorname{INDX}(8)$.EQ. 5)) THEN

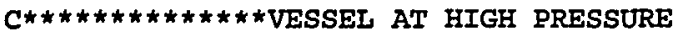

Vol. 6, Part 1 


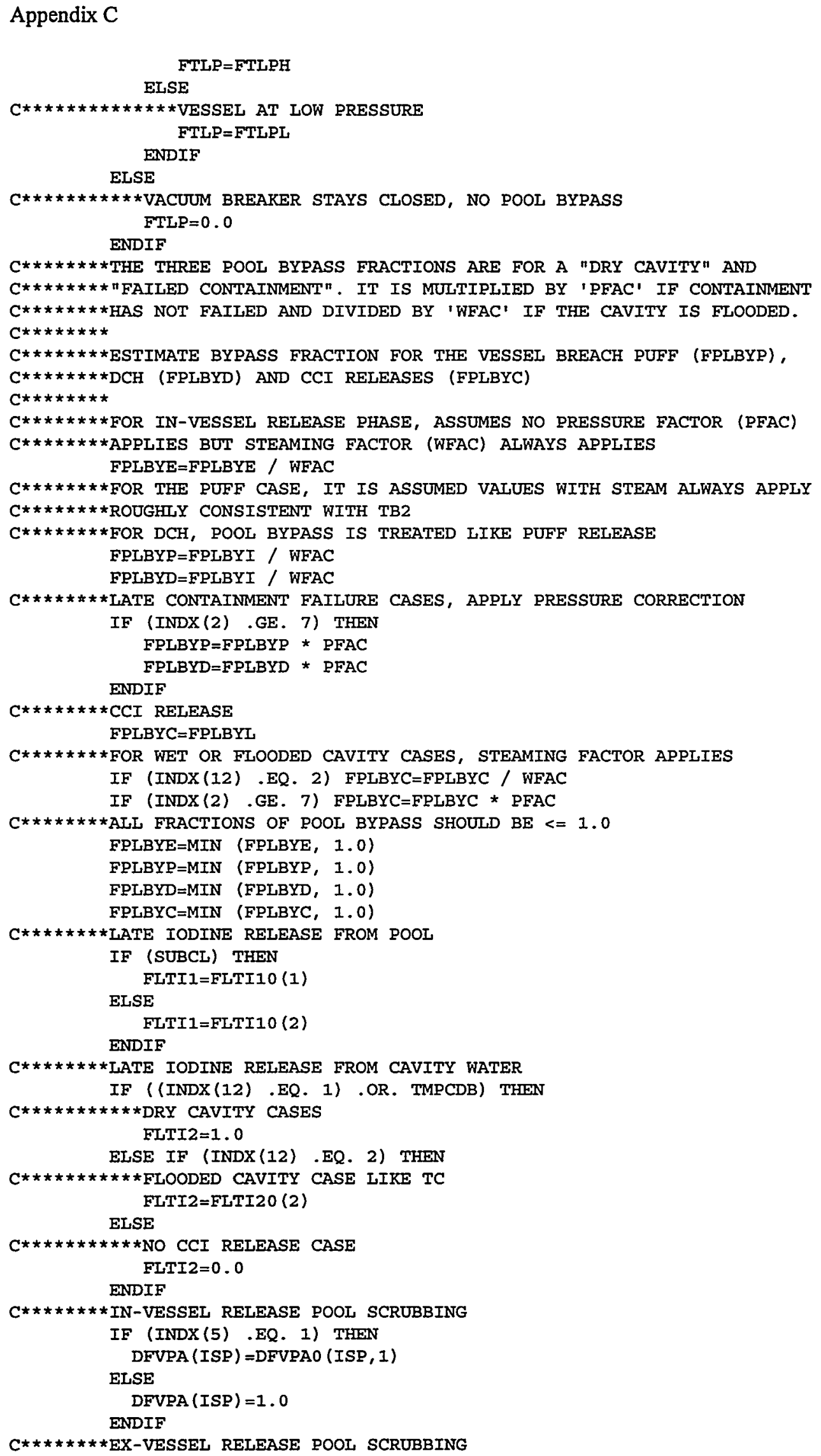

$C \star \star \star \star \star \star \star \star$ EX VESSEL RELEASE POOL SCRUBBING 


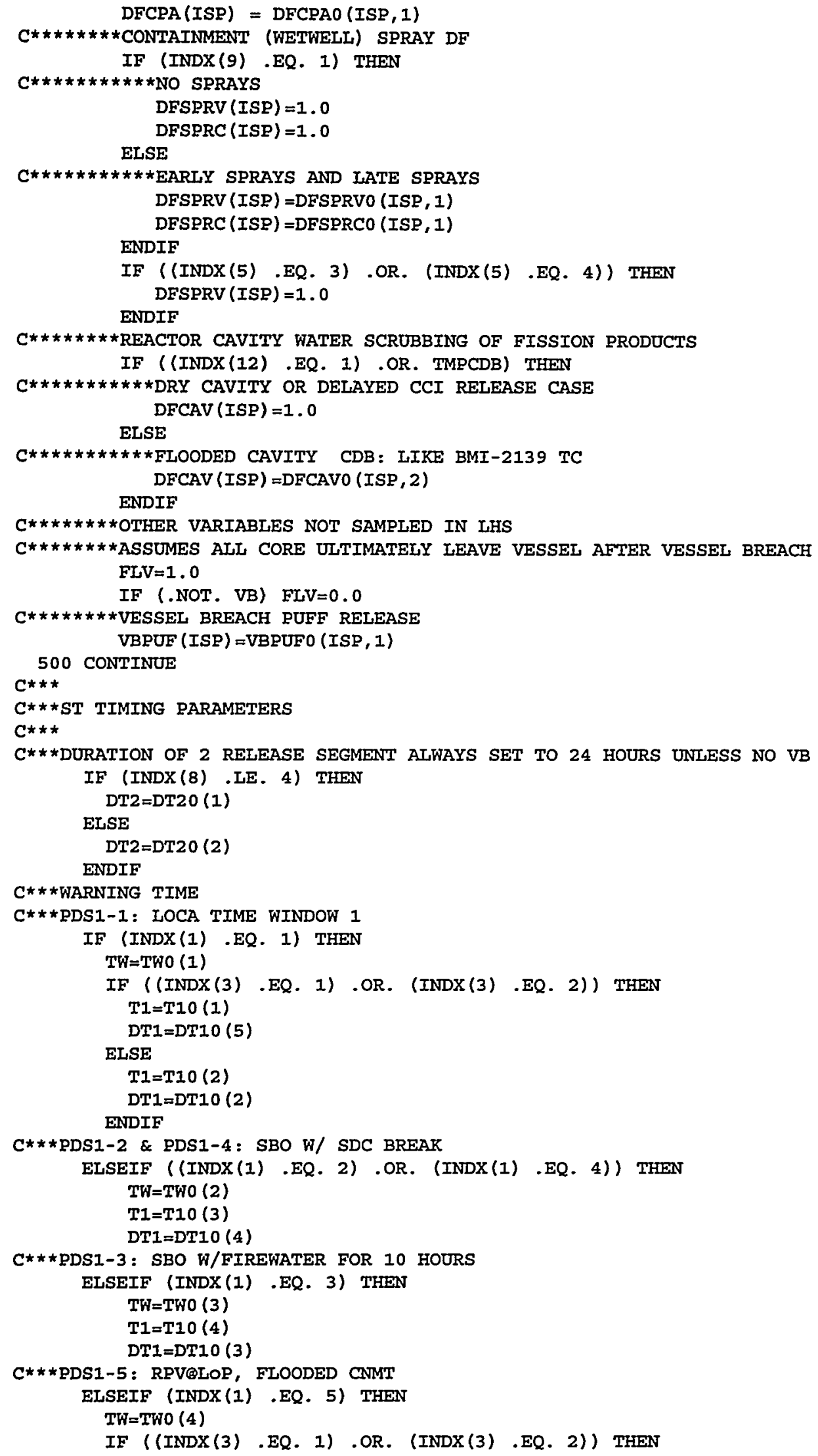


Appendix C

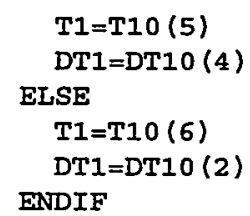


$C * \star \star C N M T$ FAILS AS A IEARK OR NOT AT ALI

ELSE

DT1 $=$ DT10 (3)

ENDIF

ENDIF

ENDIF

C $\star \star \star \star \star$ PDS2 -6 : RPVOLOP, OPEN MSIVS

ELSE IF (INDX (1) .EQ. 11) THEN

$T W=T W 0(12)$

$\mathrm{T} 1=\mathrm{T} 10(16)$

DT1 $1=\mathrm{DT} 10$ (5)

C*****DDS3-1: LOCA TIME WINDOW 3

ELSE IF (INDX(1) .EQ. 12) THEN

$T W=T W O(13)$

IF ((INDX (3) .EQ. 1) .OR. (INDX (3) .EQ. 2)) THEN

$T 1=T 10$ (17)

DT $1=D T 10(5)$

ELSE

$T 1=T 10(18)$

$\mathrm{DT} 1=\mathrm{DT} 10(2)$

ENDIF

ENDIF

$C * \star \star \star \star$ START OF SECOND RELEASE

$C \star \star \star \star \star N O$ TEMPORARY COOTABLE DEBRIS BED

$\mathrm{T} 2=\mathrm{T} 1+\mathrm{DT} 1$

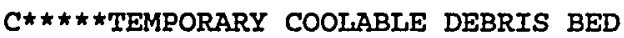

IF (TMPCDB) $T 2=T 2+$ DTCDB

C $\star \star \star \star \star$ FOR IATE CONTAINMENT FAILURE CASES, ASSIGN FRACTION

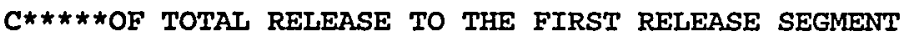

$C \star * \star * \star$ SET DEFAULT OF PUFF TO 1.0

IF ((INDX (2) .EQ. 7) .OR. (INDX (2) .EQ. 8)) THEN

$C \star \star \star \star \star \star \star \star$ LATE RUPTURE OR LATE VENT PUFF=PUFFO (1)

ELSE IF ((INDX (2) .EQ. 9) .OR. (INDX (2) .EQ. 10)) THEN

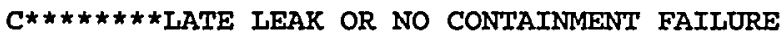
PUFF $=$ PUFFO (2)

ELSE

$C \star \star \star \star \star \star \star \star$ SET DEFAULT

$\mathrm{PUFF}=1.0$

ENDIF

RETURN

END

SUBROUTINE EXPTAB

$C \star \star \star \star \star$ SET VARIABLES IN COMMON BLOCK BINNED BY INTERPOLATION OF

$C * * \star * *$ EXPERT OPINION TABLES

PARAMETER (MAXBD=20, MAXBIN=10000, MAXSMP=300, MAXCAS=8,

1

MAXISS $=20$, MAXLEV $=10, \operatorname{MAXVAR}=100, \operatorname{MAXVAL}=13000$, MAXSPC $=10$, MAXTIM $=20$ )

COMMON /BASVAL/ FCOR (MAXSPC), FVES (MAXSPC), DFVPA (MAXSPC),

1 DFCPA (MAXSPC), FEVSE (MAXSPC), FDCH (MAXSPC), FCCI (MAXSPC), DFCAV (MAXSPC), VBPUF (MAXSPC), FCONV (MAXSPC), FCONC (MAXSPC), RBDF (MAXSPC), DFSPRV (MAXSPC)， DFSPRC (MAXSPC)， FREVO (MAXSPC), VAIISS (MAXISS), FLTI1, FLTI2, NSPEC, FLV, FHPE, EVSE, WFAC, PFAC, FPLBYE, FPLBYP, FPLBYD, FPLBYC, FILPH, FTLPL, FTLP, TC11, TC12, TB11, TB12, TB21, TB22, TBS1, TBS2, TBR1, TBR2, TW, T1, T2, DT1, DT2, DTCDB, ELEV, PUFF, HVSPLT, FCD

COMMON /BININED/ FCORO (MAXSPC,MAXCAS), FVESO (MAXSPC,MAXCAS), DFVPAO (MAXSPC, MAXCAS), DFCPAO (MAXSPC, MAXCAS), FDCHO (MAXSPC, MAXCAS), FEVSEO (MAXSPC, MAXCAS), FCCIO (MAXSPC, MAXCAS), DFCAVO (MAXSPC, MAXCAS), VBPUFO (MAXSPC, MAXCAS), FCONVO (MAXSPC, MAXCAS), 
Appendix C

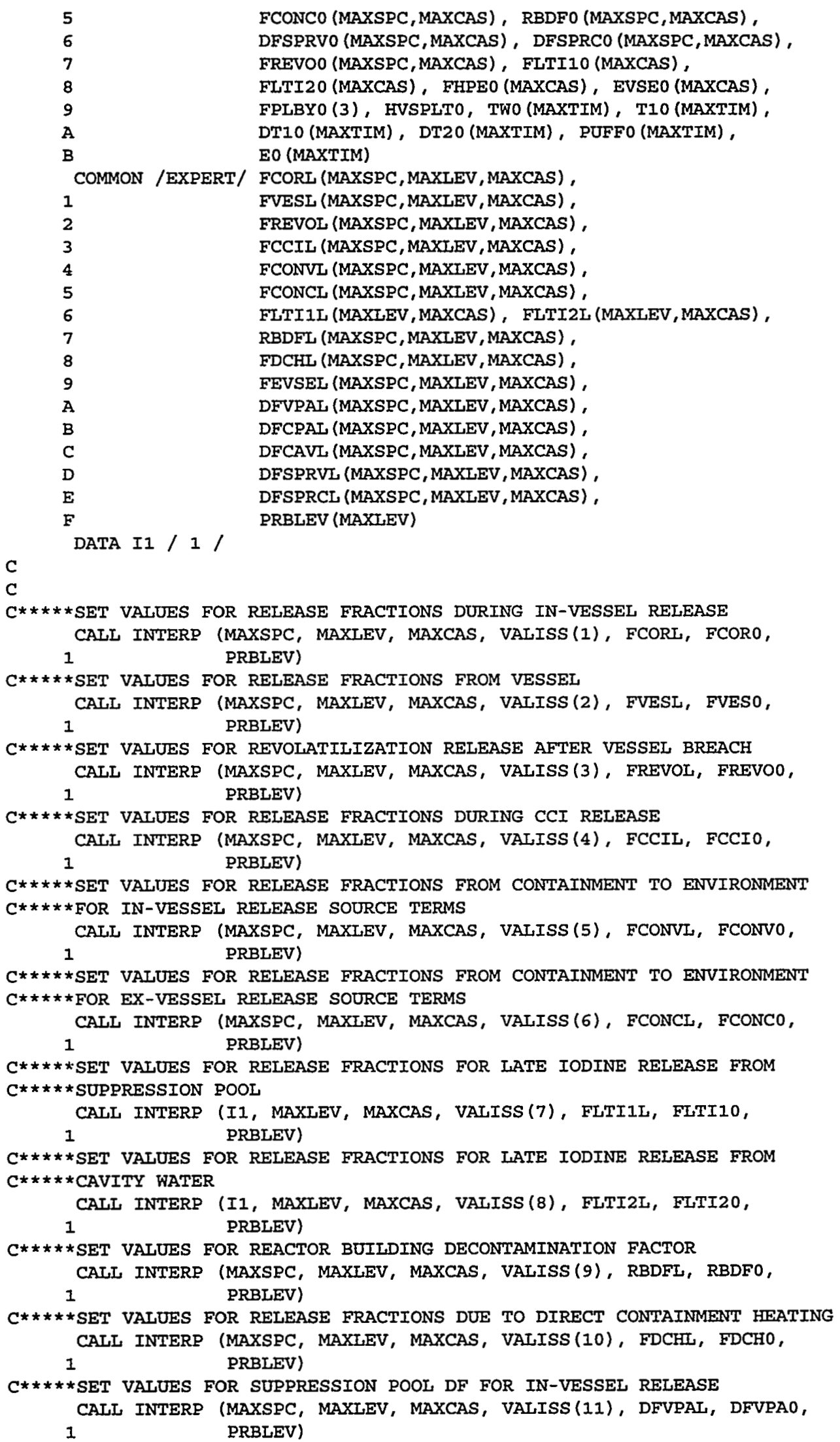


$C \star \star \star \star \star$ SET VALUES FOR SUPPRESSION POOL DF AFTER VESSEL BREACH

CALL INTERP (MAXSPC, MAXIEV, MAXCAS, VALISS(12), DFCPAL, DFCPAO,

1 PRBLEV)

$C \star \star \star \star \star$ SET VALUES FOR CAVITY WATER DF FOR CCI RELEASE

CAIL INTERP (MAXSPC, MAXIEV, MAXCAS, VALISS(13), DFCAVL, DFCAVO,

1 PRBLEV)

$C \star \star \star \star \star$ SET VALUES FOR CONTAINMENT SPRAYS DF FOR IN-VESSEL RELEASE

CAIL INTERP (MAXSPC, MAXLEV, MAXCAS, VAIISS(14), DFSPRVL, DFSPRVO,

1 PRBLEV)

$C \star \star \star * \star$ SET VALUES FOR CONTAINMENT SPRAYS DF FOR EX-VESSEL RELEASE

CALL INTERP (MAXSPC, MAXLEV, MAXCAS, VALISS(15), DFSPRCL, DFSPRCO,

1

$C \star \star \star \star *$ SET VALUES FOR EX-VESSEL STEAM EXPLOSION RELEASE

CALL INTERP (MAXSPC, MAXLEV, MAXCAS, VALISS(16), FEVSEL, FEVSE0,

1

RETURN

END

SUBROUTINE INTERP (MAXSPC, MAXLEV, MAXCAS, PROB, RL, RO, PRBLEV)

$C * \star * \star \star$ PERFORM INTERPOLATION IN SPECIFIED EXPERT OPINION TABLE

DIMENSION RL (MAXSPC, MAXIEV, MAXCAS), RO (MAXSPC, MAXCAS),

1

LOGICAL FIRST

DATA FIRST / .TRUE. /

C

C

IF (FIRST) THEFN

C $\star \star \star \star \star \star \star \star \star$ DETERMINE NUMBER OF LEVELS

DO 100 ILEV $=2$, MAXIEV

IF (PRBLEV (ILEV) .LE. 0.0) THEN

NLEV =ILEV - 1

GO TO 200

ENDIF

100 CONTINUE

NLEV $=$ MAXLEV

200 CONTINUE

IF (NLEV .LE. 1) THEN

WRITE $(6,1002)$

STOP

ENDIF

FIRST $=$. FALSE .

ENDIF

$C * \star \star * \star$ VALIDATE PROBABIIITY

IF (PROB .LT. PRBLEV(1)) THEN

WRITE $(6,1001)$ PROB， (PRBLEV (I), I=1, NLEV) STOP

ENDIF

$C \star * \star \star \star$ LOCATE PROBABILITY LEVELS TO INTERPOLATE BETWEEN

DO 1000 ILEV $=2$, NLEV

IF (RROB .IE. RRBLEV (ILEV)) THEN

JLEV=ILEV

GO TO 2000

ENDIF

1000 CONTINUE

$C \star \star \star \star \star$ PROBABILITY VALUE OUTSIDE OF TABLE RANGE

WRITE $(6,1001)$ PROB， (PRBLEV (I), I=1, NLEV)

STOP

2000 CONTINUE

$C * * * * *$ LOOP OVER CASES

DO 4000 ICAS $=1$, MAXCAS

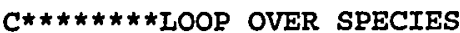

DO 3000 ISPEC $=1$, MAXSPC

C $\star \star \star \star \star \star * \star * \star * \star$ DERFORM INTERPOLATION FOR CURRENT SPECIES AND CASE

IF ((RI (ISPEC, 1, ICAS) .GT. 0.0) .AND.

Vol. 6, Part 1 


\section{Appendix C}

1 (RI (ISPEC, NLEV, ICAS)/RL (ISPEC, 1, ICAS) .GT. 10.1) THEN

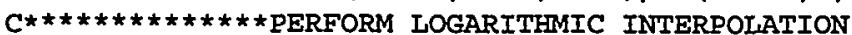

R0 (ISPEC, ICAS) $=10$ * * (LOGI0 (RL (ISPEC, JLEV-1, ICAS)) +

1 (PROB-PRBLEV (JLEV-1)) *

2 (LOG1O (RL (ISPEC, JLEV, ICAS)) -IOG10 (RL (ISPEC, JLEV-1, ICAS))) /

3 (PRBLEV (JLEV) - PRBLEV (JLEV-1))) ELSE

C $\star \star \star \star \star \star \star \star * \star * \star \star \star \star * \star$ PERFORM LINEAR INTERPOLATION

1

RO (ISPEC, ICAS) =RL (ISPEC, JLEV-1, ICAS) +

1

(PROB-PRBLEV (JLEV-1)) *

(RL (ISPEC, JLEV, ICAS) -RL (ISPEC, JLEV-1, ICAS)) /

ENDIF

(PRBLEV (JLEV) - PRBLEV (JLEV - 1))

3000 CONTINUE

4000 CONTINUE

RETURN

C $\star \star \star \star \star$ FORMAT STATEMENTS

1001 FORMAT $(/ 1 \mathrm{X}, 1 \gg \gg>$ PROBABILITY VALUE $(1, F 5.2,1)$ OUT OF RANGE FOR ',

1 'INTERPOLATION OF LEVELS'

$2 \quad(1 \mathrm{X}, 1 \gg>>\operatorname{PRBLEV}(I)=1,20 \mathrm{~F} 6.3)$

1002 FORMAT (/1X,'>>>PEWER THAN 2 PROBABILITY LEVELS (PRBLEV) ',

1 'SPECIFIED')

END

SUBROUTINE GGSORC (IOBS, IBIN)

C*****CALCULATE XXSOR TYPE OF SOURCE TERMS FOR THE GRAND GULF

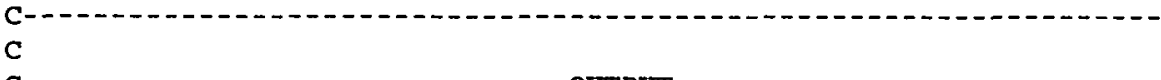

ST(ISP) == TOTAL ENVIRONMENTAL RELEASE FRACTIONS FOR SPECIES 'ISP' (EARIY + LATE)

STE (ISP) $==$ RELEASES UP THROUGH VESSEL BREACH. THE DEFINING TIME IS RELEASE TO THE CONTAINMENT; ACTUAL RELEASE TO THE ENVIRONMENT WIIL BE IATER IF CONTAINMENT FAIIIURE IS IAATER

STCCI (ISP) $==$ CCI RELEASE SOURCE TERMS

STL(ISP) == IATE RELEASE SOURCE TERMS (CCI+STIL+STRVOL)

STIL == "I.ATE" IODINE COMPONENT, TREATED AS GASEOUS (E.G., ORGANIC) IODINE RELEASED FROM POOL AND FLOODED CAVITY; NO DF'S OR CONTAINMENT RETENTION FACTORS APPLY

STRVOL (ISP) $==$ I, CS AND TE COMPONENT REVOLATILIZED FROM PRIMARY SYSTEM: TREATED AS AEROSOL; DF'S FOR SPRAYS, SUPPRESSION POOL SCRUBBING, AND CONTAINMENT RETENTION APPLY

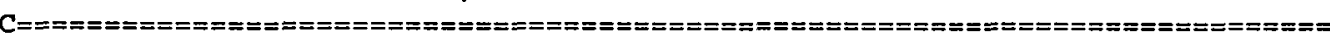

SPECIES INDEX=ISP, 1 TO NSPEC; ORDER IS NG, I, CS, TE, SR, RU, LA, CE, BA

FCOR == RELEASE FRACTION OF EACH ELEMENT GROUP FROM THE FUEL DURING DURING IN-VESSEL RELEASE

FVES $==$ RELEASE FRACTION FROM THE VESSEL (FRACTION OF FCOR)

DFVPA $==$ POOL DF'S DURING IN-VESSEL RELEASE

DFCPA $==$ POOL DF'S DURING CCI RELEASE

VBPOF $==$ PUFF RELEASE FRACTION OF THE TOTAL CORE AT VESSEL BREACH

- - - POOL BYPASS PARAMETERS - - -

FPLBYE, FPLBYP, FPLBYD, FPLBYC ==

FRACTION OF POOL BYPASS AT DIFFERENT TIME STEPS:

EARLY (BEFORE VB), PUFF SOURCE TERMS,

DCH SOURCE TERMS, AND CCI SOURCE TERMS.

THIS FRACTION DO NOT GO THROUGH SUPPRESSION POOL

FCONV $==$ FRACTIONS OF AEROSOL SPECIES RELEASED FROM THE RCS TO THE CONTAINMENT AND THEN TO THE ENVIRONMENT

FCONC $==$ FRACTIONS OF AEROSOL SPECIES RELEASED TO FROM CCI TO THE CONTAINMENT AND THEN TO THE ENVIRONMENT (INCLUDES DRYWELL RETENTION AND OUTER CONTAINMENT RETENTION) 


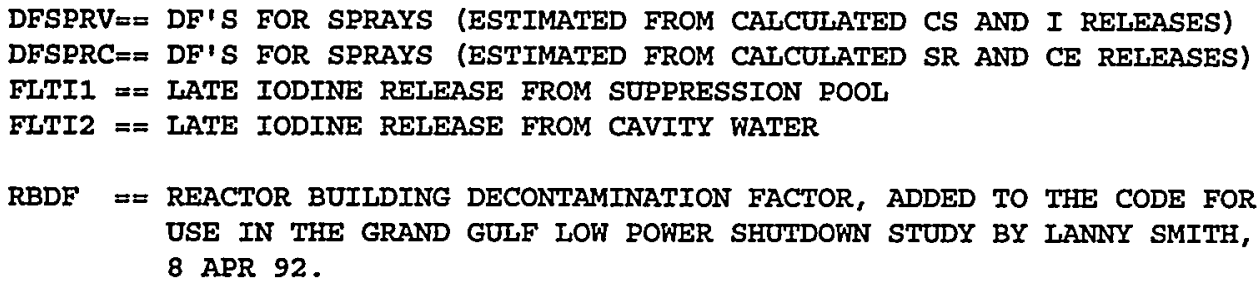


Appendix C

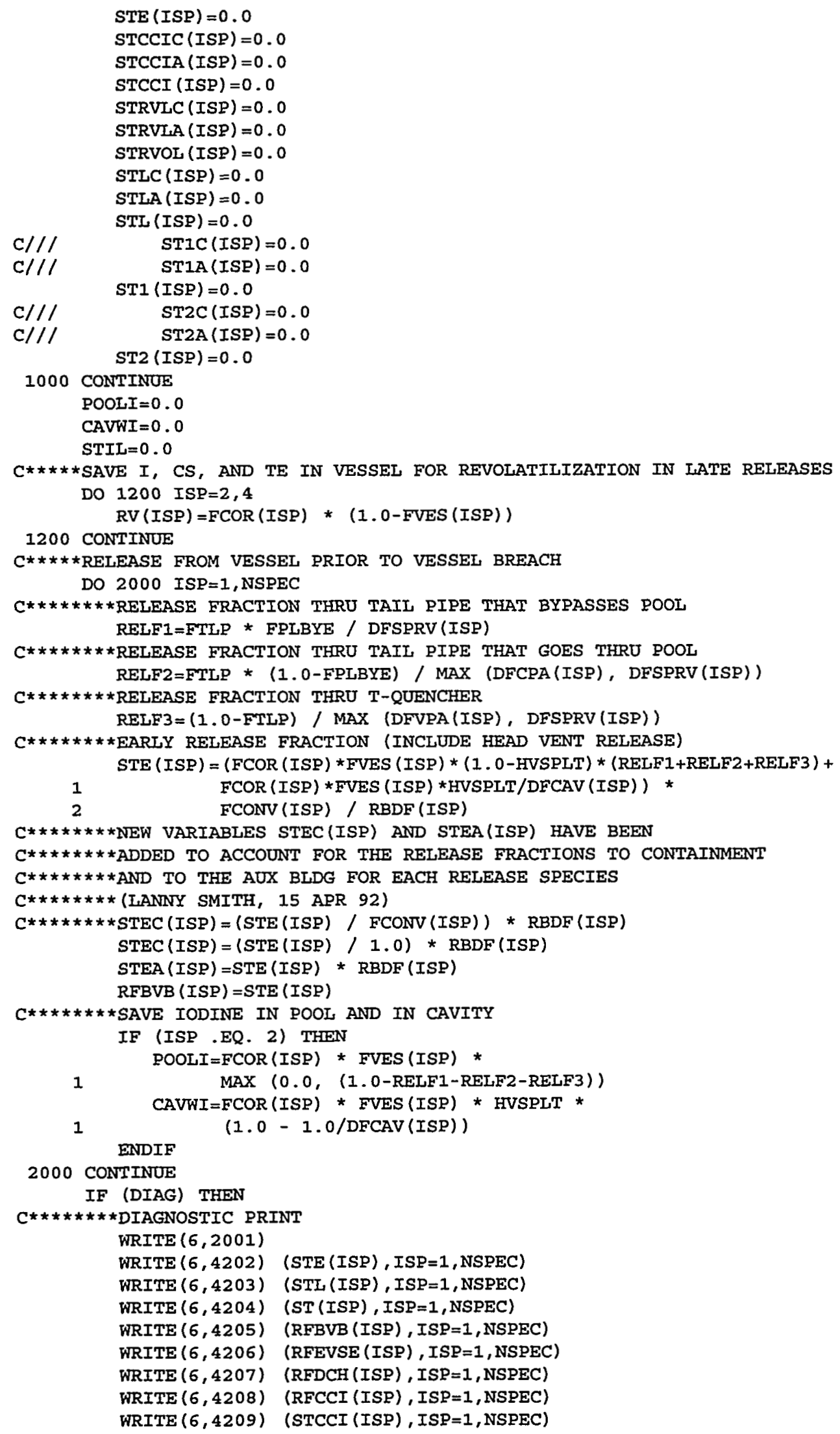


1

$\operatorname{WRITE}(6,4210)(R V(I), I=2,4),(\operatorname{STRVOL}(I), I=2,4)$, POOLI,

CAVWI, STII

ENDIF

$C \star \star \star \star \star A D D$ FOR LR\&S POS-5 ANALYSIS

$C \star \star \star \star \star I F$ CNMT CLOSE WITH ILOCA OR OPEN MSIV AND ILATE CNMT FAILURE, SET

C

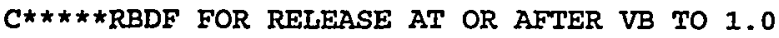

$\operatorname{IF}(((\operatorname{INDX}(2) . E Q \cdot 2)$.OR. (INDX (2) .EQ. 3) .OR. (INDX (2) .EQ. 5))

1 .AND. (INDX (5) .EQ.3)) THEN

DO 2099 ISP $=1,9$

$\operatorname{RBDF}($ ISP) $=1.0$

2099 CONTINUE

ENDIF

$C * \star \star \star \star *$

$C \star \star \star \star \star$ IF EVSE, STEAM EXPLOSIONS ARE CONSIDERED

IF ((INDX (11) .LT. 3) .OR. (INDX (11) .EQ. 5) ) GO TO 7250

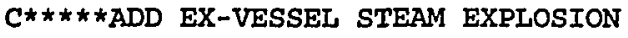

DO 4500 ISP $=1$, NSPEC

C $\star * \star * \star * \star *$ RELEASE FRACTION DUE TO EX-VESSEL STEAM EXPLOSION

$1 \quad$ EVSE $\star$ FEVSE (ISP)

IF (RFEVSE (ISP) .GT. 0.0 ) THEN

C $\star \star \star \star \star * * \star * \star *$ RELEASE FRACTION DUE TO EX-VESSEL STEAM EXPLOSION, THAT

C $\star \star \star \star \star \star \star \star \star \star \star \star \star$ BYPASSES POOL

RELF1=FPLBYD / DFSPRC (ISP)

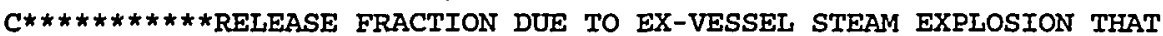

C $\star \star \star \star \star \star \star \star \star \star \star \star *$ GOES THRU POOI

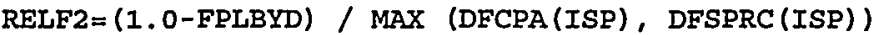

C $\star \star \star \star \star \star \star \star * \star * * \star$ EARLY RELEASE FRACTION

C $\star \star \star \star \star \star \star \star \star \star \star \star *$ NEW VARIABLES 'STEC(ISP)' AND 'STEA(ISP)' HAVE BEEN

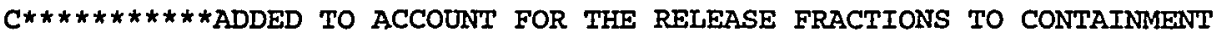

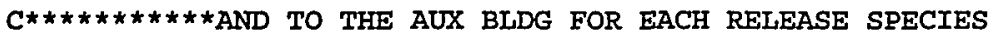

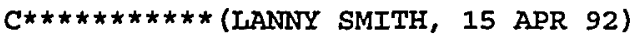

$\operatorname{STEC}($ ISP) $=\operatorname{MIN}(1.0, \operatorname{STEC}($ ISP) + RFEVSE (ISP) * (RELF1+RELF2))

1

$\operatorname{STEA}($ ISP $)=\operatorname{MIN}(1.0$, STEA $($ ISP $)+$ RFEVSE (ISP) * (RELF1+RELF2) *FCONC (ISP))

STE (ISP) $=$ MIN $(1.0, \operatorname{STE}($ ISP $)+$ RFEVSE (ISP) * (RELF1+RELF2) *FCONC (ISP) /RBDF (ISP))

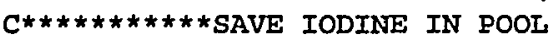

IF (ISP .EQ. 2) THEN POOLI=POOLI + RFEVSE (ISP) $*$ MAX $(0.0,(1.0-$ RELF1-RELF2) $)$ ENDIF

ENDIF

4500 CONTINUE

IF (DIAG) THEN

$C * * * * * * * *$ DIAGNOSTIC PRINT

WRITE $(6,4501)$

WRITE $(6,4202)$ (STE (ISP), ISP $=1$, NSPEC)

WRITE $(6,4203) \quad$ (STL (ISP), ISP $=1$, NSPEC)

WRITE $(6,4204)$ (ST (ISP), ISP $=1$, NSPEC)

WRITE $(6,4205)$ (RFBVB (ISP), ISP $=1$, NSPEC)

WRITE $(6,4206)$ (RFEVSE (ISP), ISP $=1$, NSPEC)

WRITE $(6,4207)$ (RFDCH (ISP) , ISP $=1$, NSPEC)

WRITE $(6,4208$ ) (RFCCI (ISP), ISP $=1$, NSPEC)

WRITE $(6,4209)$ (STCCI (ISP), ISP =1, NSPEC)

1

WRITE $(6,4210)(R V(I), I=2,4)$, (STRVOL $(I), I=2,4)$, POOLI,

ENDIF

7250 CONTINUE

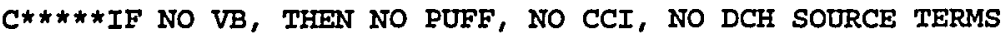

IF (.NOT. VB) GO TO 7500

$C \star \star \star \star \star A D D$ VESSEL BREACH PUFF RELEASE TO EARLY SOURCE TERM

DO 3000 ISP $=1$, NSPEC

Vol. 6, Part 1

C-47

NUREG/CR-6143 


\section{Appendix C}

C $\star \star \star \star \star \star \star \star$ RELEASE FRACTION DUE TO VESSEL BREACH PUFF THAT BYPASSES POOI RELFI=FPLBYP / DFSPRC (ISP)

C $\star \star \star \star \star \star \star \star$ RELEASE FRACTION DUE TO VESSEL BREACH PUFF THAT GOES THRO POOL RELF2 $=(1.0-$ FPLBXP) / MAX (DFCPA (ISP), DFSPRC (ISP))

$C \star \star \star \star \star \star \star \star \star$ EARLY RELEASE FRACTION

C $\star \star \star \star \star \star \star \star$ NEW VARIABLES STEC (ISP) AND STEA (ISP) HAVE BEEN

$C \star \star \star \star \star \star \star \star A$ ADDED TO ACCOUNT FOR THE RELEASE FRACTIONS TO CONTAINMENT

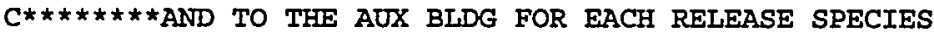

$C \star \star \star \star \star \star \star \star \star$ (IAANNY SMITH, 15 APR 92)

$\operatorname{STEC}($ ISP) $=\mathrm{MIN}(1.0, \operatorname{STEC}($ ISP) $+\operatorname{VBPUF}($ ISP $) *($ RELF1+RELF2) $)$

$S T E A(I S P)=M I N(1.0, S T E A$ (ISP) + VBPUF (ISP) * (RELF1+RELF2) *FCONC (ISP))

$S T E(I S P)=M I N \quad(1.0, \operatorname{STE}($ ISP $)+$ VBPUF (ISP) * (RELF1+RELF2) *FCONC (ISP) /RBDF (ISP))

$C * * \star * \star * * *$ SAVE IODINE IN POOL

IF (ISP .EQ. 2) THEN

POOLI $=$ POOLI + VBPUF (ISP) *MAX $(0.0,(1.0-$ RELF1-RELF2) ENDIF

3000 CONTINUE

IF (DIAG) THEN

$C * * * * * * * *$ DIAGNOSTIC PRINT

WRITE $(6,3001)$

WRITE $(6,4202)$ (STE (ISP), ISP $=1, \operatorname{NSPEC)}$

WRITE $(6,4203)$ (STL (ISP), ISP $=1$, NSPEC)

WRITE $(6,4204)$ (ST (ISP), ISP $=1$, NSPEC)

WRITE $(6,4205) \quad$ (RFBVB (ISP), ISP $=1$, NSPEC)

WRITE $(6,4206$ ) (RFEVSE (ISP), ISP $=1$, NSPEC)

WRITE $(6,4207)$ (RFDCH (ISP) , ISP $=1$, NSPEC)

$\operatorname{WRITE}(6,4208) \quad$ (RFCCI (ISP), ISP $=1, \operatorname{NSPEC}$ )

WRITE $(6,4209)$ (STCCI (ISP), ISP $=1$, NSPEC)

$\operatorname{WRITE}(6,4210)(R V(I), I=2,4),(\operatorname{STRVOL}(I), I=2,4)$, POOLI,

ENDIF

CAVWI, STIL

$C * \star \star \star \star A D D$ DIRECT CONTAINMENT HEATING RELEASE TO EARLY SOURCE TERM

DO 4000 ISP $=1$, NSPEC

C********RELEASE FRACTION DUE TO DIRECT CONTAINMENT HEATING

1

$R F D C H(I S P)=\operatorname{MAX}(0.0,(1.0-F C O R($ ISP) $-\mathrm{VBPOF}($ ISP) $))$ * FLV *

FHPE * FDCH(ISP)

IF (RFDCH (ISP) .GT. 0.0) THEN

$C \star \star \star \star \star \star \star \star \star \star \star \star *$ RELEASE FRACTION DUE TO DIRECT CONTAINMENT HEATING THAT

$C \star \star \star \star \star \star \star \star \star \star \star \star \star *$ BYPASSES POOL RELFI=FPLBYD / DFSPRC (ISP)

C $\star \star \star \star \star \star \star \star \star \star \star \star$ RELEASE FRACTION DUE TO DIRECT CONTAINMENT HEATING THAT

C $\star \star \star \star \star \star \star \star \star \star \star \star \star$ GOES THRU POOI

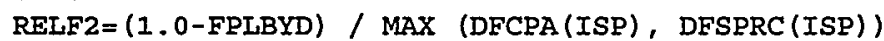

$C \star \star \star \star \star \star \star \star \star * \star * *$ EARLY RELEASE FRACTION

$C \star \star \star \star \star \star \star \star \star \star \star \star *$ NEW VARIABLES STEC (ISP) AND STEA (ISP) HAVE BEEN

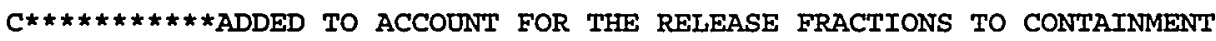

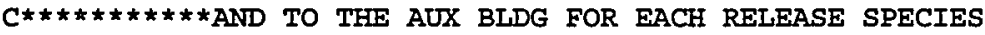

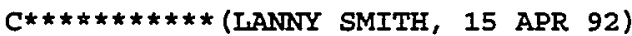
$\operatorname{STEC}($ ISP) $=\operatorname{MIN}(1.0, \operatorname{STEC}($ ISP $)+\operatorname{RFDCH}($ ISP $) *($ RELF1+RELF2) $)$

STEA (ISP) $=$ MIN $(1.0$, STEA $($ ISP $)+$

1 RFDCH (ISP) * (RELFI+RELF2) *FCONC (ISP) )

STE (ISP) $=$ MIN $(1.0, \operatorname{STE}($ ISP $)+$

$1 \quad$ RFDCH (ISP) * (RELF1+RELF2) *FCONC (ISP) /RBDF (ISP))

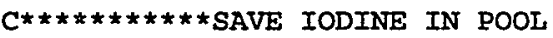

IF (ISP .EQ. 2) THEN ENDIF

POOLI $=$ POOLI + RFDCH $(I S P) *$ MAX $(0.0,(1.0-$ RELF1-RELF2)

ENDIF

4000 CONTINUE

IF (DIAG) THEN

C********DIAGNOSTIC PRINT 
WRITE $(6,4201)$

WRITE $(6,4202)$ (STE (ISP), ISP $=1$, NSPEC)

WRITE $(6,4203)$ (STL (ISP), ISP $=1$, NSPEC)

WRITE $(6,4204)$ (ST (ISP), ISP $=1, \mathrm{NSPEC}$ )

WRITE $(6,4205)$ (RFBVB (ISP), ISP=1, NSPEC)

WRITE $(6,4206)$ (RFEVSE (ISP), ISP $=1$, NSPEC)

WRITE $(6,4207)$ (RFDCH (ISP), ISP $=1$, NSPEC)

WRITE $(6,4208)$ (RFCCI (ISP), ISP $=1$, NSPEC)

WRITE $(6,4209)$ (STCCI (ISP), ISP $=1$, NSPEC)

1

$\operatorname{WRITE}(6,4210)(R V(I), I=2,4),($ STRVOL $(I), I=2,4)$, POOLI,

ENDIF

CAVWI, STII

$C \star \star \star \star \star$ EX-VESSEL STEAM EXPLOSION

IF (EVSE .GT. 0.0) THEN

$X C C I=1.0$ - EVSE

ELSE IF (FHPE .GT. 0.0) THEN XCCI $=1.0$ - FHPE

ELSE

$X C C I=1.0$

ENDIF

IF (.NOT. CDB) THEN

$C * \star \star * \star * * *$ CORE-CONCRETE INTERACTION RELEASES AND CAVITY SCRUBBING DO 5000 ISP $=1$, NSPEC

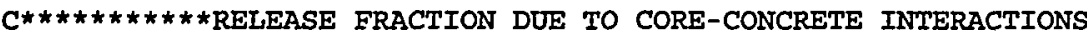
$\operatorname{RFCCI}(I S P)=\operatorname{MAX}(0.0,(1.0-F C O R($ ISP $)-V B P U F($ ISP $))) *$ FLV *

1 XCCI * FCCI (ISP)

C $\star \star \star \star \star \star \star \star \star \star \star \star$ RELEASE FRACTION DUE TO CORE-CONCRETE INTERACTIONS THAT

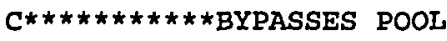
RELF1=FPLBYC / MAX (DFCAV(ISP), DFSPRC(ISP))

C $\star \star \star \star \star \star \star \star \star \star \star \star *$ RELEASE FRACTION DUE TO CORE-CONCRETE INTERACTIONS THAT

C $\star \star \star \star \star \star \star \star \star \star \star \star \star$ GOES THRU POOL

RELF2 $=(1.0-$ FPIBYC $) /$

1 MAX (DFCAV(ISP), DFCPA(ISP), DFSPRC(ISP))

$C \star \star \star \star \star \star \star \star \star \star \star * \star$ CORE-CONCRETE RELEASE FRACTION $\operatorname{STCCI}($ ISP $)=$ RFCCI (ISP) * (RELF1+RELF2) *

1 FCONC(ISP) / RBDF(ISP)

$C \star \star \star \star \star \star * \star \star \star \star \star$ NEW VARIABLES STCCIC(ISP) AND STCCIA(ISP) HAVE

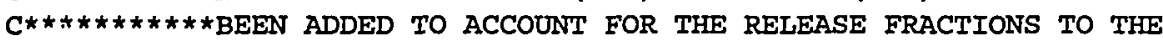

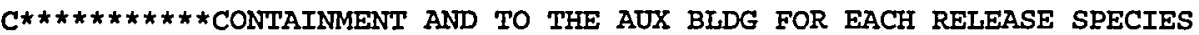

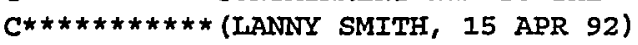

IF (FCONC(ISP) .GT. 0.0) THEN $\operatorname{STCCIC}($ ISP $)=\operatorname{STCCI}($ ISP) $/ \mathrm{FCONC}($ ISP $) * \operatorname{RBDF}($ ISP $)$ ELSE $\operatorname{STCCIC}($ ISP $)=0.0$

ENDIF $\operatorname{STCCIA}($ ISP) $=\operatorname{STCCI}($ ISP) $* \operatorname{RBDF}($ ISP)

$C \star * \star * * * * * * * *$ SAVE IODINE IN CAVITY WATER AND IN POOL

IF (ISP . EQ. 2) THEN

CAVWI2 $=1.0-1.0 /$ DFCAV (ISP)

POOLI $=$ POOLI + RFCCI (ISP) *

$1 \operatorname{MAX}(0.0,(1.0-R E L F 1-R E L F 2-C A V W I 2))$

ENDIF

CAVWI $=$ CAVWI + RFCCI (ISP) * CAVWI2

5000 CONTINUE

ENDIF

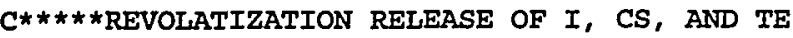

$C \star \star \star \star \star$ (SIMILAR TO VESSEL BREACF PUFF RELEASE)

DO 6000 ISP $=2,4$

C $* * * * * * *$ RELEASE FRACTION DUE TO REVOLATILIZATION THAT BYPASSES POOL RELF1=FPLBYC / DFSPRC (ISP)

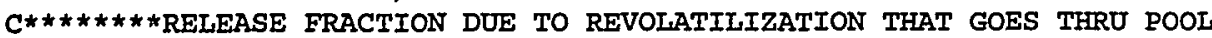
RELF2 $=(1.0-F P L B Y C) / \operatorname{MAX}$ (DFCPA (ISP), DFSPRC (ISP))

$C \star * \star * \star * \star *$ REVOLATIIIZATION RELEASE FRACTION 
Appendix C

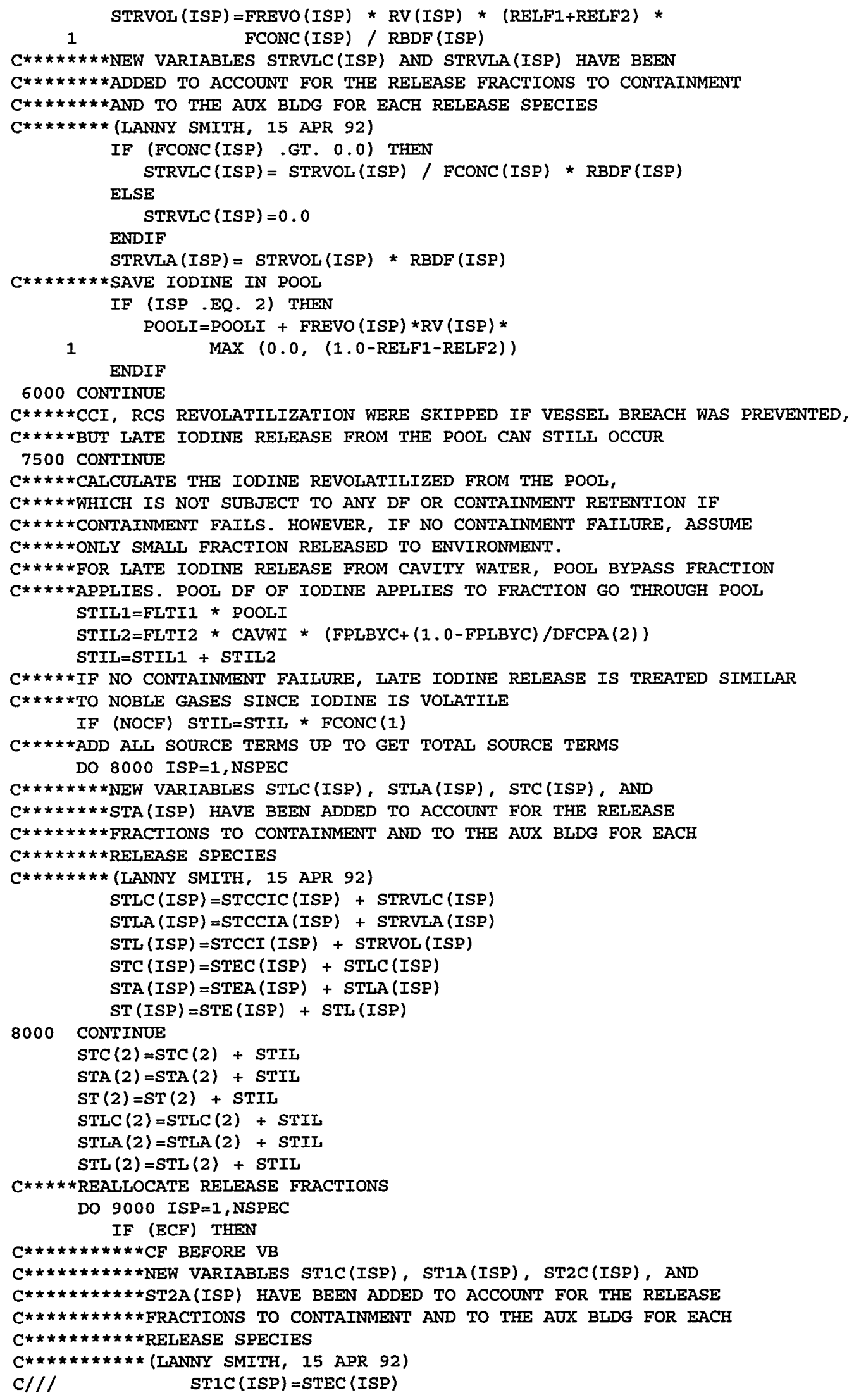




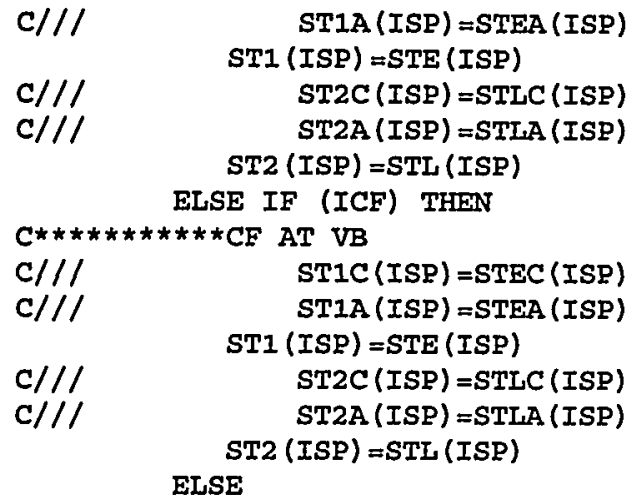


Appendix C

ENDIF

$\operatorname{WRITE}(9,1004) \quad \mathrm{ER} 2, \quad(\mathrm{ST} 2$ (ISP) , ISP $=1, \mathrm{NSPEC})$

$C \star \star \star \star \star N E W$ WRITE STATEMENTS HAVE BEEN ADDED TO ACCOUNT FOR THE

$C \star \star \star \star \star$ RELEASE FRACTIONS TO CONTAINMENT, THE AUX BLDG, AND THE

$C \star \star \star \star \star$ ENVIRONMENT FOR EACH RELEASE SPECIES

$C \star \star \star \star \star$ (LANRY SMITH, 15 APR 92)

IF (CONSFL) THEN

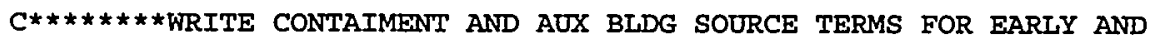

C $\star \star \star \star \star \star * * *$ LATE SEGMENTS

C $/ / /$ WRITE $(10,1004) \quad$ (ST1C (ISP), ISP $=1$, NSPEC)

$\mathrm{C} / / / \quad$ WRITE $(10,1004) \quad$ (STIA (ISP), ISP=1, NSPEC)

C/// WRITE $(10,1004) \quad$ (ST2C (ISP), ISP=1, NSPEC)

$\mathrm{C} / / /$ WRITE $(10,1004) \quad$ (ST2A (ISP), ISP $=1$, NSPEC)

ENDIF

RETURN

C*****FORMAT STATEMENTS

1003 FORMAT (I4, 2X, A)

1004 FORMAT (1P10E9.2)

2001 FORMAT $(/ / 5 \mathrm{X}, \cdot \star \star \star \star \star$ DIAGNOSTIC PRINT $\star \star \star \star \star \star 1$,

$1 / 10 X, 1=====$ PARAMETER VALUES UP TO VESSEL BREACH $=====^{\prime}$ )

3001 FORMAT $(/ / 5 \mathrm{X}, 1 * \star \star \star *$ DIAGNOSTIC PRINT $* * * * * 1$,

$1 / 10 \mathrm{X}, 1=====$ PARAMETER VALUES AFTER VESSEL BREACH $=====^{\prime}$ )

4201 FORMAT $(/ / 5 X, 1 * \star \star \star *$ DIAGNOS IIC PRINT $\star \star \star \star \star \star 1$,

$1 / 10 \mathrm{X},{ }^{\prime}=====$ PARAMETER VALUES AFTER DCH $=====1$ )

4202 FORMAT (/5X, 'STE: ' , 1P, / (5X, 10E10.2))

4203 FORMAT (5X, 'STL: ', 1P, /(5X,10E10.2))

4204 FORMAT (5X,'ST : ', 1P, /(5X,10E10.2))

4205 FORMAT (5X, 'RFBVB: ' , 1P, / (5X, 10E10.2))

4206 FORMAT (5X, 'RFEVSE : ' , 1P, / (5X, 10E10.2))

4207 FORMAT (5X, 'RFDCH: ' , 1P, /(5X, 10E10.2))

4208 FORMAT (5X, 'RFCCI : ' , IP, /(5X, 10E10.2))

4209 FORMAT (5X, 'STCCI : ', 1P, /(5X, 10E10.2))

4210 FORMAT $(5 X, 1 P, ' R V I=', E 10.2,5 X, '$ RVCS $=1, E 10.2,5 X, ' R V T E=1, E 10.2$,

$1 / 5 \mathrm{X}$, 'STRVOL $(2)=1, \mathrm{E} 10.2,5 \mathrm{X}, \cdot \operatorname{STRVOL}(3)=1, \mathrm{E} 10.2$,

$25 \mathrm{X}$, 'STRVOL $(4)=1$, E10.2,

$3 \quad / 5 \mathrm{X}$, ' POOLI $=1, \mathrm{E} 10.2,5 \mathrm{X}, \mathrm{CAVWI}=1, \mathrm{E} 10.2$,

$45 \mathrm{X}$, 'STIL $=1, \mathrm{E} 10.2)$

4211 FORMAT (/5X, 'SOURCE TERM INFORMATION: ',

$1 \quad / 5 \mathrm{X}, 1 \mathrm{P},{ }^{\prime} \mathrm{TW}={ }^{\prime}, \mathrm{E} 10.2,5 \mathrm{X},{ }^{\prime} \mathrm{T} 1={ }^{\prime}, \mathrm{E} 10.2,5 \mathrm{X},{ }^{\prime} \mathrm{DT} 1={ }^{\prime}, \mathrm{E} 10.2,5 \mathrm{X}$, 'T2 $=1, \mathrm{E} 10.2,5 \mathrm{X}$, 'DT2 $=1, \mathrm{E} 10.2$,

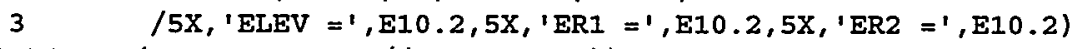

4212 FORMAT (5X, ' ST1: ' , 1P, /(5X, 10E10.2))

4213 FORMAT (5X, ' ST2 : ', 1P, /(5X, 10E10.2))

4501 FORMAT $(/ / 5 X, 1 * \star \star \star \star$ DIAGNOSTIC PRINT $* \star \star * \star * 1$,

$1 / 10 \mathrm{X}, 1=====$ PARAMETER VALUES AFTER EVSE $=====1$ )

5002 FORMAT ( $1 \mathrm{X}$, ' OBS: ' , I $4,2 \mathrm{X}$, ' BIN : ' , $\mathrm{A}, /$ )

5003 FORMAT (1X,'T1 =', 1PE12.4,' DT1 =', 1PE12.4,' T2 =', 1PE12.4,'

$1 \mathrm{DT} 2=1,1 \mathrm{PE} 12.4, /)$

5004 FORMAT (1X, 'EARLY CONT', 1P9E12.4)

5005 FORMAT (1X, 'EARLY AUX ', 1P9E12.4)

5006 FORMAT (1X, 'EARLY ENV ', 1P9E12.4)

5007 FORMAT (1X, 'LATE CONT', 1P9E12.4)

5008 FORMAT (IX,' IATE AUX', 1P9E12.4)

5009 FORMAT (IX, 'ILATE ENV ', 1P9E12.4,/)

8001 FORMAT $(/ / 5 \mathrm{X}, 1 * * \star * \star$ DIAGNOSTIC PRINT $* * \star * * 1$,

$1 \quad / 10 \mathrm{X}, 1=====$ PARAMETER VALUES AT END OF GGSORC $=====1$ )

END

SUBROUTINE ENERGY (EARLY, TAIL)

C $\star \star \star \star \star$ ESTIMATE ENERGY RETEASES FOR BOTH EARLY PUFF AND LATE

C $* * \star * *$ TAIL. DATA BASE ARE TAKEN FROM RESULTS OF MELCOR CALCULATIONS

$C \star \star \star \star \star$ FOR GRAND GULF (ENERGIES ARE IN JOULES).

$C \star \star \star \star \star$ EARLY : CALCULATED PUFF ENERGY RELEASE (JOULES)

C*****TAIL: CALCULATED ENERGY RELEASE AFTER PUFF (JOULES) 
C*****RLATCF: CORRECTION FACTOR FOR IATE CONTAINMENT FAILURE, $C \star \star \star \star \star$ NOT USED FOR POS 5

C $\star \star \star \star \star$ SPRFAC: CONTAINMENT SPRAY FACTOR FOR BOTH EARLY AND TAIL $C * * \star * \star \quad$ NOT USED FOR POS 5

PARAMETER (MAXBD $=20, M A X B I N=10000, \operatorname{MAXSMP}=300$, MAXCAS $=8$, 1 MAXISS $=20, M A X I E V=10, \operatorname{MAXVAR}=100, M A X V A L=13000$, 2 MAXSPC $=10$, MAXTIM $=20$ )

COMMON /BASVAI/ FCOR (MAXSPC), FVES (MAXSPC), DFVPA (MAXSPC), 1 DFCPA (MAXSPC), FEVSE (MAXSPC), FDCH (MAXSPC), 2 FCCI (MAXSPC), DFCAV (MAXSPC), VBPUF (MAXSPC),

3 FCONV (MAXSPC), FCONC (MAXSPC), RBDF (MAXSPC),

4 DFSPRV (MAXSPC), DFSPRC (MAXSPC), FREVO (MAXSPC),

5 VALISS (MAXISS), FLTI1, FLTI2, NSPEC, FLV, FHPE,

6 EVSE, WFAC, PFAC, FPLBYE, FPLBYP, FPLBYD,

7 FPLBYC, FTLPH, FTLPL, FTLP, TC11, TC12, TB11,

8 TB12, TB21, TB22, TBS1, TBS2, TBR1, TBR2, TW,

9 T1, T2, DT1, DT2, DTCDB, ELEV, PUFF, HVSPLT, FCD

C

COMMON /BININD/ INDX (MAXBD)

C

RIATCF $=1.0$

SPRFAC $=1.0$

SPRAYV $=1.0$

SPRAYC $=1.0$

C*****IF CONTAINMENT DOES NOT FAIL, BYPASS CALCULATION

IF (INDX (3) .NE. 4) THEN

$C * \star \star$ BASE CASE ENERGIES-LATER CASES WILL OVER WRITE IF NECESSARY

EARLY $=$ TC12

TAIL $=$ TB2I

$C * * *$ CNMT FLOODED SCENARIOS

IF ( $(\operatorname{INDX}(1)$.EQ. 1) .OR. ( $\operatorname{INDX}(1)$.EQ. 5) .OR.

$+\quad(\operatorname{INDX}(1) . E Q .6)$.OR. (INDX (1) .EQ. 9) .OR.

$+\quad($ INDX (1) .EQ. 12)) THEN

IF (INDX (3) .EQ. 3) THEN

EARLY $=$ TC11

TAIL $=$ TBII

ELSE

EARLY $=$ TB11

TAIL $=$ TB21

ENDIF

ENDIF

$C * \star *$ SBO SCENARIOS

IF ( $(\operatorname{INDX}(1), E Q .2)$.OR. (INDX (1) .EQ. 3) .OR.

$+\quad(\operatorname{INDX}(1)$.EQ. 4) .OR. (INDX (1) .EQ. 7) .OR.

+ (INDX (1) .EQ. 8)) THEN

EARLY $=$ TC12

ENDIF

TAIL $=$ TB21

$C * \star *$ OPEN MSIV SCENARIO

IF (INDX(1) .EQ. 11) THEN

EARLY $=$ TB21

ENDIF

$C \star \star \star$ CNMT CLOSED

ELSE

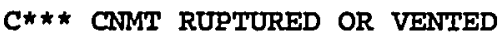

IF (( INDX (2) .EQ. 2) .OR. (INDX (2) .EQ. 3) .OR

$+\quad(\operatorname{INDX}(2)$.EQ. 5) .OR. (INDX(2) .EQ. 7) .OR.

$+\quad($ INDX (2) .EQ. 8)) THEN

EARLY $=$ TBII

TAIL $=$ TB21

$C * * *$ CNMT DOES NOT FAIL

Vol. 6, Part 1 
Appendix C

ELSEIF (INDX (2) .EQ. 10) THEN

EARIY $=0.0$

TAII $=0.0$

$C * * *$ CNMT IEARS

ELSE

EARLY $=$ TC12

TAIL $=$ TB21

ENDIF

ENDIF

$C \star \star \star$ NO VESSEL FAILURE

IF ((INDX (8) .EQ. 5) .OR. (INDX (8) .EQ. 6)) THEN TAIL $=0.0$

ENDIF

C $* \star * \star *$ CONVERT BTU TO JOULES

C EARLY $=1055$. - EARLY

C TAIL $=1055$. * TAIL

RETURN

END 
Appendix C

\section{C.2 Input Data for GGSORP5}

The following is the input file to GGSORP5 that provides the values used to quantify the parameters in the XSOR expression.

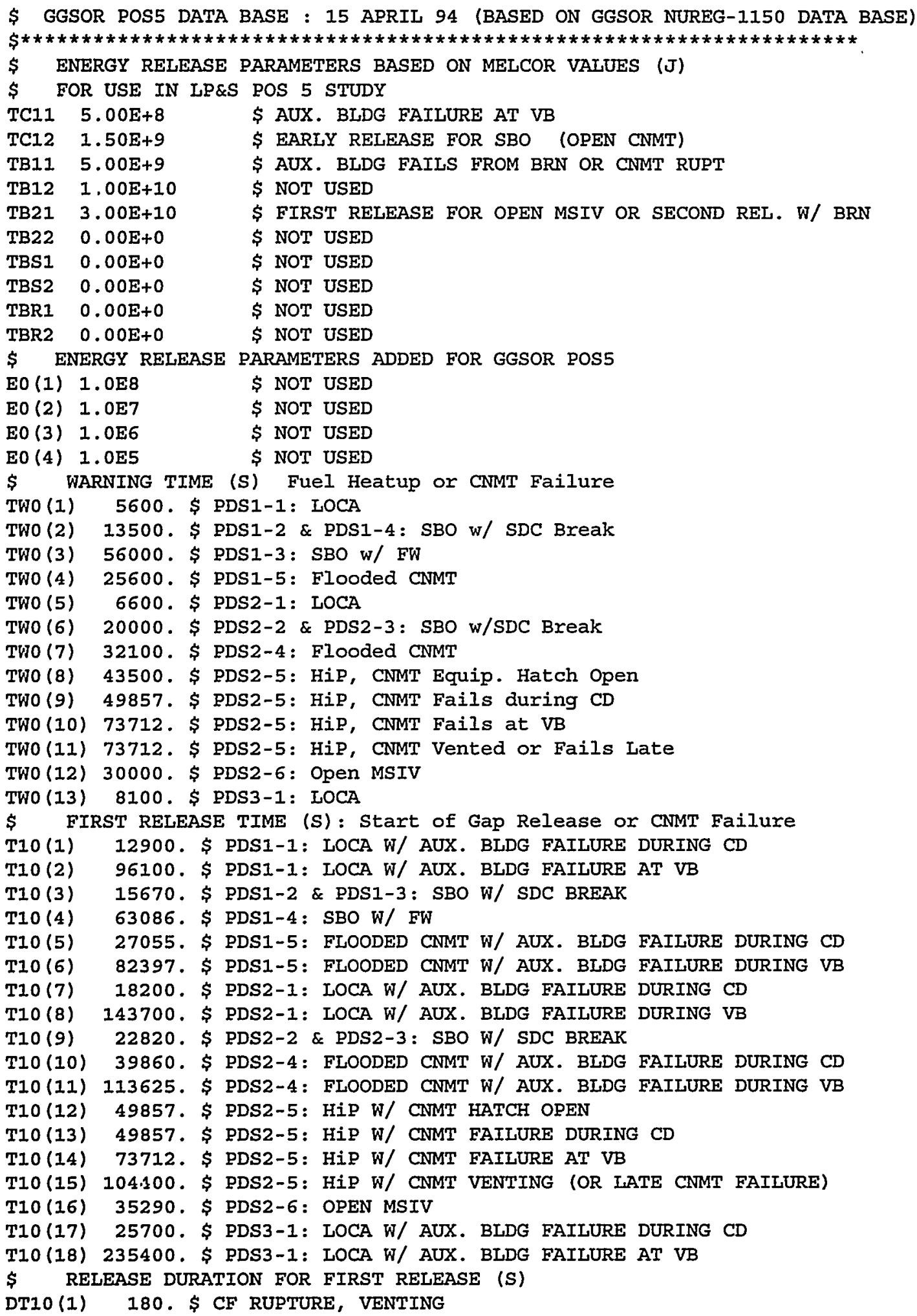


Appendix C

DT10 (2) 1800. \$ AUX. BLDG FAILURE AT VB

DT10 (3) 21600. \$ SHORT DURATION (6 HRS)

DT10 (4) 36000. \$ MEDIUM DURATION (10 HRS)

DT10 (5) 72000. \$ LONG DURATION (20 HRS)

$\$$ RELEASE DURATION FOR SECOND RELEASE (S)

DT20 (1) 86400. \$ VB

DT20 (2) 0 . \$ NO VB

$\$$ DELAY TIME FOR SECOND RELEASE (S) FOR TEMPORARY COOLABLE DEBRIS BED

DTCDB 10800 .

$\$$ FIRST RELEASE (PUFF) FRACTION FOR LATE CONTAINMENT FAILURE

PUFFO (1) 0.90 \$ LATE CONTAINMENT FAILURE

PUFFO (2) 0.50 \$ LATE LEAK OR NO CONTAINMENT FAILURE

$\$$ RELEASE ELEVATION (M)

ELEV 32 .

S

$\$$ FPLBYO : FRACTION OF POOL BYPASS HAS THREE CASES

FPLBYO (1) $\quad 0.0564 \quad 1.32 \quad 1 . E+06$

\$ DRY CAVITY AND CONTAINMENT FAILURE CASES DERIVED FROM BMI-2139 GG STCP CALC

$\$$ IF CAVITY IS WET, DIVIDED BY WEAC

$\$$ IF LATE CF, MULTIPLIED BY PFAC

\$ STEAMING CORRECTION FACTOR FOR FPLBYO IF CAVITY IS NOT DRY

WFAC 3.1

\$ PRESSURE CORRECTION FACTOR FOR FPLBYO IF LATE CONTAINMENT FAILURE

PFAC 3.9

$\$$ SPLIT FRACTION BETWEEN TAIL PIPE VACUUM BREAKER OPENING AND T-QUENCHER

$\$$ HIGH PRESSURE SEQUENCES

FTLPH 0.39

$\$$ LOW PRESSURE SEQUENCES

FTLPL 1.0

$\$$ SPLIT FRACTION TO REACTOR HEAD VENT VS PIPE (ADDED FOR GGSOR POS5)

HVSPLTO 1.0

\$ FRACTION CORE DAMAGE (ADDED FOR GGSOR POS5)

FCD 1.0

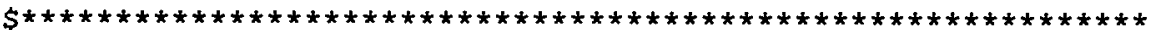

$\$$ FHPE: FRACTION OF CORE PARTICIPATING IN DCH OR STEAM EXPLOSION

$\$$ TWO CASES: (1) HIGH, (2) LOW

FHPEO (1) $\quad 0.4 \quad 0.1$

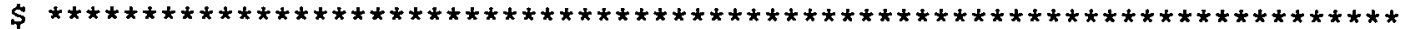

\$ EVSE: FRACTION OF CORE PARTICIPATING IN EX-VESSEL STEAM EXPLOSION

EVSE0 (1) $\quad 0.2 \quad 0.05$

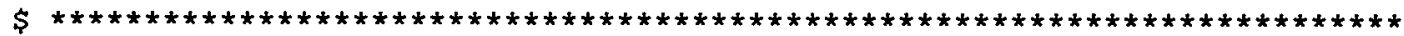

\$ PUFE RELEASE AT VESSEL BREACH: ONE SET FOR ALI $=>$ USE GG TB1/TB2

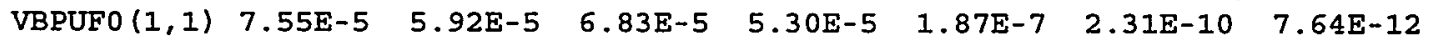

$$
0.0 \quad 5.63 \mathrm{E}-6
$$

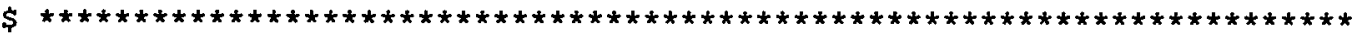

$\$$ THE FOLLOWING DATA BLOCKS WHICH HAVE VARIABLES ENDING WITH "O"

$\$$ ARE TAKEN FROM MEDIAN VALUES FROM EXPERT OPINION VALUES FOR GRAND GULF

$\$$ UNLESS OTHERWISE NOTED.

$\$$ (1) FIRST DIMENSION IS CHEMICAL SPECIES

$\$$ (2) SECOND DIMENSION IS CASE

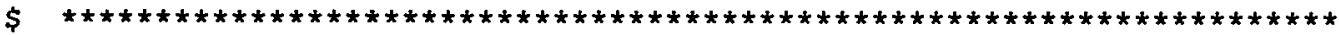

$\$$ NUMBER OF CHEMICAL SPECIES (NG, I, CS, TE, SR, RU, LA, CE, BA)

NSPEC 9

\$

\$ FCORO : IN-VESSEL RELEASE FRACTION FROM CORE TO RPV ATMOS.

$\$$ BWR CASE 1: HIGH ZR OXIDATION

$\begin{array}{llllllllll}\text { FCORO }(1,1) & .9 & .74 & .59 & .15 & 6.4 \mathrm{E}-3 & 4.6 \mathrm{E}-3 & 1.0 \mathrm{E}-4 & 1.5 \mathrm{E}-4 & 8.6 \mathrm{E}-3\end{array}$

$\$$ BWR CASE 2: LOW ZR OXIDATION 


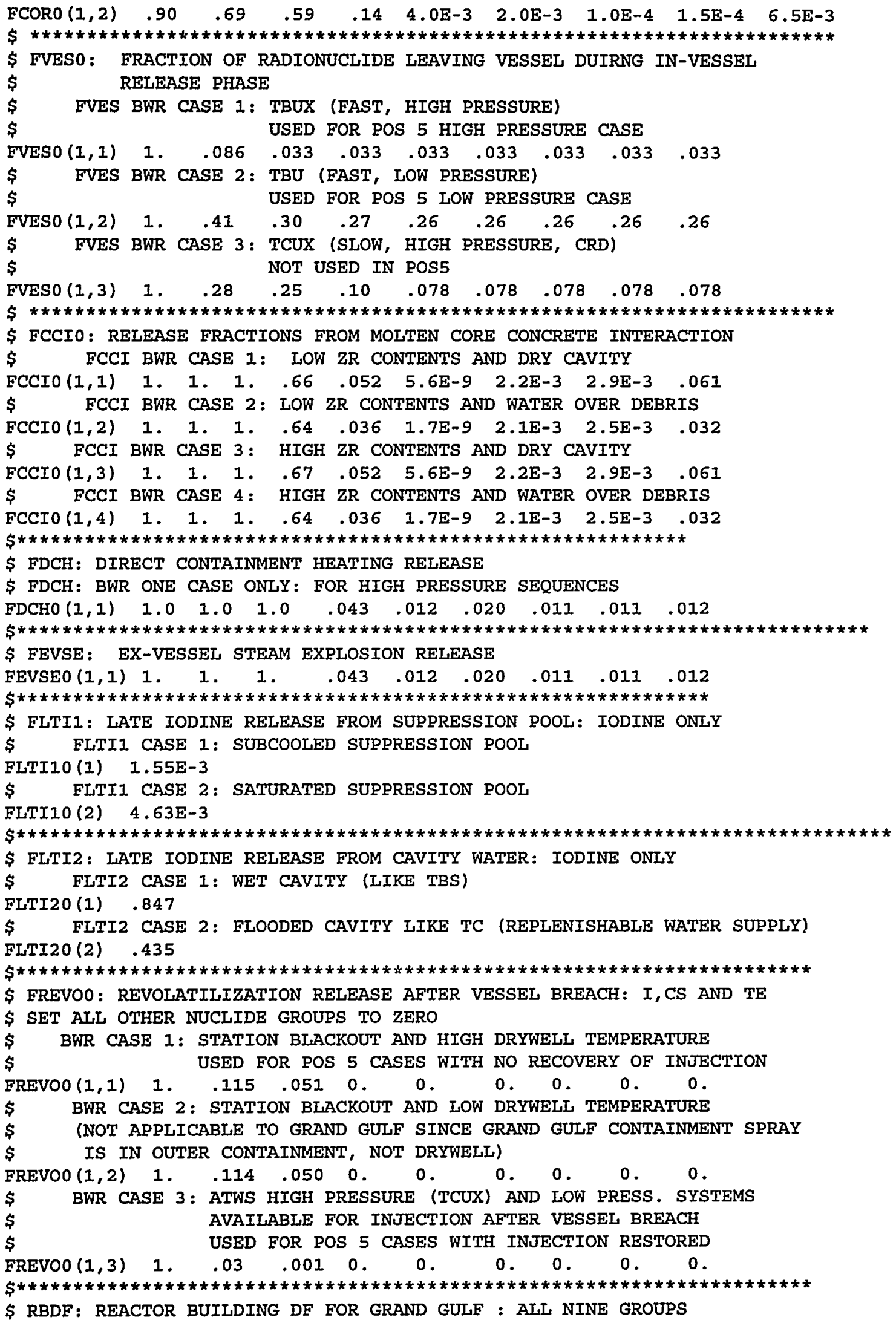


Appendix C

\$ DF FROM PEACH BOTTOM-DW SHELI FAILURE INTO REACTOR BUILDING SAT. POOL. $\$$ GG CASE 1:

$\operatorname{RBDFO}(1,1) \quad 1 . \quad 4.05 \quad 4.05 \quad 4.02 \quad 4.02 \quad 4.02 \quad 4.02 \quad 4.02 \quad 4.02$

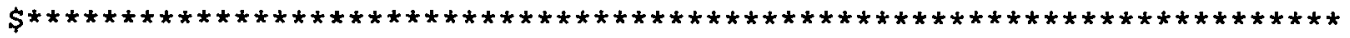

\$ FCONV : CONTAINMENT RELEASE FRACTION BEFORE VESSEL BREACH

$\$$ FCONV GG CASE 1: EARLY LEAK SUBCOOLED POOL

$\begin{array}{lllllllllll}\text { FCONVO }(1,1) & 1 & 1 & .233 & .233 & .233 & .233 & .233 & .233 & .233 & .233\end{array}$

$\$$ FCONV GG CASE 2: EARLY LEAK SATUARATED POOL

$\begin{array}{llllllllll}\text { FCONVO }(1,2) & 1 . & .245 & .245 & .245 & .245 & .245 & .245 & .245 & .245\end{array}$

$\$$ FCONV GG CASE 3: EARLY RUPTURE SUBCOOLED POOL

$\$$ (ALSO USED FOR POS 5 CASES WITH OPEN OPEN CNMT)

$\begin{array}{lllllllllll}\text { FCONVO }(1,3) & 1 & .639 & .639 & .639 & .639 & .639 & .639 & .639 & .639\end{array}$

\$ FCONV GG CASE 4: EARLY RUPTURE SATURATED POOL

$\$$ (ALSO USED FOR POS 5 CASES WITH OPEN OPEN CNMT)

$\begin{array}{lllllllllll}\text { FCONVO }(1,4) & 1 . & .639 & .639 & .639 & .639 & .639 & .639 & .639 & .639\end{array}$

$\$$ FCONV GG CASE 5: LATE LEAK

$\begin{array}{llllllllll}\text { FCONVO }(1,5) & 1 . & .052 & .052 & .052 & .052 & .052 & .052 & .052 & .052\end{array}$

$\$$ FCONV GG CASE 6: LATE RUPTURE

$\begin{array}{lllllllllll}\text { FCONVO }(1,6) & 1 . & .084 & .084 & .084 & .084 & .084 & .084 & .084 & .084\end{array}$

$\$$ NO CONTAINMENT FAILURE CASE

FCONVO $(1,7) \quad 0.005$ 1.0E-6 1.0E-6 1.0E-6 1.0E-6 1.0E-6 $1.0 \mathrm{E}-6 \quad 1.0 \mathrm{E}-6 \quad 1.0 \mathrm{E}-6$

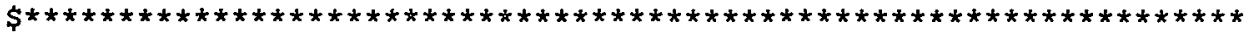

\$ FCONC: CONTAINMENT RELEASE FRACTION AFTER VESSEL BREACH

$\$$ FCONC GG CASE 1: EARLY LEAK SUBCOOLED POOL

$\begin{array}{lllllllllll} & \mathrm{FCONCO}(1,1) & 1 & .280 & .280 & .251 & .251 & .251 & .251 & .251 & .251\end{array}$

$\$$ FCONC GG CASE 2: EARLY IEAK SATURATED POOL

$\begin{array}{lllllllllll}\text { FCONCO }(1,2) & 1 & .251 & .251 & .231 & .231 & .231 & .231 & .231 & .231\end{array}$

$\$$ FCONC GG CASE 3: EARLY RUPTURE SUBCOOLED POOL

$\$$ (ALSO USED FOR POS 5 CASES WITH OPEN OPEN CNMT)

$\begin{array}{lllllllllll}\text { FCONCO }(1,3) & 1 & 1 & .743 & .743 & .720 & .720 & .720 & .720 & .720 & .720\end{array}$

$\$$ FCONC GG CASE 4: EARLY RUPTURE SATURATED POOL

$\$$ (ALSO USED FOR POS 5 CASES WITH OPEN OPEN CNMT)

$\begin{array}{lllllllllll}\text { FCONCO }(1,4) & 1 & .719 & .719 & .675 & .675 & .675 & .675 & .675 & .675\end{array}$

$\$$ FCONC GG CASE 5: LATE LEAK

$\begin{array}{lllllllllll}\text { FCONCO }(1,5) & 1 . & .052 & .052 & .082 & .063 & .082 & .063 & .072 & .072\end{array}$

$\$ \quad$ FCONC GG CASE 6 : LATE RUPTURE

$\begin{array}{lllllllllll}\text { FCONCO }(1,6) & 1 . & .084 & .084 & .107 & .094 & .107 & .094 & .094 & .094\end{array}$

$\$$ NO CONTAINMENT FAILURE CASE

FCONCO $(1,7) \quad 0.005$ 1.0E-6 $1.0 \mathrm{E}-6$ 1.0E-6 $1.0 \mathrm{E}-6 \quad 1.0 \mathrm{E}-6 \quad 1.0 \mathrm{E}-6 \quad 1.0 \mathrm{E}-6 \quad 1.0 \mathrm{E}-6$

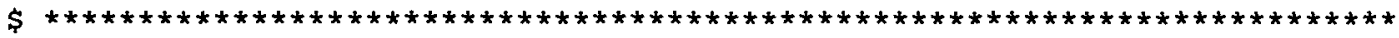

\$ SUPPRESSION POOL DF VALUES BASED ON VALUES FROM DRAFT NUREG/CR-4551

$\$$ EXPERT MEDIAN VALUES

$\$$ SUPPRESSION POOL DF THROUGH SRV T-QUENCHERS

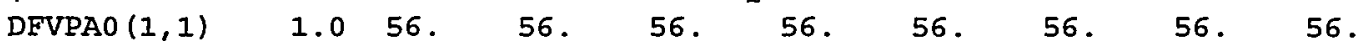

$\begin{array}{llllllllll}\$ & \text { SUPPRESSION POOL DF THROUGH } & \text { DOWNCOMERS } \\ \text { DFCPAO }(1,1) & 1.0 & 6.8 & 6.8 & 6.8 & 6.8 & 6.8 & 6.8 & 6.8 & 6.8\end{array}$

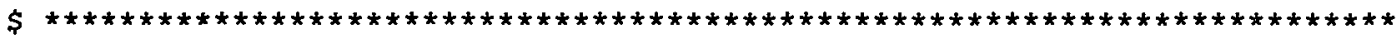

$\$$ CONTAINMENT SPRAYS DF BASED ON VALUES FROM DRAFT NUREG/CR-4551

DFSPRVO $(1,1) \quad 1.0$ 11. 11. 11. 11. 11. 11. 11. 11.

$\operatorname{DFSPRCO}(1,1) \quad 1.0$ 17. 17. 17. 17. 17. 17. 17. 17.

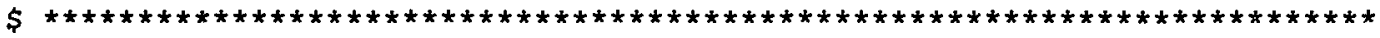

$\$$ CAVITY WATER DF VALUES BASED ON VALUES FROM DRAFT NUREG/CR-4551

$\$$ EXPERT MEDIAN VALUES

$\$$ CASE 1: WET CAVITY LIKE GRAND GULF TBS CASE

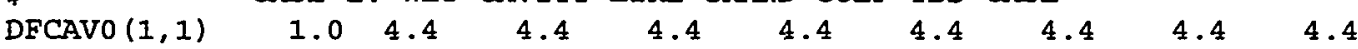

$\$$ CASE 2: FLOODED CAVITY LIKE GRAND GULF TC CASE

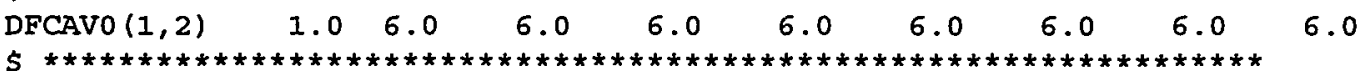


Appendix C

\begin{tabular}{|c|c|c|c|c|c|c|c|c|c|}
\hline $\operatorname{FVESL}(1,8,2)$ & 1. & 1. & 1. & 1. & 1 . & 1. & 1. & 1. & 1. \\
\hline FVESL $(1,9,2)$ & 1. & 1. & 1. & 1. & 1. & 1. & 1. & 1 . & 1. \\
\hline FVESL & BWR CASE & $\begin{aligned} 3: & \text { TCUX } \\
& \text { NOT }\end{aligned}$ & $\begin{array}{l}X \text { (SLOW, } \\
\text { USED IN }\end{array}$ & $\begin{array}{l}\text { HIGH } \\
\mathrm{N} \text { POS } 5\end{array}$ & PRESSURE & ;, CRD) & & & \\
\hline FVESL $(1,1,3)$ & 1. & 0 . & $1.0 \mathrm{E}-5$ & 0 . & 0 . & 0 . & 0. & 0 . & 0. \\
\hline $\operatorname{FVESL}(1,2,3)$ & 1. & $8.0 E-5$ & $8.0 E-5$ & $2.0 \mathrm{E}-5$ & $2.0 \mathrm{E}-5$ & $2.0 \mathrm{E}-5$ & $2.0 \mathrm{E}-5$ & $2.0 \mathrm{E}-5$ & $2.0 \mathrm{E}-5$ \\
\hline FVESI $(1,3,3)$ & 1. & .018 & $7.6 E-3$ & $1.0 \mathrm{E}-4$ & $1.0 \mathrm{E}-4$ & 1. $0 E-4$ & $1.0 \mathrm{E}-4$ & $1.0 \mathrm{E}-4$ & $1.0 \mathrm{E}-$ \\
\hline FVESL $(1,4,3)$ & 1. & .089 & .052 & $4.9 E-3$ & $4.8 E-3$ & $4.8 \mathrm{E}-3$ & $4.8 E-3$ & $4.8 \mathrm{E}-3$ & $4.8 E-$ \\
\hline FVESL $(1,5,3)$ & 1. & .28 & .25 & .10 & .078 & .078 & .078 & .078 & .078 \\
\hline FVESL $(1,6,3)$ & 1 . & .75 & .63 & .39 & .29 & .29 & .29 & .29 & .29 \\
\hline $\operatorname{FVESL}(1,7,3)$ & 1. & .95 & .9 & .7 & .7 & .7 & .7 & .7 & .7 \\
\hline FVESI $(1,8,3)$ & 1. & .99 & .99 & .88 & .88 & .88 & .88 & .88 & .88 \\
\hline $\operatorname{VVESL}(1,9,3)$ & 1. & 1. & 1. & .98 & .98 & .98 & .98 & .98 & .98 \\
\hline
\end{tabular}

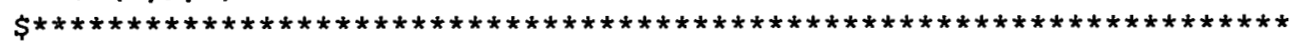

\$ FCCIL: RELEASE FRACTIONS FROM MOLTEN CORE CONCRETE INTERACTION

$\$$ FCCI GG CASE 1: LOW ZR CONTENTS AND DRY CAVITY

\begin{tabular}{|c|c|c|c|c|c|c|c|c|c|}
\hline $\operatorname{FCCII}(1,1,1)$ & 1 . & 1. & 1. & $4 \cdot 4 \mathrm{E}-3$ & 0 . & $1.0 \mathrm{E}-9$ & 0 . & 0 . & $3.0 \mathrm{E}-5$ \\
\hline $\operatorname{FCCIL}(1,2,1)$ & 1. & 1. & 1. & .012 & $5.0 E-5$ & 1. $0 E-9$ & 0 . & 0 . & 1. $2 E-4$ \\
\hline $\operatorname{ECCIL}(1,3,1)$ & 1. & 1. & 1. & .069 & $3.1 \mathrm{E}-4$ & $1.2 \mathrm{E}-9$ & $1.0 E-5$ & $3.0 \mathrm{E}-5$ & $4 \cdot 9 E-4$ \\
\hline $\operatorname{FCCIL}(1,4,1)$ & 1. & 1. & 1 . & .32 & $2.6 E-3$ & $2.4 E-9$ & $2.1 E-4$ & $3.2 E-4$ & $3.2 E-3$ \\
\hline $\operatorname{ECCIL}(1,5,1)$ & 1. & 1. & 1. & .66 & .052 & $5.6 E-9$ & $2.2 \mathrm{E}-3$ & $2.9 E-3$ & .061 \\
\hline $\operatorname{FCCIL}(1,6,1)$ & 1. & 1. & 1. & .76 & .62 & $5.0 E-6$ & .013 & .026 & .45 \\
\hline $\operatorname{FCCIL}(1,7,1)$ & 1. & 1. & 1. & .94 & .95 & $7 \cdot 3 E-3$ & .086 & .018 & .88 \\
\hline $\operatorname{FCCII}(1,8,1)$ & 1. & 1. & 1. & .99 & .99 & $9.7 E-2$ & .1 & .2 & .98 \\
\hline $\operatorname{FCCIL}(1,9,1)$ & 1. & 1. & 1. & 1. & 1. & .25 & .1 & .2 & 1. \\
\hline
\end{tabular}

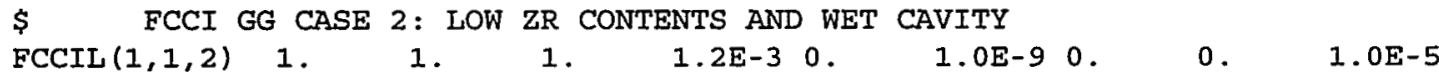

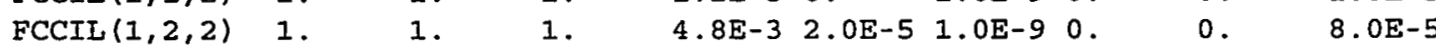
$\operatorname{FCCII}(1,3,2) \quad 1 . \quad 1 . \quad 1 . \quad .032 \quad 2.7 \mathrm{E}-41.1 \mathrm{E}-90 . \quad 1.0 \mathrm{E}-5 \quad 3.6 \mathrm{E}-4$ FCCIL $(1,4,2) \quad 1 . \quad 1 . \quad 1 . \quad .26 \quad 2.0 \mathrm{E}-3 \quad 1.3 \mathrm{E}-9$ 1.9E-4 2.6E-4 2.3E-3 $\operatorname{FCCIL}(1,5,2) \quad 1 . \quad 1 . \quad 1 . \quad .64 \quad .036 \quad 1.7 \mathrm{E}-92.1 \mathrm{E}-32.5 \mathrm{E}-3 \quad .032$

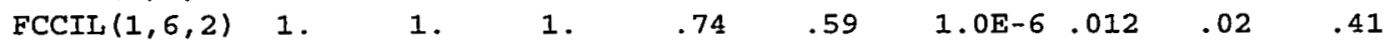
$\begin{array}{lllllllll}\operatorname{FCCIL}(1,7,2) & 1 . & 1 . & 1 . & .93 & .94 & 2.5 \mathrm{E}-3.084 & .087 & .87\end{array}$

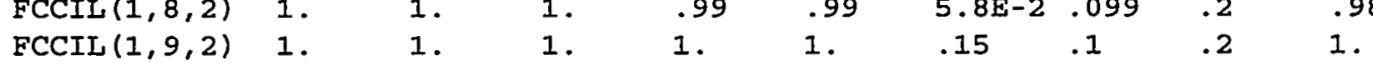
$\$$ FCCI GG CASE 3: HIGH ZR CONTENTS AND DRY CAVITY

\begin{tabular}{|c|c|c|c|c|c|c|c|c|c|}
\hline $\operatorname{FCCII}(1,1,3)$ & 1. & 1. & 1. & $4.4 E-3$ & 0. & 1. $0 E-9$ & 0 . & 0 & $3.0 \mathrm{E}-5$ \\
\hline $\operatorname{FCCIL}(1,2,3)$ & 1. & I. & 1 . & .012 & $5.0 \mathrm{E}-5$ & I. OE-9 & 0 . & 0 & 1. $2 \mathrm{E}-4$ \\
\hline $\operatorname{FCCIL}(1,3,3)$ & 1. & 1. & 1. & .069 & $3.1 E-4$ & $1.2 \mathrm{E}-9$ & $1.0 E-5$ & $3.0 \mathrm{E}-5$ & $4 \cdot 9 E-4$ \\
\hline $\operatorname{FCCIL}(1,4,3)$ & 1. & 1 . & 1. & .40 & $2.6 \mathrm{E}-3$ & $2.4 E-9$ & $2.1 \mathrm{E}-4$ & $3.2 E-4$ & $3.2 E-3$ \\
\hline $\operatorname{FCCIL}(1,5,3)$ & 1. & 1 . & 1. & .67 & .052 & $5.6 \mathrm{E}-9$ & $2 \cdot 2 E-3$ & $2.9 \mathrm{E}-3$ & .061 \\
\hline $\operatorname{FCCIL}(1,6,3)$ & 1. & I. & 1. & .79 & .65 & $5.0 E-6$ & .02 & .031 & .51 \\
\hline $\operatorname{FCCIL}(1,7,3)$ & 1. & 1. & 1. & .96 & .97 & $7.3 E-3$ & .11 & .18 & .9 \\
\hline $\operatorname{FCCIL}(1,8,3)$ & 1. & 1 . & 1. & .99 & 1. & $9.7 E-2$ & .15 & .2 & .98 \\
\hline $\operatorname{FCCIL}(1,9,3)$ & 1. & 1. & 1. & 1 . & 1. & .25 & .16 & .2 & 1. \\
\hline
\end{tabular}
$\$$ FCCI GG CASE 4: HIGH ZR CONTENTS AND WATER OVER DEBRIS

$\begin{array}{llllllllll}\operatorname{FCCII}(1,1,4) & 1 . & 1 . & 1 . & 1.2 \mathrm{E}-3 & 0 . & 1.0 \mathrm{E}-90 . & 0 . & 1.0 \mathrm{E}-5 \\ \operatorname{FCCIL}(1,2,4) & 1 . & 1 . & 1 . & 4.8 \mathrm{E}-3 & 2.0 \mathrm{E}-5 & 1.0 \mathrm{E}-90 . & 0 . & 8.0 \mathrm{E}-5 \\ \operatorname{FCCIL}(1,3,4) & 1 . & 1 . & 1 . & .032 & 2.7 \mathrm{E}-4 & 1.1 \mathrm{E}-90 . & 1.0 \mathrm{E}-5 & 3.6 \mathrm{E}-4 \\ \operatorname{FCCIL}(1,4,4) & 1 . & 1 . & 1 . & .26 & 2.0 \mathrm{E}-3 & 1.3 \mathrm{E}-9 & 1.9 \mathrm{E}-4 & 2.6 \mathrm{E}-4 & 2.3 \mathrm{E}-3 \\ \operatorname{FCCIL}(1,5,4) & 1 . & 1 . & 1 . & .64 & .036 & 1.7 \mathrm{E}-92.1 \mathrm{E}-3 & 2.5 \mathrm{E}-3.032 \\ \operatorname{FCCIL}(1,6,4) & 1 . & 1 . & 1 . & .74 & .59 & 1.0 \mathrm{E}-6.012 & .02 & .41 \\ \operatorname{FCCII}(1,7,4) & 1 . & 1 . & 1 . & .93 & .94 & 2.5 \mathrm{E}-3.084 & .17 & .87 \\ \operatorname{FCCIL}(1,8,4) & 1 . & 1 . & 1 . & .99 & .99 & 5.8 E-2.099 & .2 & .98 \\ \operatorname{FCCIL}(1,9,4) & 1 . & 1 . & 1 . & 1 . & 1 . & .15 & .1 & .2 & 1 .\end{array}$

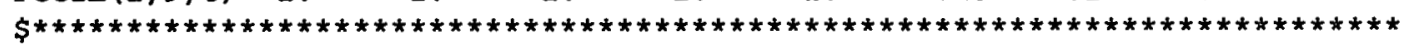
\$ FDCH: BWR ONE CASE ONLY: FOR HIGH PRESSURE SEQUENCES

$\$$ FISRT DIMENSION = RADIONUCLIDE GROUP 
$\$ \quad$ SECOND DIMENSION = PROBABILITY POINTS

\begin{tabular}{|c|c|c|c|c|c|c|c|c|c|}
\hline $\operatorname{FDCHL}(1,1,1)$ & 1. & .063 & .063 & 0 . & 0 . & 0. & 0 . & 0. & 0. \\
\hline $\operatorname{FDCHL}(1,2,1)$ & 1. & .15 & .15 & 0 . & 0 . & 0 . & 0 . & 0 . & 0 . \\
\hline $\operatorname{FDCHL}(1,3,1)$ & 1 . & .50 & .50 & .001 & .001 & .001 & .001 & .001 & .001 \\
\hline $\operatorname{FDCHL}(1,4,1)$ & 1. & 1 . & 1. & .008 & .002 & .007 & .002 & .002 & .004 \\
\hline FDCHL $(1,5,1)$ & 1. & 1. & 1 . & .043 & .012 & .020 & .011 & .011 & .012 \\
\hline $\operatorname{FDCHI}(1,6,1)$ & 1 . & 1. & 1. & .600 & .030 & .063 & .040 & .040 & .067 \\
\hline $\operatorname{FDCHL}(1,7,1)$ & 1. & 1. & 1. & .975 & .751 & .700 & .087 & .087 & .863 \\
\hline $\operatorname{FDCHL}(1,8,1)$ & 1. & 1 . & 1. & 1 . & .980 & .900 & .200 & .280 & .980 \\
\hline $\operatorname{FDCHL}(1,9,1)$ & 1. & 1. & 1 . & 1. & 1 . & .950 & .230 & .330 & 1. \\
\hline \multicolumn{10}{|c|}{ 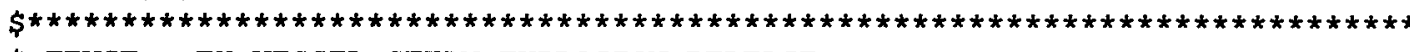 } \\
\hline \multicolumn{10}{|c|}{ \$ FEVSE: EX-VESSEL STEAM EXPLOSION RELEASE } \\
\hline FEVSEL $(1,1,1)$ & 1. & .063 & .063 & 0. & 0 . & 0 . & 0 . & 0 . & 0 . \\
\hline FEVSEL $(1,2,1)$ & 1. & .15 & .15 & 0 . & 0 . & 0 . & 0 . & 0 . & 0 . \\
\hline $\operatorname{EEVSEL~}(1,3,1)$ & 1 . & .50 & .50 & .001 & .001 & .001 & .001 & .001 & .001 \\
\hline FEVSEL $(1,4,1)$ & 1. & 1. & 1. & .008 & .002 & .007 & .002 & .002 & .004 \\
\hline $\operatorname{FEVSEL}(1,5,1)$ & 1. & 1. & 1. & .043 & .012 & .020 & .011 & .011 & .012 \\
\hline $\operatorname{FEVSEL~}(1,6,1)$ & 1. & 1. & 1 & .600 & .030 & .063 & .040 & .040 & .067 \\
\hline $\operatorname{FEVSEL}(1,7,1)$ & 1. & 1. & 1. & .975 & .751 & .700 & .087 & .087 & .863 \\
\hline $\operatorname{FEVSEL~}(1,8,1)$ & 1. & 1. & 1. & 1 . & .980 & .900 & .200 & .280 & .980 \\
\hline $\operatorname{FEVSEL}(1,9,1)$ & 1. & 1. & 1. & 1. & 1. & .950 & .230 & .330 & 1. \\
\hline
\end{tabular}

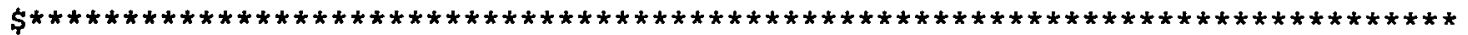

\$ FLTI1: LATE IODINE RELEASE FROM SUPPRESSION POOL: IODINE ONLY

$\$$ THEREFORE, PROBABILITY GOING ACROSS

$\$$ FIRST DIMENSION = PROBABILITY POINTS

$\$ \quad$ SECOND DIMENSION = CASES

$\$$ FLTI1 CASE 1: SUBCOOLED SUPPRESSION POOL

$\begin{array}{llllllllll}\text { FLTIL }(1,1) & 0 . & 0 . & 0 . & 5.00 \mathrm{E}-4 & 1.55 \mathrm{E}-3 & .0278 & .085 & .097 & .10\end{array}$

$\$$ FLTI1 CASE 2: SATURATED SUPPRESSION POOL

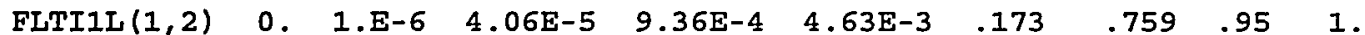

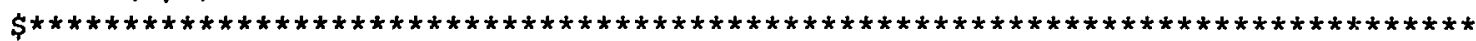

\$ FLTI2: IATE IODINE RELEASE FROM CAVITY WATER: IODINE ONLY

$\$$ FLTI2 CASE 1: WET CAVITY (LIKE TBS)

$\begin{array}{llllllllllll}\operatorname{FLTILL}(1,1) & .080 & .109 & .153 & .365 & .847 & .957 & 1 . & 1 . & 1 .\end{array}$

$\$$ FLTI2 CASE 2: FLOODED CAVITY LIKE TC (REPLENISHABLE WATER SUPPLY)

$\begin{array}{lllllllllll}\text { FLTILI }(1,2) & .004 & .04 & .109 & .247 & .435 & .670 & .936 & .985 & 1 .\end{array}$

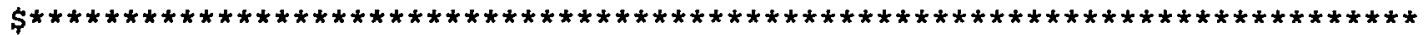

\$ FREVOL: REVOLATILIZATION RELEASE AFTER VESSEL BREACH: I,CS AND TE

$\$$ SET ALL OTHER NUCLIDE GROUPS TO ZERO

\$ BWR CASE 1: SBO AND HIGH DW TEMP

$\$$

USED FOR POS 5 CASES WITH NO LPI RECOVERED

$\begin{array}{llllllllll}\text { FREVOL }(1,1,1) & 1 . & 0 . & 0 . & 0 . & 0 . & 0 . & 0 . & 0 . & 0 . \\ \text { FREVOL }(1,2,1) & 1 . & 0 . & 0 . & 0 . & 0 . & 0 . & 0 . & 0 . & 0 . \\ \text { FREVOL }(1,3,1) & 1 . & 0 . & 0 . & 0 . & 0 . & 0 . & 0 . & 0 . & 0 . \\ \text { FREVOL }(1,4,1) & 1 . & .03 & .001 & 0 . & 0 . & 0 . & 0 . & 0 . & 0 . \\ \text { FREVOL }(1,5,1) & 1 . & .115 & .051 & 0 . & 0 . & 0 . & 0 . & 0 . & 0 . \\ \text { FREVOL }(1,6,1) & 1 . & .306 & .132 & .024 & 0 . & 0 . & 0 . & 0 . & 0 . \\ \text { FREVOL }(1,7,1) & 1 . & .557 & .284 & .224 & 0 . & 0 . & 0 . & 0 . & 0 . \\ \text { FREVOL }(1,8,1) & 1 . & .800 & .535 & .413 & 0 . & 0 . & 0 . & 0 . & 0 . \\ \text { FREVOL }(1,9,1) & 1 . & 1 . & .750 & .800 & 0 . & 0 . & 0 . & 0 . & 0 .\end{array}$

$\$$ BWR CASE 2: STATION BLACKOUT AND LOW DRYWELL TEMPERATURE

$\$$ (NOT APPLICABLE TO GRAND GULF SINCE GRAND GULF CONTAINMENT SPRAY

$\$$ IS IN OUTER CONTAINMENT, NOT DRYWELL)

$\begin{array}{llllllllll}\text { FREVOL }(1,1,2) & 1 . & 0 . & 0 . & 0 . & 0 . & 0 . & 0 . & 0 . & 0 . \\ \text { FREVOL }(1,2,2) & 1 . & 0 . & 0 . & 0 . & 0 . & 0 . & 0 . & 0 . & 0 . \\ \text { FREVOL }(1,3,2) & 1 . & 0 . & 0 . & 0 . & 0 . & 0 . & 0 . & 0 . & 0 . \\ \text { FREVOL }(1,4,2) & 1 . & .03 & .001 & 0 . & 0 . & 0 . & 0 . & 0 . & 0 . \\ \text { FREVOL }(1,5,2) & 1 . & .114 & .050 & 0 . & 0 . & 0 . & 0 . & 0 . & 0 .\end{array}$


Appendix $\mathrm{C}$

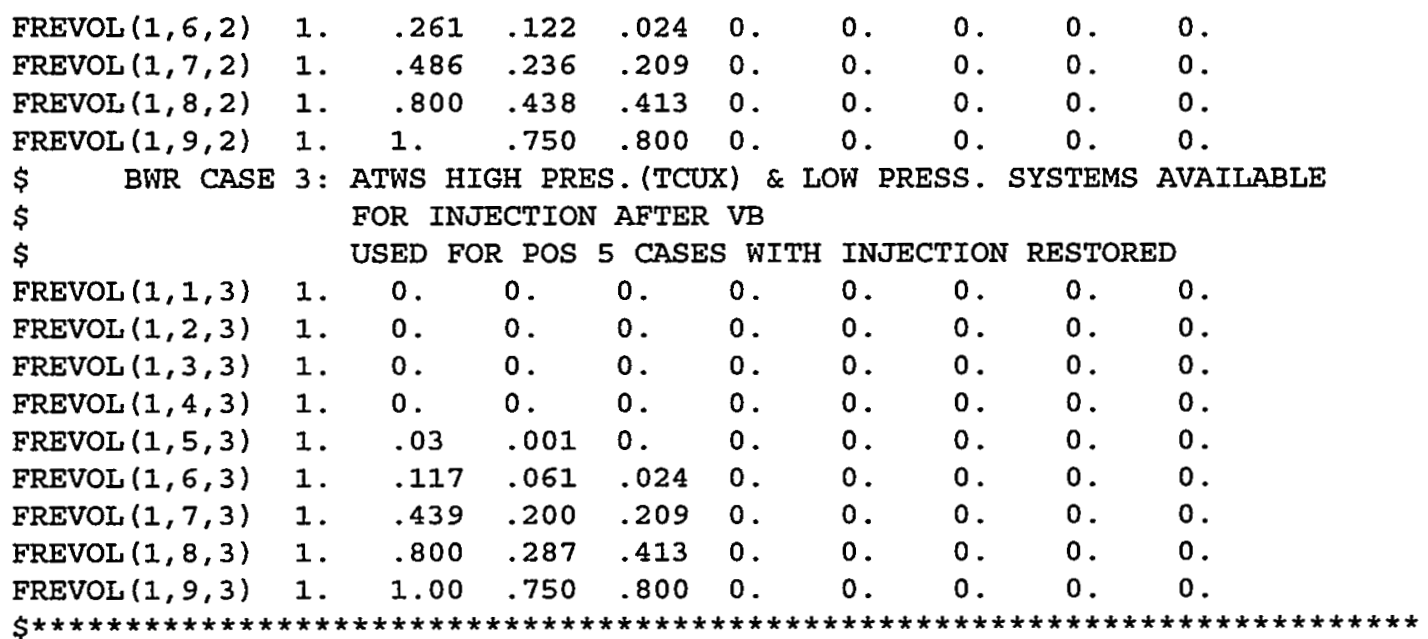

\$ RBDF: REACTOR BUILDING DF FOR GRAND GULF : ALL NINE GROUPS

$\$$ DF FROM PEACH BOTTOM-DW SHELL FAILURE INTO REACTOR BUILDING SAT. POOL.

$\begin{array}{llllllllll}\$ \quad G G \text { CASE } 1: & & & & & & \\ \text { RBDFL }(1,1,1) & 1 . & 1 . & 1 . & 1 . & 1 . & 1 . & 1 . & 1 . & 1 . \\ \operatorname{RBDFL}(1,2,1) & 1 . & 1.06 & 1.06 & 1.08 & 1.08 & 1.08 & 1.08 & 1.08 & 1.08 \\ \operatorname{RBDFL}(1,3,1) & 1 . & 1.23 & 1.23 & 1.25 & 1.25 & 1.25 & 1.25 & 1.25 & 1.25 \\ \operatorname{RBDFL}(1,4,1) & 1 . & 2.29 & 2.29 & 2.22 & 2.22 & 2.22 & 2.22 & 2.22 & 2.22 \\ \operatorname{RBDFL}(1,5,1) & 1 . & 4.05 & 4.05 & 4.02 & 4.02 & 4.02 & 4.02 & 4.02 & 4.02 \\ \operatorname{RBDFL}(1,6,1) & 1 . & 6.86 & 6.86 & 6.86 & 6.86 & 6.86 & 6.86 & 6.86 & 6.86 \\ \operatorname{RBDFL}(1,7,1) & 1 . & 14.5 & 14.5 & 17.0 & 17.0 & 17.0 & 17.0 & 17.0 & 17.0 \\ \operatorname{RBDFL}(1,8,1) & 1 . & 87.9 & 87.9 & 92.1 & 92.1 & 92.1 & 92.1 & 92.1 & 92.1 \\ \operatorname{RBDFL}(1,9,1) & 1 . & 551 . & 551 . & 590 . & 590 . & 590 . & 590 . & 590 . & 590 .\end{array}$

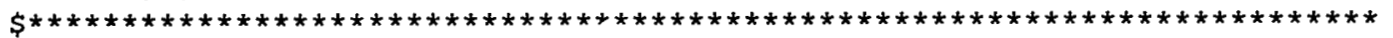

$\$$ FCONV: CONTAINMENT RELEASE FRACTION BEFORE VESSEL BREACH: ALL NINE GROUPS

$\$$ FCONVL GG CASE 1: EARLY LEAK, SUBCOOLED POOL

\begin{tabular}{|c|c|c|c|c|c|c|c|c|c|}
\hline CONVL $(1,1,1)$ & 1 . & .001 & 001 & 001 & 01 & .001 & .001 & .001 & .001 \\
\hline CONVL $(1,2,1)$ & 1 & .003 & .003 & .003 & .003 & .003 & .003 & .003 & .003 \\
\hline CONVL $(1,3,1)$ & 1. & .012 & .012 & .012 & .012 & .012 & .012 & .012 & .012 \\
\hline ONVL $(1,4,1)$ & 1. & .117 & .117 & .117 & .117 & & 117 & 17 & \\
\hline ONVL $(1,5,1)$ & 1 & .233 & .233 & .233 & .233 & .233 & .233 & .233 & .233 \\
\hline CONVL $(1,6,1)$ & 1. & .417 & .417 & .417 & .417 & .417 & .417 & .417 & .417 \\
\hline $\operatorname{ONVL}(1,7,1)$ & 1. & .676 & .676 & .676 & .676 & .676 & .676 & .676 & .676 \\
\hline $\operatorname{CONVL}(1,8,1)$ & 1. & .784 & .784 & .784 & .784 & .784 & .784 & .784 & .784 \\
\hline FCONVL $(1,9,1)$ & 1. & .949 & .949 & .949 & .949 & .949 & .949 & .949 & .949 \\
\hline FCONVL GG & CASE & $2: E A$ & RLY LE & $A K, S F$ & IURATEI & POOL & & & \\
\hline $\operatorname{CONVL}(1,1,2)$ & 1 . & .002 & .002 & .002 & .002 & .002 & .002 & .002 & .002 \\
\hline $\operatorname{CONVL}(1,2,2)$ & 1. & .008 & .008 & .008 & .008 & .008 & .008 & .008 & .008 \\
\hline$, 3,2)$ & 1. & .030 & .030 & .030 & .030 & .030 & .030 & .030 & .030 \\
\hline CONVL $(1,4,2)$ & 1. & .151 & .151 & .151 & .151 & .151 & .151 & .151 & .151 \\
\hline $\operatorname{FCONVL}(1,5,2)$ & 1 . & .245 & .245 & .245 & .245 & .245 & .245 & .245 & .245 \\
\hline 6,2$)$ & 1 . & .447 & .447 & .447 & .447 & .447 & .447 & .447 & .447 \\
\hline $\operatorname{CONVL}(1,7,2)$ & 1. & .695 & .695 & .695 & .695 & .695 & .695 & .695 & .695 \\
\hline FCONVL $(1,8,2)$ & 1. & .792 & .792 & .792 & .792 & .792 & .792 & .792 & .792 \\
\hline $\operatorname{CONVL}(1,9,2)$ & 1. & .953 & .953 & .953 & .953 & .953 & .953 & .953 & .953 \\
\hline$\$ \quad$ FCONVL GG & 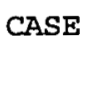 & & $\begin{array}{l}\mathrm{R} L \mathrm{R} \\
\mathrm{CSO} \\
\mathrm{LSO}\end{array}$ & $\begin{array}{l}\text { PTURE, } \\
\text { ED FOR }\end{array}$ & $\begin{array}{l}\text { SUBCC } \\
\text { POS }\end{array}$ & LED & $\begin{array}{l}\text { DOL } \\
\text { CNMT C }\end{array}$ & SES) & \\
\hline $\mathrm{NV}$ & 1. & .021 & .021 & .021 & .021 & .021 & .021 & .021 & .021 \\
\hline FCONV & 1 & .090 & .090 & .090 & .090 & .090 & .090 & .090 & .090 \\
\hline CONVL $(1,3,3)$ & 1. & .197 & .197 & .197 & .197 & .197 & .197 & .197 & .197 \\
\hline$a_{0}$ & 1. & .437 & .437 & .437 & .437 & .437 & .437 & .437 & .437 \\
\hline $\operatorname{CONVL}(1,5,3)$ & 1 & .639 & .639 & .639 & .639 & .639 & .639 & .639 & .639 \\
\hline
\end{tabular}




\begin{tabular}{|c|c|c|c|c|c|c|c|c|c|}
\hline FCONVL $(1,6,3)$ & 1. & .790 & .790 & .770 & .770 & .770 & .770 & .770 & .770 \\
\hline $\operatorname{FCONVL}(1,7,3)$ & 1. & .915 & .915 & .892 & .892 & .892 & .892 & .892 & .892 \\
\hline $\operatorname{FCONVL}(1,8,3)$ & 1. & .966 & .966 & .966 & .966 & .966 & .966 & .966 & .966 \\
\hline FCONVL $(1,9,3)$ & 1. & .996 & .996 & .996 & .996 & .996 & .996 & .996 & .996 \\
\hline FCONVL GG & CASE & 4: EF & $\begin{array}{ll}\mathrm{IY} & \mathrm{RU} \\
\mathrm{SO} & \mathrm{US}\end{array}$ & $\begin{array}{l}\text { TTURE, } \\
\text { ED FOR }\end{array}$ & $\begin{array}{l}\text { SATUR } \\
\text { POS } 5\end{array}$ & $\begin{array}{l}\text { ATED } \\
\text { OPEN }\end{array}$ & $\begin{array}{l}\text { OOL } \\
\text { CNMT }\end{array}$ & ASES) & \\
\hline FCONVL $(1,1,4)$ & 1. & .021 & .021 & .021 & .021 & .021 & .021 & .021 & .021 \\
\hline FCONVL $(1,2,4)$ & 1. & .090 & .090 & .090 & .090 & .090 & .090 & .090 & .090 \\
\hline FCONVL $(1,3,4)$ & 1. & .197 & .197 & .197 & .197 & .197 & .197 & .197 & .197 \\
\hline $\operatorname{FCONVL~}(1,4,4)$ & 1. & .437 & .437 & .437 & .437 & .437 & .437 & .437 & .437 \\
\hline $\operatorname{ECONVL}(1,5,4)$ & 1. & .639 & .639 & .639 & .639 & .639 & .639 & .639 & .639 \\
\hline FCONVL $(1,6,4)$ & 1. & .790 & .790 & .770 & .770 & .770 & .770 & .770 & .770 \\
\hline $\operatorname{FCONVL}(1,7,4)$ & 1. & .915 & .915 & .892 & .892 & .892 & .892 & .892 & .892 \\
\hline FCONVL $(1,8,4)$ & 1. & .966 & .966 & .966 & .966 & .966 & .966 & .966 & .966 \\
\hline FCONVL $(1,9,4)$ & 1. & .996 & .996 & .996 & .996 & .996 & .996 & .996 & .996 \\
\hline FCONVI, GG & CAS & $5:$ & ATE LE & & & & & & \\
\hline FCONVL $(1,1,5)$ & 1. & 0 & 0 & 0. & 0 . & 0 . & 0 . & 0 . & 0 . \\
\hline FCONVI $(1,2,5)$ & 1. & 0 . & 0 . & 0 . & 0 . & 0 & 0 . & 0 . & 0 . \\
\hline FCONVI $(1,3,5)$ & 1. & .001 & .001 & .001 & .001 & .001 & .001 & .001 & .001 \\
\hline FCONVL $(1,4,5)$ & 1. & .008 & .008 & .008 & .008 & .008 & .008 & .008 & .008 \\
\hline FCONVL $(1,5,5)$ & 1. & .052 & .052 & .052 & .052 & .052 & .052 & .052 & .052 \\
\hline FCONVL $(1,6,5)$ & 1. & .128 & .128 & .128 & .128 & .128 & .128 & .128 & .128 \\
\hline FCONVL $(1,7,5)$ & 1. & .330 & .330 & .330 & .330 & .330 & .330 & .330 & .330 \\
\hline FCONVL $(1,8,5)$ & 1. & .510 & .510 & .510 & .510 & .510 & .510 & .510 & .510 \\
\hline FCONVL $(1,9,5)$ & 1. & .814 & .814 & .814 & .814 & .814 & .814 & .814 & .814 \\
\hline FCONVL & $\mathrm{GCl}$ & E 6: & LATE & JPTURE & & & & & \\
\hline FCONVL $(1,1,6)$ & 1. & 0 . & 0 & 0 . & 0 . & 0 . & 0. & 0 . & 0 . \\
\hline FCONVL $(1,2,6)$ & 1. & 0 . & 0 & 0 . & 0 . & 0 . & 0 . & 0 . & 0 . \\
\hline FCONVL $(1,3,6)$ & 1. & .002 & .002 & .002 & .002 & .002 & .002 & .002 & .002 \\
\hline FCONVL $(1,4,6)$ & 1. & .017 & .017 & .017 & .017 & .017 & .017 & .017 & .017 \\
\hline FCONVL $(1,5,6)$ & 1. & .084 & .084 & .084 & .084 & .084 & .084 & .084 & .084 \\
\hline ECONVL $(1,6,6)$ & 1. & .186 & .186 & .186 & .186 & .186 & .186 & .186 & .186 \\
\hline $\operatorname{FCONVL}(1,7,6)$ & 1. & .338 & .338 & .338 & .338 & .338 & .338 & .338 & .338 \\
\hline FCONVL $(1,8,6)$ & 1. & .540 & .540 & .540 & .540 & .540 & .540 & .540 & .540 \\
\hline FCONVL $(1,9,6)$ & 1. & .969 & .969 & .969 & .969 & .969 & .969 & .969 & .969 \\
\hline
\end{tabular}

\section{$\$$ FCONVL: NO CONTAINMENT FAILURE CASE}

FCONVL $(1,1,7) \quad 0.005$ 1.0E-6 1.0E-6 1.0E-6 1.0E-6 1.0E-6 1.0E-6 $1.0 \mathrm{E}-6$ 1.0E-6 FCONVL $(1,2,7) \quad 0.0051 .0 \mathrm{E}-6 \quad 1.0 \mathrm{E}-6 \quad 1.0 \mathrm{E}-6 \quad 1.0 \mathrm{E}-6 \quad 1.0 \mathrm{E}-6 \quad 1.0 \mathrm{E}-6 \quad 1.0 \mathrm{E}-6 \quad 1.0 \mathrm{E}-6$ FCONVL $(1,3,7) \quad 0.005$ 1.0E-6 $1.0 \mathrm{E}-6 \quad 1.0 \mathrm{E}-6 \quad 1.0 \mathrm{E}-6 \quad 1.0 \mathrm{E}-6 \quad 1.0 \mathrm{E}-6 \quad 1.0 \mathrm{E}-6 \quad 1.0 \mathrm{E}-6$ FCONVL $(1,4,7) \quad 0.005$ 1.0E-6 $1.0 \mathrm{E}-6$ 1.0E-6 $1.0 \mathrm{E}-6$ 1.0E-6 $1.0 \mathrm{E}-6$ 1.0E-6 $1.0 \mathrm{E}-6$ FCONVL $(1,5,7) \quad 0.0051 .0 \mathrm{E}-6 \quad 1.0 \mathrm{E}-6 \quad 1.0 \mathrm{E}-6 \quad 1.0 \mathrm{E}-6 \quad 1.0 \mathrm{E}-6 \quad 1.0 \mathrm{E}-6 \quad 1.0 \mathrm{E}-6 \quad 1.0 \mathrm{E}-6$ FCONVL $(1,6,7) \quad 0.005$ 1.0E-6 $1.0 \mathrm{E}-6$ 1.0E-6 $1.0 \mathrm{E}-6$ 1.0E-6 $1.0 \mathrm{E}-6$ 1.0E-6 $1.0 \mathrm{E}-6$ FCONVL $(1,7,7) \quad 0.005 \quad 1.0 \mathrm{E}-6 \quad 1.0 \mathrm{E}-6 \quad 1.0 \mathrm{E}-6$ 1.0E-6 $1.0 \mathrm{E}-6 \quad 1.0 \mathrm{E}-6 \quad 1.0 \mathrm{E}-6 \quad 1.0 \mathrm{E}-6$ FCONVL $(1,8,7) \quad 0.005 \quad 1.0 \mathrm{E}-6 \quad 1.0 \mathrm{E}-6 \quad 1.0 \mathrm{E}-6 \quad 1.0 \mathrm{E}-6 \quad 1.0 \mathrm{E}-6 \quad 1.0 \mathrm{E}-6 \quad 1.0 \mathrm{E}-6 \quad 1.0 \mathrm{E}-6$ FCONVL $(1,9,7) \quad 0.0051 .0 \mathrm{E}-6$ 1.0E-6 $1.0 \mathrm{E}-6$ 1.0E-6 $1.0 \mathrm{E}-6$ 1.0E-6 $1.0 \mathrm{E}-6$ 1.0E-6

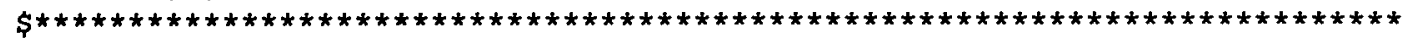
\$ FCONC: CONTAINMENT RELEASE FRACTION AFTER VESSEL BREACH: ALI NINE GROUPS $\$$ FCONC GG CASE 1: EARLY LEAK, SUBCOOLED POOL

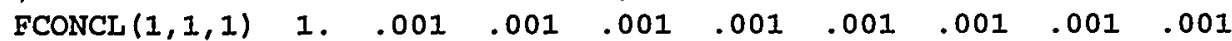
$\begin{array}{lllllllllll}\text { FCONCL }(1,2,1) & 1 & .003 & .003 & .003 & .003 & .003 & .003 & .003 & .003\end{array}$ $\begin{array}{lllllllllll}\text { FCONCL }(1,3,1) & 1 & .012 & .012 & .012 & .012 & .012 & .012 & .012 & .012\end{array}$ $\begin{array}{llllllllllll}\text { FCONCL }(1,4,1) & 1 . & .115 & .115 & .088 & .088 & .088 & .088 & .088 & .088\end{array}$ $\begin{array}{llllllllll}\operatorname{FCONCL}(1,5,1) & 1 . & .280 & .280 & .251 & .251 & .251 & .251 & .251 & .251\end{array}$ $\begin{array}{llllllllll}\operatorname{FCONCL}(1,6,1) & 1 . & .461 & .461 & .428 & .428 & .428 & .428 & .428 & .428\end{array}$ $\begin{array}{llllllllll}\text { FCONCL }(1,7,1) & 1 . & .672 & .672 & .672 & .672 & .672 & .672 & .672 & .672\end{array}$ $\begin{array}{llllllllll}\text { FCONCL }(1,8,1) & 1 . & .779 & .779 & .779 & .779 & .779 & .779 & .779 & .779\end{array}$ $\begin{array}{lllllllllll}\operatorname{FCONCL}(1,9,1) & 1 . & .876 & .876 & .876 & .876 & .876 & .876 & .876 & .876\end{array}$ $\$$ FCONC GG CASE 2: EARLY LEAK, SATURATED POOL 
Appendix C

$\begin{array}{llllllllll}\text { FCONCL }(1,1,2) & 1 . & .002 & .002 & .002 & .002 & .002 & .002 & .002 & .002 \\ \text { FCONCL }(1,2,2) & 1 . & .008 & .008 & .008 & .008 & .008 & .008 & .008 & .008 \\ \text { FCONCL }(1,3,2) & 1 . & .030 & .030 & .024 & .024 & .024 & .024 & .024 & .024 \\ \text { FCONCL }(1,4,2) & 1 . & .141 & .141 & .115 & .115 & .115 & .115 & .115 & .115 \\ \text { FCONCL }(1,5,2) & 1 . & .251 & .251 & .231 & .231 & .231 & .231 & .231 & .231 \\ \text { FCONCL }(1,6,2) & 1 . & .449 & .449 & .405 & .405 & .405 & .405 & .405 & .405 \\ \text { FCONCL }(1,7,2) & 1 . & .689 & .689 & .689 & .689 & .689 & .689 & .689 & .689 \\ \text { FCONCL }(1,8,2) & 1 . & .789 & .789 & .789 & .789 & .789 & .789 & .789 & .789 \\ \text { FCONCL }(1,9,2) & 1 . & .892 & .892 & .892 & .892 & .892 & .892 & .892 & .892\end{array}$
$\begin{array}{llll}892 & 892 & .892\end{array}$ $\$$ FCONC GG CASE 3: EARLY RUPTURE, SUBCOOLED POOL $\$$ (ALSO USED FOR POS 5 OPEN CNMT CASES)

$\begin{array}{llllllllll}\text { FCONCL }(1,1,3) & 1 . & .038 & .038 & .015 & .015 & .015 & .015 & .015 & .015 \\ \text { FCONCL }(1,2,3) & 1 . & .148 & .148 & .054 & .054 & .054 & .054 & .054 & .054 \\ \text { FCONCL }(1,3,3) & 1 . & .218 & .218 & .169 & .169 & .169 & .169 & .169 & .169 \\ \text { FCONCL }(1,4,3) & 1 . & .512 & .512 & .451 & .451 & .451 & .451 & .451 & .451 \\ \text { FCONCL }(1,5,3) & 1 . & .743 & .743 & .720 & .720 & .720 & .720 & .720 & .720 \\ \text { FCONCL }(1,6,3) & 1 . & .882 & .882 & .855 & .855 & .855 & .855 & .855 & .855 \\ \text { FCONCL }(1,7,3) & 1 . & .985 & .985 & .985 & .985 & .985 & .985 & .985 & .985 \\ \text { FCONCL }(1,8,3) & 1 . & .990 & .990 & .990 & .990 & .990 & .990 & .990 & .990 \\ \text { FCONCL }(1,9,3) & 1 . & 1 . & 1 . & 1 . & 1 . & 1 . & 1 . & 1 . & 1 .\end{array}$
$\$$ FCONC GG CASE 4: EARLY RUPTURE, SATURATED POOL $\$$ (ALSO USED FOR POS 5 OPEN CNMT CASES)

\begin{tabular}{|c|c|c|c|c|c|c|c|c|c|}
\hline FCONCL $(1,1,4)$ & 1. & .038 & 38 & .0 & & .013 & .013 & 13 & - \\
\hline FCONCL $(1,2,4)$ & 1 . & .148 & .148 & .042 & .042 & .042 & .042 & .042 & .042 \\
\hline $\operatorname{CONCL}(1,3,4)$ & 1. & 218 & 218 & 153 & 153 & 153 & 153 & 153 & . \\
\hline$L(1,4,4)$ & 1 & .491 & .491 & 435 & 35 & 35 & 35 & 35 & 435 \\
\hline $\operatorname{CONCL}(1,5,4)$ & 1. & .719 & .719 & .675 & 675 & 675 & 675 & 675 & 675 \\
\hline $\operatorname{CONCL}(1,6,4)$ & 1. & .859 & .859 & .828 & .828 & .828 & .828 & 28 & 828 \\
\hline$L(1,7,4)$ & 1. & .940 & .940 & .936 & .936 & .936 & .936 & 936 & 936 \\
\hline $\operatorname{CONCL}(1,8,4)$ & 1. & .974 & .974 & .974 & .974 & .974 & .974 & .974 & .974 \\
\hline $\operatorname{CONCL}(1,9,4)$ & 1. & .994 & .994 & .994 & .994 & & & & 39 \\
\hline FCONC GG & CASE & 5: LA & CE IJEA & & & & & & \\
\hline $\operatorname{CONCL}(1,1,5)$ & 1. & 0 . & 0 & 0 . & 0. & . & r. & $\cdot$ & • \\
\hline CONCL $(1,2,5)$ & 1. & 0 . & 0 . & .001 & .001 & 01 & 001 & 001 & .001 \\
\hline $\operatorname{CONCL}(1,3,5)$ & 1. & .001 & .001 & .002 & .002 & .002 & .002 & 02 & 002 \\
\hline $\operatorname{CONCL}(1,4,5)$ & 1. & .008 & .008 & .023 & .014 & 23 & .014 & 4 & 014 \\
\hline $\operatorname{CONCL}(1,5,5)$ & 1. & .052 & .052 & .082 & .063 & 082 & .063 & 072 & .072 \\
\hline $\operatorname{CONCL}(1,6,5)$ & 1. & .128 & .128 & .183 & .149 & .183 & .149 & 64 & .164 \\
\hline $\operatorname{CONCL}(1,7,5)$ & 1. & .330 & .330 & .423 & .392 & & 32 & 4 & 404 \\
\hline $\operatorname{CONCL}(1,8,5)$ & 1. & .510 & .510 & .595 & .510 & .595 & .510 & 510 & .510 \\
\hline $\operatorname{CONCL}(1,9,5)$ & 1. & .814 & .814 & .820 & 010 & & 1 & 4 & .814 \\
\hline FCONC GG & CASE & 6: LA & CE RUP & IURE & & & & & \\
\hline $\operatorname{CONCL}(1,1,6)$ & 1 & 0 . & 0 & 0 . & 0 . & 0 & 0 . & 0 . & 0. \\
\hline $\operatorname{CONCL}(1,2,6)$ & 1. & 0 . & 0 . & .001 & & & & & .001 \\
\hline $\operatorname{CONCL}(1,3,6)$ & 1. & .002 & .002 & .003 & .003 & .003 & .003 & .003 & .003 \\
\hline $\operatorname{CONCL}(1,4,6)$ & 1 . & .017 & .017 & .037 & .020 & .037 & .020 & .020 & .020 \\
\hline $\operatorname{CONCL}(1,5,6)$ & 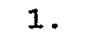 & .084 & .084 & .107 & .094 & & .094 & .094 & .094 \\
\hline $\operatorname{CONCL}(1,6,6)$ & & .186 & .186 & .256 & .226 & .256 & .226 & .226 & .226 \\
\hline $\operatorname{CONCL}(1,7,6)$ & 1. & .338 & .338 & .775 & .771 & .775 & .771 & .771 & .771 \\
\hline $\operatorname{ONCL}(1,8,6)$ & 1 . & .540 & .540 & .920 & 920 & .920 & .920 & .920 & .920 \\
\hline $\operatorname{CONCL}(1,9,6)$ & 1 & .969 & 969 & 73 & 973 & .973 & .973 & .973 & .973 \\
\hline
\end{tabular}
$\$$ FCONCL: NO CONTAINMENT FAILURE CASE

FCONCL $(1,1,7) \quad 0.005$ 1.0E-6 $1.0 \mathrm{E}-6$ 1.0E- 6 1.0E-6 $1.0 \mathrm{E}-6 \quad 1.0 \mathrm{E}-6 \quad 1.0 \mathrm{E}-6 \quad 1.0 \mathrm{E}-6$ FCONCL $(1,2,7) \quad 0.0051 .0 \mathrm{E}-6 \quad 1.0 \mathrm{E}-6 \quad 1.0 \mathrm{E}-6 \quad 1.0 \mathrm{E}-6 \quad 1.0 \mathrm{E}-6 \quad 1.0 \mathrm{E}-6 \quad 1.0 \mathrm{E}-6 \quad 1.0 \mathrm{E}-6$ FCONCL $(1,3,7) \quad 0.0051 .0 \mathrm{E}-6 \quad 1.0 \mathrm{E}-6$ 1.0E-6 $1.0 \mathrm{E}-6 \quad 1.0 \mathrm{E}-6 \quad 1.0 \mathrm{E}-6 \quad 1.0 \mathrm{E}-6 \quad 1.0 \mathrm{E}-6$ FCONCL $(1,4,7) \quad 0.005$ 1.0E-6 $1.0 \mathrm{E}-6 \quad 1.0 \mathrm{E}-6 \quad 1.0 \mathrm{E}-6 \quad 1.0 \mathrm{E}-6 \quad 1.0 \mathrm{E}-6 \quad 1.0 \mathrm{E}-6 \quad 1.0 \mathrm{E}-6$ FCONCL $(1,5,7) \quad 0.005 \quad 1.0 \mathrm{E}-6 \quad 1.0 \mathrm{E}-6 \quad 1.0 \mathrm{E}-6 \quad 1.0 \mathrm{E}-6 \quad 1.0 \mathrm{E}-6 \quad 1.0 \mathrm{E}-6 \quad 1.0 \mathrm{E}-6 \quad 1.0 \mathrm{E}-6$ FCONCL $(1,6,7) \quad 0.0051 .0 \mathrm{E}-6 \quad 1.0 \mathrm{E}-6 \quad 1.0 \mathrm{E}-6 \quad 1.0 \mathrm{E}-6 \quad 1.0 \mathrm{E}-6 \quad 1.0 \mathrm{E}-6 \quad 1.0 \mathrm{E}-6 \quad 1.0 \mathrm{E}-6$ 
FCONCL $(1,7,7) \quad 0.005$ 1.0E-6 $1.0 \mathrm{E}-6 \quad 1.0 \mathrm{E}-6 \quad 1.0 \mathrm{E}-6 \quad 1.0 \mathrm{E}-6 \quad 1.0 \mathrm{E}-6 \quad 1.0 \mathrm{E}-6 \quad 1.0 \mathrm{E}-6$ FCONCL $(1,8,7) \quad 0.0051 .0 \mathrm{E}-6 \quad 1.0 \mathrm{E}-6 \quad 1.0 \mathrm{E}-6 \quad 1.0 \mathrm{E}-6 \quad 1.0 \mathrm{E}-6 \quad 1.0 \mathrm{E}-6 \quad 1.0 \mathrm{E}-6 \quad 1.0 \mathrm{E}-6$ FCONCL $(1,9,7) \quad 0.005$ 1.0E-6 1.0E-6 1.0E-6 1.0E-6 1.0E-6 1.0E-6 1.0E-6 $1.0 \mathrm{E}-6$

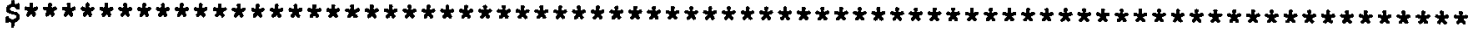
\$ DFVPA: SUPPRESSION POOL DF DURING IN-VESSEL RELEASE PHASE $\$$ (THROUGH T-QUENCHER) $\$$ DFVRA GG CASE 1: DRAFT NUREG/CR-4551

$\begin{array}{llllllllll}\text { DFVPAL }(1,1,1) & 1.0 & 1.0 & 1.0 & 1.0 & 1.0 & 1.0 & 1.0 & 1.0 & 1.0 \\ \text { DFVPAL }(1,2,1) & 1.0 & 1.1 & 1.1 & 1.1 & 1.1 & 1.1 & 1.1 & 1.1 & 1.1 \\ \text { DFVPAL }(1,3,1) & 1.0 & 1.8 & 1.8 & 1.8 & 1.8 & 1.8 & 1.8 & 1.8 & 1.8 \\ \text { DFVPAL }(1,4,1) & 1.0 & 16 . & 16 . & 16 . & 16 . & 16 . & 16 . & 16 . & 16 . \\ \text { DFVPAL }(1,5,1) & 1.0 & 56 . & 56 . & 56 . & 56 . & 56 . & 56 . & 56 . & 56 . \\ \text { DFVPAL }(1,6,1) & 1.0 & 180 . & 180 . & 180 . & 180 . & 180 . & 180 . & 180 . & 180 . \\ \text { DFVPAL }(1,7,1) & 1.0 & 2500 . & 2500 . & 2500 . & 2500 . & 2500 . & 2500 . & 2500 . & 2500 . \\ \text { DFVPAL }(1,8,1) & 1.0 & 4300 . & 4300 . & 4300 . & 4300 . & 4300 . & 4300 . & 4300 . & 4300 . \\ \text { DFVPAL }(1,9,1) & 1.0 & 5000 . & 5000 . & 5000 . & 5000 . & 5000 . & 5000 . & 5000 . & 5000 .\end{array}$

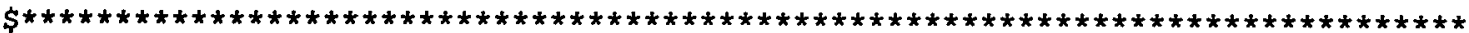
$\$$ DFCPA: SUPPRESSION POOL DF THRU VENT PIPES

$\$$ DFCPA GG CASE 1: DRAFT NUREG/CR-455I

$\begin{array}{llllllllll}\operatorname{DFCPAI}(1,1,1) & 1.0 & 1.0 & 1.0 & 1.0 & 1.0 & 1.0 & 1.0 & 1.0 & 1.0 \\ \operatorname{DFCPAI}(1,2,1) & 1.0 & 1.0 & 1.0 & 1.0 & 1.0 & 1.0 & 1.0 & 1.0 & 1.0 \\ \operatorname{DFCPAI}(1,3,1) & 1.0 & 1.2 & 1.2 & 1.2 & 1.2 & 1.2 & 1.2 & 1.2 & 1.2 \\ \operatorname{DFCPAI}(1,4,1) & 1.0 & 2.6 & 2.6 & 2.6 & 2.6 & 2.6 & 2.6 & 2.6 & 2.6 \\ \operatorname{DFCPAL}(1,5,1) & 1.0 & 6.8 & 6.8 & 6.8 & 6.8 & 6.8 & 6.8 & 6.8 & 6.8 \\ \operatorname{DFCPAI}(1,6,1) & 1.0 & 20 . & 20 . & 20 . & 20 . & 20 . & 20 . & 20 . & 20 . \\ \operatorname{DFCPAI}(1,7,1) & 1.0 & 72 . & 72 . & 72 . & 72 . & 72 . & 72 . & 72 . & 72 . \\ \operatorname{DFCPAL}(1,8,1) & 1.0 & 94 . & 94 . & 94 . & 94 . & 94 . & 94 . & 94 . & 94 . \\ \operatorname{DFCPAL}(1,9,1) & 1.0 & 100 . & 100 . & 100 . & 100 . & 100 . & 100 . & 100 . & 100 .\end{array}$

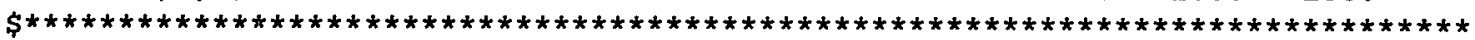
\$ DFCAV: CAVITY WATER DF FOR CCI RELEASE

$\$$ DFCAV GG CASE 1: WET CAVITY SIMILAR TO BMI-2139 GG TBS $\$$

\begin{tabular}{|c|c|c|c|c|c|c|c|c|c|}
\hline $\operatorname{JCAVL}(1,1,1)$ & 1.0 & 1.0 & 1.0 & 1.0 & 1.0 & 1.0 & 1.0 & 1.0 & 1.0 \\
\hline $\operatorname{DFCAVL}(1,2,1)$ & 1.0 & 1.0 & 1.0 & 1.0 & 1.0 & 1.0 & 1.0 & 1.0 & 1.0 \\
\hline $\operatorname{DECAVL}(1,3,1)$ & 1.0 & 1.1 & 1.1 & 1.1 & 1.1 & 1.1 & 1.1 & 1.1 & 1.1 \\
\hline $\operatorname{FCAVL}(1,4,1)$ & 1.0 & 2.0 & 2.0 & 2.0 & 2.0 & 2.0 & 2.0 & 2.0 & 2.0 \\
\hline $\operatorname{FCAVL}(1,5,1)$ & 1.0 & 4.4 & 4.4 & 4.4 & 4.4 & 4.4 & 4.4 & 4.4 & 4.4 \\
\hline $\operatorname{FCAVL}(1,6,1)$ & 1.0 & 11. & 11. & 11. & 11. & 11. & 11. & 11. & 11. \\
\hline $\operatorname{FCAVL}(1,7,1)$ & 1.0 & 41. & 41. & 41. & 41. & 41. & 41. & 41. & 41. \\
\hline $\operatorname{FCAVL}(1,8,1)$ & 1.0 & 65. & 65. & 65. & 65 & 65 & 65. & 65 . & 65. \\
\hline FCAVL $(1,9,1)$ & 1.0 & 73. & 73. & 73. & 73. & 73. & 73. & 73 & 73 \\
\hline DFCAV G & \& CAS & E 2: & FLOODED & CAVITY & SIMILAR & TO BMI & -2139 & GG TC & \\
\hline $\operatorname{FCAVu}(1,1,2)$ & 1.0 & 1.0 & 1.0 & 1.0 & 1.0 & 1.0 & 1.0 & 1.0 & 1.0 \\
\hline ECAVL $(1,2,2)$ & 1.0 & 1.0 & 1.0 & 1.0 & 1.0 & 1.0 & 1.0 & 1.0 & 1.0 \\
\hline $\operatorname{FAVI}(1,3,2)$ & 1.0 & 1.2 & 1.2 & 1.2 & 1.2 & 1.2 & 1.2 & 1.2 & 1.2 \\
\hline $\operatorname{CAVL}(1,4,2)$ & 1.0 & 2.8 & 2.8 & 2.8 & 2.8 & 2.8 & 2.8 & 2.8 & 2.8 \\
\hline $\operatorname{ECAVL}(1,5,2)$ & 1.0 & 6.0 & 6.0 & 6.0 & 6.0 & 6.0 & 6.0 & 6.0 & 6.0 \\
\hline FCAVL $(1,6,2)$ & 1.0 & 15 . & 15 . & 15 . & 15 . & 15 . & 15 . & 15 . & 15. \\
\hline $\operatorname{FCAVL}(1,7,2)$ & 1.0 & 56. & 56. & 56. & 56. & 56. & 56. & 56. & 56. \\
\hline $\operatorname{GAVI}(1,8,2)$ & 1.0 & 89. & 89. & 89. & 89. & 89. & 89. & 89. & 89 \\
\hline $\operatorname{FCAVL}(1,9,2)$ & 1.0 & 100 & 100 . & 100 . & 100 . & 100 & 100. & 100 & 100 \\
\hline 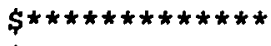 & $* * * * *$ & $\star * * * * *$ & 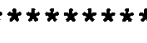 & 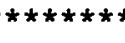 & 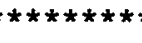 & & + & du & \\
\hline DFSPRV: SP & 2AY DI & FOR & IN-VES & RE & is & & & & \\
\hline DFSPRV & \pm G CAS & SE 1 & AFT & $G / C$ & 51 & Y) & & & \\
\hline $\operatorname{SPRVL}(1,1,1)$ & 1.0 & 1.0 & 1.0 & 1.0 & 1.0 & 1.0 & 1.0 & 1.0 & 1.0 \\
\hline $\operatorname{SPRVL}(1,2,1)$ & 1.0 & 1.1 & 1.1 & 1.1 & 1.1 & 1.1 & 1.1 & 1.1 & 1.1 \\
\hline $\operatorname{SPRVL}(1,3,1)$ & 1.0 & 1.3 & 1.3 & 1.3 & 1.3 & 1.3 & 1.3 & 1.3 & 1.3 \\
\hline $\operatorname{FSPRVL}(1,4,1)$ & 1.0 & 4.2 & 4.2 & 4.2 & 4.2 & 4.2 & 4.2 & 4.2 & 4. \\
\hline
\end{tabular}


Appendix C

\begin{tabular}{|c|c|c|c|c|c|c|c|c|c|}
\hline $\operatorname{DFSPRVL}(1,5,1)$ & 1.0 & 11. & 11. & 11. & 11. & 11. & 11. & 11. & 11. \\
\hline $\operatorname{DFSPRVL}(1,6,1)$ & 1.0 & 29. & 29. & 29. & 29. & 29. & 29. & 29 . & 29. \\
\hline $\operatorname{DFSPRVL}(1,7,1)$ & 1.0 & 78 . & 78 . & 78 . & 78 . & 78 . & 78 . & 78 . & 78 . \\
\hline $\operatorname{DFSPRVL}(1,8,1)$ & 1.0 & 95. & 95. & 95. & 95. & 95. & 95. & 95. & 95. \\
\hline $\operatorname{DFSPRVL}(1,9,1)$ & 1.0 & 100 & 100 . & 100 . & 100 & 100. & 100. & 100 . & 100 \\
\hline \multicolumn{10}{|c|}{$\$$} \\
\hline \multicolumn{10}{|c|}{ \$ DFSPRC: SPRAY DF FOR CCI RELEASES } \\
\hline \multicolumn{10}{|c|}{$\$ \quad$ DFSPRC GG CASE 1 DRAFT NUREG/CR-4551 (SURRY) } \\
\hline DFSPRCL $(1,1,1)$ & 1.0 & 1.0 & 1.0 & 1.0 & 1.0 & 1.0 & 1.0 & 1.0 & 1.0 \\
\hline DFSPRCL $(1,2,1)$ & 1.0 & 1.1 & 1.1 & 1.1 & 1.1 & 1.1 & 1.1 & 1.1 & 1.1 \\
\hline $\operatorname{DFSPRCL}(1,3,1)$ & 1.0 & 1.5 & 1.5 & 1.5 & 1.5 & 1.5 & 1.5 & 1.5 & 1.5 \\
\hline DFSPRCL $(1,4,1)$ & 1.0 & 7.8 & 7.8 & 7.8 & 7.8 & 7.8 & 7.8 & 7.8 & 7.8 \\
\hline DFSPRCL $(1,5,1)$ & 1.0 & 17 . & 17 . & 17. & 17. & 17 . & 17 . & 17. & 17. \\
\hline $\operatorname{DFSPRCL}(1,6,1)$ & 1.0 & 29 . & 29 . & 29. & 29. & 29. & 29. & 29. & 29. \\
\hline $\operatorname{DFSPRCL}(1,7,1)$ & 1.0 & 480 & 480 & 480 & 480 & 480 & 480 & 480 & 480 \\
\hline DFSPRCL $(1,8,1)$ & 1.0 & 860 . & 860 . & 860 & 860 & 860 . & 860. & 860 . & 860 . \\
\hline $\operatorname{DFSPRCL}(1,9,1)$ & 1.0 & 1000 . & 1000. & 1000 . & 1000. & 1000. & 1000 . & 1000 & 1000 . \\
\hline
\end{tabular}




\section{C.3 Partitioned Source Terms}

The source term groups were defined using the PARTITION algorithm that was first developed in the NUREG-1150 program and then modified for the LaSalle probabilistic risk assessment (PRA) that was performed in the PRUEP program. The modified version of PARTITION was used in this study. A $5 \times 5$ early health effect versus chronic health effect partition grid was used in this analysis. Radionuclide inventories specific to these shutdown accidents (i.e., presented in Appendix D) were provided as input to the PARTITION code. The partitioning process generated 55 source term groups (STGs). The source terms associated with each STG are provided in Table C.3-1. A 55 ${ }^{\text {th }}$ STG is the special case where there is no release. The source terms, as listed in Table C.3-1, provide the following information about the release:

$$
\mathrm{ST}=\left[S T G, \text { Freq, TW, T1, D1, EL, EVT, E1 }\left(R F 1_{i} \mathrm{i}=1, \ldots 9\right), \mathrm{T} 2, \mathrm{D} 2, \mathrm{E2},\left(\mathrm{RF} 2_{\mathrm{i}} \mathrm{i}=1, \ldots 9\right)\right] \text {, }
$$

where

$$
\begin{aligned}
& \text { STG }=\text { source term group } \\
& \text { Freq }=\text { frequency }(1 / \mathrm{yr}) \text { of source term group } \\
& \text { TW = the time (seconds), relative to the start of the accident, when a general emergency is declared, } \\
& \mathrm{T} 1 \text { = the time (seconds), relative to the start of the accident, when the first release segment begins, } \\
& \text { D1 = the duration (seconds) of the first release segment, } \\
& \mathrm{EL} \quad=\text { the elevation (meters), relative to ground level, from which the radionuclides are released from the containment, } \\
& \text { EVT = the event type which identifies the time window in which the accident occurs and is used to select the appropriate }
\end{aligned}
$$


Table C.3-1

Source Terms for Partitioned Source Term Groups

RGY RFI RF

TBRM (/YR) (S) (S)

(W) $\quad \mathrm{XE} / \mathrm{KR} \quad \mathrm{I}$

CS

TE SR

RF6
RU

RF7
LA

RF8

RF9

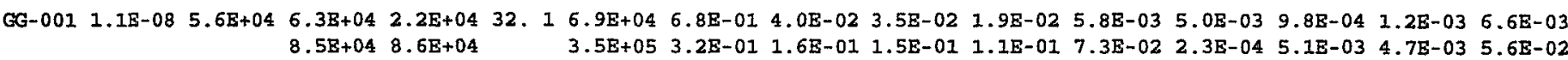

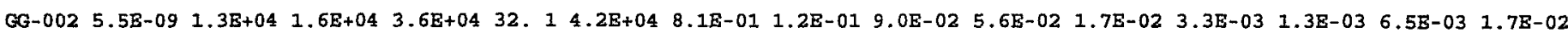

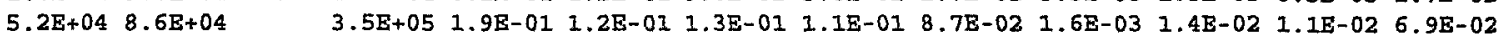

$\begin{array}{lllllllllllllllll}G \mathrm{G}-003 & 6.5 \mathrm{E}-09 & 1.3 \mathrm{E}+04 & 1.6 \mathrm{E}+04 & 3.6 \mathrm{E}+04 & 32 . & 1 & 4.2 \mathrm{E}+04 & 4.6 \mathrm{E}-01 & 4.3 \mathrm{E}-02 & 2.7 \mathrm{E}-02 & 1.2 \mathrm{E}-02 & 1.7 \mathrm{E}-03 & 3.7 \mathrm{E}-04 & 7.1 \mathrm{E}-05 & 2.6 \mathrm{E}-04 & 2.0 \mathrm{E}-03\end{array}$

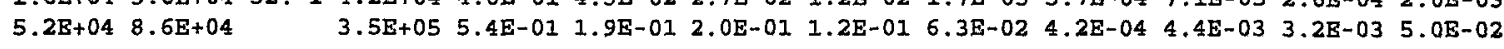

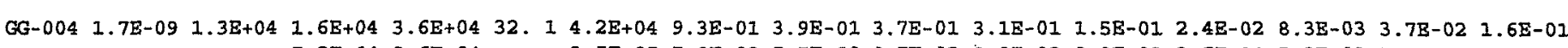

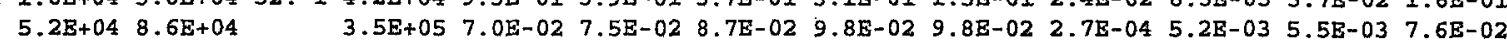

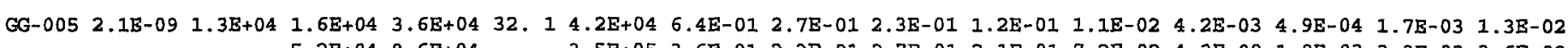

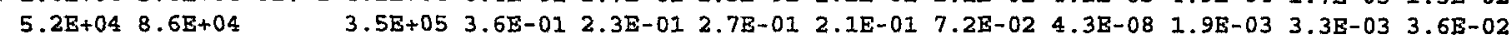

$\begin{array}{lllllllllllllllllllllll}G \mathrm{G}-006 & 8.7 \mathrm{E}-09 & 5.6 \mathrm{E}+03 & 1.3 \mathrm{E}+04 & 7.2 \mathrm{E}+04 & 32 . & 1 & 6.9 \mathrm{E}+04 & 6.5 \mathrm{E}-01 & 4.2 \mathrm{E}-02 & 3.1 \mathrm{E}-02 & 1.3 \mathrm{E}-02 & 4.2 \mathrm{E}-03 & 2.8 \mathrm{E}-03 & 5.8 \mathrm{E}-04 & 9.7 \mathrm{E}-04 & 4.6 \mathrm{E}-03\end{array}$

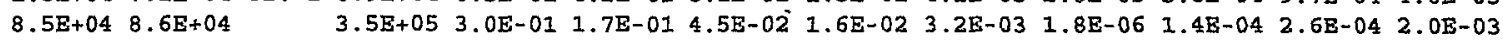

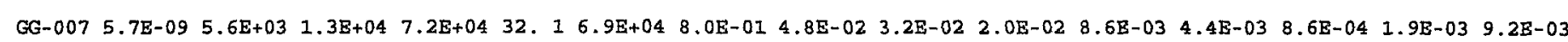

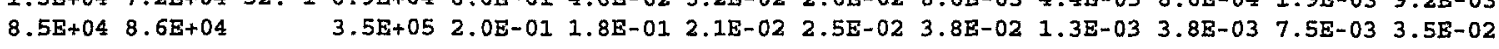

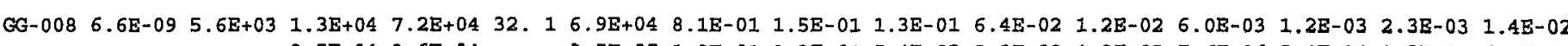

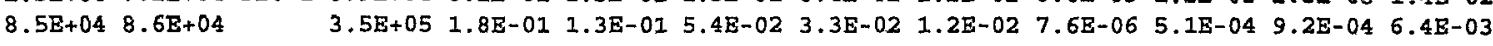

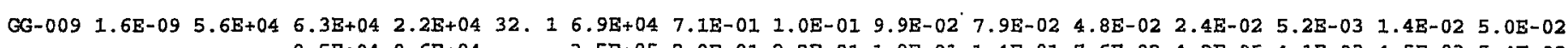

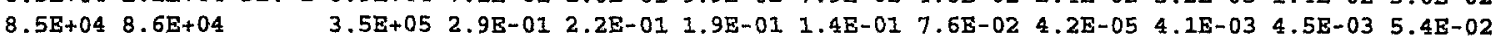

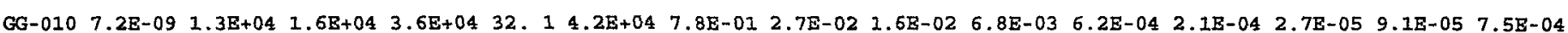

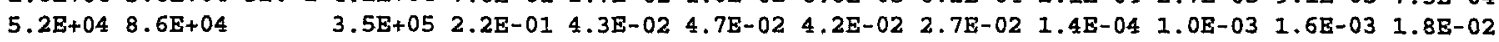

$\begin{array}{llllllllllllllllllll}G G-011 & 2.4 \mathrm{E}-09 & 1.3 \mathrm{~B}+04 & 1.6 \mathrm{E}+04 & 3.6 \mathrm{E}+04 & 32 . & 1 & 4.2 \mathrm{E}+04 & 9.9 \mathrm{E}-01 & 8.1 \mathrm{E}-02 & 5.6 \mathrm{E}-02 & 3.8 \mathrm{E}-02 & 1.6 \mathrm{E}-02 & 2.8 \mathrm{E}-03 & 1.5 \mathrm{E}-03 & 7.6 \mathrm{E}-03 & 1.6 \mathrm{E}-02\end{array}$

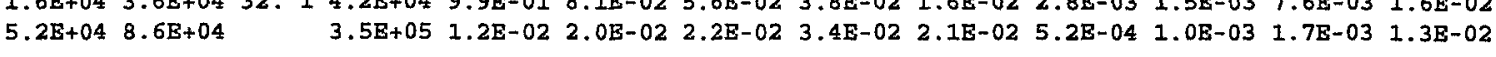

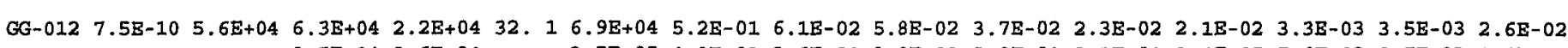

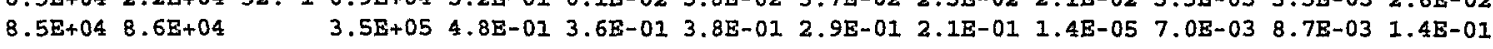

GG-013 $7.6 \mathrm{E}-10 \quad 5.6 \mathrm{E}+03 \quad 1.3 \mathrm{E}+04 \quad 7.2 \mathrm{E}+04 \quad 32.1 \quad 6.9 \mathrm{E}+04 \quad 8.0 \mathrm{E}-01 \quad 1.7 \mathrm{E}-01 \quad 1.6 \mathrm{E}-01 \quad 1.3 \mathrm{E}-01 \quad 7.8 \mathrm{E}-02 \quad 1.9 \mathrm{E}-02 \quad 6.5 \mathrm{E}-03 \quad 2.5 \mathrm{E}-02 \quad 7.9 \mathrm{E}-02$

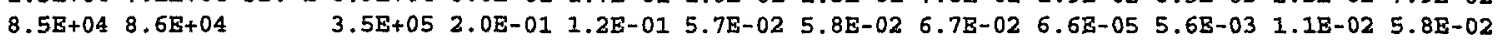

$\begin{array}{llllllllllllllllll}G G-014 & 4.3 \mathrm{E}-10 & 5.6 \mathrm{E}+03 & 1.3 \mathrm{E}+04 & 7.2 \mathrm{E}+04 & 32 . & 1 & 6.9 \mathrm{E}+04 & 1.0 \mathrm{E}+00 & 2.6 \mathrm{E}-01 & 2.5 \mathrm{~B}-01 & 2.4 \mathrm{E}-01 & 2.2 \mathrm{E}-01 & 3.2 \mathrm{E}-02 & 2.1 \mathrm{E}-02 & 1.1 \mathrm{E}-01 & 2.2 \mathrm{E}-01\end{array}$

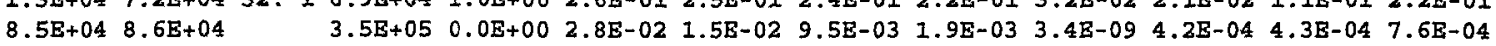

$\begin{array}{llllllllllllllllllllll}6 \mathrm{G}-015 & 4.5 \mathrm{E}-09 & 5.6 \mathrm{~B}+04 & 6.3 \mathrm{E}+04 & 2.2 \mathrm{~B}+04 & 32 . & 1 & 6.9 \mathrm{E}+04 & 9.1 \mathrm{E}-01 & 1.8 \mathrm{E}-02 & 1.6 \mathrm{E}-02 & 1.1 \mathrm{E}-02 & 4.0 \mathrm{E}-03 & 2.2 \mathrm{~B}-03 & 4.4 \mathrm{E}-04 & 7.2 \mathrm{E}-04 & 4.4 \mathrm{~B}-03\end{array}$

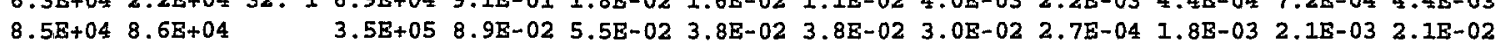

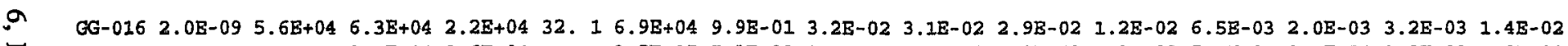




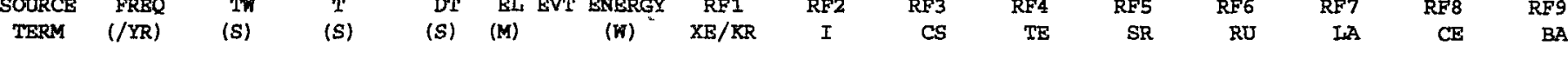

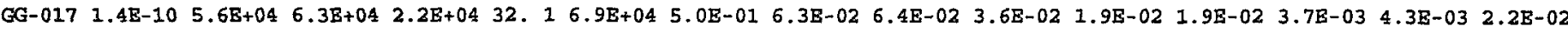

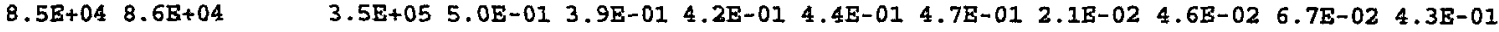

$\begin{array}{lllllllllllllllll}\text { GG-018 } & 1.3 \mathrm{E}-08 & 1.4 \mathrm{E}+04 & 2.0 \mathrm{E}+04 & 5.2 \mathrm{E}+04 & 32 . & 1 & 7.7 \mathrm{E}+04 & 8.0 \mathrm{E}-01 & 1.7 \mathrm{E}-02 & 1.2 \mathrm{E}-02 & 7.2 \mathrm{E}-03 & 2.2 \mathrm{E}-03 & 1.6 \mathrm{E}-03 & 3.5 \mathrm{E}-04 & 5.5 \mathrm{E}-04 & 2.5 \mathrm{~B}-03\end{array}$

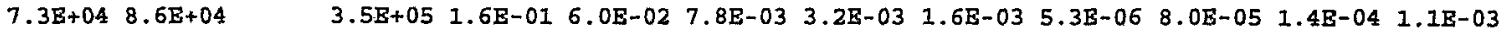

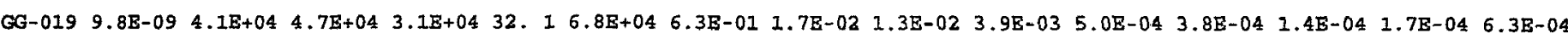

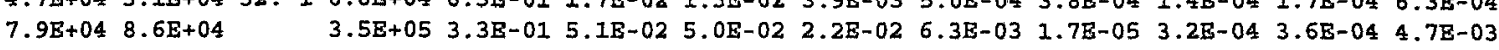

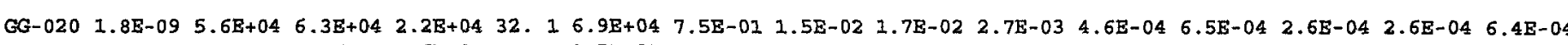

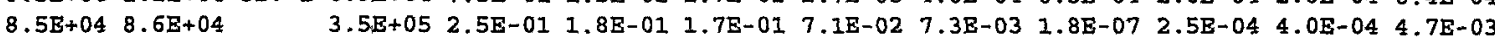

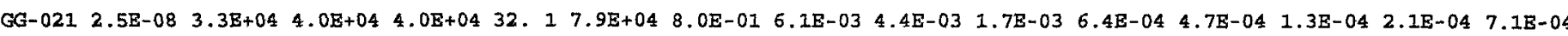

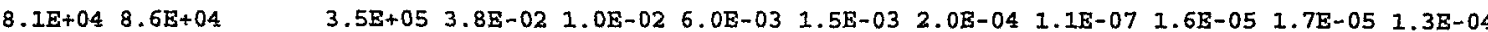

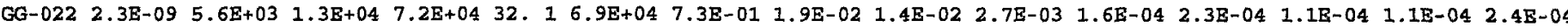

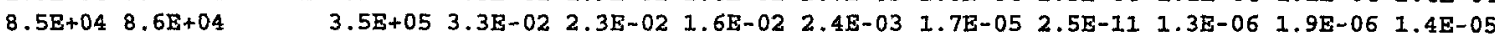

$\begin{array}{lllllllllllllllllll}G G-023 & 2.6 \mathrm{~B}-09 & 5.6 \mathrm{E}+04 & 6.3 \mathrm{E}+04 & 2.2 \mathrm{E}+04 & 32 . & 1 & 6.9 \mathrm{E}+04 & 1.0 \mathrm{E}+00 & 3.0 \mathrm{E}-04 & 1.7 \mathrm{E}-04 & 6.3 \mathrm{E}-05 & 4.8 \mathrm{E}-05 & 2.3 \mathrm{E}-05 & 9.0 \mathrm{E}-06 & 2.2 \mathrm{E}-05 & 5.0 \mathrm{E}-05\end{array}$

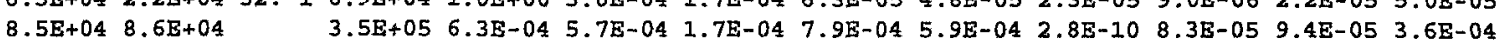

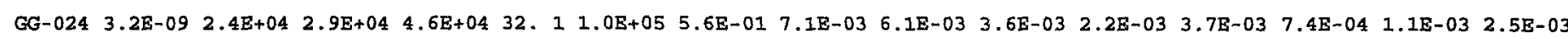

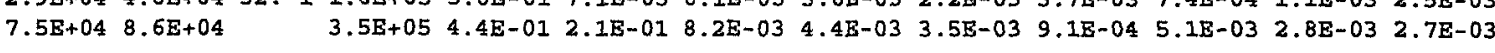

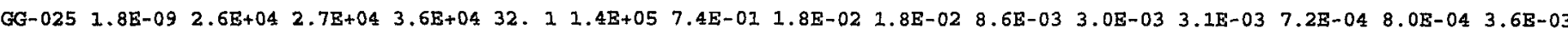

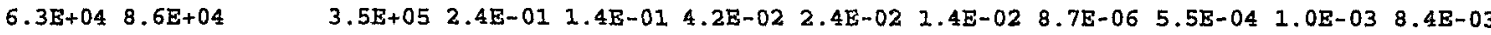

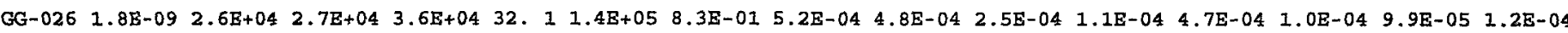

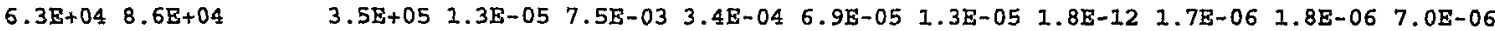

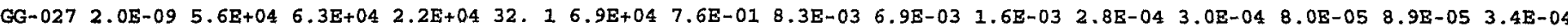

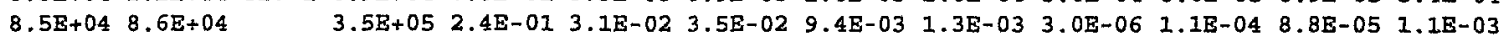

$\begin{array}{llllllllllllllllll}\mathrm{GG}-028 & 1.4 \mathrm{E}-07 & 2.0 \mathrm{E}+04 & 2.3 \mathrm{E}+04 & 2.2 \mathrm{E}+04 & 32 . & 2 & 6.9 \mathrm{E}+04 & 9.9 \mathrm{E}-01 & 2.1 \mathrm{E}-01 & 1.9 \mathrm{E}-01 & 1.6 \mathrm{~B}-01 & 8.5 \mathrm{E}-02 & 1.3 \mathrm{~B}-02 & 5.6 \mathrm{E}-03 & 2.6 \mathrm{E}-02 & 8.6 \mathrm{E}-02\end{array}$

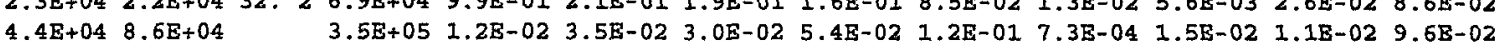

$\begin{array}{llllllllllllllllllll}G \mathrm{G}-029 & 2.6 \mathrm{E}-07 & 2.0 \mathrm{E}+04 & 2.3 \mathrm{E}+04 & 2.2 \mathrm{E}+04 & 32.2 & 6.9 \mathrm{E}+04 & 4.8 \mathrm{E}-02 & 6.2 \mathrm{E}-02 & 4.5 \mathrm{E}-02 & 2.4 \mathrm{E}-02 & 4.4 \mathrm{E}-03 & 1.1 \mathrm{E}-03 & 2.1 \mathrm{E}-04 & 8.5 \mathrm{E}-04 & 4.7 \mathrm{E}-03\end{array}$

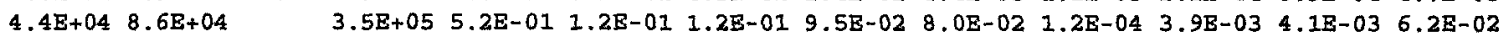

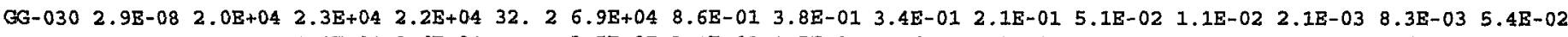

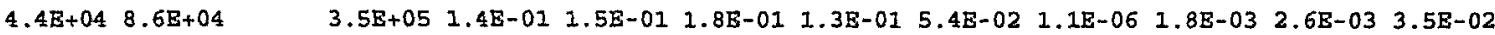

$\begin{array}{llllllllllllllll}G \mathrm{G}-031 & 8.4 \mathrm{E}-08 & 6.6 \mathrm{E}+03 & 1.8 \mathrm{E}+04 & 7.2 \mathrm{E}+04 & 32.2 & 6.9 \mathrm{E}+04 & 7.1 \mathrm{E}-01 & 4.3 \mathrm{E}-02 & 3.1 \mathrm{E}-02 & 1.6 \mathrm{E}-02 & 5.6 \mathrm{E}-03 & 2.9 \mathrm{E}-03 & 6.4 \mathrm{E}-04 & 1.4 \mathrm{E}-03 & 6.1 \mathrm{E}-03\end{array}$

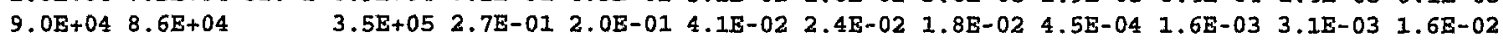

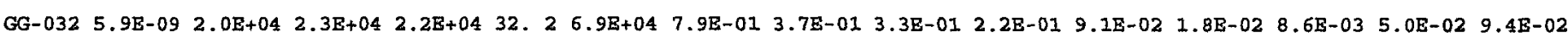

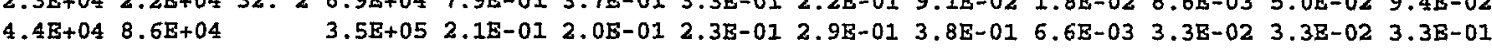


Table C.3-1 (continued)

GG POS5 PARTITION DEFINITIONS
FREO TW T T

SOURCB

$\begin{array}{cc}\text { TW } & T \\ \text { (S) } & \text { (S) }\end{array}$

$\begin{array}{ll}\text { DT } & \text { EL } \\ \text { (S) } & \text { (M) }\end{array}$

(M)

$\begin{array}{ccc}\text { ENERGY } & \text { RF1 } & \text { RF2 } \\ \text { (W) } & X E / K R & I\end{array}$

RF3
CS

RF4
TE

RF5
SR

RF6
RU

RF7
LA

RF8
CE

RF9
BA

$\begin{array}{lllllllllllllllll}\text { GG-033 } & 4.0 \mathrm{E}-08 & 6.6 \mathrm{E}+03 & 1.8 \mathrm{E}+04 & 7.2 \mathrm{E}+04 & 32.2 & 6.9 \mathrm{E}+04 & 8.2 \mathrm{E}-01 & 1.7 \mathrm{E}-01 & 1.5 \mathrm{E}-01 & 7.7 \mathrm{E}-02 & 1.5 \mathrm{E}-02 & 7.0 \mathrm{E}-03 & 1.4 \mathrm{E}-03 & 2.8 \mathrm{E}-03 & 1.7 \mathrm{E}-02\end{array}$

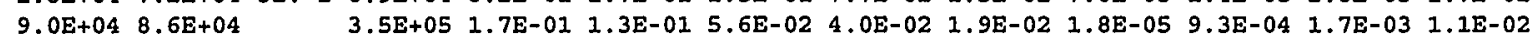

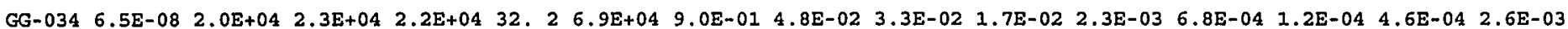

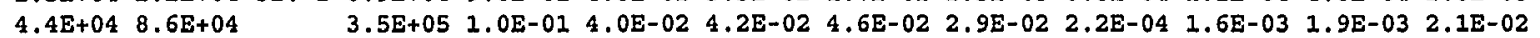

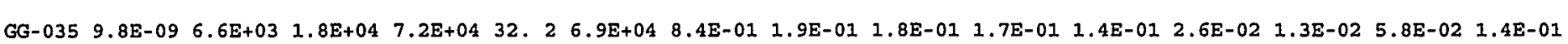

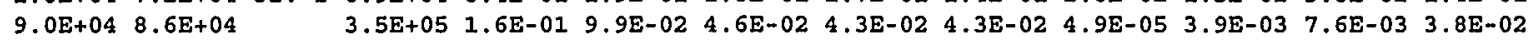

$\begin{array}{lllllllllllllllll}G G-036 & 2.1 E-08 & 2.0 E+04 & 2.3 E+04 & 2.2 E+04 & 32 . & 2 & 6.9 E+04 & 7.6 E-01 & 5.0 E-02 & 2.9 E-02 & 9.8 E-03 & 3.2 E-04 & 2.1 E-04 & 9.2 E-06 & 1.8 E-05 & 5.0 E-04\end{array}$

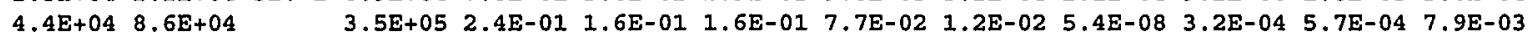

$\begin{array}{llllllllllllllllll}G G-037 & 8.1 E-08 & 2.0 E+04 & 2.3 E+04 & 2.2 E+04 & 32 . & 2 & 6.9 E+04 & 6.2 E-01 & 2.4 E-02 & 1.6 E-02 & 6.7 E-03 & 8.1 E-04 & 2.1 E-04 & 3.3 E-05 & 1.2 E-04 & 9.3 E-04\end{array}$

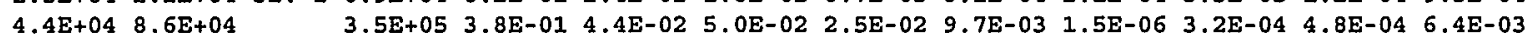

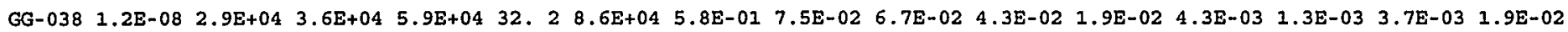
$\begin{array}{lllllllllllllll}1.1 \mathrm{E}+05 & 8.6 \mathrm{E}+04 & 3.4 \mathrm{E}+05 & 4.1 \mathrm{E}-01 & 2.4 \mathrm{E}-01 & 8.4 \mathrm{E}-02 & 7.0 \mathrm{E}-02 & 5.2 \mathrm{E}-02 & 2.9 \mathrm{E}-04 & 2.2 \mathrm{E}-03 & 4.2 \mathrm{E}-03 & 3.7 \mathrm{E}-02\end{array}$

$\begin{array}{lllllllllllllllll}G G-039 & 1.7 E-08 & 1.6 E+04 & 2.6 E+04 & 5.9 E+04 & 32 . & 2 & 1.1 E+06 & 8.8 E-01 & 5.9 E-02 & 4.3 E-02 & 3.8 E-02 & 2.8 E-02 & 9.4 E-03 & 2.9 E-03 & 9.2 E-03 & 2.9 E-02\end{array}$

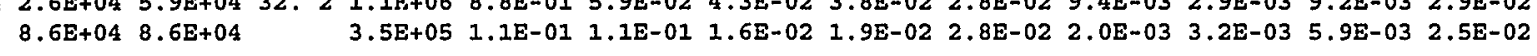

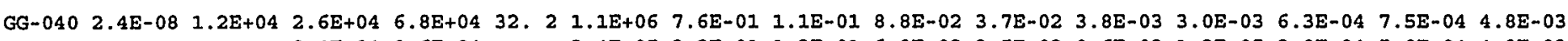

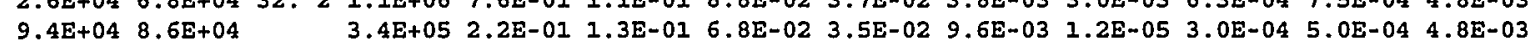

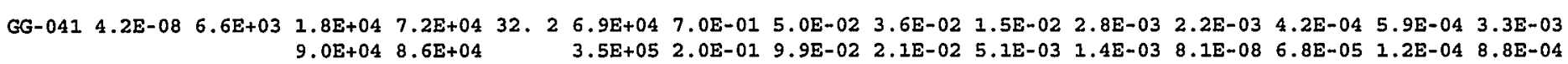

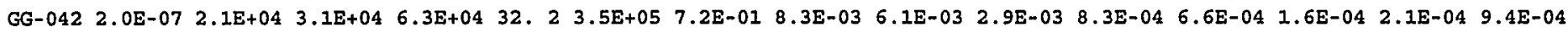

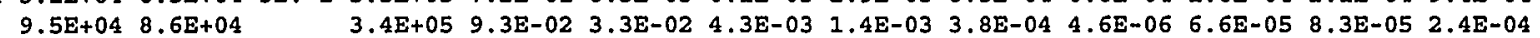

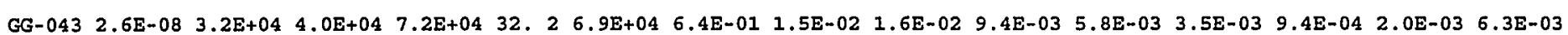

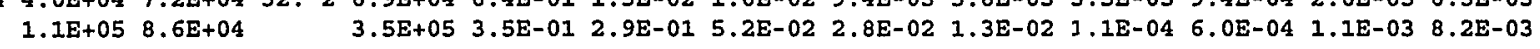

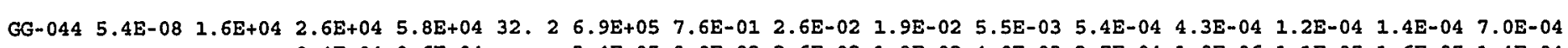

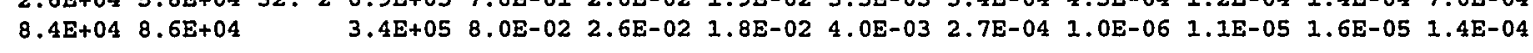

$\begin{array}{lllllllllllllllllllllll}G G-045 & 4.2 E-08 & 6.6 E+03 & 1.8 E+04 & 7.2 E+04 & 32 . & 2 & 6.9 E+04 & 6.2 E-01 & 1.7 E-02 & 1.1 E-02 & 6.9 E-03 & 2.7 E-03 & 2.2 E-03 & 4.0 E-04 & 5.1 E-04 & 3.0 E-03\end{array}$ $\begin{array}{llllllllllll}9.0 \mathrm{E}+04 & 8.6 \mathrm{E}+04 & 3.5 \mathrm{E}+05 & 3.3 \mathrm{E}-01 & 1.3 \mathrm{E}-01 & 8.8 \mathrm{E}-03 & 3.6 \mathrm{E}-03 & 1.6 \mathrm{E}-03 & 1.4 \mathrm{E}-05 & 1.1 \mathrm{E}-04 & 2.1 \mathrm{E}-04 & 1.2 \mathrm{E}-03\end{array}$

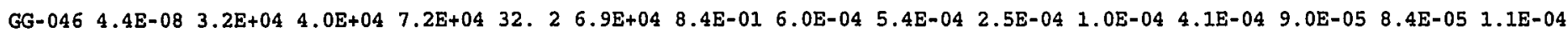

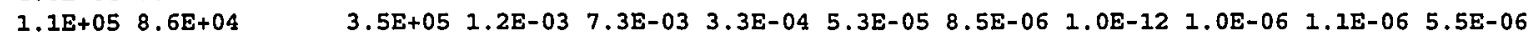

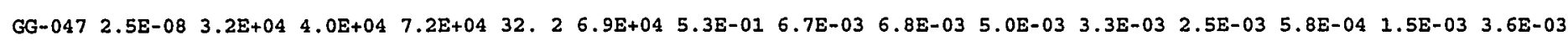

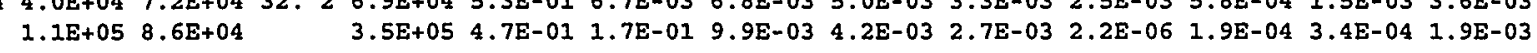

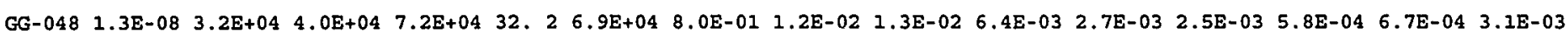

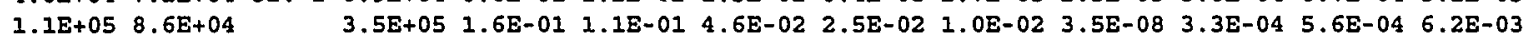


GG POS5 PARTITION DEFINITIONS

Table c.3-1 (continued)

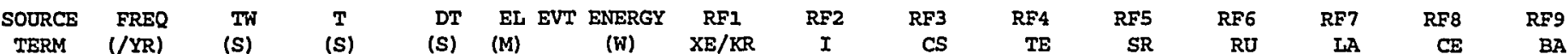

GG-049 4.9E-09 $8.1 \mathrm{E}+03 \quad 2.6 \mathrm{E}+04 \quad 7.2 \mathrm{E}+04 \quad 32,3 \quad 6.9 \mathrm{E}+04 \quad 8.1 \mathrm{E}-01 \quad 2.1 \mathrm{E}-01 \quad 2.0 \mathrm{E}-01 \quad 1.9 \mathrm{E}-01 \quad 1.8 \mathrm{E}-01 \quad 4.4 \mathrm{E}-02 \quad 2.0 \mathrm{E}-02 \quad 1.1 \mathrm{E}-01 \quad 1.8 \mathrm{E}-01$

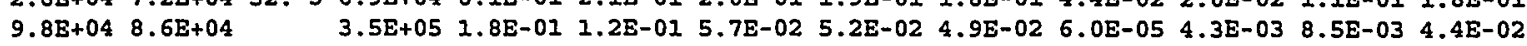

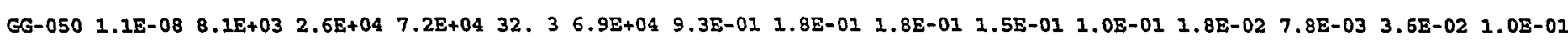

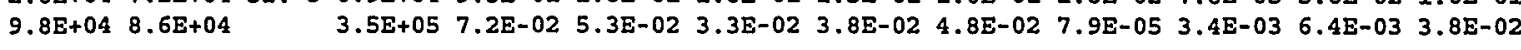

$\begin{array}{lllllllllllllllllllllll}G G-051 & 2.4 \mathrm{E}-09 & 8.2 \mathrm{E}+03 & 2.6 \mathrm{E}+04 & 7.2 \mathrm{E}+04 & 32 . & 3 & 6.9 \mathrm{E}+04 & 4.9 \mathrm{E}-01 & 9.1 \mathrm{E}-02 & 7.2 \mathrm{E}-02 & 4.7 \mathrm{E}-02 & 4.0 \mathrm{E}-02 & 3.7 \mathrm{E}-02 & 7.9 \mathrm{E}-03 & 1.1 \mathrm{E}-02 & 4.1 \mathrm{E}-02\end{array}$

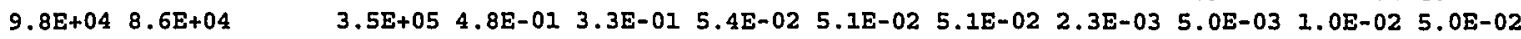

GG-052 3.8E-07 $8.1 \mathrm{E}+03 \quad 2.6 \mathrm{E}+04 \quad 7.2 \mathrm{E}+04 \quad 32.3 \quad 6.9 \mathrm{E}+04 \quad 7.5 \mathrm{E}-01 \quad 8.0 \mathrm{E}-02 \quad 6.2 \mathrm{E}-02 \quad 3.0 \mathrm{E}-02 \quad 7.0 \mathrm{E}-03 \quad 3.8 \mathrm{E}-03 \quad 8.2 \mathrm{E}-04 \quad 1.5 \mathrm{E}-03 \quad 7.8 \mathrm{E}-03$ $\begin{array}{llllllllllllll}9.8 \mathrm{E}+04 & 8.6 \mathrm{E}+04 & 3.5 \mathrm{E}+05 & 2.2 \mathrm{E}-01 & 1.3 \mathrm{E}-01 & 4.6 \mathrm{E}-02 & 2.1 \mathrm{E}-02 & 1.0 \mathrm{E}-02 & 1.4 \mathrm{E}-04 & 6.2 \mathrm{E}-04 & 1.2 \mathrm{E}-03 & 7.0 \mathrm{E}-03\end{array}$

$\begin{array}{lllllllllllllllllllll}G & 053 & 3.0 E-07 & 8.1 E+03 & 2.6 E+04 & 7.2 E+04 & 32 . & 3 & 6.9 E+04 & 6.7 E-01 & 1.7 E-02 & 1.2 E-02 & 4.9 E-03 & 1.2 E-03 & 8.7 E-04 & 1.9 E-04 & 2.7 E-04 & 1.4 E-03\end{array}$

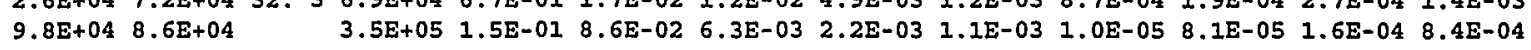

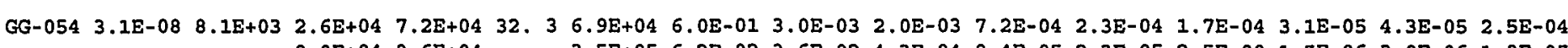

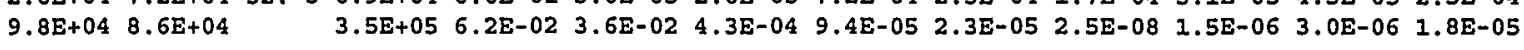

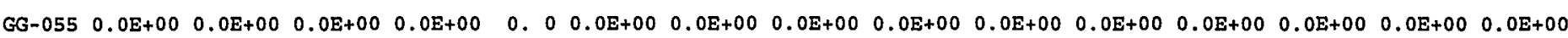
$\begin{array}{llllllllllllll}0.0 \mathrm{E}+00 & 0.0 \mathrm{E}+00 & 0.0 \mathrm{E}+00 & 0.0 \mathrm{E}+00 & 0.0 \mathrm{E}+00 & 0.0 \mathrm{E}+00 & 0.0 \mathrm{E}+00 & 0.0 \mathrm{E}+00 & 0.0 \mathrm{E}+00 & 0.0 \mathrm{E}+00 & 0.0 \mathrm{E}+00 & 0.0 \mathrm{E}+00\end{array}$ 
Appendix C 


\section{Appendix D Supporting Information for the Consequence Analysis}

Supporting information for the consequence analysis is provided in this appendix. Section D.1 lists the radionuclide inventories that were used in this study. Section D.2 provides a listing of the FORTRAN computer code that was used to estimate the onsite doses and dose rates. This code was written specifically for this study; the documentation of the code is limited to what is presented in this report. The onsite doses and dose rates for each source term group, calculated with the code listed in Section D.2, are tabulated in Section D.3. The mean offsite consequences calculated for each source term group using the MACCS code are tabulated in Section D.4.

\section{D.1 Radionuclide Inventories}

A unique radionuclide inventory was generated with ORIGEN2 for each time window. The inventory for Time Window 1 corresponds to 7 hours after shutdown; the inventory for Time Window 2 corresponds to 24 hours after shutdown; and the inventory for Time Window 3 corresponds to 40 days after shutdown. The inventories, in the form of a input file for MACCS, for the sixty radionuclides considered in the MACCS code for Time Windows 1, 2, and 3 are presented in Appendix subsections D.1.1, D.1.2, and D.1.3, respectively.

\section{D.1.1 Inventory for Time Window 1}

\begin{tabular}{|c|c|c|}
\hline $\begin{array}{l}\text { * GRAND GULF I } \\
\text { * Grand Gulf } \\
\text { * Inventories }\end{array}$ & $\begin{array}{l}\text { LOW POWER } \\
\text { Inventory } \\
3 \text { obtained }\end{array}$ & $\begin{array}{l}\text { AND SHUTDOWN PRA } \\
\text { for Time Window } 1 \text { ( } 7 \text { hrs after Shutdown) } \\
\text { from ORIGENPC; } \quad \text { L. A. Milier }(4 / 05 / 94)\end{array}$ \\
\hline $\begin{array}{l}\star \\
\star\end{array}$ & NUCNAM & CORINV (BECQUERALS) \\
\hline RDCORINVOOI & $\mathrm{co}-58$ & $1.207 \mathrm{E}+17$ \\
\hline RDCORINVOO2 & $\mathrm{CO}-60$ & $8.362 E+14$ \\
\hline RDCORINV003 & $\mathrm{KR}-85$ & $2.656 \mathrm{E}+16$ \\
\hline RDCORINV004 & $\mathrm{KR}-85 \mathrm{M}$ & $3.884 E+17$ \\
\hline RDCORINV005 & $K R-87$ & $4.952 \mathrm{E}+16$ \\
\hline RDCORINV006 & $\mathrm{KR}-88$ & $5.692 \mathrm{E}+17$ \\
\hline RDCORINV007 & $\mathrm{RB}-86$ & $3.235 E+15$ \\
\hline RDCORINVOOB & $S R-89$ & $4.106 \mathrm{E}+18$ \\
\hline RDCORINVOO9 & $S R-90$ & $2.082 \mathrm{E}+17$ \\
\hline RDCORINVO 10 & SR-91 & $3.123 E+18$ \\
\hline RDCORINV011 & SR-92 & $9.185 \mathrm{E}+17$ \\
\hline RDCORINV012 & $Y-90$ & $2.199 E+17$ \\
\hline RDCORINV013 & $Y-91$ & $5.491 E+18$ \\
\hline RDCORINVO14 & $y-92$ & $2.966 E+18$ \\
\hline RDCORINVO 15 & $y-93$ & $3.895 E+18$ \\
\hline RDCORINV016 & $\mathrm{ZR}-95$ & $7.042 \mathrm{E}+18$ \\
\hline RDCORINV017 & $\mathrm{ZR}-97$ & $5.053 \mathrm{E}+18$ \\
\hline RDCORINV018 & $\mathrm{NB}-95$ & $7.060 E+18$ \\
\hline RDCORINV019 & MO-99 & $6.408 E+18$ \\
\hline RDCORINV020 & TC-99M & $5.902 \mathrm{E}+18$ \\
\hline RDCORINVO21 & $R U-103$ & $5.109 \mathrm{E}+18$ \\
\hline RDCORINVO22 & RU-105 & $1.057 \mathrm{E}+18$ \\
\hline RDCORINVO23 & $R U-106$ & $1.080 E+18$ \\
\hline RDCORINV024 & RH-I05 & $2.761 E+18$ \\
\hline RDCORINV025 & SB-127 & $3.268 E+17$ \\
\hline RDCORINV026 & SB-129 & $3.602 \mathrm{E}+17$ \\
\hline RDCORINVO27 & TE- 127 & 3. $336 E+17$ \\
\hline RDCORINV028 & $\mathrm{TE}=127 \mathrm{M}$ & $4.399 \mathrm{E}+16$ \\
\hline RDCORINVO29 & $\mathrm{TE}-129$ & $5.278 \mathrm{E}+17$ \\
\hline RDCORINV030 & $T E-129 M$ & 1. $614 \mathrm{E}+17$ \\
\hline RDCORINV031 & $\mathrm{TE}-131 \mathrm{M}$ & 4. $375 E+17$ \\
\hline RDCORINV0 32 & $T E-132$ & $4.893 E+18$ \\
\hline RDCORINV0 33 & $I-131$ & $3.564 E+18$ \\
\hline RDCORINV034 & $I-132$ & $5.029 E+18$ \\
\hline RDCORINV0 35 & $I-133$ & $6.228 \mathrm{E}+18$ \\
\hline RDCORINV036 & $I=134$ & $1.213 E+17$ \\
\hline RDCORINV037 & $I-135$ & $3.437 \mathrm{E}+18$ \\
\hline
\end{tabular}




\section{Appendix D}

$\begin{array}{lll}\text { RDCORINV038 } & \text { XE-133 } & 7.652 E+18 \\ \text { RDCORINV039 } & \text { XE-135 } & 3.401 E+18 \\ \text { RDCORINV040 } & \text { CS-134 } & 1.831 E+17 \\ \text { RDCORINV041 } & \text { CS-136 } & 1.000 E+17 \\ \text { RDCORINV042 } & \text { CS-137 } & 2.544 E+17 \\ \text { RDCORINV043 } & \text { BA-139 } & 2.341 E+17 \\ \text { RDCORINV044 } & \text { BA-140 } & 6.630 E+18 \\ \text { RDCORINV045 } & \text { LA-140 } & 6.808 E+18 \\ \text { RDCORINV046 } & \text { LA-141 } & 2.026 E+18 \\ \text { RDCORINV047 } & \text { LA-142 } & 3.049 E+17 \\ \text { RDCORINV048 } & \text { CE-141 } & 6.388 E+18 \\ \text { RDCORINV049 } & \text { CE-143 } & 5.298 E+18 \\ \text { RDCORINV050 } & \text { CE-144 } & 4.425 E+18 \\ \text { RDCORINV051 } & \text { PR-143 } & 6.062 E+18 \\ \text { RDCORINV052 } & \text { ND-147 } & 2.472 E+18 \\ \text { RDCORINV053 } & \text { NP-239 } & 5.852 E+19 \\ \text { RDCORINV054 } & \text { PU-238 } & 1.428 E+15 \\ \text { RDCORINV055 } & \text { PU-239 } & 1.234 E+15 \\ \text { RDCORINV056 } & \text { PU-240 } & 1.032 E+15 \\ \text { RDCORINV057 } & \text { PU-241 } & 2.661 E+17 \\ \text { RDCORINV058 } & \text { AM-241 } & 1.584 E+14 \\ \text { RDCORINV059 } & \text { CM-242 } & 2.371 E+16 \\ \text { RDCORINV060 } & \text { CM-244 } & 1.764 E+14\end{array}$

\section{D.1.2 Inventory for Time Window 2}

$$
\text { * }
$$

- gRand GULF low power AND shutdown PRA

* Grand Gulf Inventory for Time Window 2 (24 hrs after shutdown)

* Inventories obtained from ORIGENPC; L. A. Miller (4/05/94)

- NUCNAM CORINV (BECQUERALs)

RDCORINVDO1 CO-58 $1.199 \mathrm{E}+17$

RDCORINV002 CO-60 $8.359 \mathrm{E}+14$

RDCORINV003 KR-85 $2.656 \mathrm{E}+16$

RDCORINV004 KR-85M $2.799 E+16$

RDCORINV005 KR-87 4.686E+12

RDCORINV006 KR-88 $8.966 E+15$

RDCORINV007 RB-86 $3.149 E+15$

RDCORINV008 SR-89 4.064E+18

RDCORINV009 SR-90 $2.082 \mathrm{E}+17$

RDCORINV010 SR-91 $9.034 \mathrm{E}+17$

RDCORINV011 SR-92 $1.188 \mathrm{E}+16$

RDCORINV012 Y-90 2.180E+17

RDCORINV013 Y-91 $5.461 E+18$

RDCORINV014 Y-92 $1.749 E+17$

RDCORINV015 Y-93 $1.213 E+18$

RDCORINV016 ZR-95 6.989E+18

RDCORINVOI7 ZR-97 2.517E+18

RDCORINV018 NB-95 7.057E+18

RDCORINV019 MO-99 5.361E+18

RDCORINV020 TC-99M 5.127E+18

RDCORINV021 RU-103 5.047E+18

RDCORINV022 RU-I05 7.433E+16

RDCORINV023 RU-106 1.079E+18

RDCORINV024 RH-105 2.076E+18

RDCORINV025 SB-127 2.881E+17

RDCORINV026 SB-129 2.353E+16

RDCORINV027 TE-127 3.123E+17

RDCORINV028 TE-127M 4.399E+16

RDCORINV029 TE-129 1.317E+17

RDCORINV030 TE-129M 1.593E+17

RDCORINV03I TE-13IM 2.954E+17

RDCORINV032 TE-132 4.209E+18

RDCORINV0 $33 \quad \mathrm{I}-131 \quad 3.374 \mathrm{E}+18$

RDCORINV034 I-I32 $4.336 \mathrm{E}+18$

RDCORINV035 I-133 $3.534 E+18$

RDCORINV036 I-134 $1.961 \mathrm{E}+11$

RDCORINV037 I-135 $5.781 \mathrm{E}+17$ 


$\begin{array}{lll}\text { RDCORINV038 } & \text { XE-133 } & 7.400 \mathrm{E}+18 \\ \text { RDCORINV039 } & \text { XE-135 } & 1.899 \mathrm{E}+18 \\ \text { RDCORINV040 } & \mathrm{CS}-134 & 1.830 \mathrm{E}+17 \\ \text { RDCORINV041 } & \mathrm{CS}-136 & 9.632 \mathrm{E}+16 \\ \text { RDCORINV042 } & \mathrm{CS}-137 & 2.544 \mathrm{E}+17 \\ \text { RDCORINV043 } & \text { BA-139 } & 4.535 \mathrm{E}+13 \\ \text { RDCORINV044 } & \text { BA-140 } & 6.382 \mathrm{E}+18 \\ \text { RDCORINV045 } & \mathrm{LA}-140 & 6.731 \mathrm{E}+18 \\ \text { RDCORINV046 } & \mathrm{LA}-141 & 1.011 \mathrm{E}+17 \\ \text { RDCORINV047 } & \mathrm{LA}-142 & 1.486 \mathrm{E}+14 \\ \text { RDCORINV048 } & \mathrm{CE}-141 & 6.302 \mathrm{E}+18 \\ \text { RDCORINV049 } & \mathrm{CE}-143 & 3.706 \mathrm{E}+18 \\ \text { RDCORINV050 } & \mathrm{CE}-144 & 4.419 \mathrm{E}+18 \\ \text { RDCORINV051 } & \text { PR-143 } & 6.003 \mathrm{E}+18 \\ \text { RDCORINV052 } & \mathrm{ND}-147 & 2.364 \mathrm{E}+18 \\ \text { RDCORINV053 } & \text { NP-239 } & 4.751 \mathrm{E}+19 \\ \text { RDCORINV054 } & \text { PU-238 } & 1.432 \mathrm{E}+15 \\ \text { RDCORINV055 } & \text { PU-239 } & 1.237 \mathrm{E}+15 \\ \text { RDCORINV056 } & \text { PU-240 } & 1.032 \mathrm{E}+15 \\ \text { RDCORINV057 } & \text { PU-241 } & 2.661 \mathrm{E}+17 \\ \text { RDCORINV058 } & \text { AM-241 } & 1.592 \mathrm{E}+14 \\ \text { RDCORINV059 } & \mathrm{CM}-242 & 2.373 \mathrm{E}+16 \\ \text { RDCORINV060 } & \mathrm{CM}-244 & 1.764 \mathrm{E}+14\end{array}$

\section{D.1.3 Inventory for Time Window 3}

* gRand gulf low power and shutdown pRa

* Grand Gulf Inventory for Time Window 3 (40 days after shutdown)

- Time Window 3 is after refueling, thus, only $2 / 3$ of core used.

- Inventories obtained from ORIGENPC; L. A. Miller (4/05/94)

-

NUCNAM CORINV (BECQUERALS)

RDCORINVO0I

RDCORINVO02

RDCORINV003

RDCORINV004

RDCORINVO05

RDCORINV006

RDCORINV007

RDCORINVO08

RDCORINV009

RDCORINV010

RDCORINV011

RDCORINV012

RDCORINV013

RDCORINV014

RDCORINV015

RDCORINVO16

RDCORINV017

RDCORINV018

RDCORINV019

RDCORINV020

RDCORINV02

RDCORINV022

RDCORINV023

RDCORINV024

RDCORINV025

RDCORINV026

RDCORINV027

RDCORINV028

RDCORINV029

RDCORINV030

RDCORINV03

RDCORINV032

RDCORINV033

RDCORINV034

RDCORINV035

Co-58

$5.461 E+16$

CO- 60

$5.503 E+14$

$\mathrm{KR}-85$

1. $761 \mathrm{E}+16$

KR-85M $0.000 \mathrm{E}+00$

KR-87 $0.000 \mathrm{E}+00$

KR-88 $0.000 \mathrm{E}+00$

RB-86 4.938E+14

SR-89 1.589E+18

SR-90 1.386E+17

SR-91 $\quad 0.000 E+00$

SR-92 $0.000 E+00$

Y-90 1.387E+I7

Y-91 2.300E+18

Y-92 $0.000 \mathrm{E}+00$

$Y-93 \quad 0.000 E+00$

2R-95 3.059E+18

ZR-97 3.572E+01

NB-95 4.171E+18

MO-99 $1.926 \mathrm{E}+14$

TC-99M 1.855E+14

RU-103 1. $692 \mathrm{E}+18$

RU-105 0.000E+00

RU-106 6.692E+17

RH-105 $1.499 \mathrm{E}+10$

SB-127 1. $715 E+14$

SB-129 $\quad 0.000 E+00$

TE-127 2.335E+16

TE-127M $2.367 \mathrm{E}+16$

TE- $129 \quad 3.096 E+16$

TE-129M 4.758E+16

TE-131M 7.994E+07

TE- $1327.000 E+14$

I-131 7.931E+16

I-132 $\quad 7.214 \mathrm{E}+14$

$I-133 \quad 6.706 E+04$

$I-134 \quad 0.000 \mathrm{E}+00$

Vol. 6, Part 1 
Appendix D

RDCORINV037

RDCORINV038

I-135

$0.000 \mathrm{E}+00$

RDCORINV0 39

$\mathrm{XE}-133$

$3.189 \mathrm{E}+16$

$\mathrm{XE}-135$

$0.000 \mathrm{E}+00$

RDCORINV0 40

RDCORINV0 4

RDCORINVO 4

RDCORINV043

CS-134

$1.178 \mathrm{E}+17$

CS-136 8.168E+15

CS-137 $1.694 \mathrm{E}+17$

BA-139 $0.000 \mathrm{E}+00$

$B A-140 \quad 5.145 \mathrm{E}+17$

RDCORINVO

RDCORINV04 5

LA-140 $5.921 E+17$

IA $-141 \quad 0.000 \mathrm{E}+00$

RDCORIN

$\begin{array}{ll}I A-142 & 0.000 E+00 \\ C E-141 & 1.832 E+18\end{array}$

$\mathrm{CE}-143 \quad 7.162 \mathrm{E}+09$

$\mathrm{CE}-144 \quad 2.681 \mathrm{E}+18$

PR-143 $5.842 \mathrm{E}+17$

ND-147 1.370E+17

NP-239 3.317E+14

PU-238 9.780E+14

PU-239 8.344E+14

PU-240 $6.886 \mathrm{E}+14$

PU-241 $1.767 E+17$

AM-241 $1.366 \mathrm{E}+14$

CM-242 1.347E+16

CM-244 $1.172 \mathrm{E}+14$

\section{D.2 Listing of Code Used to Estimate Onsite Consequences}

PROGRAM ONSITE5

C CALCULATE PARKING LOT DOSES DUE TO IMMERSION AND INHALATION AT

C VARIOUS DISTANCES AT GRAND GULF BASED ON SOURCE TERMS PRODUCED

C BY GGSOR FOLLOWED BY PARTITION

C

C NTOT = NUMBER OF SOURCE TERMS EVALUATED

C $R F(I, J)$ = RELEASE FRACTION TO ENVIRONMENT FOR ITH CHEMICAL GROUP AND

C JTH SEGMENT

C TI = TIME AT WHICH FIRST RELEASE TO ENVIRONMENT BEGINS

DT1 = DURATION OF FIRST RELEASE

C T2 = TIME AT WHICH SECOND RELEASE TO ENVIRONMENT BEGINS

C DT2 = DURATION OF SECOND RELEASE

C BR = BREATHING RATE (CURRENTLY HARD-WIRED, NUMBER OBTAINED FROM 4551 FOR A MAN BREATHING HEAVILY)

AREA = MINIMUM AREA OF BUILDING, TAKEN AS MINIMUM WIDTH

OF AUX BUILDING TIMES HEIGHT OF CONTAINMENT $\left(M^{\wedge} 2\right)$

ISTAB $=$ STABIIITY CLASS $(1=A, 2=B, \ldots, 6=E)$

$U=$ WIND SPEED AT $10 \mathrm{M}$ HEIGHT (M/S) (CURRENTLY HARD-WIRED)

ICHEM(I) = CHEMICAL GROUP THAT ITH RADIONUCLIDE BELONGS TO

$\operatorname{XINV}(I, J)=$ INVENTORY OF ITH RADIONUCLIDE AND JTH EVENT TYPE (SHUTDOWN TIME) AS DETERMINED WITH ORIGEN2

$\operatorname{NUCNAM}(I)=$ NUCLIDE NAME

CHIQ $(I, J, K)=$ RELATIVE CONCENTRATION CORRESPONDING TO ITH DISTANCE, JTH MET, AND KTH MODEL (1=RAMSDELL MODEL, 2=WILSON/REG GUIDE),

DCFIMM(I) = DOSE CONVERSION FACTOR FOR IMMERSION FOR ITH NUCLIDE IN $(S V / D A Y) /\left(B Q / M^{\wedge} 3\right)$

DCFINH(I) = DOSE CONVERSION FACTOR FOR INHALATION FOR ITH NUCLIDE IN $(\mathrm{SV} / \mathrm{BQ})$

DCFGRD(I) = DOSE CONVERSION FACTOR FOR GROUNDSHINE FOR ITH NUCLIDE IN (SV/DAY) $/\left(\mathrm{BQ} / \mathrm{M}^{\wedge} 2\right)$

DCFING (I) $=$ DOSE CONVERSION FACTOR FOR INGESTION FOR ITH NUCLIDE IN (SV/BQ)

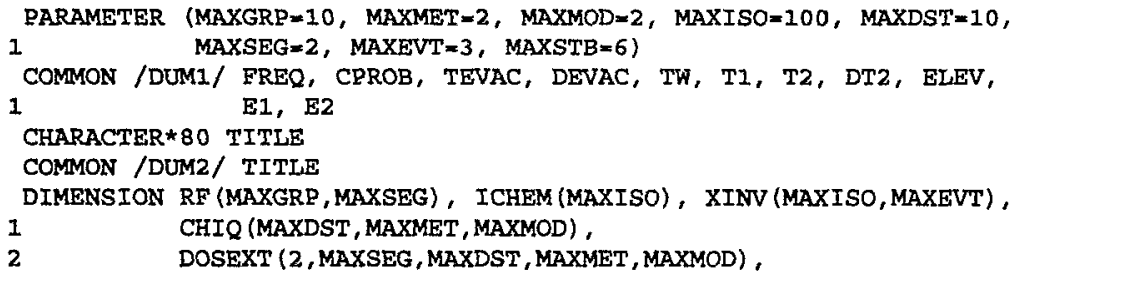




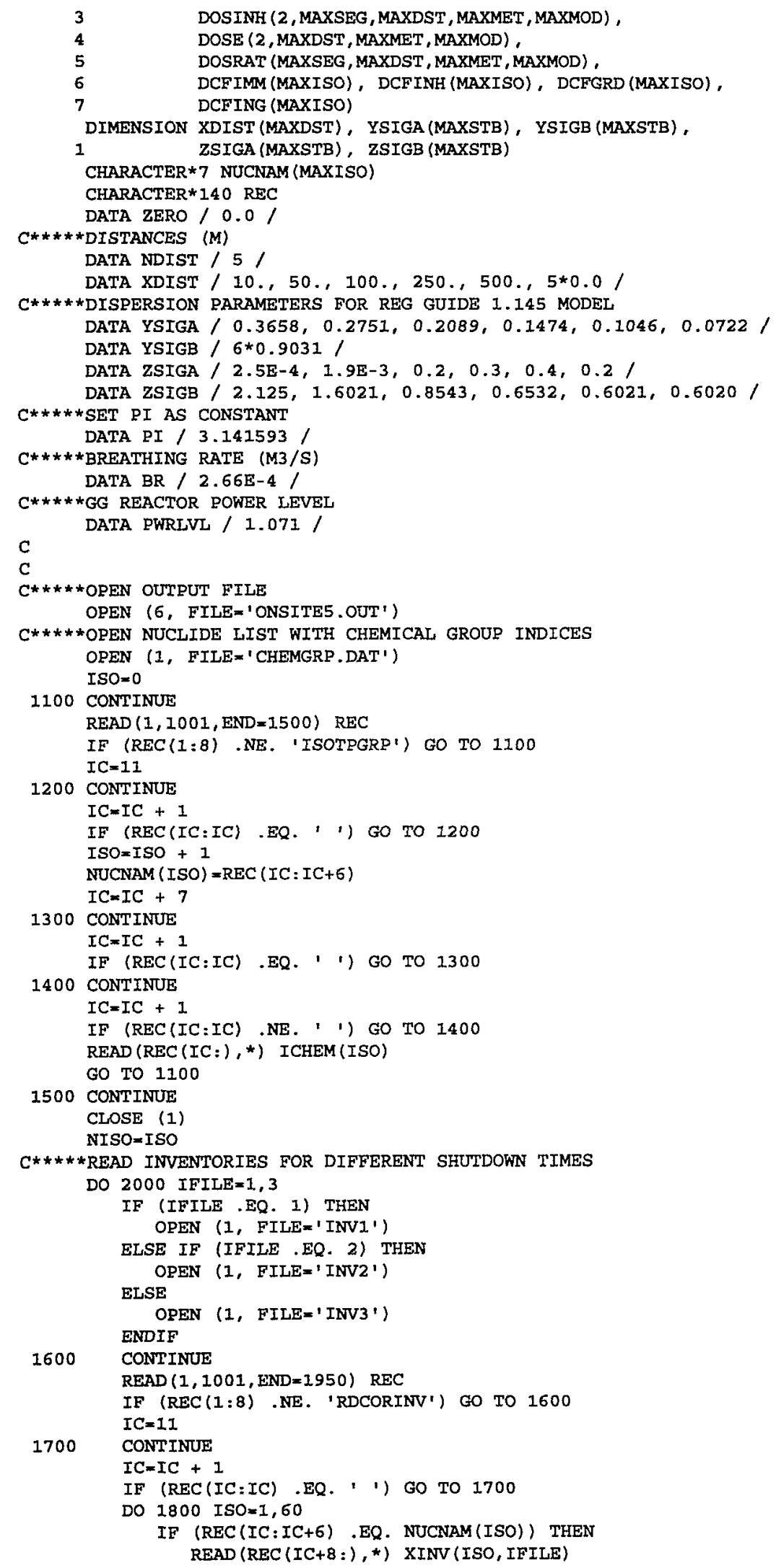




\section{Appendix D}

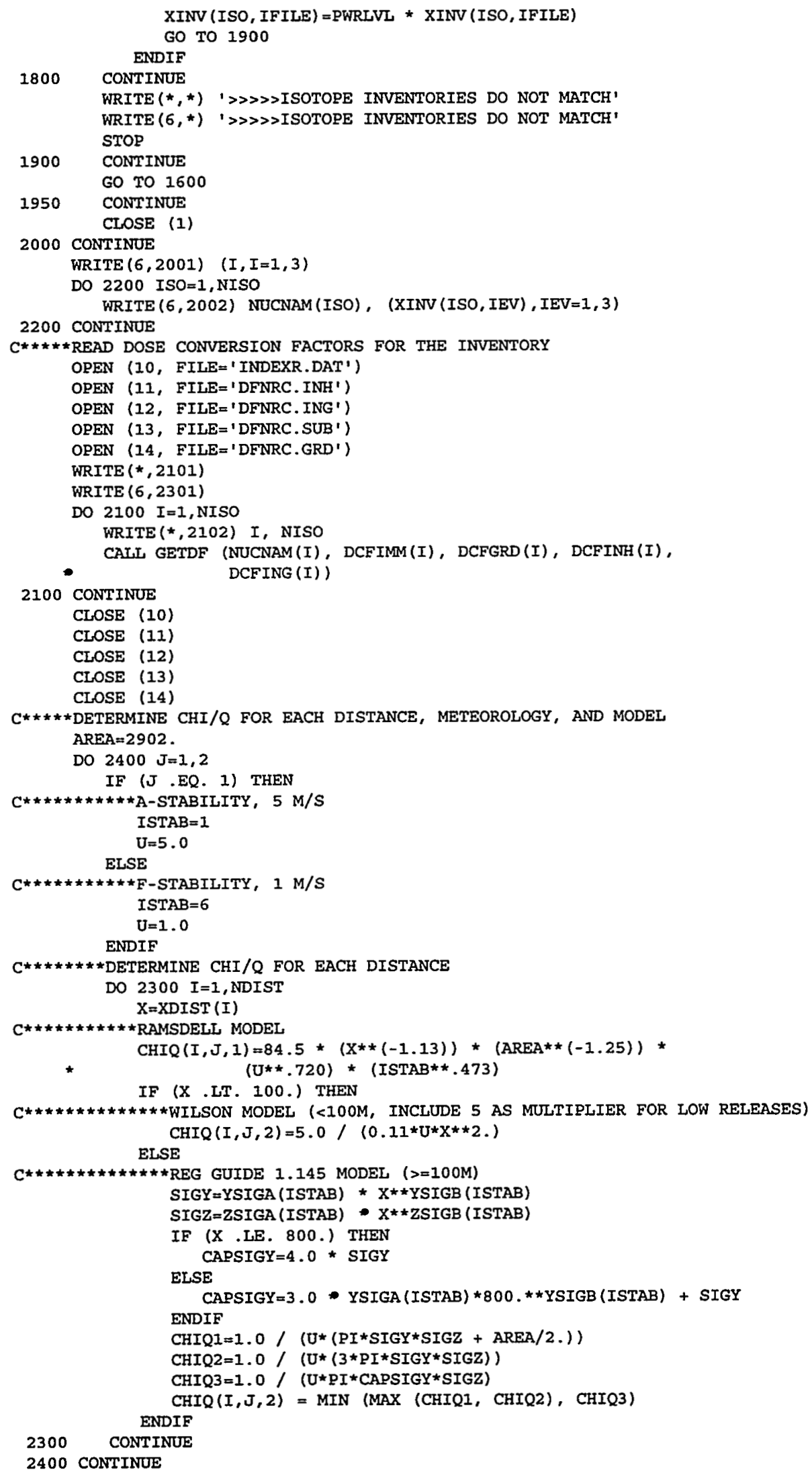


WRITE $(6,2501)$

DO $2500 \quad \mathrm{~K}=1$, NDIST

$\operatorname{WRITE}(6,2502) \operatorname{NINT}(\operatorname{XDIST}(K)),((\mathrm{CHIQ}(K, L, M), M=1,2), L=1,2)$

2500 CONTINUE

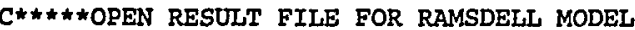

OPEN ( 1, FILE $=$ 'GGR. OUT' $)$

OPEN (11, FILE='GGRCON.OUT')

WRITE $(1,3001)$ 'RAMSDELL'

GRITE $(11,4001)$ 'RAMSDELI'

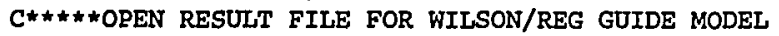

OPEN (2, FILE='GGW. OUT')

OPEN (12, FILE='GGWCON.OUT')

WRITE $(2,3001)$ 'WILSON/REG GUIDE'

WRITE $(12,4001)$ 'WILSON/REG GUIDE'

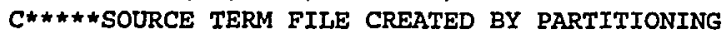

OPEN (3, FILEF'MACCS.INP')

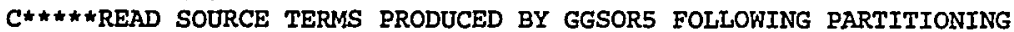

$\operatorname{READ}(3,1002)$ TITLE

$\operatorname{READ}(3, \star)$ NFRC, NSEG, NPAR

WRITE $(*, 2401)$

DO 9000 IPAR=1, NPAR

WRITE $(*, 2402)$ IPAR, NPAR

READ $(3, \star)$ IPART, FREQ, CPROB

IF (IPAR .NE. IPART) THEN

WRITE $(*, *)$ ' $\gg \gg \gg$ ERROR IN READING PARTITION SOURCE TERMS'

WRITE $(6, *)$ ' $\gg \gg \gg \gg$ ERROR IN READING PARTITION SOURCE TERMS' STOP

ENDIF

$\operatorname{READ}(3, \star)$ TEVAC, DEVAC, TW, T1, DT1, T2, DT2, ELEV, EVTYPE, IEV =NINT (EVTYPE)

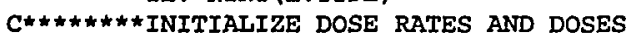

DO $3000 M=1,2$

DO $2900 \quad L=1,2$

DO $2800 \mathrm{~K}=1$, NDIST

DO $2700 \mathrm{~J}=1$, NSEG

DO $2600 \quad I=1,2$

$\operatorname{DOSEXT}(I, J, K, L, M)=0.0$

2600 $\operatorname{DOSINH}(I, J, K, L, M)=0.0$

2700

2800

2900

3000

CONTINUE

CONTINUE

CONTINUE

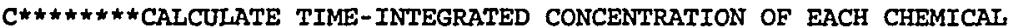

C $\star * \star \star \star \star \star \star$ GROUP AT THE VARIOUS DISTANCES

$C \star \star \star \star \star \star \star \star$ LOOP OVER MODELS

DO $3500 \mathrm{M=1,2}$

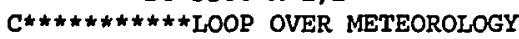

DO $3400 \mathrm{~L}=1,2$

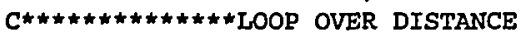

DO $3300 \quad \mathrm{~K}=1$, NDIST

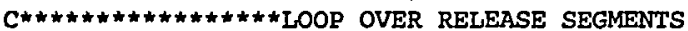

DO $3200 \mathrm{~J}=1$, NSEG

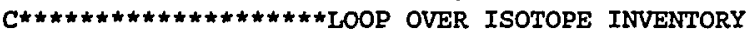

DO $3100 \quad I=1$, NISO

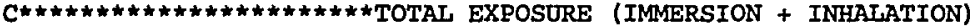

$\operatorname{RF}(I C H E M(I), J) \star X I N V(I, I E V) * C H I Q(K, L, M) \star D C F I M M(I) / 86400$ $\operatorname{DOSINH}(2, J, K, L, M)=\operatorname{DOSINH}(2, J, K, L, M)+$

1 RF $(I C H E M(I), J) \star X I N V(I, I E V) \star C H I Q(K, L, M) * D C F I N H(I) * B R$ CONTINUE

$3200 \quad$ CONTINUE

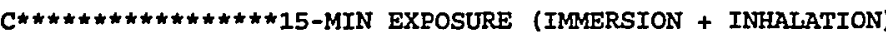

IF (DTI .GE. 900.) THEN

$\operatorname{DOSEXT}(1,1, K, L, M)=\operatorname{DOSEXT}(2,1, K, L, M) * 900 . / D T I$

$\operatorname{DOSINH}(1,1, K, I, M)=\operatorname{DOSINH}(2,1, K, L, M) * 900 . / D T 1$ ELSE

IF (DT1 .GT. 0.0) THEN

$\operatorname{DOSEXT}(1,1, K, L, M)=\operatorname{DOSEXT}(2,1, K, L, M)$

$\operatorname{DOSINH}(1,1, K, I, M)=\operatorname{DOSINH}(2,1, K, L, M)$

Vol. 6, Part 1

D-7 
Appendix D

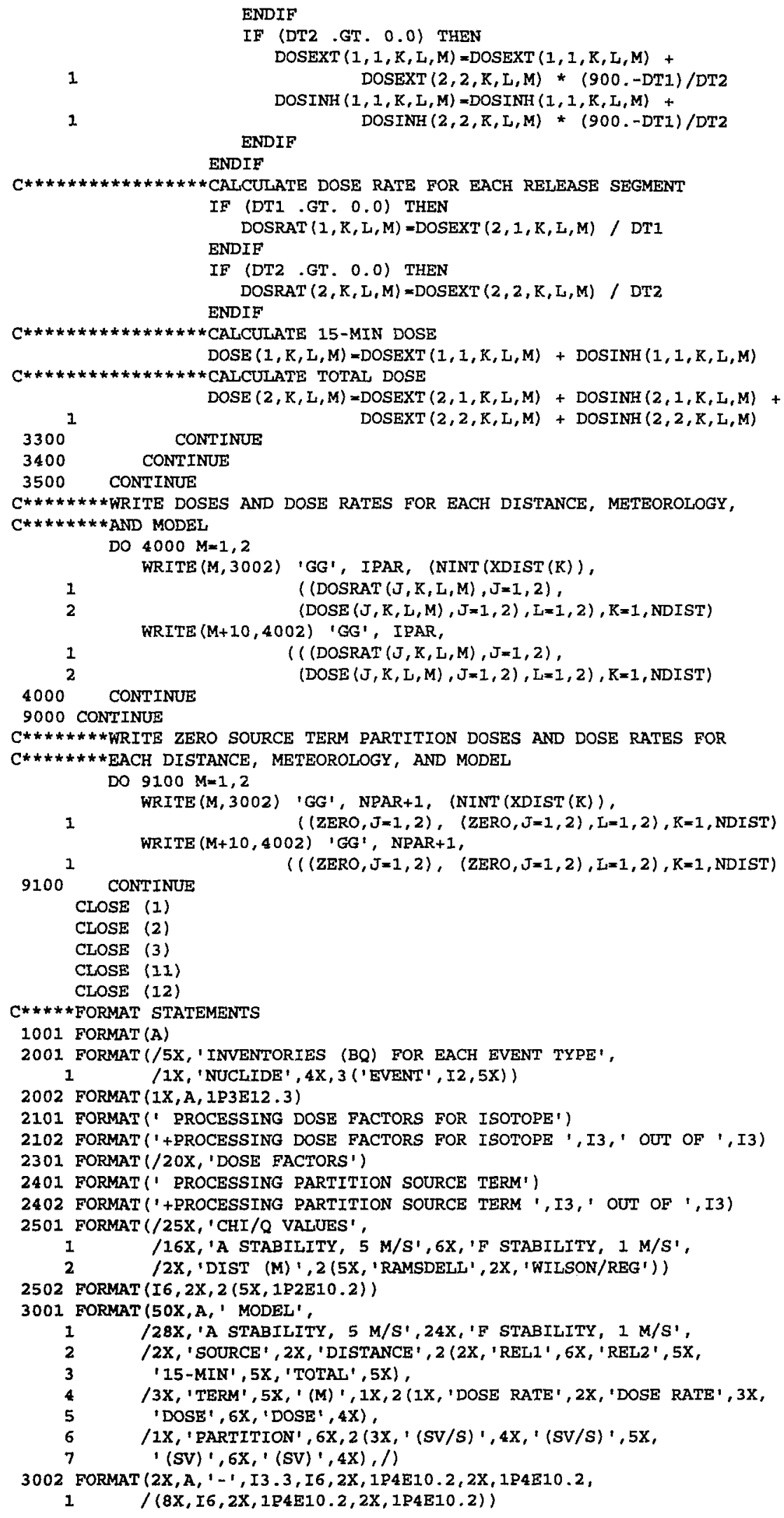


4001 FORMAT (A,' MODEL')

4002 FORMAT (A, '-1, I3.3,

1

(1PE10.2))

SUBROUTINE GETDF (NUCNAM, DCLD, DGRD, DINH, DING)

CHARACTER* $(*)$ NUCNAM

CHARACTER 7 NEWNAM

CHARACTER*26 UPPER, LOWER

DATA UPPER / ABCDEFGHIJKLMNOPQRSTUVWXYZ'/

c

DATA LOWER /'abcdefghijklmnopqrstuvwxyz'/

NEWNAM $=$ NUCNAM

DO $1000 \quad I C=2,7$

IP $=$ INDEX (UPPER, NEWNAM (IC: IC))

IF (IP .GT. O) NEWNAM (IC:IC) =LOWER (IP : IP)

1000 CONTINUE

DCLD $=0.0$

DGRD $=0.0$

$\mathrm{DINH}=0.0$

DING $=0.0$

CALL READIT (NEWNAM, DCLD, DGRD, DINH, DING)

RETURN

END

$\mathrm{C}$

SUBROUTINE READIT (NEWNAM, DCLD, DGRD, DINH, DING)

* Main Readid.For

* Program illustrates the use of INDEXR.DAT file to coordinate the reading

* of the dose factor files DFNRC.INH, DFNRC.ING, DFNRC.SUB, and DFNRC.GRD.

* All files are direct access formatted files. The INDEXR. DAT file has

* record length 102 , the others are 80 . See the OPEN atatements below for

* other details. K.F. Eckerman 4/05/89

Last mods $5 / 22 / 90$.

* The file INDEXR.DAT contains the following information:

* Variable Description

* T

* IX

* Mode

* D1

* F1

* D2

* F2

* D3

* F3

* ID()

* ID (1)

* ID (2)

* ID (3)

Name of nuclide

A7

Halflife

Halflife units

Decay Modes

A8

Name of daughter

Branching fraction

Name of daughter

Branching fraction

Name of daughter

Branching fraction A7

Pointers into files 7 I4

Record \# of D1 in INDEXR.DAT

Record \# of D2 "

Record \# of D3 "

* ID (4)

* ID (5)

* ID (6)

Record \# of Nuke in DFNRC. INH

" DFNRC ING

" " DFNRC. SUP

* ID (7)

"DFNRC. GRD

* Local variables

CHARACTER $* 8$ T

CHARACTER*7 Nuke, D1, D2, D3, Nuke1, Nuke2, Nuke3, Nuke4

CHARACTER* 7 NEWNAM

CHARACTER $* 6$ MOde

CHARACTER *2 IX

COMMON /DUMMY1/ D1, D2, D3, NUKE1, NUKE2, NUKE3, NUKE4, MODE

COMMON /DUMYY2/ F1, F2, F3, IX, T

DIMENSION DFinh (8), DFing (8), DFsub (8), Dfgrd (8)

DIMENSION ID (7)

PARAMETER (Idev $=10, \operatorname{Idf} 1=11, \operatorname{Idf} 2=12, \operatorname{Idf} 3=13, \operatorname{Idf} 4=14, \operatorname{Igrd}=4, \operatorname{Isub}=4$ Iing $=4$, I inh $=4, \mathrm{Ngxd}=826$, Nsub $=826$, Ning $=738$, Ninh $=738$, Ifile=1, Nfile $=825$ )

Search dose factor data files for user-specified nuclide and print dose factors for inhalation, ingestion, submersion and ground plane. 


\section{Appendix D}

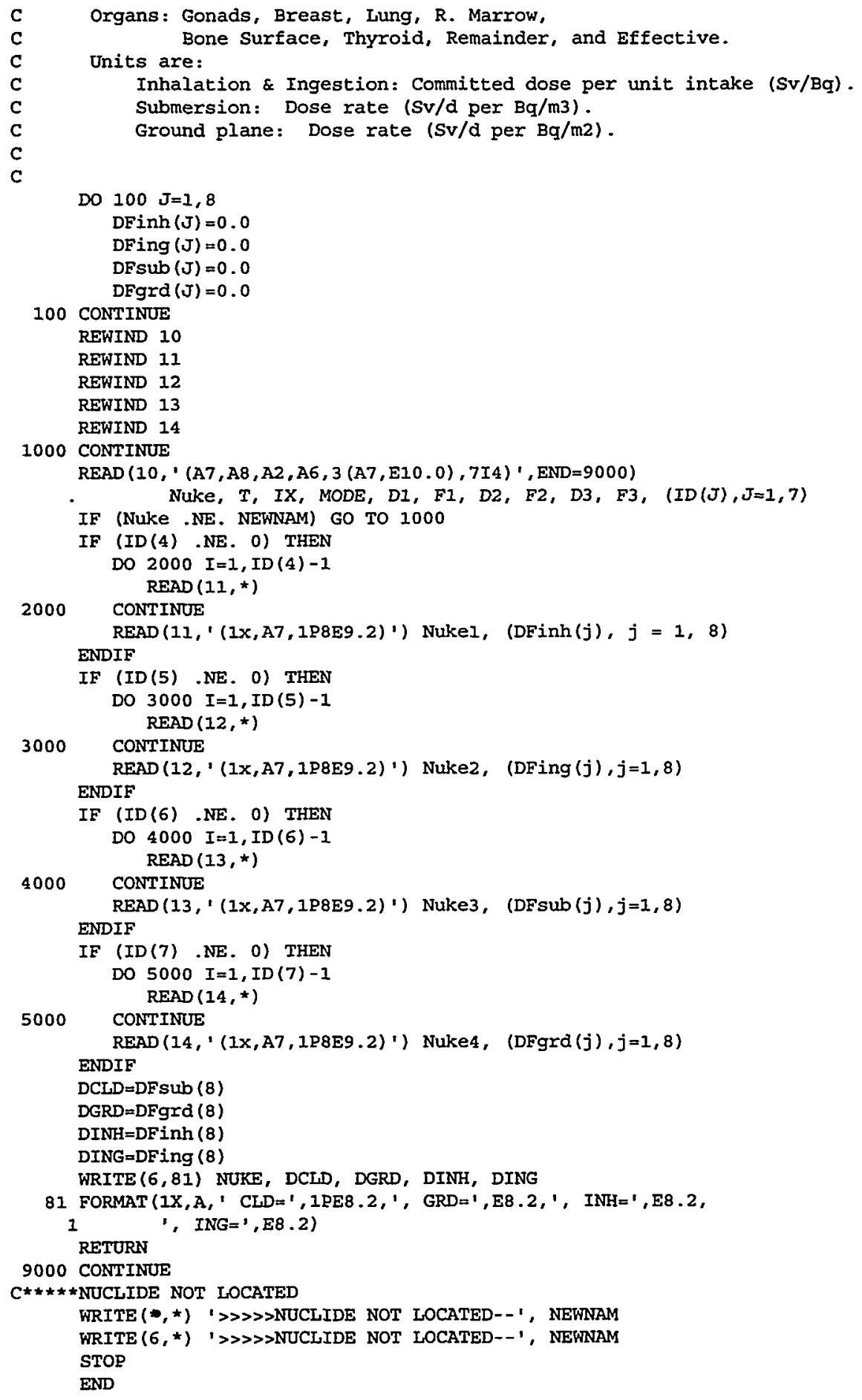




\section{D.3 Onsite Consequences for Source Term Groups}

Doses and dose rates were calculated for a variety of distances (10,50,100,250, and 500 meters) from the containment using the Wilson/Reg. Guide Model and the Ramsdell Model as described in Chapter 8 of this report. The code that was used to calculate these doses and dose rates is listed in Section D.2. These calculations were performed using the mean source term defined for each source term group. The mean source terms are tabulated in Appendix C. Two different weather scenarios were used for each set of models. The first weather scenario assumes stable conditions (stability class F) with a wind speed of $1 \mathrm{~m} / \mathrm{s}$. The second scenario assumes unstable conditions (stability class $\mathrm{A}$ ) with a wind speed of $5 \mathrm{~m} / \mathrm{s}$. The dose calculation considers exposure from both the immersion and inhalation pathways and is a 50-year committed dose. The dose rate only considered exposure from the immersion pathway. Doses were calculated assuming both exposure to the entire release and exposure to only the first 15 minutes of the release. A dose rate was calculated for each of the release segments defined in the source term analysis. The doses and dose rates calculated using the Wilson/Reg. Guide 1.145 model are presented in Table D.3-1. Similar results calculated using the Ramsdell Model are presented in Table D.3-2. 
Appendix D

\begin{tabular}{|c|c|c|c|c|c|c|c|c|c|}
\hline \multirow[b]{2}{*}{$\begin{array}{l}\text { SOURCE } \\
\text { TERM }\end{array}$} & \multicolumn{4}{|c|}{$\begin{array}{l}\text { TABLE D. } 3-1 \text {. DOse and } \\
\text { A STABILITY, } 5 \mathrm{M} / \mathrm{s}\end{array}$} & Dose Rates & Calculated & \multicolumn{3}{|c|}{$\begin{array}{l}\text { Using Filson/Reg. Guide Model } \\
\text { F STABILITY, I N/B }\end{array}$} \\
\hline & $\begin{array}{l}\text { DISTANC } \\
\text { (M) }\end{array}$ & $\begin{array}{c}\text { CE RELI } \\
\text { DOSE RATE } \\
(\mathrm{SV} / \mathrm{S})\end{array}$ & $\begin{array}{c}\text { REL2 } \\
\text { DOSE RATE } \\
(S V / S)\end{array}$ & $\begin{array}{c}\text { 15-MIN } \\
\text { DOSE } \\
(\mathrm{SV})\end{array}$ & $\begin{array}{l}\text { TOTAL } \\
\text { DOSE } \\
(8 V)\end{array}$ & $\begin{array}{c}\text { RRL1 } \\
\text { DOSE RATE } \\
(\mathrm{SV} / \mathrm{S})\end{array}$ & $\begin{array}{c}\text { REL2 } \\
\text { DOSE RATE } \\
(S V / S)\end{array}$ & $\begin{array}{c}\text { 15-MIN } \\
\text { DOSE } \\
(\mathrm{SV})\end{array}$ & $\begin{array}{l}\text { TOTAL } \\
\text { DOSE } \\
(\mathrm{SV})\end{array}$ \\
\hline \multirow[t]{5}{*}{ 96-001 } & 10 & $4.26 \mathrm{E}-01$ & $2.09 \mathrm{E}-01$ & $6.70 \mathrm{E}+03$ & $8.84 \mathrm{E}+0.5$ & $2.13 E+00$ & $1.04 E+00$ & $3.35 E+04$ & $4.42 E+06$ \\
\hline & 50 & $1.70 \mathrm{~B}-02$ & $8.36 E-03$ & $2.68 \mathrm{E}+02$ & $3.54 \mathrm{E}+04$ & $8.51 z-02$ & $4.18 \mathrm{E}-02$ & $1.34 \mathrm{E}+03$ & $1.77 E+05$ \\
\hline & 100 & $7.16 \mathrm{E}-04$ & $3.51 \mathrm{E}-04$ & $1.13 \mathrm{E}+01$ & $1.49 \mathrm{E}+03$ & $2.52 \mathrm{E}-02$ & $1.24 \mathrm{E}-02$ & $3.97 \mathrm{E}+02$ & $5.24 E+04$ \\
\hline & 250 & $4.46 \mathrm{E}-05$ & 2.19E-05 & $7.03 E-01$ & $9.28 E+01$ & $6.35 E-03$ & $3.11 E-03$ & $1.00 E+02$ & $1.32 E+04$ \\
\hline & 500 & $5.47 \mathrm{E}-06$ & $2.69 \mathrm{E}-06$ & $8.62 \mathrm{E}-02$ & $1.14 E+01$ & $2.24 E-03$ & $1.10 \mathrm{E}-03$ & $3.52 \mathrm{E}+01$ & $4.65 \mathrm{~B}+03$ \\
\hline \multirow[t]{5}{*}{ GG-002 } & 30 & $4.88 E-01$ & $1.73 E-01$ & $1.308+04$ & $1.56 \mathrm{E}+06$ & $2.44 E+00$ & $8.64 \mathrm{E}-01$ & $6.51 E+04$ & $7.82 \mathrm{E}+06$ \\
\hline & 50 & $1.95 \mathrm{E}-02$ & $6.91 E-03$ & $5.21 E+02$ & $6.25 E+04$ & $9.76 \mathrm{E}-02$ & $3.46 E-02$ & $2.61 E+03$ & $3.13 \mathrm{E}+0.5$ \\
\hline & 100 & $8.21 \mathrm{E}-04$ & $2.91 \mathrm{E}-04$ & $2.19 E+02$ & $2.63 \mathrm{E}+03$ & $2.89 \mathrm{E}-02$ & $3.02 \mathrm{E}-02$ & $7.71 E+02$ & $9.26 \mathrm{E}+04$ \\
\hline & 250 & $5.12 E-05$ & 1.81 E-05 & $1.37 \mathrm{E}+00$ & $1.64 \mathrm{E}+02$ & $7.28 \mathrm{E}-03$ & $2.58 E-03$ & $1.94 \mathrm{E}+02$ & $2.33 \mathrm{E}+04$ \\
\hline & 500 & $6.28 \mathrm{E}-06$ & $2.22 E-06$ & $1.68 \mathrm{E}-01$ & $2.01 \mathrm{E}+01$ & $2.56 \mathrm{E}-03$ & $9.08 E-04$ & $6.84 \mathbb{E}+01$ & $8.21 E+03$ \\
\hline \multirow[t]{5}{*}{ GG-003 } & 10 & $2.03 E-01$ & $2.57 \mathrm{E}-01$ & $2.05 B+03$ & $7.60 \mathrm{E}+05$ & $1.01 \mathrm{E}+00$ & $1.29 E+00$ & $1.02 E+04$ & $3.80 E+06$ \\
\hline & 50 & 8. I1E-03 & $1.03 E-02$ & $8.19 E+01$ & $3.04 E+04$ & $4.06 E-02$ & $5.15 E-02$ & $4.09 \mathrm{E}+02$ & $1.52 \mathrm{E}+0.5$ \\
\hline & 100 & $3.41 \mathrm{E}-04$ & $4.33 \mathrm{E}-04$ & $3.44 \mathrm{E}+00$ & $1.28 \mathrm{E}+03$ & $1.20 \mathrm{E}-02$ & $1.52 \mathrm{E}=02$ & $1.21 \mathrm{E}+02$ & $4.50 \mathrm{E}+04$ \\
\hline & 250 & $2.13 E-05$ & $2.70 z-05$ & $2.15 E-01$ & $7.97 E+01$ & $3.02 E-03$ & $3.84 \mathrm{E}-03$ & $3.05 \mathrm{E}+01$ & $1.13 \mathrm{E}+04$ \\
\hline & 500 & $2.618-06$ & $3.31 E-06$ & $2.63 \mathrm{E}-02$ & $9.78 \mathrm{E}+00$ & $2.07 \mathrm{E}-03$ & $1.35 \mathrm{E}-03$ & $1.08 \mathrm{E}+01$ & $3.99 E+03$ \\
\hline \multirow[t]{5}{*}{ GG-004 } & 10 & $1.29 E+00$ & $1.10 \mathrm{E}-02$ & $6.92 \mathrm{E}+04$ & $3.49 \mathrm{E}+06$ & $6.45 \mathrm{E}+00$ & $5.52 \mathrm{E}-01$ & $3.6 E+05$ & $1.74 E+07$ \\
\hline & 50 & $5.16 \mathrm{E}-02$ & $4.41 E-03$ & $2.77 \mathrm{E}+03$ & $1.40 \mathrm{E}+05$ & $2.58 E-01$ & $2.21 E-02$ & $1.38 \mathrm{E}+04$ & $6.98 \mathrm{E}+05$ \\
\hline & 100 & $2.17 E-03$ & $1.86 E-04$ & $1.16 \mathrm{E}+02$ & $5.87 E+03$ & $7.63 \mathrm{E}-02$ & $6.53 \mathrm{E}-03$ & $4.10 E+03$ & $2.07 E+05$ \\
\hline & 250 & $1.35 \mathrm{E}-04$ & $1.16 \mathrm{E}-05$ & $7.26 \mathrm{E}+00$ & $3.66 \mathrm{E}+02$ & $1.92 \mathrm{E}-02$ & $1.65 E-03$ & $1.03 E+03$ & $5.20 \mathrm{E}+04$ \\
\hline & 500 & $1.66 \mathrm{E}-05$ & $1.42 \mathrm{E}-06$ & $8.91 E-01$ & $4.49 E+01$ & $6.77 \mathrm{E}-03$ & $5.80 \mathrm{E}-04$ & $3.64 \mathrm{E}+02$ & $1.83 \mathrm{E}+04$ \\
\hline \multirow[t]{5}{*}{$00-005$} & 10 & $7.77 \mathrm{E}-01$ & $2.81 E-01$ & $1.32 E+04$ & $0 E+06$ & $3.88 E+00$ & $1.41 E+00$ & $6.62 \mathrm{E}+04$ & $6.49 E+06$ \\
\hline & 50 & $3.11 \equiv-02$ & $1.13 \mathrm{E}-02$ & $5.30 \mathrm{E}+02$ & $5.19 E+04$ & $1.55 \mathrm{E}-01$ & $5.63 E-02$ & $2.65 \mathrm{E}+03$ & $2.60 \mathrm{E}+05$ \\
\hline & 100 & $1.31 \mathrm{E}-03$ & $4.73 \mathrm{E}-04$ & $2.23 E+01$ & $2.18 \mathrm{E}+03$ & $4.60 \mathrm{E}-02$ & $1.67 \mathrm{E}-02$ & $7.84 E+02$ & $7.69 \mathrm{E}+04$ \\
\hline & 250 & $8.15 z-05$ & $2.95 E-05$ & $1.39 \mathrm{E}+00$ & $1.36 \mathrm{E}+02$ & $1.16 \mathrm{E}-02$ & $4.20 E-03$ & $1.97 \mathrm{E}+02$ & $1.94 \mathrm{E}+04$ \\
\hline & 500 & $9.99 \mathrm{E}-06$ & $3.62 E-06$ & $1.70 \mathrm{E}-01$ & $1.678+01$ & $4.08 \mathrm{E}-03$ & $1.48 E-03$ & $6.96 \mathrm{E}+01$ & $6.82 E+03$ \\
\hline \multirow[t]{5}{*}{ GG-006 } & 10 & $1.24 \mathrm{E}-01$ & $1.80 \mathrm{E}-01$ & $1.65 E+03$ & $3.66 E+05$ & $6.21 \mathrm{E}-01$ & $8.99 \mathrm{E}-01$ & $8.27 E+03$ & $1.83 E+06$ \\
\hline & 50 & $4.97 \mathrm{E}-03$ & $7.19 \mathrm{E}-03$ & $6.62 \mathrm{E}+01$ & $1.46 \mathrm{E}+04$ & $2.48 \mathrm{E}-02$ & $3.608-02$ & $3.31 E+02$ & $7.32 \mathrm{E}+04$ \\
\hline & 100 & $2.09 \mathrm{~F}-04$ & $3.03 E-04$ & $2.78 \mathrm{E}+00$ & $6.15 E+02$ & $7.35 E-03$ & $1.06 \mathrm{E}-02$ & $9.80 \mathrm{E}+01$ & $2.17 E+04$ \\
\hline & 250 & $1.30 \mathrm{E}-05$ & $1.89 \mathrm{E}-05$ & $1.74 \mathrm{E}-01$ & $3.84 E+01$ & $1.85 E-03$ & $2.68 \mathrm{E}-03$ & $2.47 \mathrm{E}+01$ & $5.45 E+03$ \\
\hline & 500 & $1.60 \mathrm{E}-06$ & $2.31 E-06$ & $2.13 E-02$ & $4.71 E+00$ & $6.52 \mathrm{E}-04$ & $9.45 \mathrm{E}-04$ & $8.69 E+00$ & $1.92 \mathrm{E}+03$ \\
\hline \multirow[t]{5}{*}{ GG-007 } & 10 & $1.51 E-01$ & $2.01 E-01$ & $2.54 E+03$ & $8.94 E+05$ & $7.54 \mathrm{E}-01$ & $1.01 \mathrm{E}+00$ & $1.27 \mathrm{E}+04$ & $4.47 E+06$ \\
\hline & 50 & $6.03 \mathrm{E}-03$ & $8.05 \mathrm{E}-03$ & $1.02 E+02$ & $3.58 E+04$ & $3.02 \mathrm{E}-02$ & $4.03 \mathrm{E}-02$ & $5.08 \mathrm{E}+02$ & $1.79 \mathrm{E}+05$ \\
\hline & 100 & $2.54 E-04$ & $3.39 Z-04$ & $4.27 E+00$ & $1.50 \mathrm{E}+03$ & $8.93 E-03$ & $1.19 \mathrm{E}-02$ & $1.50 \mathrm{E}+02$ & $5.30 \mathrm{E}+04$ \\
\hline & 250 & $1.58 \mathrm{E}-05$ & $2.11 \mathrm{E}-05$ & $2.66 \mathrm{E}-01$ & $9.38 \mathrm{E}+01$ & $2.25 E-03$ & $3.00 \mathrm{E}-03$ & $3.79 \mathrm{E}+01$ & $1.33 E+04$ \\
\hline & 500 & $1.94 \mathrm{E}-06$ & $2.59 \mathrm{E}-06$ & $3.27 \mathrm{E}-02$ & $1.15 E+01$ & $7.92 \mathrm{E}-04$ & $1.06 \mathrm{E}-03$ & $1.33 \mathrm{E}+01$ & $4.70 E+03$ \\
\hline GG-008 & 10 & $2.74 \mathrm{E}-01$ & $1.39 \mathrm{E}-01$ & $4.93 E+03$ & $6.48 E+05$ & $1.37 \mathrm{E}+00$ & $6.97 E-01$ & $2.47 E+04$ & $3.24 E+06$ \\
\hline & 50 & $1.10 \mathrm{E}-02$ & $5.58 E-03$ & $1.97 \mathrm{E}+02$ & $2.59 \mathrm{E}+04$ & $5.49 \mathrm{E}-02$ & $2.79 E-02$ & $9.86 \mathrm{E}+02$ & $1.30 \mathrm{E}+05$ \\
\hline & 100 & $4.62 \mathrm{E}-04$ & $2.35 E-04$ & $8.29 E+00$ & $1.09 E+03$ & $1.63 \mathrm{E}-02$ & $8.26 \mathrm{E}-03$ & $2.92 \mathrm{E}+02$ & $3.83 E+04$ \\
\hline & 250 & $2.88 \mathrm{E}-05$ & $1.46 \mathrm{E}-0.5$ & 5.17E-01 & $6.79 \mathrm{E}+01$ & $4.095-03$ & $2.08 \mathrm{E}-03$ & $7.35 E+01$ & $9.65 E+03$ \\
\hline & 500 & $3.53 \mathrm{E}=06$ & $1.79 \mathrm{E}-06$ & $6.34 \mathrm{E}-02$ & $8.33 E+00$ & 1. 44E-03 & $7.32 \mathrm{E}-04$ & $2.59 E+01$ & $3.40 E+03$ \\
\hline$G G$ & 10 & $7.84 \mathrm{E}-01$ & $2.60 \mathrm{E}-01$ & $4.26 E+04$ & $1.82 \mathrm{E}+06$ & $3.92 \mathrm{E}+00$ & $1.30 \mathrm{E}+00$ & $2.23 \mathrm{E}+05$ & $9.11 \mathrm{~B}+06$ \\
\hline & 50 & $3.14 \mathrm{E}-02$ & $1.04 E-02$ & $1.70 B+03$ & $7.29 E+04$ & $1.57 \mathrm{E}-01$ & $5.20 \mathrm{~B}-02$ & $8.51 E+03$ & $3.64 E+05$ \\
\hline & 100 & $1.32 \mathrm{E}-03$ & $4.37 \mathrm{E}-04$ & $7.16 \mathrm{E}+01$ & $3.07 E+03$ & $4.64 \mathrm{E}-02$ & $1.54 \mathrm{E}-02$ & $2.52 \mathrm{E}+03$ & $1.08 \mathrm{E}+05$ \\
\hline & 250 & $8.23 \mathrm{E}-05$ & $2.73 \mathrm{E}-05$ & $4.47 E+00$ & $1.91 \mathrm{E}+02$ & $1.17 \mathrm{E-02}$ & $3.87 \mathrm{E}-03$ & $6.35 E+02$ & $2.72 \mathrm{~B}+04$ \\
\hline & 500 & $1.01 \mathrm{E}-05$ & $3.34 \mathrm{E}-06$ & $5.47 E-01$ & $2.34 E+01$ & $4.12 \mathrm{E}-03$ & $1.37 \mathrm{E}-03$ & $2.24 \mathrm{E}+02$ & $9.57 \mathrm{E}+03$ \\
\hline 10 & 10 & $.38 \mathrm{E}-01$ & $7.07 \mathrm{E}-02$ & $1.24 E+03$ & $2.83 E+05$ & $1.29 \mathrm{E}+00$ & $3.53 E-01$ & $6.22 E+03$ & $1.41 E+06$ \\
\hline & 50 & $9.54 E-03$ & $2.83 E-03$ & $4.98 E+01$ & $1.13 \mathrm{E}+04$ & $4.77 \mathrm{E}-02$ & $1.41 \mathrm{E}-02$ & $2.49 E+02$ & $5.66 \mathrm{E}+04$ \\
\hline & 100 & $4.01 \mathrm{E}-04$ & $1.19 \mathrm{E}-04$ & $2.09 \mathrm{E}+00$ & $4.76 \mathrm{E}+02$ & $1,41 \mathrm{E}-02$ & $4.18 \mathrm{E}-03$ & $7.37 \mathrm{E}+01$ & $1.67 E+04$ \\
\hline & 250 & $2.50 E-05$ & $7.41 \mathrm{E}-06$ & $1.31 \mathrm{E}-01$ & $2.97 E+01$ & $3.56 \mathrm{E}-03$ & $1.05 \mathrm{~B}-03$ & $1.86 \mathrm{E}+01$ & $4.22 E+03$ \\
\hline & 500 & $3.07 E-06$ & $9.09 \mathrm{E}-07$ & $1.60 \mathrm{E}-02$ & $3.64 \mathrm{E}+00$ & $1.25 E-03$ & $3.71 \mathrm{E}-04$ & $6.54 \mathrm{E}+00$ & $1.49 \mathrm{E}+03$ \\
\hline GS-011 & 10 & *3UK-01 & $2.84 \mathrm{E}-02$ & $1.25 E+04$ & $6.83 E+05$ & $2.15 E+00$ & $1.42 \mathrm{E}-01$ & $6.24 E+04$ & $3.42 \mathrm{E}+06$ \\
\hline & 50 & $.72 E-02$ & $1.14 \mathrm{E}-03$ & $4.99 E+02$ & $2.73 E+04$ & $8.61 \mathrm{E}-02$ & $5.68 \mathrm{E}-03$ & $2.50 \mathrm{E}+03$ & $1.37 \mathrm{E}+05$ \\
\hline & 100 & $.24 \mathrm{E}-04$ & $4.77 \mathrm{E}-0.5$ & $2.10 \mathrm{E}+01$ & $1.1 .5 E+03$ & $2.55 \mathrm{E}-02$ & $1.68 \mathrm{E}-03$ & $7.39 E+02$ & $4.05 \mathrm{E}+04$ \\
\hline & 250 & $4.52 \mathrm{E}-05$ & $2.98 \mathrm{E}-06$ & $1.31 \Xi+00$ & $7.17 E+01$ & $6.42 \mathrm{E}-03$ & $4.23 \mathrm{E}-04$ & $1.86 \mathrm{E}+02$ & $1.02 \mathrm{E}+04$ \\
\hline & 500 & $5.54 \mathrm{E}-06$ & $3.65 \mathrm{E}-07$ & $1.61 \mathrm{E}-01$ & $8.79 \mathrm{~B}+00$ & $2.26 \mathrm{E}-03$ & $1.49 \mathrm{E}-04$ & $6.56 \mathrm{E}+01$ & $3.59 \mathrm{E}+03$ \\
\hline 3-012 & 10 & -01 & $4.48 E-01$ & $1.78 \mathrm{E}+04$ & $2.09 E+06$ & $2.16 \mathrm{E}+00$ & $2.24 E+00$ & $8.92 \mathrm{E}+04$ & $1.04 E+07$ \\
\hline & 50 & $1.97 \mathrm{E}-02$ & $1.79 \mathrm{E}-02$ & $7.13 E+02$ & $8.35 \mathrm{E}+04$ & $9.85 \mathrm{E}-02$ & $8.95 \mathrm{E}-02$ & $3.57 \mathrm{E}+03$ & $4.18 \mathrm{E}+05$ \\
\hline & 100 & $8.28 \mathrm{E}-04$ & $7.53 \mathrm{~B}-04$ & $3.00 \mathrm{E}+02$ & $.51 E+03$ & $2.92 \mathrm{E}-02$ & $2.65 \mathrm{E}-02$ & $1.06 E+03$ & $1.24 E+05$ \\
\hline & 250 & $5.17 \mathrm{E}-05$ & $4.70 \mathrm{E}-05$ & $1.878+00$ & $9 \mathrm{E}+02$ & 7.34 & $6.67 \mathrm{E}-03$ & $2.66 \mathrm{E}+02$ & $3.11 E+04$ \\
\hline & 500 & $6.33 E-06$ & $5.76 \mathrm{E}-06$ & $2.29 \mathrm{E}-01$ & $2.69 E+01$ & $2.59 E-03$ & $2.35 \mathrm{E}-03$ & $9.37 E+01$ & $1.10 \mathrm{E}+0 \mathrm{~A}$ \\
\hline
\end{tabular}


Appendix D

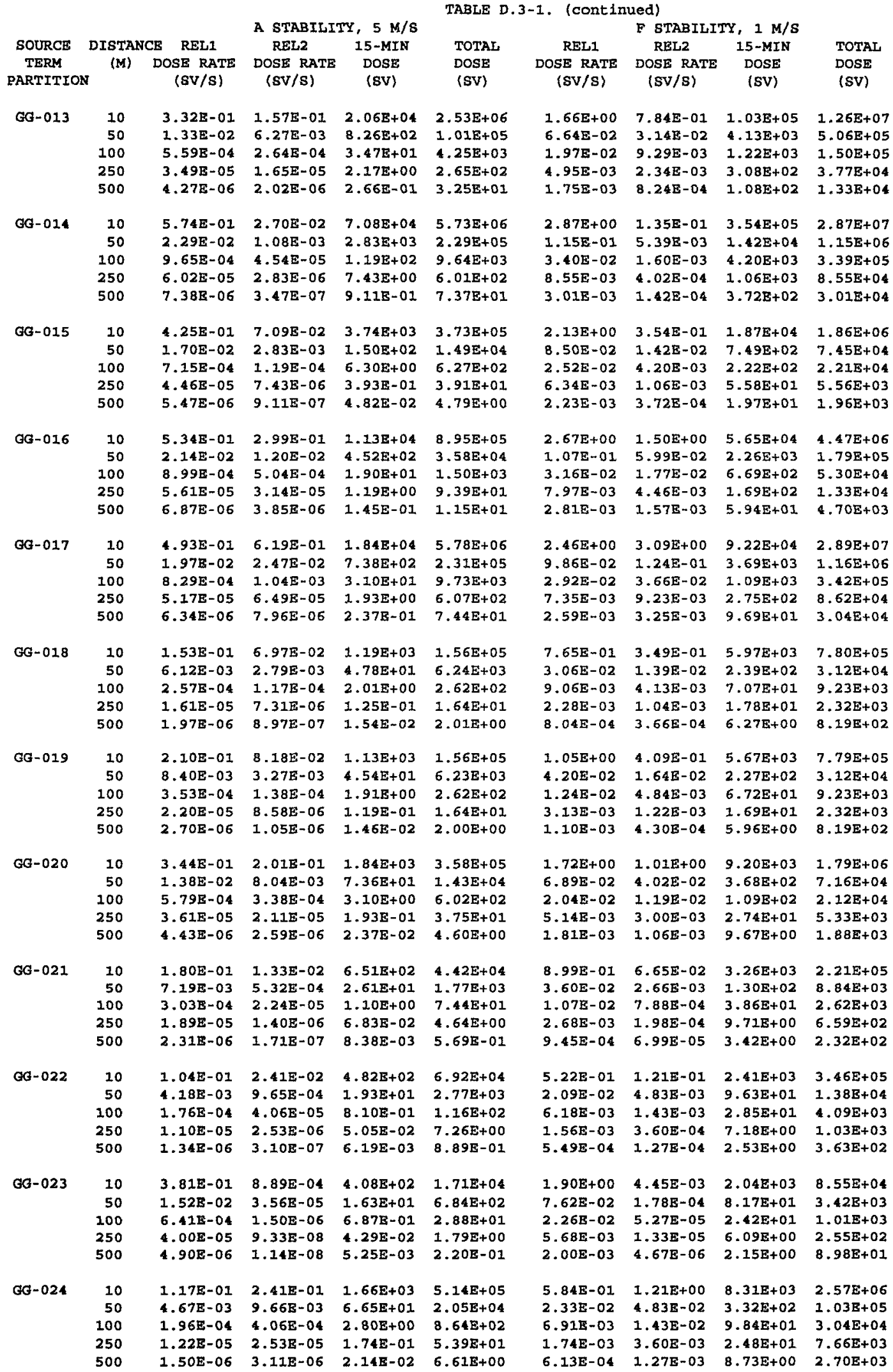

Vol. 6, Part 1

D-13

NUREG/CR-6143 
Appendix D

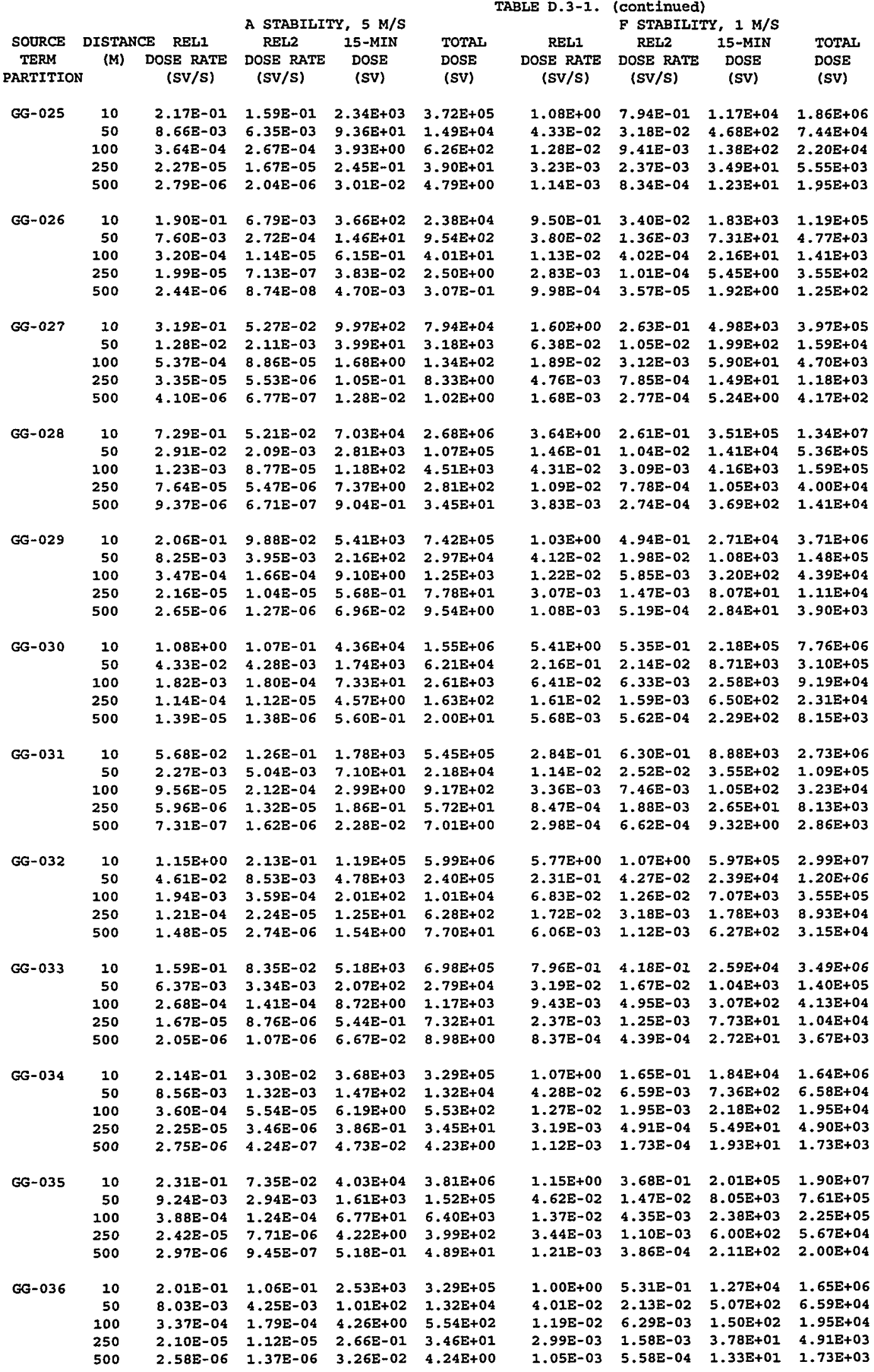




\begin{tabular}{|c|c|c|c|c|c|c|c|c|c|}
\hline & \multirow[b]{2}{*}{ DISTANCE } & \multicolumn{8}{|c|}{ TABLE D.3-1. (continued) } \\
\hline & & DE REL1 & REL2 & I5-MIN & TOTAL & RELI & REL2 & 15-MIN & TOTAI \\
\hline $\begin{array}{c}\text { TERM } \\
\text { EARTITION }\end{array}$ & (M) & $\begin{array}{c}\text { DOSE RATE } \\
(\mathrm{SV} / \mathrm{S})\end{array}$ & $\begin{array}{c}\text { DOSE RATE } \\
(\mathrm{SV} / \mathrm{S})\end{array}$ & $\begin{array}{l}\text { DOSE } \\
\text { (SV) }\end{array}$ & $\begin{array}{l}\text { DOSE } \\
\text { (SV) }\end{array}$ & $\begin{array}{c}\text { DOSE RATE } \\
(S V / S)\end{array}$ & $\begin{array}{c}\text { DOSE RATE } \\
(\mathrm{SV} / \mathrm{S})\end{array}$ & $\begin{array}{c}\text { DOSE } \\
(S V)\end{array}$ & $\begin{array}{l}\text { DOSE } \\
\text { (SV) }\end{array}$ \\
\hline \multirow[t]{5}{*}{ GG-037 } & 10 & $1.25 E-01$ & $3.89 \mathrm{E}-02$ & $1.58 \mathrm{E}+03$ & $1.50 E+05$ & $6.27 E-01$ & $1.95 \mathrm{E}-01$ & $7.92 \mathrm{E}+03$ & $7.49 \mathrm{E}+05$ \\
\hline & 50 & $5.01 E-03$ & $1.56 \mathrm{E}-03$ & $6.34 E+01$ & $5.99 E+03$ & $2.51 E-02$ & $7.78 \mathrm{E}-03$ & $3.17 \mathrm{E}+02$ & $3.00 E+04$ \\
\hline & 100 & $2.11 \mathrm{E}-04$ & $6.54 \mathrm{E}=05$ & $2.66 \mathrm{E}+00$ & $2.52 E+02$ & $7.42 \mathrm{E}-03$ & $2.30 E-03$ & $9.38 E+01$ & $8.87 \mathrm{E}+03$ \\
\hline & 250 & $1.31 \mathrm{E}-05$ & $4.08 E-06$ & $1.66 \mathrm{E}-01$ & $1.57 \mathrm{E}+01$ & $1.87 \mathrm{E}-03$ & $5.80 E-04$ & $2.36 E+01$ & $2.23 E+03$ \\
\hline & 500 & $1.61 \mathrm{E}-06$ & $5.00 \mathrm{E}-07$ & $2.04 \mathrm{E}-02$ & $1.93 \mathrm{E}+00$ & $6.58 \mathrm{E}-04$ & $2.04 \mathrm{E}-04$ & $8.32 E+00$ & $7.87 E+02$ \\
\hline \multirow[t]{5}{*}{ GG-038 } & 10 & $9.73 E-02$ & $1.62 \mathrm{E}-01$ & $5.02 \mathrm{E}+03$ & $9.54 \mathrm{E}+05$ & $4.86 E-01$ & $8.08 \mathrm{E}-01$ & $2.51 E+04$ & $4.77 E+06$ \\
\hline & 50 & $3.89 \mathrm{E}-03$ & $6.47 \mathrm{E}-03$ & $2.01 E+02$ & $3.82 \mathrm{E}+04$ & $1.95 \mathrm{E}-02$ & $3.23 \mathrm{E}-02$ & $1.00 \mathrm{E}+03$ & $1.91 E+05$ \\
\hline & 100 & $1.64 \mathrm{E}-04$ & $2.72 \mathrm{E}-04$ & $8.44 E+00$ & $1.60 \mathrm{E}+03$ & $5.76 \mathrm{E}-03$ & $9.57 \mathrm{E}-03$ & $2.97 \mathrm{E}+02$ & $5.65 \mathrm{E}+04$ \\
\hline & 250 & $1.02 \mathrm{E}-05$ & $1.70 \mathrm{E}-05$ & $5.27 \mathrm{E}-01$ & $1.00 \mathrm{E}+02$ & $1.45 E-03$ & $2.41 \mathrm{E}-03$ & $7.48 E+01$ & $1.42 \mathrm{E}+04$ \\
\hline & 500 & $1.25 \mathrm{E}-06$ & $2.08 \mathrm{E}-06$ & $6.46 \mathrm{E}-02$ & $1.23 \mathrm{E}+01$ & $5.11 E-04$ & $8.49 \mathrm{E}-04$ & $2.64 E+01$ & $5.01 E+03$ \\
\hline \multirow[t]{5}{*}{$G G-039$} & 10 & $1.03 \mathrm{E}-01$ & $7.26 \mathrm{E}-02$ & $9.06 \mathrm{E}+03$ & $2.06 \mathrm{E}+06$ & $5.14 E-01$ & $3.63 \mathrm{E}-01$ & $4.53 E+04$ & $5.29 E+06$ \\
\hline & 50 & $4.11 E-03$ & $2.90 \mathrm{E}-03$ & $3.63 E+02$ & $4.23 E+04$ & $2.06 \mathrm{E}-02$ & $1.45 \mathrm{E}-02$ & $1.81 E+03$ & $2.12 E+05$ \\
\hline & 100 & $1.73 E-04$ & $1.22 \mathrm{E}-04$ & $1.52 \mathrm{E}+01$ & $1.78 \mathrm{E}+03$ & $6.08 \mathrm{E}-03$ & $4.30 \mathrm{E}-03$ & $5.37 \mathrm{E}+02$ & $6.27 E+04$ \\
\hline & 250 & $1.08 \mathrm{E}-05$ & $7.61 \mathrm{E}-06$ & $9.51 E-01$ & $1.11 E+02$ & $1.53 \mathrm{E}-03$ & $1.08 \mathrm{E}-03$ & $1.35 E+02$ & $1.58 \mathrm{E}+04$ \\
\hline & 500 & $1.32 \mathrm{E}-06$ & $9.33 \mathrm{E}-07$ & 1.17 E-01 & $1.36 \mathrm{E}+01$ & $5.40 \mathrm{E}-04$ & $3.81 E-04$ & $4.76 \mathrm{E}+01$ & $5.56 \mathrm{E} \div 03$ \\
\hline \multirow[t]{5}{*}{ GG-040 } & 10 & $1.14 E-0 I$ & $8.33 E-02$ & $2.58 E+03$ & $3.94 E+05$ & $5.70 \mathrm{E}-01$ & $4.17 E-01$ & $1.29 \mathrm{E}+04$ & $1.97 \mathrm{E}+06$ \\
\hline & 50 & $4.56 \mathrm{E}-03$ & $3.33 E-03$ & $1.03 E+02$ & $1.58 \mathrm{E}+04$ & $2.28 \mathrm{E}-02$ & $1.67 E-02$ & $5.16 \mathrm{E}+02$ & $7.88 E+04$ \\
\hline & 100 & $1.92 \mathrm{E}-04$ & $1.40 E-04$ & $4.34 E+00$ & $6.63 E+02$ & $6.75 E-03$ & $4.93 E-03$ & $1.53 \mathrm{E}+02$ & $2.33 E+04$ \\
\hline & 250 & $1.20 \mathrm{E}-05$ & $8.74 E-06$ & $2.70 \mathrm{E}-01$ & $4.14 E+01$ & 1.70E-03 & $1.24 E-03$ & $3.84 E+01$ & $5.88 E+03$ \\
\hline & 500 & $1.47 \mathrm{E}-06$ & $1.07 \mathrm{E}-06$ & $3.32 E-02$ & $5.07 E+00$ & $5.99 \mathrm{E}-04$ & $4.38 \mathrm{E}-04$ & $1.35 E+01$ & $2.07 E+03$ \\
\hline \multirow[t]{5}{*}{ GG-041 } & 10 & $6.02 \mathrm{E}-02$ & $6.24 \mathrm{E}-02$ & $1.31 E+03$ & $2.17 E+05$ & $3.01 \mathrm{E}-01$ & $3.12 E-01$ & $6.56 \mathrm{E}+03$ & $1.09 E+06$ \\
\hline & 50 & $2.41 E-03$ & $2.50 \mathrm{E}-03$ & $5.25 E+01$ & $8.69 E+03$ & $1.20 \mathrm{E}-02$ & $1.25 \mathrm{E}-02$ & $2.62 E+02$ & $4.34 \mathrm{E}+04$ \\
\hline & 100 & $1.01 \mathrm{E}-04$ & $1.05 E-04$ & $2.21 \mathrm{E}+00$ & $3.65 \mathrm{E}+02$ & $3.56 \mathrm{E}-03$ & $3.708-03$ & $7.77 \mathrm{E}+01$ & $1.29 E+04$ \\
\hline & 250 & $6.32 \mathrm{E}-06$ & $6.55 \mathrm{E}-06$ & $1.38 \mathrm{E}-01$ & $2.28 E+01$ & $8.98 \mathrm{E}-04$ & $9.31 E-04$ & $1.96 \mathrm{E}+01$ & $3.24 E+03$ \\
\hline & 500 & $7.74 \mathrm{E}-07$ & $8.03 E-07$ & $1.69 E-02$ & $2.79 E+00$ & $3.16 \mathrm{E}-04$ & $3.28 E-04$ & $6.89 \mathrm{E}+00$ & $1.14 \mathrm{E}+03$ \\
\hline \multirow[t]{5}{*}{ GG-042 } & 10 & $3.39 \mathrm{E}-02$ & $2.13 E-02$ & $3.78 \mathrm{E}+02$ & $6.49 \mathrm{E}+04$ & $1.69 \mathrm{E}-01$ & $1.06 \mathrm{E}-01$ & $1.89 \mathrm{E}+03$ & $3.24 E+05$ \\
\hline & 50 & $1.36 \mathrm{E}-03$ & $8.52 \mathrm{E}-04$ & $1.51 E+01$ & $2.60 \mathrm{E}+03$ & $6.78 \mathrm{E}-03$ & $4.26 \mathrm{E}-03$ & $7.56 \mathrm{E}+01$ & $1.30 E+04$ \\
\hline & 100 & $5.70 \mathrm{E}-05$ & $3.58 \mathrm{E}-05$ & $6.35 E-01$ & $1.09 \mathrm{E}+02$ & $2.01 \mathrm{E}-03$ & $1.26 \mathrm{E}-03$ & $2.24 E+01$ & $3.84 E+03$ \\
\hline & 250 & $3.56 \mathrm{E}-06$ & $2.23 E-06$ & $3.96 \mathrm{E}-02$ & $6.81 E+00$ & $5.05 E-04$ & $3.13 \mathrm{E}-04$ & $5.63 \mathrm{E}+00$ & $9.68 \mathrm{E}+02$ \\
\hline & 500 & $4.36 \mathrm{E}-07$ & $2.74 \mathrm{E}-07$ & $4.86 \mathrm{E}-03$ & $8.35 E-01$ & $1.78 E-04$ & $1.12 \mathrm{E}-04$ & $1.98 \mathrm{E}+00$ & $3.41 E+02$ \\
\hline \multirow[t]{5}{*}{ GG-043 } & 10 & $3.55 \mathrm{E}-02$ & $1.81 \mathrm{E}-01$ & $1.78 \mathrm{E}+03$ & $5.41 E+05$ & 1.78E-01 & $9.05 \mathrm{E}-01$ & $8.88 E+03$ & $2.70 E+06$ \\
\hline & 50 & $1.42 \mathrm{E}-03$ & $7.24 \mathrm{E}-03$ & $7.10 \mathrm{E}+01$ & $2.16 E+04$ & $7.11 \mathrm{E}-03$ & $3.62 \mathrm{E}-02$ & $3.55 E+02$ & $1.08 \mathrm{E}+05$ \\
\hline & 100 & $5.98 \mathrm{E}-05$ & $3.04 \mathrm{E}-04$ & $2.99 E+00$ & $9.10 \mathrm{E}+02$ & $2.10 \mathrm{E}-03$ & $1.07 \pm-02$ & $1.05 E+02$ & $3.20 E+04$ \\
\hline & 250 & $3.73 E-06$ & $1.90 \mathrm{E}-05$ & $1.86 \mathrm{E}-01$ & $5.67 E+01$ & $5.30 E-04$ & $2.70 E-03$ & $2.65 E+01$ & $8.06 E+03$ \\
\hline & 500 & $4.57 \mathrm{E}-07$ & $2.33 E-06$ & $2.28 \mathrm{E}-02$ & $6.96 \mathrm{E}+00$ & $1.87 \mathrm{E}-04$ & $9.50 \mathrm{E}-04$ & $9.33 \mathrm{E}+00$ & $2.84 E+03$ \\
\hline \multirow[t]{5}{*}{ GG-044 } & 10 & $5.46 \mathrm{E}-02$ & $1.74 E-02$ & $6.52 \mathrm{E}+02$ & $7.25 \mathrm{E}+04$ & $2.73 E-01$ & 8.71E-02 & $3.26 E+03$ & $3.63 \mathrm{E}+05$ \\
\hline & 50 & $2.18 \mathrm{E}-03$ & $6.96 \mathrm{E}-04$ & $2.61 E+01$ & $2.90 \mathrm{E}+03$ & $1.09 \mathrm{E}-02$ & $3.48 \mathrm{E}-03$ & $1.30 E+02$ & $1.45 \mathrm{E}+04$ \\
\hline & 100 & $9.19 \mathrm{E}-05$ & $2.93 E-05$ & $1.10 \mathrm{E}+00$ & $1.22 \mathrm{E}+02$ & $3.23 E-03$ & $1.03 \mathrm{E}-03$ & $3.86 \mathrm{E}+01$ & $4.29 E+03$ \\
\hline & 250 & $5.73 \mathrm{E}-06$ & $1.83 E-06$ & $6.84 \mathrm{E}-02$ & $7.61 E+00$ & $8.14 E-04$ & $2.60 E-04$ & $9.73 E \div 00$ & $1.08 E+03$ \\
\hline & 500 & $7.02 \mathrm{E}-07$ & $2.24 E-07$ & $8.39 E-03$ & $9.33 \mathrm{E}-01$ & $2.87 E-04$ & $9.15 E-05$ & $3.43 E+00$ & $3.81 E+02$ \\
\hline GG-045 & 10 & $3.35 E-02$ & $8.36 \mathrm{E}-02$ & $7.84 E+02$ & $2.09 E+05$ & $1.68 \mathrm{E}-01$ & $4.18 \mathrm{E}-01$ & $3.92 \mathrm{E}+03$ & $1.05 \mathrm{E}+06$ \\
\hline & 50 & $1.34 \mathrm{E}-03$ & $3.35 \mathrm{E}-03$ & $3.14 E+01$ & $8.36 E+03$ & $6.70 \mathrm{E}-03$ & $1.67 E-02$ & $1.57 E+02$ & $4.18 E+04$ \\
\hline & 100 & $5.64 \mathrm{E}-05$ & $1.41 E-04$ & $1.32 \mathrm{E}+00$ & $3.52 \mathrm{E}+02$ & $1.98 \mathrm{E}-03$ & $4.95 \mathrm{E}-03$ & $4.64 E+01$ & $1.24 \mathrm{E}+04$ \\
\hline & 250 & $3.52 \mathrm{E}-06$ & $8.77 E-06$ & $8.23 E-02$ & $2.19 E+01$ & $5.00 \mathrm{E}-04$ & $1.25 \mathrm{E}-03$ & $1.17 \mathrm{E}+01$ & $3.12 \mathrm{E}+03$ \\
\hline & 500 & $4.31 E-07$ & $1.08 \mathrm{E}-06$ & $1.01 E-02$ & $2.69 \mathrm{E}+00$ & $1.76 \mathrm{E}-04$ & $4.39 E-04$ & $4.12 \mathrm{E}+00$ & $1.10 \mathrm{E}+03$ \\
\hline GG-046 & 10 & $2.79 \mathrm{E}-02$ & $4.18 \mathrm{E}-03$ & $1.09 \mathrm{E}+02$ & $1.61 \mathrm{E}+04$ & $40 E-01$ & $2.09 \mathrm{E}-02$ & $5.46 E+02$ & $8.04 \mathrm{E}+04$ \\
\hline & 50 & $1.12 \mathrm{E}-03$ & $1.67 \mathrm{E}-04$ & $4.37 E+00$ & $6.43 E+02$ & $5.59 E-03$ & B. 36E-04 & $2.18 E+01$ & $3.21 E+03$ \\
\hline & 100 & $4.70 \mathrm{E}-05$ & $7.03 E-06$ & $1.84 \mathrm{E}-01$ & $2.70 \mathrm{E}+01$ & $1.65 \mathrm{E}-03$ & $2.47 \mathrm{E}-04$ & $6.47 E+00$ & $9.52 E+02$ \\
\hline & 250 & $2.93 E-06$ & $4.38 \mathrm{E}-07$ & $1.15 \mathrm{E}-02$ & $1.69 \mathrm{E}+00$ & $4.17 E-04$ & $6.23 E-05$ & $1.63 \mathrm{E}+00$ & $2.40 E+02$ \\
\hline & 500 & $3.59 \mathrm{E}-07$ & $5.37 \mathrm{E}-08$ & $1.40 \mathrm{E}-03$ & $2.07 E-01$ & $1.47 \mathrm{E}-04$ & $2.19 E-05$ & $5.74 E-01$ & $8.44 \mathrm{E}+01$ \\
\hline GG-047 & 10 & $.43 \mathrm{E}-02$ & $1.10 \mathrm{E}-01$ & $1.18 \mathrm{E}+03$ & $2.90 \mathrm{E}+05$ & $1.22 \mathrm{E}-01$ & $5.49 \mathrm{E}-01$ & $5.92 \mathrm{E}+03$ & $1.45 \mathrm{E}+06$ \\
\hline & 50 & $9.74 E-04$ & $4.39 \mathrm{E}-03$ & $4.74 E+01$ & $1.16 \mathrm{E}+04$ & $4.87 E-03$ & $2.20 \mathrm{E}-02$ & $2.37 \mathrm{E}+02$ & $5.79 E+04$ \\
\hline & 100 & $4.09 \mathrm{E}-05$ & $1.85 \mathrm{E}-04$ & $1.99 E+00$ & $4.87 \mathrm{E}+02$ & $1.44 \mathrm{E}-03$ & $6.50 \mathrm{E}-03$ & $7.01 \mathrm{E}+01$ & $1.72 E+04$ \\
\hline & 250 & $2.55 E-06$ & $1.15 \mathrm{E}-05$ & $1.24 E-01$ & $3.04 E+01$ & $3.63 \mathrm{E}-04$ & $1.64 \mathrm{E}-03$ & $1.77 \mathrm{E}+01$ & $4.32 E+03$ \\
\hline & 500 & $3.13 \mathrm{E}-07$ & $1.41 E-06$ & $1.52 E-02$ & $3.73 \mathrm{E}+00$ & $1.28 \mathrm{E}-04$ & $5.77 E-04$ & $6.22 E+00$ & $1.52 \mathrm{E}+03$ \\
\hline GG-048 & 10 & $3.68 \mathrm{E}-02$ & $7.22 \mathrm{E}-02$ & $8.47 E+02$ & $2.52 \mathrm{E}+05$ & $1.84 \mathrm{E}-01$ & $3.61 \mathrm{E}-01$ & $4.24 E+03$ & $1.26 E+06$ \\
\hline & 50 & $1.47 \mathrm{E}-03$ & $2.89 \mathrm{E}-03$ & $3.39 E+01$ & $1.01 \mathrm{E}+04$ & $7.37 \mathrm{E}-03$ & $1.44 \mathrm{E}-02$ & $1.69 \mathrm{E}+02$ & $5.04 \mathrm{E}+04$ \\
\hline & 100 & $6.19 \mathrm{E}-05$ & $1.21 E-04$ & $1.42 \mathrm{E}+00$ & $4.23 E+02$ & $2.18 E-03$ & $4.27 \mathrm{E}-03$ & $5.02 E+01$ & $1.49 E+04$ \\
\hline & 250 & $3.86 \mathrm{E}-06$ & $7.57 \mathrm{E}-06$ & $8.89 E-02$ & $2.64 E+01$ & $5.49 \mathrm{E}-04$ & $1.08 \mathrm{E}-03$ & $1.26 \mathrm{E}+01$ & $3.75 E+03$ \\
\hline & 500 & $4.74 E-07$ & $9.28 \mathrm{E}-07$ & $1.09 \mathrm{E}-02$ & $3.24 \mathrm{E}+00$ & $1.93 \mathrm{E}-04$ & $3.79 E-04$ & $4.45 E+00$ & $1.32 \mathrm{E}+03$ \\
\hline
\end{tabular}


Appendix D

TABLE D.3-1. (continued)

\begin{tabular}{|c|c|c|c|c|c|c|c|c|c|}
\hline & & & & & TABL & $3-1 . \quad(c$ & inued) & & \\
\hline & & & A $\mathrm{STA}$ & ABILITY, 5 & $\mathrm{M} / \mathrm{s}$ & & F STR & ABILITY, & $4 / 8$ \\
\hline SOURCE & DISTANCE & REL1 & REL2 & 15 -MIN & TOTAL & REL1 & REL2 & 15-MIN & TOTAL \\
\hline TERM & (M) & DOSE RATE & DOSE RATE & DOSE & DOSE & SE RATE & OSE RATE & DOSE & DOSE \\
\hline PARTITION & & $(\mathrm{SV} / \mathrm{s})$ & $(\mathrm{sv} / \mathrm{s})$ & (SV) & $(s v)$ & (sv/s) & $(s v / s)$ & (sv) & (sv) \\
\hline GG-049 & 10 & $1.22 \mathrm{E}-02$ & $2.02 \mathrm{E}-03$ & $4.08 E+04$ & $3.58 \mathrm{E}+06$ & $6.08 \mathrm{E}-02$ & $1.01 \mathrm{E}-02$ & $2.04 E+05$ & $1.79 \mathrm{E}+07$ \\
\hline & 50 & $4.86 \mathrm{E}-04$ & 8.08E-05 & $1.63 E+03$ & $1.43 E+05$ & $2.43 E-03$ & $4.04 E-04$ & $8.15 E+03$ & $7.16 \mathrm{E}+05$ \\
\hline & 100 & $2.04 \mathrm{E}-05$ & $3.40 \mathrm{E}-06$ & $6.86 \mathrm{E}+01$ & $6.02 \mathrm{E}+03$ & $7.20 E-04$ & $1.20 \mathrm{E}-04$ & $2.41 \mathrm{E}+03$ & $2.12 E+05$ \\
\hline & 250 & $1.28 \mathrm{E}-06$ & $2.12 \mathrm{E}-07$ & $4.28 E+00$ & $3.76 \mathrm{E}+02$ & $1.81 \mathrm{E}-04$ & $3.01 \mathrm{E}-0.5$ & $6.08 E+02$ & $5.34 E+04$ \\
\hline & 500 & $1.56 \mathrm{E}-07$ & $2.608-08$ & $5.24 \mathrm{E}-02$ & $4.61 \mathrm{E}+01$ & $6.38 \mathrm{E}-0.5$ & $1.06 \mathrm{E}-05$ & $2.14 E+02$ & $1.8 B E+04$ \\
\hline GG-050 & 10 & $6.05 E-03$ & $1.45 \mathrm{E}-03$ & $1.44 E+04$ & $1.41 E+06$ & $3.03 E-02$ & $7.27 E-03$ & $7.20 \mathrm{E}+04$ & $7.05 E+06$ \\
\hline & 50 & $2.42 \mathrm{E}-04$ & $5.82 \mathrm{E}-05$ & $5.76 E+02$ & $5.64 \mathrm{E}+04$ & $1.21 E-03$ & $2.91 E-04$ & $2.88 \mathrm{E}+03$ & $2.82 \mathrm{~B}+05$ \\
\hline & 100 & $1.02 E-05$ & $2.45 E-06$ & $2.42 E+01$ & $2.37 E+03$ & $3.58 \mathrm{E}-04$ & $8.61 E-05$ & $8.53 E+02$ & $8.35 E+04$ \\
\hline & 250 & $6.35 E-07$ & $1.53 \mathrm{E}-07$ & $1.51 E+00$ & $1.48 E+02$ & $9.02 \mathrm{E}-05$ & $2.17 E-05$ & $2.15 E+02$ & $2.10 E+04$ \\
\hline & 500 & $7.78 \mathrm{E}-08$ & $1.87 \mathrm{E}-08$ & $1.85 \mathrm{E}-01$ & $1.81 E+01$ & $3.18 \mathrm{E}-05$ & $7.64 \mathrm{E}-06$ & $7.57 \mathrm{E}+01$ & $7.40 \mathrm{E}+03$ \\
\hline GG-051 & 10 & $5.16 \mathrm{E}-03$ & $2.56 \mathrm{E}-03$ & $5.72 E+0.3$ & $8.32 \mathrm{E}+0.5$ & $2.58 \mathrm{E}-02$ & $1.28 \mathrm{E}-02$ & $2.86 \mathrm{E}+04$ & $4.16 \mathrm{E}+06$ \\
\hline & 50 & $2.06 \mathrm{E}-04$ & $1.03 E-04$ & $2.29 E+02$ & $3.33 \mathrm{E}+04$ & $1.03 E-03$ & $5.13 \mathrm{E}-04$ & $1.14 \mathrm{E}+03$ & $1.66 \mathrm{E}+05$ \\
\hline & 100 & $8.67 \mathrm{E}-06$ & $4.32 \mathrm{~s}-06$ & $9.61 \mathrm{E}+00$ & $1.40 \mathrm{E}+03$ & $3.05 \mathrm{E}-04$ & $1.52 \mathrm{E}-04$ & $3.38 \mathrm{E}+02$ & $4.93 E+04$ \\
\hline & 250 & $5.41 E-07$ & $2.69 \mathrm{E}-07$ & $5.99 E-01$ & $8.73 E+01$ & $7.69 \mathrm{E}-0.5$ & $3.82 \mathrm{E}-05$ & 8. $52 \mathrm{E}+01$ & $1.24 E+04$ \\
\hline & 500 & $6.63 E-08$ & $3.30 \mathrm{E}-08$ & $7.35 E-02$ & $1.07 \mathrm{E}+01$ & $2.71 E-05$ & $1.35 \mathrm{E}-05$ & $3.00 E+0 I$ & $4.37 E+03$ \\
\hline GG-052 & 10 & $1.22 E-03$ & $7.47 \mathrm{E}-04$ & $8.41 E+02$ & $1.22 \mathrm{E}+05$ & $6.07 E-03$ & $3.74 \mathrm{E}-03$ & $4.20 \mathrm{E}+03$ & $6.12 \mathrm{E}+05$ \\
\hline & 50 & $4.86 E-05$ & $2.99 E-05$ & $3.36 E+01$ & $4.90 \mathrm{E}+03$ & $2.43 \mathrm{E}-04$ & $1.49 E-04$ & $1.68 \mathrm{E}+02$ & $2.45 E+04$ \\
\hline & 100 & $2.04 E-06$ & $1.26 \mathrm{E}-06$ & $1.41 \mathrm{E}+00$ & $2.06 E+02$ & $7.19 \mathrm{E}-05$ & $4.42 E-05$ & $4.98 E+01$ & $7.25 E+03$ \\
\hline & 250 & $1.27 E-07$ & $7.84 \mathrm{E}-08$ & 8.82E-02 & 1. $28 \mathrm{E}+01$ & $1.81 E-05$ & $1.11 E-0.5$ & $1.25 \mathrm{E}+01$ & $1.82 \mathrm{E}+03$ \\
\hline & 500 & $1.56 \mathrm{E}-08$ & $9.61 \mathrm{E}-09$ & $1.08 \mathrm{E}-02$ & $1.57 E+00$ & $6.38 \mathrm{E}-06$ & $3.93 E-06$ & $4.42 \mathrm{E}+00$ & $6.43 \mathrm{E}+02$ \\
\hline GG-053 & 10 & $2.69 E-04$ & $1.87 E-04$ & 1. $58 \mathrm{E}+02$ & $2.08 \mathrm{E}+04$ & $1.35 E-03$ & $9.36 \mathrm{E}-04$ & $7.92 \mathrm{E} \div 02$ & $1.04 \mathrm{E}+05$ \\
\hline & 50 & $1.08 \mathrm{E}-05$ & $7.48 \mathrm{E}-06$ & $6.34 \mathrm{E}+00$ & $8.34 \mathrm{E}+02$ & $5.38 \mathrm{E}-0.5$ & $3.74 \mathrm{E}-05$ & $3.17 \mathrm{E}+01$ & $4.17 E+03$ \\
\hline & 100 & $4.53 E-07$ & $3.15 \mathrm{E}-07$ & $2.66 \mathrm{E}-01$ & $3.51 E+01$ & $1.59 E-05$ & $1.11 E-05$ & $9.38 \mathrm{E}+00$ & $1.23 E+03$ \\
\hline & 250 & $2.82 E-08$ & $1.96 E-08$ & $1.6 .6 \mathrm{~B}-02$ & $2.19 \mathrm{E}+00$ & 4.01E-06 & $2.79 \mathrm{E}-06$ & $2.36 E \div 00$ & 3.11E+02 \\
\hline & 500 & $3.46 E-09$ & $2.41 \mathrm{E}-09$ & $2.04 \mathrm{E}-0.3$ & $2.68 \mathrm{E}-01$ & $1.41 E-06$ & $9.83 E-07$ & $8.32 \mathrm{E}-01$ & $1.09 \mathrm{E}+02$ \\
\hline GG-054 & 10 & $6.87 E-05$ & $4.96 \mathrm{~g}-05$ & $2.74 E+O I$ & $3.00 \mathrm{E}+03$ & $3.44 \mathrm{E}-04$ & $2.48 \mathrm{E}-04$ & $1.37 \mathrm{E}+02$ & $1.50 \mathrm{E}+04$ \\
\hline & 50 & $2.75 E-06$ & $1.98 \mathrm{~B}-06$ & $1.10 E+00$ & $1.20 \mathrm{E}+02$ & $1.37 E-05$ & $9.91 \mathrm{E}-06$ & $5.48 \mathrm{E}+00$ & $6.00 E+02$ \\
\hline & 100 & $1.16 \mathrm{E}-07$ & $8.33 E-08$ & $4.61 \mathrm{E}-02$ & $5.05 \mathrm{E}+00$ & $4.07 \mathrm{E}-06$ & $2.93 \mathrm{E}-06$ & $1.62 \mathrm{E}+00$ & 1. $78 \mathrm{E}+02$ \\
\hline & 250 & $7.21 E-09$ & $5.20 E-09$ & $2.87 \mathrm{E}-0.3$ & $3.15 E-01$ & $1.02 \mathrm{E}-06$ & $7.39 E-07$ & $4.08 \mathrm{E}-01$ & $4.47 \mathrm{E}+01$ \\
\hline & 500 & $8.84 E-10$ & $6.378-10$ & $3.52 E-04$ & $3.86 \mathrm{E}-02$ & $3.61 \mathrm{E}-07$ & $2.60 E-07$ & $1.44 E-01$ & $1.58 E+01$ \\
\hline GG-055 & 10 & $0.00 E+00$ & $0.00 \mathrm{E}+00$ & $0.00 \mathrm{E}+00$ & $0.00 \mathrm{E}+00$ & $0.00 E+00$ & $0.00 E+00$ & $0.00 E+00$ & $0.00 \mathrm{~B}+00$ \\
\hline & 50 & $0.00 \mathrm{E}+00$ & $0.00 \mathrm{E}+00$ & $0.00 E+00$ & $0.00 \mathrm{E}+00$ & $0.00 E+00$ & $0.00 \mathrm{E}+00$ & $0.00 \mathrm{E}+00$ & $0.00 E+00$ \\
\hline & 100 & $0.00 \mathrm{E}+00$ & $0.00 \mathrm{E}+00$ & $0.00 \mathrm{E}+00$ & $0.00 \mathrm{E}+00$ & $0.00 \mathrm{E}+00$ & $0.00 E+00$ & $0.00 \mathrm{E}+00$ & $0.00 E+00$ \\
\hline & 250 & $0.00 E+00$ & $0.008+00$ & $0.00 \mathrm{E}+00$ & $0.00 E+00$ & $0.00 E+00$ & $0.00 E+00$ & $0.00 E+00$ & $0.00 \mathrm{E}+00$ \\
\hline & 500 & $0.00 E+00$ & $0.00 \mathrm{E}+00$ & $0.00 E+00$ & $0.00 E+00$ & $0.00 \mathrm{E}+00$ & $0.00 \mathrm{E}+00$ & $0.00 \mathrm{E}+00$ & $0.00 \mathrm{E}+00$ \\
\hline
\end{tabular}

NUREG/CR-6143

D-16

Vol. 6, Part 1 
Appendix D

TABLE D.3-2. Dose and Dose Rates Calculated Using Ramsdell Model

A STABILITY, $5 \mathrm{M} / \mathrm{S}$

\begin{tabular}{|c|c|c|c|c|c|c|c|c|c|}
\hline \multirow{2}{*}{\multicolumn{2}{|c|}{ SOURCE DISTANCE }} & \multicolumn{4}{|c|}{ A STABILITX, $5 \mathrm{M} / \mathrm{E}$} & & \multicolumn{3}{|c|}{ F STABILITY, $1 \mathrm{M} / \mathrm{S}$} \\
\hline & & CE RELI & REL2 & 15-MIN & TOTAL & REL, 1 & REL2 & 15-MIN & TOTAL \\
\hline \multirow{2}{*}{\multicolumn{2}{|c|}{ PARTITION }} & DOSE RATE & DOSE RATE & & DOSE & DOSE RATE & DOSE RATE & DOSE & DOSE \\
\hline & & & & & & & & & \\
\hline \multirow[t]{5}{*}{ GG-001 } & 20 & $4.398-03$ & $2.15 \mathrm{E}-03$ & $6.91 E+02$ & $9.12 \mathrm{E}+03$ & $3.21 \mathrm{E}-03$ & 1. $58 E-03$ & $5.06 \mathrm{E}+01$ & $6.68 \mathrm{E}+03$ \\
\hline & 50 & $7.12 \mathrm{E}-04$ & $3.49 \mathrm{E}-04$ & $1.12 \mathrm{E}+01$ & $1.48 \mathrm{E}+03$ & $5.21 \mathrm{E}-04$ & $2.56 \mathrm{E}-04$ & $8.21 E+00$ & $1.08 \mathrm{E}+03$ \\
\hline & 100 & $3.25 E-04$ & $1.60 \mathrm{E}-04$ & $5.12 \mathrm{E}+00$ & $6.76 \mathrm{E}+02$ & $2.38 \mathrm{~B}-04$ & $1.17 \mathrm{E}-04$ & $3.75 \mathrm{E}+00$ & $4.95 \mathrm{E}+02$ \\
\hline & 250 & $1.15 E-04$ & $5.67 \mathrm{E}-0.5$ & $1.82 \mathrm{E}+00$ & $2.40 E+02$ & $8.46 \mathrm{E}-05$ & $4.15 \mathrm{E}-05$ & $1.33 E+00$ & $1.76 \mathrm{E}+02$ \\
\hline & 500 & $5.28 \mathrm{E}-05$ & $2.59 E-05$ & $8.31 \mathrm{E}-01$ & $1.20 \mathrm{E}+02$ & $3.86 \mathrm{E}-05$ & $1.90 \mathrm{E}-05$ & $6.095-01$ & $8.03 E+01$ \\
\hline \multirow[t]{5}{*}{ GG-002 } & 10 & $5.03 \mathrm{E}-03$ & $1.78 \mathrm{E}-03$ & $1.34 \mathrm{E}+02$ & $1.61 \mathrm{E}+04$ & $3.68 \mathrm{E}-03$ & $1.31 \mathrm{E}-03$ & $9.84 \mathrm{E}+01$ & $1.18 \mathrm{E}+04$ \\
\hline & 50 & $8.16 \mathrm{E}-04$ & $2.89 \mathrm{E}-04$ & $2.18 E+01$ & $2.61 E+03$ & $5.98 E-04$ & $2.12 \mathrm{E}-04$ & $1.60 \mathrm{E}+01$ & $1.91 \mathrm{E}+03$ \\
\hline & 100 & $3.73 \mathrm{E}-04$ & $1.32 \mathrm{E}-04$ & $9.95 \mathrm{E}+00$ & $1.19 \mathrm{E}+03$ & $2.73 E-04$ & $9.67 \mathrm{E}-05$ & $7.29 \mathrm{E}+00$ & $8.75 \mathrm{E}+02$ \\
\hline & 250 & $1.32 \mathrm{E}-04$ & $4.69 \mathrm{E}-05$ & $3.53 \mathrm{E}+00$ & $4.24 E+02$ & $9.70 \mathrm{E}-05$ & $3.44 E-05$ & $2.595+00$ & $3.11 E+02$ \\
\hline & 500 & $6.05 \mathrm{E}-05$ & $2.14 \mathrm{E}-05$ & $1.62 \mathrm{E}+00$ & $1.94 \mathrm{E}+02$ & $4.43 E-05$ & $1.57 \mathrm{E}-0.5$ & $1.18 \mathrm{E}+00$ & $1.42 \mathrm{E}+02$ \\
\hline \multirow[t]{5}{*}{ GG-003 } & 10 & $2.09 \mathrm{E}-03$ & $2.65 E-03$ & $2.11 \mathrm{E}+01$ & $7.83 \mathrm{E}+03$ & $1.53 \mathrm{E}-03$ & $1.94 \mathrm{E}-03$ & $1.55 \mathrm{E}+01$ & $5.74 E+03$ \\
\hline & 50 & $3.39 E-04$ & $4.30 \mathrm{E}-04$ & $3.42 \mathrm{E}+00$ & $1.27 \mathrm{E}+03$ & $2.48 E-04$ & $3.25 \mathrm{E}-04$ & $2.51 E+00$ & $9.31 \mathrm{E}+02$ \\
\hline & 100 & $1.55 \mathrm{E}-04$ & $1.97 \mathrm{E}-04$ & $1.56 \mathrm{E}+00$ & $5.81 \mathrm{E}+02$ & $1.13 \mathrm{E}-04$ & $1.44 \mathrm{E}-04$ & $1.15 \mathrm{E}+00$ & $4.25 E+02$ \\
\hline & 250 & $5.50 \mathrm{E}-05$ & $6.98 E-05$ & $5.55 \mathrm{E}-01$ & $2.06 E+02$ & $4.03 E-05$ & $5.21 \mathrm{E}-05$ & $4.07 E-01$ & 1. $51 \mathrm{E}+02$ \\
\hline & 500 & $2.51 E-05$ & $3.29 \mathrm{E}-05$ & $2.54 \mathrm{E}-01$ & $9.42 E+01$ & $1.84 \mathrm{E}-05$ & $2.34 E-05$ & $1.86 \mathrm{E}-01$ & $6.90 \mathrm{E}+\mathrm{OI}$ \\
\hline \multirow[t]{5}{*}{$G G-004$} & 10 & $1.33 \mathrm{E}-02$ & $1.245-03$ & $7.14 E+02$ & $3.60 \mathrm{E}+04$ & $9.73 \mathrm{E}-03$ & $8.33 E-04$ & $5.23 \mathrm{E}+02$ & $2.63 E+04$ \\
\hline & 50 & $2.16 \mathrm{E}-03$ & $1.85 E-04$ & $1.16 \mathrm{E}+02$ & $5.83 \mathrm{E}+03$ & $1.58 \mathrm{E}-03$ & $1.35 E-04$ & $8.48 \mathrm{E}+01$ & $4.27 E+03$ \\
\hline & 100 & $9.85 \mathrm{E}-04$ & $8.43 E-05$ & $5.29 \mathrm{E}+01$ & $2.67 \mathrm{E}+03$ & $7.21 \mathrm{E}-04$ & $6.28 \mathrm{E}-05$ & $3.88 E+0.1$ & $1.95 E+03$ \\
\hline & 250 & $3.50 \mathrm{E}-04$ & $2.99 E-05$ & $1.88 E+01$ & $9.46 \mathrm{E}+02$ & $2.56 E-04$ & $2.19 \mathrm{E}-05$ & $1.38 E+01$ & $6.93 E+02$ \\
\hline & 500 & $1.60 \mathrm{E}-04$ & $1.37 \mathrm{E}-05$ & $8.58 E+00$ & $4.32 \mathrm{E}+02$ & $1.17 E-04$ & $1.00 \mathrm{E}-05$ & $6.29 E+00$ & $3.17 \mathrm{E}+02$ \\
\hline \multirow[t]{5}{*}{ GG-005 } & 10 & $8.018-03$ & $2.90 \mathrm{E}-03$ & $1.37 \mathrm{E}+02$ & $1.34 \mathrm{E}+04$ & $5.86 \mathrm{E}-03$ & $2.13 E-03$ & $1.00 \mathrm{E}+02$ & $9.80 \mathrm{~B}+03$ \\
\hline & 50 & $1.30 E-03$ & $4.71 E-04$ & $2.21 E+01$ & $2.17 E+03$ & $9.51 \mathrm{E}-04$ & $3.45 \mathrm{E}-04$ & $1.62 \mathrm{E}+01$ & $1.59 \mathrm{E} \div 03$ \\
\hline & 100 & $5.93 \mathrm{E}-0 \mathrm{E}$ & $2.15 E-04$ & $1.01 E+0.1$ & $9.92 \mathrm{E}+02$ & $4.35 E-04$ & $1.58 \mathrm{E}-04$ & $7.41 \mathrm{E}+00$ & $7.27 \mathrm{E}+02$ \\
\hline & 250 & $2.11 \mathrm{E}-04$ & $7.64 \mathrm{E}-05$ & $3.59 E+00$ & $3.52 \mathrm{E}+02$ & $1.54 \mathrm{E}-04$ & $5.59 \mathrm{E}-05$ & $2.63 \mathrm{E}+00$ & $2.58 \mathrm{E}+02$ \\
\hline & 500 & $9.63 \mathrm{E}-05$ & $3.49 E-05$ & $1.64 E+00$ & $1.61 \mathrm{E}+02$ & $7.05 \mathrm{E}-0.05$ & $2.56 \mathrm{E}-0.05$ & $1.20 E+00$ & $1.18 \mathrm{E}+02$ \\
\hline \multirow[t]{5}{*}{ GG-006 } & 10 & $1.28 \mathrm{E} \sim 03$ & $1.85 E-03$ & $1.71 E+01$ & $3.77 E+03$ & $9.38 \mathrm{E}-04$ & $1.36 \mathrm{E}-03$ & $1.25 \mathrm{E}+01$ & $2.76 \mathrm{E}+03$ \\
\hline & 50 & $2.08 E-04$ & $3.02 E-04$ & $2.77 E+00$ & $6.12 \mathrm{E}+02$ & $1.52 \mathrm{E}-04$ & $2.20 E-04$ & $2.03 E+00$ & $4.48 \mathrm{E}+02$ \\
\hline & 100 & $9.49 \mathrm{E}-05$ & $1.37 \mathrm{~B}-04$ & $1.26 E+00$ & $2.80 \mathrm{E}+02$ & $6.95 E-05$ & $1.01 E-04$ & $9.26 E-01$ & $2.05 \mathrm{E}+02$ \\
\hline & 250 & $3.37 \mathrm{E}-05$ & $4.88 E-05$ & $4.49 E-01$ & $9.93 \mathrm{E}+01$ & $2.47 E-05$ & $3.57 \mathrm{E}-05$ & $3.29 E-01$ & $7.27 \mathrm{E}+01$ \\
\hline & 500 & $1.54 \mathrm{E}-05$ & $2.23 \mathrm{~B}-0.05$ & $2.05 \mathrm{E}-01$ & $4.54 \mathrm{E}+01$ & $1.13 \mathrm{E}-05$ & $1.63 E-05$ & $1.50 E-01$ & $3.32 \mathrm{E}+01$ \\
\hline \multirow[t]{5}{*}{ GG-007 } & 10 & $1.55 \mathrm{E}-03$ & $2.08 E-03$ & $2.62 E+01$ & $9.22 \mathrm{E}+03$ & $1.14 \mathrm{E}-03$ & $1.52 E-03$ & $1.92 \mathrm{E}+01$ & $6.75 E+03$ \\
\hline & 50 & $2.52 \mathrm{E}-04$ & $3.37 \mathrm{E}-04$ & $4.25 E+00$ & $1.50 \mathrm{E}+03$ & $1.85 E-04$ & $2.47 E-04$ & $3.11 E+00$ & $1.10 \mathrm{E}+03$ \\
\hline & 100 & $1.15 E-04$ & $1.54 \mathrm{E}-04$ & $1.94 E+00$ & $6.83 \mathrm{E}+02$ & $8.44 \mathrm{E}-0.5$ & $1.13 \mathrm{~F}-04$ & $1.42 E+00$ & $5.01 \mathrm{E}+02$ \\
\hline & 250 & $4.09 \mathrm{E}-05$ & $5.46 \mathrm{E}-05$ & $6.89 E-01$ & $2.43 E+02$ & $3.00 \mathrm{E}-05$ & $4.00 E-05$ & $5.05 E-01$ & $1.78 \mathrm{E}+02$ \\
\hline & 500 & $1.87 \mathrm{E}-05$ & $2.50 \mathrm{E}-05$ & $3.15 \mathrm{E}-01$ & $1.11 E+02$ & $1.37 \mathrm{E}-05$ & $1.83 \mathrm{E}-05$ & $2.31 E-01$ & $8.12 \mathrm{E}+01$ \\
\hline \multirow[t]{5}{*}{ GG-008 } & 10 & $2.83 \mathrm{E}-03$ & $2.44 E-03$ & $5.08 E+01$ & $6.67 E+03$ & $2.07 \mathrm{E}-03$ & $2.05 E-03$ & $3.72 \mathrm{E}+01$ & $4.89 E+03$ \\
\hline & 50 & $4.59 \mathrm{E}-04$ & $2.33 \mathrm{E}-04$ & $8.25 \mathrm{E}+00$ & $2.08 \mathrm{E}+03$ & $3.36 \mathrm{E}-04$ & $1.71 \mathrm{E}-04$ & $6.04 E+00$ & $7.93 \mathrm{E}+02$ \\
\hline & 100 & $2.10 E-04$ & $1.07 E-04$ & $3.77 E+00$ & $4.95 \mathrm{E}+02$ & $1.54 \mathrm{E}-04$ & $7.80 \mathrm{E}-05$ & $2.76 \mathrm{E}+00$ & $3.62 \mathrm{E}+02$ \\
\hline & 250 & $7.45 \mathrm{E}-0.5$ & $3.78 \mathrm{E}-05$ & $1.34 E+00$ & $1.76 \mathrm{E}+02$ & $5.45 \mathrm{E}-05$ & $2.77 \mathrm{~B}-05$ & $9.80 \mathrm{E}-01$ & $1.29 \mathrm{E} \div 02$ \\
\hline & 500 & $3.40 E-05$ & $1.73 E-05$ & $6.11 E-01$ & $8.03 E+01$ & $2.49 \mathrm{E}-05$ & $1.27 E-05$ & $4.48 E-01$ & $5.88 \mathrm{E}+01$ \\
\hline GG-009 & 10 & $.08 E-03$ & $2.68 \mathrm{E}-03$ & $4.39 \mathrm{E}+02$ & $1.68 E+04$ & $5.92 \mathrm{E}-03$ & $1.96 \mathrm{E}-03$ & $3.21 E+02$ & $1.38 E+04$ \\
\hline & 50 & $.31 z-03$ & $4.35 E-04$ & $7.12 E+01$ & $3.05 \mathrm{E}+03$ & $9.60 \mathrm{E}-04$ & $3.18 E-04$ & $5.21 E+01$ & $2.23 \mathrm{~B}+03$ \\
\hline & 100 & $5.99 \mathrm{~F}-04$ & $1.99 \mathrm{E}-04$ & $3.25 E+01$ & $1.39 \mathrm{E}+03$ & $4.39 \mathrm{E}-04$ & $1.45 \mathrm{E}-04$ & $2.38 \mathrm{E}+01$ & $1.02 \mathrm{E}+03$ \\
\hline & 250 & $2.13 \mathrm{E}-04$ & $7.05 E-05$ & $1.1 .5 \mathrm{E}+0.1$ & $4.94 \mathrm{E}+02$ & $1.56 E-04$ & $5.16 \mathrm{E}-05$ & $8.46 E+00$ & $3.62 \mathrm{E}+02$ \\
\hline & 500 & $9.72 \mathrm{E}-05$ & $3.22 \mathrm{E}-05$ & $5.28 \mathrm{E}+00$ & $2.26 \mathrm{E}+02$ & $7.12 \mathrm{E}-05$ & $2.36 \mathrm{E}-0.5$ & $3.87 \mathrm{E}+00$ & $1.65 \mathrm{E}+02$ \\
\hline$G G-020$ & 10 & $2.46 \mathrm{E}-03$ & $7.28 \mathrm{E}-04$ & $1.28 \mathrm{E}+01$ & $2.92 \mathrm{E}+03$ & $1.80 E-03$ & $5.34 E-04$ & $9.40 E+00$ & $2.14 \mathrm{E}+03$ \\
\hline & 50 & $3.99 \mathrm{E}-04$ & $1.18 \mathrm{E}-04$ & $2.08 E+00$ & $4.73 \mathrm{E}+02$ & $2.92 \mathrm{E}-04$ & B. $66 \mathrm{E}-05$ & $1.52 \mathrm{E}+00$ & $3.47 E+02$ \\
\hline & 100 & $1.82 \mathrm{E}-04$ & $5.40 E-05$ & $9.51 \mathrm{E}-01$ & $2.16 \mathrm{E}+02$ & $1.33 \mathrm{E}-04$ & $3.96 \mathrm{E}-05$ & $6.97 E-01$ & $1.58 \mathrm{E}+02$ \\
\hline & 250 & $6.47 \mathrm{E}-05$ & $1.92 \mathrm{E}-05$ & $3.38 E-01$ & $7.67 \mathrm{E}+0.1$ & $4.74 \mathrm{E}-05$ & $1.40 E-05$ & $2.47 \mathrm{E}-01$ & $5.62 \mathrm{E}+01$ \\
\hline & 500 & $2.96 E-05$ & $8.76 \mathrm{E}-06$ & $1.54 \mathrm{E}-01$ & $3.51 \mathrm{E}+01$ & $2.17 \mathrm{E}-05$ & $6.42 \mathrm{E}-06$ & $1.33 \mathrm{~B}-01$ & $2.57 E+01$ \\
\hline GG-011 & 10 & $4.44 \mathrm{E}-03$ & $2.92 \mathrm{E}-04$ & $1.29 E+02$ & $7.04 \mathrm{E}+03$ & $3.25 E-03$ & $2.14 \mathrm{E}-04$ & $9.43 \mathrm{E}+01$ & $5.16 \mathrm{E}+03$ \\
\hline & 50 & $7.20 \mathrm{E}-04$ & $4.75 \mathrm{~B}-05$ & $2.09 E+01$ & $1.14 \mathrm{E}+03$ & $5.27 E-04$ & $3.48 E-05$ & $1.53 \mathrm{E}+01$ & $8.37 E+02$ \\
\hline & 100 & $3.29 \mathrm{E}-04$ & $2.17 E-05$ & $9.54 \mathrm{E}+00$ & $5.22 \mathrm{E}+02$ & $2.41 E-04$ & $2.59 E-05$ & $6.99 \mathrm{E}+00$ & $3.83 \mathrm{E}+02$ \\
\hline & 250 & $1.17 \mathrm{E}-04$ & $7.70 \mathrm{E}-06$ & $3.39 E+00$ & $1.85 \mathrm{E}+02$ & $8.55 \mathrm{E}-05$ & $5.64 \mathrm{E}-06$ & $2.48 E+00$ & $1.36 \mathrm{E}+02$ \\
\hline & 500 & $5.34 E-05$ & $3.52 E-06$ & $1.55 \mathrm{E}+00$ & $8.47 \mathrm{E}+01$ & $3.91 E-0.5$ & $2.58 E-06$ & $1.13 E+00$ & $6.218+01$ \\
\hline 3-012 & 10 & $.08 E-03$ & $.61 \mathrm{E}-03$ & $1.84 \mathrm{E}+02$ & $2.15 E+04$ & $3.72 \mathrm{E}-03$ & $3.38 \mathrm{E}-03$ & $1.35 \mathrm{E}+02$ & $1.58 E+04$ \\
\hline & 50 & $8.24 \mathrm{~g}-04$ & $7.49 \mathrm{E}-04$ & $2.98 E+01$ & $3.49 \mathrm{E}+03$ & $6.03 \mathrm{E}-04$ & $5.48 \mathrm{E}-04$ & $2.18 \mathrm{E}+01$ & $2: 56 \mathrm{E}+03$ \\
\hline & 100 & $3.76 \mathrm{E}-04$ & $3.42 \mathrm{E}-04$ & $1.36 E+01$ & $1.60 \mathrm{E}+03$ & $2.76 \mathrm{E}-04$ & $2.51 \mathrm{E}-04$ & $9.98 \mathrm{E}+00$ & $1.17 E+03$ \\
\hline & 250 & $1.34 \mathrm{E}-04$ & $1.21 \mathrm{E}-04$ & $4.84 E+00$ & $5.67 \mathrm{E}+02$ & $9.79 \mathrm{E}-05$ & $8.90 \mathrm{E}-05$ & $3.54 \mathrm{E}+00$ & $4.15 E+02$ \\
\hline & 500 & $6.11 \mathrm{E}-05$ & $5.55 E-05$ & $2.21 E+00$ & $2.59 \mathrm{E}+02$ & 4.47E-0.5 & $4.06 \mathrm{E}-05$ & $1.62 E+00$ & $1.90 \mathrm{E}+02$ \\
\hline
\end{tabular}


Appendix D

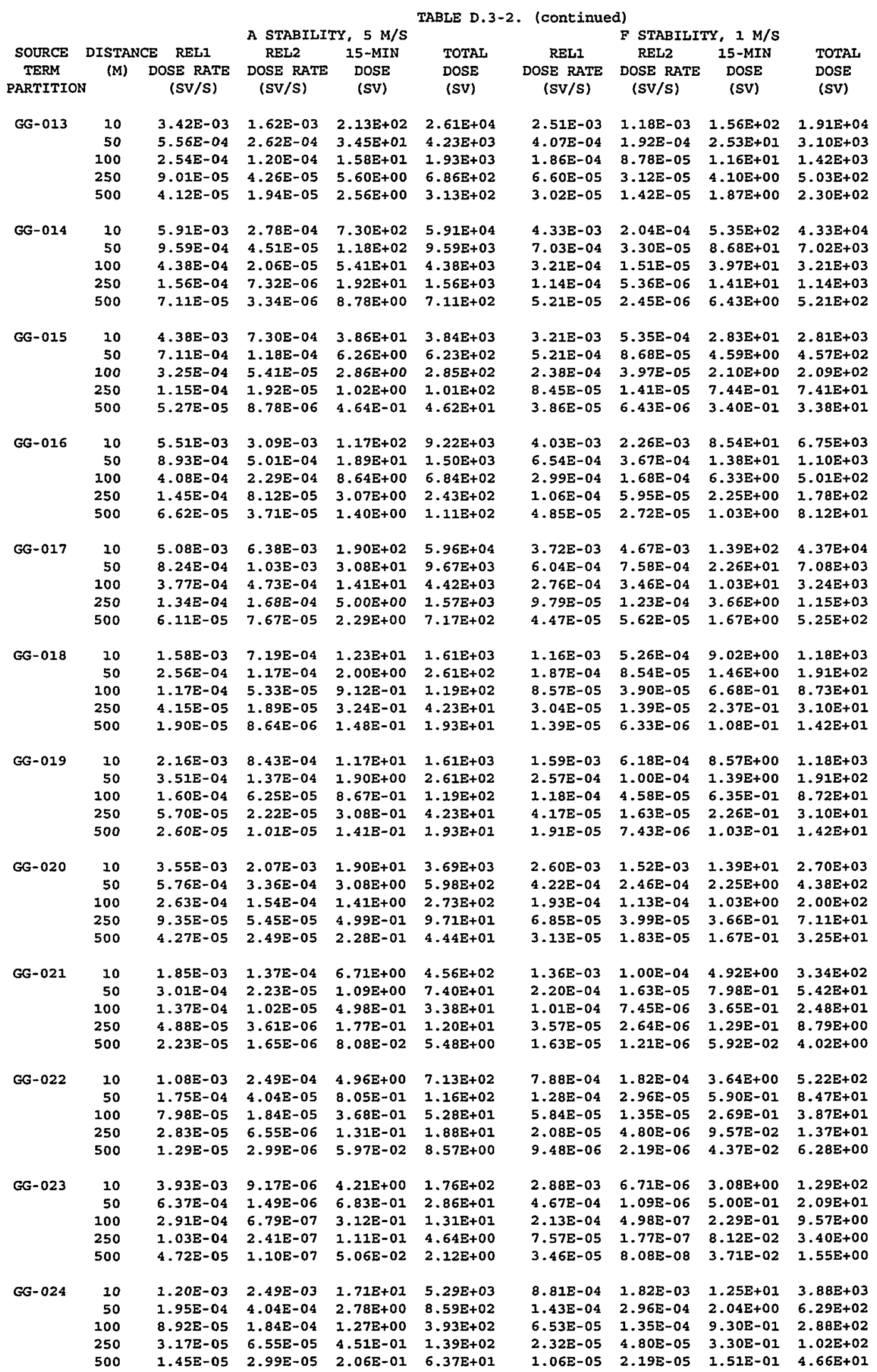




\begin{tabular}{|c|c|c|c|c|c|c|c|c|c|}
\hline & A STP & ABILITY， 5 & $M / S$ & & $\begin{array}{c}\text { TABI } \\
\text { F }\end{array}$ & $\begin{array}{l}3-2, \text {, con } \\
\text { ILITY, I }\end{array}$ & $\begin{array}{l}\text { inued) } \\
/ \mathrm{s}\end{array}$ & & \\
\hline $\begin{array}{l}\text { SOURCE } \\
\text { TERM } \\
\text { PARTITION }\end{array}$ & $\begin{array}{c}\text { DISTANC } \\
\text { (M) }\end{array}$ & $\begin{array}{l}\text { CE RELI } \\
\text { DOSE RATE } \\
\text { (SV/S) }\end{array}$ & $\begin{array}{c}\text { REL2 } \\
\text { DOSE RATE } \\
\text { (SV/S) }\end{array}$ & $\begin{array}{c}\text { 15-MIN } \\
\text { DOSE } \\
\text { (SV) }\end{array}$ & $\begin{array}{l}\text { TOTAL } \\
\text { DOSE } \\
\text { (SV) }\end{array}$ & $\begin{array}{c}\text { RELI } \\
\text { DOSE RATE } \\
(S V / S)\end{array}$ & $\begin{array}{c}\text { REL2 } \\
\text { DOSE RATE } \\
\text { (SV/S) }\end{array}$ & $\begin{array}{c}\text { 15-MIN } \\
\text { DOSE } \\
\text { (SV) }\end{array}$ & $\begin{array}{l}\text { TOTAL } \\
\text { DOSE } \\
\text { (SV) }\end{array}$ \\
\hline GG-025 & 10 & $2.23 \mathrm{E}-03$ & $1.64 \mathrm{E}-03$ & $2.41 E+01$ & $3.84 E+03$ & $1.64 \mathrm{E}-03$ & $1.20 E-03$ & $1.77 \mathrm{E}+01$ & $2.81 E+03$ \\
\hline & 50 & $3.62 E-04$ & $2.66 E-04$ & $3.91 E+00$ & $6.22 \mathrm{E}+02$ & $2.65 E-04$ & $1.95 \mathrm{E}-04$ & $2.86 \mathrm{E}+00$ & $4.56 E+02$ \\
\hline & 100 & $1.66 \mathrm{E}-04$ & $1.21 E-04$ & $1.79 E+00$ & $2.84 E+02$ & $1.21 E-04$ & $8.89 \mathrm{E}-05$ & $1.31 \mathrm{E}+00$ & $2.08 \mathrm{E}+02$ \\
\hline & 250 & $5.88 \mathrm{E}-05$ & $4.31 \mathrm{E}-05$ & $6.35 E-01$ & $1.01 E+02$ & $4.30 E-05$ & $3.16 \mathrm{E}-05$ & $4.65 E-01$ & $7.40 \mathrm{E}+01$ \\
\hline & 500 & $2.69 \mathrm{E}-05$ & $1.97 \mathrm{E}-05$ & $2.90 E-01$ & $4.61 \mathrm{E}+01$ & $1.97 \mathrm{E}-05$ & $1.44 \mathrm{E}-05$ & $2.12 \mathrm{E}-01$ & $3.38 E+01$ \\
\hline GG-026 & 10 & $1.96 \mathrm{E}-03$ & $7.00 \mathrm{E}-05$ & $3.77 E+00$ & $2.46 \mathrm{E}+02$ & $1.44 \mathrm{E}-03$ & $5.13 E-05$ & $2.76 E+00$ & $1.80 E+02$ \\
\hline & 50 & $3.18 \mathrm{E}-04$ & $1.14 \mathrm{E}-05$ & $6.11 E-01$ & $3.99 \mathrm{E}+01$ & $2.33 \mathrm{E}-04$ & 8.32E-06 & $4.48 \mathrm{E}-01$ & $2.92 E+01$ \\
\hline & 100 & $1.45 \mathrm{E}-04$ & $5.19 \mathrm{E}-06$ & $2.79 \mathrm{E}-01$ & $1.82 \mathrm{E}+01$ & $1.06 \mathrm{E}-04$ & $3.80 \mathrm{E}-06$ & $2.05 \mathrm{E}-01$ & $1.33 E+01$ \\
\hline & 250 & $5.16 \mathrm{E}-05$ & $1.84 E-06$ & $9.92 \mathrm{E}-02$ & $6.47 E+00$ & $3.78 \mathrm{E}-05$ & $1.35 \mathrm{E}-06$ & $7.26 \mathrm{E}-02$ & $4.74 \mathrm{E}+00$ \\
\hline & 500 & $2.36 \mathrm{E}-05$ & $8.42 \mathrm{E}-07$ & $4.53 E-02$ & $2.96 \mathrm{E}+00$ & $1.73 \mathrm{E}-05$ & $6.17 \mathrm{E}-07$ & $3.32 \mathrm{E}-02$ & $2.16 E+00$ \\
\hline GG-027 & 10 & $3.29 \mathrm{E}-03$ & $5.43 \mathrm{E}-04$ & $1.03 E+01$ & $8.18 E+02$ & $2.41 \mathrm{E}-03$ & $3.98 \mathrm{E}-04$ & $7.52 \mathrm{E}+00$ & $5.99 E+02$ \\
\hline & 50 & $5.34 E-04$ & $8.81 E-05$ & $1.67 \mathrm{E}+00$ & $1.33 E+02$ & $3.91 E-04$ & $6.45 \mathrm{E}-05$ & $1.22 E+00$ & $9.73 \mathrm{E}+01$ \\
\hline & 100 & $2.44 \mathrm{E}-04$ & $4.02 \mathrm{E}-05$ & $7.62 \mathrm{E}-01$ & $6.07 \mathrm{E}+01$ & $1.79 E-04$ & $2.95 \mathrm{E}-05$ & $5.58 \mathrm{E}-01$ & $4.44 E+01$ \\
\hline & 250 & $8.66 E-05$ & $1.43 E-05$ & $2.70 E-01$ & $2.15 E+01$ & $6.34 \mathrm{E}-05$ & $1.05 \mathrm{E}-05$ & $1.98 \mathrm{E}-01$ & $1.58 \mathrm{E}+01$ \\
\hline & 500 & $3.96 \mathrm{E}-05$ & $6.53 \mathrm{E}-06$ & $1.24 E-01$ & $9.84 E+00$ & $2.90 E-05$ & $4.78 E-06$ & $9.05 \mathrm{E}-02$ & $7.21 E+00$ \\
\hline GG-028 & 10 & $7.51 \mathrm{E}-03$ & $5.37 \mathrm{E}-04$ & $7.24 E+02$ & $2.76 \mathrm{E}+04$ & $5.50 E-03$ & $3.94 \mathrm{E}-04$ & $5.31 E+02$ & $2.03 E+04$ \\
\hline & 50 & $1.22 E-03$ & $8.72 E-05$ & $1.18 E+02$ & $4.49 E+03$ & $8.93 E-04$ & $6.39 E-05$ & $8.61 E+01$ & $3.29 E+03$ \\
\hline & 100 & $5.57 E-04$ & $3.98 E-05$ & $5.37 E+01$ & $2.05 E+03$ & $4.08 \mathrm{E}-04$ & $2.92 \mathrm{E}-05$ & $3.93 E+0 I$ & $1.50 \mathrm{E}+03$ \\
\hline & 250 & $1.98 E-04$ & $1.41 E-05$ & $1.91 E+01$ & $7.28 E+02$ & $1.45 \Xi-04$ & $1.04 \mathrm{E}-05$ & $1.40 \mathrm{E}+01$ & $5.33 E+02$ \\
\hline & 500 & $9.03 E-05$ & $6.46 \mathrm{E}-06$ & $8.71 E+00$ & $3.32 E+02$ & $6.62 \mathrm{E}-0.5$ & $4.74 \mathrm{E}-06$ & $6.38 E+00$ & $2.44 E+02$ \\
\hline GG-029 & 10 & $2.12 E-03$ & $1.02 \mathrm{E}-03$ & $5.58 \mathrm{E}+01$ & $7.65 \mathrm{E}+03$ & $1.56 \mathrm{E}-03$ & $7.46 \mathrm{E}-04$ & $4.09 \mathrm{E}+01$ & $5.60 \Xi+03$ \\
\hline & 50 & $3.45 E-04$ & $1.65 \mathrm{E}-04$ & $9.05 E+00$ & $1.24 \mathrm{E}+03$ & $2.53 E-04$ & $1.21 \mathrm{E}-04$ & $6.63 \mathrm{E}+00$ & $9.09 \mathrm{E}+02$ \\
\hline & 100 & $1.58 \mathrm{E}-04$ & $7.55 \mathrm{E}-05$ & $4.13 E+00$ & $5.67 \mathrm{E}+02$ & $1.15 E-04$ & $5.53 E-05$ & $3.03 E+00$ & $4.15 E+02$ \\
\hline & 250 & $5.59 \mathrm{E}-05$ & $2.68 \mathrm{E}-05$ & $1.47 \mathrm{E}+00$ & $2.01 \mathrm{E}+02$ & $4.10 \mathrm{E}-05$ & $1.96 \mathrm{E}-05$ & $1.08 \mathrm{E}+00$ & $1.47 \mathrm{E}+02$ \\
\hline & 500 & $2.56 E-05$ & $1.23 \mathrm{E}-05$ & $6.71 \mathrm{E}-01$ & $9.20 \mathrm{E}+01$ & $1.87 E-05$ & $8.98 E-06$ & $4.91 E-01$ & $6.74 E+01$ \\
\hline GG-030 & 10 & $1.12 \mathrm{E}-02$ & $1.10 \mathrm{E}-03$ & $4.49 \mathrm{E}+02$ & $1.60 \mathrm{E}+04$ & $8.17 \mathrm{E}-03$ & $8.07 \mathrm{E}-04$ & $3.29 \mathrm{E}+02$ & $1.17 \mathrm{E}+04$ \\
\hline & 50 & 1.81E-03 & $1.79 E-04$ & $7.29 \mathrm{E}+01$ & $2.59 E+03$ & $1.33 \mathrm{E}-03$ & $1.31 \mathrm{E}-04$ & $5.34 \mathrm{E}+01$ & $1.90 E+03$ \\
\hline & 100 & $8.27 E-04$ & $8.17 \mathrm{E}-05$ & $3.33 E+01$ & $1.19 E+03$ & $6.06 \mathrm{E}-04$ & $5.99 E-05$ & $2.44 E+01$ & $8.68 \mathrm{E}+02$ \\
\hline & 250 & 2. $94 \mathrm{E}-04$ & $2.90 E-05$ & $1.18 \mathrm{E}+01$ & $4.21 E+02$ & $2.15 E-04$ & $2.13 E-05$ & $8.66 \mathrm{E}+00$ & $3.08 E+02$ \\
\hline & 500 & $1.34 \mathrm{E}-04$ & $1.33 \mathrm{E}-05$ & $5.40 \mathrm{E}+00$ & $1.92 E+02$ & $9.83 E-05$ & $9.71 E-06$ & $3.96 E+00$ & $1.41 \mathrm{E}+02$ \\
\hline GG-031 & 10 & $5.86 \mathrm{E}-04$ & $1.30 \mathrm{E}-03$ & $1.83 E+01$ & $5.62 \mathrm{E}+03$ & $4.29 \mathrm{E}-04$ & $9.51 E-04$ & $1.34 \mathrm{E}+01$ & $4.12 \mathrm{E}+03$ \\
\hline & 50 & $9.50 E-05$ & $2.11 E-04$ & $2.97 \mathrm{E}+00$ & $9.12 \mathrm{E}+02$ & $6.96 E-05$ & $1.54 \mathrm{E}-04$ & $2.17 \mathrm{E}+00$ & $6.68 E+02$ \\
\hline & 100 & $4.34 \mathrm{E}-05$ & $9.62 \mathrm{E}-05$ & $1.36 \mathrm{E}+00$ & $4.17 \mathrm{E}+02$ & $3.18 E-05$ & $7.05 \mathrm{E}-05$ & $9.94 E-01$ & $3.05 E+02$ \\
\hline & 250 & $1.54 \mathrm{E}-05$ & $3.42 \mathrm{E}-05$ & $4.82 E-01$ & $1.48 \mathrm{E}+02$ & $1.13 E-05$ & $2.50 \mathrm{E}-05$ & $3.53 E-01$ & $1.08 E+02$ \\
\hline & 500 & $7.04 \mathrm{E}-06$ & $1.56 \mathrm{E}-05$ & $2.20 E-01$ & $6.76 \mathrm{E}+01$ & $5.16 \mathrm{E}-06$ & $1.14 \mathrm{E}-05$ & $1.61 E-01$ & $4.95 E+01$ \\
\hline GG-032 & 10 & $1.19 E-02$ & $2.20 E-03$ & $1.23 E+03$ & $6.17 \mathrm{E}+04$ & $8.71 E-03$ & $1.61 \mathrm{E}-03$ & $9.02 E+02$ & $4.52 \mathrm{E}+04$ \\
\hline & 50 & $1.93 E-03$ & $3.57 E-04$ & $2.00 E+02$ & $1.00 E+04$ & $1.41 \mathrm{E}-03$ & $2.61 E-04$ & $1.46 \mathrm{E}+02$ & $7.34 \mathrm{E}+03$ \\
\hline & 100 & 8.81E-04 & $1.63 \mathrm{E}-04$ & $9.13 E+01$ & $4.58 \mathrm{E}+03$ & $6.45 \mathrm{E}-04$ & $1.19 \mathrm{E}-04$ & $6.68 \mathrm{E}+01$ & $3.35 \mathrm{E}+03$ \\
\hline & 250 & $3.13 \mathrm{E}-04$ & $5.79 \mathrm{E}-05$ & $3.24 E+01$ & $1.62 \mathrm{E}+03$ & $2.29 E-04$ & $4.24 E-05$ & $2.37 E+01$ & $1.19 E+03$ \\
\hline & 500 & $1.43 E-04$ & $2.64 E-05$ & $1.48 \mathrm{E}+01$ & $7.42 \mathrm{E}+02$ & $1.05 E-04$ & $1.94 \mathrm{E}-05$ & $1.08 E+01$ & $5.44 E+02$ \\
\hline GG-033 & 10 & E-03 & $8.61 E-04$ & $5.34 E+01$ & $7.19 \mathrm{E}+03$ & $1.20 \mathrm{E}-03$ & $6.31 E-04$ & $3.91 E+01$ & $5.27 E+03$ \\
\hline & 50 & $2.66 \mathrm{E}-04$ & $1.40 \mathrm{E}-04$ & $8.67 E+00$ & $1.17 \mathrm{E}+03$ & $1.95 \mathrm{E}-04$ & $1.02 \mathrm{E}-04$ & $6.35 E+00$ & $8.55 E+02$ \\
\hline & 100 & $1.22 \mathrm{E}-04$ & $6.38 \mathrm{E}-05$ & $3.96 \mathrm{E}+00$ & $5.33 E+02$ & $8.91 \mathrm{E}-05$ & $4.68 \mathrm{E}-05$ & $2.90 E+00$ & $3.91 E+02$ \\
\hline & 250 & 4.32E-05 & $2.27 \mathrm{E}-05$ & $1.41 E+00$ & $1.89 \mathrm{E}+02$ & $3.17 \mathrm{E}-05$ & $1.66 \mathrm{E}-05$ & $1.03 E+00$ & $1.39 \mathrm{E}+02$ \\
\hline & 500 & $1.97 E-05$ & $1.04 \mathrm{E}-05$ & $6.43 \mathrm{E}-01$ & $8.65 \mathrm{E}+01$ & $1.45 \mathrm{E}-05$ & $7.59 \mathrm{E}-06$ & $4.71 E-01$ & $6.34 \mathrm{E}+01$ \\
\hline GG-034 & 10 & $2.21 E-03$ & $3.40 \mathrm{E}-04$ & $3.79 \mathrm{E}+01$ & $3.39 E+03$ & $1.62 \mathrm{E}-03$ & $2.49 \mathrm{E}-04$ & $2.78 E+01$ & $2.48 E+03$ \\
\hline & 50 & $3.58 \mathrm{E}-04$ & $5.51 E-05$ & $6.15 \mathrm{E}+00$ & $5.50 \mathrm{E}+02$ & $2.62 E-04$ & $4.04 \mathrm{E}-05$ & $4.51 E+00$ & $4.03 E+02$ \\
\hline & 100 & $1.64 \mathrm{E}-04$ & $2.52 E-05$ & $2.81 E+00$ & $2.51 E+02$ & $1.20 \mathrm{E}-04$ & 1.84 E-05 & $2.06 E+00$ & $1.84 E+02$ \\
\hline & 250 & $5.81 E-05$ & $8.94 E-06$ & $9.98 \mathrm{E}-01$ & $8.92 E+01$ & $4.25 \mathrm{E}-05$ & $6.55 \mathrm{E}-06$ & $7.31 \mathrm{E}-01$ & $6.54 \mathrm{E}+01$ \\
\hline & 500 & $2.65 \mathrm{E}-05$ & $4.09 \mathrm{E}-06$ & $4.56 E-01$ & $4.08 E+01$ & $1.94 \mathrm{E}-05$ & $2.99 E-06$ & $3.34 E-01$ & $2.99 E+02$ \\
\hline GG-035 & 10 & $2.38 \mathrm{E}-03$ & $7.58 \mathrm{E}-04$ & $4.15 E+02$ & $3.92 \mathrm{E}+04$ & $1.74 \mathrm{E}-03$ & $5.55 E-04$ & $3.04 \mathrm{E}+02$ & $2.87 E+04$ \\
\hline & 50 & $3.86 \mathrm{E}-04$ & $1.23 E-04$ & $6.73 E+01$ & $6.36 E+03$ & $2.83 E-04$ & $9.00 \mathrm{E}-05$ & $4.93 E+01$ & $4.66 \mathrm{E}+03$ \\
\hline & 100 & $1.76 \mathrm{E}-04$ & $5.62 \mathrm{E}-05$ & $3.08 E+01$ & $2.91 \mathrm{E}+03$ & $1.29 \mathrm{E}-04$ & $4.11 E-05$ & $2.25 E+01$ & $2.13 E+03$ \\
\hline & 250 & $6.27 \mathrm{E}-05$ & $1.99 E-05$ & $1.09 E+01$ & $1.03 \mathrm{E}+03$ & $4.59 E-05$ & $1.46 \mathrm{E}-05$ & $8.00 \mathrm{E}+00$ & $7.56 \mathrm{E}+02$ \\
\hline & 500 & $2.86 \mathrm{E}-05$ & $9.11 \mathrm{E}-06$ & $4.99 E+00$ & $4.72 \mathrm{E}+02$ & $2.10 E-05$ & $6.67 \mathrm{E}-06$ & $3.66 \mathrm{E}+00$ & $3.46 \mathrm{E}+02$ \\
\hline GG-036 & 10 & $.075-03$ & $1.105-03$ & $2.61 E+01$ & $3.40 E+03$ & 1.51E-03 & $8.02 \mathrm{E}-04$ & 1.91E+01 & $2.49 E+03$ \\
\hline & 50 & $3.35 E-04$ & $1.78 \mathrm{E}-04$ & $4.24 E+00$ & $5.51 E+02$ & $2.46 \mathrm{E}-04$ & $1.30 \mathrm{E}-04$ & $3.10 \mathrm{E}+00$ & $4.04 \mathrm{E}+02$ \\
\hline & 100 & $1.53 \mathrm{E}-04$ & 8.12E-05 & $1.94 \mathrm{E}+00$ & $2.52 E+02$ & $1.12 \mathrm{E}-04$ & $5.95 \mathrm{E}-05$ & $1.42 E+00$ & $1.84 E+02$ \\
\hline & 250 & $5.44 \mathrm{E}-05$ & $2.88 E-05$ & $6.87 E-01$ & $8.94 E+01$ & 3.99E-05 & $2.11 E-05$ & 5.03E-01 & $6.55 E \div 01$ \\
\hline & 500 & $2.49 \mathrm{E}-05$ & $1.32 \mathrm{E}-05$ & $3.14 \mathrm{E}-01$ & $4.08 E+01$ & $1.82 \mathrm{E}-05$ & $9.65 \mathrm{E}-06$ & $2.30 E-01$ & $2.99 E+01$ \\
\hline
\end{tabular}


Appendix D

A STABILITY, $5 \mathrm{M} / \mathrm{S}$

\begin{tabular}{|c|c|c|c|c|c|c|c|c|c|}
\hline \multirow{3}{*}{ SOURCE $_{\text {TERM }}^{\text {A }}$} & \multicolumn{2}{|c|}{ STABILITY, $5 \mathrm{M} / \mathrm{s}$} & \multicolumn{7}{|c|}{ F STABILITY, I $\mathrm{M} / \mathrm{s}$} \\
\hline & \multicolumn{2}{|c|}{ DISTANCE RELI } & REL2 & 15-MIN & TOTAL & RELI & REL2 & \multirow{2}{*}{$\begin{array}{c}15-\mathrm{MIN} \\
\text { DOSE } \\
(\mathrm{SV})\end{array}$} & \multirow{2}{*}{$\begin{array}{l}\text { TOTAL } \\
\text { DOSE } \\
\text { (SV) }\end{array}$} \\
\hline & (M) & $\begin{array}{c}\text { DOSE RATE } \\
(\mathrm{SV} / \mathrm{S})\end{array}$ & $\begin{array}{c}\text { DOSE RATE } \\
(\mathrm{SV} / \mathrm{S})\end{array}$ & $\begin{array}{l}\text { DOSE } \\
\text { (sV) }\end{array}$ & $\begin{array}{l}\text { DOSE } \\
\text { (SV) }\end{array}$ & $\begin{array}{c}\text { DOSE RATE } \\
(\mathrm{SV} / \mathrm{S})\end{array}$ & $\begin{array}{c}\text { DOSE RATE } \\
(\mathrm{SV} / \mathrm{s})\end{array}$ & & \\
\hline \multirow[t]{5}{*}{ Go-037 } & 10 & $1.29 \mathrm{E}-03$ & $4.02 E-04$ & $1.63 E+01$ & $1.54 E+03$ & $9.46 E-04$ & $2.94 \mathrm{E}-04$ & $1.20 \mathrm{E}+01$ & $1.13 E+03$ \\
\hline & 50 & $2.10 \mathrm{E}-04$ & $6.51 \mathrm{E}-05$ & $2.65 \mathrm{E}+00$ & $2.52 \mathrm{E}+02$ & $1.54 \mathrm{E}-04$ & 4. $77 E-05$ & $1.94 \mathrm{E}+00$ & $1.84 \mathrm{E}+02$ \\
\hline & 300 & $9.58 \mathrm{E}-05$ & $2.97 \mathrm{E}-05$ & $1.21 E+00$ & $1.15 \mathrm{E}+02$ & $7.01 E-05$ & $2.18 \mathrm{E}-05$ & $8.87 \mathrm{E}-01$ & $8.39 E+01$ \\
\hline & 250 & $3.40 \mathrm{~s}-05$ & $1.06 \mathrm{E}-05$ & $4.30 \mathrm{E}-01$ & $4.07 \mathrm{E}+01$ & $2.49 \mathrm{E}-05$ & $7.73 \mathrm{E}-06$ & $3.15 \mathrm{E}-01$ & $2.98 E+01$ \\
\hline & 500 & $1.55 E-05$ & $4.82 \mathrm{E}-06$ & $1.96 \mathrm{E}-01$ & $1.86 \mathrm{E} \div 01$ & $1.14 E-05$ & $3.53 \mathrm{E}-06$ & $1.44 \mathrm{E}-01$ & $1.36 \mathrm{E}+01$ \\
\hline \multirow[t]{5}{*}{ GG-03B } & 10 & $1.00 E-03$ & $1.67 E-03$ & $5,17 E+01$ & $9.83 \mathrm{E}+03$ & $7.34 E-04$ & $1.22 \mathrm{E}-03$ & $3.79 \mathrm{E}+01$ & $7.20 \mathrm{E}+03$ \\
\hline & 50 & $1.63 E-04$ & $2.70 E-04$ & $8.398+00$ & $1.60 \mathrm{E}+03$ & $1.19 \mathrm{E}-04$ & $1.98 \mathrm{E}-04$ & $6.15 \mathrm{E}+00$ & $1.17 \mathrm{E}+03$ \\
\hline & 100 & $7.43 \mathrm{E}-05$ & $1.24 \mathrm{E}-04$ & $3.84 \mathrm{E}+00$ & $7.29 \mathrm{E}+02$ & $5.44 \mathrm{E}-05$ & $9.05 \mathrm{E}-05$ & $2.8 I E+00$ & $5.34 E+02$ \\
\hline & 250 & $2.64 \mathrm{E}-05$ & $4.39 \mathrm{E}-05$ & $1.36 \mathrm{E}+00$ & $2.59 \mathrm{E}+02$ & $2.93 E-05$ & $3.21 \mathrm{E}-0.5$ & $9.98 \mathrm{E}-0.1$ & $1.90 \mathrm{E}+02$ \\
\hline & 500 & $1.21 \mathrm{E}-05$ & $2.00 \mathrm{E}-05$ & $6.22 E-01$ & $1.18 \mathrm{E}+02$ & $8.83 \mathrm{E}-06$ & $1.47 \mathrm{E}-05$ & $4.56 \mathrm{E}-01$ & $8.66 \mathrm{E}+01$ \\
\hline GG-039 & 10 & $1.06 \mathrm{E}-03$ & $7.48 \mathrm{E}-0.4$ & $9.34 \mathrm{E}+01$ & $1.09 \mathrm{E}+04$ & $7.76 \mathrm{E}-04$ & $5.48 \mathrm{E}-04$ & $6.84 \mathrm{E}+01$ & $7.99 \mathrm{E}+03$ \\
\hline & 50 & $1.72 E-04$ & $1.21 \mathrm{E}-04$ & $1.52 \mathrm{E}+01$ & $1.77 E+03$ & $1.26 \mathrm{E}-04$ & $8.89 \mathrm{E}-0.5$ & $1.11 \mathrm{E}+01$ & $1.30 \mathrm{E}+03$ \\
\hline & 200 & $7.85 E-05$ & $5.55 \mathrm{E}-05$ & $6.93 E+00$ & $8.09 E+02$ & $5.75 E-05$ & $4.06 \mathrm{E}-05$ & $5.07 \mathrm{E}+00$ & $5.93 \mathrm{E}+02$ \\
\hline & 250 & $2.79 \mathrm{~g}-05$ & $1.97 E-05$ & $2.46 \mathrm{E}+00$ & $2.87 E+02$ & $2.04 \mathrm{E}-05$ & $1.44 \mathrm{E}-05$ & $1.80 \mathrm{E}+00$ & $2.10 E+02$ \\
\hline & 500 & $1.27 \mathrm{E}-05$ & $9.00 \mathrm{E}-06$ & 1. $12 \mathrm{E}+00$ & $1.31 \mathrm{E}+02$ & $9.33 \mathrm{E}-06$ & $6.59 \mathrm{E}-06$ & 8.23E-01 & $9.61 E+01$ \\
\hline GG-040 & 10 & $1.18 \mathrm{E}-03$ & $8.59 \mathrm{E}-04$ & $2.66 \mathrm{E}+01$ & $4.06 E+03$ & $8.61 \mathrm{E}-04$ & $6.29 \mathrm{E}-04$ & $1.95 \mathrm{E}+01$ & $2.98 E+03$ \\
\hline & 50 & $1.912-04$ & $1.39 E-04$ & $4.32 E+00$ & $6.59 E+02$ & $1.40 \mathrm{E}-04$ & $1.02 \mathrm{E}-04$ & $3.16 E+00$ & $4.83 E+02$ \\
\hline & 100 & $8.72 \mathrm{E}-05$ & $6.37 E-05$ & $1.97 \mathrm{E}+00$ & $3.01 E+02$ & $6.38 \mathrm{E}-05$ & $4.66 \mathrm{E}-05$ & $1.44 \mathrm{E}+00$ & $2.21 E+02$ \\
\hline & 250 & $3.098-05$ & $2.26 \mathrm{E} \cdot 05$ & $6.99 E-01$ & $1.07 \mathrm{E}+02$ & $2.27 \mathrm{E}-05$ & $1.56 \mathrm{E}-05$ & $5.12 \mathrm{E}-01$ & $7.83 E+01$ \\
\hline & 500 & $1.42 \mathrm{E}-05$ & $1.03 E-05$ & $3.20 \mathrm{~F}-01$ & $4.89 \mathrm{E}+01$ & $1.04 \mathrm{E}-05$ & $7.56 \mathrm{E}-06$ & $2.34 \mathrm{E}-01$ & $3.58 \mathrm{E}+01$ \\
\hline GG-041 & 10 & $6.20 \mathrm{E}-04$ & $6.43 E-04$ & $1.35 E+01$ & $2.24 \mathrm{E}+03$ & $4.54 E-04$ & $4.71 \mathrm{E}-04$ & $9.91 \mathrm{E}+00$ & $1.64 \mathrm{E}+03$ \\
\hline & 50 & $1.01 E-04$ & $1.04 \mathrm{E}-04$ & $2.19 \mathrm{E}+00$ & $3.63 \mathrm{E}+02$ & $7.37 E-05$ & $7.65 \mathrm{E}-05$ & $1.61 \mathrm{E}+00$ & $2.66 E+02$ \\
\hline & 100 & $4.60 E-05$ & $4.77 E-05$ & $1.00 \mathrm{E}+00$ & $1.66 \mathrm{E}+02$ & $3.37 \mathrm{~g}-05$ & $3.49 \mathrm{E}-05$ & $7.34 E-01$ & $1.22 \mathrm{E}+02$ \\
\hline & 250 & $1.63 E-05$ & $1.69 E-05$ & $3.56 \mathrm{E}-01$ & $5.89 E+01$ & $1.20 \mathrm{E}-05$ & $1.24 \mathrm{E}-05$ & $2.6 I E-0 I$ & $4.32 E+01$ \\
\hline & 500 & $7.46 \mathrm{E}-06$ & $7.74 \mathrm{E}-06$ & $1.63 \mathrm{E}-01$ & $2.69 \mathrm{E}+01$ & $5.47 \mathrm{~B}-06$ & $5.67 \mathrm{E}-06$ & $1.19 E-01$ & $1.97 \mathrm{E}+01$ \\
\hline GG-042 & 10 & $3.49 z-04$ & $2.20 \mathrm{E}-04$ & $3.89 \mathrm{E}+00$ & $6.69 \mathrm{E}+02$ & $2.56 \mathrm{E}-04$ & $1.61 E-04$ & $2.85 E+00$ & $4.90 \mathrm{E}+02$ \\
\hline & 50 & $5.67 \mathrm{E}-05$ & $3.56 \mathrm{E}-05$ & $6.32 \mathrm{E}-01$ & $1.09 E+02$ & $4.15 \mathrm{E}-05$ & $2.61 E-05$ & $4.63 E-01$ & $7.95 \mathrm{E}+01$ \\
\hline & 100 & $2.59 \mathrm{E}-05$ & $1.63 \mathrm{~F}-05$ & $2.89 E-01$ & $4.96 E+01$ & $1.90 E-05$ & $1.19 \mathrm{E}-05$ & $2.11 \mathrm{E}-01$ & $3.63 \mathrm{E}+01$ \\
\hline & 250 & $9.20 \mathrm{E}-06$ & $5.78 E-06$ & $1.02 \mathrm{E}-01$ & $1.76 \mathrm{E}+01$ & $6.74 E-06$ & $4.23 E-06$ & $7.51 E-02$ & $1.29 E+01$ \\
\hline & 500 & $4.20 E-06$ & $2.64 E-06$ & $4.68 E-02$ & 8. $04 E+00$ & $3.08 E-06$ & $1.93 E-06$ & $3.43 \mathrm{E}-02$ & $5.898+00$ \\
\hline$G G-043$ & 10 & $3.66 \mathrm{E}-04$ & $1.86 \mathrm{E}-03$ & $1.83 E+01$ & $5.57 E+03$ & $2.68 \mathrm{E}-04$ & $1.37 E-03$ & $1.34 \mathrm{E}+01$ & $4.08 \mathrm{E}+03$ \\
\hline & 50 & $5.94 E-05$ & $3.03 \mathrm{E}-04$ & $2.97 \mathrm{E}+00$ & $9.04 \mathrm{E}+02$ & $4.35 E-05$ & $2.22 \mathrm{E}-04$ & $2.18 \mathrm{E}+00$ & $6.62 \mathrm{E}+02$ \\
\hline & 100 & $2.72 \mathrm{E}-05$ & $1.38 \mathrm{E}-04$ & $1.36 \mathrm{E}+00$ & $4.13 \mathrm{E}+02$ & $1.99 \mathrm{E}-05$ & $1.0 I E-04$ & $9.94 \mathrm{E}-01$ & $3.03 E+02$ \\
\hline & 250 & $9.64 E-06$ & $4.91 E-05$ & $4.82 \mathrm{E}-01$ & $1.47 E+02$ & $7.06 \mathrm{E}-06$ & $3.60 \mathrm{E}-05$ & $3.53 \mathrm{E}-01$ & $1.07 E+02$ \\
\hline & 500 & $4.418-06$ & $2.24 \mathrm{E}-05$ & $2.20 \mathrm{~B}-01$ & $6.70 \mathrm{E}+01$ & $3.23 \mathrm{E}-06$ & $1.64 \mathrm{E}-05$ & $1.61 \mathrm{E}-01$ & $4.91 \mathrm{E}+01$ \\
\hline GG-044 & 10 & $5.63 \mathrm{E}-04$ & $1.79 \mathrm{E}-04$ & $6.72 E+00$ & $7.47 E+02$ & $4.12 \mathrm{E}-04$ & $1.31 \mathrm{E}-04$ & $4.93 \mathrm{E}+00$ & $5.48 \mathrm{E}+02$ \\
\hline & 50 & $9.13 \mathrm{E}-05$ & $2.91 \mathrm{E}-05$ & $1.09 \mathrm{E}+00$ & $1.21 E+02$ & $6.69 \mathrm{E}-05$ & $2.13 \mathrm{E}-05$ & $7.99 E-01$ & $8.88 E+01$ \\
\hline & 100 & $4.17 \mathrm{E}-05$ & $1.33 E-05$ & $4.98 \mathrm{E}-01$ & $5.54 \mathrm{E}+01$ & $3.06 \mathrm{E}-05$ & $9.74 E=06$ & $3.65 \mathrm{E}-01$ & $4.06 E+01$ \\
\hline & 250 & $1.48 \mathrm{E}-05$ & $4.72 \mathrm{E}-06$ & $1.77 \mathrm{E}-01$ & $1.97 \mathrm{E}+01$ & $1.09 \mathrm{E}-0.5$ & $3.46 E-06$ & $1.30 \mathrm{E}-01$ & $1.44 E+01$ \\
\hline & 500 & $6.77 \mathrm{E}-06$ & $2.16 \mathrm{E}-06$ & $8.09 \mathrm{E}-02$ & $8.99 \mathrm{E}+00$ & $4.96 \mathrm{E}-06$ & $1.58 \mathrm{E}-06$ & $5.92 \mathrm{E}-02$ & $6.59 \mathrm{E}+00$ \\
\hline GG-045 & 10 & $3.45 E-04$ & $8.62 E-04$ & $8.08 \mathrm{E}+00$ & $2.15 E+03$ & $2.53 \mathrm{E}-04$ & $6.31 \mathrm{E}-04$ & $5.92 \mathrm{E}+00$ & $1.58 E+03$ \\
\hline & 50 & $5.60 \mathrm{E}-05$ & $1.40 \mathrm{E}-04$ & $1.31 \mathrm{E}+00$ & $3.50 \mathrm{E}+02$ & $4.10 \mathrm{E}-05$ & $1.02 \mathrm{E}-04$ & $9.60 \mathrm{z}-01$ & $2.56 \mathrm{E}+02$ \\
\hline & 100 & $2.56 \mathrm{E}-05$ & $6.39 \mathrm{E}-05$ & $5.99 \mathrm{E}-01$ & $1.60 \mathrm{E}+02$ & $1.88 \mathrm{E}-05$ & $4.68 E-05$ & $4.39 \mathrm{E}-01$ & $1.17 \mathrm{E}+02$ \\
\hline & 250 & 9.09 E-06 & $2.27 E-05$ & $2.13 E-01$ & $5.67 \mathrm{E}+01$ & $6.66 \mathrm{E}-06$ & $1.66 \mathrm{E}-0.5$ & $1.56 \mathrm{E}-01$ & $4.15 \mathrm{E}+01$ \\
\hline & 500 & $4.15 \mathrm{E}-06$ & $1.04 \mathrm{E}-05$ & $9.72 \mathrm{E}-02$ & $2.59 \mathrm{E}+01$ & $3.04 \mathrm{E}-06$ & $7.59 \mathrm{E}-06$ & $7.12 \mathrm{E}-02$ & $1.90 \mathrm{E}+01$ \\
\hline GG-046 & 10 & $2.88 \mathrm{E}-04$ & $4.31 \mathrm{E}-05$ & $1.13 \mathrm{E}+00$ & $1.66 \mathrm{E}+02$ & $2.11 E-04$ & $3.15 \mathrm{E}-05$ & $8.24 \mathrm{E}-01$ & $1.21 E+02$ \\
\hline & 50 & $4.67 \mathrm{E}-05$ & $6.99 \mathrm{~F}-06$ & 1. B3E-01 & $2.69 E+01$ & $3-42 \mathrm{~B}-05$ & $5.12 \mathrm{E}-06$ & $1.34 \mathrm{E}-01$ & $1.97 \mathrm{E}+01$ \\
\hline & 100 & $2.13 E-05$ & $3.19 E-06$ & $8.34 \mathrm{E}-02$ & $1.23 E+01$ & $1.56 \mathrm{E}-0.5$ & $2.34 \mathrm{E}-06$ & $6.11 \mathrm{E}-02$ & $8.99 E+00$ \\
\hline & 250 & $7.58 \equiv-06$ & $1.13 z-06$ & $2.96 \mathrm{E}-02$ & $4.36 \mathrm{E}+00$ & $5.55 \mathrm{E}-06$ & $8.30 \mathrm{E}-07$ & $2.17 \underline{-02}$ & $3.19 \mathrm{E}+00$ \\
\hline & 500 & $3.46 z-06$ & $5.18 \mathrm{E}-07$ & $1.35 \mathrm{E}-02$ & $1.99 \mathrm{E}+00$ & $2.54 \mathrm{E}-06$ & $3.79 \mathrm{E}-07$ & $9.92 \mathrm{E}-03$ & $1.46 \mathrm{E}+00$ \\
\hline GG-047 & 10 & $2.51 E-04$ & $1.13 E-03$ & $1.22 \mathrm{E}+01$ & $2.99 \mathrm{E}+03$ & 1. $84 \mathrm{E}-04$ & $8.29 \mathrm{E}-04$ & $8.94 E+00$ & $2.19 \mathrm{E}+03$ \\
\hline & 50 & $4.07 \mathrm{E}-05$ & $1.84 \mathrm{E}-04$ & $1.98 \mathrm{E}+00$ & $4.84 \mathrm{E}+02$ & $2.98 \mathrm{E}-05$ & $1.35 \mathrm{E}-04$ & $1.45 \mathrm{E}+00$ & $3.55 E+02$ \\
\hline & 100 & $1.86 \mathrm{E}-05$ & $8.39 E-05$ & $9.05 \mathrm{E}-01$ & $2.21 E+02$ & $1.36 \mathrm{E}-05$ & $6.15 \mathrm{E}-05$ & $6.63 \mathrm{E}-01$ & $1.62 \mathrm{E}+02$ \\
\hline & 250 & $6.61 E-06$ & $2.98 E-05$ & $3.21 \mathrm{E}-01$ & $7.86 E+01$ & $4.84 E-06$ & $2.18 \mathrm{E}-05$ & $2.35 \mathrm{E}-01$ & $5.76 \mathrm{E}+01$ \\
\hline & 500 & $3.02 \mathrm{E}-06$ & $1.36 \mathrm{E}-0.5$ & $1.47 E-01$ & $3.59 \mathrm{E}+01$ & $2.21 E-06$ & $9.97 E-06$ & $1.08 \mathrm{E}-01$ & $2.63 \mathrm{E}+01$ \\
\hline GO-048 & 10 & $3.80 \mathrm{~B}-04$ & $7.44 E-04$ & $8.73 \mathrm{E}+00$ & $2.60 \mathrm{E}+03$ & $2.78 \mathrm{E}-04$ & $5.45 E-04$ & $6.40 \mathrm{E}+00$ & $1.90 \mathrm{E}+03$ \\
\hline & 50 & $6.16 \mathrm{E}-05$ & $1.21 E-04$ & $1.42 \mathrm{E}+00$ & $4.21 E+02$ & $4.51 \mathrm{E}-05$ & $8.84 \mathrm{E}-05$ & $1.04 \mathrm{E}+00$ & $3.08 \mathrm{E}+02$ \\
\hline & 100 & $2.81 \mathrm{E}-05$ & $5.51 E-05$ & $6.47 E-01$ & $1.92 \mathrm{E}+02$ & $2.06 \mathrm{E}-0.5$ & $4.04 \mathrm{E}-05$ & $4.74 E-01$ & $1.41 E+02$ \\
\hline & 250 & $9.99 E-06$ & $1.96 \mathrm{E}-05$ & $2.30 \mathrm{E}-01$ & $6.83 \mathrm{E}+01$ & 7. $32 \mathrm{E}-06$ & $1.43 E-0.5$ & $1.68:-01$ & $5.00 \mathrm{E}+01$ \\
\hline & 500 & $4.57 \mathrm{E}-06$ & $8.95 E-06$ & $1.05 \mathrm{E}-01$ & $3.12 \mathrm{E}+01$ & $3.34 \mathrm{E}-06$ & $6.55 \mathrm{E}-06$ & $7.69 \mathrm{E}-02$ & $2.29 \mathrm{E}+01$ \\
\hline
\end{tabular}


A STABTLITY, $5 \mathrm{M} / \mathrm{S}$ SOURCE DISTANCE REL1 TERM (M) DOSE RATE RARTITION (M) DOSB RATE)

REL2

(SV)

(continued)

TOT

(Sv)

$\begin{array}{llll}1.25 \mathrm{E}-04 & 2.08 \mathrm{E}-05 & 4.20 \mathrm{E}+02 & 3.69 \mathrm{E}+04\end{array}$

$\begin{array}{llll}2.03 \mathrm{E}-05 & 3.38 \mathrm{E}-06 & 6.82 \mathrm{E}+01 & 5.99 \mathrm{E}+03\end{array}$

$\begin{array}{llll}9.29 \mathrm{E}-06 & 1.54 \mathrm{E}-06 & 3.12 \mathrm{E}+01 & 2.74 \mathrm{E}+03\end{array}$

3.30E-06 5.48E-07 $1.11 \mathrm{E}+01 \quad 9.72 \mathrm{E}+02$

$\begin{array}{llll}1.51 \mathrm{E}-06 & 2.50 \mathrm{E}-07 & 5.05 \mathrm{E}+00 & 4.44 \mathrm{E}+02\end{array}$

GG-050

$\begin{array}{llll}6.24 \mathrm{E}-05 & 1.50 \mathrm{E}-05 & 1.48 \mathrm{E}+02 & 1.45 \mathrm{E}+04\end{array}$

$\begin{array}{llll}1.01 E-05 & 2.43 E-06 & 2.41 E+01 & 2.36 E+03\end{array}$

4.62E-06 $1.12 \mathrm{E}-06 \quad 1.10 \mathrm{E}+01 \quad 1.08 \mathrm{E}+03$

$\begin{array}{llll}1.64 \mathrm{E}-06 & 3.94 \mathrm{E}-07 & 3.91 \mathrm{E}+00 & 3.82 \mathrm{E}+02\end{array}$

7.50E-07 1.80E-07 $1.79 \mathrm{E}+00 \quad 1.75 \mathrm{E}+02$

GG-051

10

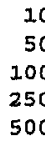

$5.32 \mathrm{E}-05$

$1.75 \mathrm{E}+02$

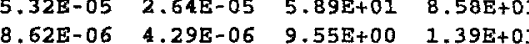

$3.94 \mathrm{E}-06 \quad 1.96 \mathrm{E}-06 \quad 4.37 \mathrm{E}+00 \quad 6.36 \mathrm{E}+02$

$\begin{array}{llll}1.40 \mathrm{E}-06 & 6.96 \mathrm{E}-07 & 1.55 \mathrm{E}+00 & 2.26 \mathrm{E}+02\end{array}$

$\begin{array}{llll}6.39 E-07 & 3.18 E-07 & 7.08 E-01 & 1.03 E+02\end{array}$

GQ-052

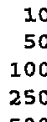

$7.70 \mathrm{E}-06 \quad 8.67 \mathrm{E}+00 \quad 1.26 \mathrm{E}+03$

$2.03 \mathrm{E}-06 \quad 2.25 \mathrm{E}-06 \quad 1.41 \mathrm{E}+00 \quad 2.05 \mathrm{E}+02$

$\begin{array}{llll}9.27 \mathrm{E}-07 & 5.71 \mathrm{E}-07 & 6.42 \mathrm{E}-01 & 9.35 \mathrm{E}+01\end{array}$

$\begin{array}{llll}3.29 \mathrm{E}-07 & 2.03 \mathrm{E}-07 & 2.28 \mathrm{E}-01 & 3.32 \mathrm{E}+01\end{array}$

$\begin{array}{llll}1.50 \mathrm{E}-07 & 9.26 \mathrm{E}-08 & 1.04 \mathrm{E}-01 & 1.52 \mathrm{E}+01\end{array}$

GG-053

10
50

$2.78 \mathrm{E}-06 \quad 1.93 \mathrm{E}-06 \quad 1.63 \mathrm{E}+00 \quad 2.15 \mathrm{E}+02$

$\begin{array}{lllll}4.50 \mathrm{E}-07 & 3.13 \mathrm{E}-07 & 2.65 \mathrm{E}-01 & 3.49 \mathrm{E}+01\end{array}$

$\begin{array}{llll}2.06 E-07 & 1.43 E-07 & 1.21 E-01 & 1.59 E+01\end{array}$

$\begin{array}{llll}7.30 \mathrm{E}-08 & 5.08 \mathrm{E}-08 & 4.30 \mathrm{E}-02 & 5.66 \mathrm{E}+00\end{array}$

$\begin{array}{llll}3.34 E-08 & 2.32 E-08 & 1.96 \mathrm{E}-02 & 2.58 \mathrm{E}+00\end{array}$

GG-054

$\begin{array}{llll}7.08 \mathrm{E}-07 & 5.11 \mathrm{E}-07 \quad 2.82 \mathrm{E}-01 & 3.09 \mathrm{E}+01\end{array}$

$\begin{array}{llll}1.15 E-07 & 8.29 E-08 & 4.58 E-02 & 5.02 E+00\end{array}$

$\begin{array}{llll}5.25 E-08 & 3.79 E-08 & 2.09 \mathrm{E}-02 & 2.29 \mathrm{E}+00\end{array}$

$\begin{array}{llll}1.86 \mathrm{E}-08 & 1.34 \mathrm{E}-08 & 7.43 \mathrm{E}-03 & 8.14 \mathrm{E}-01\end{array}$

8.52E-09 6.14E-09 3.40E-03 3.72E-01

GG-055

10
50

$\begin{array}{lllll}0.00 \mathrm{E}+00 & 0.00 \mathrm{E}+00 & 0.00 \mathrm{E}+00 & 0.00 \mathrm{E}+00\end{array}$

$\begin{array}{lllll}0.00 \mathrm{E}+00 & 0.00 \mathrm{E}+00 & 0.00 \mathrm{E}+00 & 0.00 \mathrm{E}+00\end{array}$

$\begin{array}{lllll}0.00 E+00 & 0.00 E+00 & 0.00 E+00 & 0.00 E+00\end{array}$

$\begin{array}{lllll}0.00 \mathrm{E}+00 & 0.00 \mathrm{E}+00 & 0.00 \mathrm{E}+00 & 0.00 \mathrm{E}+00\end{array}$

$\begin{array}{llll}0.00 \mathrm{E}+00 & 0.00 \mathrm{E}+00 & 0.00 \mathrm{E}+00 & 0.00 \mathrm{E}+00\end{array}$
$\mathrm{Y}, 1 \mathrm{M} / \mathrm{s}$

REL2

15-MIN

DOSE

TOTAL

DOSE

(SV/S)

(SV/S)

(SV)

(SV)

$9.18 \mathrm{E}-05 \quad 1.53 \mathrm{E}-05 \quad 3.08 \mathrm{E}+02 \quad 2.70 \mathrm{E}+0$

$\begin{array}{lllll}1.49 \mathrm{E}-05 & 2.47 \mathrm{E}-06 & 4.99 \mathrm{E}+01 & 4.39 \mathrm{~B}+03\end{array}$

$\begin{array}{llll}6.80 E-06 & 1.13 \mathrm{E}-06 & 2.28 \mathrm{E}+01 & 2.00 \mathrm{E}+03\end{array}$

$\begin{array}{llll}2.42 E-05 & 4.01 E-07 & 8.108+00 & 7.12 E+02\end{array}$

$\begin{array}{llll}1.10 \mathrm{E}-06 & 1.83 \mathrm{E}-07 & 3.70 \mathrm{E}+00 & 3.25 \mathrm{E}+02\end{array}$

$\begin{array}{llll}4.57 \mathrm{E}-05 & 1.10 \mathrm{E}-05 & 2.09 \mathrm{E}+02 & 1.06 \mathrm{E}+04\end{array}$

$\begin{array}{llll}7.41 \mathrm{E}-06 & 1.78 \mathrm{E}-06 & 1.76 \mathrm{E}+01 & 1.73 \mathrm{E}+03\end{array}$

$\begin{array}{llll}3.39 E-06 & 8.14 \mathrm{E}-07 & 8.06 \mathrm{E}+00 & 7.89 \mathrm{E}+02\end{array}$

$\begin{array}{lllll}1.20 \mathrm{E}-06 & 2.89 \mathrm{~B}-07 & 2.86 \mathrm{E}+00 & 2.80 \mathrm{E}+02\end{array}$

5.49E-07 1.32E-07 1.31E+00 1.28E+02

$3.89 \mathrm{E}-05 \quad 1.94 \mathrm{E}-05 \quad 4.31 \mathrm{E}+01 \quad 6.28 \mathrm{E}+03$

$\begin{array}{llll}6.32 \mathrm{E}-06 & 3.14 \mathrm{E}-06 & \mathbf{7 . 0 0 \mathrm { E }}+00 & 1.02 \mathrm{E}+03\end{array}$

$2.89 E-06 \quad 1.44 E-06 \quad 3.20 \mathrm{E}+00 \quad 4.66 \mathrm{E}+02$

$\begin{array}{lllll}1.02 \mathrm{E}-06 & 5.10 \mathrm{E}-07 & 1.24 \mathrm{E}+00 & 1.65 \mathrm{E}+02\end{array}$

$\begin{array}{llll}4.68 \mathrm{E}-07 & 2.33 \mathrm{E}-07 & 5.19 \mathrm{E}-01 & 7.56 \mathrm{E}+01\end{array}$

$9.16 \mathrm{E}-06 \quad 5.64 \mathrm{E}-05 \quad 6.35 \mathrm{E}+00 \quad 9.24 \mathrm{E}+02$

$\begin{array}{llll}1.49 \mathrm{E}-06 & 9.15 \mathrm{E}-07 & 1.03 \mathrm{E}+00 & 1.50 \mathrm{E}+02\end{array}$

$\begin{array}{llll}6.79 \mathrm{E}-07 & 4.18 \mathrm{E}-07 & 4.71 \mathrm{E}-01 & 6.85 \mathrm{E}+01\end{array}$

$\begin{array}{lllll}2.41 \mathrm{E}-07 & 1.49 \mathrm{E}-07 & 1.67 \mathrm{E}-01 & 2.43 \mathrm{E}+01\end{array}$

$\begin{array}{llll}1.10 \mathrm{E}-07 & 6.79 \mathrm{E}-08 & 7.63 \mathrm{E}-02 & 1.11 \mathrm{E}+01\end{array}$

2.03E-06 $\quad 1.41 \mathrm{E}-06 \quad 1.20 \mathrm{E}+00 \quad 1.57 \mathrm{E}+02$

$\begin{array}{llll}3.30 \mathrm{E}-07 & 2.29 \mathrm{E}-07 & 1.94 \mathrm{E}-01 & 2.55 \mathrm{E}+01\end{array}$

$\begin{array}{llll}1.51 \mathrm{E}-07 & 3.05 \mathrm{E}-07 & 8.87 \mathrm{E}-02 & 1.17 \mathrm{E}+01\end{array}$

$\begin{array}{llll}5.35 \mathrm{E}-08 & 3.72 \mathrm{E}-08 & 3.15 \mathrm{E}-02 & 4.14 \mathrm{E}+00\end{array}$

$2.44 \mathrm{E}-08 \quad 1.70 \mathrm{E}-08 \quad 1.44 \mathrm{E}-02 \quad 1.89 \mathrm{E}+00$

$\begin{array}{lllll}5.19 \mathrm{E}-07 & 3.74 \mathrm{E}-07 & 2.07 \mathrm{E}-01 & 2.27 \mathrm{E}+01\end{array}$

$\begin{array}{llll}8.42 E-08 & 6.07 E-08 & 3.36 \mathrm{E}-02 & 3.68 \mathrm{E}+00\end{array}$

$\begin{array}{llll}3.85 \mathrm{E}-08 & 2.77 \mathrm{E}-08 & 1.53 \mathrm{E}-02 & 1.68 \mathrm{E}+00\end{array}$

$\begin{array}{lllll}1.37 \mathrm{E}-08 & 9.85 \mathrm{E}-09 & 5.44 \mathrm{E}-03 & 5.96 \mathrm{E}-01\end{array}$

$\begin{array}{llll}6.24 \mathrm{E}-09 & 4.50 \mathrm{E}-09 & 2.49 \mathrm{E}-03 & 2.72 \mathrm{E}-01\end{array}$

$\begin{array}{llll}0.00 \mathrm{E}+00 & 0.00 \mathrm{E}+00 & 0.00 \mathrm{E}+00 \quad 0.00 \mathrm{~B}+00\end{array}$

$\begin{array}{lllll}0.00 \mathrm{E}+00 & 0.00 \mathrm{E}+00 & 0.00 \mathrm{E}+00 & 0.00 \mathrm{E}+00\end{array}$

$\begin{array}{lllll}0.00 \mathrm{E}+00 & 0.00 \mathrm{E}+00 & 0.00 \mathrm{E}+00 & 0.00 \mathrm{E}+00\end{array}$

$\begin{array}{lllll}0.00 E+00 & 0.00 E+00 & 0.00 E+00 & 0.00 E+00\end{array}$

$\begin{array}{llll}0.00 \mathrm{E}+00 & 0.00 \mathrm{E}+00 & 0.00 \mathrm{E}+00 & 0.00 \mathrm{E}+00\end{array}$ 
Appendix D

\section{D.4 Offsite Consequences for Source Term Groups}

The offsite consequences were estimated using Version 1.5.11.1 of the MACCS code. The mean (over weather variation) consequences for the 54 source term groups defined in Table C.3-1 are reported in Table D.4-1. The $55^{\text {th }}$ source term group in Table D.4-1 is present in case any source terms fall into the special case of no release; the consequences for this case are 0.0. The remaining results given in this table are conditional on the occurrence of a release. That is, given that a release takes place, with release fractions and other characteristics as defined by one of the source term groups, then the consequences reported in this section are calculated. An early fatality consequence value less than 1.0 may be interpreted as the probability of obtaining one death. The population dose is the effective dose equivalent to the whole body for the population in the region indicated.

The only uncertainty included in the consequence analysis was the variability in the weather at the time of the accident. Hence, for each source term group, a distribution of consequences that result from the various weather scenarios that are possible during the accident were calculated. For each source term group, a mean consequence was then calculated from this distribution. It is this mean consequence that is used in the aggregate risk calculation and that is reported in Table D.4-1. Table D.4-1 contains no information about the frequency with which these consequences may be expected. Information about the frequencies of consequences of various magnitudes is contained in the risk results (Chapter 9). 
Appendix D

Table D.4-1

Mean Offsite Consequence Results

\begin{tabular}{|c|c|c|c|c|c|c|}
\hline $\begin{array}{l}\text { Source } \\
\text { Term } \\
\text { Group }\end{array}$ & $\begin{array}{c}\text { Early } \\
\text { Fatalities }\end{array}$ & $\begin{array}{c}\text { Total Latent } \\
\text { Cancer Fatalities }\end{array}$ & $\begin{array}{c}\text { Population } \\
\text { Dose (rem) } \\
<50 \text { miles }\end{array}$ & $\begin{array}{c}\text { Population } \\
\text { Dose (rem) } \\
<1000 \text { miles }\end{array}$ & $\begin{array}{c}\text { Individual } \\
\text { Early Fatality } \\
\text { Risk }\end{array}$ & $\begin{array}{c}\text { Individual } \\
\text { Total Latent } \\
\text { Cancer Risk }\end{array}$ \\
\hline GG-001 & $1.24 \mathrm{E}-02$ & $3.11 E+03$ & $7.38 E+05$ & $7.26 \mathrm{E}+06$ & $1.08 \mathrm{E}-04$ & $7.20 \mathrm{E}-04$ \\
\hline GG-002 & $9.65 \mathrm{E}-03$ & $3.39 \mathrm{E}+03$ & $7.98 \mathrm{E}+05$ & $7.83 E+06$ & $9.30 \mathrm{E}-05$ & 8.43E-04 \\
\hline GG-003 & $1.20 \mathrm{E}-02$ & $3.57 \mathrm{E}+03$ & $8.54 E+05$ & $8.28 \mathrm{E}+06$ & $1.06 \mathrm{E}-04$ & $6.72 \mathrm{E}-04$ \\
\hline GG-004 & $1.08 \mathrm{E}-01$ & $5.83 E+03$ & $1.30 \mathrm{E}+06$ & $1.27 \mathrm{E}+07$ & 4.79E-04 & $1.84 \mathrm{E}-03$ \\
\hline GG-005 & $1.77 \mathrm{E}-02$ & $4.78 E+03$ & $9.83 E+05$ & $1.11 E+07$ & $1.41 \mathrm{E}-04$ & $9.00 \mathrm{E}-04$ \\
\hline GG-006 & $1.79 \mathrm{E}-03$ & $2.06 \mathrm{E}+03$ & $5.71 E+05$ & $4.79 \mathrm{E}+06$ & $2.24 \mathrm{E}-05$ & $9.01 \mathrm{E}-04$ \\
\hline GG-007 & $1.35 \mathrm{E}-03$ & $1.85 \mathrm{E}+03$ & $5.48 \mathrm{E}+05$ & $4.43 E+06$ & $1.70 \mathrm{E}-05$ & 7.22E-04 \\
\hline GG-008 & $3.14 \mathrm{E}-03$ & $2.73 E+03$ & $6.55 \mathrm{E}+05$ & $6.30 \mathrm{E}+06$ & $3.80 \mathrm{E}-05$ & $1.01 \mathrm{E}-03$ \\
\hline GG-009 & $3.31 \mathrm{E}-02$ & $4.38 \mathrm{E}+03$ & $9.76 \mathrm{E}+05$ & $1.00 \mathrm{E}+07$ & $1.99 \mathrm{E}-04$ & $8.46 \mathrm{E}-04$ \\
\hline GG-010 & $2.41 \mathrm{E}-03$ & $1.69 \mathrm{E}+03$ & $4.66 \mathrm{E}+05$ & $3.90 \mathrm{E}+06$ & $2.96 \mathrm{E}-05$ & $6.61 \mathrm{E}-04$ \\
\hline GG-011 & $4.38 \mathrm{E}-03$ & $1.73 E+03$ & $4.13 E+05$ & $3.90 \mathrm{E}+06$ & $5.05 \mathrm{E}-05$ & $8.26 \mathrm{E}-04$ \\
\hline GG-012 & $2.98 \mathrm{E}-02$ & $5.12 \mathrm{E}+03$ & $1.21 E+06$ & $1.20 \mathrm{E}+07$ & $1.92 \mathrm{E}-04$ & 7.70E-04 \\
\hline GG-013 & $2.20 \mathrm{E}-02$ & $4.16 E+03$ & $9.69 \mathrm{E}+05$ & $9.39 \mathrm{E}+06$ & 1.62E-04 & 9.47E-04 \\
\hline GG-014 & $8.20 \mathrm{E}-02$ & $5.37 E+03$ & $1.12 E+06$ & $1.10 \mathrm{E}+07$ & $3.14 \mathrm{E}-04$ & $1.11 \mathrm{E}-03$ \\
\hline GG-015 & $1.56 \mathrm{E}-03$ & $1.54 \mathrm{E}+03$ & $4.13 E+05$ & $3.56 \mathrm{E}+06$ & $1.95 \mathrm{E}-05$ & 7.69E-04 \\
\hline GG-016 & $2.33 \mathrm{E}-03$ & $1.59 \mathrm{E}+03$ & $4.34 \mathrm{E}+05$ & $3.82 E+06$ & 2.87E-05 & 7.57E-04 \\
\hline GG-017 & $3.94 \mathrm{E}-02$ & $6.10 \mathrm{E}+03$ & $1.55 \mathrm{E}+06$ & $1.41 \mathrm{E}+07$ & $2.60 \mathrm{E}-04$ & 7.64E-04 \\
\hline GG-018 & $9.40 \mathrm{E}-04$ & $9.02 \mathrm{E}+02$ & $2.98 \mathrm{E}+05$ & $2.13 E+06$ & $1.18 \mathrm{E}-05$ & $5.81 \mathrm{E}-04$ \\
\hline GG-019 & $7.25 \mathrm{E}-03$ & $1.78 \mathrm{E}+03$ & $4.78 \mathrm{E}+05$ & $4.06 E+06$ & $7.30 \mathrm{E}-05$ & $5.95 \mathrm{E}-04$ \\
\hline GG-020 & $6.65 \mathrm{E}-03$ & $2.87 E+03$ & $6.15 \mathrm{E}+05$ & $6.59 \mathrm{E}+06$ & $6.75 \mathrm{E}-05$ & $6.12 \mathrm{E}-04$ \\
\hline GG-021 & $1.05 \mathrm{E}-05$ & $4.41 E+02$ & $1.74 \mathrm{E}+05$ & $1.01 E+06$ & $1.33 \mathrm{E}-07$ & $4.63 \mathrm{E}-04$ \\
\hline GG-022 & $1.64 \mathrm{E}-07$ & $8.22 \mathrm{E}+02$ & $2.80 \mathrm{E}+05$ & $1.86 \mathrm{E}+06$ & $2.08 \mathrm{E}-09$ & 7.37E-04 \\
\hline GG-023 & $0.00 \mathrm{E}+00$ & $4.99 \mathrm{E}+01$ & $2.88 \mathrm{E}+04$ & $1.19 E+05$ & $0.00 \mathrm{E}+00$ & $1.26 \mathrm{E}-04$ \\
\hline GG-024 & 7.35E-03 & $1.35 \mathrm{E}+03$ & $4.70 \mathrm{E}+05$ & $3.30 E+06$ & $7.10 \mathrm{E}-05$ & $4.33 \mathrm{E}-04$ \\
\hline GG-025 & $3.17 \mathrm{E}-03$ & $1.72 E+03$ & $4.74 \mathrm{E}+05$ & $4.03 E+06$ & $3.72 E-05$ & $7.11 \mathrm{E}-04$ \\
\hline GG-026 & $0.00 \mathrm{E}+00$ & $6.45 \mathrm{E}+01$ & $4.19 E+04$ & $1.79 E+05$ & $0.00 \mathrm{E}+00$ & $1.66 \mathrm{E}-04$ \\
\hline GG-027 & $6.00 \mathrm{E}-03$ & $1.43 E+03$ & $4.14 \mathrm{E}+05$ & $3.23 E+06$ & $6.25 \mathrm{E}-05$ & $6.83 \mathrm{E}-04$ \\
\hline GG-028 & $4.12 \mathrm{E}-02$ & $3.94 \mathrm{E}+03$ & $9.53 E+05$ & $8.74 E+06$ & $2.25 \mathrm{E}-04$ & $1.31 \mathrm{E}-03$ \\
\hline GG-029 & $1.54 \mathrm{E}-02$ & $3.16 \mathrm{E}+03$ & $7.78 \mathrm{E}+05$ & $7.37 \mathrm{E}+06$ & $1.23 \mathrm{E}-04$ & $7.88 \mathrm{E}-04$ \\
\hline GG-030 & $2.76 \mathrm{E}-02$ & $4.95 \mathrm{E}+03$ & $9.89 \mathrm{E}+05$ & $1.13 \mathrm{E}+07$ & $1.80 \mathrm{E}-04$ & 1.19E-03 \\
\hline GG-031 & $1.44 \mathrm{E}-03$ & $2.00 \mathrm{E}+03$ & $5.71 E+05$ & $4.71 E+06$ & $1.81 E-05$ & 8.21E-04 \\
\hline GG-032 & $6.92 \mathrm{E}-02$ & $6.52 E+03$ & $1.61 \mathrm{E}+06$ & $1.47 \mathrm{E}+07$ & 3.27E-04 & $1.49 \mathrm{E}-03$ \\
\hline GG-033 & $2.54 \mathrm{E}-03$ & $2.89 E+03$ & $6.85 E+05$ & $6.67 E+06$ & $3.14 \mathrm{E}-05$ & $9.95 \mathrm{E}-04$ \\
\hline GG-034 & $1.08 \mathrm{E}-03$ & $1.70 E+03$ & $4.29 E+05$ & $3.89 E+06$ & $1.36 \mathrm{E}-05$ & $8.22 \mathrm{E}-04$ \\
\hline GG-035 & 3.93E-02 & $4.86 \mathrm{E}+03$ & $1.06 \mathrm{E}+06$ & $1.06 \mathrm{E}+07$ & $2.24 \mathrm{E}-04$ & $1.02 \mathrm{E}-03$ \\
\hline GG-036 & $3.88 \mathrm{E}-03$ & $2.89 \mathrm{E}+03$ & $6.13 E+05$ & $6.64 \mathrm{E}+06$ & $4.42 \mathrm{E}-05$ & 7.33E-04 \\
\hline GG-037 & 8.10E-03 & $1.97 \mathrm{E}+03$ & $5.07 \mathrm{E}+05$ & $4.48 \mathrm{E}+06$ & 7.85E-05 & $7.88 \mathrm{E}-04$ \\
\hline
\end{tabular}


Appendix D

Table D.4-1 (continued)

Mean Offsite Consequence Results

\begin{tabular}{c|cccccc}
\hline $\begin{array}{c}\text { Source } \\
\text { Term } \\
\text { Group }\end{array}$ & $\begin{array}{c}\text { Early } \\
\text { Fatalities }\end{array}$ & $\begin{array}{c}\text { Total Latent } \\
\text { Cancers }\end{array}$ & $\begin{array}{c}\text { Population } \\
\text { Dose (rem) } \\
<50 \text { miles }\end{array}$ & $\begin{array}{c}\text { Population } \\
\text { Dose (rem) } \\
<1000 \text { miles }\end{array}$ & $\begin{array}{c}\text { Individual } \\
\text { Early Fatality } \\
\text { Risk }\end{array}$ & $\begin{array}{c}\text { Individual } \\
\text { Total Latent } \\
\text { Cancer Risk }\end{array}$ \\
\hline GG-038 & $8.05 \mathrm{E}-03$ & $3.26 \mathrm{E}+03$ & $8.21 \mathrm{E}+05$ & $7.62 \mathrm{E}+06$ & $7.95 \mathrm{E}-05$ & $8.60 \mathrm{E}-04$ \\
GG-039 & $9.35 \mathrm{E}-04$ & $2.31 \mathrm{E}+03$ & $5.70 \mathrm{E}+05$ & $5.28 \mathrm{E}+06$ & $1.15 \mathrm{E}-05$ & $6.76 \mathrm{E}-04$ \\
GG-040 & $8.75 \mathrm{E}-05$ & $2.79 \mathrm{E}+03$ & $6.39 \mathrm{E}+05$ & $6.39 \mathrm{E}+06$ & $1.11 \mathrm{E}-06$ & $8.95 \mathrm{E}-04$ \\
GG-041 & $6.80 \mathrm{E}-04$ & $1.44 \mathrm{E}+03$ & $4.32 \mathrm{E}+05$ & $3.38 \mathrm{E}+06$ & $8.60 \mathrm{E}-06$ & $8.85 \mathrm{E}-04$ \\
GG-042 & $3.72 \mathrm{E}-07$ & $5.82 \mathrm{E}+02$ & $2.29 \mathrm{E}+05$ & $1.39 \mathrm{E}+06$ & $4.72 \mathrm{E}-09$ & $4.44 \mathrm{E}-04$ \\
GG-043 & $2.87 \mathrm{E}-03$ & $2.16 \mathrm{E}+03$ & $6.24 \mathrm{E}+05$ & $5.11 \mathrm{E}+06$ & $3.50 \mathrm{E}-05$ & $8.24 \mathrm{E}-04$ \\
GG-044 & $2.97 \mathrm{E}-07$ & $9.85 \mathrm{E}+02$ & $3.20 \mathrm{E}+05$ & $2.24 \mathrm{E}+06$ & $3.76 \mathrm{E}-09$ & $6.65 \mathrm{E}-04$ \\
GG-045 & $1.58 \mathrm{E}-03$ & $1.22 \mathrm{E}+03$ & $4.15 \mathrm{E}+05$ & $2.93 \mathrm{E}+06$ & $1.98 \mathrm{E}-05$ & $6.37 \mathrm{E}-04$ \\
GG-046 & $0.00 \mathrm{E}+00$ & $6.72 \mathrm{E}+01$ & $4.92 \mathrm{E}+04$ & $1.88 \mathrm{E}+05$ & $0.00 \mathrm{E}+00$ & $1.79 \mathrm{E}-04$ \\
GG-047 & $4.03 \mathrm{E}-03$ & $1.37 \mathrm{E}+03$ & $4.84 \mathrm{E}+05$ & $3.28 \mathrm{E}+06$ & $4.64 \mathrm{E}-05$ & $6.17 \mathrm{E}-04$ \\
GG-048 & $4.96 \mathrm{E}-04$ & $1.76 \mathrm{E}+03$ & $4.79 \mathrm{E}+05$ & $4.07 \mathrm{E}+06$ & $6.25 \mathrm{E}-06$ & $8.05 \mathrm{E}-04$ \\
GG-049 & $1.20 \mathrm{E}-02$ & $4.06 \mathrm{E}+03$ & $8.64 \mathrm{E}+05$ & $8.82 \mathrm{E}+06$ & $1.08 \mathrm{E}-04$ & $1.02 \mathrm{E}-03$ \\
GG-050 & $3.61 \mathrm{E}-03$ & $3.08 \mathrm{E}+03$ & $6.47 \mathrm{E}+05$ & $6.76 \mathrm{E}+06$ & $4.27 \mathrm{E}-05$ & $9.24 \mathrm{E}-04$ \\
GG-051 & $6.35 \mathrm{E}-04$ & $2.21 \mathrm{E}+03$ & $5.37 \mathrm{E}+05$ & $5.04 \mathrm{E}+06$ & $8.05 \mathrm{E}-06$ & $8.31 \mathrm{E}-04$ \\
GG-052 & $0.00 \mathrm{E}+00$ & $1.64 \mathrm{E}+03$ & $3.94 \mathrm{E}+05$ & $3.60 \mathrm{E}+06$ & $0.00 \mathrm{E}+00$ & $9.60 \mathrm{E}-04$ \\
GG-053 & $0.00 \mathrm{E}+00$ & $4.46 \mathrm{E}+02$ & $1.74 \mathrm{E}+05$ & $9.75 \mathrm{E}+05$ & $0.00 \mathrm{E}+00$ & $5.62 \mathrm{E}-04$ \\
GG-054 & $0.00 \mathrm{E}+00$ & $7.47 \mathrm{E}+01$ & $5.77 \mathrm{E}+04$ & $1.70 \mathrm{E}+05$ & $0.00 \mathrm{E}+00$ & $2.04 \mathrm{E}-04$ \\
GG-055 & $0.00 \mathrm{E}+00$ & $0.00 \mathrm{E}+00$ & $0.00 \mathrm{E}+00$ & $0.00 \mathrm{E}+00$ & $0.00 \mathrm{E}+00$ & $0.00 \mathrm{E}+00$ \\
\hline \hline
\end{tabular}




\section{Appendix E Supporting Information for the Risk Analysis}

Table E-1 contains the descriptive statistics for the risk distributions for each plant damage state. The distributions were generated using a Latin hypercube sampling (LHS) size of 200 observations. The risk measures are described in Chapter 8 of the main report.

Table E-1

Plant Damage State Risk Results*

\begin{tabular}{|c|c|c|c|c|c|c|}
\hline \multirow[t]{2}{*}{ PDS } & \multirow[t]{2}{*}{ Consequence Measure } & \multicolumn{5}{|c|}{ Descriptive Statistics } \\
\hline & & 5th PCT & 50th PCT & 95th PCT & MEAN & STD DEV. \\
\hline Total & Frequency & $4.1 E-07$ & $1.4 \mathrm{E}-06$ & $5.6 \mathrm{E}-06$ & $2.1 E-06$ & 2.7E-06 \\
\hline Total & Early Fatality & $3.7 \mathrm{E}-11$ & 2.8E-09 & $3.9 \mathrm{E}-08$ & $1.4 \mathrm{E}-08$ & $5.4 \mathrm{E}-08$ \\
\hline Total & Total Lat. Cancer & 4.3E-04 & $1.9 \mathrm{E}-03$ & $1.2 \mathrm{E}-02$ & $3.8 \mathrm{E}-03$ & 7.6E-03 \\
\hline Total & Pop. Dose within 50 miles & $1.3 \mathrm{E}-03$ & $5.3 \mathrm{E}-03$ & $3.1 \mathrm{E}-02$ & $9.9 E-03$ & $1.9 \mathrm{E}-02$ \\
\hline Total & Pop. Dose within 1000 miles & $9.9 \mathrm{E}-03$ & $4.4 \mathrm{E}-02$ & $2.8 \mathrm{E}-01$ & 8.7E-02 & $1.7 \mathrm{E}-01$ \\
\hline Total & Indiv. Early Fatalities within 1 mile & $4.2 \mathrm{E}-13$ & $2.6 \mathrm{E}-11$ & $3.0 \mathrm{E}-10$ & $9.6 \mathrm{E}-11$ & $3.4 \mathrm{E}-10$ \\
\hline Total & Indiv. Lat. Cancers with in 10 miles & $2.5 \mathrm{E}-10$ & $9.4 \mathrm{E}-10$ & $4.8 \mathrm{E}-09$ & $1.6 \mathrm{E}-09$ & $2.4 \mathrm{E}-09$ \\
\hline PDS1-1 & Frequency & $1.6 \mathrm{E}-09$ & $1.4 \mathrm{E}-08$ & $1.9 \mathrm{E}-07$ & $4.1 \mathrm{E}-08$ & 8.3E-08 \\
\hline PDS1-1 & Early Fatality & $5.3 E-13$ & $2.7 \mathrm{E}-11$ & $5.6 \mathrm{E}-10$ & $1.2 \mathrm{E}-10$ & $5.0 \mathrm{E}-10$ \\
\hline PDS1-1 & Total Lat. Cancer & $1.7 \mathrm{E}-06$ & $1.9 \mathrm{E}-05$ & $3.3 E-04$ & $6.7 \mathrm{E}-05$ & $1.4 \mathrm{E}-04$ \\
\hline PDS1-1 & Pop. Dose within 50 miles & $5.2 \mathrm{E}-06$ & $5.5 \mathrm{E}-05$ & $8.3 E-04$ & $1.9 \mathrm{E}-04$ & $4.0 \mathrm{E}-04$ \\
\hline PDS1-1 & Pop. Dose within 1000 miles & $4.1 E-05$ & 4.3E-04 & $7.4 \mathrm{E}-03$ & $1.6 \mathrm{E}-03$ & 3.3E-03 \\
\hline PDS1-1 & Indiv. Early Fatalities within 1 mile & $6.4 \mathrm{E}-15$ & $3.0 \mathrm{E}-13$ & $6.9 \mathrm{E}-12$ & $1.1 \mathrm{E}-12$ & $2.6 \mathrm{E}-12$ \\
\hline PDS1-1 & Indiv. Lat. Cancers within 10 miles & $1.0 \mathrm{E}-12$ & $1.0 \mathrm{E}-11$ & $1.5 \mathrm{E}-10$ & $3.0 \mathrm{E}-11$ & $6.3 \mathrm{E}-11$ \\
\hline PDS1-2 & Frequency & $1.4 \mathrm{E}-10$ & 4.3E-09 & $1.3 \mathrm{E}-07$ & $2.3 \mathrm{E}-08$ & $4.8 \mathrm{E}-08$ \\
\hline PDS 1-2 & Early Fatality & $1.1 \mathrm{E}-13$ & $2.9 E-11$ & $1.3 \mathrm{E}-09$ & $3.3 \mathrm{E}-10$ & $1.6 \mathrm{E}-09$ \\
\hline PDSI-2 & Total Lat. Cancer & $3.4 \mathrm{E}-07$ & $9.6 \mathrm{E}-06$ & $3.4 \mathrm{E}-04$ & $6.5 \mathrm{E}-05$ & $1.5 \mathrm{E}-04$ \\
\hline PDS1-2 & Pop. Dose within 50 miles & $9.6 \mathrm{E}-07$ & $2.5 \mathrm{E}-05$ & $8.0 \mathrm{E}-04$ & $1.6 \mathrm{E}-04$ & $3.6 \mathrm{E}-04$ \\
\hline PDS1-2 & Pop. Dose within 1000 miles & $7.8 \mathrm{E}-06$ & $2.2 \mathrm{E}-04$ & 7.7E-03 & $1.5 \mathrm{E}-03$ & $3.5 \mathrm{E}-03$ \\
\hline PDS1-2 & Indiv. Early Fatalities within 1 mile & $1.4 \mathrm{E}-15$ & $2.9 \mathrm{E}-13$ & $1.1 \mathrm{E}-11$ & $2.3 \mathrm{E}-12$ & $7.6 \mathrm{E}-12$ \\
\hline PDS1-2 & Indiv. Lat. Cancers within 10 miles & $1.1 \mathrm{E}-13$ & $2.9 \mathrm{E}-12$ & $9.7 \mathrm{E}-11$ & $1.8 \mathrm{E}-11$ & $4.2 \mathrm{E}-11$ \\
\hline PDS1-3 & Frequency & $2.9 \mathrm{E}-09$ & $1.7 \mathrm{E}-08$ & $1.6 \mathrm{E}-07$ & $4.4 \mathrm{E}-08$ & $1.2 \mathrm{E}-07$ \\
\hline PDS1-3 & Early Fatality & $1.4 \mathrm{E}-13$ & $1.2 \mathrm{E}-10$ & $1.4 \mathrm{E}-09$ & 3.0E-10 & $5.7 \mathrm{E}-10$ \\
\hline PDS1-3 & Total Lat. Cancer & $3.1 \mathrm{E}-06$ & 3.4E-05 & $2.9 \mathrm{E}-04$ & $7.9 \mathrm{E}-05$ & $1.3 \mathrm{E}-04$ \\
\hline PDS1-3 & Pop. Dose within 50 miles & $1.0 \mathrm{E}-05$ & 9.3E-05 & $7.6 \mathrm{E}-04$ & $2.0 \mathrm{E}-04$ & $3.5 \mathrm{E}-04$ \\
\hline PDS1-3 & Pop. Dose within 1000 miles & 7.3E-05 & $7.9 \mathrm{E}-04$ & $6.7 \mathrm{E}-03$ & $1.8 \mathrm{E}-03$ & $3.0 \mathrm{E}-03$ \\
\hline PDS1-3 & Indiv. Early Fatalities within 1 mile & $1.8 \mathrm{E}-15$ & $1.1 \mathrm{E}-12$ & $1.0 \mathrm{E}-11$ & $2.5 \mathrm{E}-12$ & $4.5 \mathrm{E}-12$ \\
\hline PDS1-3 & Indiv. Lat. Cancers within 10 miles & $1.7 \mathrm{E}-12$ & 1.1E-11 & $9.2 \mathrm{E}-11$ & $2.7 \mathrm{E}-11$ & $5.9 \mathrm{E}-11$ \\
\hline PDS1-4 & Frequency & 4.7E-11 & $2.0 \mathrm{E}-09$ & $3.5 \mathrm{E}-08$ & $9.1 \mathrm{E}-09$ & $2.9 \mathrm{E}-08$ \\
\hline PDS1-4 & Early Fatality & $2.9 \mathrm{E}-14$ & $1.0 \mathrm{E}-11$ & $3.2 \mathrm{E}-10$ & $6.5 \mathrm{E}-11$ & $1.9 \mathrm{E}-10$ \\
\hline PDS1-4 & Total Lat. Cancer & $5.3 \mathrm{E}-08$ & 3.7E-06 & 8.1E-05 & $1.9 \mathrm{E}-05$ & $5.4 \mathrm{E}-05$ \\
\hline PDS1-4 & Pop. Dose within 50 miles & $1.7 \mathrm{E}-07$ & $1.0 \mathrm{E}-05$ & $1.8 \mathrm{E}-04$ & $4.8 \mathrm{E}-05$ & $1.4 \mathrm{E}-04$ \\
\hline PDS1-4 & Pop. Dose within 1000 miles & $1.2 \mathrm{E}-06$ & 8.5E-05 & $1.8 \mathrm{E}-03$ & 4.4E-04 & $1.3 \mathrm{E}-03$ \\
\hline PDS1-4 & Indiv. Early Fatalities within 1 mile & 3.7E-16 & $9.8 \mathrm{E}-14$ & $2.5 \mathrm{E}-12$ & $5.3 \mathrm{E}-13$ & $1.4 \mathrm{E}-12$ \\
\hline PDS1-4 & Indiv. Lat. Cancers within 10 miles & $3.9 \mathrm{E}-14$ & $1.3 \mathrm{E}-12$ & $2.4 \mathrm{E}-11$ & $6.1 \mathrm{E}-12$ & $1.9 \mathrm{E}-11$ \\
\hline
\end{tabular}


Appendix E

Table E-1 (continued)

Plant Damage State Risk Results*

\begin{tabular}{|c|c|c|c|c|c|c|}
\hline \multirow[t]{2}{*}{ PDS } & \multirow[t]{2}{*}{ Consequence Measure } & \multicolumn{5}{|c|}{ Descriptive Statistics } \\
\hline & & 5th PCT & 50th PCT & 95th PCT & MEAN & STD DEV. \\
\hline PDS1-5 & Frequency & $4.4 \mathrm{E}-10$ & $6.8 \mathrm{E}-09$ & $4.8 \mathrm{E}-08$ & $1.4 \mathrm{E}-08$ & $2.4 \mathrm{E}-08$ \\
\hline PDS1-5 & Early Fatality & $1.2 \mathrm{E}-14$ & $5.9 \mathrm{E}-12$ & $1.4 \mathrm{E}-10$ & $3.0 \mathrm{E}-11$ & $5.8 \mathrm{E}-11$ \\
\hline PDS1-5 & Total Lat. Cancer & 2.8E-07 & $5.8 \mathrm{E}-06$ & $5.8 \mathrm{E}-05$ & $1.4 \mathrm{E}-05$ & 2.3E-05 \\
\hline PDS1-5 & Pop. Dose within 50 miles & $1.1 \mathrm{E}-06$ & $1.9 \mathrm{E}-05$ & $1.5 \mathrm{E}-04$ & 4.2E-05 & 7.1E-05 \\
\hline PDS1-5 & Pop. Dose within 1000 miles & $6.6 \mathrm{E}-06$ & $1.4 \mathrm{E}-04$ & 1.3E-03 & 3.3E-04 & $5.4 \mathrm{E}-04$ \\
\hline PDS1-5 & Indiv. Early Fatalities within 1 mile & $1.6 \mathrm{E}-16$ & $6.9 \mathrm{E}-14$ & $1.5 \mathrm{E}-12$ & $3.1 \mathrm{E}-13$ & $5.6 \mathrm{E}-13$ \\
\hline PDS1-5 & Indiv. Lat. Cancers within 10 miles & $1.9 \mathrm{E}-13$ & $3.3 \mathrm{E}-12$ & $2.4 \mathrm{E}-11$ & $7.2 \mathrm{E}-12$ & $1.3 \mathrm{E}-11$ \\
\hline PDS2-1 & Frequency & $1.3 \mathrm{E}-08$ & $1.4 \mathrm{E}-07$ & $1.5 \mathrm{E}-06$ & $3.5 \mathrm{E}-07$ & $6.6 \mathrm{E}-07$ \\
\hline PDS2-1 & Early Fatality & $2.3 \mathrm{E}-14$ & $8.6 \mathrm{E}-11$ & $2.5 \mathrm{E}-09$ & $6.6 \mathrm{E}-10$ & $2.6 \mathrm{E}-09$ \\
\hline PDS2-1 & Total Lat. Cancer & $1.6 \mathrm{E}-05$ & $1.8 \mathrm{E}-04$ & $3.0 \mathrm{E}-03$ & $5.8 E-04$ & $1.2 \mathrm{E}-03$ \\
\hline PDS2-1 & Pop. Dose within 50 miles & $4.8 \mathrm{E}-05$ & $5.2 \mathrm{E}-04$ & $7.9 \mathrm{E}-03$ & $1.6 \mathrm{E}-03$ & $3.2 \mathrm{E}-03$ \\
\hline PDS2-1 & Pop. Dose within 1000 miles & $3.8 \mathrm{E}-04$ & 4.2E-03 & $6.9 E-02$ & $1.4 \mathrm{E}-02$ & $2.8 \mathrm{E}-02$ \\
\hline PDS2-1 & Indiv. Early Fatalities within 1 mile & $3.0 \mathrm{E}-16$ & $1.0 \mathrm{E}-12$ & $2.9 \mathrm{E}-11$ & $6.0 \mathrm{E}-12$ & $1.7 \mathrm{E}-11$ \\
\hline PDS2-1 & Indiv. Lat. Cancers within 10 miles & $7.8 \mathrm{E}-12$ & $9.0 \mathrm{E}-11$ & $1.3 \mathrm{E}-09$ & $2.6 \mathrm{E}-10$ & $5.0 \mathrm{E}-10$ \\
\hline PDS2-2 & Frequency & 2.2E-08 & $1.5 \mathrm{E}-07$ & $1.6 \mathrm{E}-06$ & $5.5 \mathrm{E}-07$ & $1.9 \mathrm{E}-06$ \\
\hline PDS2-2 & Early Fatality & $4.6 \mathrm{E}-14$ & $1.1 \mathrm{E}-09$ & $2.5 \mathrm{E}-08$ & $1.0 \mathrm{E}-08$ & $5.0 \mathrm{E}-08$ \\
\hline PDS2-2 & Total Lat. Cancer & 4.4E-05 & $3.5 \mathrm{E}-04$ & $4.3 \mathrm{E}-03$ & $1.7 \mathrm{E}-03$ & $6.4 \mathrm{E}-03$ \\
\hline PDS2-2 & Pop. Dose within 50 miles & $1.0 \mathrm{E}-04$ & $9.1 E-04$ & $1.1 \mathrm{E}-02$ & $4.0 \mathrm{E}-03$ & $1.5 \mathrm{E}-02$ \\
\hline PDS2-2 & Pop. Dose within 1000 miles & $1.0 \mathrm{E}-03$ & $8.1 \mathrm{E}-03$ & $9.8 \mathrm{E}-02$ & $3.8 \mathrm{E}-02$ & $1.5 \mathrm{E}-01$ \\
\hline PDS2-2 & Indiv. Early Fatalities within 1 mile & $5.8 \mathrm{E}-16$ & $9.9 \mathrm{E}-12$ & $1.6 \mathrm{E}-10$ & $6.8 \mathrm{E}-11$ & $3.0 \mathrm{E}-10$ \\
\hline PDS2-2 & Indiv. Lat. Cancers within 10 miles & $1.7 \mathrm{E}-11$ & $1.1 \mathrm{E}-10$ & $1.7 \mathrm{E}-09$ & $5.1 \mathrm{E}-10$ & $1.8 \mathrm{E}-09$ \\
\hline PDS2-3 & Frequency & 2.7E-09 & $2.9 E-08$ & $4.5 \mathrm{E}-07$ & $1.1 \mathrm{E}-07$ & $3.7 \mathrm{E}-07$ \\
\hline PDS2-3 & Early Fatality & $1.8 \mathrm{E}-14$ & $2.1 \mathrm{E}-10$ & $4.9 \mathrm{E}-09$ & $1.3 \mathrm{E}-09$ & $4.9 \mathrm{E}-09$ \\
\hline PDS2-3 & Total Lat. Cancer & $3.7 \mathrm{E}-06$ & $6.4 \mathrm{E}-05$ & $1.1 \mathrm{E}-03$ & $2.8 \mathrm{E}-04$ & $1.0 \mathrm{E}-03$ \\
\hline PDS2-3 & Pop. Dose within 50 miles & $1.2 \mathrm{E}-05$ & $1.5 \mathrm{E}-04$ & $2.5 \mathrm{E}-03$ & $6.9 \mathrm{E}-04$ & $2.5 \mathrm{E}-03$ \\
\hline PDS2-3 & Pop. Dose within 1000 miles & 8.7E-05 & $1.5 \mathrm{E}-03$ & $2.5 \mathrm{E}-02$ & $6.4 \mathrm{E}-03$ & 2.3E-02 \\
\hline PDS2-3 & Indiv. Early Fatalities within 1 mile & $2.3 \mathrm{E}-16$ & $1.9 \mathrm{E}-12$ & $3.6 \mathrm{E}-11$ & $9.9 \mathrm{E}-12$ & $3.8 \mathrm{E}-11$ \\
\hline PDS2-3 & Indiv. Lat. Cancers within 10 miles & $1.8 \mathrm{E}-12$ & $2.2 \mathrm{E}-11$ & 3.3E-10 & 8.5E-11 & $2.6 \mathrm{E}-10$ \\
\hline PDS2-4 & Frequency & 7.7E-09 & $8.8 \mathrm{E}-08$ & 7.5E-07 & $2.0 \mathrm{E}-07$ & $3.0 \mathrm{E}-07$ \\
\hline PDS2-4 & Early Fatality & $2.0 \mathrm{E}-15$ & $3.2 E-11$ & $1.4 \mathrm{E}-09$ & $2.7 \mathrm{E}-10$ & $7.4 \mathrm{E}-10$ \\
\hline PDS2-4 & Total Lat. Cancer & $4.8 \mathrm{E}-06$ & $9.4 \mathrm{E}-05$ & 8.3E-04 & $2.1 \mathrm{E}-04$ & $3.6 \mathrm{E}-04$ \\
\hline PDS2-4 & Pop. Dose within 50 miles & $1.8 \mathrm{E}-05$ & $3.0 \mathrm{E}-04$ & $2.6 \mathrm{E}-03$ & $6.8 \mathrm{E}-04$ & 1.1E-03 \\
\hline PDS2-4 & Pop. Dose within 1000 miles & $1.1 \mathrm{E}-04$ & $2.2 \mathrm{E}-03$ & $2.0 \mathrm{E}-02$ & $5.0 \mathrm{E}-03$ & $8.4 \mathrm{E}-03$ \\
\hline PDS2-4 & Indiv. Early Fatalities within 1 mile & $2.5 \mathrm{E}-17$ & $3.9 \mathrm{E}-13$ & $1.6 \mathrm{E}-11$ & $2.8 \mathrm{E}-12$ & $6.9 \mathrm{E}-12$ \\
\hline PDS2-4 & Indiv. Lat. Cancers within 10 miles & $3.4 \mathrm{E}-12$ & $5.1 \mathrm{E}-11$ & 4.4E-10 & $1.1 \mathrm{E}-10$ & $1.5 \mathrm{E}-10$ \\
\hline
\end{tabular}


Table E-1 (concluded)

Plant Damage State Risk Results*

\begin{tabular}{|c|c|c|c|c|c|c|}
\hline \multirow[t]{2}{*}{ PDS } & \multirow[t]{2}{*}{ Consequence Measure } & \multicolumn{5}{|c|}{ Descriptive Statistics } \\
\hline & & 5th PCT & 50th PCT & 95th PCT & MEAN & STD DEV. \\
\hline PDS2-5 & Frequency & 7.7E-11 & 2.7E-09 & $5.3 \mathrm{E}-08$ & $1.3 \mathrm{E}-08$ & $3.4 \mathrm{E}-08$ \\
\hline PDS2-5 & Early Fatality & $1.2 \mathrm{E}-14$ & $6.0 \mathrm{E}-12$ & $2.3 \mathrm{E}-10$ & $9.4 \mathrm{E}-11$ & $4.8 \mathrm{E}-10$ \\
\hline PDS2-5 & Total Lat. Cancer & $1.2 \mathrm{E}-07$ & $4.4 \mathrm{E}-06$ & $1.2 \mathrm{E}-04$ & $2.7 \mathrm{E}-05$ & $8.3 \mathrm{E}-05$ \\
\hline PDS2-5 & Pop. Dose within 50 miles & 3.5E-07 & $1.3 \mathrm{E}-05$ & $2.9 \mathrm{E}-04$ & $6.9 \mathrm{E}-05$ & $2.1 \mathrm{E}-04$ \\
\hline PDS2-5 & Pop. Dose within 1000 miles & 2.7E-06 & $1.0 \mathrm{E}-04$ & $2.8 \mathrm{E}-03$ & 6.1E-04 & $1.9 \mathrm{E}-03$ \\
\hline PDS2-5 & Indiv. Early Fatalities within 1 mile & $1.2 \mathrm{E}-16$ & $5.2 \mathrm{E}-14$ & $2.0 \mathrm{E}-12$ & $6.5 \mathrm{E}-13$ & $3.1 \mathrm{E}-12$ \\
\hline PDS2-5 & Indiv. Lat. Cancers within 10 miles & $6.5 \mathrm{E}-14$ & $1.9 \mathrm{E}-12$ & $3.9 \mathrm{E}-11$ & $9.5 \mathrm{E}-12$ & $2.7 \mathrm{E}-11$ \\
\hline PDS2-6 & Frequency & $1.7 \mathrm{E}-11$ & 1.1E-09 & $2.8 \mathrm{E}-08$ & 7.4E-09 & $2.2 \mathrm{E}-08$ \\
\hline PDS2-6 & Early Fatality & 8.7E-18 & $9.9 \mathrm{E}-13$ & $1.0 \mathrm{E}-10$ & $2.9 \mathrm{E}-11$ & $1.4 \mathrm{E}-10$ \\
\hline PDS2-6 & Total Lat. Cancer & $1.2 \mathrm{E}-08$ & $1.3 \mathrm{E}-06$ & $5.7 \mathrm{E}-05$ & $1.2 \mathrm{E}-05$ & $3.0 \mathrm{E}-05$ \\
\hline PDS2-6 & Pop. Dose within 50 miles & $4.3 \mathrm{E}-08$ & $4.0 \mathrm{E}-06$ & $1.5 E-04$ & $3.3 \mathrm{E}-05$ & $8.2 \mathrm{E}-05$ \\
\hline PDS2-6 & Pop. Dose within 1000 miles & $2.7 \mathrm{E}-07$ & $3.1 \mathrm{E}-05$ & $1.3 \mathrm{E}-03$ & 2.8E-04 & $6.8 \mathrm{E}-04$ \\
\hline PDS2-6 & Indiv. Early Fatalities within 1 mile & $1.1 \mathrm{E}-19$ & $9.9 \mathrm{E}-15$ & $6.9 \mathrm{E}-13$ & $2.1 \mathrm{E}-13$ & $9.1 \mathrm{E}-13$ \\
\hline PDS2-6 & Indiv. Lat. Cancers within 10 miles & $1.0 \mathrm{E}-14$ & $6.8 \mathrm{E}-13$ & $2.4 \mathrm{E}-11$ & $5.2 \mathrm{E}-12$ & $1.4 \mathrm{E}-11$ \\
\hline PDS3-1 & Frequency & $6.2 \mathrm{E}-08$ & 3.7E-07 & $2.4 \mathrm{E}-06$ & 7.3E-07 & $1.2 \mathrm{E}-06$ \\
\hline PDS3-1 & Early Fatality & $0.0 \mathrm{E}+00$ & $0.0 \mathrm{E}+00$ & $8.6 \mathrm{E}-11$ & $1.0 \mathrm{E}-10$ & $6.5 \mathrm{E}-10$ \\
\hline PDS3 -1 & Total Lat. Cancer & $2.8 \mathrm{E}-05$ & $3.6 \mathrm{E}-04$ & $3.5 \mathrm{E}-03$ & 8.2E-04 & $1.5 \mathrm{E}-03$ \\
\hline PDS3-1 & Pop. Dose within 50 miles & $1.2 \mathrm{E}-04$ & $1.0 \mathrm{E}-03$ & $8.6 \mathrm{E}-03$ & $2.2 \mathrm{E}-03$ & $3.8 \mathrm{E}-03$ \\
\hline PDS3-1 & Pop. Dose within 1000 miles & $6.3 \mathrm{E}-04$ & $7.8 \mathrm{E}-03$ & $7.8 \mathrm{E}-02$ & $1.8 \mathrm{E}-02$ & $3.3 \mathrm{E}-02$ \\
\hline PDS3-1 & Indiv. Early Fatalities within 1 mile & $0.0 \mathrm{E}+00$ & $0.0 \mathrm{E}+00$ & $1.1 \mathrm{E}-12$ & $1.0 \mathrm{E}-12$ & $6.6 \mathrm{E}-12$ \\
\hline PDS3-1 & Indiv. Lat. Cancers within 10 miles & $3.4 \mathrm{E}-11$ & $2.6 \mathrm{E}-10$ & $2.0 \mathrm{E}-09$ & $5.6 \mathrm{E}-10$ & $9.6 \mathrm{E}-10$ \\
\hline
\end{tabular}

* All values are per calendar year; population doses are in sieverts; statistics based on an LHS size of 200 observations. 
Appendix E 


\section{Appendix F Summary Report for Abridged Study of POS 6}

For the sake of completeness, this appendix contains a letter report entitled "Summary Report: Grand Gulf Low Power and Shutdown Abridged Risk Analysis, POS 6: Early Refueling." This report documents the abridged study of the early portion of the refueling mode of operation, referred to as POS 6, which was performed under FIN L1679 during the Spring of 1992. The reader is cautioned that there are many differences between the study of POS 5 and the abridged study of POS 6; the two studies should not be viewed as equal in scope or approach. The abridged study of POS 6 used a much more abbreviated version of the NUREG-1150 methodology. Some of the more important differences include:

- The results from POS 6 are conditional on the occurrence of the plant damage states (PDSs) defined in the analysis. Only two PDSs were defined: loss of offsite power (LOSP) and non-LOSP. These PDSs were based on information from a Level 1 coarse screening study of the Grand Gulf low power and shutdown modes of operation; the screening study did not provide core damage frequencies. Because core damage frequencies for the PDSs were not available, the analysis of POS 6 provides no information on the likelihood of the accidents.

- Estimates of the risk associated with POS 6 were not calculated because core damage frequencies for POS 6 were not available.

- The accident progression event tree (APET) used in the POS 6 study did not address as many events, and the events it did include were treated in less detail as compared to the POS 5 APET. Also, the POS 6 analysis included fewer events in its uncertainty analysis as compared to the POS 5 analysis.

- In the calculation of offsite consequences, the POS 6 analysis used a Latin hypercube sampling (LHS) size of only 12 observations as opposed to the 200 observations used in the POS 5 analysis. Thus, while the results from the 12 observations can be used to provide an indication of the range of expected results, meaningful statistics cannot be calculated based on this limited sample.

- The onsite consequence analysis for POS 6 was based on a slightly different set of assumptions as compared to the onsite POS 5 analysis. In both studies, the dose was based on exposure from both the immersion and the inhalation pathways. In the POS 6 analysis, the dose rate was also based on both the immersion and the inhalation pathways (very conservative to include inhalation pathway in the calculation of the dose rate), whereas in the POS 5 analysis the dose rate was only based on exposure from the immersion pathway. The dose and dose rates calculated in the two studies were based on different weather scenarios. Also, dose and dose rates in the containment and auxiliary building were calculated in the POS 6 study; similar calculations were not performed in the POS 5 study. 


\title{
SUMMARY REPORT OF: \\ GRAND GULF LOW POWER AND SHUTDOWN \\ ABRIDGED RISK ANALYSIS
}

POS 6: Early Refueling

FINAL LETTER REPORT

\author{
Thomas D. Brown ${ }^{1}$ \\ LeAnn A. Miller ${ }^{1}$ \\ Lubomyra N. Kmetyk ${ }^{1}$ \\ Lanny N. Smith ${ }^{2}$ \\ Donnie W. Whitehead ${ }^{1}$ \\ John Darby ${ }^{3}$ \\ John Forester ${ }^{2}$
}

October 1993

Sandia National Laboratories

Albuquerque, NM 87185

Operated for the

U.S. Department of Energy

Contract DE-AC04-76DP00789

Prepared for

Division of Safety Issue Resolution

Office of Nuclear Regulatory Research

U.S. Nuclear Regulatory Commission

Washington, DC 20555

NRC FIN L1679

${ }^{1}$ Sandia National Laboratories

${ }^{2}$ Science Application International Corporation

${ }^{3}$ Science \& Engineering Associates, Inc. 


\section{CONTENTS}

$1.0 \quad$ INTRODUCTION $\ldots \ldots \ldots \ldots \ldots \ldots \ldots \ldots \ldots \ldots \ldots \ldots$

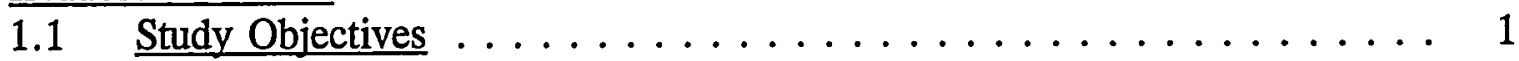

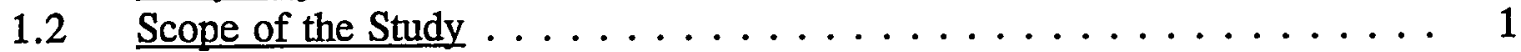

1.3 Methods ........................ 1

1.4 Limitations and Strengths of the Study . . . . . . . . . . . 4

$2.0 \quad$ ACCIDENT PROGRESSION ANALYSIS $\ldots \ldots \ldots \ldots \ldots \ldots \ldots$

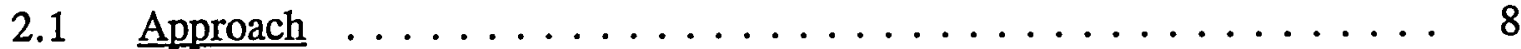

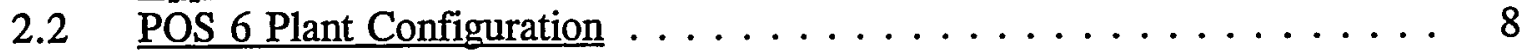

$2.3 \quad$ Level 1 Sequence Description $\ldots \ldots \ldots \ldots \ldots \ldots \ldots$

2.3.1 Sequence Description . . . . . . . . . . . . . . 9

2.3.2 Plant Damage State Description $\ldots \ldots \ldots \ldots \ldots \ldots \ldots$

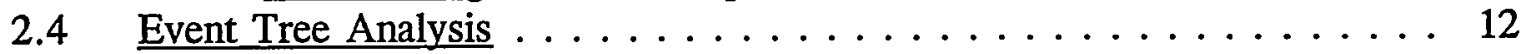

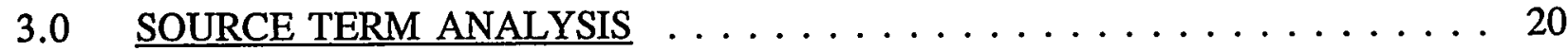

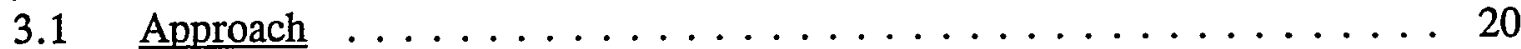

3.2 Description of Parametric Model ............... 20

3.3 Results ......................... 21

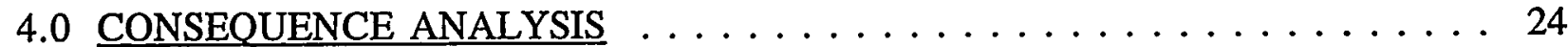

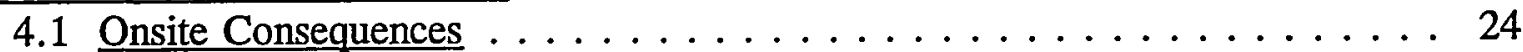

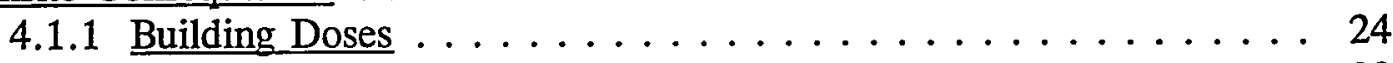

4.1 .2 Parking Lot Doses . . . . . . . . . . . . . . . . 28

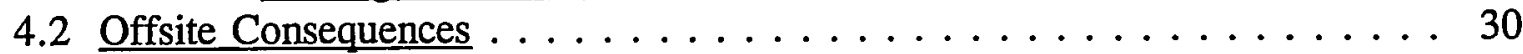

5.0 INTEGRATED RESULTS CONDITIONAL ON CORE DAMAGE $\ldots \ldots \ldots$. . 34

$6.0 \quad$ INSIGHTS AND CONCLUSIONS $\ldots \ldots \ldots \ldots \ldots \ldots \ldots \ldots \ldots$

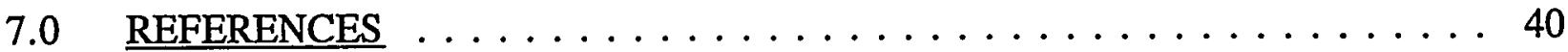




\section{FIGURES}

1-1. Scope of abridged study $\ldots \ldots \ldots \ldots \ldots \ldots \ldots \ldots \ldots \ldots \ldots \ldots$

1-2. Summary of abridged methodology . . . . . . . . . . . 7

2.4-1. Grand Gulf POS 6 abridged accident progression event tree (APET) . . . . 19

4.1.1-1. Containment and auxiliary building dose rates for selected paths . . . . . 28

4.1.2-1. Parking Lot dose rates for Ramsdell and Wilson/Regulatory Guide models for distances from 10 - 500 meters from the reactor: First Release Segment . . 32

4.1.2-2. Parking Lot dose rates for Ramsdell and Wilson/Regulatory Guide models for distances from 10 - 500 meters from the reactor: Second Release Segment 32

5-1. Grand Gulf POS 6 offsite consequences for LOSP and nonLOSP PDSs . . . . 36

\section{TABLES}

2.3-1 Grand Gulf LP\&S POS 6 Initiating Events $\ldots \ldots \ldots \ldots \ldots 11$

2.3-2 Grand Gulf LP\&S POS 6 Plant Damage State Attributes . . . . . . . . . . . . 12

2.4-1 Accident Progression Timing . . . . . . . . . . . . . . . . . 13

3.3-1 Mean Source Terms for Accident Progression Paths (Total Release) . . . . . 23

4.1.1-1. Transit times through the containment and auxiliary building for Grand Gulf POS $6 . \ldots \ldots \ldots \ldots \ldots \ldots$

4.1.1-2. Grand Gulf POS 6 mean containment doses and dose rates. . . . . . . . . . 26

4.1.1-3. Grand Gulf POS 6 mean auxiliary building doses and dose rates. . . . . . . 27

4.1.2-1. Grand Gulf POS 6 mean doses and dose rates at $100 \mathrm{~m}$ based on the Ramsdell building wake effect model. . . . . . . . . . . . . . . . . 29

4.1.2-2. Grand Gulf POS 6 mean doses and dose rates at $100 \mathrm{~m}$ based on the Wilson building wake effect model. . . . . . . . . . 30

4.2-1 Grand Gulf POS 6 Offsite Mean Consequences . . . . . . . . . . . . 33 


\section{ACKNOWLEDGMENTS}

We wish to thank the many people who worked in various capacities to support this analysis: Fred T. Harper (SNL) who provided many helpful technical suggestions in the development and application of methods and who provided a much needed sanity check on the analysis and results; Jay Johnson (GRAM) who performed the MACCS calculations for the offsite consequences; David I. Chanin (Technadyne Engineering Consultants) who provided helpful ideas for assessing onsite consequences; and Ann W. Shiver (SNL) who provided support in the data preparation for the uncertainty analysis.

We would also like to thank Chris Ryder (NRC) for his program and management support and for his words that form the introduction of this report.

Finally, we would like to thank the Source Term Advisory Group, which consisted of John E. Kelly (SNL), Hossein P. Nourbakhsh (BNL), Dana A. Powers (SNL), and Trevor Pratt (BNL), for their review of the source term analysis and their many helpful suggestions. 


$\begin{array}{ll}\text { ADHR } & \text { Alternate Decay Heat Removal System } \\ \text { APET } & \text { Accident Progression Event Tree } \\ \text { BNL } & \text { Brookhaven National Laboratory } \\ \text { BWR } & \text { Boiling Water Reactor } \\ \text { CCI } & \text { Core Concrete Interactions } \\ \text { CDS } & \text { Condensate System } \\ \text { CF } & \text { Containment Failure } \\ \text { CNT } & \text { Containment } \\ \text { CRD } & \text { Control Rod Drive System } \\ \text { ECCS } & \text { Emergency Core Cooling System } \\ \text { FW } & \text { Firewater System } \\ \text { HIS } & \text { Hydrogen Ignition System } \\ \text { HPCS } & \text { High Pressure Core Spray System } \\ \text { HRA } & \text { Human Reliability Analysis } \\ \text { LHS } & \text { Latin Hypercube Sample } \\ \text { LOCA } & \text { Loss of Coolant Accident } \\ \text { LOSP } & \text { Loss of Offsite Power } \\ \text { LP\&S } & \text { Low Power and Shutdown } \\ \text { NRC } & \text { Nuclear Regulatory Commission } \\ \text { PDS } & \text { Plant Damage State } \\ \text { POS } & \text { Plant Operating State } \\ \text { PRA } & \text { Probabilistic Risk Assessment } \\ \text { PSW } & \text { Plant Service Water } \\ \text { PWR } & \text { Pressurized Water Reactor } \\ \text { RFO } & \text { Refueling Outage } \\ \text { RHR } & \text { Residual Heat Removal System } \\ \text { RPV } & \text { Reactor Pressure Vessel } \\ \text { SBGT } & \text { Standby Gas Treatment System } \\ \text { SBO } & \text { Station Blackout } \\ \text { SDC } & \text { Shutdown Cooling System } \\ \text { SPC } & \text { Suppression Pool Cooling System } \\ \text { SPMU } & \text { Suppression Pool Makeup System } \\ \text { SRV } & \text { Safety Relief Valve } \\ \text { SSW } & \text { Standby Service Water } \\ \text { TAF } & \text { Top of Active Fuel } \\ \text { TBCW } & \text { Turbine Building Cooling Water } \\ \text { VB } & \text { Vessel Breach } \\ & \end{array}$




\section{$1.0 \quad$ INTRODUCTION}

\subsection{Study Objectives}

The Office of Nuclear Regulatory Research at the U. S. Nuclear Regulatory Commission established programs to investigate postulated accidents during low power and shutdown (LP\&S) operations of a BWR (Grand Gulf) and a PWR (Surry). One such program is a risk study of accident progressions and consequences.

The objective of this study is to make a preliminary risk determination of the progressions (Level 2 analysis) and the consequences (Level 3 analysis) of accidents during low power and shutdown operations in the Grand Gulf plant. The study was designed to obtain results for regulatory decisions. This letter report documents the methods, findings, and implications of the study done under NRC FIN L1679. A sister study of the Surry plant is reported separately by the staff at Brookhaven National Laboratory (BNL) under NRC FIN L1680.

\subsection{Scope of the Study}

The abbreviated risk analysis took place from January through April 1992. The study has been referred to as an abridged risk analysis. The term abridged means that simple event trees (about nine top event questions) were developed and used with assumptions and other approximate methods to compute rough estimates. The term risk means conditional consequences (probability of the various events during the accident progressions multiplied by the consequences), given that core damage has occurred. Traditional risk estimates, computed by multiplying the conditional consequences and the frequency of the sequences, could not be made at this time because the core damage frequencies have yet to be determined in companion Level 1 and HRA studies. Uncertainty has been taken into account in a manner consistent with the detail of the abridged study.

This study investigated the possible accident progressions and the associated consequences of a single plant operating state, POS 6 , an early stage of refueling, where the reactor vessel head is removed, the steam dryers and separators are removed, the drywell is open, and the containment is open. The sister study at BNL investigated mid-loop operation. The scope of both studies is illustrated in Figure 1-1.

\section{$1.3 \quad$ Methods}

The abridged process of computing conditional consequences is shown in Figure 1-2. In general, both the study reported here and the study done at BNL follow this scheme. Some differences in the details of the procedure exist and are noted at the end of Section 1.3. The process used here is an abbreviated form of the NUREG-1150 study [1]. 


\section{Accident progressions}

The calculations begin with the assumption that core damage has occurred. Given core damage, the reasonable accident progressions are delineated with the accident progression event tree (APET). Much of the delineation is based on information obtained from PRAs of full power operation, knowledge of severe accident phenomena, and deterministic calculations with codes used to compute source terms, such as MELCOR [2]. The likelihood of the various accident progressions is reflected vis-a-vis branch point probabilities.

Branch point probabilities were assigned to reflect the likelihood of various pathways thought to exist. In large scale risk studies, the assignment can be done by groups of experts knowledgeable in severe accident issues. Here, because of resource limitations, most of the assignments were done by the project staff. The probabilities are not as rigorous as they could be but this is one of many limitations of the study to be discussed. Some lack of rigor in determining the probabilities is taken into account by repeating the calculations with other possible probabilities; taken together, the repeated calculations constitute an uncertainty analysis.

Through the uncertainty analysis, distributions, instead of point values, were assigned to selected branch points. The distributions are subjective but account for many possible values of the branch points. Point values are selected from the distributions with a form of Monte Carlo sampling known as Latin Hypercube Sampling (LHS) [3]. After making sets of inputs, each set, consisting of point values, is assigned to the branch points and multiplied through to the ends of the APET. The calculations are repeated using the sets of inputs, building a probability distribution at the end of each pathway.

\section{$\underline{\text { Source terms }}$}

Having delineated accident progressions with the APET, the source terms of the progressions were calculated with a parametric code [4]. The parametric code is a collection of simple massbalance equations designed to mimic detailed source term codes. The parametric approach is not meant to be a substitute for detailed, mechanistic computer simulations codes. Rather, it is a framework for integrating the results of these codes together with experimental results and expert judgment.

The parametric code determines source terms, given the characteristics of the accident progression and other inputs (e.g., fraction of the inventory a) leaving the reactor vessel; b) involved in core concrete interactions; c) entering the containment). Because these other variables are imprecisely known, many reasonable values can be assigned to the inputs. As in the APET calculations, distributions are assigned to the variables and sampled with LHS to form many sets of input values for repeated calculations. The result is a distribution of source terms for each accident progression pathway.

Because the estimation of the source terms is a critical component of this study, an internal advisory group, call the Source Term Advisory Group, was formed to support this study. The 
members of the advisory group included: John E. Kelly (SNL), Hossein P. Nourbakhsh (BNL), Dana A. Powers (SNL), and Trevor Pratt (BNL). The role of the Source Term Advisory Group was to 1) provide guidance on the identification of phenomena that may be important to the formation of the source term during these modes of operation, and 2) assess the adequacy, relative to the study's objectives and scope, of the assumptions, methods and data used in this study. The results of the accident progression and source term analysis were presented to and discussed with the advisory group in two meetings during the course of this analysis.

\section{Consequences}

Three sets of radiological consequences were determined: building dose, onsite dose (so called parking lot dose), and offsite consequences.

- Building dose was determined based on source terms derived from the parametric source term expressions. Doses in the containment and auxiliary building were estimated.

- Parking lot dose was based on relative concentrations computed with the Ramsdell model [11], in which the release concentration is somewhat proportional to wind speed, and a combination of the Wilson model [12] and the model in Regulatory Guide 1.145 [13], in which the concentration is inversely proportional to wind speed.

o Offsite consequences were computed using the MACCS code $[5,6,7]$.

Uncertainty was not propagated through the consequence analysis as it was through the APET and the source term calculations. While a sample size of 100 was used in the onsite analysis to propagate accident progression and source term uncertainties, a reduced sample size of 12 was used in the determination of offsite consequences.

\section{Conditional offsite consequences}

Conditional risk was computed by multiplying the offsite consequences by their associated accident probability that was determined with the APET. This product of probability and consequences was computed for each accident progression pathway. The products of the pathways were summed. This process was repeated for each of the few samples of the source terms. Then, high, medium, and low results were reported.

\section{Differences}

This study differs slightly from its sister program at BNL in three ways. (1) Here, one hundred samples from the uncertainty distributions were propagated through the accident progression and source term analyses whereas, in the BNL study, two hundred samples were taken. (2) Here, twelve samples were propagated through the APET to offsite consequences whereas, in the BNL study, twenty samples from the source term distributions were used in consequence calculations and traced back through the APET for the probabilities needed to compute conditional risk. 
(3) Here, doses in the containment and auxiliary building were calculated whereas, in the BNL study, these calculations were thought unnecessary since the releases were assumed to pass from the containment directly into the environment.

\subsection{Limitations and Strengths of the Study}

In order to place the calculations in proper context, it is necessary to understand the strengths and limitations of the study.

\section{Limitations}

o The subject of the study is one POS, early refueling. This POS was selected for study because it was identified in a preliminary Level 1 study, known as a coarse screening analysis [8], as potentially occurring at a relatively high frequency. Also, the POS had characteristics (i.e., reactor vessel head removed) of interest to the staff in the Office of Nuclear Reactor Regulations at the NRC.

o The abridged study is based on the coarse screening analysis where accident sequences potentially having high frequencies were identified. The consequences of these sequences were determined in the Level 2 and 3 abridged study reported here. The frequency is not merged with the Level 2 and 3 calculations to determine risk because the numerical value of the frequency estimate is believed to be too rough for such use.

o The simple APET accounts for a limited number of factors. The APET consisted of nine top event questions, compared to about one hundred questions in a large scale PRA.

o The onsite dose estimates stem from simple equations yielding rough estimates.

- Variables were selected and assigned distributions for the uncertainty analysis by the project staff.

o Because of gaps in knowledge of the plant configuration and operator actions, assumptions were necessary. The assumptions are documented in the sections to follow.

\section{Strengths}

o Even with the limitations noted above, the abridged study is a systematic evaluation, which includes a limited treatment of the uncertainty in severe accident progressions.

o The source term analysis was reviewed by an internal advisory group.

o The project staff and the NRC project staff believe that the APET represents the occurrence of key events during accident progressions. 
o The relationship and timing of accident progression events and factors have been determined to at least a first approximation.

o Estimates of both onsite and offsite conditional consequences were made.

The sections to follow document the abridged study of the Grand Gulf plant. The discussion above is expanded, providing important details and results. 


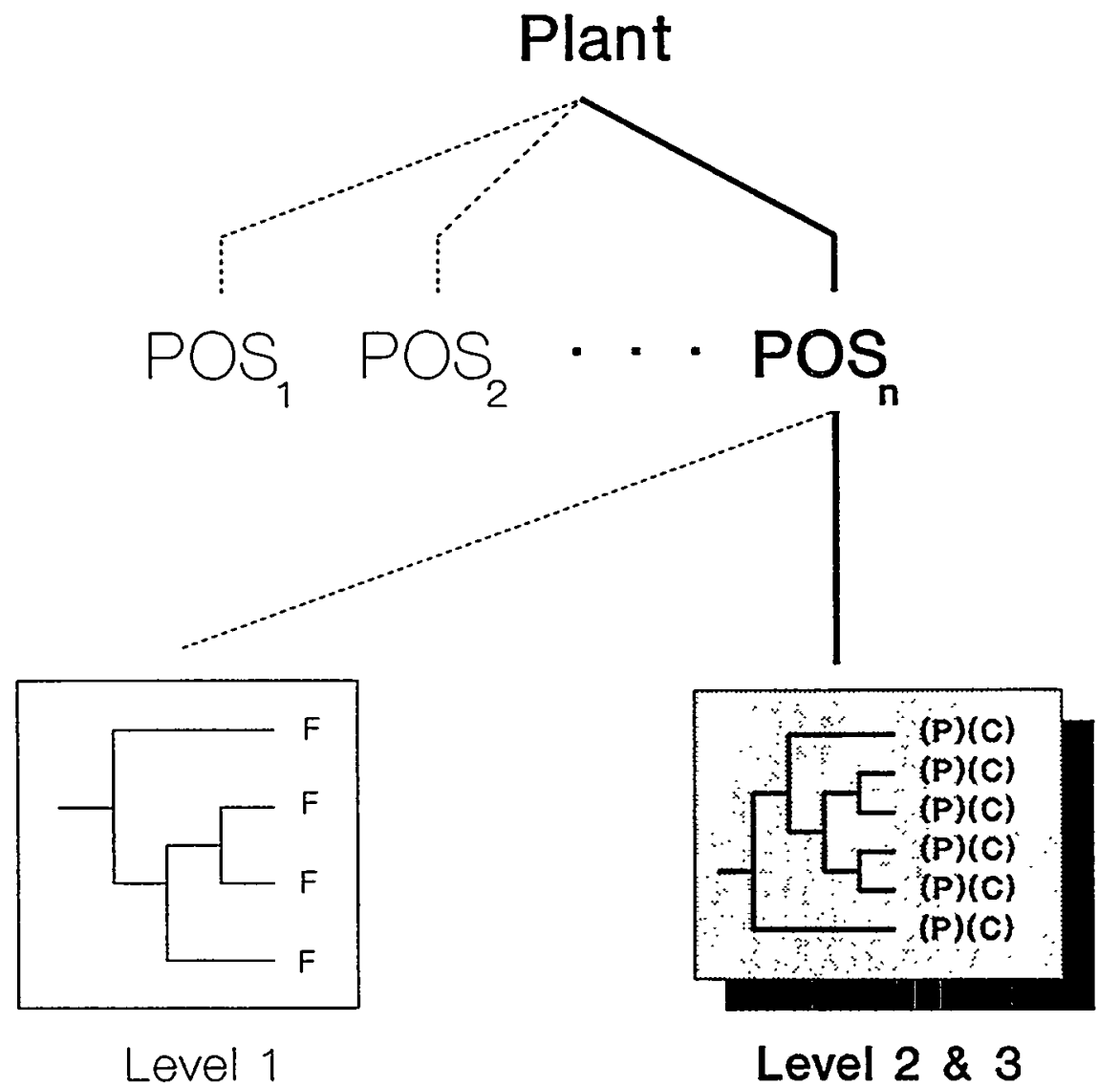

$$
\begin{aligned}
& R=[F][P][C] \\
& R=\text { risk } \\
& F \text { - sequence frequency } \\
& P=\text { accident progression } \\
& \text { probability } \\
& \mathrm{C}=\text { consequences }
\end{aligned}
$$

Figure 1-1. Scope of abridged study 


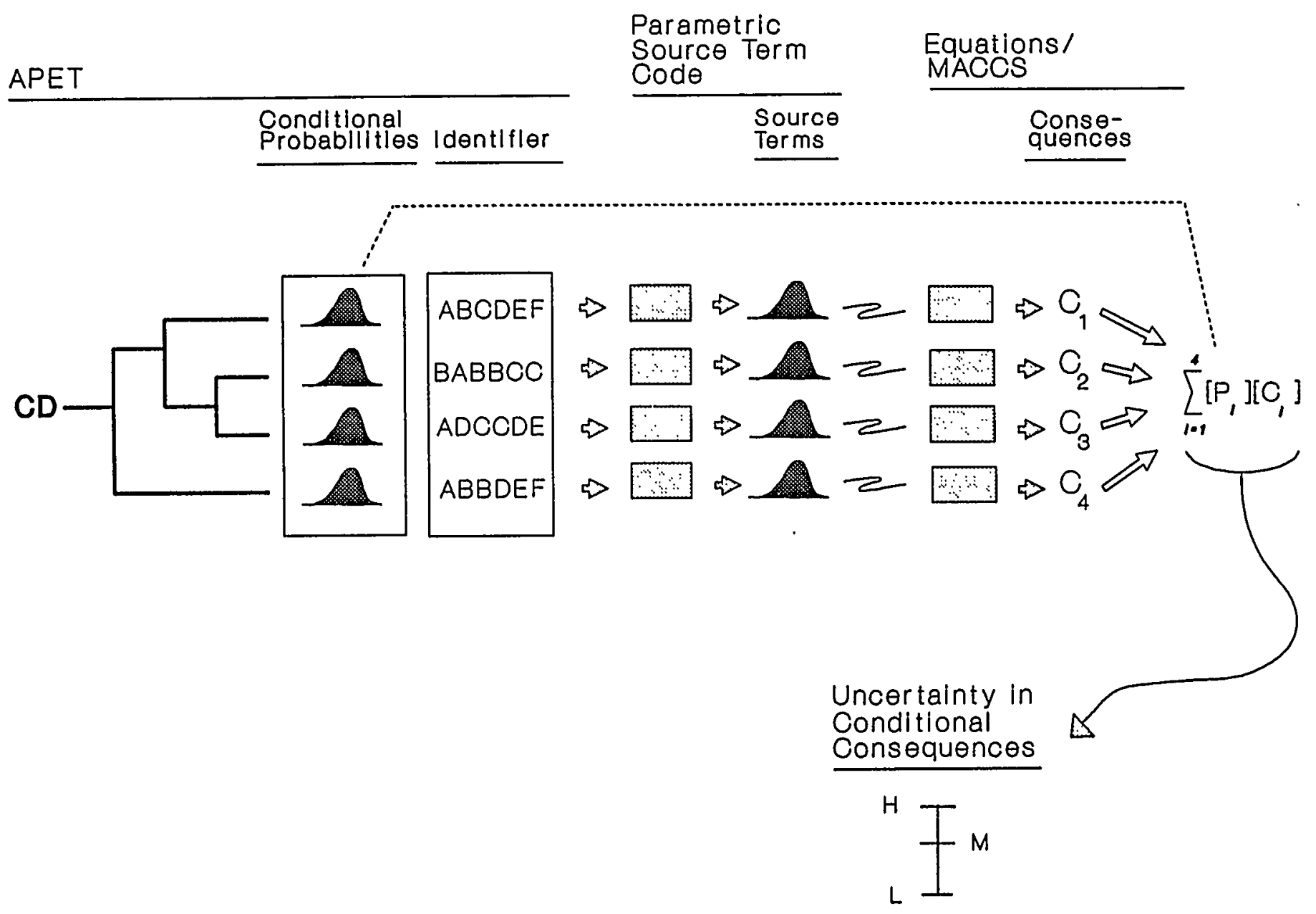

Figure 1-2. Summary of abridged methodology 


\subsection{ACCIDENT PROGRESSION ANALYSIS}

\section{$2.1 \quad$ Approach}

The progression of accidents following core damage are analyzed in the Level 2 portion of the PRA. In this chapter the development and quantification of the accident progression scenarios will be presented. The input to the accident progression analysis is the core damage sequence definitions developed in the Level 1 analysis [8]. The core damage sequences define the successes and failures of equipment and human actions that have resulted in the loss of core cooling and the onset of core damage. The sequence definitions provide information on the status of core cooling systems, containment cooling systems, and containment integrity at the time of core damage. From this information the possible accident progressions, which identify the response of the core and the containment following core damage, are determined. These accident progressions are developed and displayed using an event tree. In this abridged analysis only the most important events that affect the timing and the magnitude of the radionuclide release are addressed. The outputs from the accident progression analysis are the accident progression path definitions and the likelihood, conditional on core damage having occurred, of each path. In the source term analysis, the fission product release associated with each path is estimated. The estimation of the source term is addressed in Chapter 3 and the resulting consequences are presented in Chapter 4.

In the following subsections the configuration of the plant during POS 6 will be presented, the important characteristics of the Level 1 core damage sequences will be identified, and the development of the accident progression paths will be discussed.

\section{$2.2 \quad$ POS 6 Plant Configuration}

The configuration of the plant at the onset of core damage is important because it will determine the framework within which the accident will unfold. That is, the plant configuration will define the boundary conditions for the analysis. For example, it will define the mitigative features of the plant that will be available during the accident (e.g., containment, suppression pool, containment sprays).

The abridged risk analysis was performed on the early portion of the refueling mode of operation, referred to as plant operating state 6 (POS 6). During a refueling outage the plant will enter POS 6 prior to loading fresh fuel (i.e., going down) and then following fuel transfer on the way back up to power conditions (i.e., going up). In the Level 1 analysis, the sequence definitions are based on the "going down" phase because (1) more systems are likely to be unavailable (i.e., on the way back up, maintenance and repairs may already have been performed on many systems) and (2) the decay heat levels are higher and, therefore, there is less time to respond to events in the going down phase versus the going up phase. Thus, in this study only the "going down" phase is analyzed. POS 6 begins when the vessel head is detached and ends when the upper reactor cavity has been filled with water. During this POS the following tasks are performed: 
1. Steam dryers are removed,

2. Vessel water level is lowered to the bottom of the steam lines and the steam lines are plugged,

3. Water level is raised and the steam separators are removed, and

4. Vessel water level is raised to flood the upper reactor cavity.

Prior to this mode of operation, the containment equipment hatch and personnel locks have been opened, the drywell head has been removed, and the drywell equipment hatch and personnel locks have been opened. Thus, the suppression pool is effectively bypassed both from the vessel and from the drywell (i.e., steam lines are plugged and the drywell is open).

Timing information for the initiation of the accident in POS 6 is based on Grand Gulf refueling outage (RFO) data. Information was available for the first four RFOs. However, because of the number of special tests that were conducted during the first refueling outage, RFO-1 was considered atypical and, therefore, data from this outage was excluded from the analysis. Thus, only RFO 2,3, and 4 data were used in this study. Based on this data the fastest the plant will enter POS 6 from full power is approximately four days after shutdown and the longest the plant has been in POS 6 (in the "going down" phase) is approximately 12 days (i.e., 16 days from shutdown). In the Level 1 analysis the time window from the initiating event to core damage was based on the decay heat at four days. This assumption is carried through the Level $2 / 3$ analyses.

\subsection{Level 1 Sequence Description}

\subsubsection{Sequence Description}

The initial conditions for the accident progression analysis are the core damage sequence descriptions from the Level 1 analysis [8]. That is, a list of attributes that describe the status of systems that can be used to mitigate the accident and the configuration of the plant at the time of core damage. In the Level 1 coarse screening analysis the sequences were placed into three groups: potentially high likelihood group, potentially medium likelihood group, and potentially low likelihood group. Only sequences from the high likelihood group were analyzed in this study. Fourteen different initiating events are associated with these sequences. A list of these 14 initiating events is presented in Table 2.3-1. The initiating events can be divided into four major groups: Loss of Offsite Power (LOSP) Transients, Loss of Support System Transients, Loss of Coolant Accidents (LOCAs), and Decay Heat Removal Challenges. The accident sequences that form the input to this study all progress to core damage in the following manner. The initiating event leads to the loss of the operating shutdown cooling system, subsequent random failures and unavailabilities complete the loss of core cooling and injection. Without a means to keep the core cool, the vessel inventory is lost via boiling and core damage ensues.

In the Level 1 screening analysis both the emergency core cooling system (ECCS) and Makeup (i.e., CRD and CDS) were assumed to be unavailable or unable, due to some postulated failure, 
to prevent core damage. Thus, only the firewater system (FW) and the standby service water (SSW) cross-tie were considered as potential injection systems.

In POS 6 the suppression pool can be either at its normal level, partially drained, or empty. Furthermore, the suppression pool makeup system (SPMU) is not available. Because a supply of water to the SP is not available, ECCS systems that draw water from the SP could not be used in a continuous mode and, therefore, it was assumed in the Level 1 analysis that these systems were not available to cool the core. Because the containment spray system is one mode of the residual heat removal system (i.e., part of ECCS) and draws water from the SP, it is also unavailable during these postulated accidents.

The CRD system has insufficient capacity to prevent the core inventory from boiling and, therefore, was not considered as a means to cool the core in the Level 1 screening study. (It should be noted, however, that if this system was used, the energy removed from the core via steaming would be sufficient to prevent core damage.) While CDS has more than enough capacity to cool the core, its unavailability due to random failures and maintenance precludes its use as a means to cool the core.

A general description of the core damage sequences for each class of initiators is presented below.

\section{$\underline{\text { LOSP Transients }}$}

The LOSP initiating event leads directly to the loss of the alternate decay heat removal system (ADHR). Subsequent random failures lead to the complete loss of shutdown cooling (SDC), makeup, the standby service water and the firewater system. With ECCS unavailable in this POS, as a result of support system failures, the accident proceeds to core damage because of the lack of core cooling.

\section{Loss of Support System Transients}

In these sequences the initiating event leads directly to the loss of ADHR, makeup, and the firewater system. Subsequent random failures lead to the complete loss of SDC and the SSW system.

\section{Decay Heat Removal Challenges}

In these sequences the initiating event leads to the loss of the operating shutdown cooling system. In some of these sequences this system is recovered. However, subsequent random failures lead to the complete loss of SDC, the firewater system, and SSW. 


\section{LOCAs That Can Be Isolated}

In these sequences the isolation of the LOCA also isolates the SDC systems. Subsequent random failures lead to the loss of both the firewater system and the standby service water cross-tie system.

Table 2.3-1 Grand Gulf LP\&S POS 6 Initiating Events

\begin{tabular}{|c|c|c|}
\hline $\begin{array}{l}\text { Initiating } \\
\text { Event } \\
\text { Group }\end{array}$ & $\begin{array}{c}\text { Initiating } \\
\text { Event } \\
\text { Nomenclature }\end{array}$ & Description \\
\hline LOSP & $\mathrm{T} 1$ & Loss of Offsite Power (LOSP) Transient \\
\hline \multirow{3}{*}{$\begin{array}{l}\text { Loss of } \\
\text { Support } \\
\text { System } \\
\text { Transient }\end{array}$} & $\mathrm{T} 5 \mathrm{~B}$ & Loss of all TBCW \\
\hline & $\mathrm{T} 5 \mathrm{C}$ & Loss of all PSW (includes Radial Well) \\
\hline & TIA & Loss of all Instrument Air \\
\hline \multirow{8}{*}{$\begin{array}{c}\text { Decay } \\
\text { Heat } \\
\text { Removal } \\
\text { Challenge }\end{array}$} & E1B & Isolation of SDC Loop B only \\
\hline & E2B & Loss of SDC Loop B only \\
\hline & E1D & Isolation of ADHRS \\
\hline & E2D & Loss of ADHRS only \\
\hline & E1T & Isolation of SDC Common Suction Line \\
\hline & $\mathrm{E} 2 \mathrm{~T}$ & Loss of SDC Common Suction Line \\
\hline & E1V & Isolation of Common Suction Line for ADHRS \\
\hline & E2V & Loss of Common Suction Line for ADHRS \\
\hline \multirow{2}{*}{$\begin{array}{l}\text { Isolated } \\
\text { LOCAs }\end{array}$} & H1 & Diversion to Suppression Pool via RHR \\
\hline & $\mathrm{J} 2$ & LOCA in Connected System (RHR) \\
\hline
\end{tabular}

\subsubsection{Plant Damage State Description}

The Level 1 sequences were divided into two plant damage state (PDS) groups: LOSP and nonLOSP. This distinction is made because of the effect that the LOSP has on injection recovery and containment closure. In the analysis of the nonLOSP PDS it is assumed that if injection is not recovered prior to core damage, it will not be recovered during core damage. The reason for this assumption is that there is a considerable amount of time from the initiating event to core damage for the operators to align and use injection systems to cool the core. If this has not been done by the time of core damage, there is no reason to believe that they will recover core cooling during core damage. Recovery of injection is considered in the LOSP PDS. In these sequences offsite power is unavailable and, therefore, non-emergency systems are unavailable to provide injection to the core. Thus, for the LOSP PDS it is assumed that if 
offsite power is recovered, injection can be recovered. The availability of ac power also affects the likelihood that the containment is closed prior to core damage. The crane that is used to position the equipment hatch is powered with offsite ac power and, therefore, without offsite ac power the containment cannot be closed. If offsite power is available during the accident, closure of the containment prior to core damage is addressed in the event tree analysis. The key attributes associated with these two PDSs are presented in Table 2.3-2.

Table 2.3-2 Grand Gulf LP\&S POS 6 Plant Damage State Attributes

\begin{tabular}{||l||c|c|}
\hline \multicolumn{1}{|c|}{ PDS Attributes } & \multicolumn{2}{c||}{ Plant Damage States (PDS) } \\
\cline { 2 - 3 } & LOSP & nonLOSP \\
\hline \hline Offsite Power & Not Available & Available \\
\hline Vessel Head & Off & Off \\
\hline Containment Integrity & Open & Open \\
\hline Drywell Integrity & Open & Open \\
\hline Suppression Pool Makeup & Not Available & Not Available \\
\hline Containment Sprays & Not Available & Not Available \\
\hline Containment Closure Possible? & No & Yes \\
\hline Injection Recovery Possible? & Yes & No \\
\hline \hline
\end{tabular}

From Table 2.3-2 it can be seen that the main differences between the LOSP and nonLOSP PDSs are 1) the containment can be closed only in the nonLOSP PDS and 2) injection can be recovered only in the LOSP PDS. Because sequence frequencies are unavailable from the Level 1 screening analysis, the relative likelihood of the two PDSs is unavailable. The remaining analysis that is presented in this report is conditional on the occurrence of these PDSs.

\subsection{Event Tree Analysis}

A simplified APET was used in this analysis to delineate and quantify the likelihood of the possible accident progression paths. The selection of events to include in the accident progression analysis was based on (1) insights gained from the NUREG-1150 full power PRAs [1,9], (2) results from MELCOR calculations specifically performed for this analysis, and (3) the plant configuration during POS 6 . Events deemed important for inclusion in the APET were events that related to containment performance and the estimation of the radionuclide release.

The APET addresses three general time regimes: prior to core damage, during core damage, and following vessel failure. In the first time regime the issue of containment closure is addressed. Injection recovery, core damage arrest, in-vessel steam explosions and early containment failure are all addressed in the second time regime. The characteristics of the interaction between the core debris release from the vessel and the reactor pedestal are addressed in the last time regime. 
The times associated with these time regimes are based on results from a series of MELCOR calculations that were performed to support this analysis. The timing of key events in the accident progression analysis is presented in Table 2.4-1.

Table 2.4-1 Accident Progression Timing

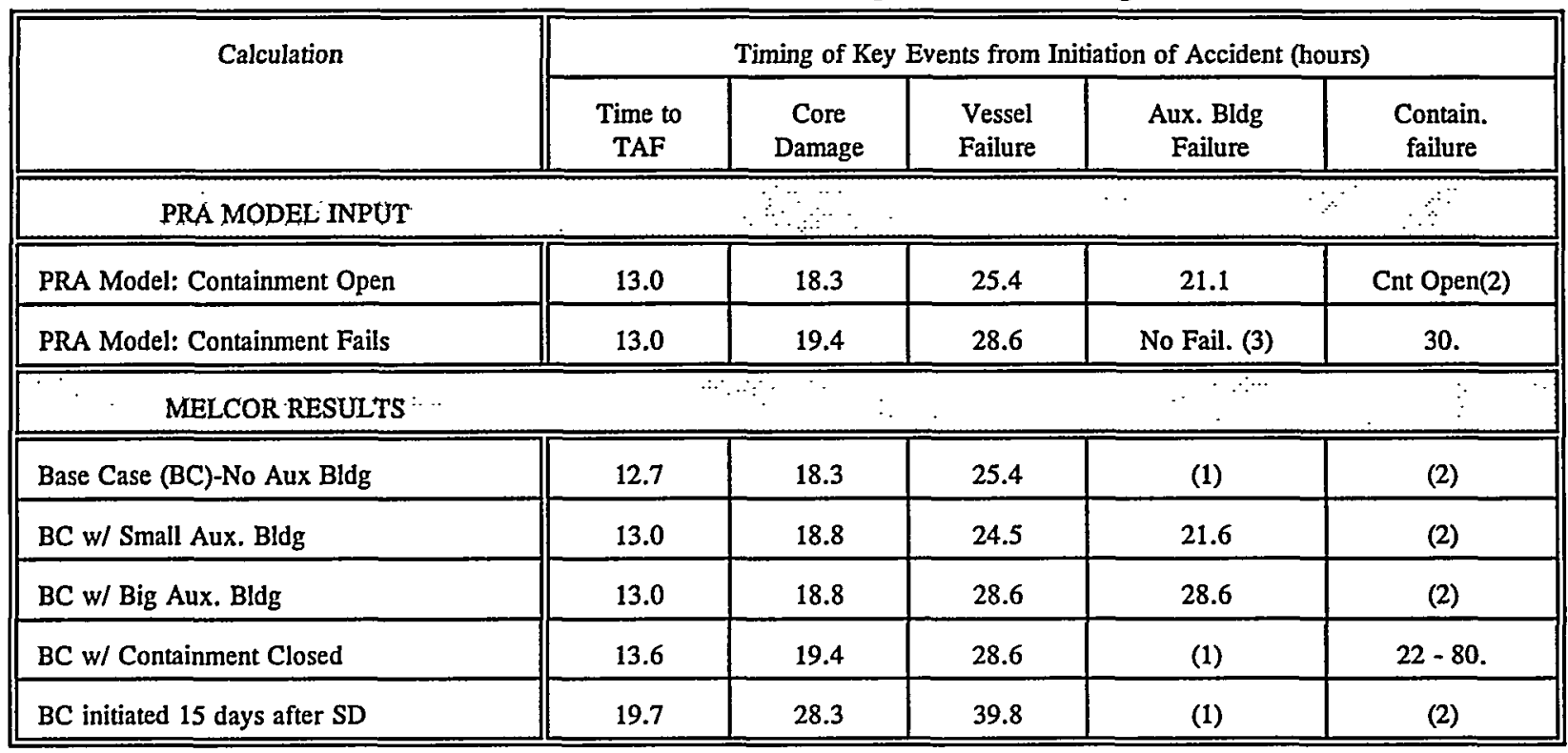

Notes:

1. Auxiliary building model not included

2. Containment is open during the accident

3. Containment failure bypasses the auxiliary building

4. MELCOR POS 6 BC Calculation:

- Accident Initiated 4 days after shutdown

- Containment is open (i.e, equipment hatch and both personnel locks)

- Injection, shutdown cooling, and containment sprays are all unavailable

5. Core damage is defined as the first gap release

6. $\mathrm{TAF}=$ Collapsed water level at the top of the active fuel

In this table both the times estimated with MELCOR and the times assumed in this PRA are presented. From this table it is apparent that the timings of these accidents are quite different from accidents initiated at full power. For example, it takes approximately 18 hours to progress from the initiation of the accident to the onset of core damage. In comparison, a fast station blackout initiated from full power progresses to a similar point in approximately 1 hour. Another notable entry in this table is the predicted time of auxiliary building failure for cases with the containment open. The building is predicted to overpressurize and fail from the accumulation of steam and noncondensibles during core damage. The exact timing of building failure depends on the volume assumed for the auxiliary building (i.e., various rooms in the building can isolated) and the building failure pressure. For this abridged study, the auxiliary building is estimated to fail approximately half way through the core damage process. 
Nine events are used to characterize the accident progression. A graphical depiction of the APET is presented in Figure 2.4-1. The first nine paths are associated with the LOSP PDS and the remaining 7 paths (i.e., paths 10 through 16) are associated with the nonLOSP PDS. The mean probability for each path is also presented in this figure. The path probabilities for each PDS sum to 1.0. The nine events and a brief description of each event are presented below.

\section{Is the containment closed prior to core damage?}

The containment equipment hatch has been removed prior to entry into POS 6. For the LOSP PDS the lack of offsite ac power precludes containment closure prior to core damage. However, for the nonLOSP PDS it is possible that the plant personnel will close the containment after the initiation of the accident but prior to core damage. The containment can be closed if the operators recognize that a problem exists early in the accident and decide that containment closure would be prudent. Because it takes between 8 to 12 hours to completely close the hatch, it is necessary that the operators begin the closure tasks within the first few hours of the accident. The equipment hatch is a pressure seating hatch which requires the personnel closing the hatch to be in the containment. Thus, the environment in the containment during the boiloff is an important parameter that will affect the personnel's ability to close the containment. MELCOR calculations performed for this analysis indicate that the temperatures in the containment during this phase of the accident will be high (i.e., range from 100 to 140 degrees F) but not so high that it would preclude the personnel from carrying out their tasks. It was also assumed that the radiological environment in the containment will not preclude the closure tasks from being performed. These assumption will have to be verified in future analysis. In this analysis it was assumed that the containment was habitable up until the time of core uncovery (i.e., approximately 13 hours).

\section{If the containment is closed prior to core damage, does it fail prior to vessel failure?}

The Grand Gulf plant utilizes a Mark III containment to house its BWR-6 reactor. The containment has a volume of 1.6 million cubic feet and a design pressure of 15 psig. The mean estimated failure pressure is 56 psig [9]. Since the containment has a relatively low failure pressure, the pressure rise from the accumulation of steam and noncondensibles can pose a threat to the containment integrity. Actions must also be taken to prevent the combustion of large quantities of hydrogen. Containment venting was not considered in this analysis as a means to control pressure because venting would still result in an open containment. In POS 6 the suppression pool is bypassed and, therefore, the steam and noncondensibles are released directly into the containment atmosphere. Furthermore, the containment sprays are not available. Thus, the containment will pressurize during the core damage process. The peak pressure during this phase of the accident depends on the steam generation rate, the condensation rate in the containment, and the presence and magnitude of hydrogen burns. MELCOR calculations indicate that the containment pressure can exceed the lower range of the containment failure pressure distribution if a burst of steam 
occurs at the time of vessel failure or if discrete hydrogen burns (not diffusion flames) occur during the core damage phase of the accident. Because steam and hot hydrogen are released directly into the containment in this POS, the effectiveness of the HIS to control the accumulation of hydrogen is uncertain. Thus, it is possible that for some accident scenarios the containment will fail early in the accident. If the containment does not fail early, calculations indicate that it will take several days to reach the mean estimated failure pressure of 56 psig. Therefore, it was assumed that if the containment does not fail early, it will not fail in the time frame of this analysis.

\section{If the containment fails, is the failure in the form of a leak or rupture?}

The failure size will determine how fast the radionuclides are released from the containment and the amount of radionuclides deposited within the containment.

\section{Is the auxiliary building bypassed?}

This question distinguishes the accidents in which the releases pass through the auxiliary building from those accidents which result in a release from the containment directly into the environment. The release path is important because it will affect the amount of mitigation that the release experiences before entering the environment. Accidents in which the containment equipment hatch is off will result in a release that passes through the auxiliary building; accidents in which the containment fails bypass the auxiliary building. Based on previous structural analysis of the Grand Gulf containment, it was concluded that the most likely location for failure is the region near the junction of the dome and the cylindrical wall [9]. A failure in this location will result in a release to the enclosure building that surrounds the containment dome. The enclosure building has virtually no pressure retaining capability and is essentially isolated from the auxiliary building. Therefore, it is assumed that following containment failure, the release goes directly from the containment into the environment. The retention in the containment will be fairly small in this case because the containment fails early in the accident. The result will be essentially an unmitigated release. If the containment is open to the auxiliary building, the majority of the radionuclides will quickly enter the auxiliary building and the retention in the containment will be small. For these accidents, the only significant mitigation feature will be the auxiliary building which acts as a large holdup volume allowing time for natural processes to remove radionuclides from the building atmosphere before being released into the environment. The auxiliary building is predicted to overpressurize and fail from the accumulation of steam and noncondensibles during core damage. The exact timing of building failure depends on the volume of the auxiliary building that will pressurize (i.e., some rooms within the building can be isolated and therefore will not pressurize) and the building failure pressure. For this abridged study, the auxiliary building is estimated to fail approximately halfway through the core damage process. 


\section{Is injection recovered prior to vessel failure?}

This question is used to identify those accidents in which injection is restored to the vessel during the core damage process. The recovery of injection allows for the possibility that the core damage process will be arrested in the vessel (i.e., prevent vessel failure). Injection can only be recovered for the LOSP PDS. The probability that injection is recovered is based on the probability that offsite ac power is recovered during core damage.

\section{If injection is recovered, when is it recovered?}

The timing of injection recovery during core damage affects the likelihood that the core damage process will be arrested before the vessel fails. For this analysis, the in-vessel phase of the accident (i.e., core damage) has been divided into three time regimes: very early, early, and late. The very early time regime ranges from the initiation of core damage to the onset of autocatalytic oxidation. If injection is recovered during this phase of the accident the core damage process will be arrested in the vessel and the releases will be limited to the inventory in the gap. The early time regime ranges from onset of autocatalytic oxidation to $30 \%$ core damage. Based on extrapolation of analysis performed in NUREG-1150, if injection is recovered before $30 \%$ of the core has been damaged, it is very likely that the core damage process can be arrested. Because MELCOR calculations indicate that core damage progresses rapidly from $30 \%$ to full core damage, the late time regime is defined as $30 \%$ core damage to vessel failure. Recovery of injection during this phase of the accident will not prevent vessel failure. The time windows for each of these time regimes is based on results from MELCOR calculations. The possibility of the reactor going critical following the restoration of injection was not addressed in this abridged analysis.

\section{Does an in-vessel steam explosion occur during core damage?}

In-vessel steam explosions are treated in a very limited fashion in this abridged analysis. A primary motivation for including this question in the APET is to highlight the fact that in-vessel steam explosions are possible. The effect of the steam explosion on the accident progression can be quite different from in-vessel steam explosions that occur at full power because the steam and radionuclides that are generated during this event are released directly into the containment atmosphere. In this analysis the treatment of in-vessel steam explosions was limited to the estimation of the source term that is associated with the debris that participates in the steam explosions. Neither the pressure loading from in-vessel steam explosions nor the relocation of intact fuel from the steam explosion was addressed in this study. Both issues were beyond the scope of this abridged study. Ex-vessel steam explosions were not considered in this analysis because the pedestal cavity below the vessel will be essentially dry at the time of vessel failure. 


\section{Is the core damage process arrested in the vessel?}

This question addresses the coolability of the core debris following injection recovery. If the core damage process is arrested before the vessel fails, the core debris will remain in the vessel and core-concrete interactions (CCI) will be prevented. Because only a portion of the core is damaged and $\mathrm{CCI}$ is prevented, the source term associated with recovered accidents is typically less than the source term associated with full core damage accidents. If injection is not restored during core damage, the accident always progresses to vessel failure and the core debris relocates to the pedestal cavity below the vessel. The likelihood that the core damage process is arrested before vessel failure depends on when injection is restored during the core damage process (see question 6). If injection is restored during either the very early or early time regimes, analysis indicates that it is very likely the core damage process will be arrested. If, on the other hand, injection is not restored until the late time regime, it is very likely that the vessel will fail and the core debris will relocate to the pedestal cavity.

\section{Do core-concrete interactions occur following vessel failure?}

Core-concrete interactions consist of the thermal and chemical interactions between the core debris and the concrete pedestal. During this process the concrete is eroded and gases and radionuclides are released from the core/concrete mixture. For the accidents analyzed in this study, the vessel will fail and the core debris will enter the cavity if 1) injection is not restored to vessel during core damage or 2) injection is restored during the late time regime. The presence of water can affect $\mathrm{CCI}$ in two different ways. First, water can quench the debris and prevent CCI. Second, if the debris is not quenched, the overlying pool of water will retain some of the radionuclides released during $\mathrm{CCI}$ and thus, tend to mitigate the release. Thus, for the accidents in which injection is restored but the vessel still fails, there is some probability that the core debris will be quenched and CCI will be prevented. The probability of this occurring is based on information from the NUREG1150 study [9]. If injection is not restored during core damage, CCI will always proceed in a dry cavity.

From inspection of Figure 2.4-1 it can be seen that there are several important differences between the LOSP PDS and the nonLOSP PDS. In the LOSP PDS injection can be recovered allowing for the possibility to arrest the core damage process in the vessel. If the vessel does fail, it is still possible to quench the core debris in the cavity (i.e., no CCI). Thus, in many of the LOSP accidents the ex-vessel radionuclide release is prevented. The containment, however, cannot be closed in the LOSP PDS and, therefore, the releases always pass into the auxiliary building and then out into the environment. In the nonLOSP PDS, the containment can be closed, however, injection cannot be recovered. Thus, all of the nonLOSP accidents identified in the APET progress to full core damage, vessel failure, and involve CCI. In some of the scenarios the containment is closed. However, because containment cooling (i.e., containment sprays) is unavailable and the suppression pool is bypassed, even if the containment is closed it is possible that it will fail early in the accident from pressure transients associated with events 
accompanying vessel failure and hydrogen combustion. Based on information from NUREG1150 , it is expected that the containment will fail above the auxiliary building roof. Thus, the releases from the containment will enter the environment without first going through the auxiliary building. Because so many of the mitigative features of the plant are bypassed in this POS (e.g., suppression pool, containment sprays, containment), the auxiliary building plays an important role in reducing the amount of radionuclide material that is released into the environment. Thus, for the nonLOSP accidents there are two extremes: 1) if the containment is closed and remains intact, the releases to the environment are expected to be very small and 2) if the containment fails, the releases to the environment are expected to be quite large because all of the accidents involve full core damage and $\mathrm{CCI}$ and the releases bypass the auxiliary building. The scenarios in which the containment is not closed are very similar to the LOSP accidents in which injection is not recovered. 


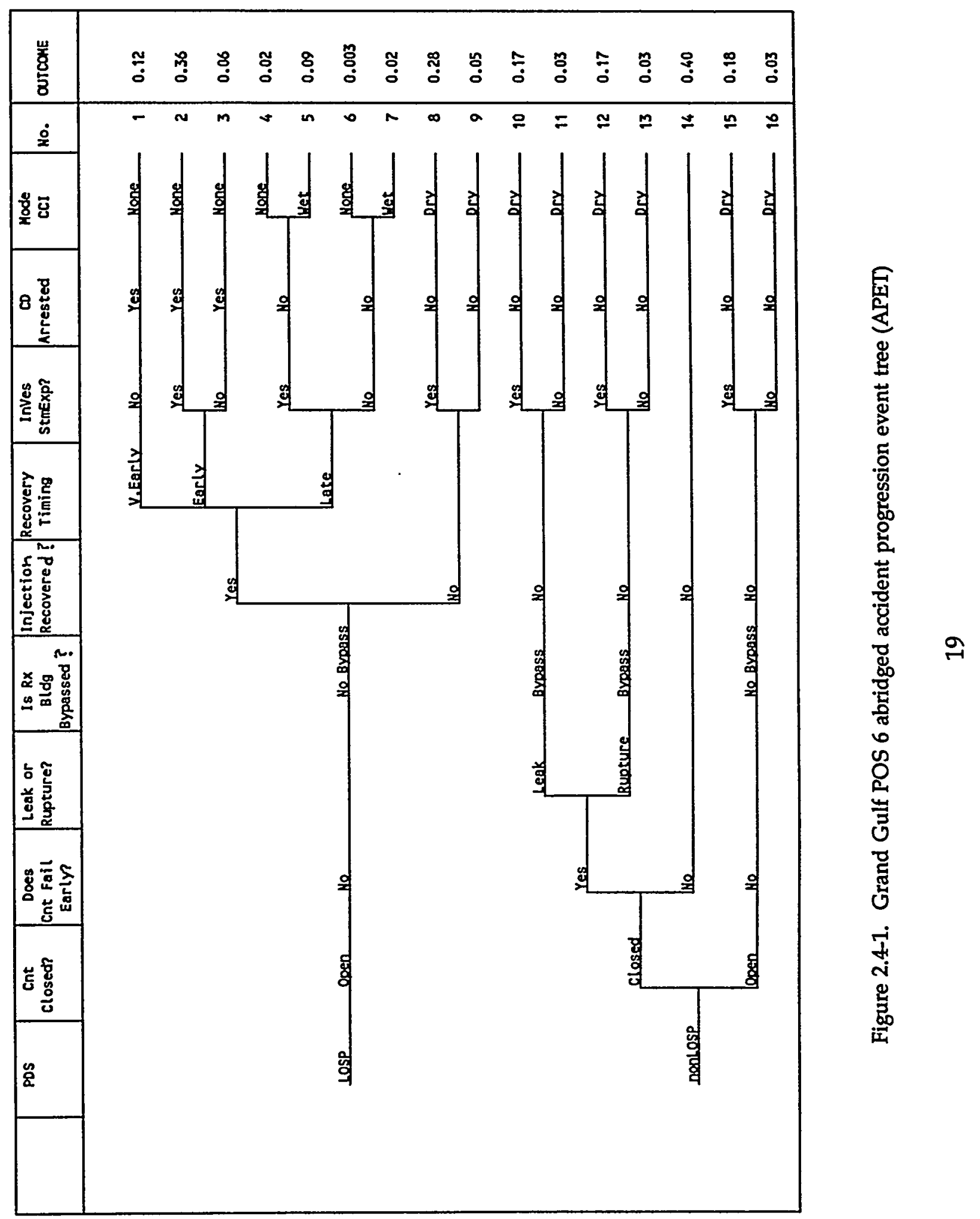




\subsection{SOURCE TERM ANALYSIS}

\subsection{Approach}

A source term is estimated for each accident progression path identified in the APET (see Figure 2.4-1). The simple parametric source term approach that was used in NUREG-1150 to estimate source terms is used in this study. The parametric source approach is used because 1) information from a wide variety of sources can be used in the model, 2) it is easily incorporated into uncertainty analysis, and 3) thousands of source terms can be estimated with this model in a very efficient manner. The parametric source term code GGSOR that was developed in NUREG-1150 [9] was modified for this analysis. The modified parametric code is called GGLPSOR. Modifications were made to the code to incorporate the unique plant configuration associated with accidents initiated in POS 6 . Wherever possible, data from NUREG-1150 was used to quantify the model. Results from MELCOR were compared with both the input distributions and the final source terms to verify that distributions developed for full power accidents could be applied to shutdown accidents.

A limited uncertainty analysis was performed in this section of the analysis. For each accident progression path, the model was repeatedly exercised with different combinations of selected input variables. The distributions for these input variables were obtained, when applicable, from NUREG-1150.

\subsection{Description of Parametric Model}

The parametric source term model GGSOR that was developed in NUREG-1150 was modified to account for unique features of POS 6 that have a strong impact on the source term. In POS 6 both the drywell head and the vessel head have been removed and the steam lines have been plugged. Thus, during the core damage process radionuclides released from the core debris will bypass the suppression pool and directly enter the containment. Furthermore, because most of the internal structures above the core (e.g., steam dryers and separators) have been removed and the steam lines are plugged, there is very little deposition of radionuclides in the vessel. Thus, the mitigative features of both the vessel and the suppression pool, which are present in many full power accident scenarios, are absent in this POS. For scenarios in which the containment hatch is open, the residence time of the radionuclides in the containment atmosphere is fairly short and, thus, there will be limited deposition (i.e., from gravitational settling) of radionuclides in the containment. In this POS the drywell is open to the containment (i.e., the drywell hatch is open) and, therefore, an ex-vessel release will also bypass the suppression pool. For these accidents, the containment sprays are not available and cannot be used to scrub the releases. Thus, the mitigative features of the vessel, suppression pool, containment sprays, and possibly the containment, are bypassed or unavailable.

For scenarios with the containment open, the only major mitigative feature of the plant is the auxiliary building. The auxiliary building encompasses a very large volume and, therefore, acts as a hold up volume for the radionuclides which allows time for the radionuclides to deposit on 
surfaces within the building. The auxiliary building can play an important role in POS 6 because so many of the other mitigative features of the plant are absent and the characteristics of the radionuclide transport to the auxiliary building are different from the transport associated with full power accidents. In full power accidents the containment pressurizes to the ultimate failure pressure and then blows down into the auxiliary/reactor building (i.e., Peach Bottom analysis in the NUREG-1150 study). Following containment failure the auxiliary building rapidly pressurizes and fails (the failure pressure of the auxiliary building is only a few psi). Thus, the releases are swept through the auxiliary building fairly rapidly. In the POS 6 accident scenarios the steam and radionuclides are released to the auxiliary building much more slowly allowing more time for condensation and deposition. The scenarios that involve containment closure followed by containment failure will not benefit from the auxiliary building because the containment failure location is assumed to be above the roof of the auxiliary building [9]. Thus, the releases will bypass the auxiliary building essentially resulting in an unmitigated release.

Neither the normal ventilation system nor the standby gas treatment system (SBGT) were modeled in this analysis. The filters and charcoal beds in the SBGT system could act to mitigate the release or at least delay the release of radionuclides. Before credit can be given to this system, the capacity of the system and the performance of the filters under severe accident conditions will have to be addressed. The analysis of this system was beyond the scope of this study.

\subsection{Results}

A source term is estimated for each path through the APET. In addition, because an uncertainty analysis was performed, a distribution of source terms is available for each path. For the sake of brevity, only the mean source terms, expressed as fractions of the core inventory, that enter the environment are presented in Table 3.3-1. When reviewing this table, it must be remembered that the initial inventory of radionuclides four days after shutdown is different from the inventory typical of full power accidents.

Inspection of Table 3.3-1 confirms that many of the releases are essentially unmitigated and, therefore, are quite large. Table 3.3-1 also highlights some of the differences between the various accident scenarios (i.e., paths). Paths 1 through 3 correspond to accidents in which injection is recovered early in the accident and the core damage process is arrested in the vessel. Thus, because only a portion of the core is damaged and there are no ex-vessel releases (i.e., no $\mathrm{CCI}$ ), the source terms associated with these accidents are relatively small compared to the other source terms presented in this table. The notable exception is Path 14 which corresponds to the scenario in which the containment is closed prior to core damage and remains intact throughout the accident. Because the containment remains intact, only nominal leakage occurs and the resulting source term is quite small. Paths 4 through 9 , on the other hand, correspond to full core damage accidents that have the containment open to the auxiliary building. The source terms associated with Paths 4 and 6 tend to be lower than the other full core damage source terms because the core debris is quenched in the pedestal cavity and, therefore, there are 
no releases associated with CCI. This difference is fairly minor, however, and the fact still remains that these are large source terms. Paths 10 through 13 are nonLOSP accidents in which the containment fails around the time of vessel failure. All of these accidents progress to full core damage and CCI. The containment fails via a leak in Paths 10 and 11; the containment ruptures in Paths 12 and 13. In all four of these scenarios the containment fails directly to the environment (i.e., the auxiliary building is bypassed). The source terms associated with the leak failure mode are similar to the source terms when the release passes through the auxiliary building. In the leakage cases, the radionuclides are held up in the containment for a period of time thus allowing a fraction of the radionuclides to settle out of the containment atmosphere. For the rupture cases, however, the containment quickly depressurizes following containment failure and considerably less deposition occurs. Thus, the source terms associated with the rupture cases are quite large. Paths 15 and 16 correspond to the nonLOSP cases where the containment is not closed prior to core damage and the radionuclides pass through the auxiliary building. These source terms are essentially the same as the LOSP full core damage source terms. 
Table 3.3-1 Mean Source Terms for Accident Progression Paths (Total Release)

\begin{tabular}{|c|c|c|c|c|c|c|c|c|c|c|c|c|c|}
\hline \multirow[t]{2}{*}{ Path } & \multicolumn{9}{|c|}{ Radionuclide Release Classes } & \multicolumn{4}{|c|}{ Timing of Release (hr.s) } \\
\hline & NG & I & Cs & $\mathrm{Te}$ & $\mathrm{Sr}$ & $\mathbf{R u}$ & $\mathrm{La}$ & $\mathrm{Ce}$ & $\mathrm{Ba}$ & TW & T1 & DT1 & DT2 \\
\hline & $\therefore$ & : & $\therefore$ & & LOSP PDS & $\because$ & & $\therefore$ & & & & & \\
\hline 1 & 0.015 & 0.002 & $5.9 \mathrm{E}-3$ & $1.2 \mathrm{E}-5$ & 0.0 & 0.0 & 0.0 & 0.0 & $1.2 \mathrm{E}-7$ & 16.3 & 21.1 & 24.0 & 0.0 \\
\hline 2 & 0.072 & 0.012 & 0.011 & $6.3 \mathrm{E}-3$ & $2.1 \mathrm{E}-3$ & $3.3 \mathrm{E}-4$ & $1.4 \mathrm{E}-4$ & $6.7 E-4$ & $2.2 \mathrm{E}-3$ & 16.3 & 21.1 & 4.3 & 0.0 \\
\hline 3 & 0.072 & 0.012 & 0.011 & $6.3 \mathrm{E}-3$ & $2.1 \mathrm{E}-3$ & $3.3 E-4$ & $1.4 \mathrm{E}-4$ & $6.7 \mathrm{E}-4$ & $2.2 \mathrm{E}-3$ & 16.3 & 21.1 & 4.3 & 0.0 \\
\hline 4 & 0.79 & 0.17 & 0.15 & 0.085 & 0.027 & 0.012 & $3.0 \mathrm{E}-3$ & 8.7E-3 & 0.033 & 16.3 & 21.1 & 4.3 & 10.0 \\
\hline 5 & 1.0 & 0.25 & 0.19 & 0.11 & 0.042 & 0.012 & $4.0 \mathrm{E}-3$ & 0.011 & 0.047 & 16.3 & 21.1 & 4.3 & 10.0 \\
\hline 6 & 0.74 & 0.15 & 0.13 & 0.075 & 0.022 & $4.9 \mathrm{E}-3$ & $1.5 \mathrm{E}-3$ & 7.1E-3 & 0.026 & 16.3 & 21.1 & 4.3 & 10.0 \\
\hline 7 & 1.0 & 0.25 & 0.18 & 0.11 & 0.041 & $4.9 \mathrm{E}-3$ & $2.7 \mathrm{E}-3$ & $9.6 \mathrm{E}-3$ & 0.042 & 16.3 & 21.1 & 4.3 & 10.0 \\
\hline 8 & 1.0 & 0.25 & 0.25 & 0.16 & 0.08 & 0.012 & $6.9 \mathrm{E}-3$ & 0.012 & 0.084 & 16.3 & 21.1 & 4.3 & 10.0 \\
\hline \multirow[t]{2}{*}{9} & 1.0 & 0.25 & 0.25 & 0.17 & 0.088 & $5.4 \mathrm{E}-3$ & $6.3 \mathrm{E}-3$ & 0.011 & 0.089 & 16.3 & 21.1 & 4.3 & 10.0 \\
\hline & \multicolumn{9}{|c|}{ nontosp PDS } & & & & $\because$ \\
\hline 10 & 1.0 & 0.27 & 0.27 & 0.18 & 0.094 & 0.013 & $6.8 \mathrm{E}-3$ & 0.011 & 0.083 & 17.4 & 30.0 & 2.0 & 10.0 \\
\hline 11 & 1.0 & 0.28 & 0.28 & 0.19 & 0.10 & $6.1 \mathrm{E}-3$ & $6.2 \mathrm{E}-3$ & 0.011 & 0.086 & 17.4 & 30.0 & 2.0 & 10.0 \\
\hline 12 & 1.0 & 0.62 & 0.63 & 0.40 & 0.22 & 0.029 & 0.016 & 0.027 & 0.19 & 17.4 & 30.0 & 0.05 & 10.0 \\
\hline 13 & 1.0 & 0.62 & 0.63 & 0.43 & 0.24 & 0.013 & 0.014 & 0.025 & 0.20 & 17.4 & 30.0 & 0.05 & 10.0 \\
\hline 14 & $5.0 \mathrm{E}-3$ & $4.1 \mathrm{E}-7$ & $4.1 \mathrm{E}-7$ & $2.9 \mathrm{E}-7$ & $1.4 \mathrm{E}-7$ & $9.4 \mathrm{E}-9$ & $9.7 \mathrm{E}-9$ & $1.9 \mathrm{E}-8$ & $1.4 \mathrm{E}-7$ & 16.3 & 21.1 & 4.3 & 10.0 \\
\hline 15 & 1.0 & 0.25 & 0.25 & 0.16 & 0.08 & 0.012 & $6.9 \mathrm{E}-3$ & 0.012 & $8.4 \mathrm{E}-2$ & 16.3 & 21.1 & 4.3 & 10.0 \\
\hline 16 & 1.0 & 0.25 & 0.25 & 0.17 & 0.088 & $5.4 \mathrm{E}-3$ & $6.3 E-3$ & 0.011 & 0.089 & 16.3 & 21.1 & 4.3 & 10.0 \\
\hline
\end{tabular}

1. $T W=$ Warning Time

2. $\quad T 1=$ Timing of first release

3. $\mathrm{DT} 1=$ Duration of first release

4. $\quad \mathrm{DT2}=$ Duration of second release (start immediately after first release ends) 


\subsection{CONSEQUENCE ANALYSIS}

The consequences of a severe accident during POS 6 were calculated as part of the abridged study. As is typically done, the offsite consequences were estimated. The onsite doses were also estimated, which is not typically done.

An important difference between this analysis and those previously performed for full power accidents is that the radionuclides in the fuel have had at least four days to decay resulting in a different inventory than that present at shutdown. ORIGEN2 [10] was used to calculate the inventory in three different fuel assemblies, one which had been irradiated for three fuel cycles, one which had been irradiated for two fuel cycles, and one which had been irradiated for one fuel cycle. All fuel assemblies were then allowed to decay for four days. Based on information from plant personnel, a fuel cycle consisted of 540 days of irradiation and 55 days of decay. The inventory for the whole core four days after shutdown was then summed. This inventory, which was reduced to include only the sixty radionuclides currently available in the MACCS code $[5,6,7]$, was then used as the basis for both the onsite and offsite consequence calculations. This inventory, which does not include short-lived radionuclides, is appropriate for both the onsite and offsite analyses since the reactor has been in shutdown for at least four days at the beginning of the accident thus allowing decay of the short-lived radionuclides.

The following sections detail the methodology and results for the onsite consequences, both in the buildings and in the parking lot, and the offsite consequences.

\subsection{Onsite Consequences}

Onsite consequences have seldom been considered in the analysis of severe accidents at nuclear power plants. During shutdown there will be hundreds of onsite personnel and, thus, onsite consequences could be large. For this reason a method for estimating the potential doses to onsite personnel had to be developed as part of this study. The primary simplifying assumption of the analysis was that radioactive decay was neglected during the exposure time. This assumption is justified by the fact that the accident under analysis typically occurs no earlier than four days after shutdown by which time the decay heat curve is fairly flat. Other assumptions were employed in the two aspects of the onsite consequences: (1) in building doses and (2) parking lot doses. The method, assumptions, and results of the analyses are discussed in the following two sections.

\subsubsection{Building Doses}

The onsite consequences for POS 6 were estimated based on the source terms to both the containment and the auxiliary building that were determined with the parametric source term code, GGLPSOR. However, since GGLPSOR calculates integral releases, the time dependence of the two release segments of the source terms was determined from MELCOR calculations. Three different sets of residence times (i.e., estimated time airborne material spends in the building) were used based on the status of the containment. The first set of residence times was 
used if the containment was open to the auxiliary building at the time of the accident. The residence times through both buildings were based on a MELCOR calculation modeling this scenario. The residence time of the radioactive material in each building was directly proportional to the volume of that building. The second set of residence times was used if the containment ruptured directly to the environment. For this case, the same residence times were used as in the previous scenario, however, the residence time in the auxiliary building was set to zero. In other words, the amount of time the material spent in the containment was the same for both of these scenarios, but in the latter scenario the material did not pass through the auxiliary building. The third set of residence times was used if the containment leaked directly to the environment. In this case, the residence time for the first release was increased by two hours, and again the residence time in the auxiliary building was set to zero. The residence times used under the various conditions are summarized in Table 4.1.1-1.

Table 4.1.1-1. Residence times through the containment and auxiliary building for Grand Gulf POS 6.

\begin{tabular}{|c|c|c|c|c|}
\hline $\begin{array}{l}\text { Accident Progression } \\
\text { Path Number }\end{array}$ & $\begin{array}{l}\text { Containment Residence } \\
\text { Time: First Segment } \\
\text { (hours) }\end{array}$ & $\begin{array}{c}\text { Containment Residence } \\
\text { Time: Second Segment } \\
\text { (min) }\end{array}$ & $\begin{array}{l}\text { Auxiliary Building } \\
\text { Residence Time: First } \\
\text { Segment (hours) }\end{array}$ & $\begin{array}{l}\text { Auxiliary Building } \\
\text { Residence Time: } \\
\text { Second Segment } \\
\text { (hours) }\end{array}$ \\
\hline$\therefore$ & \multicolumn{4}{|c|}{ LOSP PDS } \\
\hline Paths 1-9 & 3.4 & 47 & 6.1 & 1.4 \\
\hline . & \multicolumn{3}{|c|}{$\because \cdots \quad$ nonLOSP PDS } & $\because$ \\
\hline Path 10 & $4.1^{\circ}$ & 47 & 0.0 & 0.0 \\
\hline Path 11 & 4.1 & 47 & 0.0 & 0.0 \\
\hline Path 12 & 3.4 & 47 & 0.0 & 0.0 \\
\hline Path 13 & 3.4 & 47 & 0.0 & 0.0 \\
\hline Path $14^{1}$ & $\mathrm{NC}$ & $\mathrm{NC}$ & $\mathrm{NC}$ & $\mathrm{NC}$ \\
\hline Path 15 & 3.4 & 47 & 6.1 & 1.4 \\
\hline Path 16 & 3.4 & 47 & 6.1 & 1.4 \\
\hline
\end{tabular}

' Building doses were not calculated since the containment is not open.

To estimate the doses in the buildings, the average release fraction of each chemical group was determined for each building. The integrated concentration of each radionuclide in the buildings was then based on the average release fraction of its chemical group and the amount of time spent in that building. Using the integrated concentration for each radionuclide, the immersion and 50 year committed inhalation dose were calculated over the entire exposure time. In addition, the immersion and 50 year committed inhalation dose were calculated for the first 30 minutes of exposure. These doses should be viewed with caution since the integrated concentration in the building was based on an average concentration in the building and therefore the time dependence of the dose is not well represented. The final result estimated in the 
buildings was a dose rate. These results should also be viewed with caution since they are also based on average concentrations in the building. In addition, the dose rates were calculated by dividing the total dose during a release segment by the transit time through the building. This results in a conservative estimate of the inhalation dose rate. The mean dose due to the entire release, the first 30 minutes of exposure, and the mean dose rates during the first and second release segments in the containment are shown in Table 4.1.1-2 for each of the paths through the APET. Similar estimates are shown in Table 4.1.1-3 for the auxiliary building.

Table 4.1.1-2. Grand Gulf POS 6 mean containment doses and dose rates.

\begin{tabular}{|c|c|c|c|c|c|}
\hline \multirow{2}{*}{$\begin{array}{l}\text { Accident } \\
\text { Progression Path } \\
\text { Number }\end{array}$} & \multirow{2}{*}{$\begin{array}{l}\text { Path Conditional } \\
\text { Probability }\end{array}$} & \multicolumn{4}{|c|}{ Consequence Measure } \\
\hline & & Total Dose (rem) & $\begin{array}{l}30 \text { minute Dose } \\
\text { (rem) }\end{array}$ & $\begin{array}{l}\text { Dose Rate First } \\
\text { Segment (rem } / \mathrm{hr})\end{array}$ & $\begin{array}{l}\text { Dose Rate Second } \\
\text { Segment (rem/hr) }\end{array}$ \\
\hline & & \multicolumn{4}{|c|}{ LOSP PDS } \\
\hline Path 1 & 0.10 & $1.81 E+6$ & $2.69 \mathrm{E}+5$ & $5.38 E+5$ & 0.0 \\
\hline Path 2 & 0.48 & $4.27 \mathrm{E}+7$ & $6.35 \mathrm{E}+6$ & $1.27 \mathrm{E}+7$ & 0.0 \\
\hline Path 3 & 0.08 & $4.27 E+7$ & $6.35 \mathrm{E}+6$ & $1.27 \mathrm{E}+7$ & 0.0 \\
\hline Path 4 & 0.02 & $5.38 \mathrm{E}+6$ & $7.95 \mathrm{E}+7$ & $1.59 \mathrm{E}+8$ & 0.0 \\
\hline Path 5 & 0.09 & $5.69 E+8$ & $7.95 \mathrm{E}+7$ & $1.59 \mathrm{E}+8$ & $4.04 \mathrm{E}+7$ \\
\hline Path 6 & 0.003 & $4.27 \mathrm{E}+8$ & $6.35 \mathrm{E}+7$ & $1.27 \mathrm{E}+8$ & 0.0 \\
\hline Path 7 & 0.01 & $4.66 \mathrm{E}+8$ & $6.35 \mathrm{E}+7$ & $1.27 E+8$ & $5.05 \mathrm{E}+7$ \\
\hline Path 8 & 0.18 & $6.16 \mathrm{E}+8$ & $7.95 \mathrm{E}+7$ & $1.59 \mathrm{E}+8$ & $1.01 \mathrm{E}+8$ \\
\hline \multirow[t]{2}{*}{ Path 9} & 0.03 & $5.25 \mathrm{E}+8$ & $6.35 E+7$ & $1.27 \mathrm{E}+8$ & $1.26 \mathrm{E}+8$ \\
\hline & & \multicolumn{4}{|c|}{ nonLOSP Pos } \\
\hline Path 10 & 0.16 & $5.78 \mathrm{E}+8$ & $6.35 \mathrm{E}+7$ & $1.27 \mathrm{E}+8$ & $7.74 \mathrm{E}+7$ \\
\hline Path 11 & 0.02 & $4.88 \mathrm{E}+8$ & $5.05 E+7$ & $1.01 \mathrm{E}+8$ & $9.68 \mathrm{E}+7$ \\
\hline Path 12 & 0.16 & $6.16 \mathrm{E}+8$ & $7.95 \mathrm{E}+7$ & $1.59 \mathrm{E}+8$ & $1.01 \mathrm{E}+8$ \\
\hline Path 13 & 0.02 & $5.25 \mathrm{E}+8$ & $6.35 E+7$ & $1.27 \mathrm{E}+8$ & $1.26 \mathrm{E}+8$ \\
\hline Path $14^{t}$ & 0.37 & NC & NC & NC & $\mathrm{NC}$ \\
\hline Path 15 & 0.23 & $6.16 \mathrm{E}+8$ & $7.95 \mathrm{E}+7$ & $1.59 \mathrm{E}+8$ & $1.01 E+8$ \\
\hline Path 16 & 0.04 & $5.25 \mathrm{E}+8$ & $6.35 \mathrm{E}+7$ & $1.27 \mathrm{E}+8$ & $1.26 \mathrm{E}+8$ \\
\hline
\end{tabular}

\footnotetext{
${ }^{1}$ Building doses were not calculated since the containment is not open.
} 
Table 4.1.1-3. Grand Gulf POS 6 mean auxiliary building doses and dose rates.

\begin{tabular}{|c|c|c|c|c|c|}
\hline \multirow{2}{*}{$\begin{array}{c}\text { Accident } \\
\text { Progression Path } \\
\text { Number }\end{array}$} & \multirow{2}{*}{$\begin{array}{l}\text { Path Conditional } \\
\text { Probability }\end{array}$} & \multicolumn{4}{|c|}{ Consequence Measure } \\
\hline & & Total Dose (rem) & $\begin{array}{l}30 \text { minute Dose } \\
\text { (rem) }\end{array}$ & $\begin{array}{l}\text { Dose Rate First } \\
\text { Segment (rem/hr) }\end{array}$ & $\begin{array}{l}\text { Dose Rate Second } \\
\text { Segment (rem/hr) }\end{array}$ \\
\hline$\because \because \quad \vdots$ & 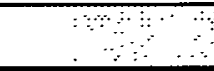 & \multicolumn{3}{|c|}{ LOSP PDS } & $\because$ \\
\hline Path 1 & 0.10 & $9.41 \mathrm{E}+5$ & $7.70 \mathrm{E}+4$ & $1.54 \mathrm{E}+5$ & 0.0 \\
\hline Path 2 & 0.48 & $2.13 E+7$ & $1.74 \mathrm{E}+6$ & $3.47 E+6$ & 0.0 \\
\hline Path 3 & 0.08 & $2.13 E+7$ & $1.74 \mathrm{E}+6$ & $3.47 E+6$ & 0.0 \\
\hline Path 4 & 0.02 & $2.80 \mathrm{E}+8$ & $2.28 \mathrm{E}+7$ & $4.56 \mathrm{E}+7$ & 0.0 \\
\hline Path 5 & 0.09 & $2.98 \mathrm{E}+8$ & $2.28 \mathrm{E}+7$ & $4.56 \mathrm{E}+7$ & $1.31 \mathrm{E}+7$ \\
\hline Path 6 & 0.003 & $2.20 \mathrm{E}+8$ & $1.79 \mathrm{E}+7$ & $3.59 \mathrm{E}+7$ & 0.0 \\
\hline Path 7 & 0.01 & $2.43 E+8$ & $1.79 \mathrm{E}+7$ & $3.59 \mathrm{E}+7$ & $1.64 \mathrm{E}+7$ \\
\hline Path 8 & 0.18 & $3.23 E+8$ & $2.28 \mathrm{E}+7$ & $4.56 \mathrm{E}+7$ & $3.06 \mathrm{E}+7$ \\
\hline \multirow[t]{2}{*}{ Path 9} & 0.03 & $2.74 \mathrm{E}+8$ & $1.79 \mathrm{E}+7$ & $3.59 \mathrm{E}+7$ & $3.83 \mathrm{E}+7$ \\
\hline & & & \multicolumn{2}{|c|}{ nonLOSP PDS } & 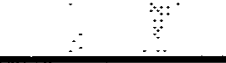 \\
\hline Path 10 & 0.16 & 0.0 & 0.0 & 0.0 & 0.0 \\
\hline Path 11 & 0.02 & 0.0 & 0.0 & 0.0 & 0.0 \\
\hline Path 12 & 0.16 & 0.0 & 0.0 & 0.0 & 0.0 \\
\hline Path 13 & 0.02 & 0.0 & 0.0 & 0.0 & 0.0 \\
\hline Path $14^{1}$ & 0.37 & $\mathrm{NC}$ & $\mathrm{NC}$ & NC & $\mathrm{NC}$ \\
\hline Path 15 & 0.23 & $3.23 E+8$ & $2.28 E+7$ & $4.56 \mathrm{E}+7$ & $3.06 \mathrm{E}+7$ \\
\hline Path 16 & 0.04 & $2.74 \mathrm{E}+8$ & $1.79 \mathrm{E}+7$ & $3.59 \mathrm{E}+7$ & $3.83 E+7$ \\
\hline
\end{tabular}

'Building doses were not calculated since the containment is not open.

To illustrate the uncertainty in the dose rate in the containment and the auxiliary building due to the uncertainty in the source term, the $5^{\text {th }}, 50^{\text {th }}$, and $95^{\text {th }}$ percentile dose rates as well as the mean dose rate for two pathways through the APET are shown in Figure 4.1.1-1. The first of these paths represents a scenario in which injection is recovered very early in the accident, thus arresting core damage. Note that in the recovered accident, CCI does not occur therefore the source term consists of only one segment and only one dose rate was calculated. The second path represents a scenario in which full core damage occurs. 
Building Dose Rates for Recovered Accident (Path 1) and Full Core Damage Accident (Path 8)

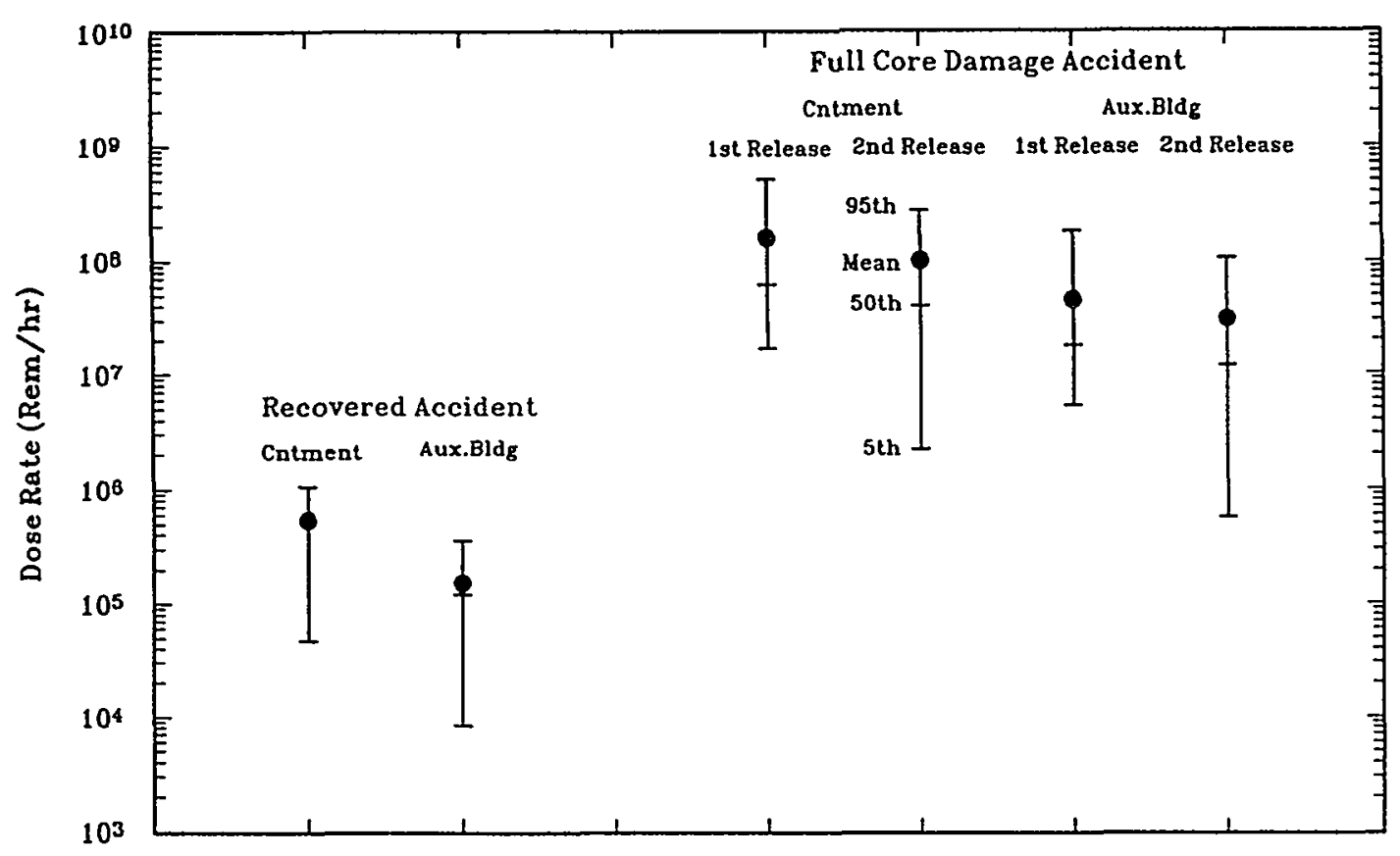

Figure 4.1.1-1. Containment and auxiliary building dose rates for selected paths

\subsubsection{Parking Lot Doses}

The dose due to immersion and inhalation was also estimated for several distances from the reactor. The source terms were obtained from the parametric source term code, GGLPSOR. In contrast to the building doses, the timing of the source terms was taken directly from GGLPSOR. For comparative purposes, three different wake effect models were used to estimate the relative concentrations downwind of the reactor. These models were developed by Ramsdell [11], Wilson [12], and the NRC [13]. For simplicity, the directional dependence of the weather was ignored and doses were calculated for several distances from the reactor. The weather used in each of the wake effect models was chosen to represent conservative values for the model. In the case of the Ramsdell model the relative concentration is somewhat proportional to the wind speed and the stability class. For this reason the highest wind speed and the corresponding stability class in a year of weather data at Grand Gulf was chosen as input to this model. In addition, the relative concentration is predicted to be somewhat inversely proportional to the area of the building, therefore, the minimum area was utilized. In the case of the Wilson and NRC models the relative concentration is predicted to be inversely proportional to the wind speed. 
Therefore, a wind speed of $1 \mathrm{~m} / \mathrm{s}$ and a stability class of $\mathrm{F}$ (i.e., moderately stable meteorological conditions) were used in these models. Using the integrated air concentrations for each building wake effect model, the dose and dose rate due to immersion and inhalation for the entire source term was determined for each of the unique accident progression paths. As with the building dose rates, the dose rates in the parking lot are very conservative since the inhalation dose rate was determined by dividing the 50 year committed dose by the exposure time. The dose due to 30 minutes of exposure was also estimated. Table 4.1.2-1 contains the mean total dose, 30 minute dose, and dose rates for each segment of the release based on the Ramsdell building wake effect model at 100 meters from the reactor. Similar estimates of the mean doses and dose rates at 100 meters based on the Wilson model is shown in Table 4.1.2-2.

Table 4.1.2-1. Grand Gulf POS 6 mean doses and dose rates at $100 \mathrm{~m}$ based on the Ramsdell building wake effect model.

\begin{tabular}{|c|c|c|c|c|c|}
\hline \multirow{2}{*}{$\begin{array}{c}\text { Accident } \\
\text { Progression Path } \\
\text { Number }\end{array}$} & \multirow{2}{*}{$\begin{array}{l}\text { Path Conditional } \\
\text { Probability }\end{array}$} & \multicolumn{4}{|c|}{ Consequence Measure } \\
\hline & & Total Dose (rem) & $\begin{array}{l}30 \text { minute Dose } \\
\text { (rem) }\end{array}$ & $\begin{array}{c}\text { Dose Rate First } \\
\text { Segment (rem/hr) }\end{array}$ & $\begin{array}{l}\text { Dose Rate Second } \\
\text { Segment (rem/hr) }\end{array}$ \\
\hline$\because y^{\prime} \quad \cdots$ & $\because$ & \multicolumn{4}{|c|}{ LOSP PDS } \\
\hline Path 1 & 0.10 & $4.23 \mathrm{E}+2$ & 8.80 & 17.6 & 0.0 \\
\hline Path 2 & 0.48 & $9.42 \mathrm{E}+3$ & $1.09 \mathrm{E}+3$ & $2.19 \mathrm{E}+3$ & 0.0 \\
\hline Path 3 & 0.08 & $9.42 \mathrm{E}+3$ & $1.09 \mathrm{E}+3$ & $2.19 \mathrm{E}+3$ & 0.0 \\
\hline Path 4 & 0.02 & $1.32 \mathrm{E}+5$ & $1.53 \mathrm{E}+4$ & $3.06 \mathrm{E}+4$ & 0.0 \\
\hline Path 5 & 0.09 & $1.73 E+5$ & $1.53 \mathrm{E}+4$ & $3.06 \mathrm{E}+4$ & $4.08 \mathrm{E}+3$ \\
\hline Path 6 & 0.003 & $1.04 \mathrm{E}+5$ & $1.21 \mathrm{E}+4$ & $2.43 E+4$ & 0.0 \\
\hline Path 7 & 0.01 & $1.55 E+5$ & $1.21 \mathrm{E}+4$ & $2.43 E+4$ & $5.10 \mathrm{E}+3$ \\
\hline Path 8 & 0.18 & $2.16 \mathrm{E}+5$ & $1.53 E+4$ & $3.06 \mathrm{E}+4$ & $8.44 E+3$ \\
\hline Path 9 & 0.03 & $2.10 \mathrm{E}+5$ & $1.21 \mathrm{E}+4$ & $2.43 \mathrm{E}+4$ & $1.05 E+4$ \\
\hline & & $\because \quad \because$ & & SPP:PDS & $\because$ \\
\hline Path 10 & 0.16 & $2.26 \mathrm{E}+5$ & $3.41 E+4$ & $6.83 E+4$ & $8.94 E+3$ \\
\hline Path 11 & 0.02 & $2.19 E+5$ & $2.68 E+4$ & $5.37 E+4$ & $1.12 \mathrm{E}+4$ \\
\hline Path 12 & 0.16 & $5.24 \mathrm{E}+5$ & $3.20 \mathrm{E}+5$ & $6.22 \mathrm{E}+6$ & $2.13 E+4$ \\
\hline Path 13 & 0.02 & $5.10 \mathrm{E}+5$ & $2.56 \mathrm{E}+5$ & $4.88 \mathrm{E}+6$ & $2.66 \mathrm{E}+4$ \\
\hline Path 14 & 0.37 & 0.899 & $5.25 \mathrm{E}-2$ & 0.105 & $4.50 \mathrm{E}-2$ \\
\hline Path 15 & 0.23 & $2.16 \mathrm{E}+5$ & $1.53 \mathrm{E}+4$ & $3.06 \mathrm{E}+4$ & $8.44 E+3$ \\
\hline Path 16 & 0.04 & $2.10 \mathrm{E}+5$ & $1.21 \mathrm{E}+4$ & $2.43 E+4$ & $1.05 \mathrm{E}+4$ \\
\hline
\end{tabular}


Table 4.1.2-2. Grand Gulf POS 6 mean doses and dose rates at $100 \mathrm{~m}$ based on the Wilson building wake effect model.

\begin{tabular}{|c|c|c|c|c|c|}
\hline \multirow{2}{*}{$\begin{array}{c}\text { Accident } \\
\text { Progression Path } \\
\text { Number }\end{array}$} & \multirow{2}{*}{$\begin{array}{l}\text { Path Conditional } \\
\text { Probability }\end{array}$} & \multicolumn{4}{|c|}{ Consequence Measure } \\
\hline & & Total Dose (rem) & $\begin{array}{l}30 \text { minute Dose } \\
\text { (rem) }\end{array}$ & $\begin{array}{l}\text { Dose Rate First } \\
\text { Segment }(\mathrm{rem} / \mathrm{hr})\end{array}$ & $\begin{array}{l}\text { Dose Rate Second } \\
\text { Segment (rem/hr) }\end{array}$ \\
\hline & & \multicolumn{4}{|c|}{ LOSP PDS } \\
\hline Path 1 & 0.10 & $9.48 \mathrm{E}+3$ & $1.97 E+2$ & $3.95 E+2$ & 0.0 \\
\hline Path 2 & 0.48 & $2.11 \mathrm{E}+5$ & $2.45 E+4$ & $4.91 \mathrm{E}+4$ & 0.0 \\
\hline Path 3 & 0.08 & $2.11 \mathrm{E}+5$ & $2.45 \mathrm{E}+4$ & $4.91 E+4$ & 0.0 \\
\hline Path 4 & 0.02 & $2.95 \mathrm{E}+6$ & $3.43 \mathrm{E}+5$ & $6.86 \mathrm{E}+5$ & 0.0 \\
\hline Path 5 & 0.09 & $3.56 \mathrm{E}+6$ & $3.43 \mathrm{E}+5$ & $6.86 \mathrm{E}+5$ & $9.14 \mathrm{E}+4$ \\
\hline Path 6 & 0.003 & $2.34 \mathrm{E}+6$ & $2.72 \mathrm{E}+5$ & $5.44 \mathrm{E}+5$ & 0.0 \\
\hline Path 7 & 0.01 & $3.48 \mathrm{E}+6$ & $2.72 \mathrm{E}+5$ & $5.44 \mathrm{E}+5$ & $1.14 \mathrm{E}+5$ \\
\hline Path 8 & 0.18 & $4.84 \mathrm{E}+6$ & $3.43 E+5$ & $6.86 \mathrm{E}+5$ & $1.89 \mathrm{E}+5$ \\
\hline \multirow[t]{2}{*}{ Path 9} & 0.03 & $4.70 \mathrm{E}+6$ & $2.72 E+5$ & $5.44 \mathrm{E}+5$ & $2.36 \mathrm{E}+5$ \\
\hline & . & \multicolumn{4}{|c|}{ nonLOSP PDS } \\
\hline Path 10 & 0.16 & $5.06 \mathrm{E}+6$ & $7.65 \mathrm{E}+5$ & $1.53 \mathrm{E}+6$ & $2.00 \mathrm{E}+5$ \\
\hline Path 11 & 0.02 & $4.91 E+6$ & $6.00 \mathrm{E}+5$ & $1.20 \mathrm{E}+6$ & $2.50 \mathrm{E}+5$ \\
\hline Path 12 & 0.16 & $1.17 \mathrm{E}+7$ & $7.16 \mathrm{E}+6$ & $1.39 E+8$ & $4.77 \mathrm{E}+5$ \\
\hline Path 13 & 0.02 & $1.14 \mathrm{E}+7$ & $5.72 \mathrm{E}+6$ & $1.09 \mathrm{E}+8$ & $5.96 \mathrm{E}+5$ \\
\hline Path 14 & 0.37 & 20.1 & 1.17 & 2.34 & 1.01 \\
\hline Path 15 & 0.23 & $4.84 \mathrm{E}+6$ & $3.43 \mathrm{E}+5$ & $6.86 E+5$ & $1.89 \mathrm{E}+5$ \\
\hline Path 16 & 0.04 & $4.70 \mathrm{E}+6$ & $2.72 \mathrm{E}+5$ & $5.44 \mathrm{E}+5$ & $2.36 \mathrm{E}+5$ \\
\hline
\end{tabular}

Figure 4.1.2-1 contains the $5^{\text {th }}, 50^{\text {th }}, 95^{\text {th }}$ percentile as well as the mean parking lot dose rates for the first release for both the Ramsdell and Wilson/Regulatory Guide models for distances of 10 - 500 meters from the reactor. A similar plot for the second release is shown in Figure 4.1.2-2. The uncertainty in both the building wake effect models and the source term is shown by the wide range of dose rates at each distance.

\subsection{Offsite Consequences}

The MACCS code $[5,6,7]$ was used to estimate the consequences to the general public. MACCS models the transport and dispersion of plumes of radioactive material released from the plant. As the plumes travel through the atmosphere, material is deposited on the ground. Several of the pathways through which the general population can be exposed are considered. Emergency 


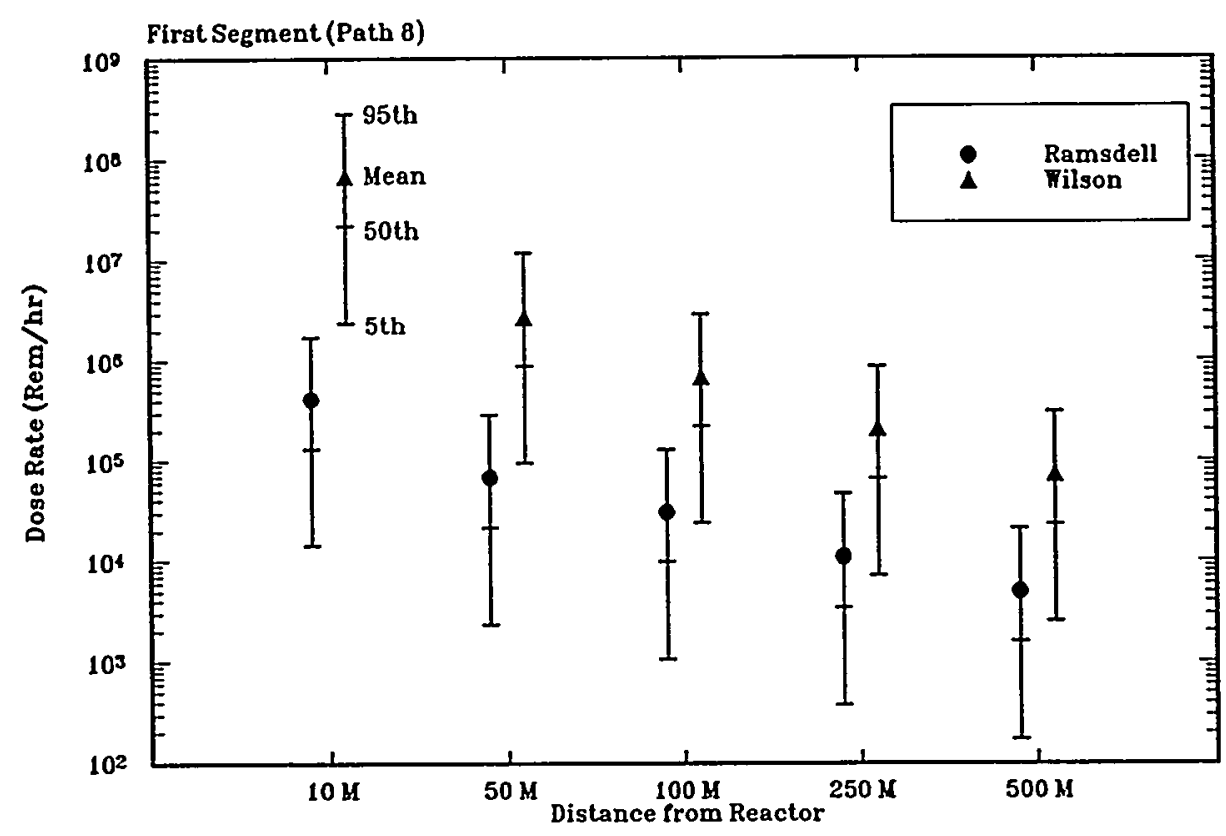

Figure 4.1.2-1. Parking Lot dose rates for Ramsdell and Wilson/Regulatory Guide models for distances from 10 - 500 meters from the reactor: First Release Segment

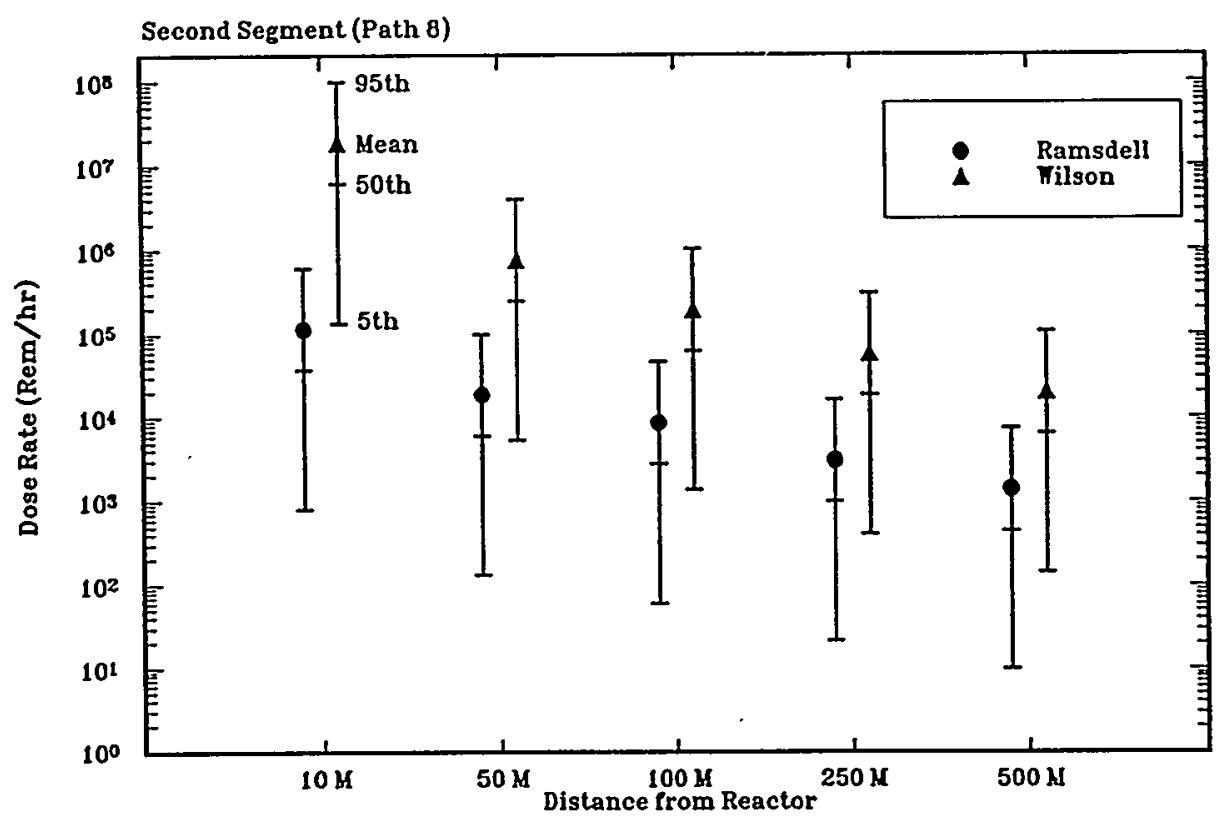

Figure 4.1.2-2. Parking Lot dose rates for Ramsdell and Wilson/Regulatory Guide models for distances from $10-500$ meters from the reactor: Second Release Segment 
response and protective action guides are also considered as means to mitigate the extent of the public exposure.

The input used in this study is identical to that used for Grand Gulf in the NUREG-1150 study [9] with the exception of the core inventory for which the inventory four days after shutdown was used and the source terms which resulted from GGLPSOR. The emergency response assumptions were not changed for this analysis.

Table 4.2-1 contains the estimated mean number of early fatalities, latent cancers, 50 mile population dose, and 1000 mile population dose for the sixteen paths through the APET along with the conditional probability of that path. The mean number of early fatalities ranged from 0 to $3.9 \times 10^{-2}$ while the mean number of latent cancers ranged from 0 to 1940 .

Table 4.2-1 Grand Gulf POS 6 Offsite Mean Consequences

\begin{tabular}{|c|c|c|c|c|c|}
\hline \multirow{2}{*}{$\begin{array}{c}\text { Accident } \\
\text { Progression } \\
\text { Path Number }\end{array}$} & \multirow{2}{*}{$\begin{array}{l}\text { Path } \\
\text { Conditional } \\
\text { Probability }\end{array}$} & \multicolumn{4}{|c|}{ Consequence Measure } \\
\hline & & $\begin{array}{c}\text { Early } \\
\text { Fatalities }\end{array}$ & $\begin{array}{l}\text { Total Latent } \\
\text { Cancers }\end{array}$ & $\begin{array}{l}50 \text { mile Population } \\
\text { Dose }^{1}\end{array}$ & $\begin{array}{l}1000 \text { Mile } \\
\text { Pop. Dose' }\end{array}$ \\
\hline & $\therefore$. & 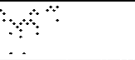 & LoSP PDS & & \\
\hline Path $1^{2}$ & 0.10 & NC & NC & NC & $\mathrm{NC}$ \\
\hline Path 2 & 0.48 & $1.3 \mathrm{E}-5$ & 102 & 77,000 & 591,000 \\
\hline Path 3 & 0.08 & $1.3 \mathrm{E}-5$ & 102 & 77,000 & 591,000 \\
\hline Path 4 & 0.02 & $4.8 \mathrm{E}-3$ & 684 & 330,000 & $4,010,000$ \\
\hline Path 5 & 0.09 & $4.8 \mathrm{E}-3$ & 984 & 496,000 & $5,800,000$ \\
\hline Path 6 & 0.003 & $4.0 \mathrm{E}-3$ & 588 & 293,000 & $3,450,000$ \\
\hline Path 7 & 0.01 & $4.0 \mathrm{E}-3$ & 940 & 479,000 & $5,560,000$ \\
\hline Path 8 & 0.18 & $5.2 \mathrm{E}-3$ & 1270 & 652,000 & $7,480,000$ \\
\hline \multirow[t]{2}{*}{ Path 9} & 0.03 & 4.7E-3 & 1260 & 662,000 & $7,460,000$ \\
\hline & \multicolumn{5}{|c|}{ nonLOSP PDS } \\
\hline Path 10 & 0.16 & $8.9 \mathrm{E}-3$ & 1190 & 624,000 & $7,090,000$ \\
\hline Path 11 & 0.02 & $9.3 \mathrm{E}-3$ & 1200 & 640,000 & $7,130,000$ \\
\hline Path 12 & 0.16 & $3.7 \mathrm{E}-2$ & 1920 & 939,000 & $11,300,000$ \\
\hline Path 13 & 0.02 & $3.9 \mathrm{E}-2$ & 1940 & 966,000 & $11,500,000$ \\
\hline Path $14^{2}$ & 0.37 & $\mathrm{NC}$ & $\mathrm{NC}$ & $\mathrm{NC}$ & $\mathrm{NC}$ \\
\hline Path 15 & 0.23 & $5.2 \mathrm{E}-3$ & 1270 & 652,000 & $7,480,000$ \\
\hline Path 16 & 0.04 & 4.7E-3 & 1260 & 662,000 & $7,460,000$ \\
\hline \multicolumn{6}{|l|}{$\begin{array}{l}\text { able Notes: } \\
\text { Dose is in Per }\end{array}$} \\
\hline
\end{tabular}




\subsection{INTEGRATED RESULTS CONDITIONAL ON CORE DAMAGE}

In the previous section the consequences associated with individual accident progression paths were presented. In this section the offsite consequences conditional on the occurrence of the LOSP PDS and the nonLOSP PDS are presented and are compared to full power PRA results extracted from the Grand Gulf analysis presented in NUREG-1150. Onsite consequences were not evaluated in NUREG-1150 and, therefore, an analogous comparison is not provided.

The consequences for a given PDS are calculated by taking a weighted average of the consequences for the individual paths. The weighted average is based on the conditional probability of each path. The PDS consequence is the sum of all of the "weighted" path consequences for the given PDS.

The offsite consequence distributions associated with the LOSP and nonLOSP PDSs are presented in Figure 5.1. Because a relatively small LHS sample was used in the evaluation of offsite consequences, the presentation of exact quantiles (i.e., $95^{\text {th }}$ ) is inappropriate. Instead of quantiles, the high, low, median, and mean values are presented in this figure. From this figure it can be seen that the consequences associated with the nonLOSP PDS tend to be higher than the consequences associated with the LOSP PDS. This stems from the assumption that injection cannot be recovered in the nonLOSP PDS and, therefore, all of these accidents proceed to full core damage and CCI. Although the probability that the containment is closed during this PDS is significant, the lack of a means to control the containment pressure results in a significant probability of early containment failure. Containment failure bypasses the auxiliary building and results in, essentially, an unmitigated release.

Also presented in Figure 5.1 are the conditional consequences from the Grand Gulf full power PRA. The full power results are for internal events and are "averaged" over all of the accidents analyzed in the study. In addition to the global consequences, the mean consequences associated with a selected full power accident are also presented (i.e., triangle on the full power distribution). This selected accident is a fast station blackout that progresses to full core damage. The containment is ruptured during core damage; the containment sprays are unavailable throughout the accident. Thus, this accident is similar to the accidents analyzed in this abridged study in that many of the mitigative features of the plant (i.e., the containment and sprays) are unavailable. In this full power accident, however, the in-vessel releases are typically scrubbed by the suppression pool. From Figure 5.1 it can be seen that the number of early fatalities associated with POS 6 are very similar to the number of early fatalities associated with full power accidents. This may seem somewhat surprising at first because the inventory of radionuclides important to early fatalities during POS 6 is less than the inventory at full power. However, this difference is compensated by the lack of mitigative features in POS 6. In POS 6 the inventory has been reduced by decay but because of the lack of mitigative features, a significant amount of the radionuclides are released to the environment. In the full power accidents, on the other hand, there is a large inventory, however, mitigative features of the plant limited the size of the release. In full power accidents, for example, a considerable fraction of these radionuclides are retained in the suppression pool. The net effect is that the number of 
early fatalities is roughly the same. The number of latent cancers associated with POS 6 accidents is greater than the number of latent cancers associated with full power accidents. The radionuclides that are important to latent health effects are long lived isotopes and, therefore, four days of decay will not have a significant impact on the radiological potential of the release to cause latent cancer fatalities. Thus, the magnitude of the release is the driving factor for latent cancer fatalities. Because in POS 6 the releases tend to be higher than the full power accidents, the number of latent cancers associated with POS 6 are greater than the number of latent cancers associated with full power accidents. The factors that influence latent cancers also affect the population dose. 

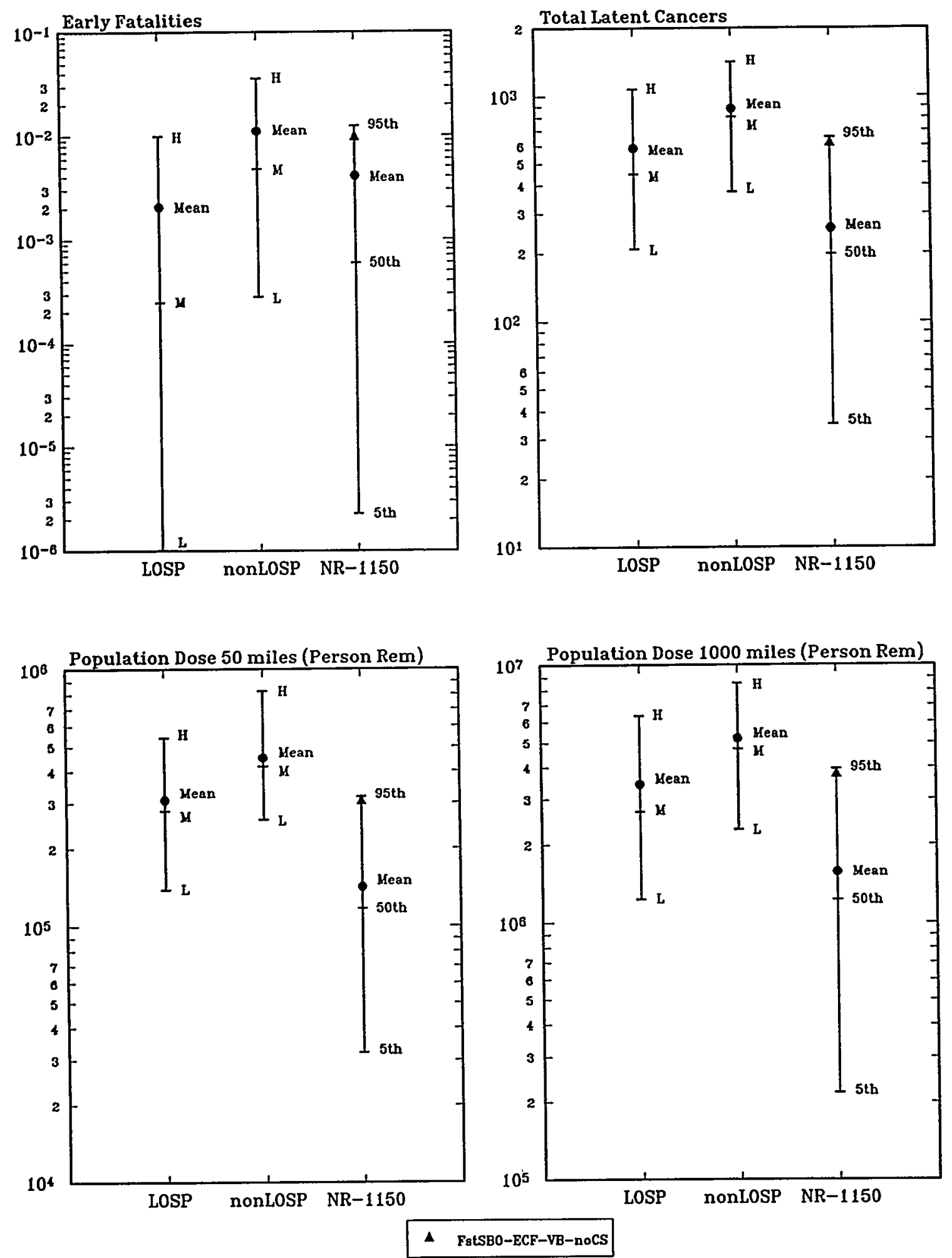

Figure 5-1. Grand Gulf POS 6 offsite consequences for LOSP and nonLOSP PDSs 


\subsection{INSIGHTS AND CONCLUSIONS}

The results and insights presented in this study are conditional on the occurrence of core damage. Thus, this study gives no indication about the likelihood of these postulated accidents, but rather what could be expected given that core damage does occur. The input to this analysis is the core damage sequence definitions from the Level 1 coarse screening analysis. In this Level 1 scoping analysis conservative assumptions were made with regard to the availability of certain systems and the performance of the plant operators. These assumptions provided the necessary simplifications such that the dominant sequences could be identified and still keep the scope of the study manageable. While the calculated frequencies from the Level 1 study are used to rank the sequences, the absolute values of these frequencies were not reported due to the conservative nature of many of the necessary simplifications. Thus, frequencies were not propagated through to the Level 2 and 3 analyses. It is within this framework that the abridged study was performed. Therefore, when interpreting these results it must be remembered that frequency information is not available to indicate the likelihood of accidents and simplifying assumptions were made in both the Level 1 and the Level $2 / 3$ studies.

The following is a list of insights obtained from this study:

o During POS 6 the majority of the mitigative features of the plant are bypassed or are unavailable. The vessel and drywell are open to the containment and, thus, the suppression pool is effectively bypassed. Furthermore, the containment spray system is unavailable during these accidents. Thus, steam and radionuclides are released directly into the containment atmosphere without being scrubbed by either the suppression pool or the containment sprays. For the accidents in which the containment hatch is removed, the only significant plant mitigative feature is the deposition that occurs in the auxiliary building. If the containment is closed but then fails during core damage, the auxiliary building is also bypassed.

o Because of the lack of mitigative features associated with these accidents, the source terms tend to be quite large.

o The consequences associated with these accidents are also significant. Offsite consequences are comparable with consequences associated with full power accidents. Onsite consequences are large.

o The time from the accident initiation to the onset of core damage is significant (i.e., from 18 to 28 hours). Thus, there is a considerable amount of time to restore core cooling and to close the containment. If offsite ac power is available, it is likely that the operators will close the containment prior to core damage.

o The pressure suppression features (i.e., suppression pool and containment sprays) of the Mark III design are bypassed during POS 6 . Since the ultimate pressure capacity of the containment is fairly low, the plant is vulnerable to pressurization events accompanying vessel failure and 
associated with hydrogen burns. Failure to avoid or mitigate pressure excursions from these events could result in early containment failure.

o Because of the large recovery potential associated with these accidents (i.e., which were not fully accounted for in either the Level 1 analysis or this abridged analysis because of simplifying assumptions), POS 6 offsite risk could be significantly lower than the risk associated with full power accidents.

o Recovered accidents can pose a significant threat to onsite personnel.

o Because of the lack of mitigation features associated with accidents initiated in this POS, the auxiliary building and the SBGTs could play a significant role in the mitigation of the release, especially for recovered accidents.

There were many issues that were identified in this study that could affect the possible accident progressions and consequences. The resolution of many of these issues was beyond the scope of this abridged analysis and will have to be addressed in any more detailed analysis that is performed in the future. The following is a list of potentially significant issues:

o Containment Closure. The effects that the temperature, humidity, and radiation have on the plant personnel's ability to close the containment needs to be addressed in more detail. Containment closure is a critical issue that will affect the consequences associated with these accidents.

o Containment Loading. Hydrogen combustion phenomena associated with this plant configuration need to be investigated. In this plant configuration steam and hot hydrogen are released directly into the containment atmosphere. The amount of steam blanketing and air ingression and the availability of ignition sources will all affect the likelihood and magnitude of hydrogen burns. The effectiveness of the hydrogen ignition system in this plant configuration also needs to be investigated. The loading from in-vessel steam explosions is another issue that needs to be addressed. With the vessel head off in this POS and the relatively low failure pressure of this containment, in-vessel steam explosions could be a significant mechanism for early containment failure.

o Source Term. There are several events that can enhance the source term that were not included in the PRA model. First, the role that air ingression plays during core damage needs to be investigated. If significant air ingression does occur, the in-vessel phase of the core damage process could be significantly altered and the release of certain radionuclides enhanced. Second, the relocation of intact fuel from an in-vessel steam explosion could also result in the enhancement of an early source term. This issue was not addressed in this analysis. Third, for recovered accidents the embrittlement and failure of the clad could lead to a release earlier than what is currently modeled. This could be particularly important for onsite consequences. 
o Auxiliary Building. For accidents in which the containment is open during core damage, the auxiliary building could play a major role in mitigating the release. The radionuclide retention capabilities of this building need to be assessed in more detail than what was done in this abridged analysis. Furthermore, the effectiveness of the SBGT system to mitigate the release, especially for recovered accidents, also needs to be assessed.

o Onsite Consequences. Only a scoping type analysis of onsite consequences was performed in this study. In the calculation of doses in the building, the integrated concentrations were based on average concentrations from GGLPSOR and on crude estimates of the residence times in the buildings. More detailed information on the concentration as a function of time and on the residence time would produce more realistic dose estimates. 


\subsection{REFERENCES}

1. U.S. Nuclear Regulatory Commission, "Severe Accident Risks: An Assessment for Five U.S. Nuclear Power Plants," NUREG-1150, Vols. 1-3, December 1990 -January 1991.

2. R. M. Summers, et al., "MELCOR 1.8.0: A Computer Code for Nuclear Reactor Severe Accident Source Term and Risk Assessment Analyses," NUREG/CR-5531, SAND90-0364, Sandia National Laboratories, January 1991.

3. R. L. Iman, "A FORTRAN 77 Program and User's Guide for the Generation of Latin Hypercube and Random Samples for Use with Computer Models, "NUREG/CR-3624, SAND83-2365, Sandia National Laboratories, 1984.

4. P. Cybulskis, "Assessment of the XSOR Codes," NUREG/CR-5346, BMI-2171, Battelle Columbus Division, November 1989.

5. D. I. Chanin, et al., "MELCOR Accident Analysis Consequence Code System," NUREG/CR-4691, SAND86-1562, Sandia National Laboratories, Vol 1, February 1990.

6. H-N Jow, et al., "MELCOR Accident Analysis Consequence Code System," NUREG/CR-4691, SAND86-1562, Sandia National Laboratories, Vol 2, February 1990.

7. J. A. Rollstin, et al., "MELCOR Accident Analysis Consequence Code System," NUREG/CR-4691, SAND86-1562, Sandia National Laboratories, Vol 3, February 1990.

8. D. W. Whitehead, et al., "BWR Low Power and Shutdown Accident Frequencies Program: Phase 1 - Coarse Screening Analysis," Vols. 1-3, Draft Letter Report, October 1991 - November 1991. Available in the NRC Public Document Room, 2120 L Street, NW.

9. T. D. Brown, et al., "Evaluation of Severe Accident Risks: Grand Gulf Unit 1," NUREG/CR-4551, SAND86-1309, Vol. 6, Rev. 1, Sandia National Laboratories, December 1990.

10. A. G. Croff, et al., "ORIGEN2: Isotope Generation and Depletion Code, " RSIC, Oak Ridge National Laboratory, Oak Ridge, TN, CCC-371, September 1989.

11. J. V. Ramsdell Jr., "Diffusion in Building Wakes for Ground-Level Releases," Atmospheric Environment, Vol. 24B, No. 3, pp 377-388, 1990. 
12. Wilson in "Atmospheric Science and Power Production," Ed. Randerson, D., DOE/TIC-27601, 299 (1984).

13. U. S. Nuclear Regulatory Commission, Regulatory Guide 1.145, Revision 1, November 1982. 


\section{Distribution}

Kiyoharu Abe

Dept. of Reactor Safety Research Nuclear Safety Research Center

Tokai Research Establishment

JAERI

Tokai-mura, Naga-gun

Ibaraki-ken,

JAPAN

Sarbes Acharya

Department of Energy

NS-1/FORS

Washington, DC 20585

Dr. Ulvi Adalioglu

Cekmece Nukleer Arastraima ve

Egitim Merekezi

P.K. 1

Havaalani/ISTANBUL

TURKEY

Dr. Eng. Kiyoto Aizawa

Senior Engineer

Reactor Eng. Dev. Department

PNC

9-13, Chome, Akasaka

Minato-K, Tokyo

JAPAN

Harry Alter

Manager Applied Tech

Nuclear Systems Tech

NE-46

US DOE

Washington, DC 20585

R.M. Andrews

Nuclear Installations Insp.

St. Peters House

Balliol Raod, Bootle

Merseyside L20 31Z

UNITED KINGDOM
George Apostolakis

UCLA

Boelter Hall, Room 5532

Los Angeles, CA 90024-1597

Director of Reactor Engineering

Argonne National Laboratory

$9700 \mathrm{~S}$ Cass Ave

Bldg 208

Argonne, IL 60439

Ephraim Asculai

Division of Nuclear Safety

Wagramestrasse, 5

P.O.-Box 100

A-1400 Wien

AUSTRIA

Vladimar Asmolov

Head, Nuclear Safety Department

I. V. Kurchatov Institute of Atomic Enegry

Moscow, 123181

RUSSIA

J. de Assuncao

Cabinete de Proteccao è Seguranca Nuclear

Ministerio da Indusstria

Ave. de Republica 45-6

1000 Lisbon

PORTUGAL

H.P. Balfanz, Head

Institute of Probabilistic

Safety Analysis

TUV Nord

Grosse Bahnstrasse 31

D-22525 Hamburg 54

GERMANY 
Pat Baranowsky

USNRC-AEOD/TPAB

MS: T-4A9

Robert A. Bari, Deputy Chairman

Dept of Nuclear Energy

Bldg 197C

Brookhaven National Laboratory

Upton, NY 11973

Librarian

Technical Information Section

Battelle Pacific Northwest Lab

P. O. Box 999

Richland, WA 99352

Dr. John Baum

Dept of Nuclear Energy

Radiological Sciences Div

Bldg $703 \mathrm{M}$

Brookhaven National Laboratory

Upton, NY 11973

Eric Beckjord

USNRC-RES/DO

MS: T-10F12

Robert Bernero

USNRC-NMSS/DO

MS: T-8A23

Andrea Besi

Institute for Systems Engineering and Informatics

CEC Joint Research Centre

CP N 1

1-21020 Ispra (Varese)

ITALY

John Bickel

Idaho National Engineering Lab.

EG\&G MS: 3850

P.O. Box 1625

Idaho Falls, ID 83415
Vicki Bier

Dept. of Industrial Engineering

University of Wisconsin-Madison

1513 University Avenue, Room 389

Wisconsin, WI 53706

Scott Bigelow

S-CUBED

2501 Yale SE, Suite 300

Albuquerque, NM 87106

Prof. Dr. Dr.-Ing. E. H. Adolf

Birkhofer

Gesellschaft für Anlagen und

Reaktorsicherheit (GRS) $\mathrm{mbH}$

Forschungsgelände

D-8046 Garching

Federal Republic of Germany

David Black

American Electric Power

1 Riverside Plaza

Columbus, $\mathrm{OH} 43215$

Harold Blackman

Idaho National Engineering Lab.

EG\&G MS: 3850

P.O. Box 1625

Idaho Falls, ID 83415-3850

Dennis Bley

Buttonwood Consulting

17291 Buttonwood St.

Fountain Valley, CA 92708

Roger Blond

Booz-Allen \& Hamilton

4330 East West Highway

Bethesda, MD 20814 
Dr. Mario Bonaca

Manager, Reactor Engineering

Northeast Utilities

P.O. Box 270

Hartford, Conn. 06141

Robert B. Borsum

Nuclear Power Division

B \& W Nuclear Tech

1700 Rockville Pike

Suite 525

Rockville, MD 20852

Stephen Boult

Electrowatt Engineering Services

(UK) Ltd.

Grandford House

16 Carfax, Horsham

West. Sussex RH12 IUP

ENGLAND

Gary Boyd

Safety \& Reliability Optimization

Services

9724 Kingston Pike, Suite 102

Knoxville, TN 37922

Brookhaven National Laboratory (2)

Attn: Lev Neymotin

Arthur Tingle

Building 130

Upton, NY 11973

David M. Brown

Paul C. Rizzo Associates, Inc.

300 Oxford Drive

Monroeville, PA 15146-2347

Tom D. Brown

Sandia National Laboratories

Dept. 6413

P.O. Box 5800

Albuquerque, NM 87185
Robert J. Budnitz

Future Resources Associates, Inc. 2039 Shattuck Avenue, Suite 402

Berkeley, CA 94704

Gary Burdick

USNRC-RES/SAIB

MS: T-10F13

Arthur Buslik

USNRC-RES/PRAB

MS: T-9F31

Edward Butcher

USNRC-NRR/SPSB

MS: O-10E4

Technical Library

B\&W Nuclear Service Co

P. O. Box 10935

Lynchburg, VA 24506

Stefaan Caeymaex

Safety \& Systems Section

Nuclear Generation Dept.

TRACTEBEL

Avenue Ariane 7

B-1200 Bruxelles

BELGIUM

Leonard Callan, Administrator

U.S. Nuclear Regulatory Commission

Harris Tower and Pavilion

611 Ryan Plaza Drive, Suite 400

Arlington, TX 76011-8064

J. Calvo

Division of PSA \& Human Factors

Consejo de Seguridad Nuclear

Calle Justo Dorado, 11

28040 Madrid

SPAIN 
John Forbes Campbel

HM Superintending Inspector

Health \& Safety Executive

St. Peter's House

Balliol Road

Bootle L20 31Z

UNITED KINGDOM

Leonel Canelas

New University of Lisbon

Quinta de Torre

2825 Monte de Caparica

PORTUGAL

Harold Careway

General Electric Co., M/C 754

175 Curtner Ave.

San Jose, CA 95129

Jose E. De Carlos

CSN International Coordinator

Consejo de Seguridad Nuclear

Calle Justo Dorado 11

28040 Madrid

SPAIN

Annick Carnino

International Atomic Energy Agency

Wagramerstrasse 5, P.O. Box 100

A-1400 Vienna

AUSTRIA

S. Chakraborty

Swiss Federal Nuclear Safety Inspectorate

Hauptabteilung für die Sicherheit der Kernanlagen

CH-5232 Villigen-HSK

SWITZERLAND

Erulappa Chelliah

USNRC-RES/PRAB

MS: T-9F31
Mike Cheok

NUS

910 Clopper Road

Gaithersburg, MD 20878

Nilesh Chokshi

USNRC-RES/SSEB

MS: T-10L1

T. L. Chu

Brookhaven National Laboratory

Department of Nuclear Energy

Bldg. 130

Upton, NY 11973

Peter Cooper

SRD/AEA Technology

Wigshaw Lane

Culcheth

Cheshire WA3 4NE

England

Susan E. Cooper

Science Applications Int'1. Corp.

11251 Roger Bacon Drive

Reston, VA 22090

Michael Corradini

University of Wisconsin

1500 Johnson Drive

Madison, WI 53706

E. R. Corran

ANSTO Reasearch Establishment

Lucas Heights Reserch Labs.

Private Mail Bag 1

Manai, NSW 2234

AUSTRALIA

Massimo Cozzone

A.N.P.A.

Via V. Brancati, 48

I-00144 Rome

ITALY

Dist-4 
George Crane

1570 E. Hobble Creek Dr.

Springville, Utah 84663

Mark Cunningham

USNRC-RES/PRAB

MS: T-9F31

S. Daggupaty

Environment Canada

4905 Dufferin Street

Downsview

Ontario, M3H ST4

CANADA

Louise Dahlerup

Inspectorate of Nuclear Inst.

Danish Civil Defense \&

Emergency Planning Agency

16, Datavej

DK-3460 Birkerod

DENMARK

John Darby

SEA, Inc.

6100 Uptown Blvd. NE

Albuquerque, NM 87110

Gerald Davidson

Fauske and Associates, Inc. $16 \mathrm{~W} 070$ West 83rd Street

Burr Ridge, IL 60521

Peter Davis

PRD Consulting

P.O. Box 2046

Sheridan, WY 82801
P. De Gelder

Secretary, BELGIAN NUCLEAR

SOCIETY (BNS)

A V Nuclear

Avenue du Roi 157

B-1060 Brussels

BELGIUM

Lennart Devell

Studsvik Nuclear

Studsvik Energiteknik AB

S-611 82 Nykoping

SWEDEN

J. Devooght

Service de la Metrologie Nucl

University Libre de Bruxelles

Faculte des Sciees Appliqu.

50 Avenue F-D Roosevelt

Bruxelles 5

BELGIUM

G. Diederick

Commonwealth Edison Co.

LaSalle County Station

RR1, Box 220

2601 North 21st Rd.

Marsielles, IL 61341

Chuck Dobbe

Idaho National Engineering Lab.

EG\&G MS: 3840

P.O. Box 1625

Idaho Falls, ID 83415

Mary Drouin

USNRC-RESISAIB

MS: T-10F13

Duke Power Co. (2)

Attn: Duncan Brewer

Steve Deskevich

422 South Church Street

Charlotte, NC 28242 
Bill Eakin

Northeast Utilities

Box 270

Hartford, CT 06141

Stewart D. Ebneter

USNRC

101 Marietta St., Suite 2900

Atlanta, GA 30323-0199

Adel A. El-Bassioni

USNRC-NRR/PRAB

MS: O-10E4

ENEA/DISP (2)

Attn: Alvaro Valeri Alfredo Bottino

Via Vitaliano Brancati, 48

00144 Roma EUR

ITALY

Walter P. Engel

PRAG MGR Analysis \& Reg Matter

NE-60

CRYCITY

US DOE

Washington, DC 20585

John Flack

USNRC-RES/SAIB

MS: T-10F13

Karl Fleming

Pickard, Lowe \& Garrick

2260 University Drive

Newport Beach, CA 92660

Terry Foppe

Safety Analysis Engineering

Rocky Flats Plant

Energy Systems Group

Rockwell International Corp

P.O. Box 464

Golden, CO 80401
R H. Gauger

Manager-Reliability Engr

A/E Div

Holmes \& Narver Inc.

R Roanne Circle

Irvine, CA 92714

Robert Gobel

Clark University

Center for Technology, Environment and Development

950 Main St.

Worcester, MA 01610-1477

Paul Govaerts

Studiecentrum voor Kernenergie

(SCK/CEN)

Boeretang, 200

B-2400 Mol

BELGIUM

Mr. Gubler

International Atomic Energy Agency

NENS/SAD BO842

Wagramerstrasse 5, P.O. Box 100

A-1140 Vienna

AUSTRIA

Paul M. Haas, President

Concord Associates, Inc.

725 Pellissippi Parkway

Suite 101, Box 6

Knoxville, TN 37933

Dr. U. Hauptmanns

Gesellschaft Für Anlagen und

Reaktorsicherheit (GRS) mgH

Schwertnergasse 1

D-5000 Köln 1

GERMANY 
Sharif Heger

UNM Chemical and Nuclear

Engineering Department

Farris Engineering, Room 209

Albuquerque, NM 87131

Jon C. Helton

Dept. of Mathematics

Arizona State University

Tempa, AZ 85287

Dr. P. M. Herttrich

Gesellschaft für Anlagen und

Reaktorsicherheit (GRS) $\mathrm{mbH}$

Schwertnergasse 1

5000 Köln 1

GERMANY

Dr. D.J. Higson

Radiological Safety Bureau

Australian Nuclear Science \&

Technology Organisation

P.O. Box 153

Roseberry, NSW 2018

AUSTRALIA

Dr. Mitsumasa Hirano

Deputy General Manager

Institute of Nuclear Safety

NUPEC

3-6-2, Toranomon, Minato-ku

Tokyo 108

JAPAN

Dr. S. Hirschberg

Paul Scherrer Institute

Vurenlingen and Villigen

CH-5232 Villigen PSI

SWITZERLAND

Steven Hodge

Oak Ridge National Laboratories

P. O. Box Y

Oak Ridge, TN 37831
Gary Holahan

USNRC-AEOD/ÖSP

MS: T-4A9

N.J. Holloway

A72.1

Atomic Weapons Establishment

Ademaston

Reading RG7 4PR

UNITED KINGDOM

Griff Holmes

Westinghouse Electric Co.

Energy Center East

Bldg. 371

P.O. Box 355

Pittsburgh, PA 15230

William Hopkins

Bechtel Power Corporation

15740 Shady Grove Road

Gaithersburg, MD 20877

Dean Houston

USNRC-ACRS

MS: P-315

Der-Yu Hsia

Institute of Nuclear Energy Research

Lung-Tan 325

TAIWAN

Alejandro Huerta-Bahena

National Commission on Nuclear

Safety and Safeguards (CNSNS)

Insurgentes Sur N. 1776

C. P. 04230 Mexico, D. F.

MEXICO

Peter Humphreys

US Atomic Energy Authority

Wigshaw Lane, Culcheth

Warrington, Cheshire

UNITED KINGDOM, WA3 4NE

Dist-7 
W. Huntington

Commonwealth Edison Co.

LaSalle County Station

RR1, Box 220

2601 North 21st Rd.

Marsielles, IL 61341

J.S. Hyslop

USNRC-RES/PRAB

MS: T-9F31

Idaho National Engineering Lab. (2)

Attn: Doug Brownson

Darrel Knudson

EG\&G MS: 3840

P.O. Box 1625

Idaho Falls, ID 83415

Idaho National Engineering Lab. (2)

Attn: Art Rood

Mike Abbott

EG\&G MS: 2110

P.O. Box 1625

Idaho Falls, ID 83415

Hanspeter Isaak

Abteilung Strahlenschutz

Hauptabteilung für die Sicherheit der Kernanlagen (HSK)

CH-5303 Wurenlingen

SWITZERLAND

Brian Ives

UNC Nuclear Industries

P. O. Box 490

Richland, WA 99352

Kamiar Jamili

DP-62/FTN

Department of Energy

Washington, D.C. 20585
Robert Jones

USNRC-NRR/DSSA

MS: O-8E1

Edward Jordan

USNRC-AEOD/DO

MS: T-4D18

Dr. H. Kalfsbeek

$\mathrm{DG} / \mathrm{XII} / \mathrm{D} / 1$

Commission of the European Communities

Rue de la Loi, 200

B-1049 Brussels

BELGIUM

Yoshio Kano

General Mngr. \& Sr. Engineer

Systems Analysis Section

O-arai Engineer. Centr, PNC

Higashi-Ibaraki-gun

Ibaraki-Ken, 133-13

JAPAN

William Kastenberg

UCLA

Boelter Hall, Room 5532

Los Angeles, CA 90024

Barry Kaufer

OECD/NEA

"Le Seine St. Germain" 12

Boulevard des Iles

92130 Issy-les-Moulineaux

FRANCE

Paul Kayser

Division de la Radioprotection

Avenue des Archiducs, 1

L-1135 Luxembourg-Belair

LUXEMBOURG

Dist-8 
Ken Keith

TVA

W 20 D 201

400 West Surmnit Hill

Knoxville, TN 37092

G. Neale Kelly

Commission of the European

Communities

Joint Research Centre

Rue de la Loi 200

B-1049 Brussels

BELGIUM

Knolls Atomic Power Laboratory (2)

Attn: Ken McDonough

Dominic Sciaudone

Box 1072

Schenectady, NY 12301

Dr. K. Koberlein

Gesellschaft für

Reaktorsicherheit $\mathrm{mbH}$

Forschungsgelände

D-8046 Garching

GERMANY

Alan Kolaczkowski

Science Applications International

Corporation

2109 Air Park Rd. S. E.

Albuquerque, NM 87106

Jim Kolanowski

Commonwealth Edison Co.

35 1st National West

Chicago, IL 60690
John G. Kollas

Institute of Nuclear Technology and

Radiation Protection

N.R.C.P.S. "Demokritos"

P.O. Box 60228

GR-153 10 Aghia Paraskevi

Attiki

GREECE

S. Kondo

Department of Nuclear Engineering

Facility of Engineering

University of Tokyo

3-1, Hongo 7, Bunkyo-ku

Tokyo

JAPAN

D. Lamy

CEN/SCK

Dept. Scientific Irradiation

Experiment \& Study BR2

Boeretang, 200

B-2400 Mol

BELGIUM

Dr. J.M. Lanore

CEA/IPSN/DAS

Centre d'Etudes Nucléaires de

Fontenay-aux-Roses

B.P. $n^{\circ} 6$

92265 Fontenay-aux-Roses CEDEX

FRANCE

Jose A. Lantaron

Consejo de Seguridad Nuclear

Sub. Analisis y Evaluaciones

Calle Justo Dorado, 11

28040 Madrid

SPAIN

Dist-9 
Josette Larchier-Boulanger

Electricte de France

Direction des Etudes Et Recherches

30, Rue de Conde

75006 Paris

FRANCE

H. Larsen

Head of Department

Riso National Laboratory

P.O. Box 49

DK-4000 Roskilde

DENMARK

Lawrence Livermore Nat'l Lab. (4)

Attn: George Greenly

Marvin Dickerson

Rolf Lange

Sandra Brereton

Livermore, CA 94550

Shengdar Lee

Yankee Atomic Electric Company

580 Main St.

Boston, MA 17407

B.T.F. Liwaang

Dept. of Plant Safety Assessment

Swedish Nuclear Power Inspec.

P.O. Box 27106

S-10252 Stockholm

SWEDEN

Peter Lohnberg

Expresswork International, Inc.

1740 Technology Drive

San Jose, CA 95110

Steven M. Long

USNRC-NRR/SPSB

MS: O-10E4
D. Eugenio Gil Lopez

Consejo de Seguridad Nuclear

Calle Justo Dorado, 11

28040 Madrid

SPAIN

Los Alamos National Laboratory (2)

Attn: Kent Sasser

N-6, K-557

Los Alamos, NM 87545

Christiana H. Lui

USNRC-RES/PRAB

MS: T-9F31

John Luke

Florida Power \& Light

P.O. Box 14000

Juno Beach, FL 33408

Daniel Manesse

ISPN

Boite Postale $n^{\circ} 6$

92265 Fontenay-aux-Roses CEDEX

FRANCE

Fred Mann

Westinghouse Hanford Co.

WIA-53

P.O. Box 1970

Richland, WA 99352

Nadia Soido Falcao Martins

Comissao Nacional de Energia Nuclear

R General Severianao 90 S/408-1

Rio de Janeiro

BRAZIL

Harry F. Martz

Analysis and Assessment Division

Los Alamos National Laboratory

Los Alamos, NM 87545 
Herbert Massin

Commonwealth Edison Co.

35 1st National West

Chicago, IL 60690

Hideo Matsuzuru

Tokai Research Establishment

Tokai-mur

Maka-gun

Ibaraki-ken, 319-11

JAPAN

Jim Mayberry

Ebasco Services

60 Chubb Ave.

Lyndhurst, NJ 07071

Andrew S. McClymont

IT-Delian Corporation

1340 Saratoga-Sunnyvale Rd.

Suite 206

San Jose, CA 95129

Michael McKay

Los Alamos National Laboratory

A-1, MS F600 Services

P.0. Box 1663

Los Alamos, NM87545

Zen Mendoza

SAIC

5150 El Camino Real

Suite C3 1

Los Altos, CA 94022

Dr. J. Mertens

Division of Risk Analysis \&

Reactor Technology

Institute of Safety Research

Research Centre Julich (KFA)

D-52425 Julich

GERMANY
Jim Meyer

Scientech

11821 Parklawn Dr.

Suite 100

Rockville, MD 20852

Joe Minarick

Science Applications Int'l Corp.

301 Laboratory Road

P.O. Box 2501

Oak Ridge, TN 37830

Jose I. Calvo Molins, Head

Division of P.S.A. and Human Factors

Consejo de Seguridad Nuclear

Calle Justo Dorado, 11

28040 Madrid

SPAIN

Ken Muramatsu

Risk Analysis Laboratory

Japan Atomic Energy Research Institute

Tokai-mura, Naka-gun

Ibaraki-ken, 319-11, Tokyo

JAPAN

Joseph A. Murphy

Division of Safety Issue Resolution

U.S. Nuclear Regulatory Commission

MS: T-10E50

Washington, DC 20555

Kenneth G. Murphy, Jr.

US Department of Energy

19901 Germantown Rd.

Germantown, MD 20545

Shankaran Nair

Central Electricity

Generating Board

Berkeley Nuclear Laboratories

Berkeley

Gloucestrshire CL13 9PB

UNITED KINGDOM

Dist-11 
Ray $\mathrm{Ng}$

NEI

1776 Eye St. N

Suite 300

Washington, DC 20006-2496

G. Niederauer

Los Alamos National Laboratory

P. O. Box 1663

MSK 575

Los Alamos, NM 87545

Oak Ridge National Laboratory (2)

Attn: Steve Fisher

Sherrel Greene

MS-8057

P.O. Box 2009

Oak Ridge, TN 37831

Ken O'Brien

University of Wisconsin

Nuclear Engineering Dept.

153 Engineering Research Blvd.

Madison, WI 53706

Theresa Oh

INEL Tech Library

EG\&G MS: 2300

P. O. Box 1625

Idaho Falls, ID 83415-2300

Robert Ostmeyer

U.S. Department of Energy

Rocky Flats Area Office

P. O. Box 928

Golden, CO 80402

Robert Palla

USNRC-NRR/SPSB

MS: O-10E4
Gareth Parry

NUS Corporation

910 Clopper Rd.

Gaithersburg, MD 20878

Vern Peterson

Building T886B

EG\&G Rocky Flats

P.O. Box 464

Golden, CO 80402

G. Petrangeli

ENEA Nuclear Energy ALT Disp

Via V. Brancati, 48

00144 Rome

ITALY

Ing. Jose Antonio Becerra Perez

Comision Nacional De Seguridad

Nuclear Y Salvaguardias

Insurgentes Sur 1806

01030 Mexico, D. F.

MEXICO

William T. Pratt

Brookhaven National Laboratory

Building 130

Upton, NY 11973

Urho Pulkkinen

Technical Research Centre of

Finland

Laboratory of Electrical \&

Automation Engineeering

Otakaari 7B, 02150 Espoo 15

FINLAND

Blake Putney

Science Applications

International Corporation

5150 El Camino Real, Suite C31

Los Altos, CA 94022 
Dr. V. M. Raina

Project Manager-Risk Assessment

Ontario Hydro H11 G1

700 University Ave.

Toronto, Ontario M5G 1X6

CANADA

William Raisin

NEI

1726 M. St. NW

Suite 904

Washington, DC 20036

Ann Ramey-Smith

USNRC-RES/PRAB

MS: T-9F31

Dale Rasmuson

USNRC-AEOD/TPAB

MS: T-4A9

John Ridgely

USNRC-RES/SAIB

MS: T-10F13

Richard Robinșon (2)

USNRC-RES/PRAB

MS: T-9F31

M. Roch

Manager of Design, Nuclear

Department

TRACTEBEL

Avenue Ariane 7

B-1200 Bruxelles

BELGIUM

A.E. Rogers

General Electric Co

175 Curtner Ave

MC-489

San Jose, CA 95125
Judy Rollstin

GRAM Inc

8500 Menual Blvd. NE

Albuquerque, NM 87112

Marc Rothschild

Halliburton NUS

1303 S. Central Ave.

Suite 202

Kent, WA 98032

Christopher Ryder

USNRC-RES/PRAB

MS: T-9F31

Takashi Sato, Deputy Manager

Nu'clear Safety Engineering Section

Reactor Design Engineering Dept.

Nuclear Energy Group, Toshiba Corp.

Isogo Engineering Center

8, Shinsugita-cho, Isogo-ku,

Yokohama 235, JAPAN

Martin Sattison

Idaho National Engineering Lab.

P. O. Box 1625

Idaho Falls, ID 83415

Dr. U. Schmocker

Hauptabteilung für die

Sicherheit der Kernanlagen

CH-5232 Villigen HSK

SWITZERLAND

A. J. Seẹbregts

ECN Nuclear Energy

Westerduinweg, 3

Postbus 1

NL-1755 Petten ZG

THE NETHERLANDS 
Dr. S. Serra

Ente Naxionale per I'Energia

Electtrica (ENEL)

via G.B. Martini 3

I-00198 Rome

ITALY

\section{H. Shapiro}

Licensing \& Risk Branch

Atomic Energy of Canada Ltd.

Sheridan Park Research Comm.

Mississauga, Ontario L5K 1B2

CANADA

Nathan O. Siu

Center for Reliability and Risk Assessment

Idaho National Engineering Lab.

EG\&G MS: 3850

P.O. Box 1625

Idaho Falls, ID 83415-3855

E. Soederman

ES-Konsult AB

Energy and Safety

P.O. Box 3096

S-16103 Bromma

SWEDEN

Desmond Stack

Los Alamos National Laboratory

Group Q-6, Mail Stop K556

Los Alamos, NM 87545

Jao Van de Steen

KEMA Laboratories

Utrechtseweg, 310

Postbus 9035

NL 800 ET Arnhem

THE NETHERLANDS
Eli Stern

Israel AEC Licensing Div.

P.O. Box 7061

Tel-Aviv 61070

ISRAEL

Dr. Egil Stokke

Advisory Group

OECD Halden Reactor Project

P.O. Box 173

N-1751 Halden

NORWAY

Stone \& Webster Engineering Corp

Technical Information Center

A. Hosford

245 Summer Street

245/01

Boston, MA 02210

Dennis Strenge

Pacific Northwest Laboratory

RTO/ 125

P.O. Box 999

Richland, WA 99352

Technadyne Engineer. Consultants (3)

Attn: David Chanin Jeffery Foster Walt Murfin

Suite A225

8500 Menual Blvd. N

Albuquerque, NM 87112

Ashok Thadani

USNRC-NRR/ADT

MS: O-12G18

T. G. Theofanous

University of California, S. B.

Department of Chemical and Nuclear

Engineering

Santa Barbara, CA 93106 
Catherine Thompson

USNRC-RES/SAIB

MS: T-10F13

Soren Thykier-Nielsen

Riso National Laboratory

Postbox 49

DK4000 Roskile

DENMARK

R. Toossi

Physical Research, Inc.

25500 Hawthorn Blvd.

Torrance, CA 90505

Ennio Traine

ENEL

Via Vialiano, 48

00144 Rome

ITALY

Ulf Tveten

Environmental Physics Section

Institutt for Energiteknikk

Postboks 40

N-2007 Kjeller

NORWAY

US Department of Energy

Energy Library

Room G 034/GTN

AD-622.1

Washington, DC 20585

US Department of Energy

NS-50 (GTN)

NS-10.1

S-161

Washington, DC 20585
U.S. Environmental

Protection Agency (2)

Attn: Allen Richardson

Joe Logsdon

Office of Radiation Programs

Environmental Analysis Division

Washington, DC 20460

Harold VanderMolen

USNRC-RES/PRAB

MS: T-9F31

Dr. A. Valeri

A.N.P.A.

Via Vitaliano Brancati, 48

I-00144 Rome

ITALY

Magiel F. Versteeg

Ministry of Social Affairs

and Employment

P.O. Box 90804

2509 LV Den Haag

THE NETHERLANDS

Martin Virgilio

USNRC-NRR/DSSA

MS: O-8E2

R. Virolainen, (Chairman PWG5)

Systems Integ. Off. (STUK)

P.O. Box 268

Kumpulanite 7

SF-60101 Helsinki

FINLAND

Seppo Vuori

Technical Research Centre of Finland

Nuclear Engineering Laboratory

Lonnrotinkatu 37

P.O. Box 169

Sf-00181 Helsinki 18

FINLAND

Dist-15 
Dr. Ian B. Wall

81 Irving Avenue

Atherton, CA 94027

Edward Warman

Stone \& Webster Engineering Corp.

P.O. Box 2325

Boston, MA 02107

J.E. Werner

Reactor Research \& Techn Division

US DOE Idaho Operations

MS: 1219

850 Energy Drive

Idaho Falls, ID 83401-1563

Dr. Wolfgang Werner

Safety Assessment Consulting $\mathrm{GmbH}$

Veilchenweg 8 .

D 83254 Breitbrunn

GERMANY

Westinghouse Electric Corp

Technical Library

P. O. Box 355

East 209

Pittsburgh, PA 15230

Westinghouse Electric Corp

NTD

Central File Nuclear Safety

P. O. Box 355

408 1-A

Pittsburgh, PA 15230

Westinghouse Electric Company (3)

Attn: John Lacovin

Burt Morris

Griff Holmes

Energy Center East, Bldg. 371

P.O. Box 355

Pittsburgh, PA 15230
Westinghouse Savannah River Co. (2)

Attn: Kevin O'Kula

Jackie East

Safety Technology Section

1991 S. Centennial Ave., Bldg. 1

Aiken, SC 29803

Keith Woodard

PLG, Inc.

7315 Wisconsin Ave.

Suite 620 East

Bethesda, MD 20814-3209

John Wreathall

John Wreathall \& Co.

4157 MacDuff Way

Dubin, OH 43017

M. K. Yeung

University of Hong Kong

Mechanical Engineering Dept.

Polfulam

HONG KONG

Bob Youngblood

Brookhaven National Laboratory

Department of Nuclear Energy

Bldg. 130

Upton, NY 11973

Carlo Zaffiro

A.N.P.A.

Directorate for Nuclear

Via Vitaliano Brancate, 48

I-00144 Rome

ITALY

Dr. X. Zikidis

Greek Atomic Energy Comm.

N.R.C.P.S. "Demokritos"

GR-153 10 Agia Paraskevi

Attiki

GREECE 


\section{INTERNAL DISTRIBUTION}

$\begin{array}{ll}\text { MS0405 } & \text { D. D. Carlson, 6411 } \\ \text { MS0747 } & \text { A. L. Camp, 6412 } \\ \text { MS0747 } & \text { V. J. Dandini, 6412 } \\ \text { MS0747 } & \text { S. L. Daniel, 6412 } \\ \text { MS0747 } & \text { S. E. Dingman, 6412 } \\ \text { MS0747 } & \text { J. A. Forrester, 6412 } \\ \text { MS0747 } & \text { K. M. Hays, 6412 } \\ \text { MS0747 } & \text { H. K. Schriner, 6412 } \\ \text { MS0747 } & \text { D. B. Mitchell, 6412 } \\ \text { MS0747 } & \text { B. D. Staple, 6412 } \\ \text { MS0747 } & \text { J. A. Lambright, 6412 } \\ \text { MS0747 } & \text { D. W. Whitehead, 6412 } \\ \text { MS0747 } & \text { G. D. Wyss, 6412 } \\ \text { MS0748 } & \text { F. T. Harper, 6413 } \\ \text { MS0748 } & \text { T. D. Brown, 6413 (10) } \\ \text { MS0737 } & \text { M. P. Bohn, 6449 } \\ \text { MS0742 } & \text { J. E. Kelly, 6414 } \\ \text { MS0745 } & \text { L. N. Kmetyk, 6418 } \\ \text { MS0736 } & \text { N. R. Ortiz, 6400 } \\ \text { MS1175 } & \text { L. A. Miller, 6513 } \\ \text { MS9018 } & \text { Central Technical Files, } \\ & \text { 8523-2 } \\ \text { MS0899 } & \text { Technical Library, 13414 } \\ \text { MS0619 } & \text { (5) } \\ & \text { Technical Publications, } \\ & \text { 12613 }\end{array}$




\section{TITLE AND SUBTITLE}

Evaluation of Potential Severe Accidents During Low Power and Shutdown Operations at Grand Gulf, Unit 1

Evaluation of Severe Accident Risks for Plant Operational State 5 During a Refueling Outage

Main Report and Appendices

5. AUTHOR(S)

T. D. Brown, L. N. Kmgtyk, D. Whitehead, J. Forester, ${ }^{1}$

L. Miller, J. Johnson

SAND93-2440

Vol. 6, Part 1

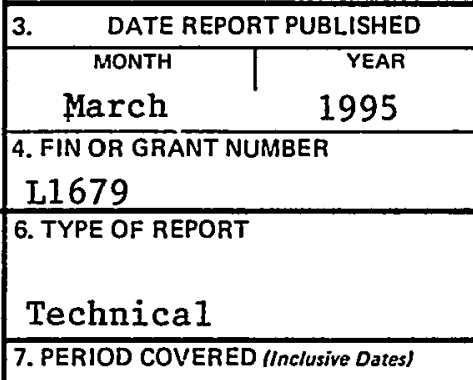

8. PERFORMING ORGANIZATION - NAME AND ADDRESS (If NRC, provide Division, Office or Region, U.S. Nuclear Regulatory Commission, and mailing address; if contractor, provide name and mailling address

Sandia National Laboratory Albuquerque, NM 87185
${ }^{1}$ Science Applications International Corporation 2109 Air Park Road, S.E. Albuquerque, NM 87106

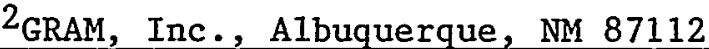

9. SPONSORING ORGANIZATION - NAME AND ADDRESS (If NRC, type "Same as above"; if contractor, provide NRC Division, Office or Region, U.S. Nuclear Regulatory Commission, and malling address.)

Division of Systems Technology

Office of Nuclear Regulatory Research

U.S. Nuclear Regulatory Commission

Washington, DC 20555-0001

10. SUPPLEMENTARY NOTES

\section{ABSTRACT (200 wards or less)}

The analysis documented in this volume of the report is the Level $2 / 3$ analysis of the traditional internal events. Plant damage states, which define the configuration of the plant and its systems at the onset of core damage for the accident scenarios developed in the Level 1 analysis, were used to define the interface between the Level 1 and Level $2 / 3$ analyses. In the Level $2 / 3$ analysis, the possible progressions of the accident following the onset of core damage were delineated and the amount of radioactive material released to the environment was estimated. Based on the amount of radioactive material released to the environment, health effects to the general public were estimated. In addition to the offsite consequences, a scoping analysis of the potential doses and dose rates within the site were also estimated. The final product of the analysis was the: integration of the accident frequencies with the consequences of the accidents to form an expression for aggregate risk.

\begin{tabular}{|c|}
\hline $\begin{array}{l}\text { 13. AVAILABILITY STATEMENT } \\
\text { Unlimited }\end{array}$ \\
\hline 14. SECURITY CLASSIFICATION \\
\hline $\begin{array}{l}\text { (This Page) } \\
\text { Unclassified }\end{array}$ \\
\hline $\begin{array}{l}\text { (This Report) } \\
\text { Unclassified }\end{array}$ \\
\hline 15. NUMBER OF PAGES \\
\hline 16. PRICE \\
\hline
\end{tabular}

Probabilistic Risk Assessment, Low Power and Shutdown Operations Boiling Water Reactor, Accident Progression and Risk Analysis 